\title{
Simulation study of estimating between-study variance and overall effect in meta-analyses of log-response-ratio for normal data
}

\author{
Ilyas Bakbergenuly, David C. Hoaglin, and Elena Kulinskaya
}

October 23, 2019

\begin{abstract}
Methods for random-effects meta-analysis require an estimate of the betweenstudy variance, $\tau^{2}$. The performance of estimators of $\tau^{2}$ (measured by bias and coverage) affects their usefulness in assessing heterogeneity of study-level effects, and also the performance of related estimators of the overall effect. For the effect measure log-response-ratio (LRR, also known as the logarithm of the ratio of means, RoM), we review four point estimators of $\tau^{2}$ (the popular methods of DerSimonian-Laird (DL), restricted maximum likelihood, and Mandel and Paule (MP), and the less-familiar method of Jackson), four interval estimators for $\tau^{2}$ (profile likelihood, Q-profile, Biggerstaff and Jackson, and Jackson), five point estimators of the overall effect (the four related to the point estimators of $\tau^{2}$ and an estimator whose weights use only study-level sample sizes), and seven interval estimators for the overall effect (four based on the point estimators for $\tau^{2}$, the Hartung-Knapp-Sidik-Jonkman (HKSJ) interval, a modification of HKSJ that uses the MP estimator of $\tau^{2}$ instead of the DL estimator, and an interval based on the sample-size-weighted estimator). We obtain empirical evidence from extensive simulations of data from normal distributions. Simulations from lognormal distributions are in a separate report Bakbergenuly et al. $2019 \mathrm{~b}$.
\end{abstract}

Keywords between-study variance, heterogeneity, random-effects model, metaanalysis, log-response-ratio, ratio of means 


\section{Introduction}

Meta-analysis is a statistical methodology for combining estimated effects from several studies in order to assess their heterogeneity and obtain an overall estimate. In this paper we focus on the log-response-ratio (LRR, also known as the logarithm of the ratio of means, RoM, in medical applications) as the effect measure. In ecology almost half of all meta-analyses use this outcome measure (Koricheva and Gurevitch 2014, Nakagawa and Santos 2012]).

The LRR was introduced by Hedges et al. 1999 and rediscovered as RoM by Friedrich et al. 2008 assuming underlying normality of the raw data. However, the LRR is not defined for negative values of the study means, and Lajeunesse 2015 modeled the data by lognormal distributions. We explore the meta-analysis of LRR under the normal distribution in this report. Our results under log-normality constitute a companion report Bakbergenuly et al. 2019b.

If the studies can be assumed to have the same true effect, a meta-analysis can use a fixed-effect (FE) model (common-effect model) to combine the estimates. Otherwise, the studies' true effects can depart from homogeneity in a variety of ways. Most commonly, a random-effects (RE) model regards those effects as a sample from a distribution and summarizes their heterogeneity via its variance, usually denoted by $\tau^{2}$. The between-studies variance, $\tau^{2}$, has a key role in estimates of the mean of the distribution of random effects; but it is also important as a quantitative indication of heterogeneity Higgins et al., 2009]. In studying estimation for meta-analysis of LRR, we focus first on $\tau^{2}$ and then proceed to the overall effect.

Veroniki et al. [2016] provide a comprehensive overview and recommendations on methods of estimating $\tau^{2}$ and its uncertainty. Their review, however, has two important limitations. First, the authors study only "methods that can be applied for any type of outcome data." However, as we show elsewhere, the performance of the methods varies widely among effect measures. Second, any review of the topic, such as Veroniki et al. 2016, currently can draw on only limited empirical information on the comparative performance of the methods. We address both issues for the effect measure LRR.

Veroniki et al. 2016] (Appendix Table 1) cite no previous simulation studies on the comparative performance of estimators of $\tau^{2}$ for LRR.

Several studies have considered the quality of estimation of LRR itself. Friedrich et al. 2008 report extensive simulations for LRR under normality, but they use only 
the DerSimonian-Laird (DL) method to estimate $\tau^{2}$ and do not report on its quality. Lajeunesse 2015] discusses bias correction for study-level LRR and its variance, and provides some simulation results for lognormal distributions, but only under the fixed-effect model. Doncaster and Spake 2018 provide some limited simulation results under normality for accuracy of estimation of the heterogeneity variance $\tau^{2}$, the overall LRR, and its variance, using the DL and restricted maximum-likelihood (REML) methods to estimate $\tau^{2}$. To assess bias of the estimators of LRR, they use mean absolute error, which is not a measure of bias; it is the linear counterpart of mean squared error.

To address this gap in information on methods of estimating the heterogeneity variance for LRR, we use simulation to study four methods recommended by Veroniki et al. 2016]. These are the well-established methods of DerSimonian and Laird [1986], restricted maximum likelihood, and Mandel and Paule [1970] (MP), and the less-familiar method of Jackson 2013. We also study coverage of confidence intervals for $\tau^{2}$ achieved by four methods: the Q-profile method of Viechtbauer 2007, the methods of Biggerstaff and Jackson [2008] and Jackson 2013, and the profilelikelihood-based interval.

For each estimator of $\tau^{2}$, we also study bias of the corresponding inverse-varianceweighted estimator of the overall effect. However, it is well known that these inversevariance-weighted estimators have unacceptable bias for some other effect measures, as Bakbergenuly et al. 2018 and Hamman et al. 2018] show for the standardized mean difference. Therefore, we added an estimator (SSW) whose weights depend only on the sample sizes of the Treatment and Control arms. We study the coverage of the confidence intervals associated with the inverse-variance-weighted estimators, and also the HKSJ interval (Hartung and Knapp 2001, Sidik and Jonkman 2002]), a modification of the HKSJ interval that uses the MP estimator of $\tau^{2}$ instead of the DL estimator, and an interval centered at SSW that uses the MP estimator of $\tau^{2}$ in estimating its variance and bases its half-width on a $t$ distribution.

\section{Study-level estimation of log-response-ratio}

We assume that each of the $K$ studies in the meta-analysis consists of two arms, Treatment and Control, with sample sizes $n_{i T}$ and $n_{i C}$. The total sample size in Study $i$ is $n_{i}=n_{i T}+n_{i C}$. The subject-level data in each arm are assumed to be 
normally distributed with means $\mu_{i T}$ and $\mu_{i C}$ and variances $\sigma_{i T}^{2}$ and $\sigma_{i C}^{2}$. The sample means are $\bar{X}_{i j}$, and the sample variances are $s_{i j}^{2}$, for $i=1, \ldots, K$ and $j=C$ or $T$.

The response ratio is usually meta-analyzed on a log scale, where the effect measure is $\lambda_{i}=\log \left(\mu_{i T} / \mu_{i C}\right)$, estimated by $\hat{\lambda}_{i}=\log \left(\bar{X}_{i T} / \bar{X}_{i C}\right)$, and the population and sample means are assumed to be positive. The within-study variance estimate of $\hat{\lambda}_{i}$, obtained by the delta method, is Hedges et al., 1999

$$
v_{i}^{2}=\frac{s_{i T}^{2}}{n_{i T} \bar{X}_{i T}^{2}}+\frac{s_{i C}^{2}}{n_{i C} \bar{X}_{i C}^{2}}=\frac{\hat{V}_{i T}^{2}}{n_{i T}}+\frac{\hat{V}_{i C}^{2}}{n_{i C}},
$$

where $\hat{V}_{i j}$ is the sample coefficient of variation $(\mathrm{CV})$. The positively skewed distribution of $\hat{\lambda}_{i}$ is not well approximated by a normal distribution when at least one of the sample means is close to zero. This results in considerable bias of $\hat{\lambda}_{i}$ and affects the quality of its variance estimator (Hedges et al. [1999]). The standard advice is to use LRR for Study $i$ only when at least one of $\sqrt{n_{i j}} \bar{X}_{i j} / s_{i j} \geq 3$. This advice is often ignored in practice (Lajeunesse 2015]). To eliminate the small-sample bias of $\hat{\lambda}_{i}$, Lajeunesse 2015] proposed two bias-corrected modifications, and he recommended

$$
\hat{\lambda}_{i}^{\Delta}=\hat{\lambda}_{i}+\frac{1}{2}\left[\frac{s_{i T}^{2}}{n_{i T} \bar{X}_{i T}^{2}}-\frac{s_{i C}^{2}}{n_{i C} \bar{X}_{i C}^{2}}\right],
$$

and estimated its variance by

$$
\widehat{\operatorname{Var}}\left(\hat{\lambda}_{i}^{\Delta}\right)=v_{i}^{2}+\frac{1}{2}\left[\frac{s_{i T}^{4}}{n_{i T}^{2} \bar{X}_{i T}^{4}}-\frac{s_{i C}^{4}}{n_{i C}^{2} \bar{X}_{i C}^{4}}\right] .
$$

However, this bias-corrected estimator and its variance would be even less stable for small values of $\bar{X}_{i j}$.

Since $\hat{\lambda}$ is defined only in the positive quadrant $\bar{X}_{T}>0, \bar{X}_{C}>0$, the standard FE and RE models of meta-analysis are not quite appropriate. Let $\bar{X}_{j} \sim N\left(\mu_{j}, \sigma_{j}^{2} / n_{j}\right)$. Then, under the fixed-effect model, the probability that $\hat{\lambda}$ is not defined is

$$
1-P\left(\bar{X}_{T}>0\right) P\left(\bar{X}_{C}>0\right)=1-\left(1-\Phi\left(-\sqrt{n_{C}} \mu_{C} / \sigma_{C}\right)\right)\left(1-\Phi\left(-\sqrt{n_{T}} \mu_{T} / \sigma_{T}\right)\right),
$$

where $\Phi(\cdot)$ is the cumulative distribution function of the standard normal distribution. This probability is a decreasing function of $n_{j}$ and an increasing function of $V_{j}=\sigma_{j} / \mu_{j}$, and it is close to 0 for large sample sizes. However, very small sample sizes, starting from $n_{T}=n_{C}=2$, are abundant in ecology, where the LRR is a measure of choice. Keeping, say, the value of $\sqrt{n_{C}} / V_{C}=3$, the probability that $\hat{\lambda}$ is not defined is 0.0027 for $\sqrt{n_{T}} / V_{T}=3,0.024$ for $\sqrt{n_{T}} / V_{T}=2,0.160$ for $\sqrt{n_{T}} / V_{T}=1$, and 0.309 for $\sqrt{n_{T}} / V_{T}=0.5$. Therefore, only large values of both $\sqrt{n_{C}} / V_{C}$ and $\sqrt{n_{T}} / V_{T}$ would avoid problems. When the population value of $\sqrt{n_{T}} / V_{T}<3$, say, studies for 
which $\hat{\lambda}$ is not defined would occur with comparatively high probability and would need to be dropped from the meta-analysis. These omissions imply that the data in each arm come from a normal distribution truncated from below at $\sqrt{n_{j}} / V_{j}$, even under the FE model. This may introduce bias in $\hat{\lambda}$.

Restrictions on the range are not unique to LRR; they apply to other effect measures such as the relative risk (Bakbergenuly et al. 2019a), where the restriction arises because the probabilities of events must be between 0 and 1 .

In the RE model, $\lambda_{i} \sim N\left(\lambda, \tau^{2}\right)$ and can be negative. If so, $\mu_{i T}\left(=\mu_{i C} \exp \left(\lambda_{i}\right)\right)$, and consequently the contribution of $n_{i T} / \hat{V}_{i T}^{2}$ to the reciprocal of $v_{i}^{2}$ in Equation 2.1), may be close to zero, further increasing the probability of truncation for the $\bar{X}_{i T}$.

But the RE model for LRR has yet another complication, involving its specification on the log scale. The expected value of $\mu_{T i}$ is not equal to $\mu_{C i} \exp (\lambda)$, since $\mathrm{E}\left(\exp \left(\lambda_{i}\right)\right)=\exp (\lambda)\left(1+\tau^{2} / 2\right)$. Bakbergenuly et al. 2016] discuss this sort of transformation bias.

Because $\hat{\lambda}$ is not defined for negative values of the study means, Lajeunesse 2015. modeled the data by lognormal distributions. In principle, log-normal distributions often make sense for non-negative data. This choice would eliminate the restrictedrange bias of LRR. Of course, the choice of model should be based on the properties of the data and not on the perceived ease of statistical modeling.

We describe simulations from normal distributions and their results in Section 6 . We study both the standard estimator $\hat{\lambda}$ and also the bias-corrected estimator $\hat{\lambda}^{\Delta}$, Equation (2.2), the latter when the data are at some distance from zero, because it is a priori clear in normal data, that it will incur considerable bias and its variance will be grossly inflated near zero. Simulations from lognormal distributions are in a separate report Bakbergenuly et al. 2019b.

\section{$3 \quad$ Standard random-effects model}

The standard random-effects model assumes that within- and between-study variabilities are accounted for by approximately normal distributions of within- and betweenstudy effects. For a generic measure of effect,

$$
\hat{\theta}_{i} \sim N\left(\theta_{i}, \sigma_{i}^{2}\right) \text { and } \theta_{i} \sim N\left(\theta, \tau^{2}\right)
$$

resulting in the marginal distribution $\hat{\theta}_{i} \sim N\left(\theta, \sigma_{i}^{2}+\tau^{2}\right) . \hat{\theta}_{i}$ is the estimate of the effect in Study $i$, and its within-study variance is $\sigma_{i}^{2}$, estimated by $\hat{\sigma}_{i}^{2}, i=1, \ldots, K$. The 
between-study variance, $\tau^{2}$, is estimated by $\hat{\tau}^{2}$. The overall effect, $\theta$, is customarily estimated by the weighted mean

$$
\hat{\theta}_{R E}=\frac{\sum_{i=1}^{K} \hat{w}_{i}\left(\hat{\tau}^{2}\right) \hat{\theta}_{i}}{\sum_{i=1}^{K} \hat{w}_{i}\left(\hat{\tau}^{2}\right)},
$$

where the $\hat{w}_{i}\left(\hat{\tau}^{2}\right)=\left(\hat{\sigma}_{i}^{2}+\hat{\tau}^{2}\right)^{-1}$ are inverse-variance weights. The FE estimate $\hat{\theta}$ uses weights $\hat{w}_{i}=\hat{w}_{i}(0)$.

If $w_{i}=1 / \operatorname{Var}\left(\hat{\theta}_{i}\right)$, the variance of the weighted mean of the $\hat{\theta}_{i}$ is $1 / \sum w_{i}$. Thus, many authors estimate the variance of $\hat{\theta}_{R E}$ by $\left[\sum_{i=1}^{K} \hat{w}_{i}\left(\hat{\tau}^{2}\right)\right]^{-1}$. In practice, however, this estimate may not be satisfactory (Sidik and Jonkman 2006, Li et al. 1994, Rukhin 2009).

\section{Methods of estimating between-study vari-}

\section{ance}

In this section we briefly list the point and interval estimators of the between-studies variance $\left(\tau^{2}\right)$ used in our study.

\subsection{Point estimators}

The most popular, but rather biased, estimator of $\tau^{2}$ is the method-of-moments estimator of DerSimonian and Laird 1986 (DL), denoted by $\hat{\tau}_{D L}^{2}$.

Assuming that the $\hat{\theta}_{i}$ are distributed as $N\left(\theta, \hat{\sigma}_{i}^{2}+\tau^{2}\right)$, the restricted-maximumlikelihood (REML) estimator $\hat{\tau}_{R E M L}^{2}$ maximizes the restricted (or residual) log-likelihood function $l_{R}\left(\theta, \tau^{2}\right)$. REML is superior to DL because of its balance between unbiasedness and efficiency Viechtbauer, 2005.

The Mandel-Paule (MP) estimator (Mandel and Paule [1970]), $\hat{\tau}_{M P}^{2}$, is another moment-based estimator of the between-study variance. It is estimated iteratively. It is known to be superior to DL (Veroniki et al. [2016]), but no simulations for LRR have been performed so far.

DerSimonian and Kacker 2007] generalized DL, replacing the weights $\hat{w}_{i}$ by arbitrary fixed positive constants, $a_{i}$. As an option when there is little a priori knowledge about the extent of heterogeneity, but some is anticipated, Jackson 2013 proposed the estimator of $\tau^{2}$ with $a_{i}=1 / \hat{\sigma}_{i}$. We refer to this method as J. 


\subsection{Interval estimators}

The $95 \%$ profile-likelihood (PL) confidence interval for $\tau^{2}$ consists of the values that are not rejected by the likelihood-ratio test with $\tau^{2}$ as the null hypothesis. This interval is usually used with $\hat{\tau}_{R E M L}^{2}$.

Similarly, the Q-profile (QP) confidence interval for $\tau^{2}$ consists of the values that are not rejected by the usual test for heterogeneity based on Cochran's $Q$ Cochran 1954). The null distribution of $Q$ is assumed (incorrectly) to be the chi-squared distribution with $K-1$ degrees of freedom.

For a generic effect measure, Biggerstaff and Jackson 2008] derived the exact distribution of a $Q$ statistic with constant weights $a_{i}$. That distribution yielded a generalized Q-profile confidence interval. We refer to this interval with $a_{i}=1 / \hat{\sigma}_{i}^{2}$ as the BJ confidence interval.

Jackson 2013] proposed another generalized Q-profile confidence interval ( $\mathrm{J}$ ) for $\tau^{2}$. The approach is the same as for the BJ interval, but with $a_{i}=1 / \hat{\sigma}_{i}$.

\section{Methods of estimating overall effect}

Most of the point estimators of the overall effect have corresponding interval estimators, but some do not. Therefore, we describe point estimators and interval estimators in separate sections.

\subsection{Point estimators}

A random-effects method that estimates $\theta$ by a weighted mean with inverse-variance weights, as in Equation 3.2, is determined by the particular $\hat{\tau}^{2}$ that it uses in $\hat{w}_{i}\left(\hat{\tau}^{2}\right)$. Because the study-level effects and their variances are related (as in Equation 2.1) for LRR), all inverse-variance-weighted estimators of $\hat{\lambda}$ may have considerable bias. For completeness, we studied DL, REML, MP, and J.

To reduce this bias in estimating $\lambda$, our experience with the bias of inversevariance-weighted estimators for standardized mean difference Bakbergenuly et al. [2018) led us to include a point estimator whose weights depend only on the studies' sample sizes (Hedges and Olkin 1985, Hunter and Schmidt 1990]). For this estimator (SSW), $w_{i}=\tilde{n}_{i}=n_{i T} n_{i C} /\left(n_{i T}+n_{i C}\right)$; that is, $w_{i}$ substitutes 1 for the estimated CVs in Equation 2.1); $\tilde{n}_{i}$ is the effective sample size in Study $i$. The estimator of 
the variance of SSW is

$$
\widehat{\operatorname{Var}}\left(\hat{\theta}_{S S W}\right)=\frac{\sum \tilde{n}_{i}^{2}\left(v_{i}^{2}+\hat{\tau}^{2}\right)}{\left(\sum \tilde{n}_{i}\right)^{2}}
$$

in which $v_{i}^{2}$ comes from Equation 2.1 and $\hat{\tau}^{2}=\hat{\tau}_{M P}^{2}$.

\section{$5.2 \quad$ Interval estimators}

The point estimators DL, REML, MP, and J have companion interval estimators of

$\theta$. The customary approach estimates the variance of $\hat{\theta}_{R E}$ by $\left[\sum_{i=1}^{K} \hat{w}_{i}\left(\hat{\tau}^{2}\right)\right]^{-1}$ and bases the half-width of the interval on the normal distribution. These intervals are usually too narrow.

Hartung and Knapp 2001] and, independently, Sidik and Jonkman 2002 developed an improved estimator for the variance of $\hat{\theta}_{R E}$. The Hartung-Knapp-SidikJonkman (HKSJ) confidence interval uses this estimator together with critical values from the $t$ distribution on $K-1$ degrees of freedom. A potential weakness is that the HKSJ interval uses $\hat{\theta}_{D L}$ as its midpoint, so it will have any bias that is present in $\hat{\theta}_{D L}$. We studied a modification of the HKSJ confidence interval that uses $\hat{\tau}_{M P}^{2}$ and $\hat{\theta}_{M P}$; we refer to this interval as the HKSJ(MP) confidence interval.

The interval estimator corresponding to SSW (SSW MP) uses the SSW point estimator as its center, and its half-width equals the estimated standard deviation of SSW under the random-effects model times the critical value from the $t$ distribution on $K-1$ degrees of freedom.

\section{Simulation study}

As mentioned in Section 1 a few studies have used simulation to examine estimators of the overall effect for LRR, but no studies have examined estimators of $\tau^{2}$.

The range of values of $R R$ may be rather wide. The empirical study by Senior et al. 2016 reports values of $\mathrm{RR}$ up to 3.72 , though the second largest value is 1.46. The simulations by Friedrich et al. 2008 used values up to 1.56 (LRR = 0.445). Lajeunesse 2015 used means between 0 and 8 in both arms and small sample sizes, starting from $n_{T}+n_{C}=4$. Our simulation study for LRR uses an interval of $0 \leq \lambda \leq 2$ (or $0 \leq \mathrm{RR} \leq 7.39$ ) as realistic for a range of applications. Unfortunately, no information is available on the accompanying range of $\tau^{2}$ values. In their simulations for SMD, Hamman et al. 2018 consider the range from 0 to 2.5 as typical for ecology. 


\subsection{Design of the simulations}

Our simulation study assesses the performance of four methods for point estimation of the between-studies variance, $\tau^{2}$ (DL, REML, J, and MP) and four methods of interval estimation of $\tau^{2}$ (the Q-profile method, the generalized Q-profile intervals of Biggerstaff and Jackson 2008] and Jackson 2013, and the profile-likelihood confidence interval based on REML).

We study bias of the inverse-variance-weighted estimator of the overall effect corresponding to each of the estimators of $\tau^{2}$ (DL, REML, J, and MP), as well as bias of SSW, whose weights depend only on the sample sizes of the Treatment and Control arms.

We also study coverage of the confidence intervals associated with those inversevariance-weighted estimators, and also the HKSJ interval (Hartung and Knapp 2001], Sidik and Jonkman 2002]), a modification of the HKSJ interval that uses the MP estimator of $\tau^{2}$ instead of the DL estimator, and an interval centered at SSW that uses the MP estimator of $\tau^{2}$ in estimating its variance and uses critical values from a $t$ distribution.

Two basic distributions serve as the source of the data in the Treatment and Control arms: the normal distribution (the subject of the present report) and the lognormal distribution (the subject of a separate report, Bakbergenuly et al. 2019b.

For the overall value of LRR, we chose $\lambda=(0,0.2,0.5,1,2)$ (corresponding to $0 \leq \mathrm{RR} \leq 7.39$ ), as realistic for a range of applications. The true values of LRR in the individual studies, $\lambda_{i}$, are generated from a normal distribution: $\lambda_{i} \sim N\left(\lambda, \tau^{2}\right)$. For a given Control mean $\mu_{i C}$, the Treatment mean is $\mu_{i T}=\mu_{i C} \exp \left(\lambda_{i}\right)$.

To evaluate performance issues related to proximity to zero, we use two values of the mean in the Control arm: $\mu_{i C}=1$ and $\mu_{i C}=4$.

All simulations use the same numbers of studies, small $(K=5,10,30)$ and large $(K=50,100,125)$, and equal numbers of observations in the Control and Treatment arms.

We study only meta-analyses in which the study size is the same in all $K$ studies. The study sizes, $n_{i}$, start from 4 , because some studies in ecology have such small sample sizes; the small sample sizes are $n_{i}=4,10,20,40$, and the large sample sizes are $n_{i}=100,250,640,1000$. By using the same set of sample sizes for each combination of the other parameters, we avoid the additional variability in the results that would arise from choosing sample sizes at random (e.g., uniformly between 100 
and 250).

We generate the within-study sample variances $s_{i j}^{2}(j=T, C)$ from chi-squared distributions as $\sigma_{i j}^{2} \chi_{n_{i j}-1}^{2} /\left(n_{i j}-1\right)$. We generate the estimated means $\bar{X}_{i j}$ from a normal distribution with mean $\mu_{i j}$ and variance $\sigma_{i j}^{2} / n_{i j}$. We obtain the estimated within-study LRR as $\hat{\lambda}_{i}=\log \left(\bar{X}_{i T} / \bar{X}_{i C}\right)$ and the estimated within-study variance from formula (2.1). Studies with at least one negative sample mean are discarded, and the value of $K$ is reduced accordingly, resulting effectively in a simulation from a truncated normal distribution in each arm.

In summary, we vary five parameters: the overall true LRR $(\lambda)$, the betweenstudies variance $\left(\tau^{2}\right)$, the mean in the Control arm $\left(\mu_{C}\right)$, the number of studies $(K)$, and the total sample size $(n)$. We set $\sigma_{C}^{2}=\sigma_{T}^{2}=1$. Table 1 lists the configurations.

We use a total of 10,000 repetitions for each combination of parameters. Thus, the simulation standard error for estimated coverage of $\tau^{2}$ or $\lambda$ at the $95 \%$ confidence level is roughly $\sqrt{0.95 \times 0.05 / 10,000}=0.00218$.

The simulations were programmed in $\mathrm{R}$ version 3.3.2 using the University of East Anglia 140-computer-node High Performance Computing (HPC) Cluster, providing a total of $2560 \mathrm{CPU}$ cores, including parallel processing and large memory resources. For each configuration, we divided the 10,000 replications into 10 parallel sets of 1000 replications.

Table 1: Configurations of parameters in the simulations for LRR.

\begin{tabular}{|l|l|l|}
\hline Parameter & Equal study sizes & $\begin{array}{l}\text { Full results in } \\
\text { Web Appendix }\end{array}$ \\
\hline$K$ (number of studies: small/large) & $(5,10,30) \&(50,100,125)$ & A \& B - small $n$ \\
$n$ (total study size: small/large) & $(4,10,20,40) \&(100,250,640,1000)$ & \\
$\sigma_{T}^{2} \& \sigma_{C}^{2}$ (within-study variances) & $1 \& 1$ & C \& D - large $n$ \\
$\lambda$ (overall value of the LRR) & $0,0.2,0.5,1,2$ & \\
$\tau^{2}$ (variance of random effect) & $0(0.1) 1$ & \\
Normal distribution & $1 \& 4$ & \\
$\mu_{C}$ (mean in Control arm) & & A \& C \\
\hline estimation of $\tau^{2}$ & & B \& D \\
estimation of $\lambda$ & & \\
\hline
\end{tabular}




\subsection{Results}

Bias and coverage in estimation of $\tau^{2}$ under normal distribution (Appendices $\mathrm{A}$ and $\mathrm{C}$ )

Bias The main feature is a negative relation between bias and $\tau^{2}$. When $\mu_{C}=1$ and $n=4$, all four estimators have definite positive bias at $\tau^{2}=0$ (e.g., 0.13 to 0.22 when $\lambda=0$ and $K=5$ ). As $\tau^{2}$ increases, the bias trends downward, roughly linearly, and it can reach substantial negative values (e.g., -0.47 to -0.64 at $\tau^{2}=1$ when $\lambda=0$ and $K=5$ ). When $n=10$, the bias at $\tau^{2}=0$ is less than 0.05 , and it goes to 0 as $n$ increases. Also, the slopes of the traces decrease in magnitude, and the traces separate. The separation is slight at $n=40$ but substantial at $n=100$. Interestingly, MP and REML improve steadily, but DL and J do not (Figure C1.1.1); when $\lambda=0, K=5$, and $n=1000$, the bias at $\tau^{2}=1$ is -0.33 for DL and -0.20 for $\mathrm{J}$, versus -0.06 for REML and -0.03 for MP. Increasing $K$ (even to 50, 100, and 125) generally does not reduce the biases, but the traces separate when $n \leq 20$, especially when $n=4$. As $\lambda$ increases, the biases at $\tau^{2}=0$ decrease slightly, and the magnitudes of the slopes decrease substantially; when $\lambda=2$ and $n \geq 40$, MP and REML are nearly unbiased (see Figure A1.1.5). The biases are much smaller when $\mu_{C}=4$ (see Figure A2.1.5 for comparison); we believe the difference is due to restricted-range issues. None of the four estimators of $\tau^{2}$ has adequately small bias overall, but (excluding $n=4$, which is generally quite challenging) MP often has the least bias, followed by REML, and J and DL have the most bias.

Bias in estimation of $\tau^{2}$ increases when the bias-corrected estimator $\lambda^{\Delta}$ is used (Appendices A3.1 and A6.1). As expected, bias-correction does not have any effect for large $n$.

Coverage When $\mu_{C}=1$, coverage of $\tau^{2}$ depends strongly on $K$. Counterintuitively, all four methods have coverage close to nominal when $K=5$, somewhat below nominal when $K=10$ (unless $n>100$ ), and often far below nominal when $K \geq 30$ (in problematic patterns when $n=4)$. At $\tau^{2}=0$ coverage is noticeably above nominal when $n \geq 10$ (e.g., Figure A1.2.1). When $n \leq 40$, coverage generally improves as $\lambda$ increases (but it deteriorates at small $\tau^{2}$ when $n=4$ ). When $n \geq 100, \mathrm{QP}$ and PL generally have coverage reasonably close to nominal, even for $K=30$, and QP is sometimes closer; when $K=30$, the coverage of BJ and $\mathrm{J}$ decreases sharply as 
$\tau^{2}$ increases (except when $\lambda=2$ ) (Figure C1.2.1 to Figure C1.2.5). When $\mu_{C}=4$, coverage of the Q-profile intervals is close to nominal for $\tau^{2} \geq 0.1$. All four methods have below-nominal coverage at $\tau^{2}=0$ when $n=4$ and $\lambda=0$, and the deficit becomes worse and affects other values of $n$ as $\lambda$ increases. When $n$ is large or $K$ is large, the coverage of $\mathrm{J}$ and especially BJ declines steeply as $\tau^{2}$ increases.

Coverage of $\tau^{2}$ is not much affected for smaller $K$ and considerably worse for larger $K$, when the bias-corrected estimator $\lambda^{\Delta}$ is used (Appendices A3.2 and A6.2).

\section{Bias and coverage in estimation of $\lambda$ under normal distribution (Appen- dices $B$ and $D$ )}

Bias All four IV-weighted estimators of $\lambda$ have practically the same bias. When $\mu_{C}=1$ and $\lambda=0$, the bias is 0 at $\tau^{2}=0$ and increases as $\tau^{2}$ increases (the traces resemble $\sqrt{\tau^{2}}$ for $\tau^{2} \leq 0.4$ and are nearly linear for $\tau^{2} \geq 0.4$ ). At $\tau^{2}=1$ it can be large when $n$ is small (e.g., around 0.40 when $K=5$ and $n \leq 20$ ). When $\lambda \geq 0.2$, the bias is negative at small $\tau^{2}$, with magnitude exceeding 0.12 when $\lambda \geq 1$ and $n \leq 10$; it becomes positive as $\tau^{2}$ increases (Figures B1.1.1, B1.1.4). The bias can be large for small sample sizes, and it decreases to zero only for very large sample sizes. The bias depends very little on $K$. The bias of SSW is positive, though considerably less than the bias of the IV-weighted methods, and it decreases much more rapidly as $n$ increases. It decreases for increasing values of $\lambda$, staying at about $5 \%$ for $n=10$ and $\lambda=2$. Interestingly, when $\lambda \geq 1$ and $n \geq 10$, the bias of SSW (when positive) is roughly constant across the range of $\tau^{2}$. The bias of the IV methods is still present, though much smaller, when $\mu_{C}=4$, whereas SSW is practically unbiased (see Figure B2.1.1). This means that bias for large $\mu_{C}$ is due to the use of the IV weights and/or to transformation bias, as restricted-range effects will not be involved.

Bias in IV estimation of $\lambda$ is not affected by the use of the bias-corrected estimator $\lambda^{\Delta}$, but SSW often generates aberrantly large values (see Figure B3.1.4). For each study, the correction terms in $\lambda^{\Delta}(2.2)$ include the reciprocals of the squared arm-level sample means, so a sample mean near zero considerably inflates a study's LRR, and results in an inflated SSW estimate of $\lambda$, especially for small to moderate $K$. Interestingly, the IV-weighted estimators are not affected, as such studies have even more inflated variances, cf Equation (2.3), and thus very small IV weights (Appendices B3.1 and B6.1). 
Coverage When $\mu_{C}=1$, coverage of all IV-weighted methods is considerably below nominal for $n \leq 40$ (with some exceptions near $\tau^{2}=0$ when $\lambda$ is small) (Figure B1.2.1. Coverage of the IV-weighted methods deteriorates substantially for larger $K$ and larger values of $\tau^{2}$, especially for small values of $\lambda$ (Figures B4.2.1. B4.2.4). The HKSJ intervals provide nominal coverage only for $n \geq 100$, and the standard IV-weighted methods never achieve the nominal level. SSW MP, with $t$ critical values, is the only alternative, but its coverage is somewhat above nominal for very small $n$. When $\mu_{C}=4$, the HKSJ intervals provide nominal coverage starting from $n=10$ when $K \leq 30$ and $\tau^{2}>0$, and the coverage of all other IV-weighted methods is considerably lower than nominal. Coverage of all except SSW MP deteriorates for larger $K$ (Figure B5.2.1), though coverage of SSW MP is above nominal at $\tau^{2}=0$. Coverage of all methods except DL and $\mathrm{J}$ improves for very large sample sizes, but it still can be extremely low at zero (Figure D4.2.4).

Coverage of $\lambda$ is not affected when the bias-corrected estimator $\lambda^{\Delta}$ is used (Appendices B3.2 and B6.2).

\section{Discussion}

The results of our simulations provide a rather disappointing picture of the current state of meta-analysis of LRR. For such effect measures as LRR and SMD, also popular in ecology, the relation between the studies' estimated effects and their estimated variances has several undesirable results: dependence of the performance of all inverse-variance-based methods on the effect sizes, biased estimation of overall effects, and below-nominal coverage of their confidence intervals, especially for small sample sizes. Our simulations show this clearly.

We found considerable biases in all methods of estimation of $\tau^{2}$ for very small $n$, but overall MP and REML are reasonable choices, especially farther from zero. QP provides reasonable, though not perfect, coverage.

Arguably, the main purpose of a meta-analysis is to provide point and interval estimates of an overall effect. For general use, the estimate of overall effect should be unbiased, and the confidence interval should have close to nominal coverage.

Usually, after estimating the between-study variance $\tau^{2}$, an inverse-varianceweighted approach is used to estimate the overall effect (and, often, its variance). The origin of the IV approach lies in the fact that, for known variances, and given 
unbiased estimators of the within-study effects, it provides a uniformly minimumvariance unbiased estimator (UMVUE) of $\theta$. However, in practice, the within-study variances are unknown, and using estimates for them leads to bias in the IV-weighted estimate of the overall effect and below-nominal coverage of the confidence interval. Thus, the IV approach is misguided; for most measures of effect, it cannot avoid these shortcomings.

The gaps in evidence include the possibility that the variances in the two arms may differ, which is rarely, if ever, reflected in simulations. Because of the sheer volume of our simulations, we did not attempt to fill this gap. However, we do not expect the performance of the IV-weighted methods to improve under morechallenging scenarios.

A pragmatic solution for unbiased estimation of $\theta$ uses weights that do not involve estimated variances (for example, weights proportional to the studies' sample sizes $n_{i}$ ). Our point estimator SSW uses weights proportional to an effective sample size, $\tilde{n}_{i}=n_{i C} n_{i T} / n_{i}$. Then the estimate of the overall effect is $\hat{\lambda}_{S S W}=\sum \tilde{n}_{i} \hat{\lambda}_{i} / \sum \tilde{n}_{i}$ and the estimate of its variance comes from Equation (5.1). Finally, the t-based confidence interval for $\lambda$ is centered at $\hat{\lambda}_{S S W}$.

Unfortunately, the use of sample-size-based weights does not avoid all the problems with bias in estimation of LRR for very small sample sizes and close to zero. The response ratio is a rather challenging effect measure, with two additional sources of possible bias: its restricted range near zero under the normal model, and transformation bias in using the log scale for specification of random effects. Both issues are inherent in the choice of the respective true model and cannot be easily resolved. SSW MP is the only feasible option for confidence intervals.

Importantly, the bias correction of $\lambda$ by the method of Lajeunesse 2015 adversely affects the quality of estimation and is not recommended for meta-analysis of LRR for normal data.

\section{Methods of estimation of $\tau^{2}$ and $\lambda$ used in simulations}

Point estimators of $\tau^{2}$

- DL - method of DerSimonian and Laird 1986 
- J - method of Jackson 2013

- MP - method of Mandel and Paule 1970

- REML - restricted maximum-likelihood method

\section{Interval estimators of $\tau^{2}$}

- BJ - method of Biggerstaff and Jackson 2008

- J - method of Jackson 2013

- PL - profile-likelihood confidence interval based on $\hat{\tau}_{R E M L}^{2}$

- QP - Q-profile confidence interval of Viechtbauer 2007

\section{Point estimators of $\lambda$}

Inverse-variance-weighted methods with $\tau^{2}$ estimated by:

- DL

- $\mathrm{J}$

- MP

- REML

and

- SSW - weighted mean with weights that depend only on studies' sample sizes

\section{Interval estimators of $\lambda$}

Inverse-variance-weighted methods using normal quantiles, with $\tau^{2}$ estimated by:

- DL

- $\mathrm{J}$

- MP

- REML

Inverse-variance-weighted methods with modified variance of $\hat{\lambda}$ and t-quantiles, as in Hartung and Knapp 2001] and Sidik and Jonkman 2002],

- HKSJ (DL) - $\tau^{2}$ estimated by DL

- HKSJ MP - $\tau^{2}$ estimated by MP 
and

- SSW MP - SSW point estimator of $\lambda$ with estimated variance given by Equation (5.1) and t-quantiles

\section{Funding}

The work by E. Kulinskaya was supported by the Economic and Social Research Council [grant number ES/L011859/1].

\section{References}

Ilyas Bakbergenuly, Elena Kulinskaya, and Stephan Morgenthaler. Inference for binomial probability based on dependent Bernoulli random variables with applications to meta-analysis and group level studies. Biometrical Journal, 58(4):896-914, 2016.

Ilyas Bakbergenuly, Elena Kulinskaya, and David C Hoaglin. Estimation in metaanalyses of mean difference and standardized mean difference. Statistics in Medicine, 2018. submitted.

Ilyas Bakbergenuly, Elena Kulinskaya, and David C Hoaglin. Pitfalls of using the risk ratio in meta-analysis. Research Synthesis Methods, 10:398-419, 2019a.

Ilyas Bakbergenuly, Elena Kulinskaya, and David C Hoaglin. Simulation study of estimating between-study variance and overall effect in meta-analyses of log-responseratio for lognormal data. In eprint arXiv:1905.01243 [stat.ME], May 2019b.

Brad J Biggerstaff and Dan Jackson. The exact distribution of Cochran's heterogeneity statistic in one-way random effects meta-analysis. Statistics in Medicine, 27(29):6093-6110, 2008.

William G Cochran. The combination of estimates from different experiments. Biometrics, 10(1):101-129, 1954.

Rebecca DerSimonian and Raghu Kacker. Random-effects model for meta-analysis of clinical trials: an update. Contemporary Clinical Trials, 28(2):105-114, 2007.

Rebecca DerSimonian and Nan Laird. Meta-analysis in clinical trials. Controlled Cinical Trials, 7(3):177-188, 1986. 
C Patrick Doncaster and Rebecca Spake. Correction for bias in meta-analysis of little-replicated studies. Methods in Ecology and Evolution, 9(3):634-644, 2018.

Jan O Friedrich, Neill KJ Adhikari, and Joseph Beyene. The ratio of means method as an alternative to mean differences for analyzing continuous outcome variables in meta-analysis: a simulation study. BMC Medical Research Methodology, 8:32, 2008 .

Elizabeth A. Hamman, Paula Pappalardo, James R. Bence, Scott D. Peacor, and Craig W. Osenberg. Bias in meta-analyses using Hedges'd. Ecosphere, 9(9):e02419, 2018 .

Joachim Hartung and Guido Knapp. A refined method for the meta-analysis of controlled clinical trials with binary outcome. Statistics in Medicine, 20(24):3875$3889,2001$.

Larry V Hedges and Ingram Olkin. Statistical Methods for Meta-Analysis. Academic Press, San Diego, CA, 1985.

Larry V Hedges, Jessica Gurevitch, and Peter S Curtis. The meta-analysis of response ratios in experimental ecology. Ecology, 80(4):1150-1156, 1999.

Julian Higgins, Simon G Thompson, and David J Spiegelhalter. A re-evaluation of random-effects meta-analysis. Journal of the Royal Statistical Society: Series A (Statistics in Society), 172(1):137-159, 2009.

John E Hunter and Frank L Schmidt. Methods of Meta-analysis: Correcting Error and Bias in Research Findings. Sage Publications, Inc, 1990.

Dan Jackson. Confidence intervals for the between-study variance in random effects meta-analysis using generalised Cochran heterogeneity statistics. Research Synthesis Methods, 4(3):220-229, 2013.

Julia Koricheva and Jessica Gurevitch. Uses and misuses of meta-analysis in plant ecology. Journal of Ecology, 102(4):828-844, 2014.

Marc J Lajeunesse. Bias and correction for the log response ratio in ecological metaanalysis. Ecology, 96(8):2056-2063, 2015. 
Yuanzhang Li, Li Shi, and H Daniel Roth. The bias of the commonly-used estimate of variance in meta-analysis. Communications in Statistics-Theory and Methods, 23(4):1063-1085, 1994.

John Mandel and Robert C Paule. Interlaboratory evaluation of a material with unequal numbers of replicates. Analytical Chemistry, 42(11):1194-1197, 1970.

Shinichi Nakagawa and Eduardo SA Santos. Methodological issues and advances in biological meta-analysis. Evolutionary Ecology, 26(5):1253-1274, 2012.

Andrew L Rukhin. Weighted means statistics in interlaboratory studies. Metrologia, 46(3):323, 2009.

Alistair M. Senior, Catherine E. Grueber, Tsukushi Kamiya, Malgorzata Lagisz, Katie O'Dwyer, Eduardo S. A. Santos, and Shinichi Nakagawa. Heterogeneity in ecological and evolutionary meta-analyses: its magnitude and implications. Ecology, 97(12):3293-3299, 2016.

K. Sidik and J. N. Jonkman. A simple confidence interval for meta-analysis. Statistics in Medicine, 21(21):3153-3159, 2002.

Kurex Sidik and Jeffrey N Jonkman. Robust variance estimation for random effects meta-analysis. Computational Statistics \& Data Analysis, 50(12):3681-3701, 2006.

Areti Angeliki Veroniki, Dan Jackson, Wolfgang Viechtbauer, Ralf Bender, Jack Bowden, Guido Knapp, Oliver Kuss, Julian P T Higgins, Dean Langan, and Georgia Salanti. Methods to estimate the between-study variance and its uncertainty in meta-analysis. Research Synthesis Methods, 7(1):55-79, 2016.

Wolfgang Viechtbauer. Bias and efficiency of meta-analytic variance estimators in the random-effects model. Journal of Educational and Behavioral Statistics, 30(3): 261-293, 2005.

Wolfgang Viechtbauer. Confidence intervals for the amount of heterogeneity in metaanalysis. Statistics in Medicine, 26(1):37-52, 2007. 


\section{Appendices}

\section{A: Plots of bias and coverage of estimators of $\tau^{2}$}

for RoM with $n=4,10,20,40$

- A1. Normal model, $\mu_{C}=1, K=5,10,30$

- A2. Normal model, $\mu_{C}=4, K=5,10,30$

- A3. Normal model, bias-corrected $\lambda, \mu_{C}=4, K=5,10,30$

- A4. Normal model, $\mu_{C}=1, K=50,100,125$

- A5. Normal model, $\mu_{C}=4, K=50,100,125$

- A6. Normal model, bias-corrected $\lambda, \mu_{C}=4, K=50,100,125$ 
A1: Normal model, $\mu_{C}=1, n=4,10,20,40, K=$ $5,10,30$

\section{A1.1 Bias of point estimators of $\tau^{2}$ when $\mu_{C}=1$}

Each figure corresponds to a value of $\lambda(=0,0.2,0.5,1,2)$, a set of values of $n(=4$, $10,20,40)$, and a set of values of $K(=5,10,30)$.

Each panel corresponds to a value of $n$ and a value of $K$ and has $\tau^{2}=0.0(0.1) 1.0$ on the horizontal axis.

The point estimators of $\tau^{2}$ are

- DL (DerSimonian-Laird)

- REML (restricted maximum likelihood)

- MP (Mandel-Paule)

- J (Jackson) 

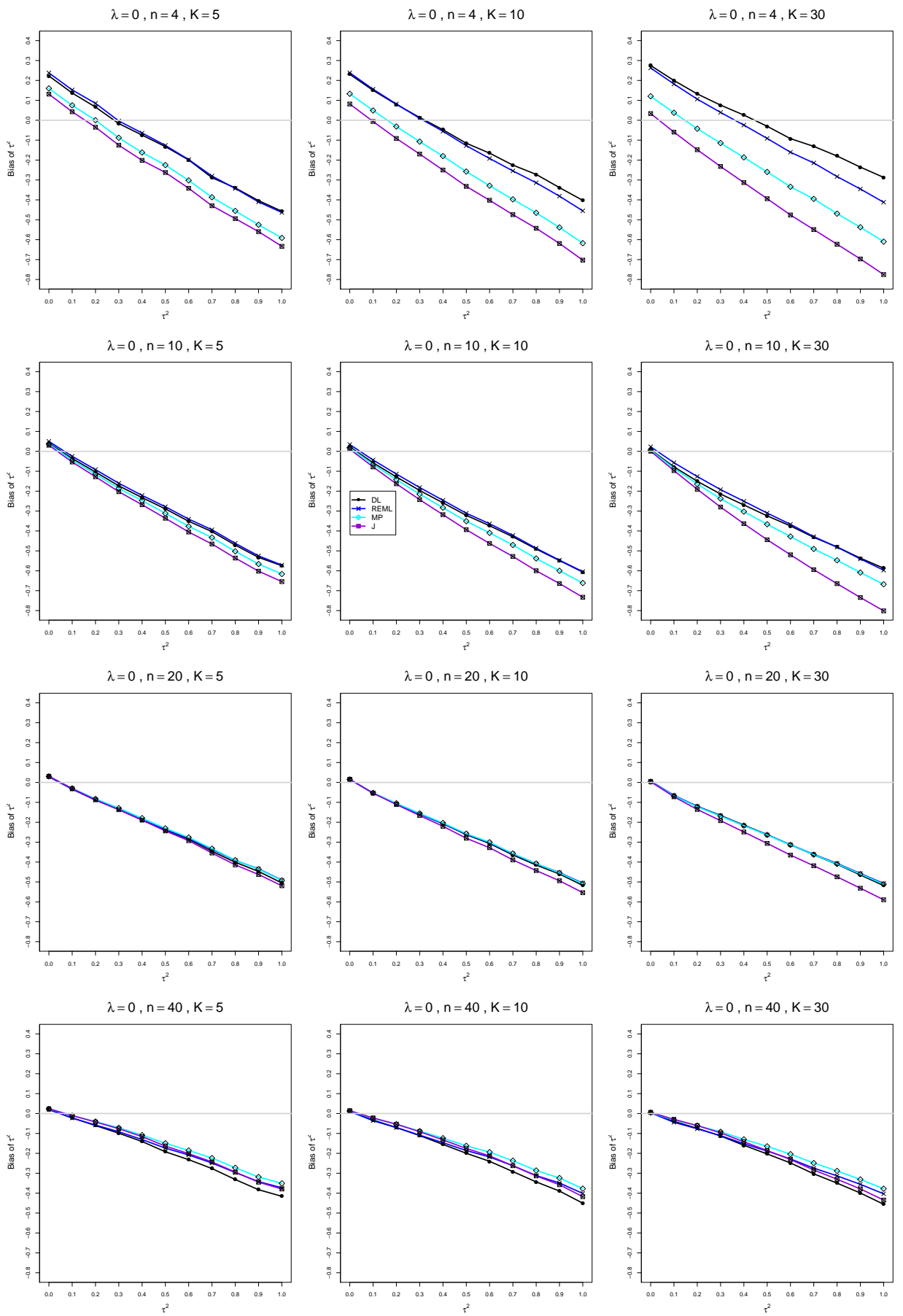

Figure A1.1.1: Bias of estimators of the between-studies variance $\tau^{2}$ when $\mu_{C}=1, \lambda=0$, $n=4,10,20,40$, and $K=5,10,30$ 

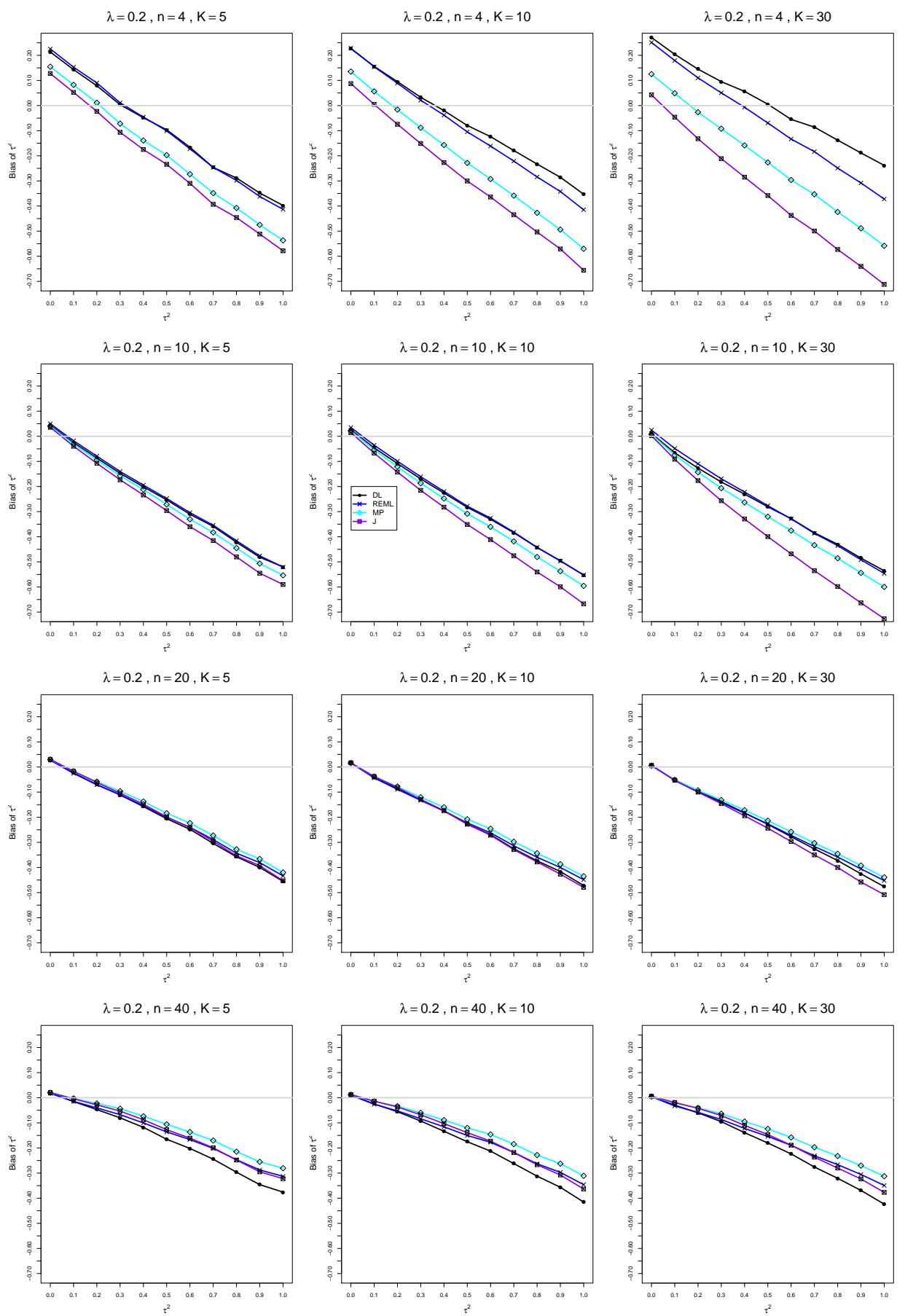

Figure A1.1.2: Bias of estimators of the between-studies variance $\tau^{2}$ when $\mu_{C}=1, \lambda=$ $0.2, n=4,10,20,40$, and $K=5,10,30$ 

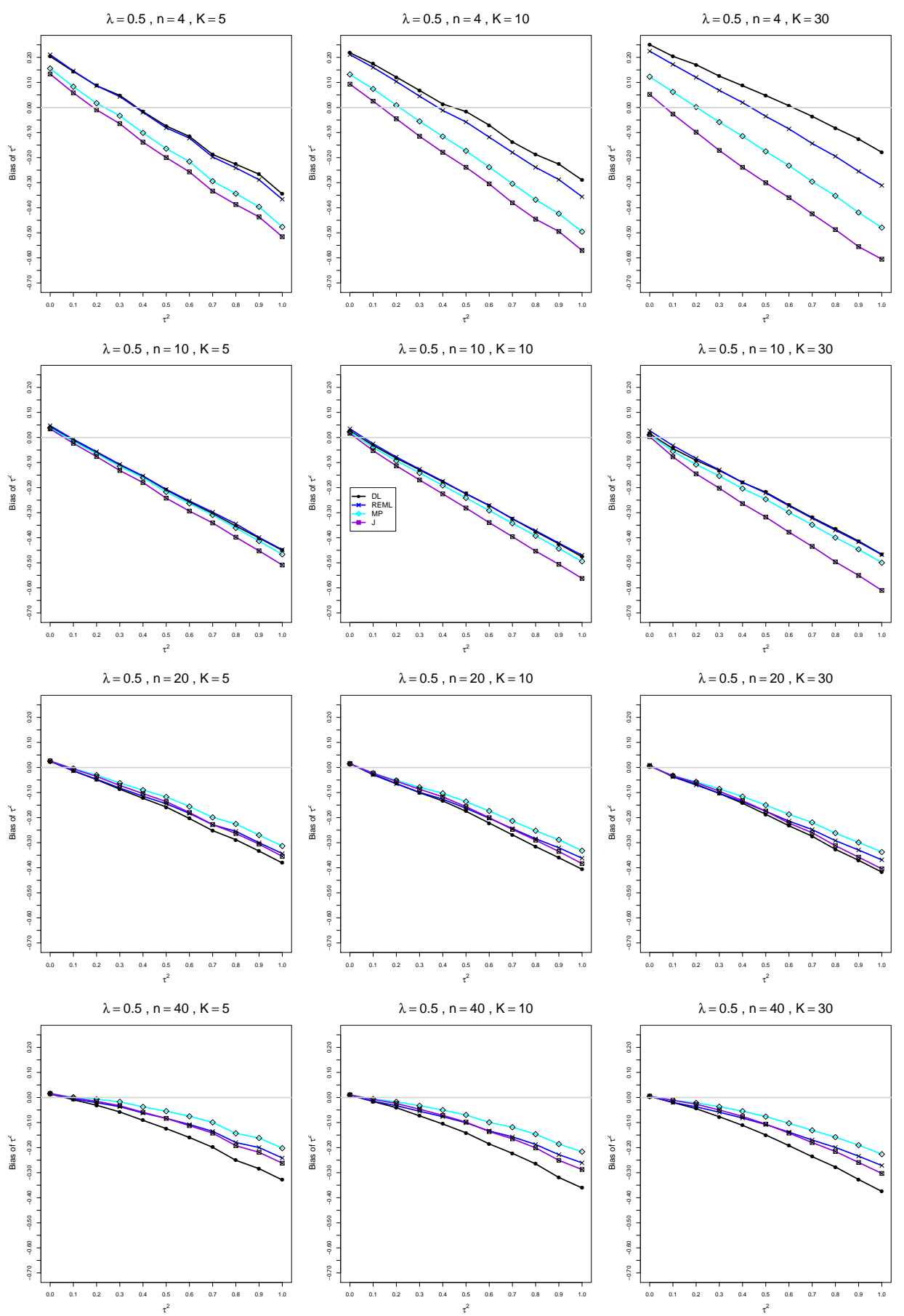

Figure A1.1.3: Bias of estimators of the between-studies variance $\tau^{2}$ when $\mu_{C}=1, \lambda=$ $0.5, n=4,10,20,40$, and $K=5,10,30$ 

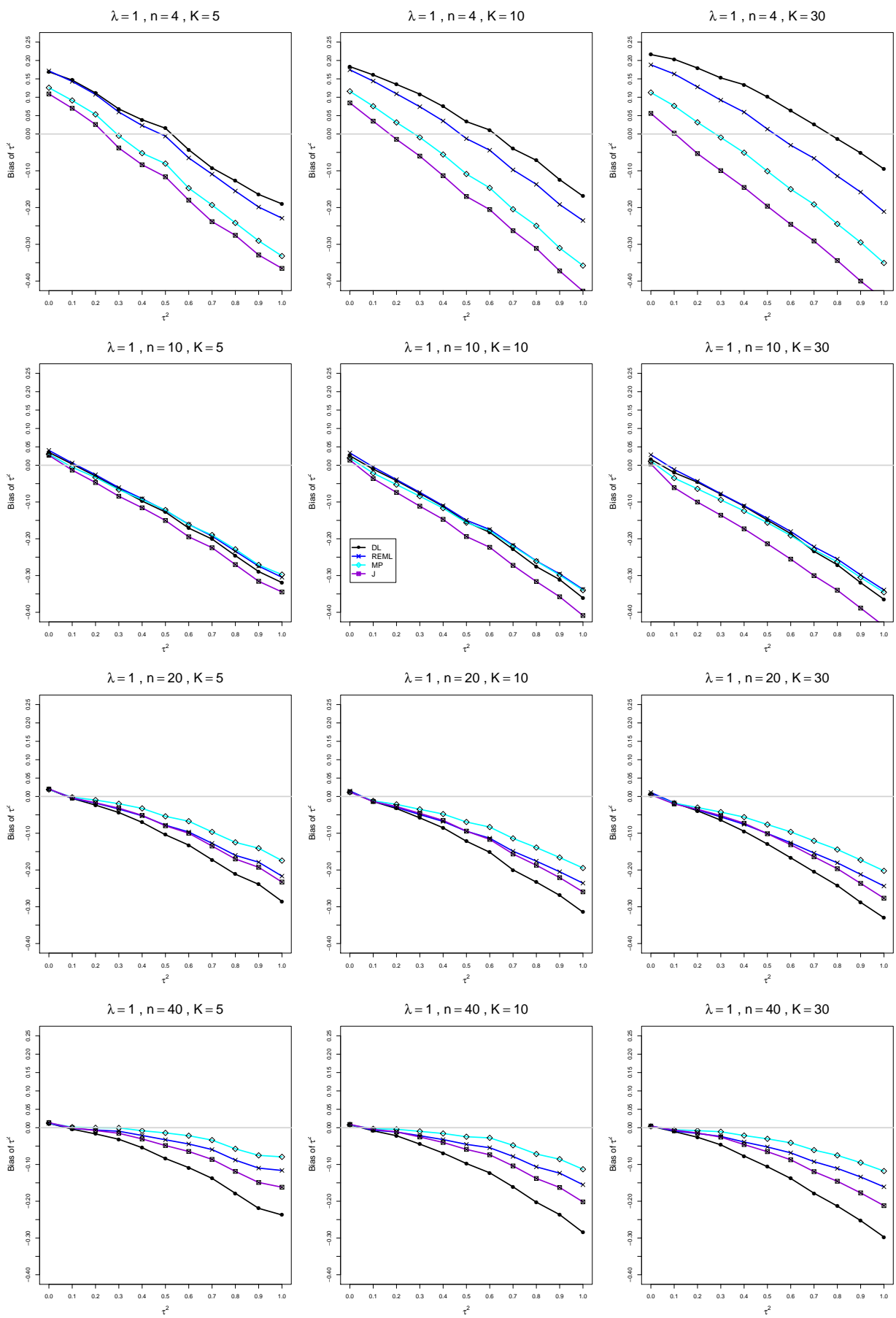

Figure A1.1.4: Bias of estimators of the between-studies variance $\tau^{2}$ when $\mu_{C}=1, \lambda=1$, $n=4,10,20,40$, and $K=5,10,30$ 

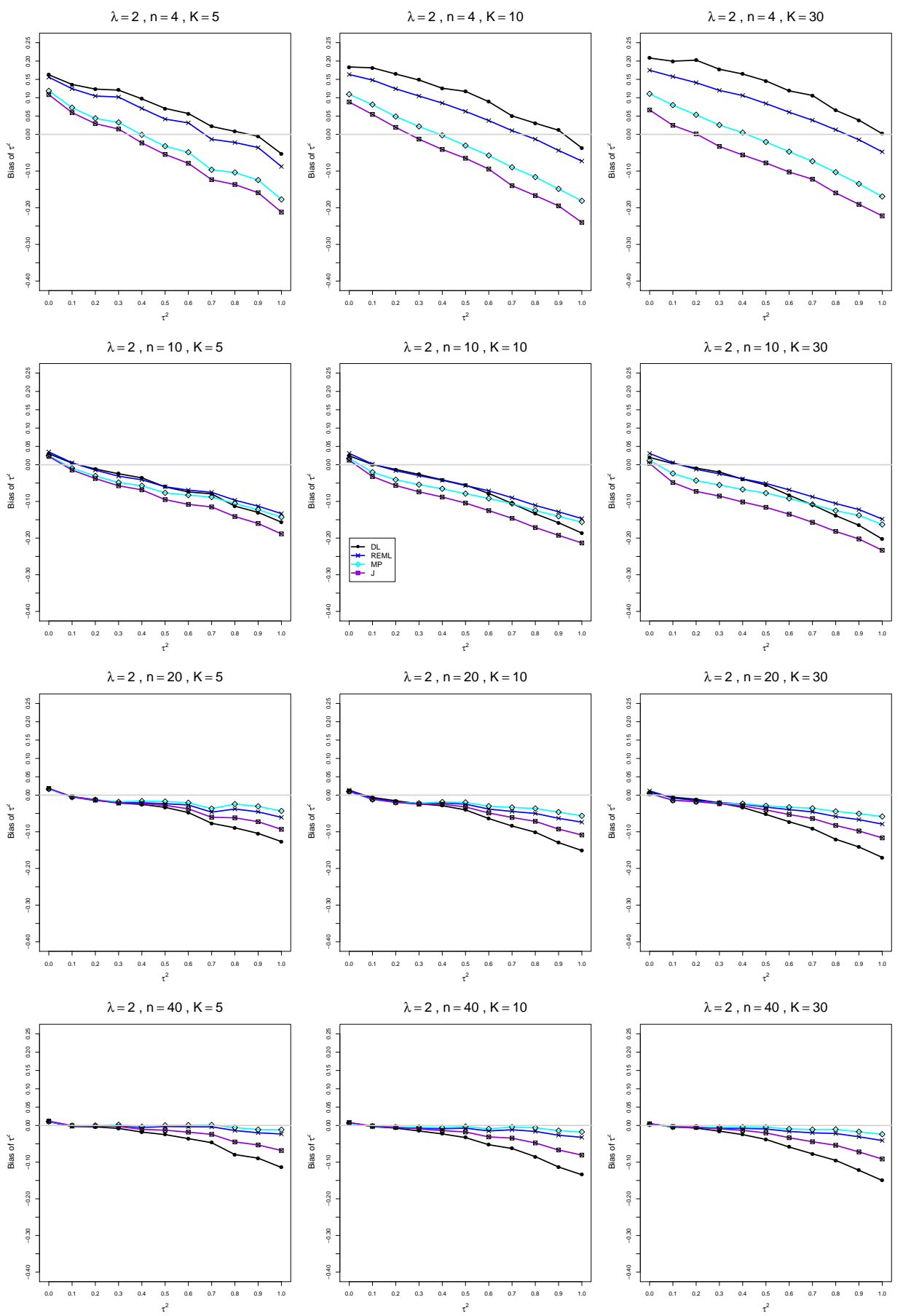

Figure A1.1.5: Bias of estimators of the between-studies variance $\tau^{2}$ when $\mu_{C}=1, \lambda=2$, $n=4,10,20,40$, and $K=5,10,30$ 


\section{A1.2 Coverage of interval estimators of $\tau^{2}$ when $\mu_{C}=1$}

Each figure corresponds to a value of $\lambda(=0,0.2,0.5,1,2)$, a set of values of $n(=4$, $10,20,40)$, and a set of values of $K(=5,10,30)$.

Each panel corresponds to a value of $n$ and a value of $K$ and has $\tau^{2}=0.0(0.1) 1.0$ on the horizontal axis.

The interval estimators of $\tau^{2}$ are

- QP (Q-profile confidence interval)

- BJ (Biggerstaff and Jackson interval)

- PL (Profile likelihood interval)

- J (Jackson's interval) 

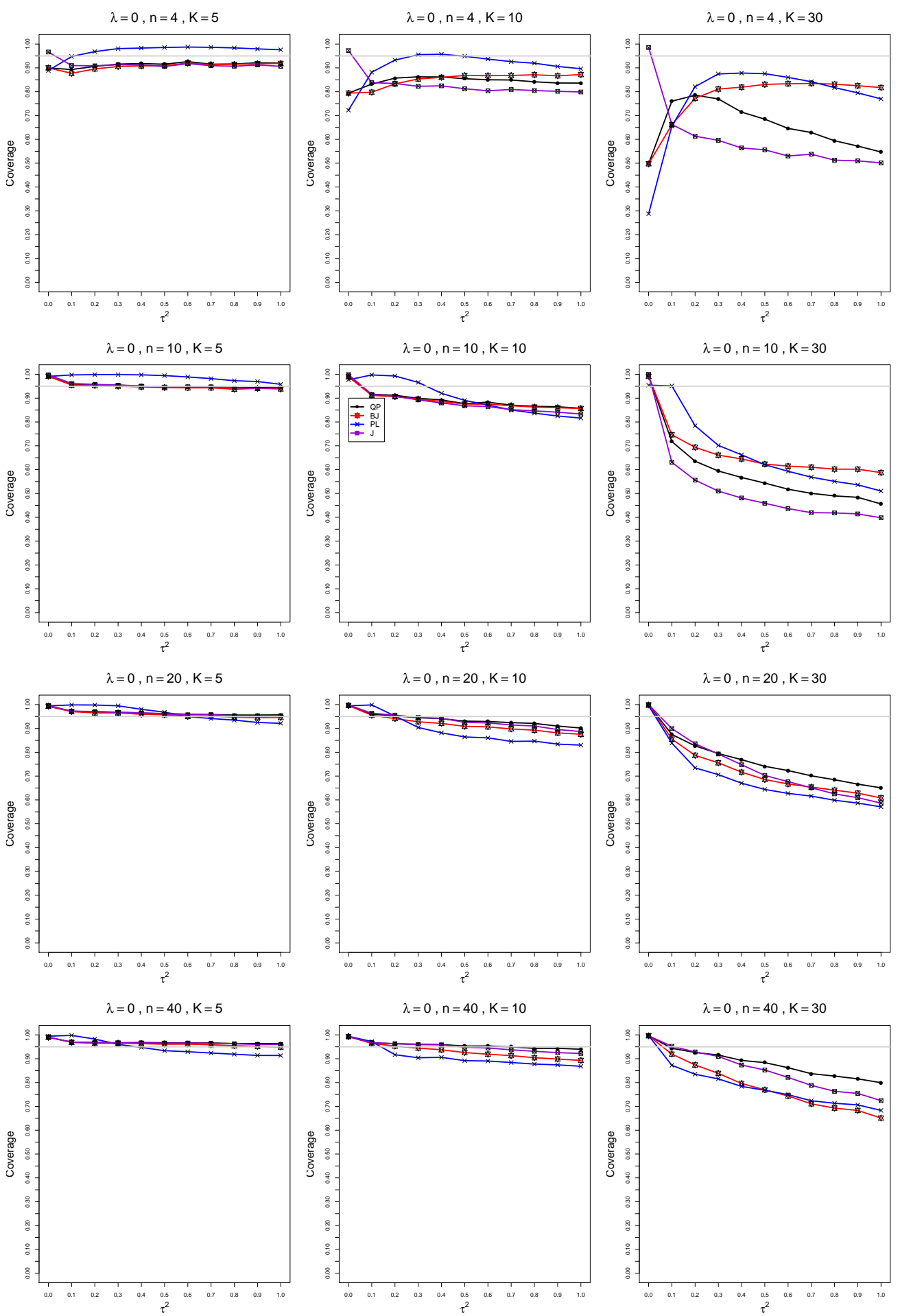

Figure A1.2.1: Coverage of 95\% confidence intervals for the between-studies variance $\tau^{2}$ when $\mu_{C}=1, \lambda=0, n=4,10,20,40$, and $K=5,10,30$ 

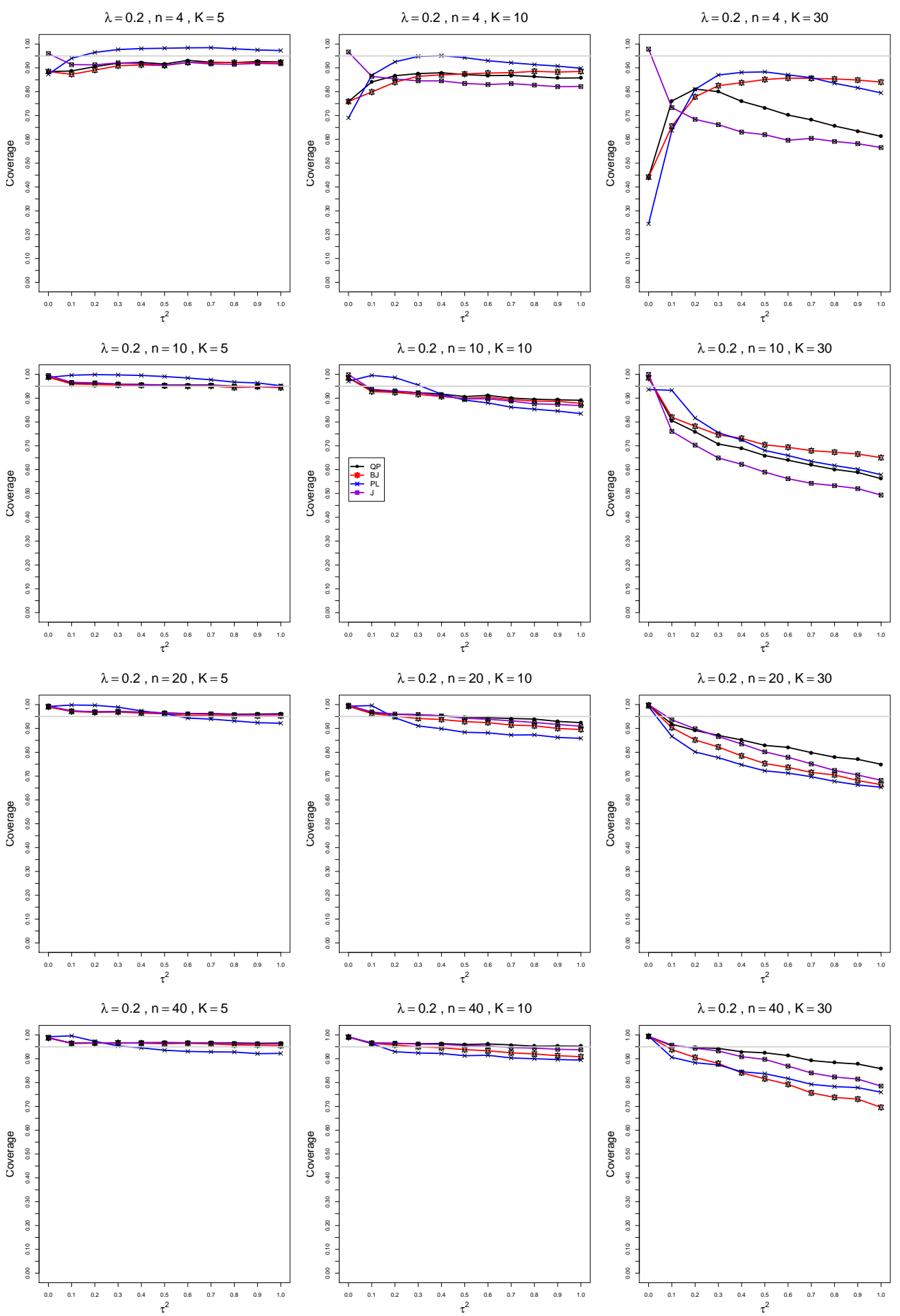

Figure A1.2.2: Coverage of 95\% confidence intervals for the between-studies variance $\tau^{2}$ when $\mu_{C}=1, \lambda=0.2, n=4,10,20,40$, and $K=5,10,30$ 

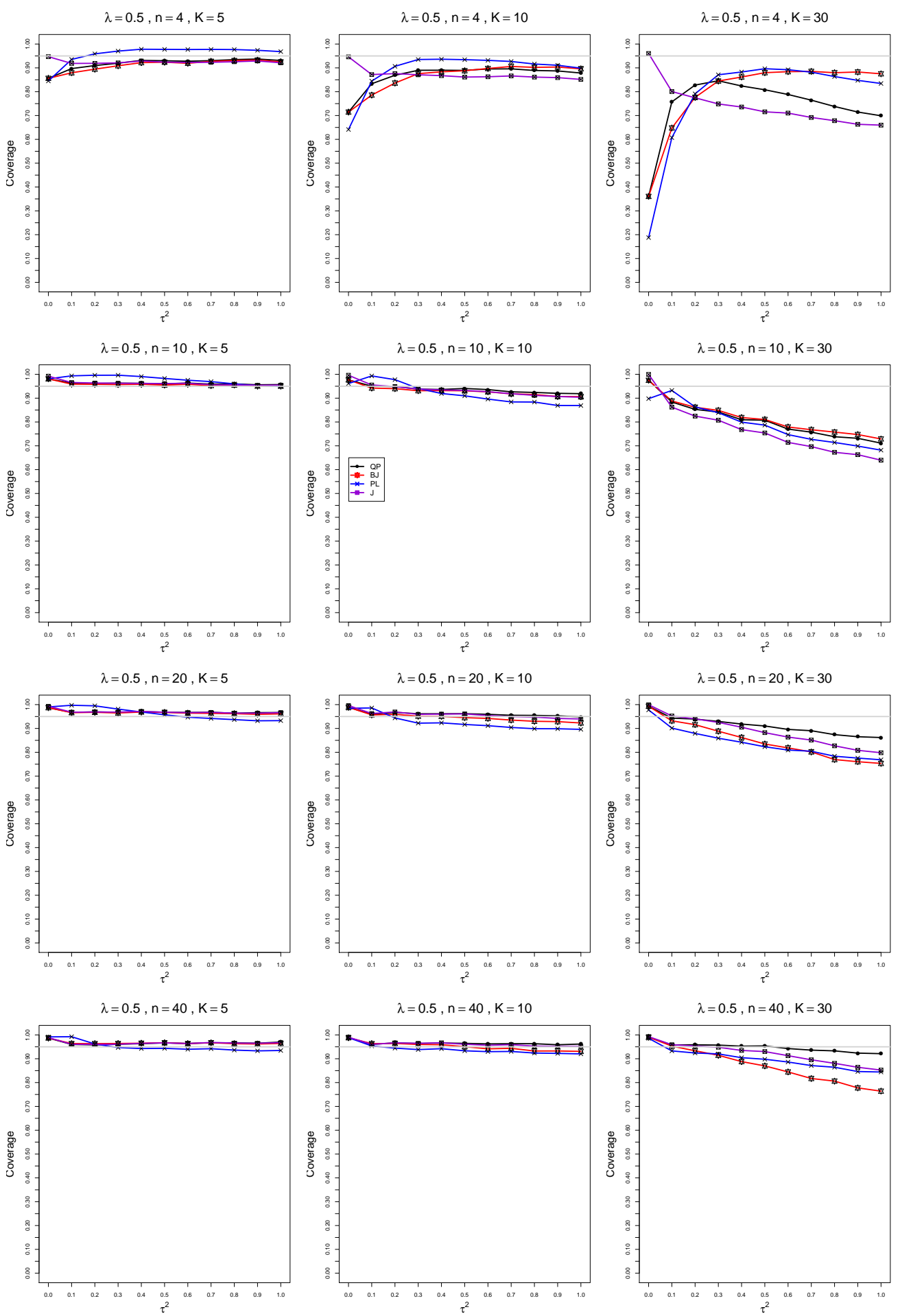

Figure A1.2.3: Coverage of 95\% confidence intervals for the between-studies variance $\tau^{2}$ when $\mu_{C}=1, \lambda=0.5, n=4,10,20,40$, and $K=5,10,30$ 

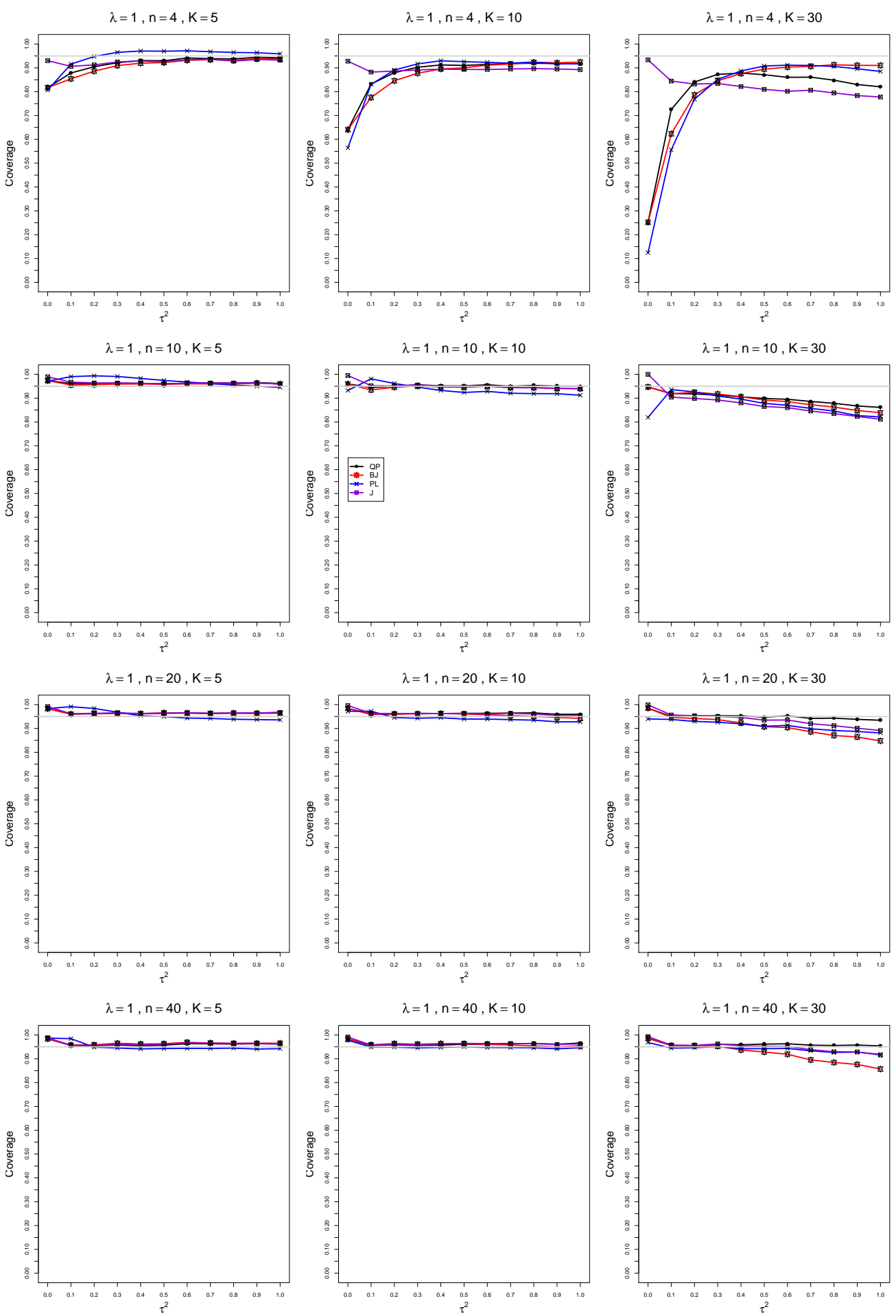

Figure A1.2.4: Coverage of 95\% confidence intervals for the between-studies variance $\tau^{2}$ when $\mu_{C}=1, \lambda=1, n=4,10,20,40$, and $K=5,10,30$ 

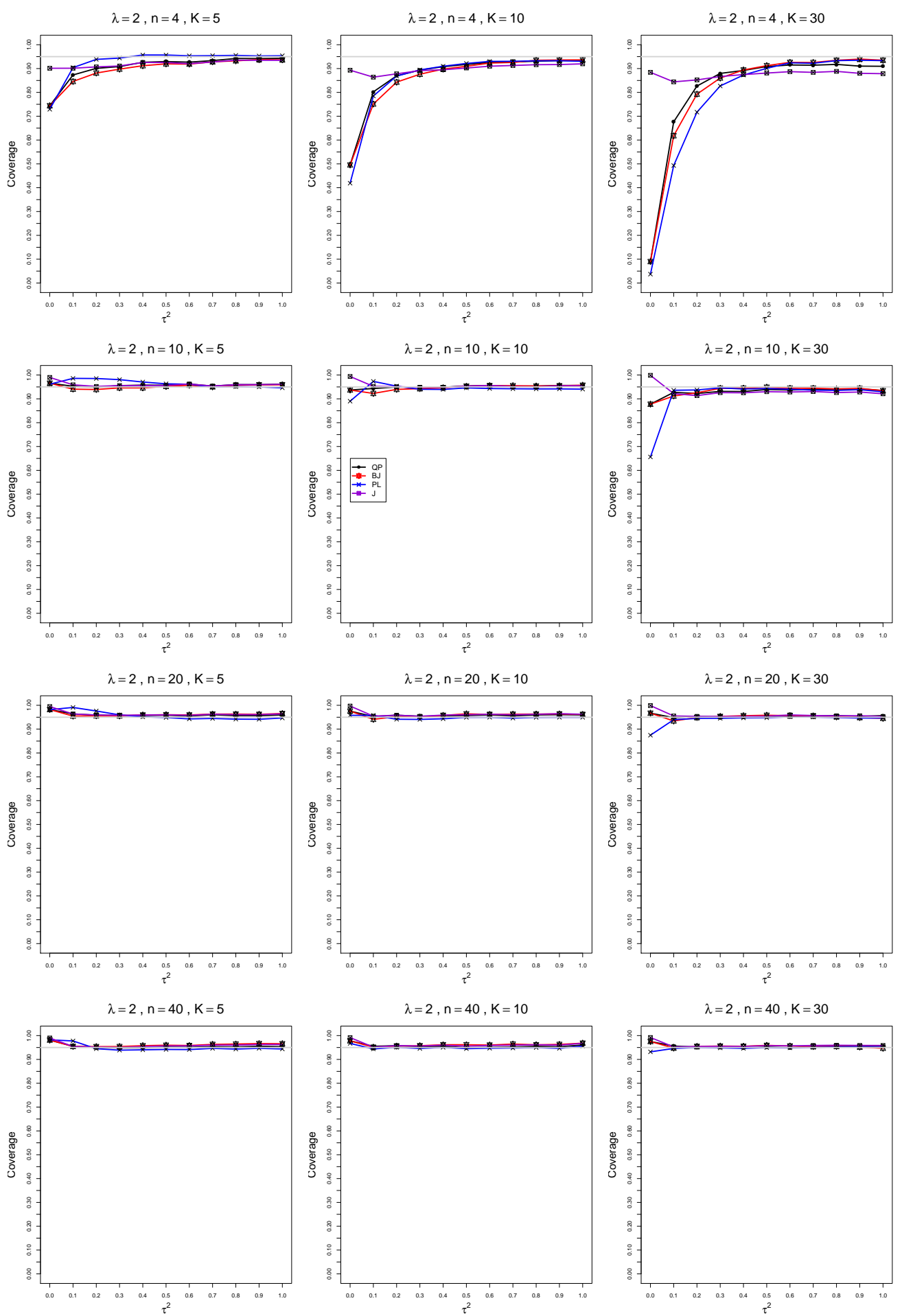

Figure A1.2.5: Coverage of 95\% confidence intervals for the between-studies variance $\tau^{2}$ when $\mu_{C}=1, \lambda=2, n=4,10,20,40$, and $K=5,10,30$ 
A2. Normal model, $\mu_{C}=4, n=4,10,20,40, K=$ $5,10,30$

\section{A2.1 Bias of point estimators of $\tau^{2}$ when $\mu_{C}=4$}

Each figure corresponds to a value of $\lambda(=0,0.2,0.5,1,2)$, a set of values of $n(=4$, $10,20,40)$, and a set of values of $K(=5,10,30)$.

Each panel corresponds to a value of $n$ and a value of $K$ and has $\tau^{2}=0.0(0.1) 1.0$ on the horizontal axis.

The point estimators of $\tau^{2}$ are

- DL (DerSimonian-Laird)

- REML (restricted maximum likelihood)

- MP (Mandel-Paule)

- J (Jackson) 

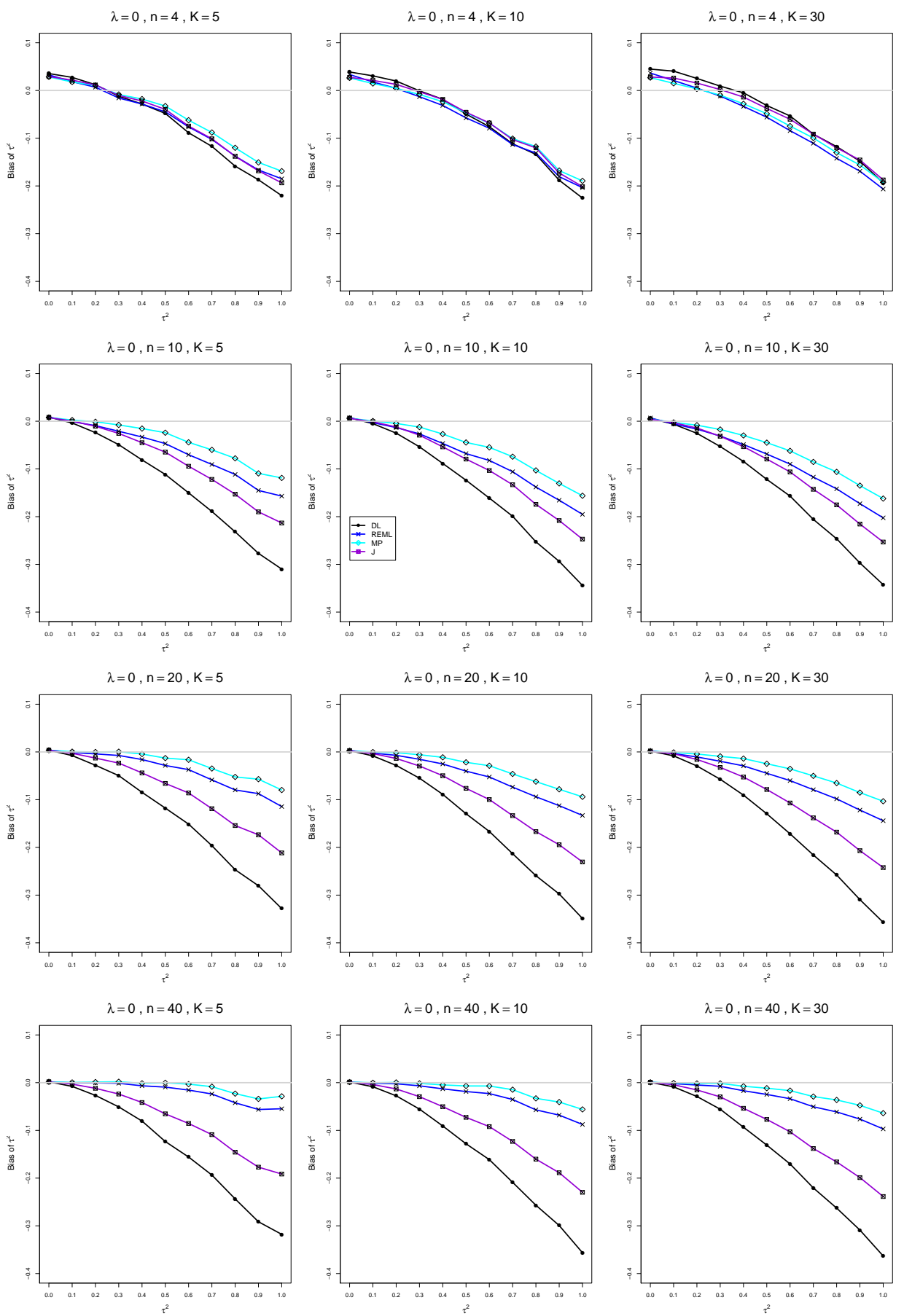

Figure A2.1.1: Bias of estimators of the between-studies variance $\tau^{2}$ when $\mu_{C}=4, \lambda=0$, $n=4,10,20,40$, and $K=5,10,30$ 

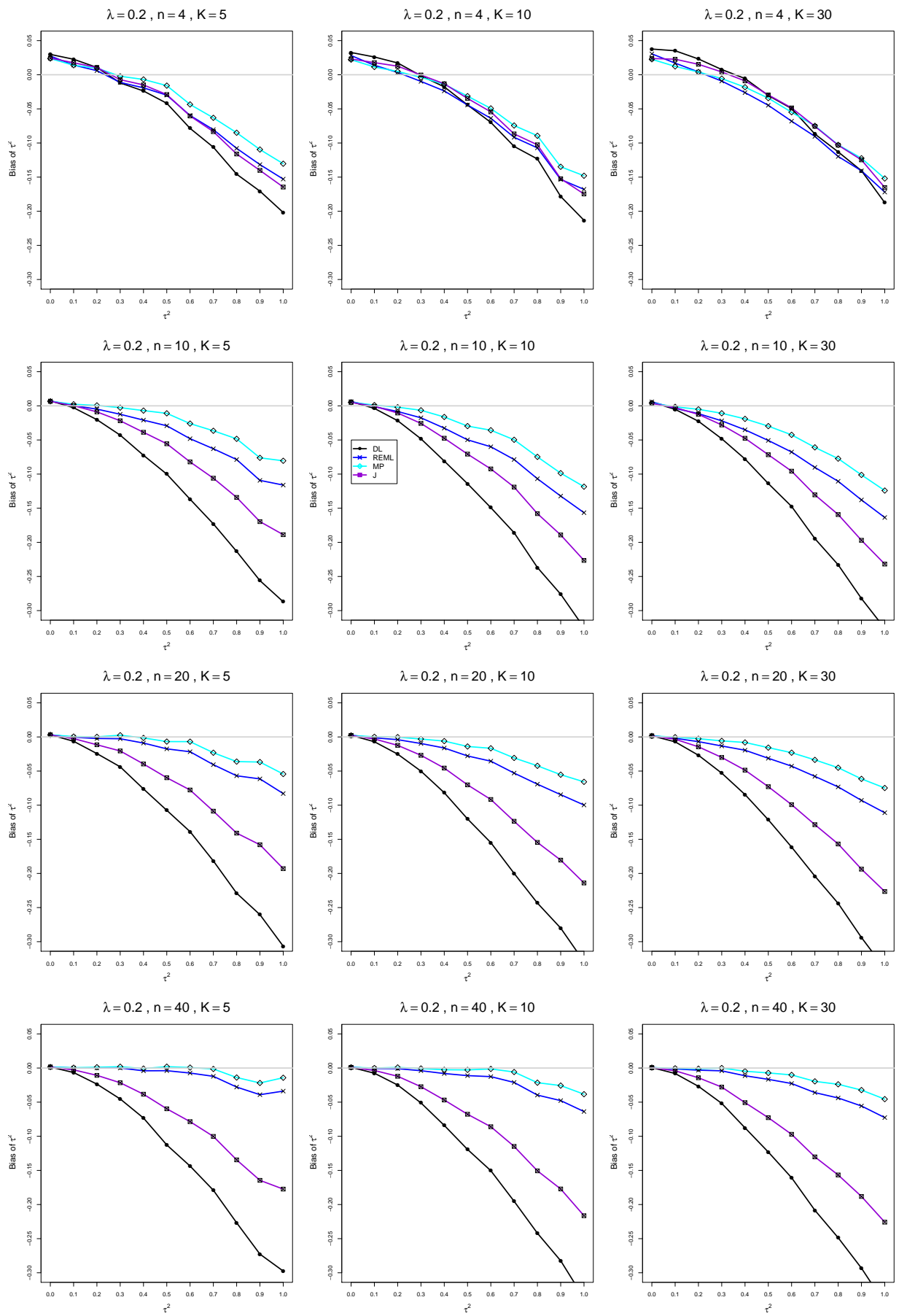

Figure A2.1.2: Bias of estimators of the between-studies variance $\tau^{2}$ when $\mu_{C}=1, \lambda=$ $0.2, n=4,10,20,40$, and $K=5,10,30$ 

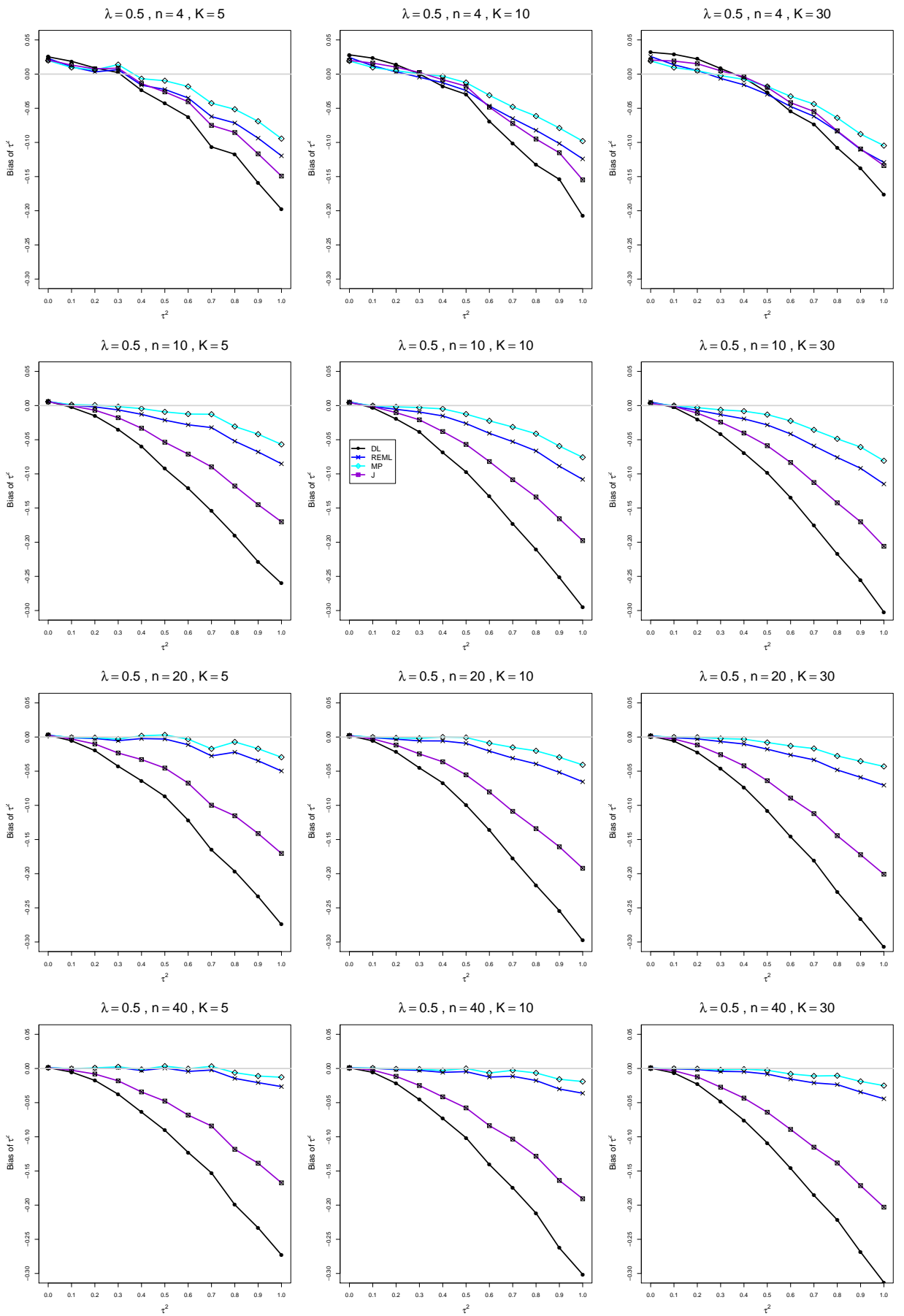

Figure A2.1.3: Bias of estimators of the between-studies variance $\tau^{2}$ when $\mu_{C}=4, \lambda=$ $0.5, n=4,10,20,40$, and $K=5,10,30$ 

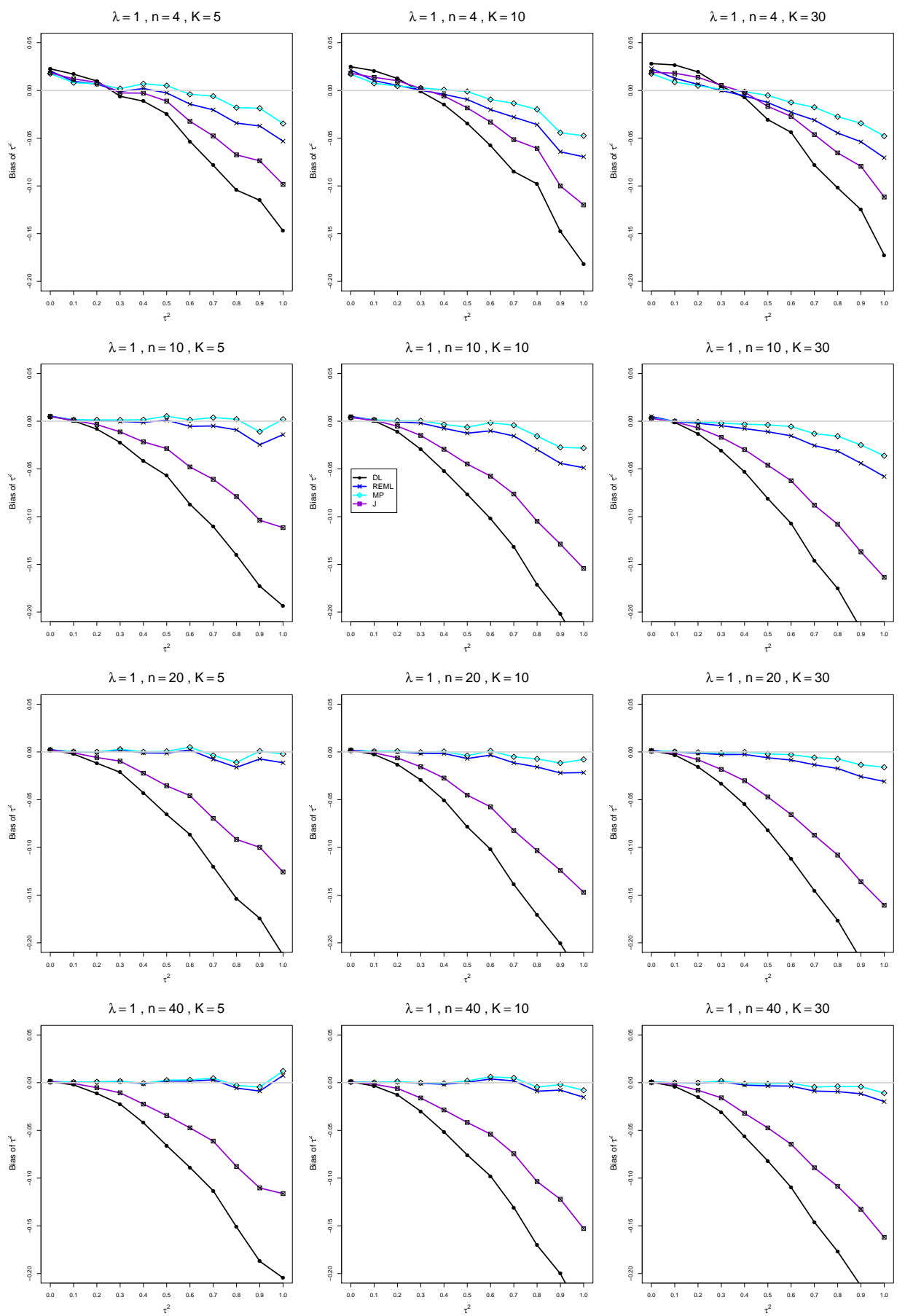

Figure A2.1.4: Bias of estimators of the between-studies variance $\tau^{2}$ when $\mu_{C}=4, \lambda=1$, $n=4,10,20,40$, and $K=5,10,30$ 

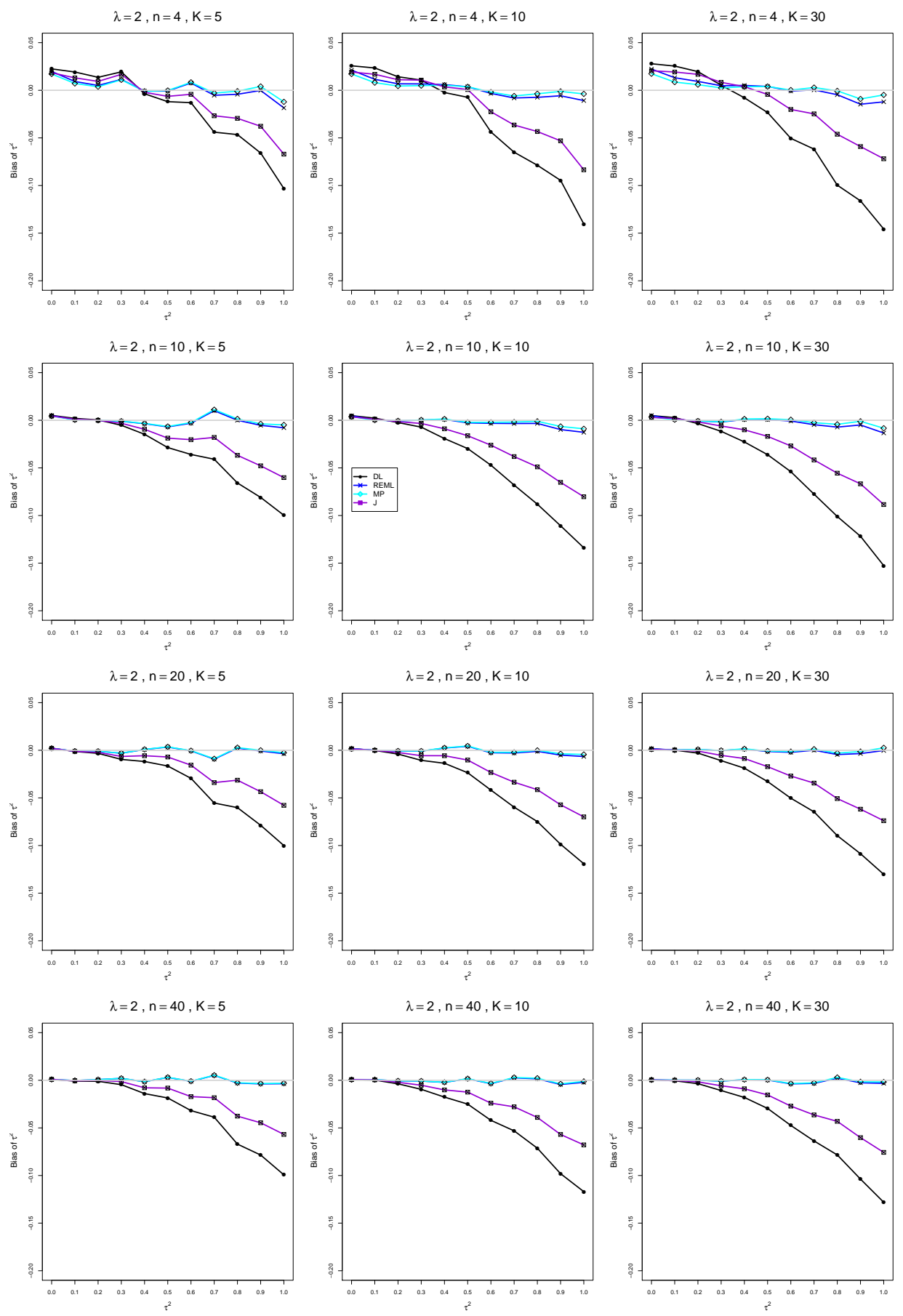

Figure A2.1.5: Bias of estimators of the between-studies variance $\tau^{2}$ when $\mu_{C}=4, \lambda=2$, $n=4,10,20,40$, and $K=5,10,30$ 


\section{A2.2 Coverage of interval estimators of $\tau^{2}$ when $\mu_{C}=4$}

Each figure corresponds to a value of $\lambda(=0,0.2,0.5,1,2)$, a set of values of $n(=4$, $10,20,40)$, and a set of values of $K(=5,10,30)$.

Each panel corresponds to a value of $n$ and a value of $K$ and has $\tau^{2}=0.0(0.1) 1.0$ on the horizontal axis.

The interval estimators of $\tau^{2}$ are

- QP (Q-profile confidence interval)

- BJ (Biggerstaff and Jackson interval)

- PL (Profile likelihood interval)

- J (Jackson's interval) 

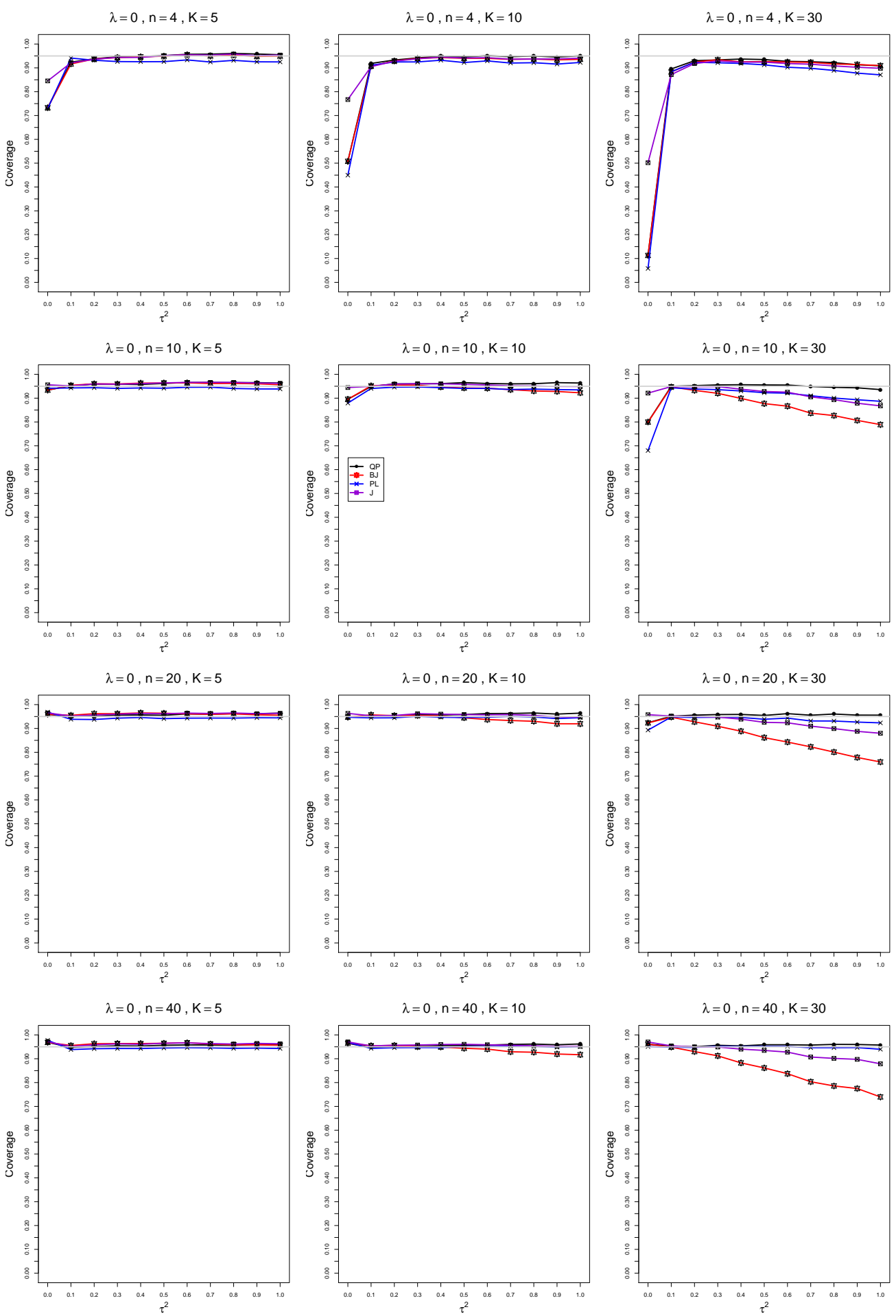

Figure A2.2.1: Coverage of 95\% confidence intervals for the between-studies variance $\tau^{2}$ when $\mu_{C}=4, \lambda=0, n=4,10,20,40$, and $K=5,10,30$ 

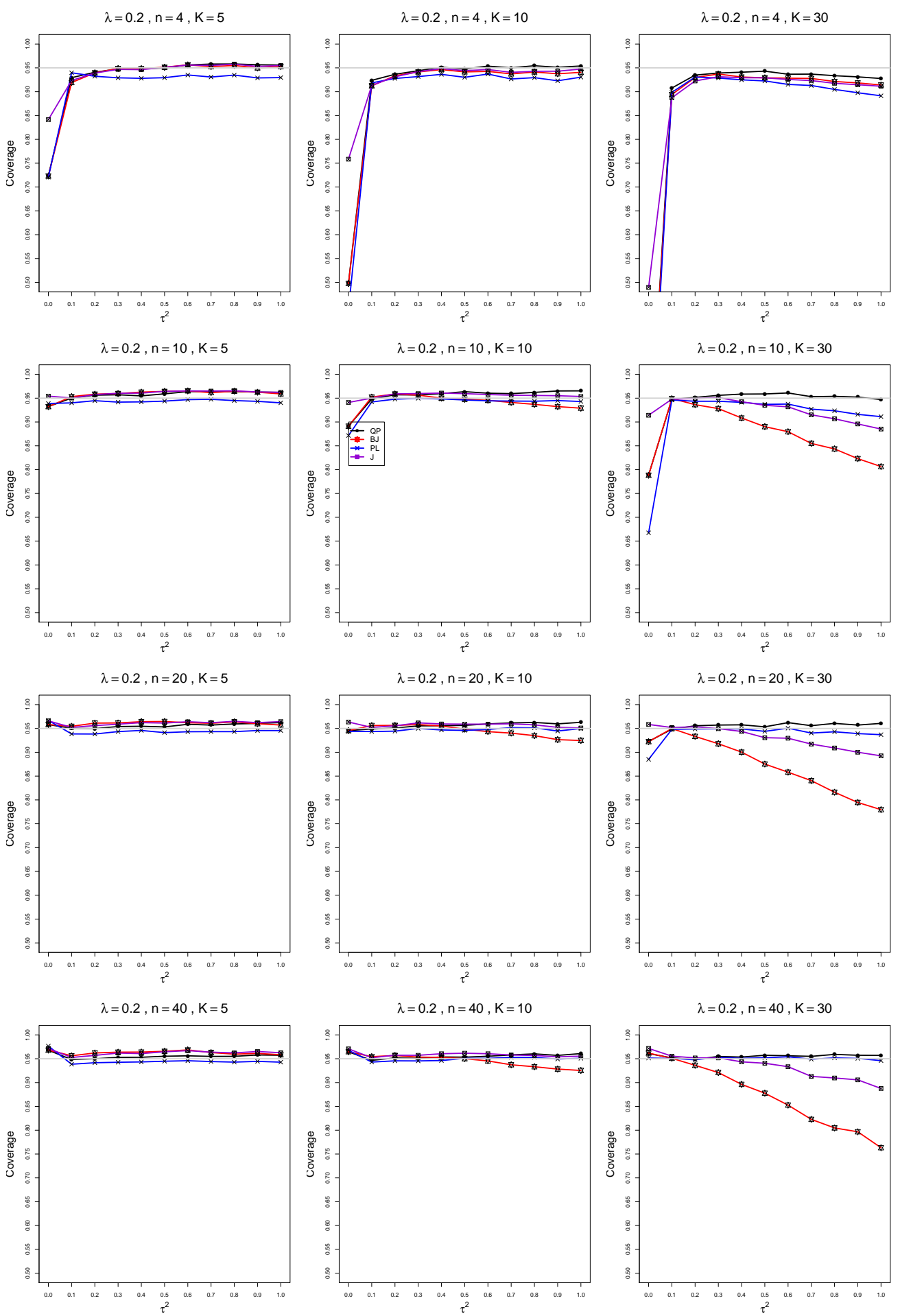

Figure A2.2.2: Coverage of 95\% confidence intervals for the between-studies variance $\tau^{2}$ when $\mu_{C}=4, \lambda=0.2, n=4,10,20,40$, and $K=5,10,30$ 

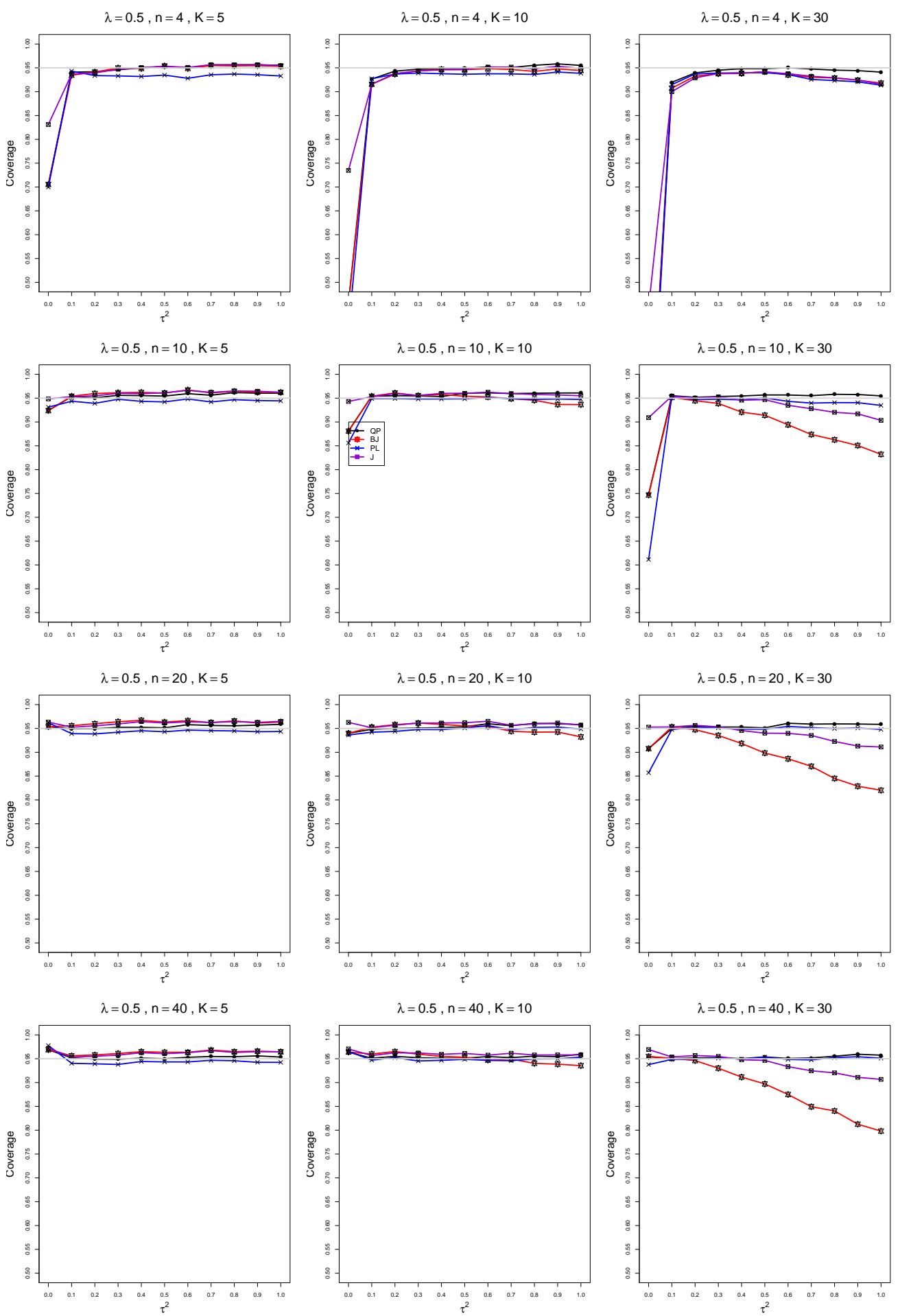

Figure A2.2.3: Coverage of 95\% confidence intervals for the between-studies variance $\tau^{2}$ when $\mu_{C}=4, \lambda=0.5, n=4,10,20,40$, and $K=5,10,30$ 

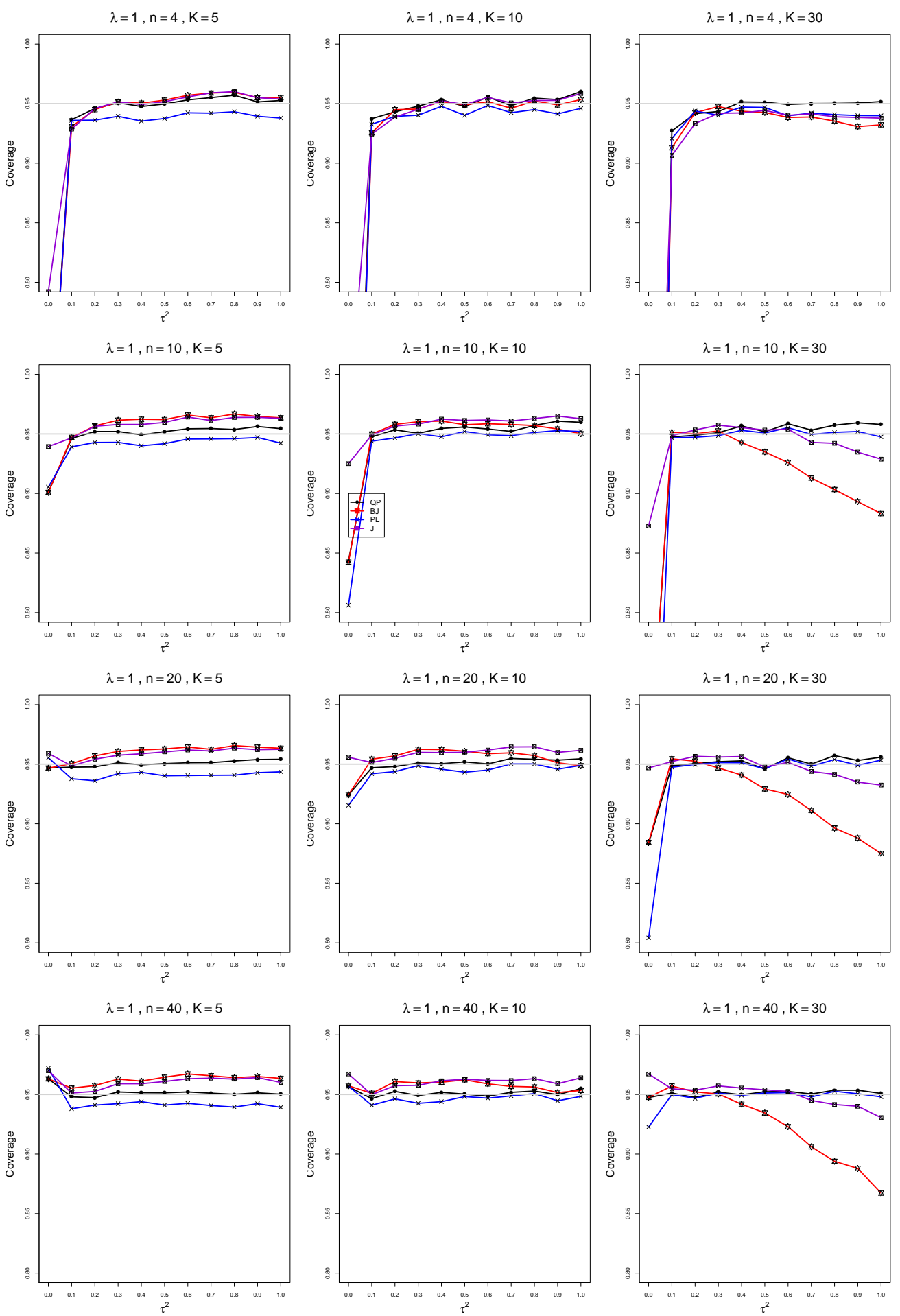

Figure A2.2.4: Coverage of 95\% confidence intervals for the between-studies variance $\tau^{2}$ when $\mu_{C}=4, \lambda=1, n=4,10,20,40$, and $K=5,10,30$ 

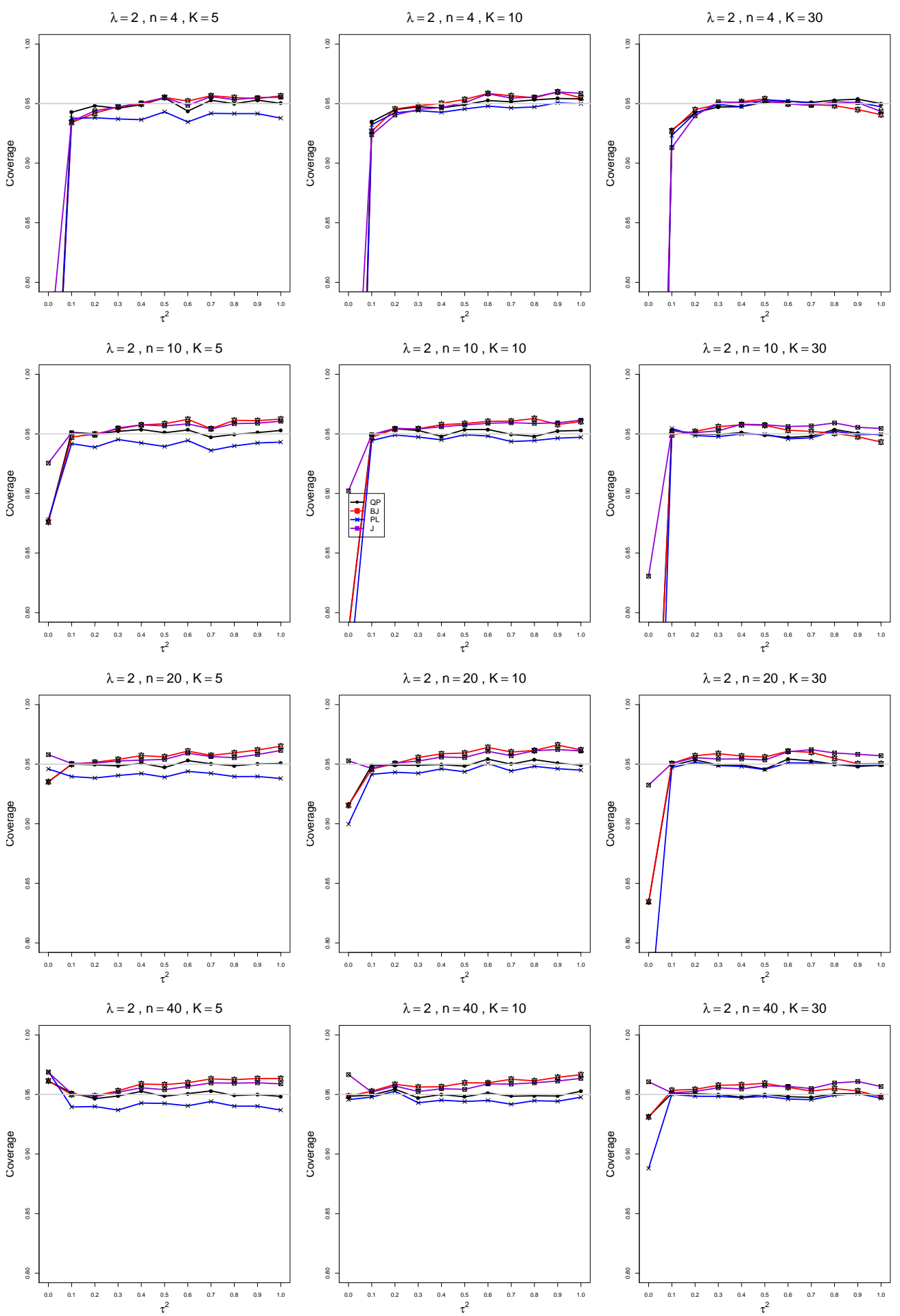

Figure A2.2.5: Coverage of 95\% confidence intervals for the between-studies variance $\tau^{2}$ when $\mu_{C}=4, \lambda=2, n=4,10,20,40$, and $K=5,10,30$ 


\section{A3. Normal model, bias-corrected estimator of}

$\lambda, \mu_{C}=4, n=4,10,20,40, K=5,10,30$

\section{A3.1 Bias of point estimators of $\tau^{2}$ when $\mu_{C}=4$}

Each figure corresponds to a value of $\lambda(=0,0.2,0.5,1,2)$, a set of values of $n(=4$, $10,20,40)$, and a set of values of $K(=5,10,30)$.

Each panel corresponds to a value of $n$ and a value of $K$ and has $\tau^{2}=0.0(0.1) 1.0$ on the horizontal axis.

The point estimators of $\tau^{2}$ are

- DL (DerSimonian-Laird)

- REML (restricted maximum likelihood)

- MP (Mandel-Paule)

- J (Jackson) 

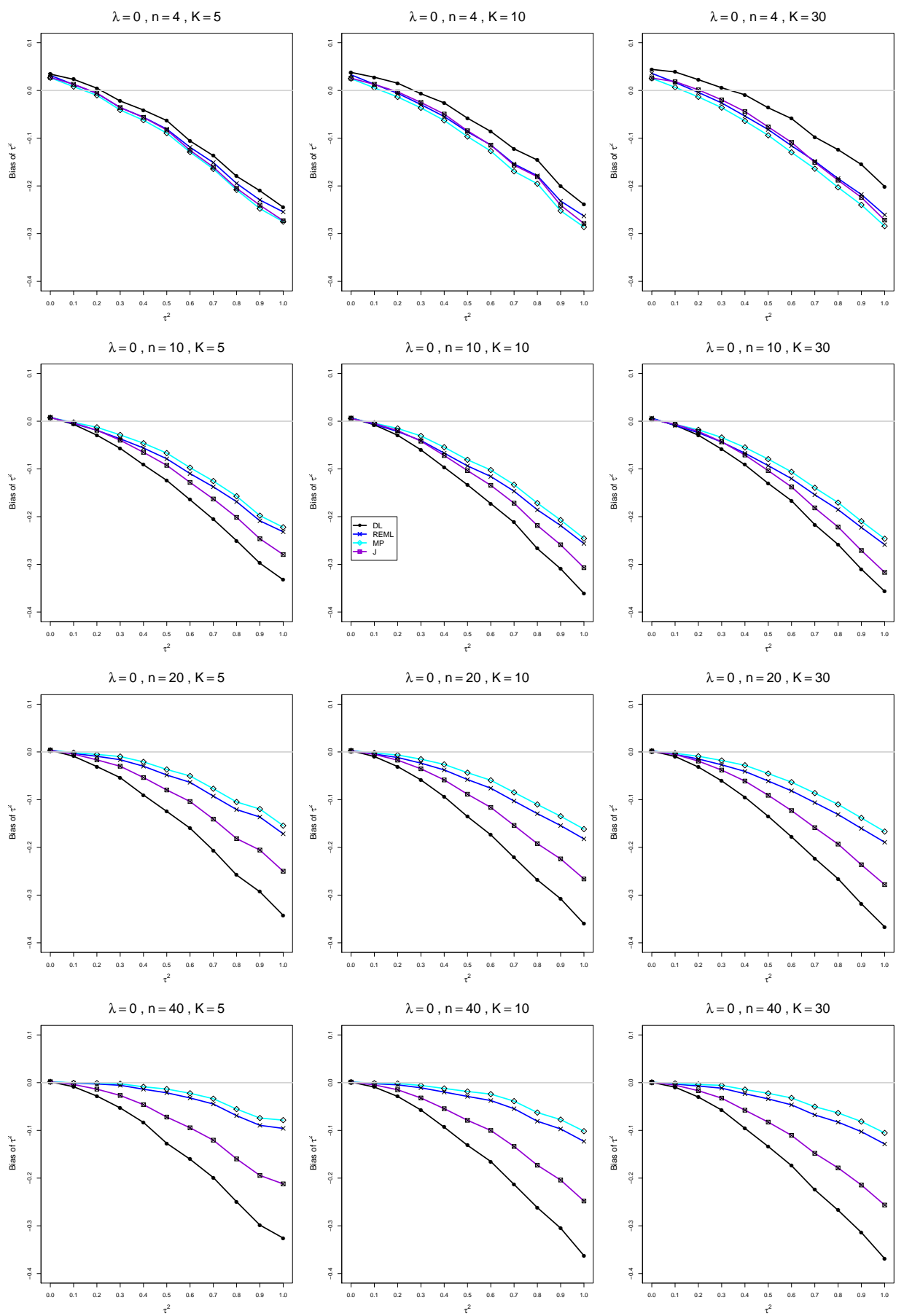

Figure A3.1.1: Bias of estimators of the between-studies variance $\tau^{2}$ when $\mu_{C}=4, \lambda=0$, $n=4,10,20,40$, and $K=5,10,30$ 

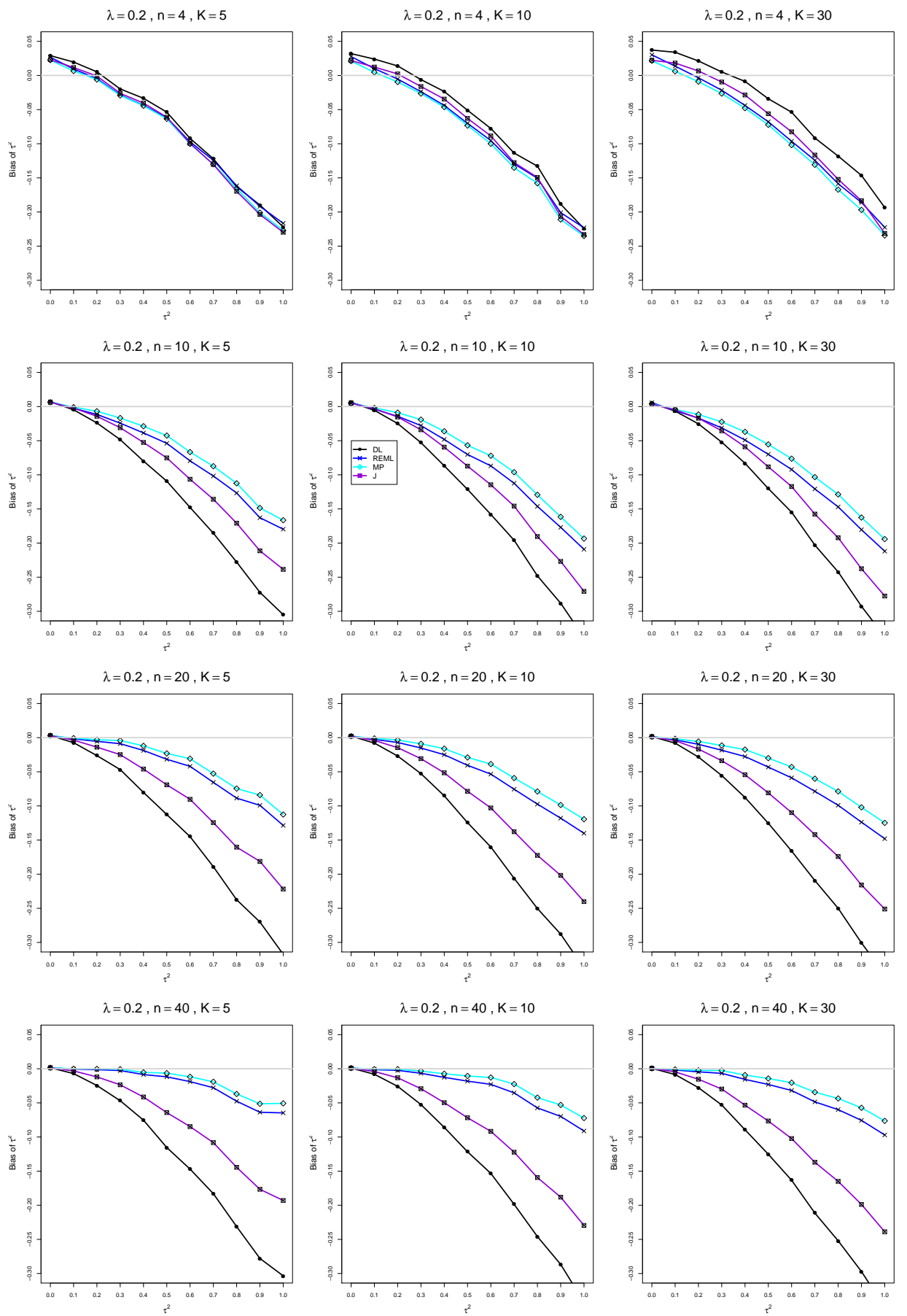

Figure A3.1.2: Bias of estimators of the between-studies variance $\tau^{2}$ when $\mu_{C}=1, \lambda=$ $0.2, n=4,10,20,40$, and $K=5,10,30$ 

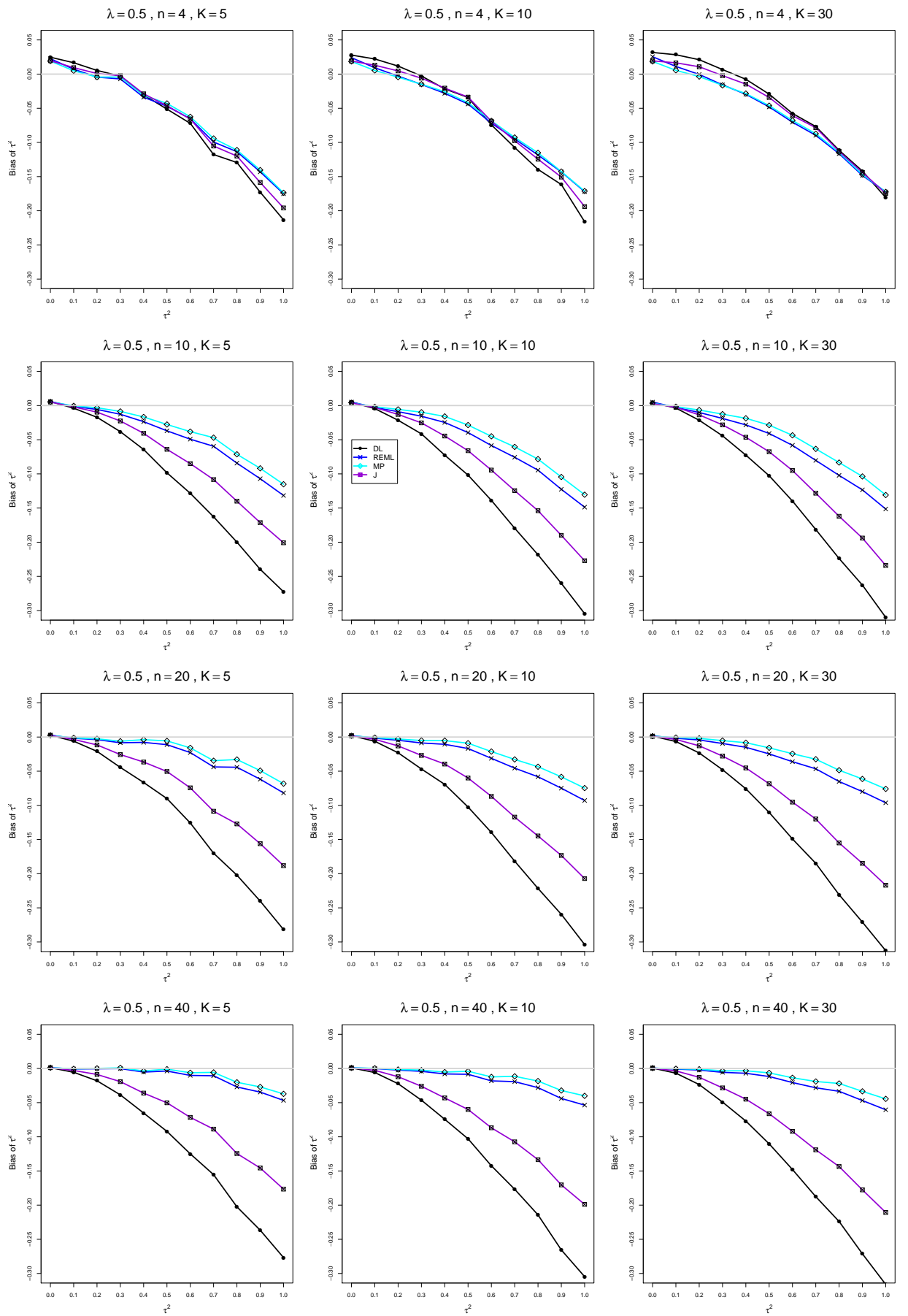

Figure A3.1.3: Bias of estimators of the between-studies variance $\tau^{2}$ when $\mu_{C}=4, \lambda=$ $0.5, n=4,10,20,40$, and $K=5,10,30$ 

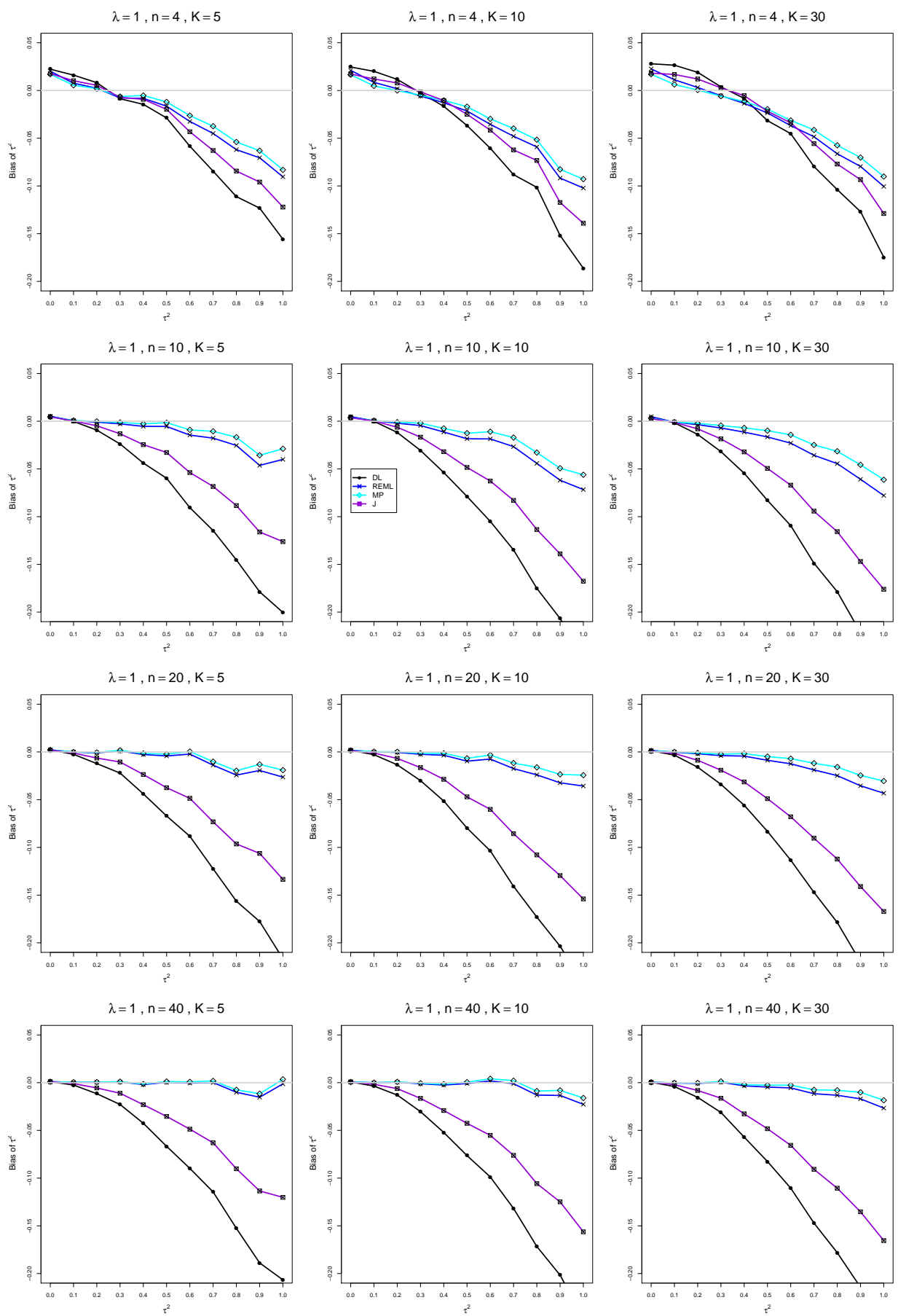

Figure A3.1.4: Bias of estimators of the between-studies variance $\tau^{2}$ when $\mu_{C}=4, \lambda=1$, $n=4,10,20,40$, and $K=5,10,30$ 

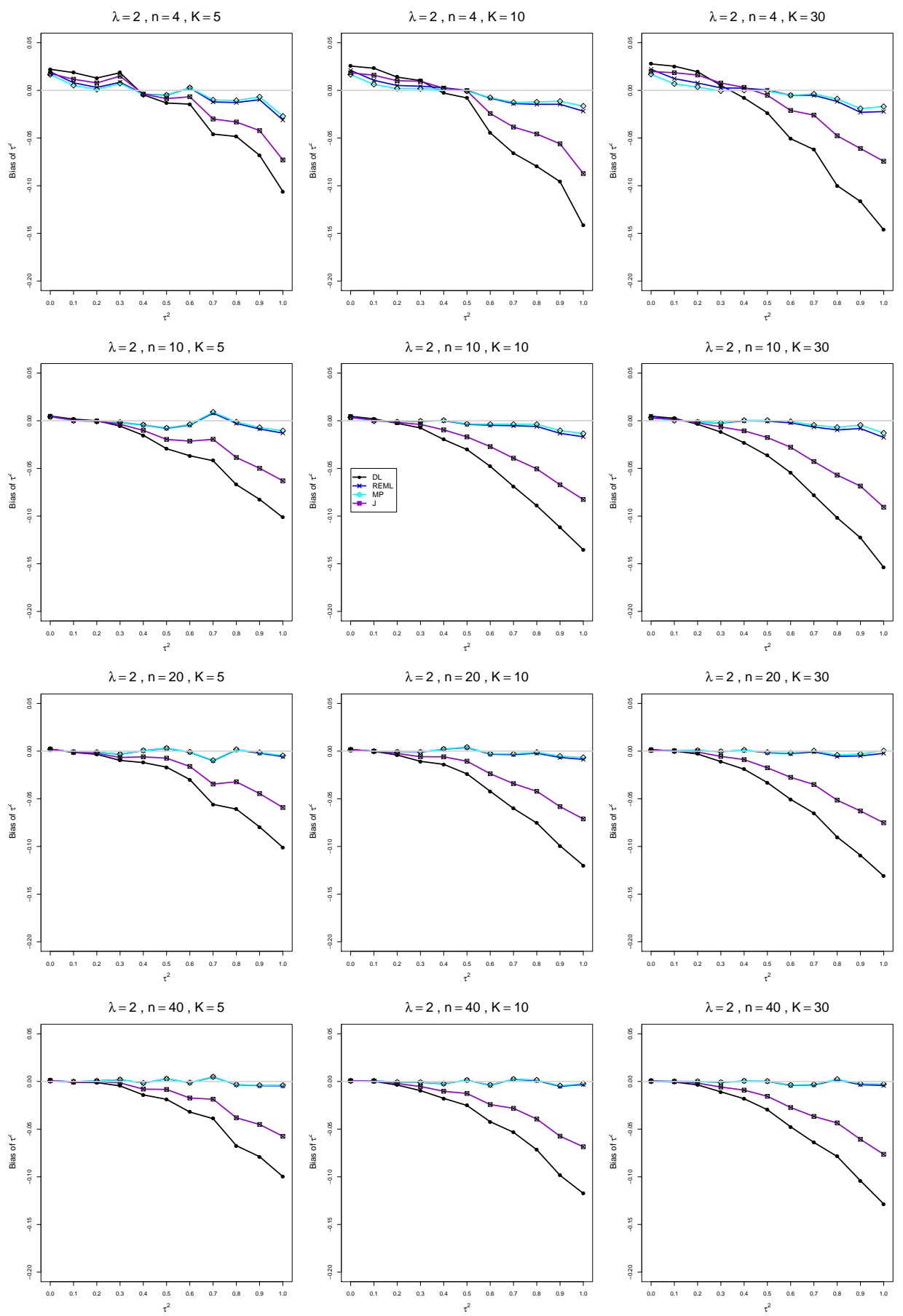

Figure A3.1.5: Bias of estimators of the between-studies variance $\tau^{2}$ when $\mu_{C}=4, \lambda=2$, $n=4,10,20,40$, and $K=5,10,30$ 


\section{A3.2 Coverage of interval estimators of $\tau^{2}$ when $\mu_{C}=4$}

Each figure corresponds to a value of $\lambda(=0,0.2,0.5,1,2)$, a set of values of $n(=4$, $10,20,40)$, and a set of values of $K(=5,10,30)$.

Each panel corresponds to a value of $n$ and a value of $K$ and has $\tau^{2}=0.0(0.1) 1.0$ on the horizontal axis.

The interval estimators of $\tau^{2}$ are

- QP (Q-profile confidence interval)

- BJ (Biggerstaff and Jackson interval)

- PL (Profile likelihood interval)

- J (Jackson's interval) 

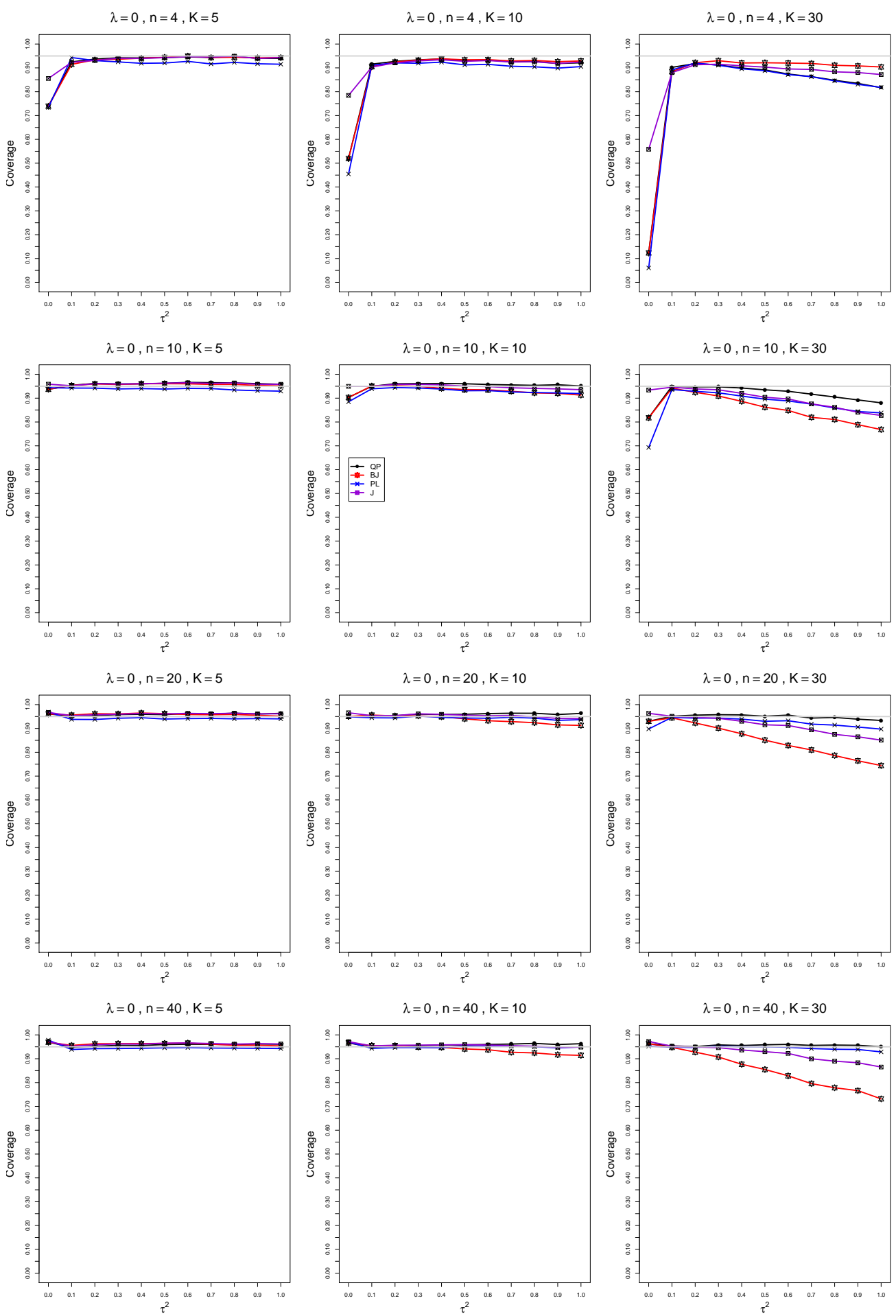

Figure A3.2.1: Coverage of 95\% confidence intervals for the between-studies variance $\tau^{2}$ when $\mu_{C}=4, \lambda=0, n=4,10,20,40$, and $K=5,10,30$ 

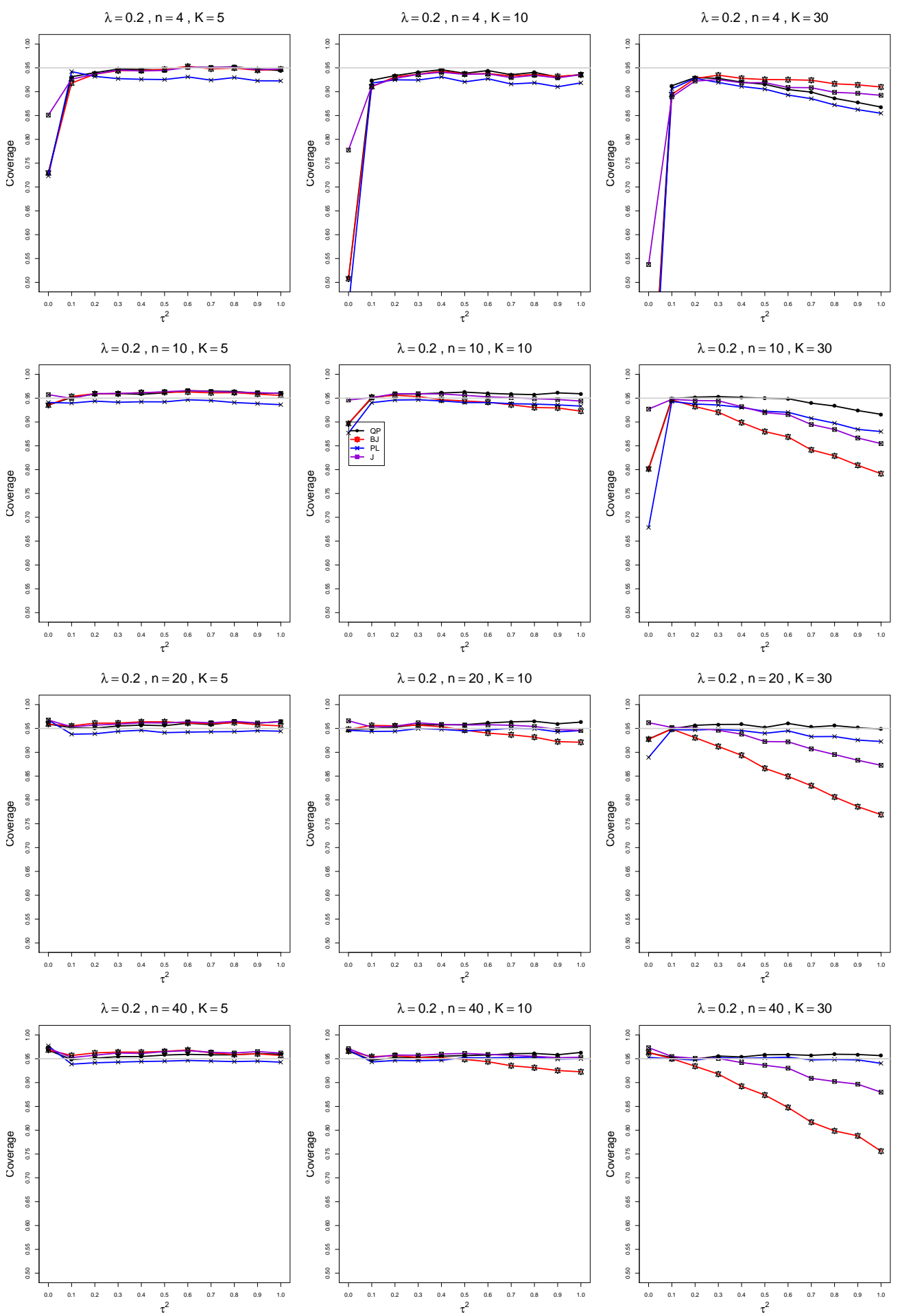

Figure A3.2.2: Coverage of 95\% confidence intervals for the between-studies variance $\tau^{2}$ when $\mu_{C}=4, \lambda=0.2, n=4,10,20,40$, and $K=5,10,30$ 

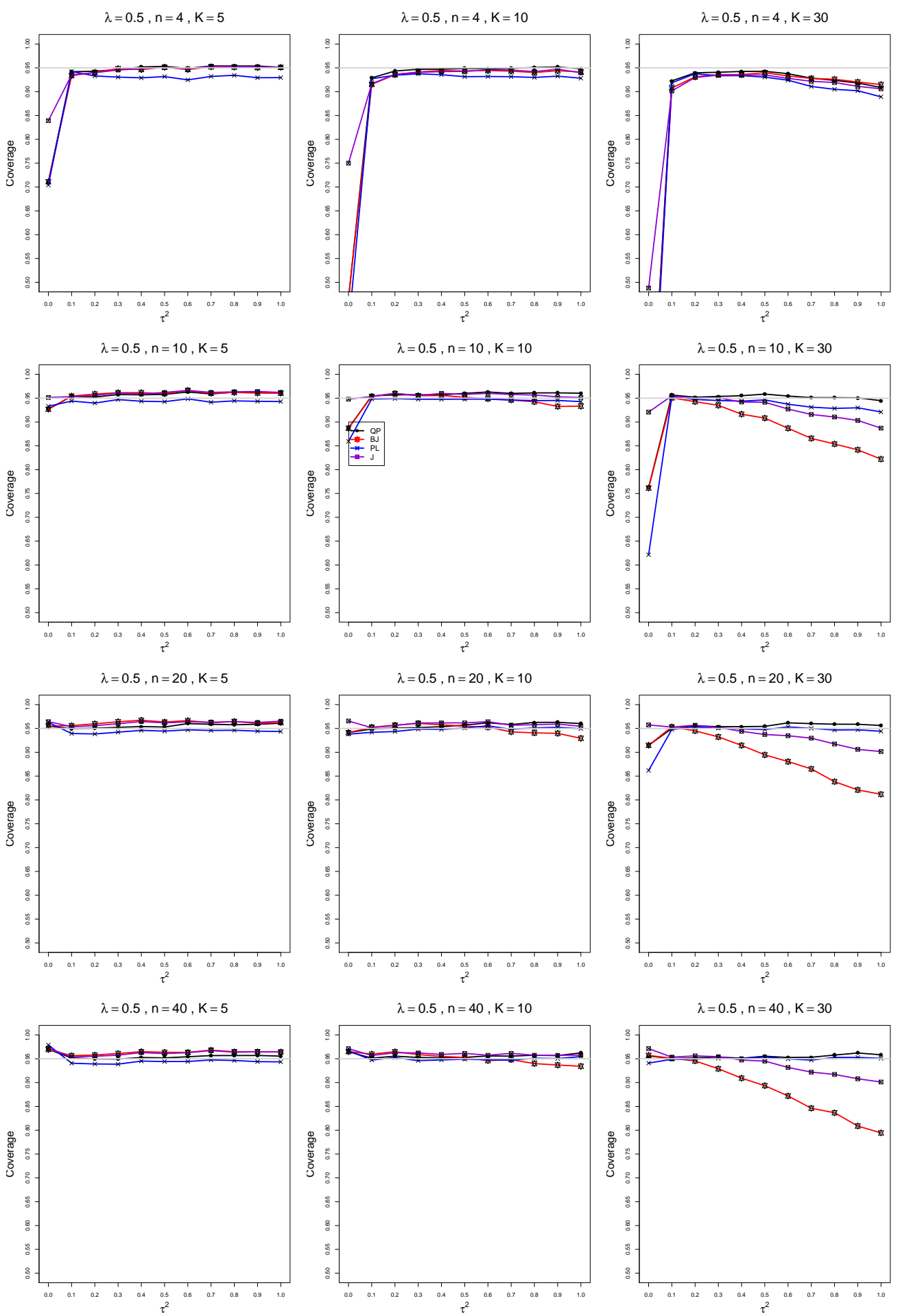

Figure A3.2.3: Coverage of 95\% confidence intervals for the between-studies variance $\tau^{2}$ when $\mu_{C}=4, \lambda=0.5, n=4,10,20,40$, and $K=5,10,30$ 

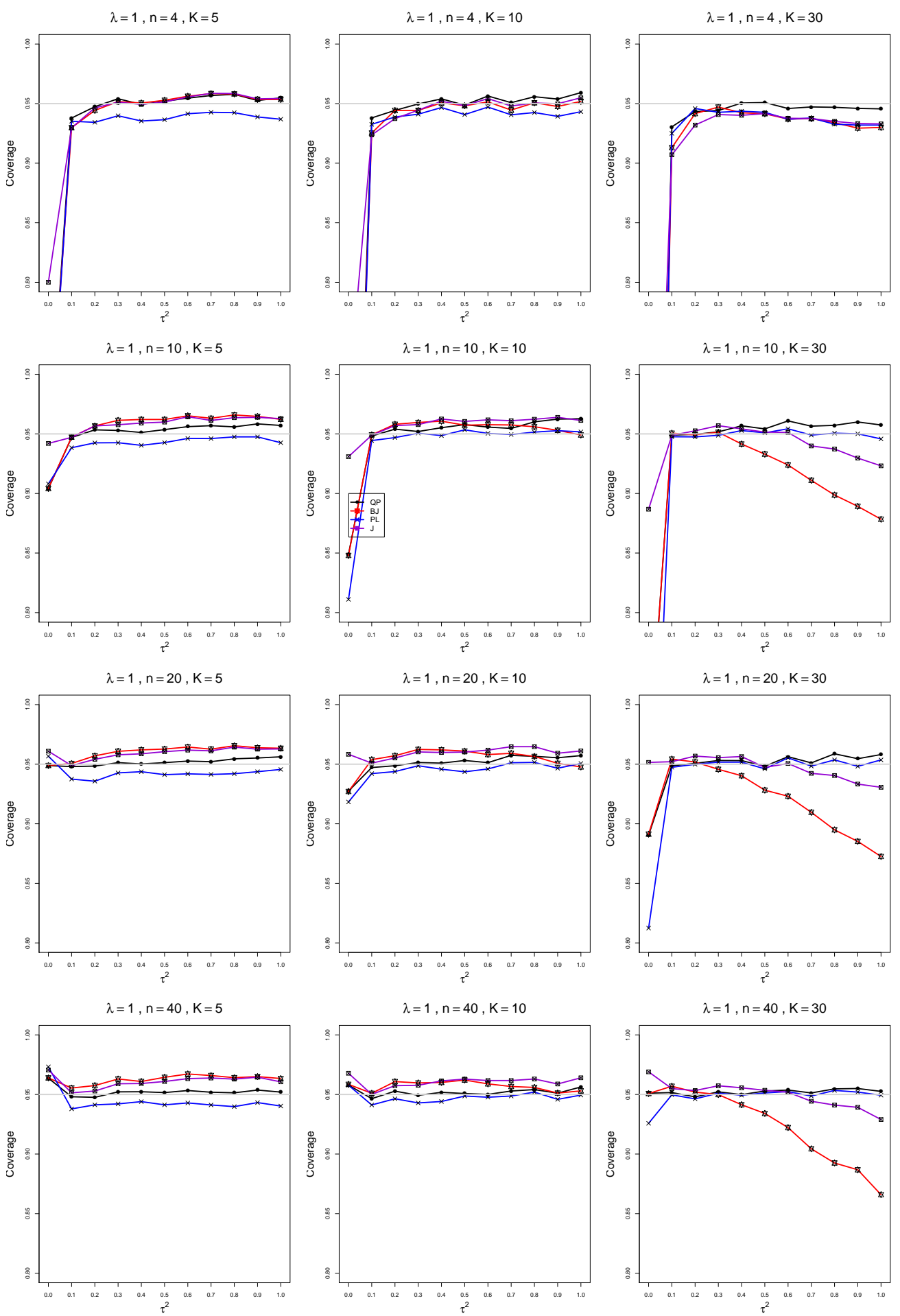

Figure A3.2.4: Coverage of 95\% confidence intervals for the between-studies variance $\tau^{2}$ when $\mu_{C}=4, \lambda=1, n=4,10,20,40$, and $K=5,10,30$ 

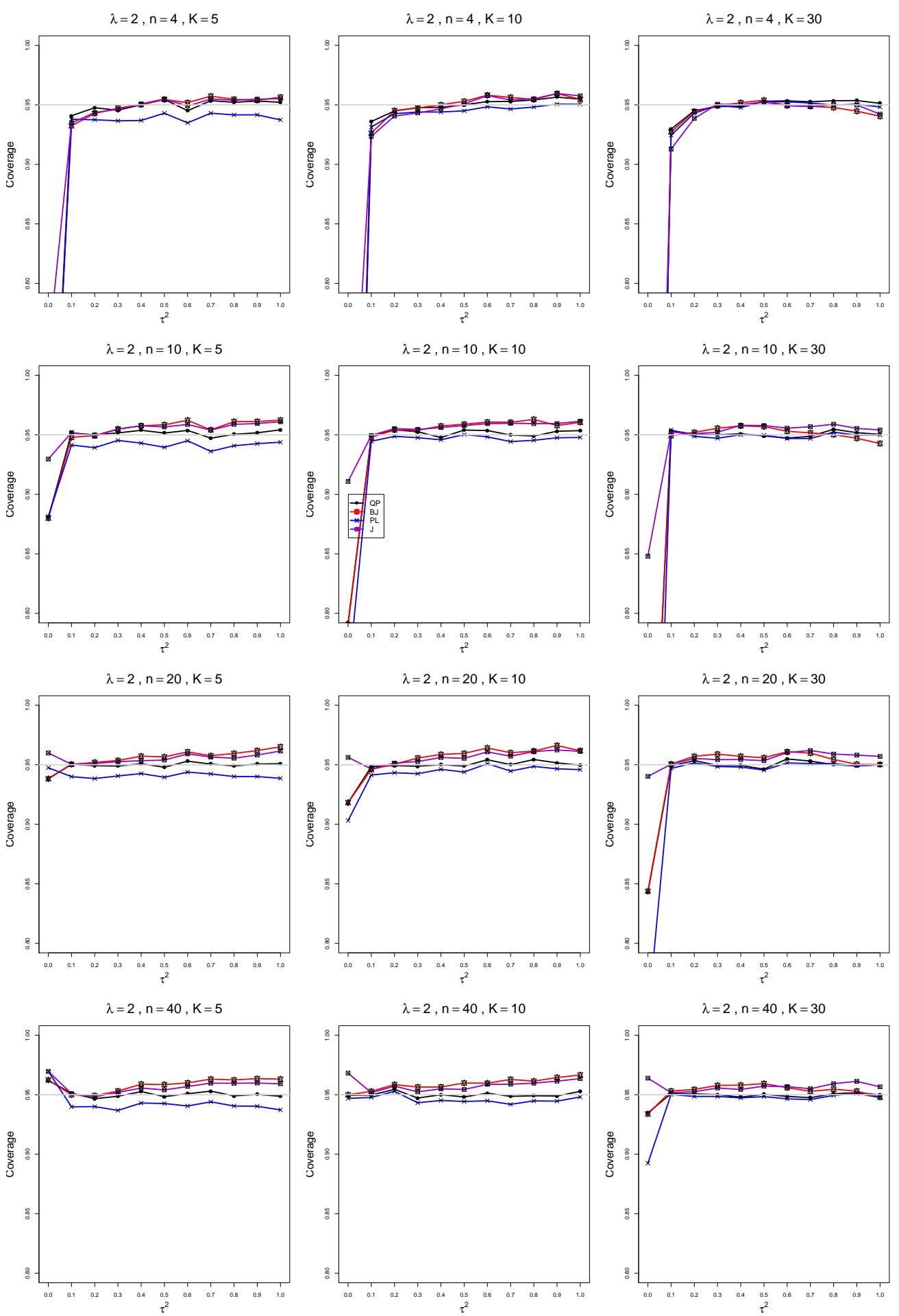

Figure A3.2.5: Coverage of 95\% confidence intervals for the between-studies variance $\tau^{2}$ when $\mu_{C}=4, \lambda=2, n=4,10,20,40$, and $K=5,10,30$ 
A4: Normal model, $\mu_{C}=1, n=4,10,20,40, K=$ $50,100,125$

\section{A4.1 Bias of point estimators of $\tau^{2}$ when $\mu_{C}=1$}

Each figure corresponds to a value of $\lambda(=0,0.2,0.5,1,2)$, a set of values of $n(=4$, $10,20,40)$, and a set of values of $K(=50,100,125)$.

Each panel corresponds to a value of $n$ and a value of $K$ and has $\tau^{2}=0.0(0.1) 1.0$ on the horizontal axis.

The point estimators of $\tau^{2}$ are

- DL (DerSimonian-Laird)

- REML (restricted maximum likelihood)

- MP (Mandel-Paule)

- J (Jackson) 

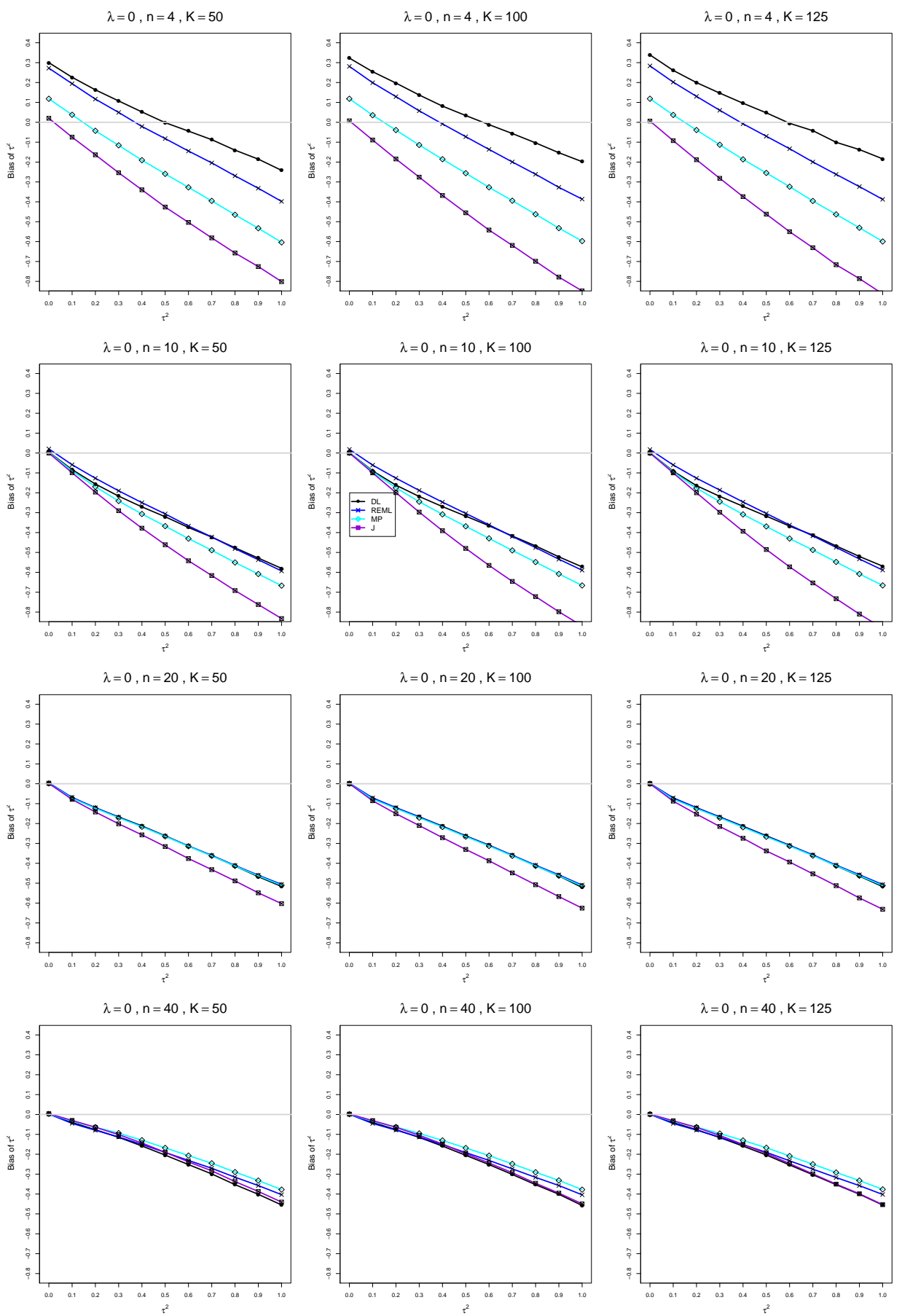

Figure A4.1.1: Bias of estimators of the between-studies variance $\tau^{2}$ when $\mu_{C}=1, \lambda=0$, $n=4,10,20,40$, and $K=50,100,125$ 

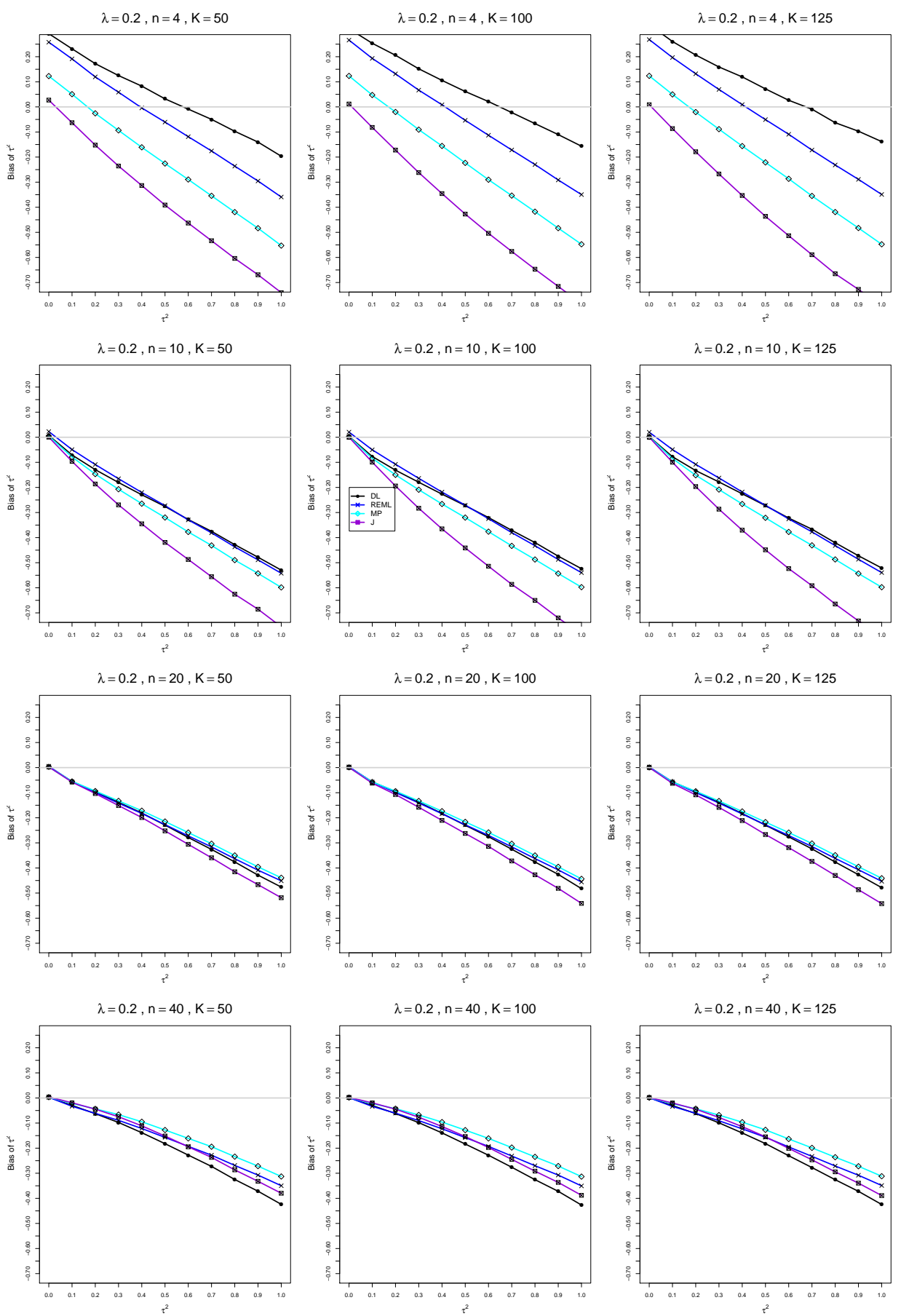

Figure A4.1.2: Bias of estimators of the between-studies variance $\tau^{2}$ when $\mu_{C}=1, \lambda=$ $0.2, n=4,10,20,40$, and $K=5,10,30$ 

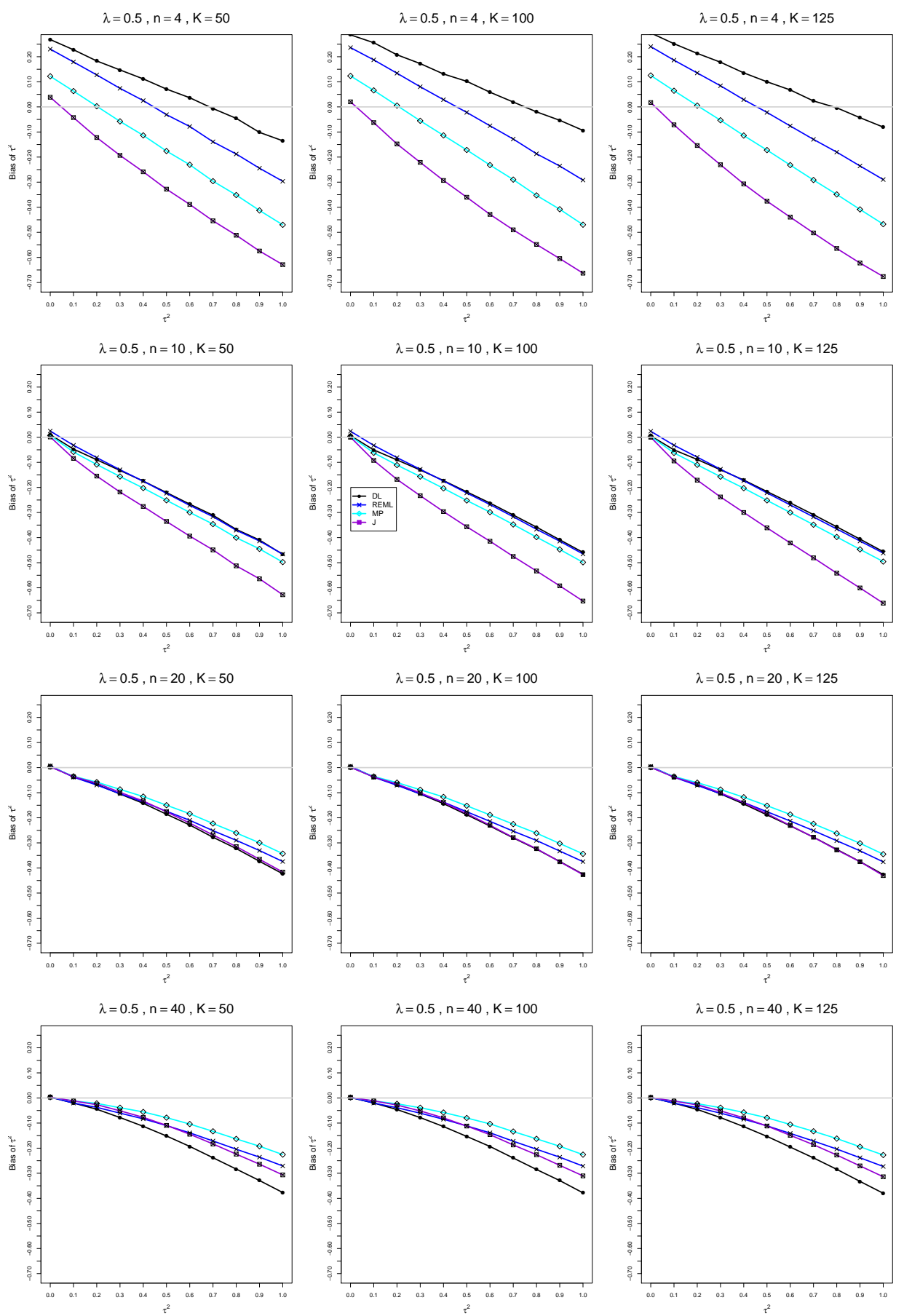

Figure A4.1.3: Bias of estimators of the between-studies variance $\tau^{2}$ when $\mu_{C}=1, \lambda=$ $0.5, n=4,10,20,40$, and $K=5,10,30$ 

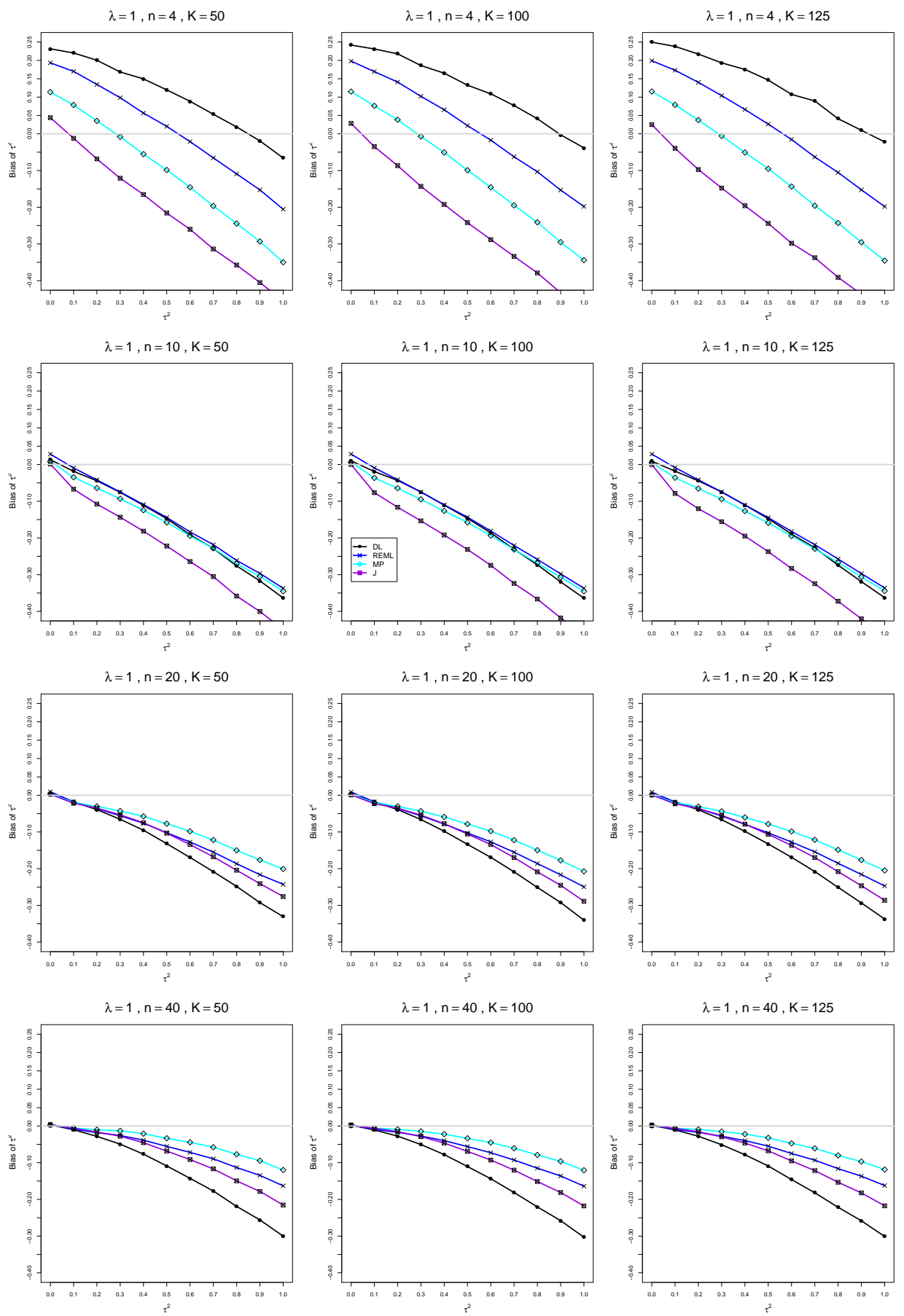

Figure A4.1.4: Bias of estimators of the between-studies variance $\tau^{2}$ when $\mu_{C}=1, \lambda=1$, $n=4,10,20,40$, and $K=5,10,30$ 

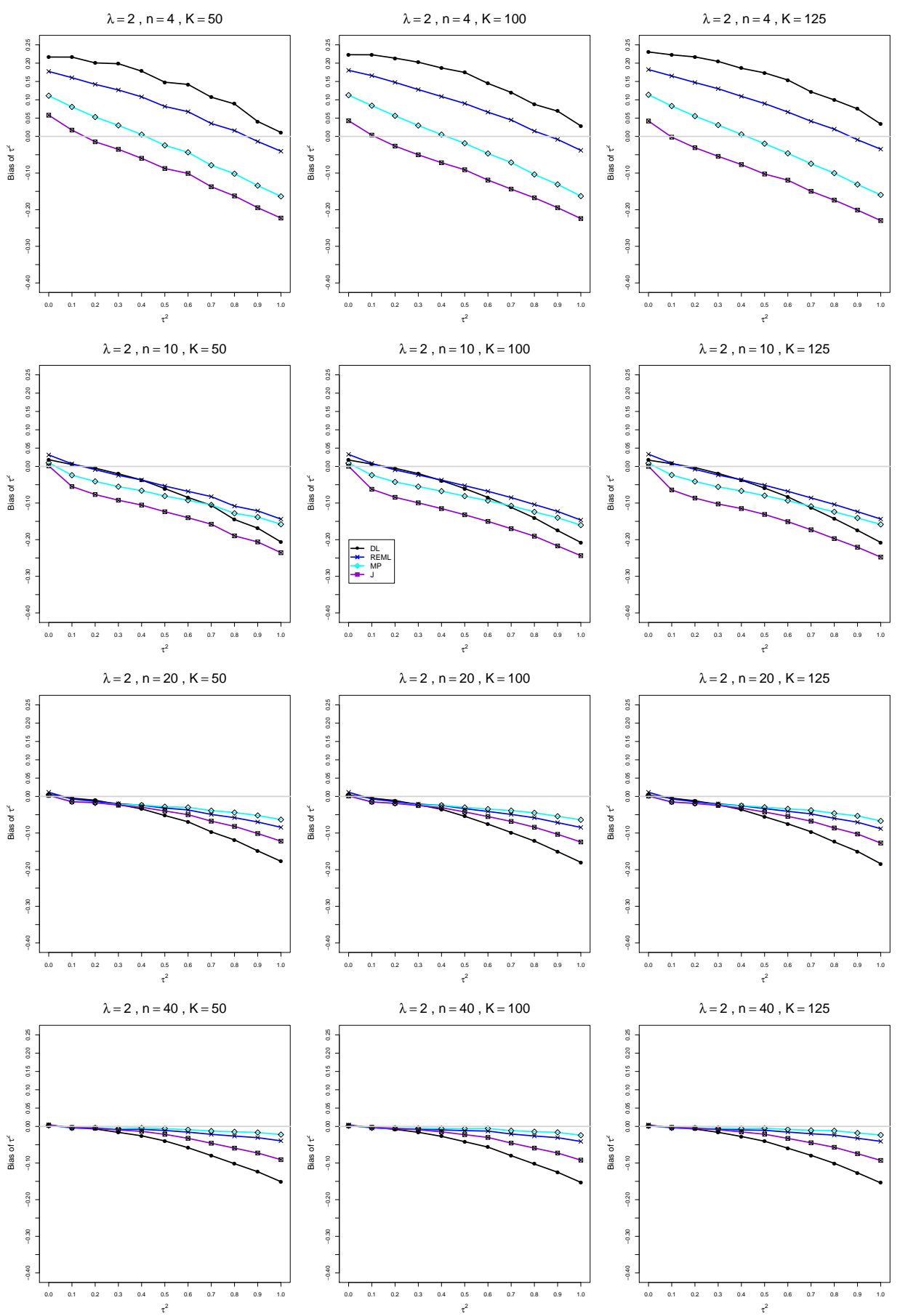

Figure A4.1.5: Bias of estimators of the between-studies variance $\tau^{2}$ when $\mu_{C}=1, \lambda=2$, $n=4,10,20,40$, and $K=5,10,30$ 


\section{A4.2 Coverage of interval estimators of $\tau^{2}$ when $\mu_{C}=1$}

Each figure corresponds to a value of $\lambda(=0,0.2,0.5,1,2)$, a set of values of $n(=4$, $10,20,40)$, and a set of values of $K(=50,100,125)$.

Each panel corresponds to a value of $n$ and a value of $K$ and has $\tau^{2}=0.0(0.1) 1.0$ on the horizontal axis.

The interval estimators of $\tau^{2}$ are

- QP (Q-profile confidence interval)

- BJ (Biggerstaff and Jackson interval)

- PL (Profile likelihood interval)

- J (Jackson's interval) 

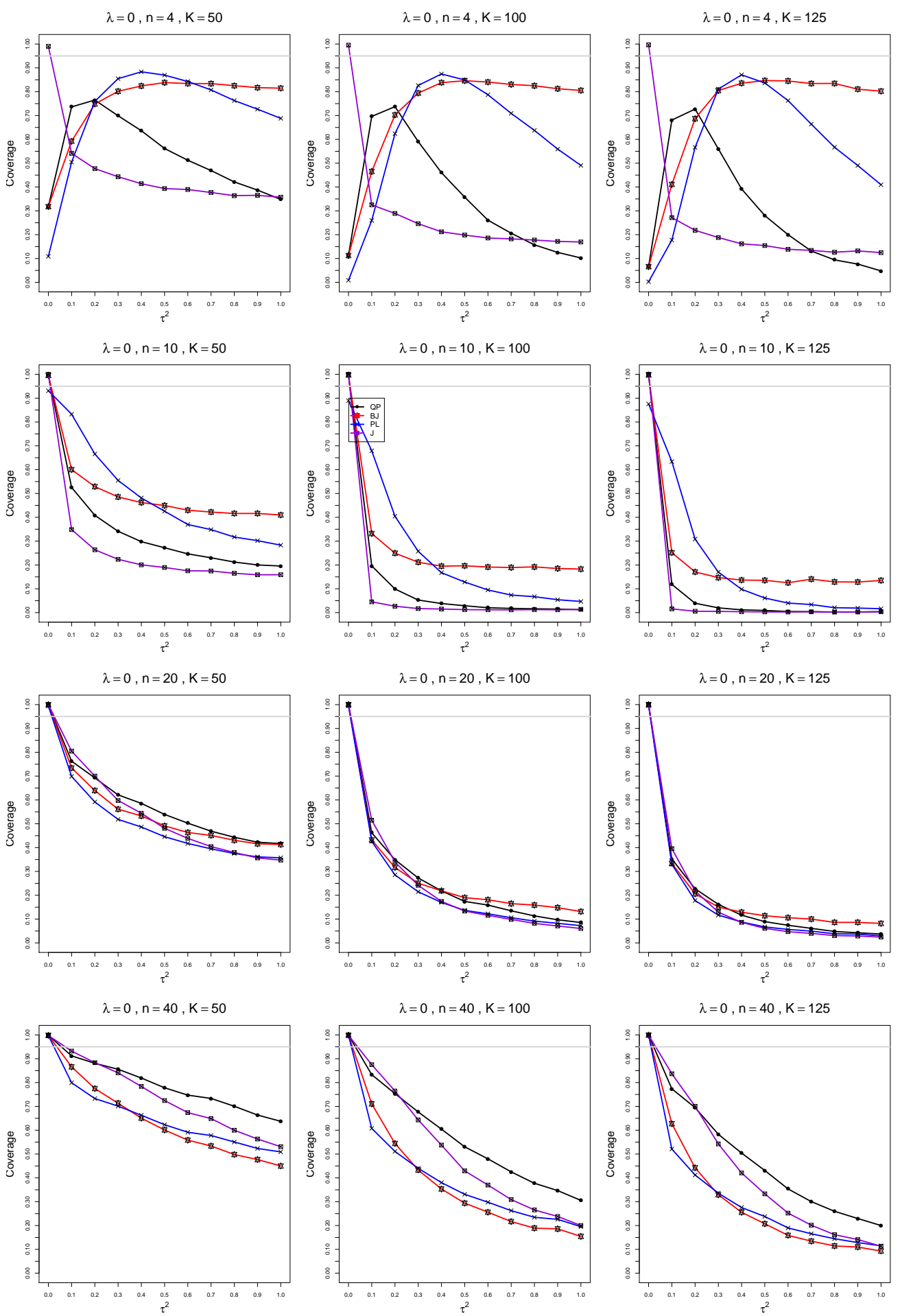

Figure A4.2.1: Coverage of 95\% confidence intervals for the between-studies variance $\tau^{2}$ when $\mu_{C}=1, \lambda=0, n=4,10,20,40$, and $K=50,100,125$ 

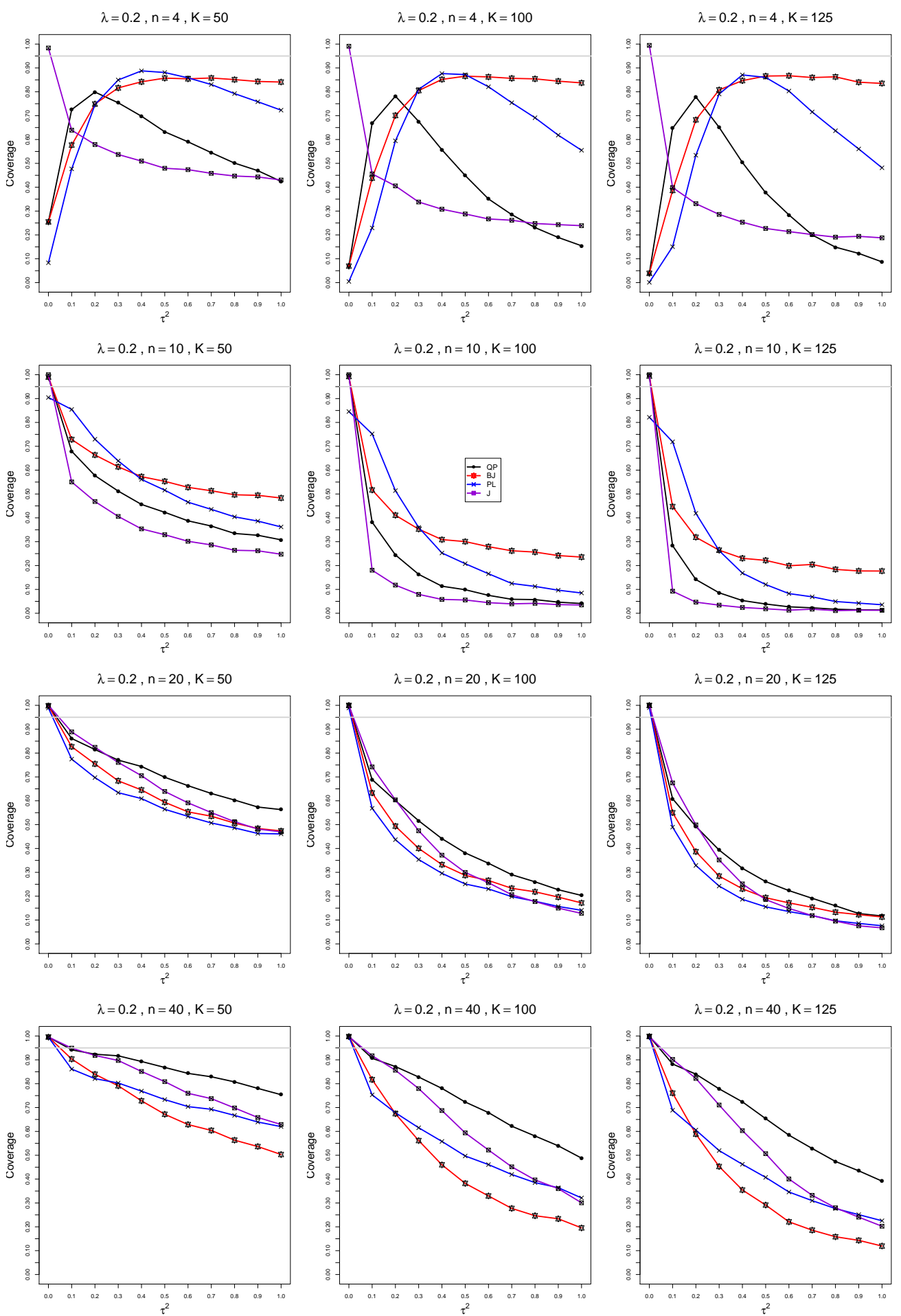

Figure A4.2.2: Coverage of 95\% confidence intervals for the between-studies variance $\tau^{2}$ when $\mu_{C}=1, \lambda=0.2, n=4,10,20,40$, and $K=50,100,125$ 

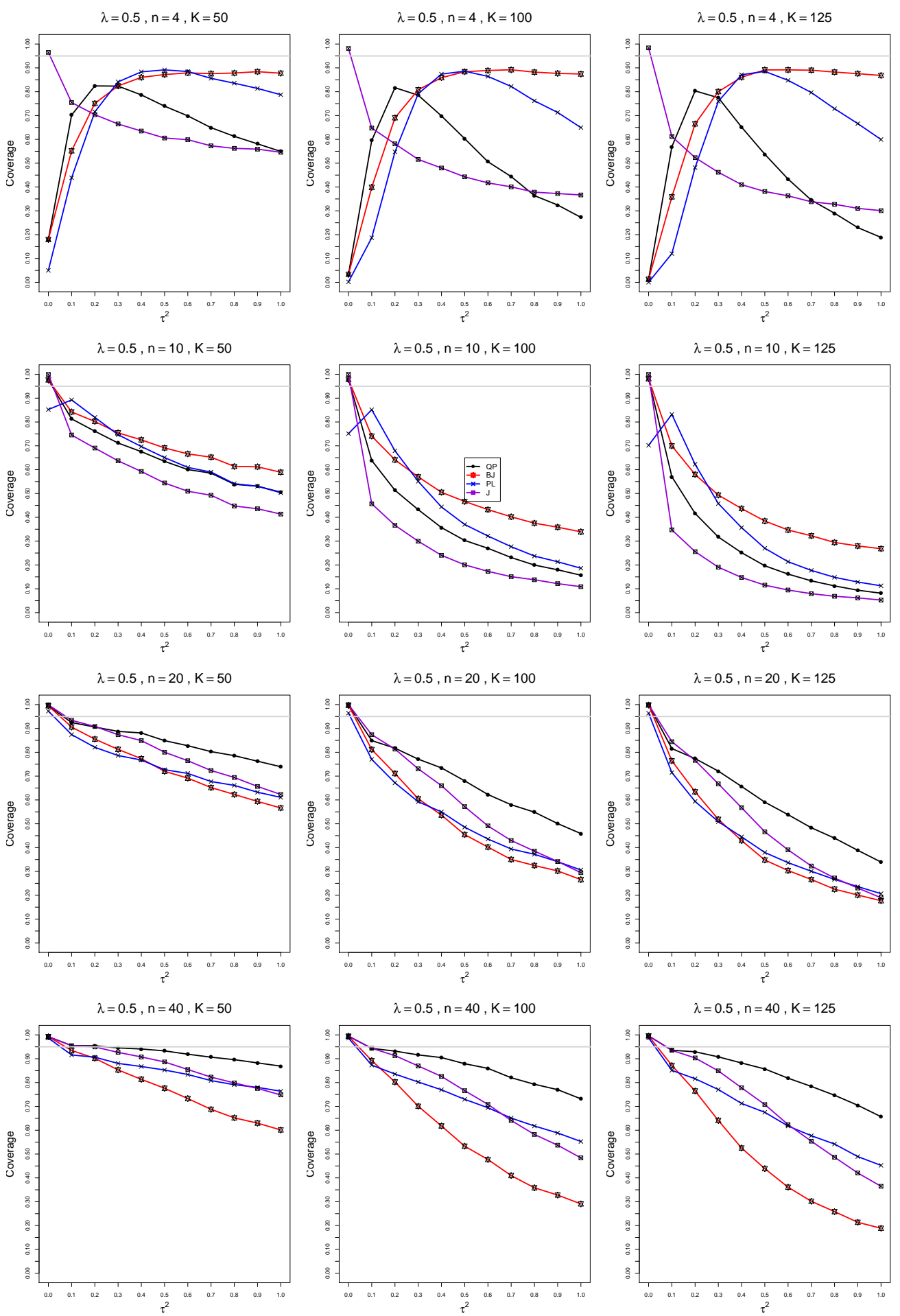

Figure A4.2.3: Coverage of 95\% confidence intervals for the between-studies variance $\tau^{2}$ when $\mu_{C}=1, \lambda=0.5, n=4,10,20,40$, and $K=50,100,125$ 

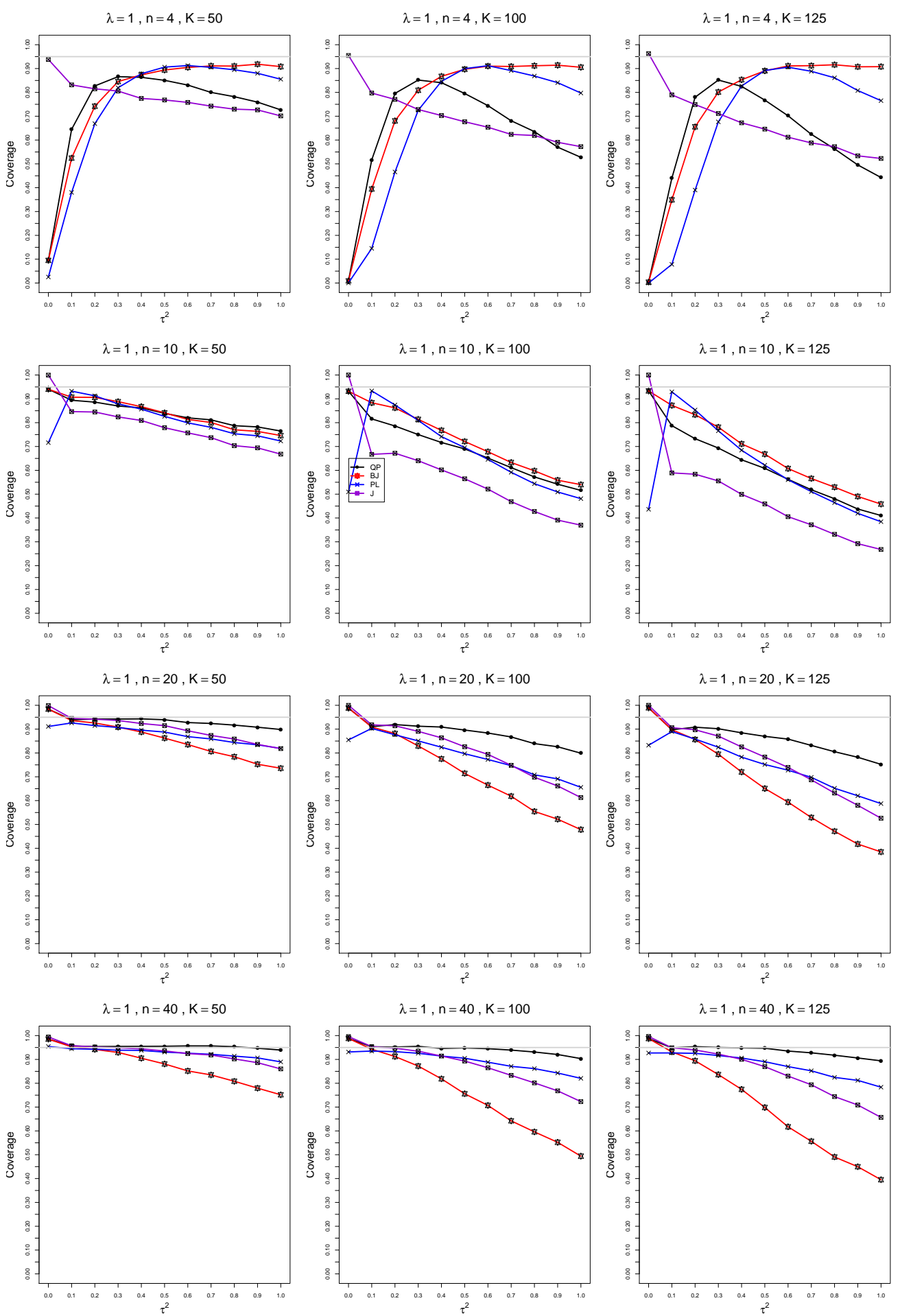

Figure A4.2.4: Coverage of 95\% confidence intervals for the between-studies variance $\tau^{2}$ when $\mu_{C}=1, \lambda=1, n=4,10,20,40$, and $K=50,100,125$ 

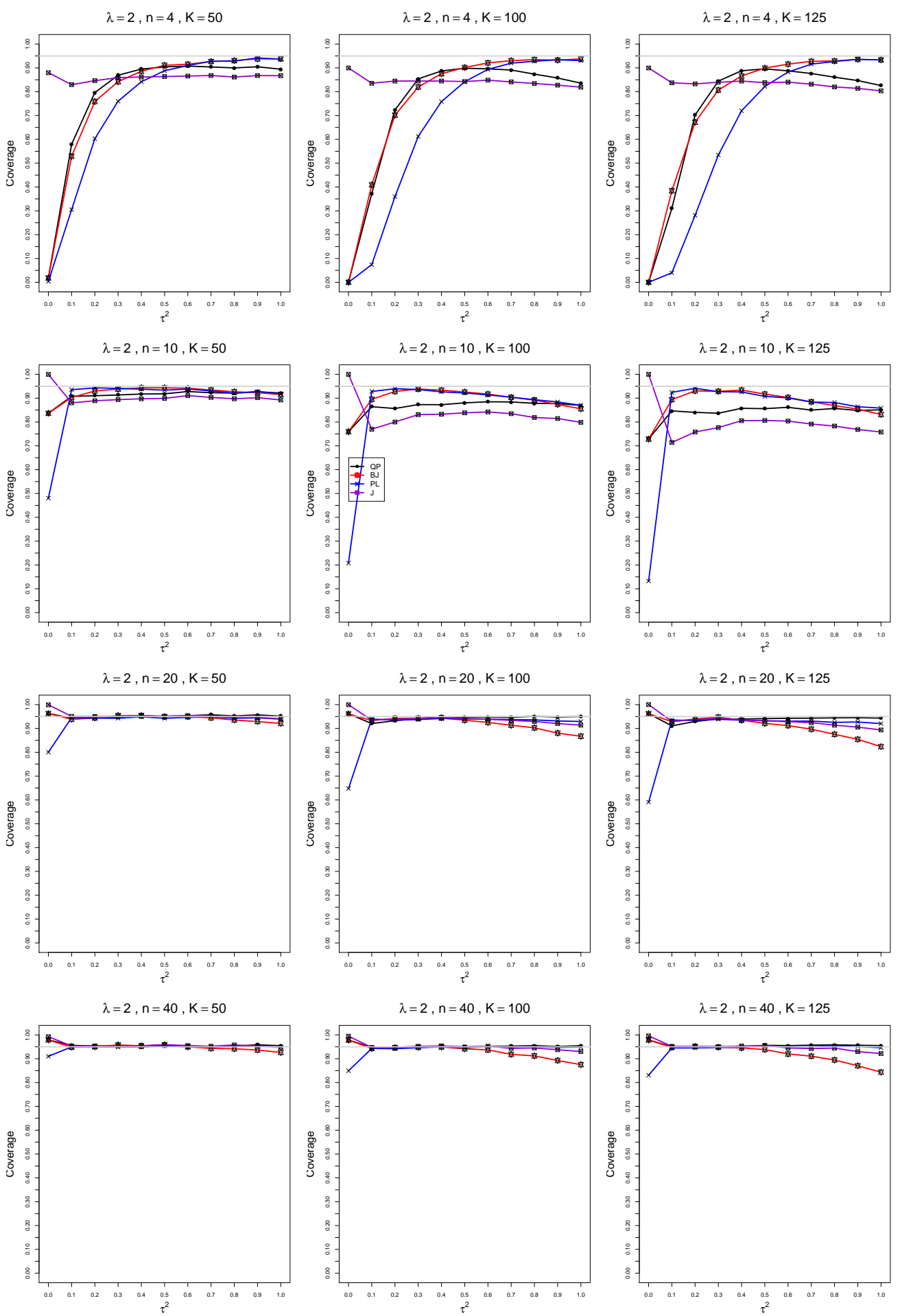

Figure A4.2.5: Coverage of 95\% confidence intervals for the between-studies variance $\tau^{2}$ when $\mu_{C}=1, \lambda=2, n=4,10,20,40$, and $K=50,100,125$ 
A5. Normal model, $\mu_{C}=4, n=4,10,20,40, K=$ $50,100,125$

\section{A5.1 Bias of point estimators of $\tau^{2}$ when $\mu_{C}=4$}

Each figure corresponds to a value of $\lambda(=0,0.2,0.5,1,2)$, a set of values of $n(=4$, $10,20,40)$, and a set of values of $K(=50,100,125)$.

Each panel corresponds to a value of $n$ and a value of $K$ and has $\tau^{2}=0.0(0.1) 1.0$ on the horizontal axis.

The point estimators of $\tau^{2}$ are

- DL (DerSimonian-Laird)

- REML (restricted maximum likelihood)

- MP (Mandel-Paule)

- J (Jackson) 

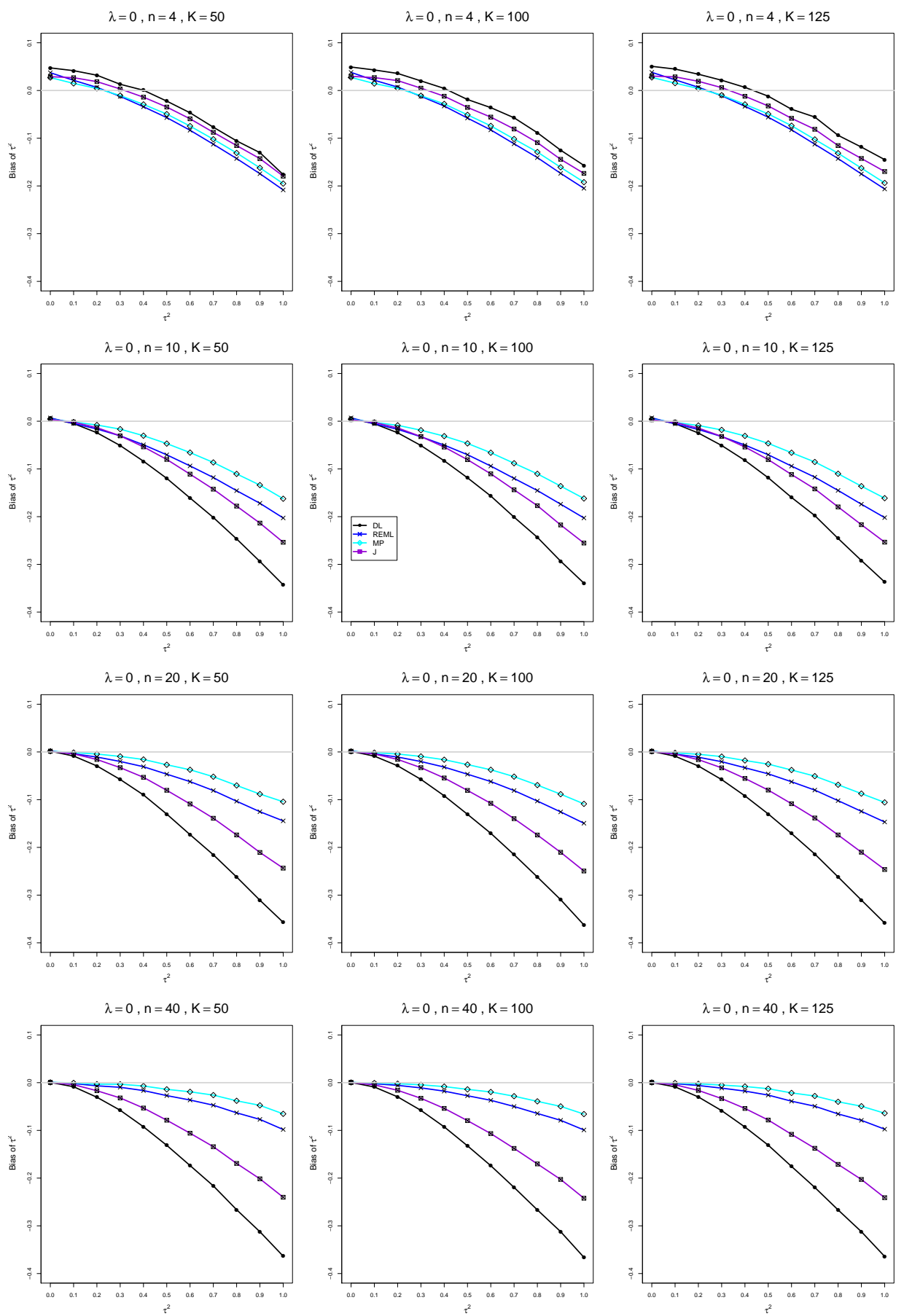

Figure A5.1.1: Bias of estimators of the between-studies variance $\tau^{2}$ when $\mu_{C}=4, \lambda=0$, $n=4,10,20,40$, and $K=50,100,125$ 

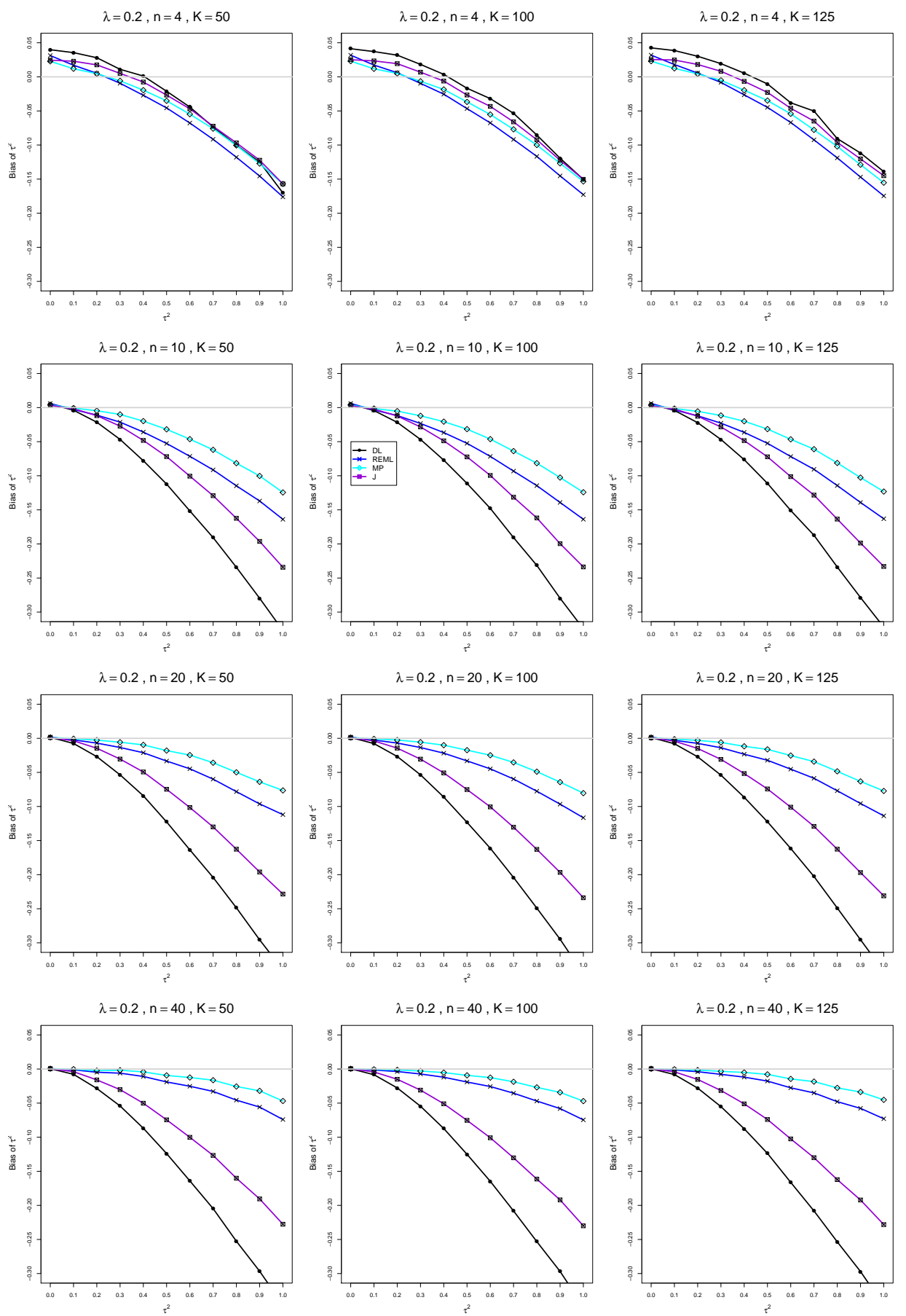

Figure A5.1.2: Bias of estimators of the between-studies variance $\tau^{2}$ when $\mu_{C}=4, \lambda=$ $0.2, n=4,10,20,40$, and $K=50,100,125$ 

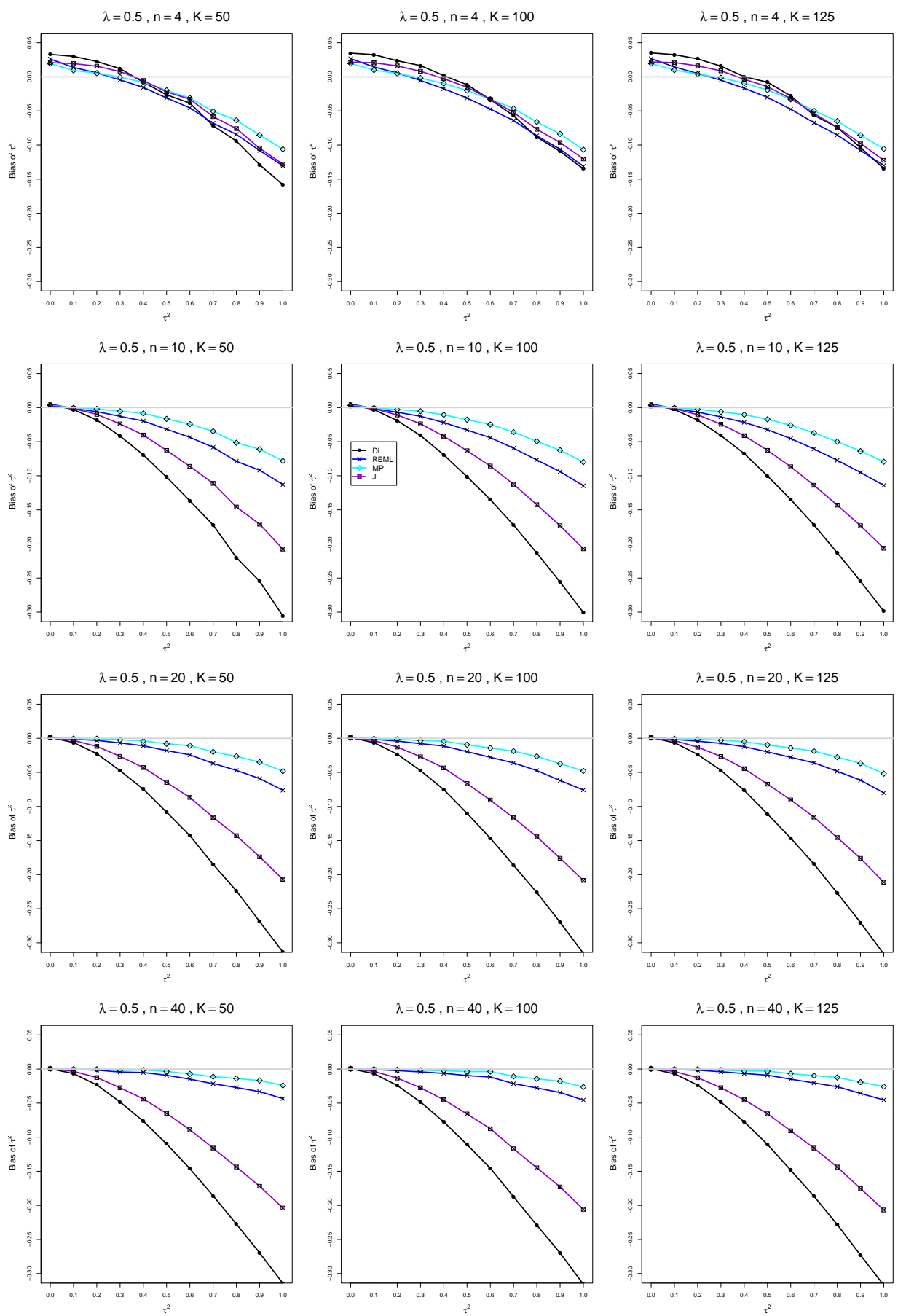

Figure A5.1.3: Bias of estimators of the between-studies variance $\tau^{2}$ when $\mu_{C}=4, \lambda=$ $0.5, n=4,10,20,40$, and $K=50,100,125$ 

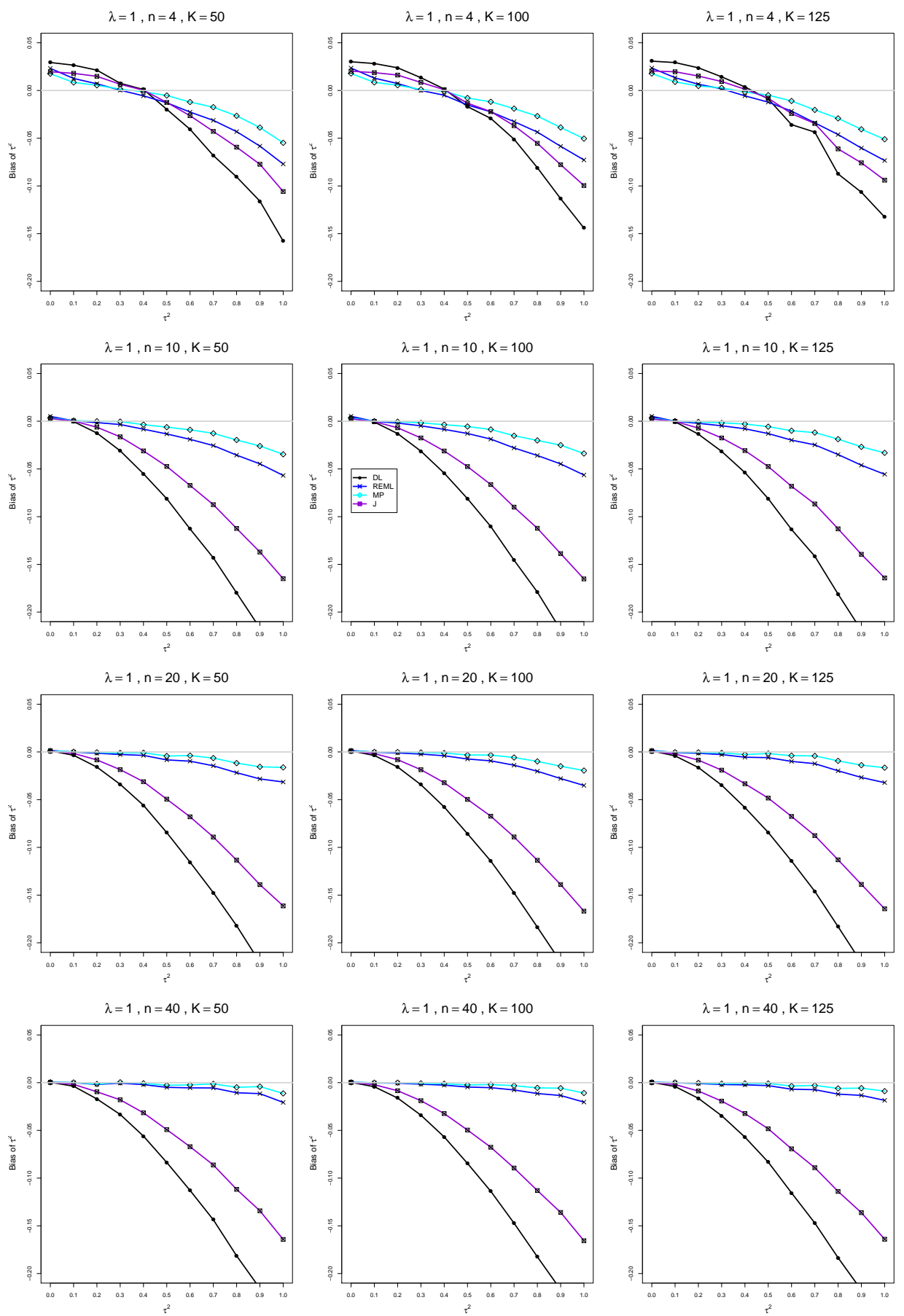

Figure A5.1.4: Bias of estimators of the between-studies variance $\tau^{2}$ when $\mu_{C}=4, \lambda=1$, $n=4,10,20,40$, and $K=50,100,125$ 

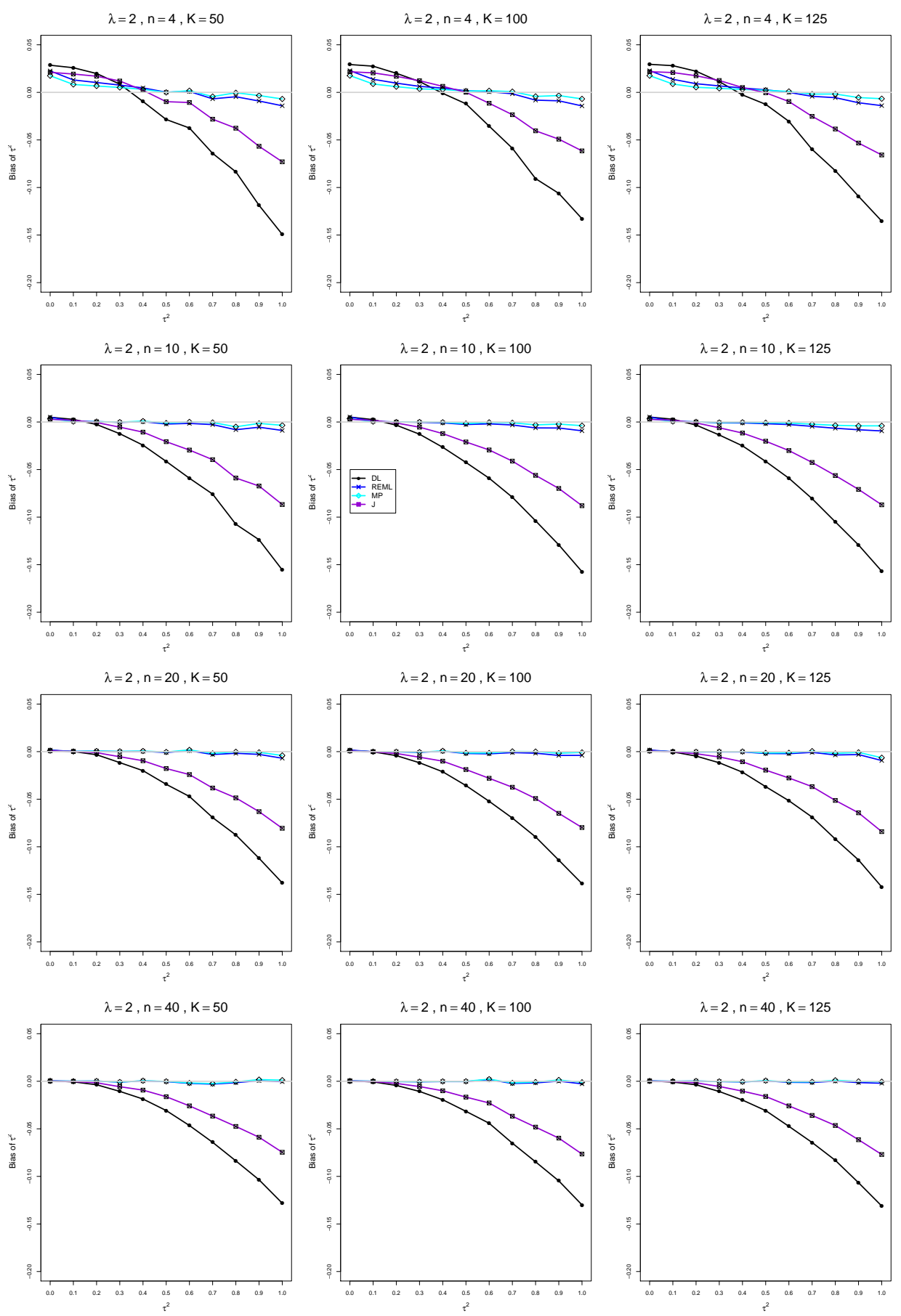

Figure A5.1.5: Bias of estimators of the between-studies variance $\tau^{2}$ when $\mu_{C}=4, \lambda=2$, $n=4,10,20,40$, and $K=50,100,125$ 


\section{A5.2 Coverage of interval estimators of $\tau^{2}$ when $\mu_{C}=4$}

Each figure corresponds to a value of $\lambda(=0,0.2,0.5,1,2)$, a set of values of $n(=4$, $10,20,40)$, and a set of values of $K(=50,100,125)$.

Each panel corresponds to a value of $n$ and a value of $K$ and has $\tau^{2}=0.0(0.1) 1.0$ on the horizontal axis.

The interval estimators of $\tau^{2}$ are

- QP (Q-profile confidence interval)

- BJ (Biggerstaff and Jackson interval)

- PL (Profile likelihood interval)

- J (Jackson's interval) 

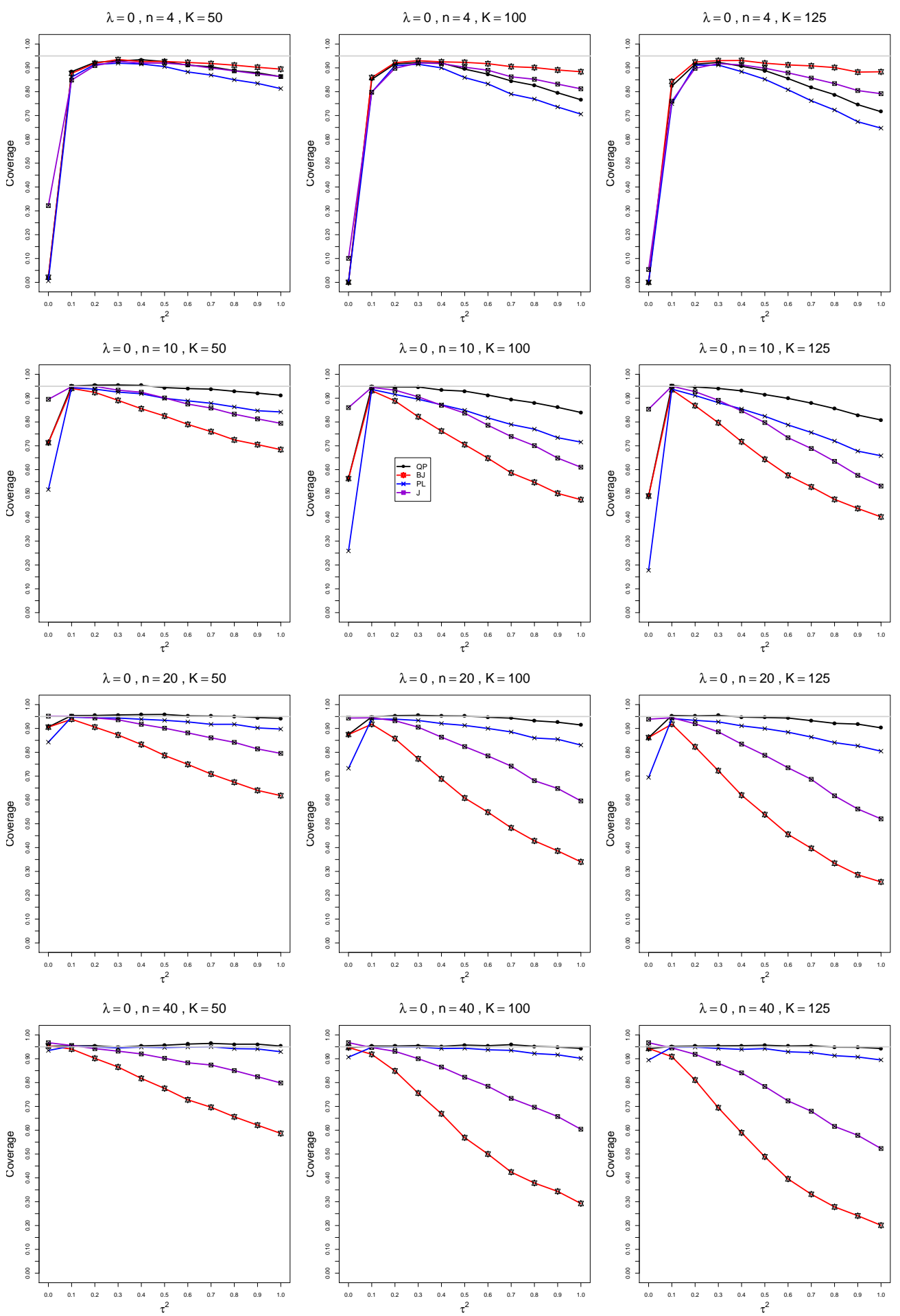

Figure A5.2.1: Coverage of 95\% confidence intervals for the between-studies variance $\tau^{2}$ when $\mu_{C}=4, \lambda=0, n=4,10,20,40$, and $K=50,100,125$ 

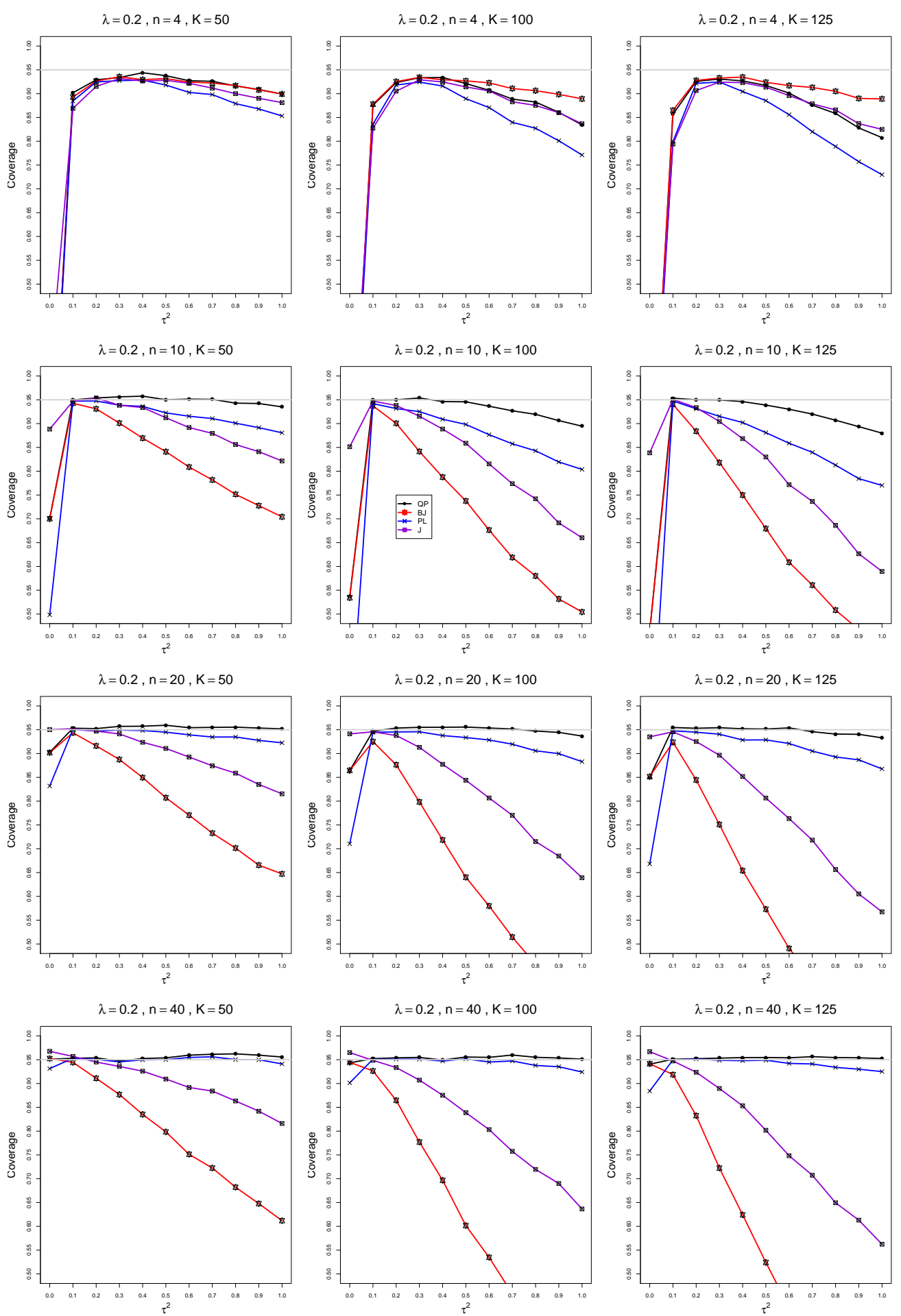

Figure A5.2.2: Coverage of 95\% confidence intervals for the between-studies variance $\tau^{2}$ when $\mu_{C}=4, \lambda=0.2, n=4,10,20,40$, and $K=50,100,125$ 

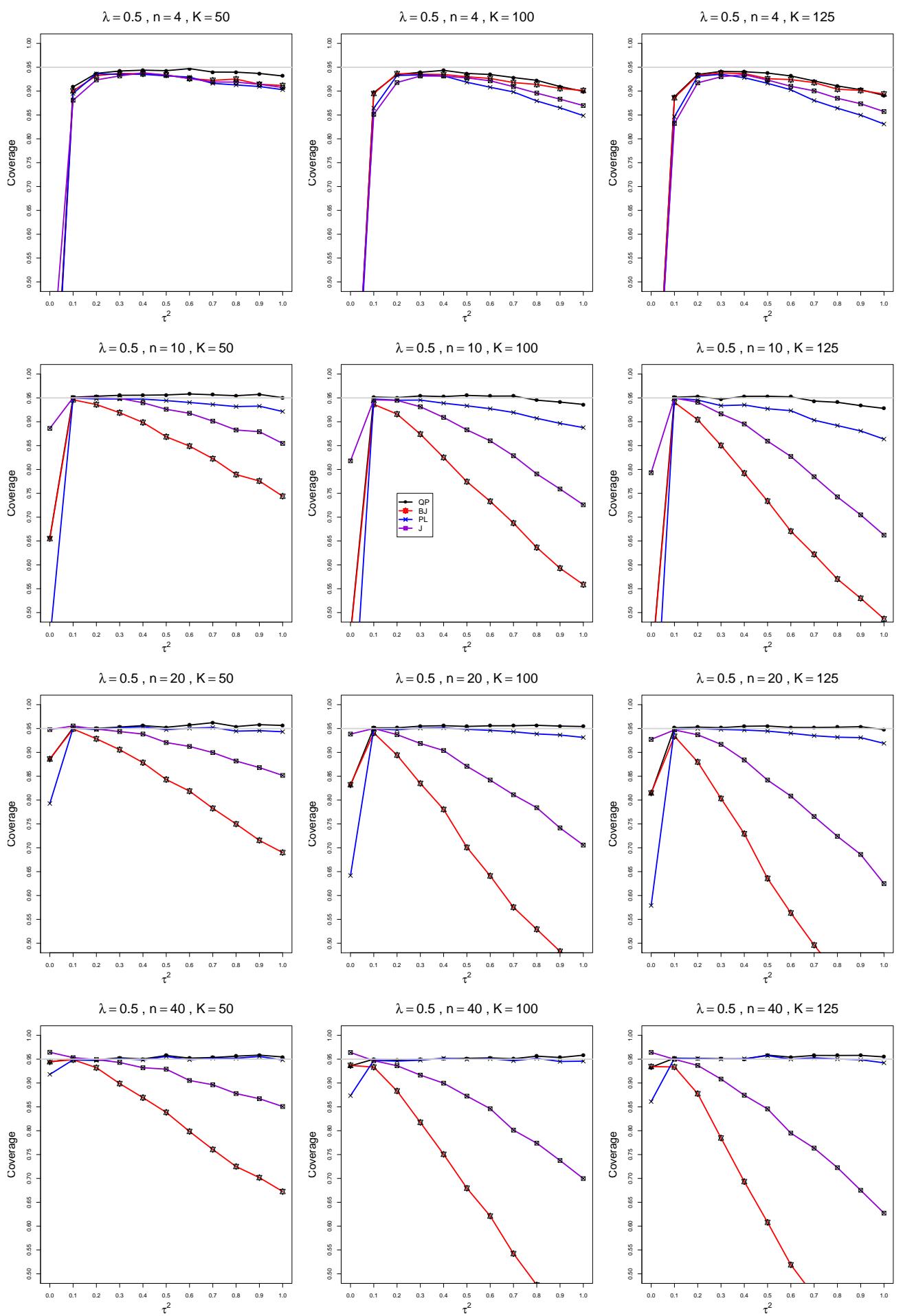

Figure A5.2.3: Coverage of 95\% confidence intervals for the between-studies variance $\tau^{2}$ when $\mu_{C}=4, \lambda=0.5, n=4,10,20,40$, and $K=50,100,125$ 

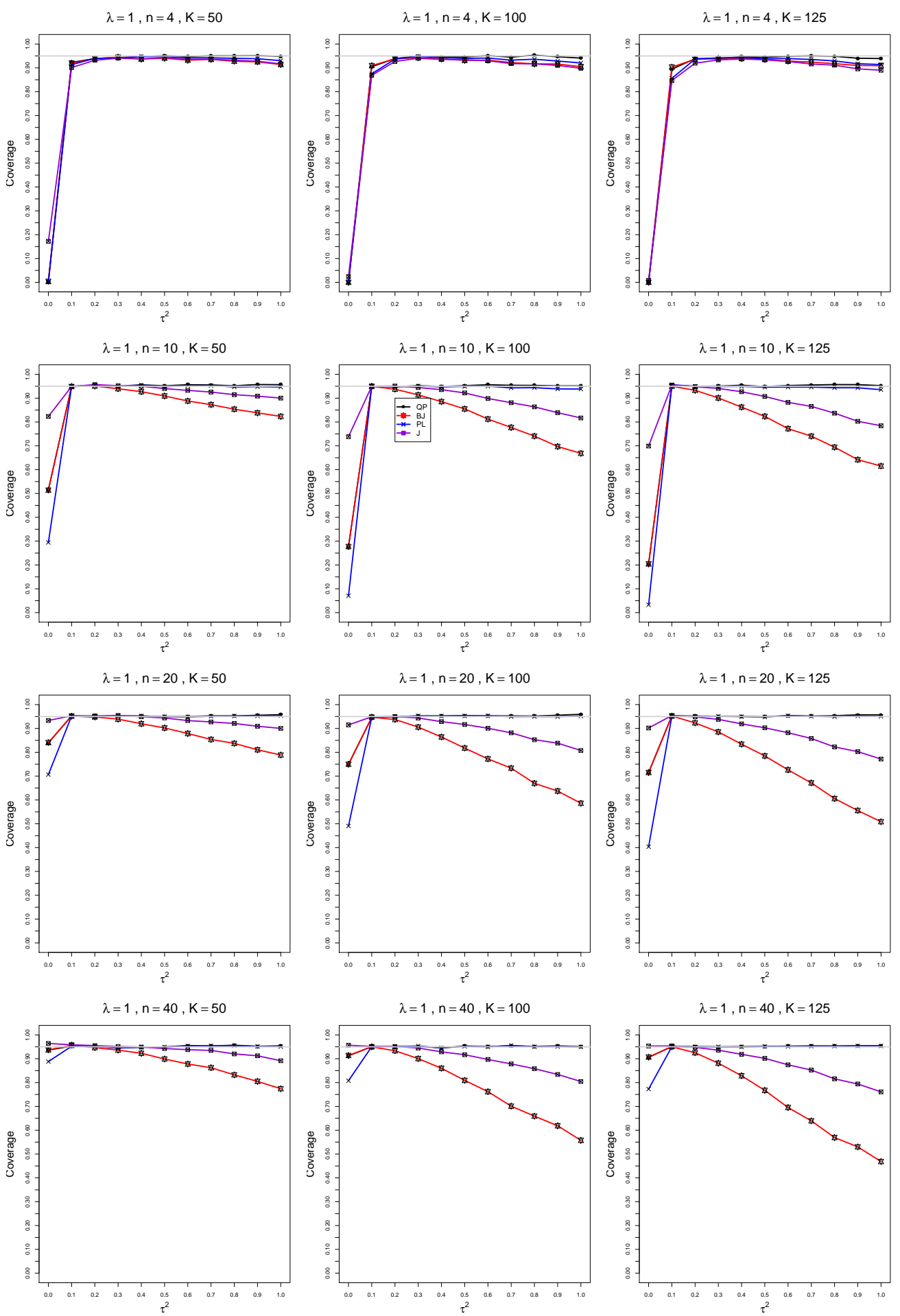

Figure A5.2.4: Coverage of 95\% confidence intervals for the between-studies variance $\tau^{2}$ when $\mu_{C}=4, \lambda=1, n=4,10,20,40$, and $K=50,100,125$ 

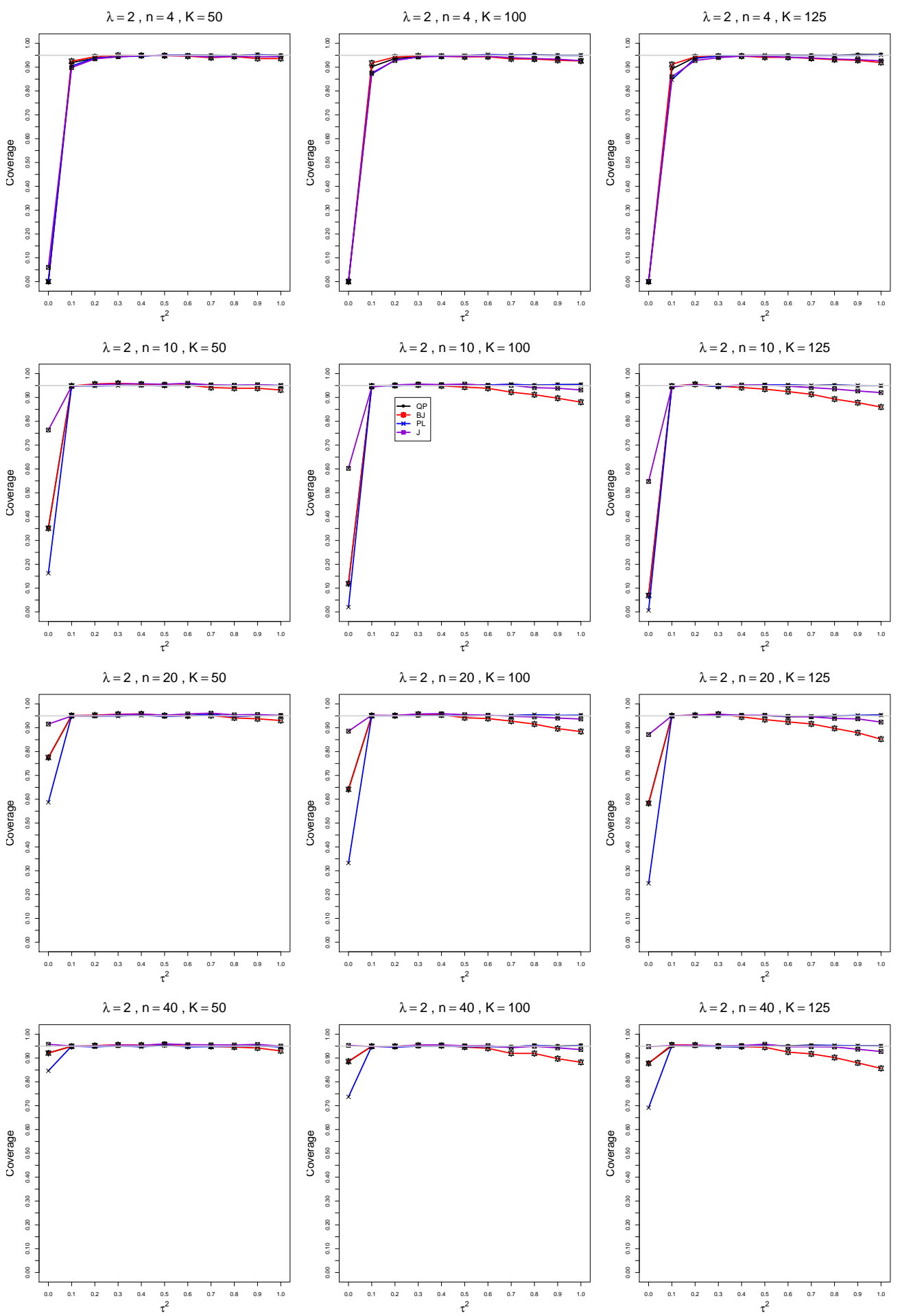

Figure A5.2.5: Coverage of 95\% confidence intervals for the between-studies variance $\tau^{2}$ when $\mu_{C}=4, \lambda=2, n=4,10,20,40$, and $K=50,100,125$ 


\section{A6. Normal model, bias-corrected estimator of}

$\lambda, \mu_{C}=4, n=4,10,20,40, K=50,100,125$

A6.1 Bias of point estimators of $\tau^{2}$ when $\mu_{C}=4$

Each figure corresponds to a value of $\lambda(=0,0.2,0.5,1,2)$, a set of values of $n(=4$, $10,20,40)$, and a set of values of $K(=50,100,125)$.

Each panel corresponds to a value of $n$ and a value of $K$ and has $\tau^{2}=0.0(0.1) 1.0$ on the horizontal axis.

The point estimators of $\tau^{2}$ are

- DL (DerSimonian-Laird)

- REML (restricted maximum likelihood)

- MP (Mandel-Paule)

- J (Jackson) 

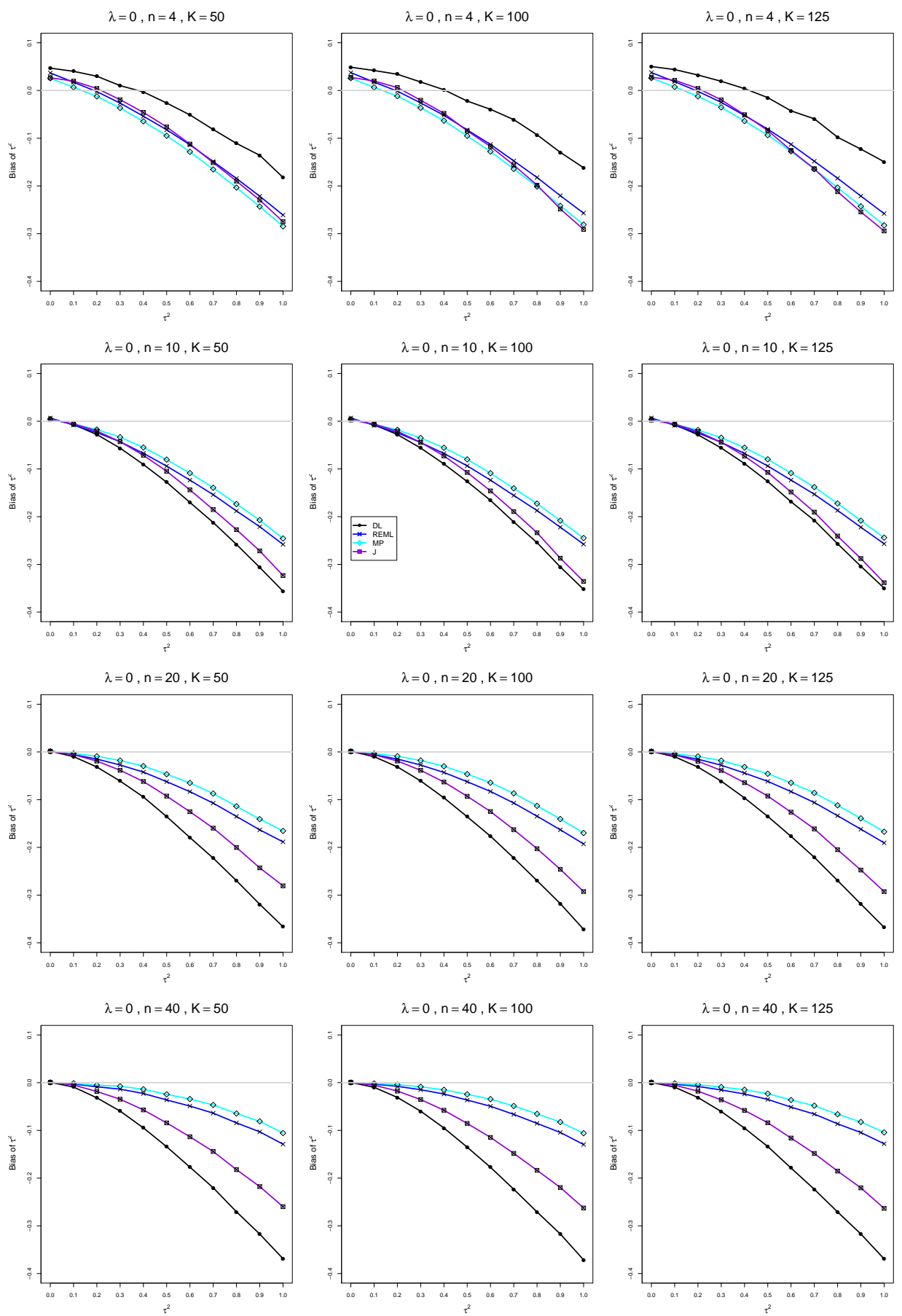

Figure A6.1.1: Bias of estimators of the between-studies variance $\tau^{2}$ when $\mu_{C}=4, \lambda=0$, $n=4,10,20,40$, and $K=50,100,125$ 

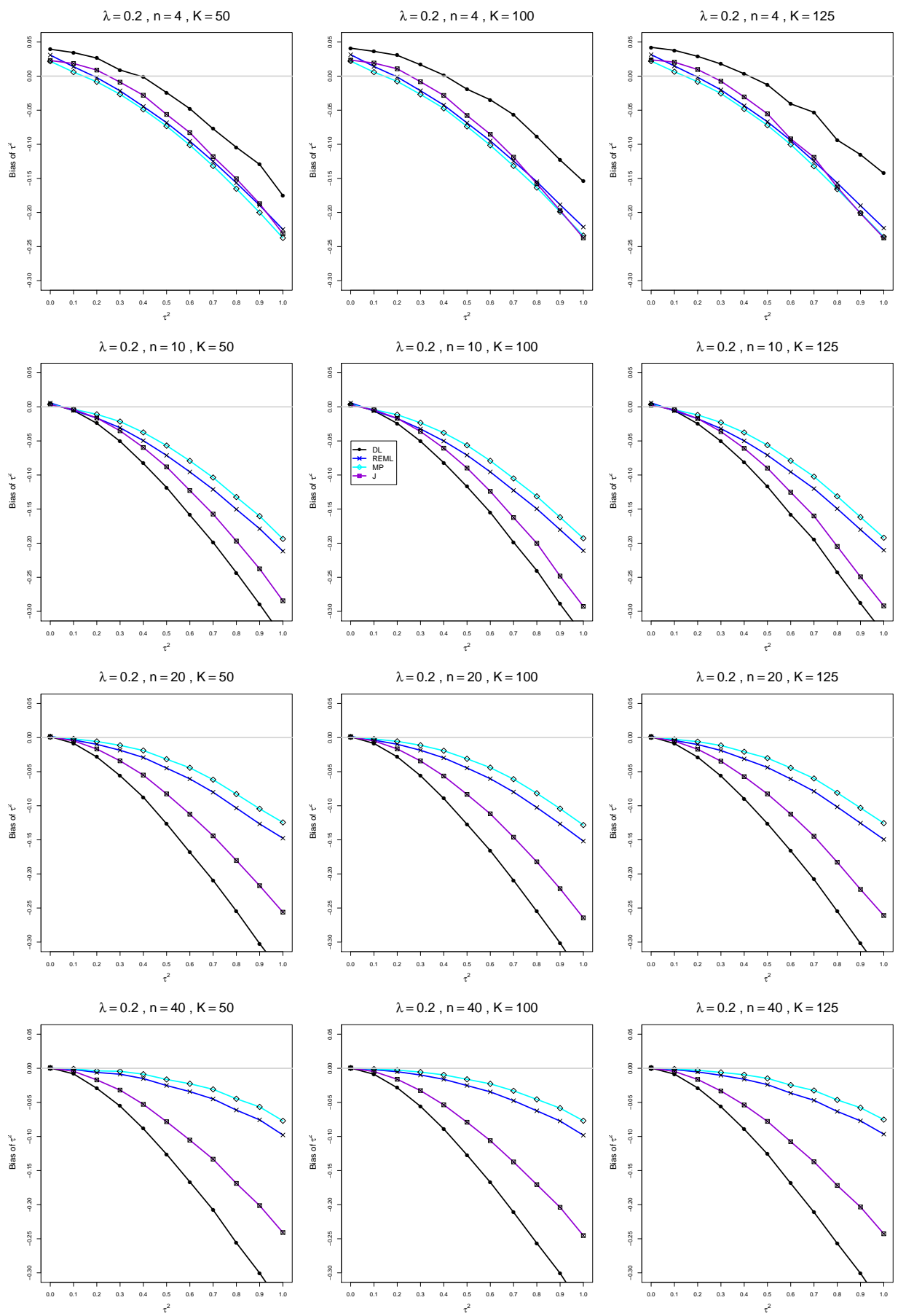

Figure A6.1.2: Bias of estimators of the between-studies variance $\tau^{2}$ when $\mu_{C}=4, \lambda=$ $0.2, n=4,10,20,40$, and $K=50,100,125$ 

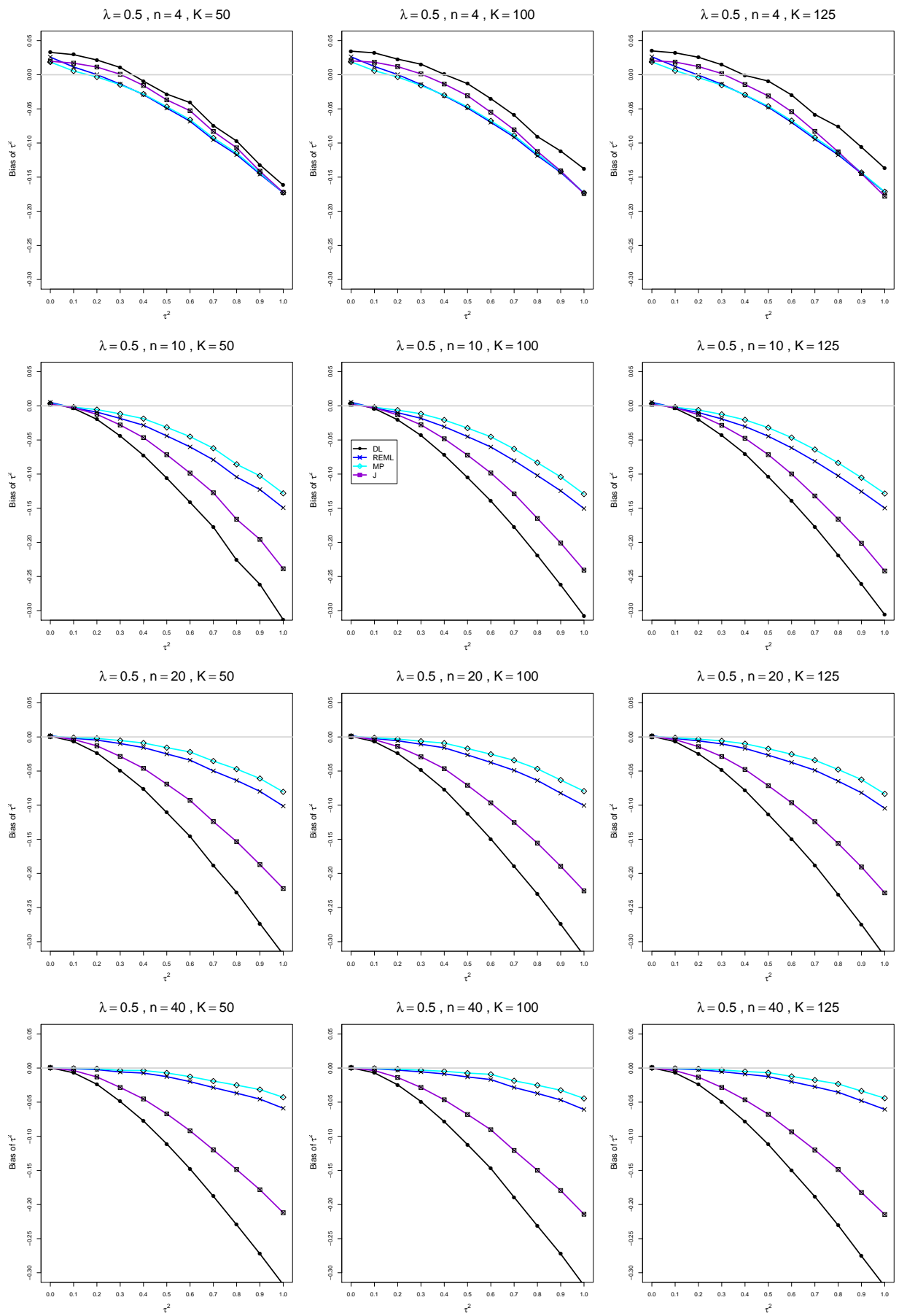

Figure A6.1.3: Bias of estimators of the between-studies variance $\tau^{2}$ when $\mu_{C}=4, \lambda=$ $0.5, n=4,10,20,40$, and $K=50,100,125$ 

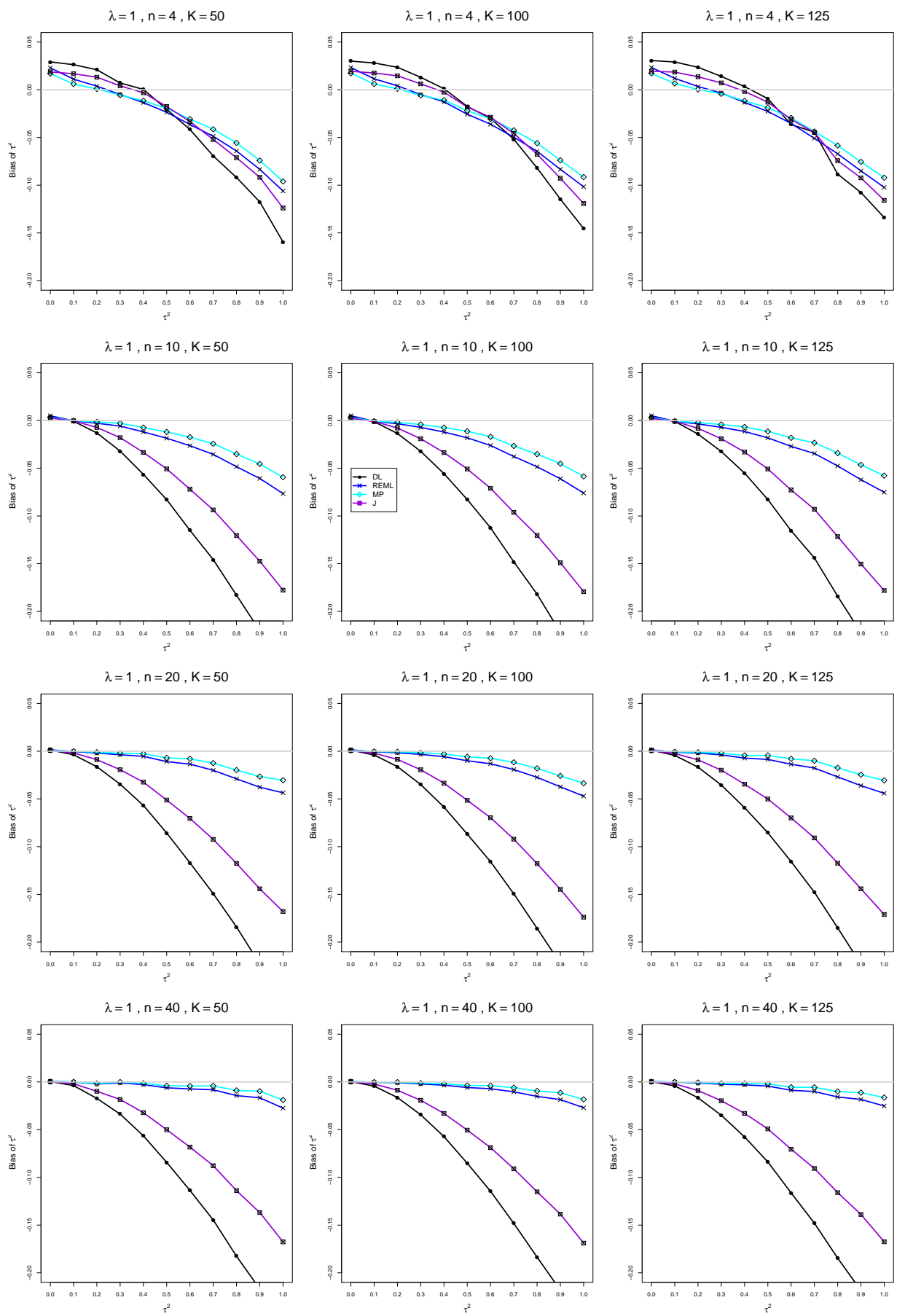

Figure A6.1.4: Bias of estimators of the between-studies variance $\tau^{2}$ when $\mu_{C}=4, \lambda=1$, $n=4,10,20,40$, and $K=50,100,125$ 

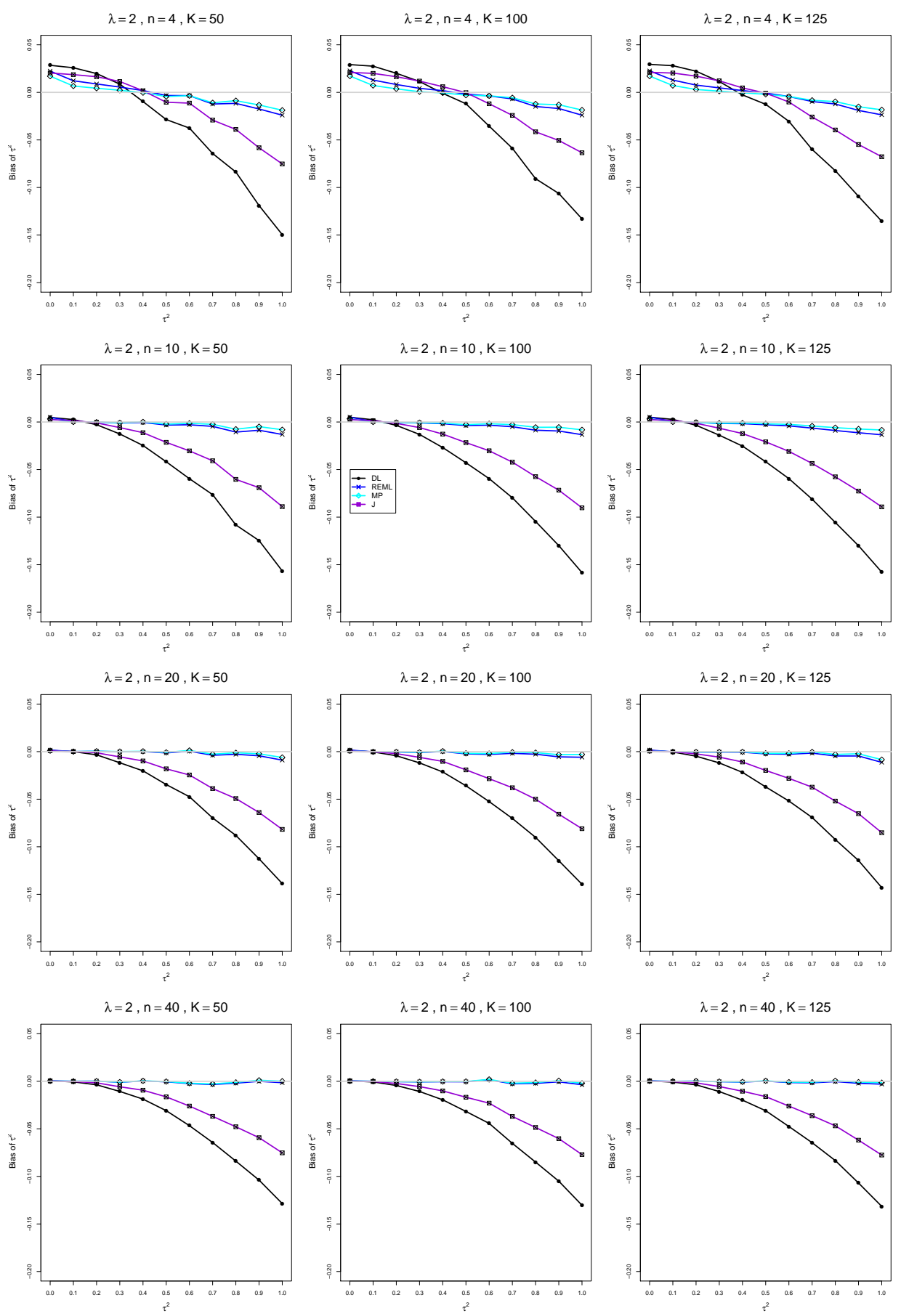

Figure A6.1.5: Bias of estimators of the between-studies variance $\tau^{2}$ when $\mu_{C}=4, \lambda=2$, $n=4,10,20,40$, and $K=50,100,125$ 


\section{A6.2 Coverage of interval estimators of $\tau^{2}$ when $\mu_{C}=4$}

Each figure corresponds to a value of $\lambda(=0,0.2,0.5,1,2)$, a set of values of $n(=4$, $10,20,40)$, and a set of values of $K(=50,100,125)$.

Each panel corresponds to a value of $n$ and a value of $K$ and has $\tau^{2}=0.0(0.1) 1.0$ on the horizontal axis.

The interval estimators of $\tau^{2}$ are

- QP (Q-profile confidence interval)

- BJ (Biggerstaff and Jackson interval)

- PL (Profile likelihood interval)

- J (Jackson's interval) 

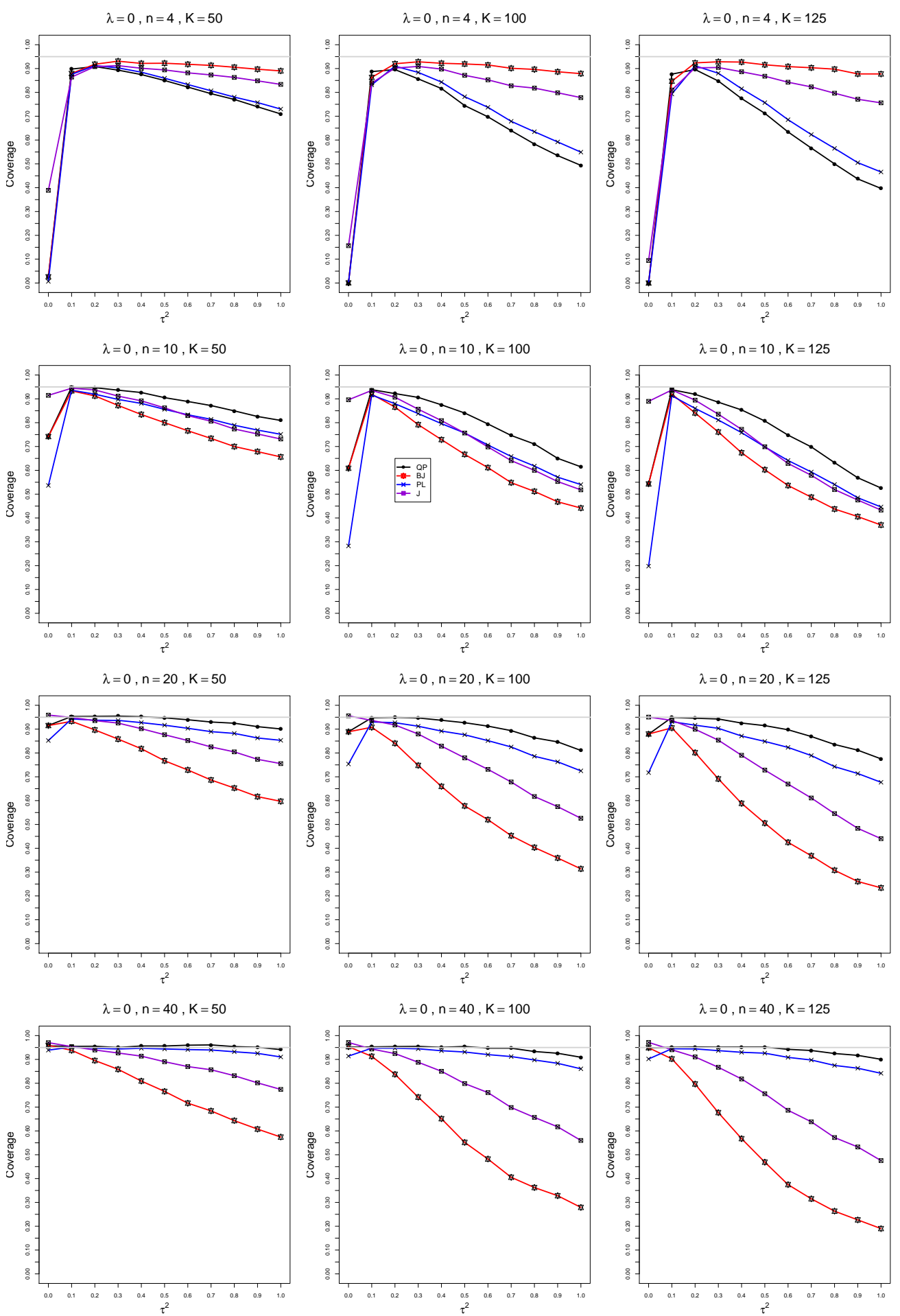

Figure A6.2.1: Coverage of 95\% confidence intervals for the between-studies variance $\tau^{2}$ when $\mu_{C}=4, \lambda=0, n=4,10,20,40$, and $K=50,100,125$ 

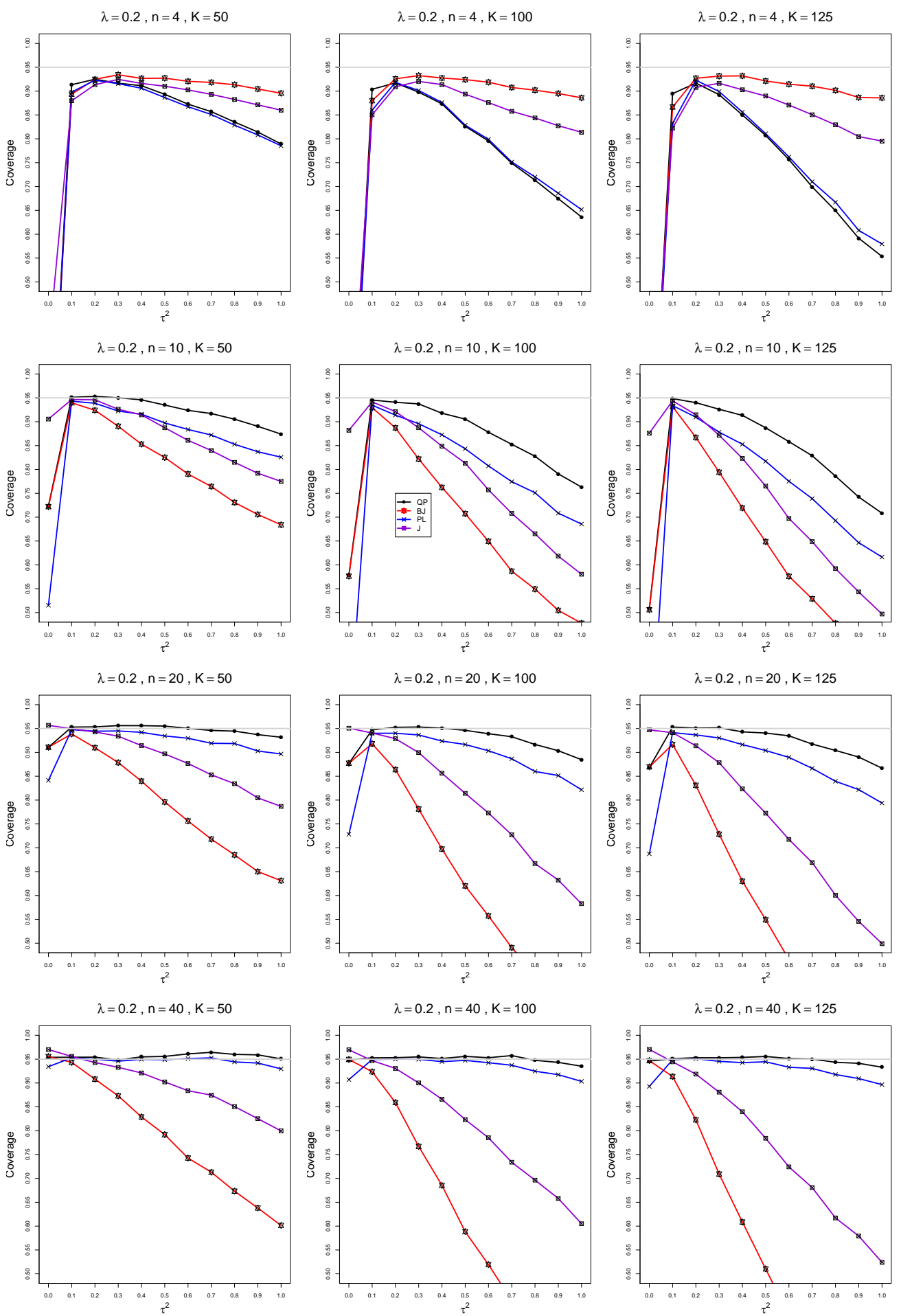

Figure A6.2.2: Coverage of 95\% confidence intervals for the between-studies variance $\tau^{2}$ when $\mu_{C}=4, \lambda=0.2, n=4,10,20,40$, and $K=50,100,125$ 

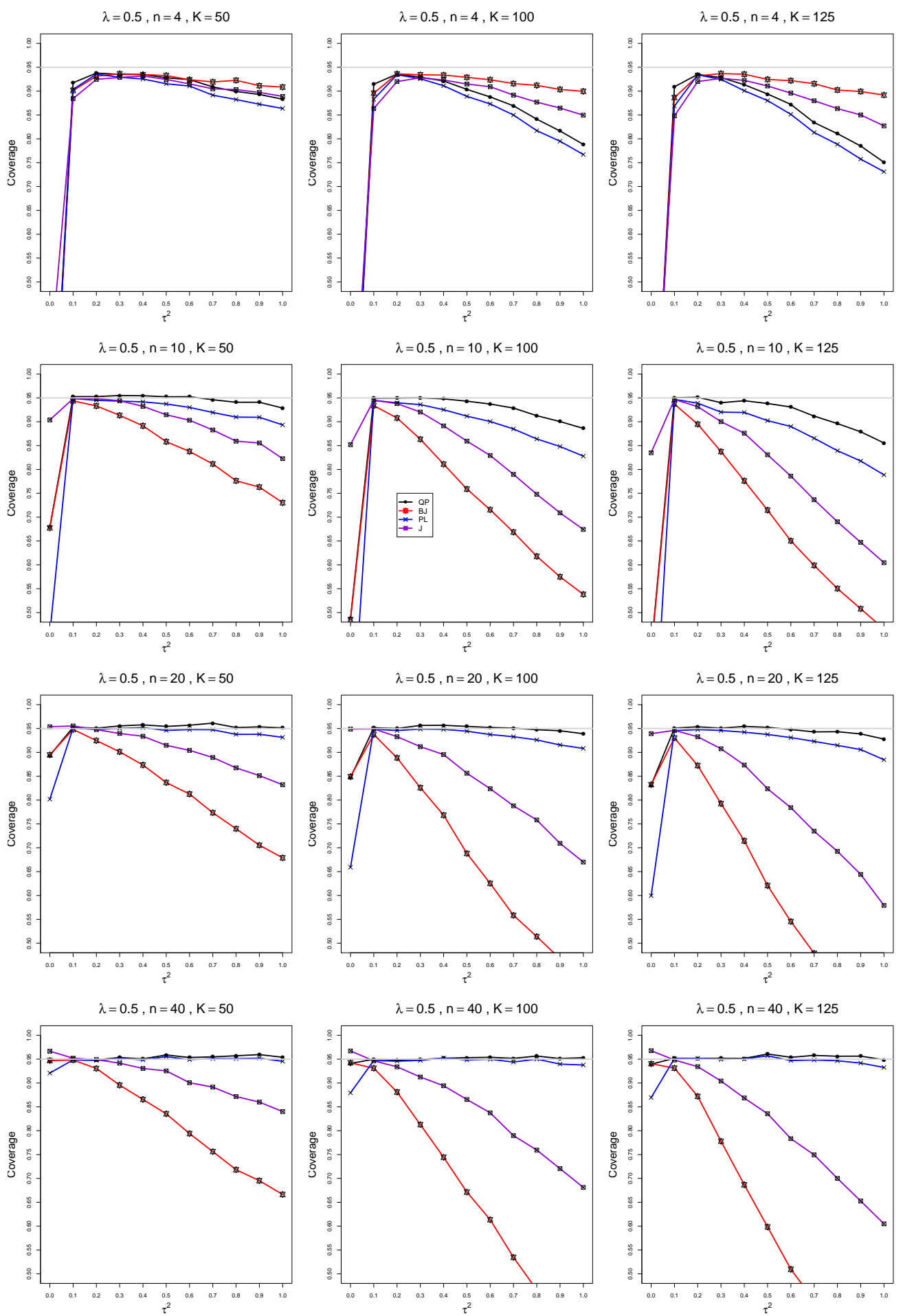

Figure A6.2.3: Coverage of 95\% confidence intervals for the between-studies variance $\tau^{2}$ when $\mu_{C}=4, \lambda=0.5, n=4,10,20,40$, and $K=50,100,125$ 

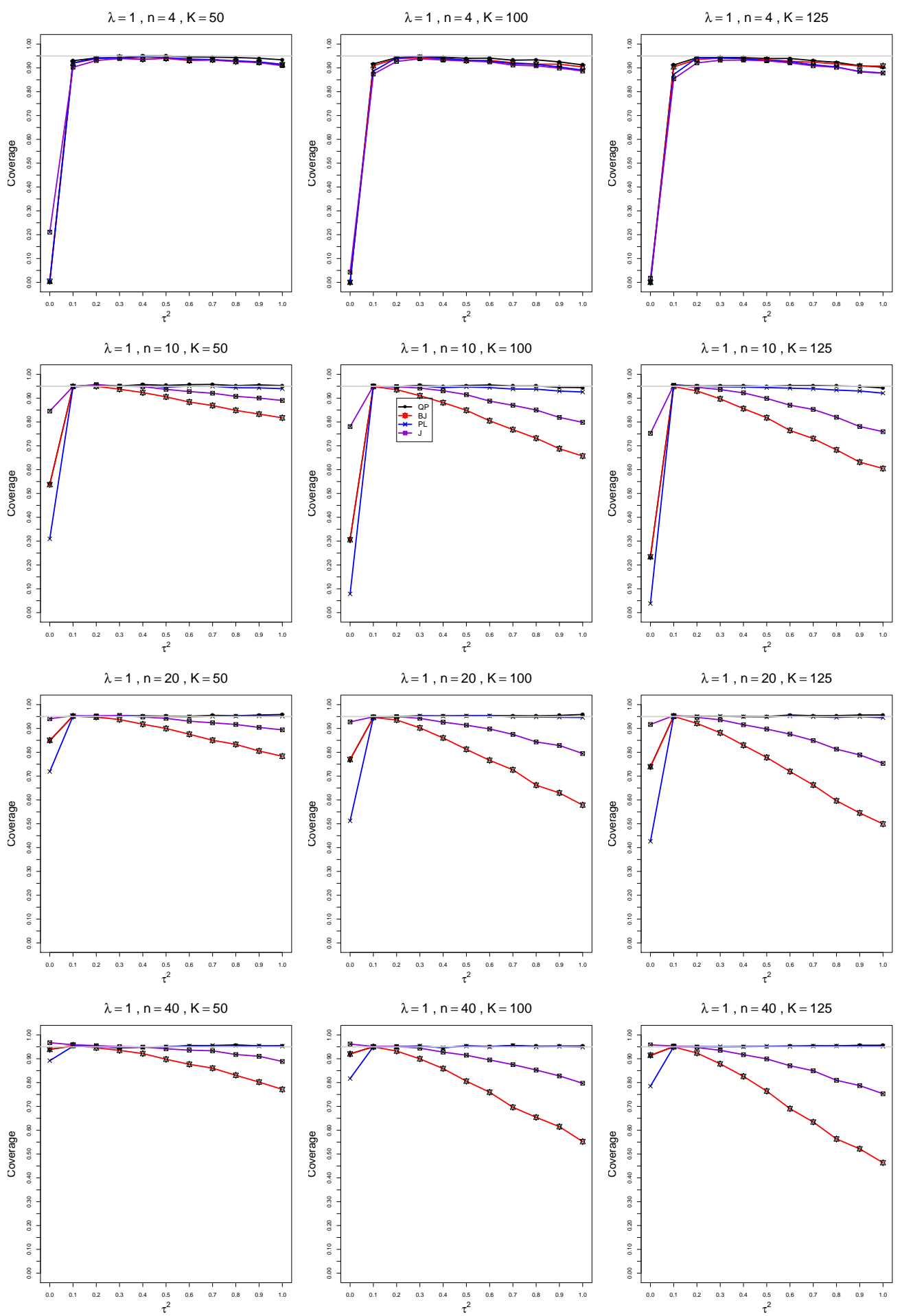

Figure A6.2.4: Coverage of 95\% confidence intervals for the between-studies variance $\tau^{2}$ when $\mu_{C}=4, \lambda=1, n=4,10,20,40$, and $K=50,100,125$ 

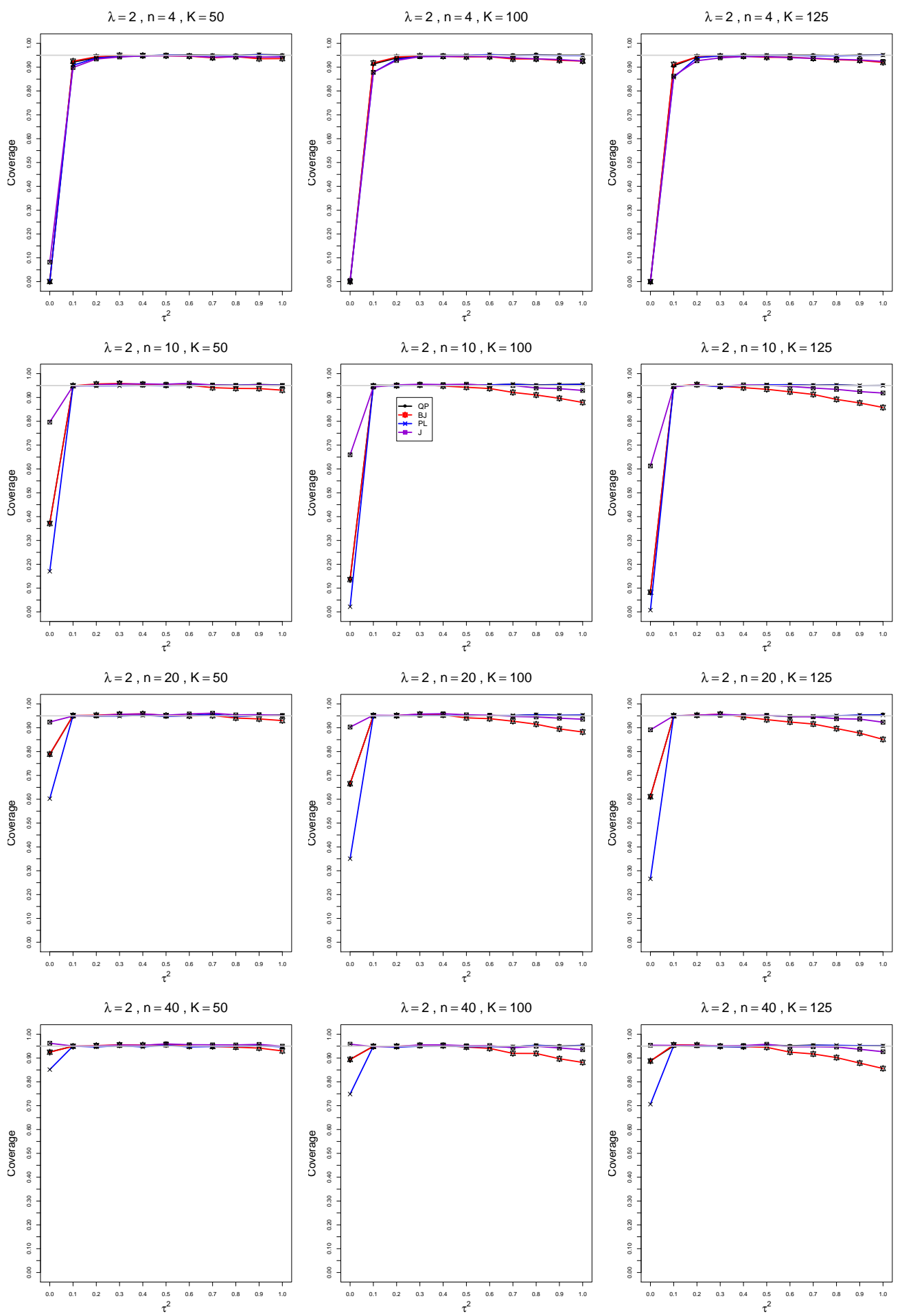

Figure A6.2.5: Coverage of 95\% confidence intervals for the between-studies variance $\tau^{2}$ when $\mu_{C}=4, \lambda=2, n=4,10,20,40$, and $K=50,100,125$ 


\section{B: Plots of bias and coverage of estimators of $\lambda$ with $n=4,10,20,40$}

- B1. Normal model, $\mu_{C}=1, K=5,10,30$

- B2. Normal model, $\mu_{C}=4, K=5,10,30$

- B3. Normal model, bias-corrected $\lambda, \mu_{C}=4, K=5,10,30$

- B4. Normal model, $\mu_{C}=1, K=50,100,125$

- B5. Normal model, $\mu_{C}=4, K=50,100,125$

- B6. Normal model, bias-corrected $\lambda, \mu_{C}=4, K=50,100,125$ 
B1. Normal model, $\mu_{C}=1, n=4,10,20,40, K=$ $5,10,30$

\section{B1.1 Bias of point estimators of $\lambda$ when $\mu_{C}=1$}

Each figure corresponds to a value of $\lambda(=0,0.2,0.5,1,2)$, a set of values of $n(=4$, $10,20,40)$, and a set of values of $K(=5,10,30)$.

Each panel corresponds to a value of $n$ and a value of $K$ and has $\tau^{2}=0.0(0.1) 1.0$ on the horizontal axis.

The point estimators of $\lambda$ are

- DL (DerSimonian-Laird)

- REML (restricted maximum likelihood)

- MP (Mandel-Paule)

- J (Jackson)

- SSW (sample-size weighted) 

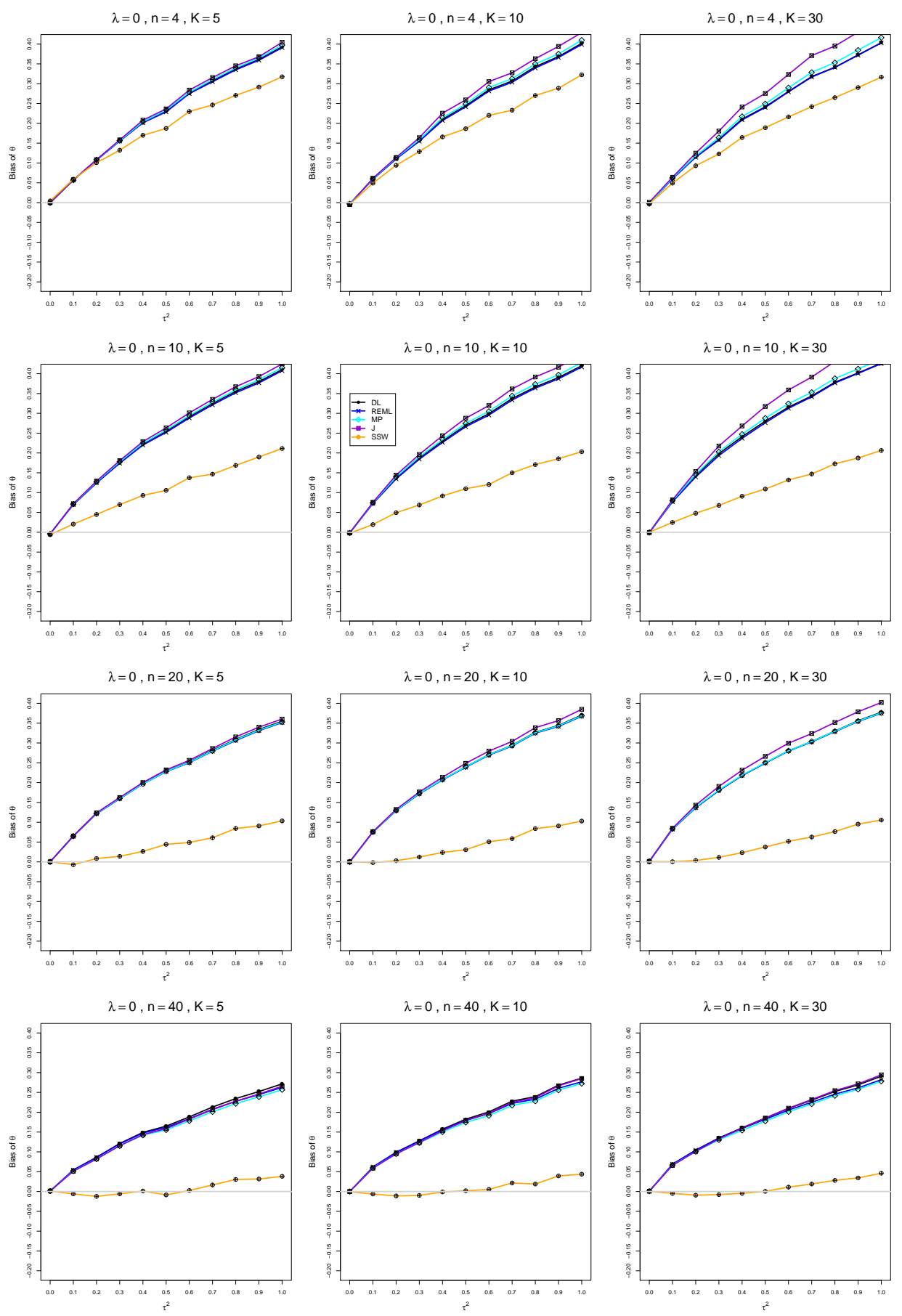

Figure B1.1.1: Bias of estimators of $\log (\operatorname{RoM}) \lambda$ when $\mu_{C}=1, \lambda=0, n=4,10,20,40$, and $K=5,10,30$ 

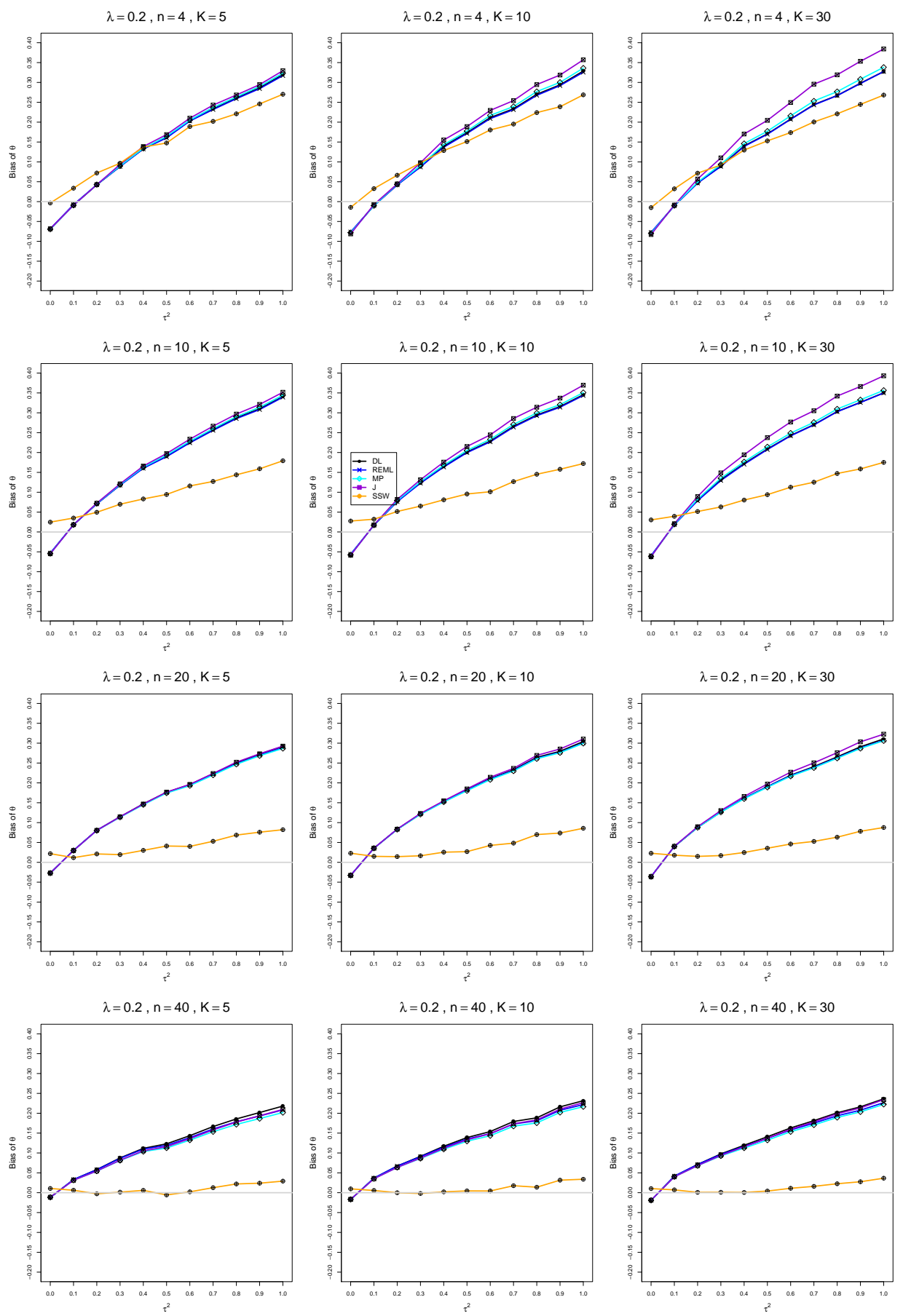

Figure B1.1.2: Bias of estimators of $\log (\operatorname{RoM}) \lambda$ when $\mu_{C}=1, \lambda=0.2, n=4,10,20,40$, and $K=5,10,30$ 

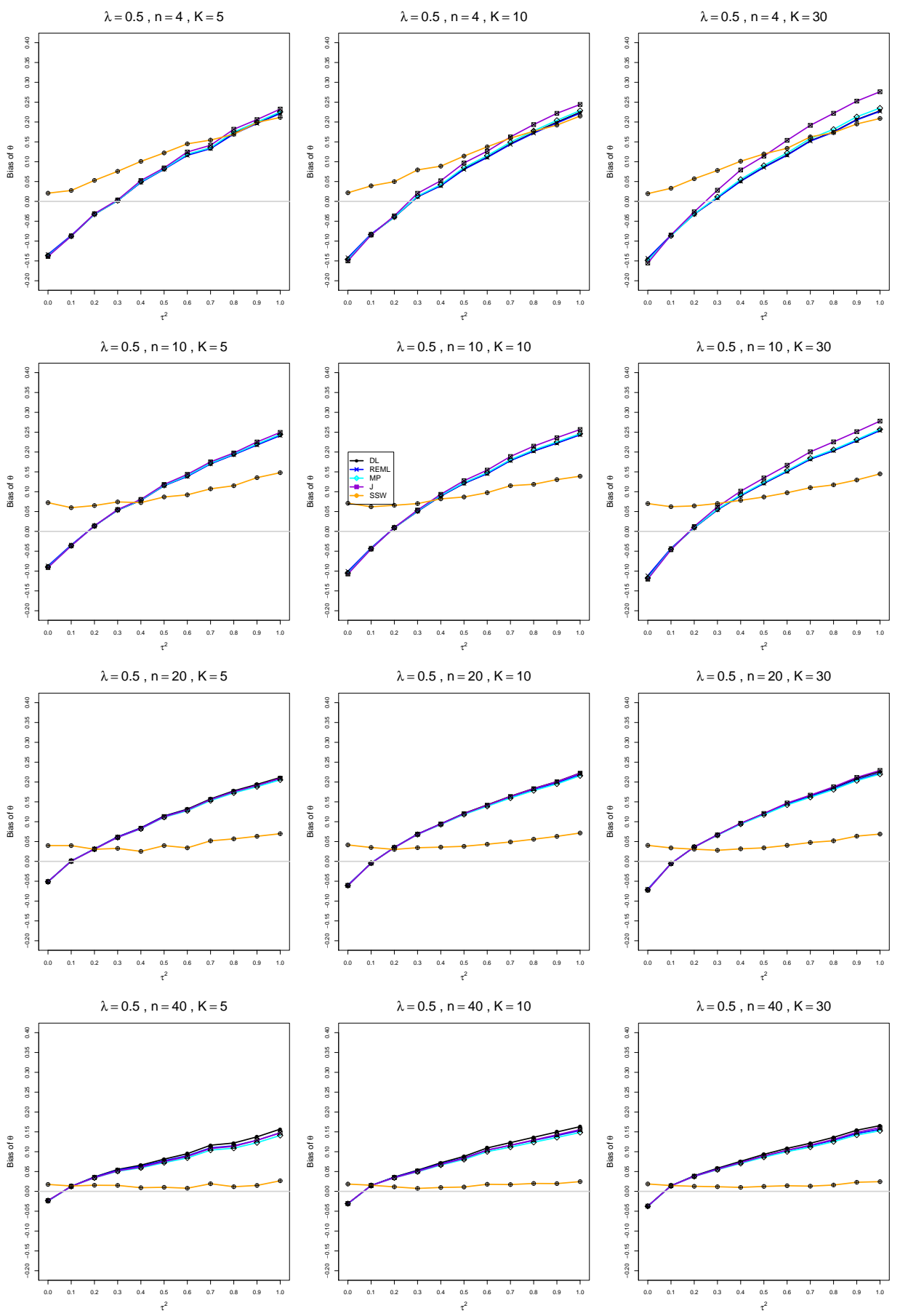

Figure B1.1.3: Bias of estimators of $\log (\operatorname{RoM}) \lambda$ when $\mu_{C}=1, \lambda=0.5, n=4,10,20,40$, and $K=5,10,30$ 

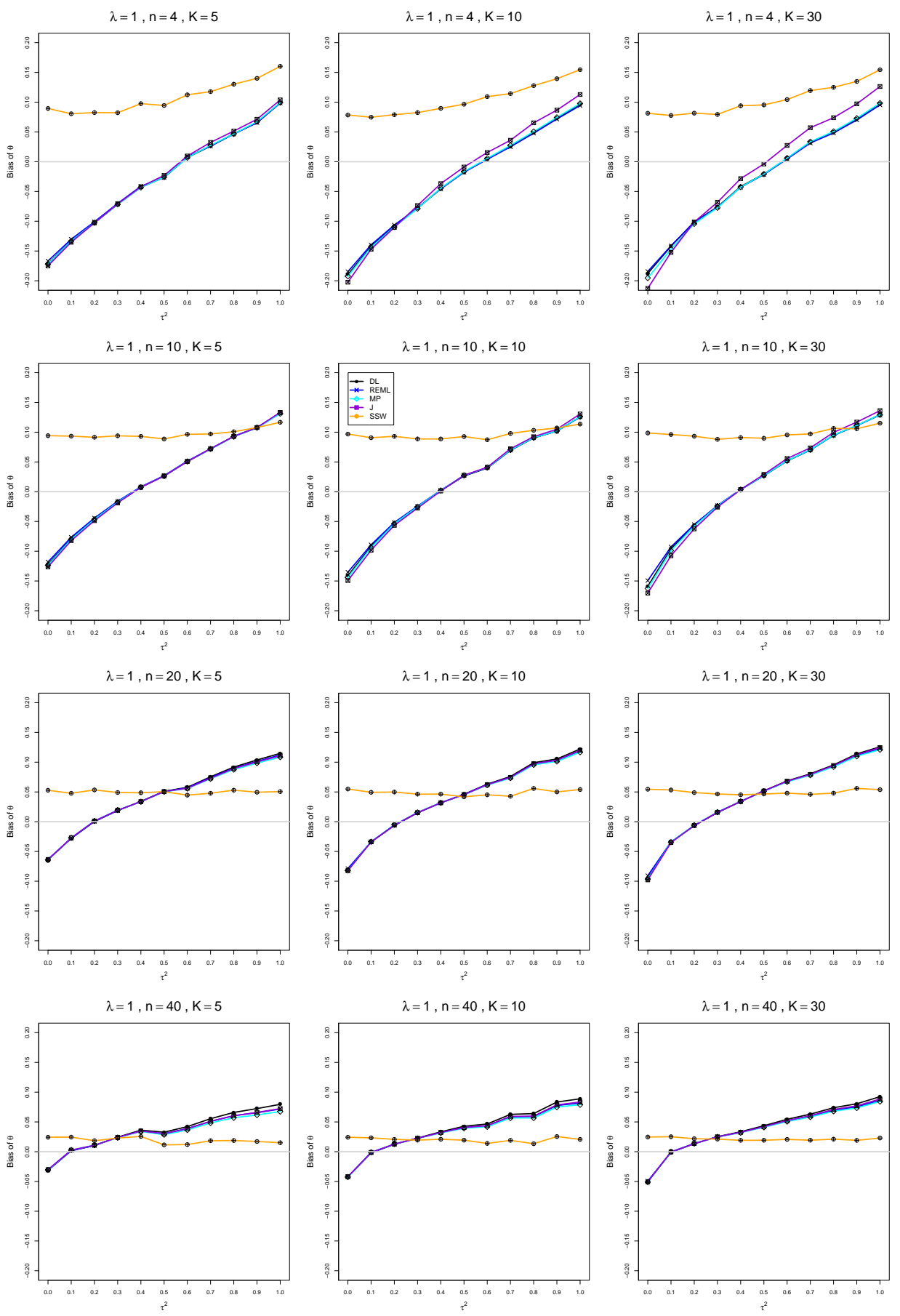

Figure B1.1.4: Bias of estimators of $\log (\operatorname{RoM}) \lambda$ when $\mu_{C}=1, \lambda=1, n=4,10,20,40$, and $K=5,10,30$ 

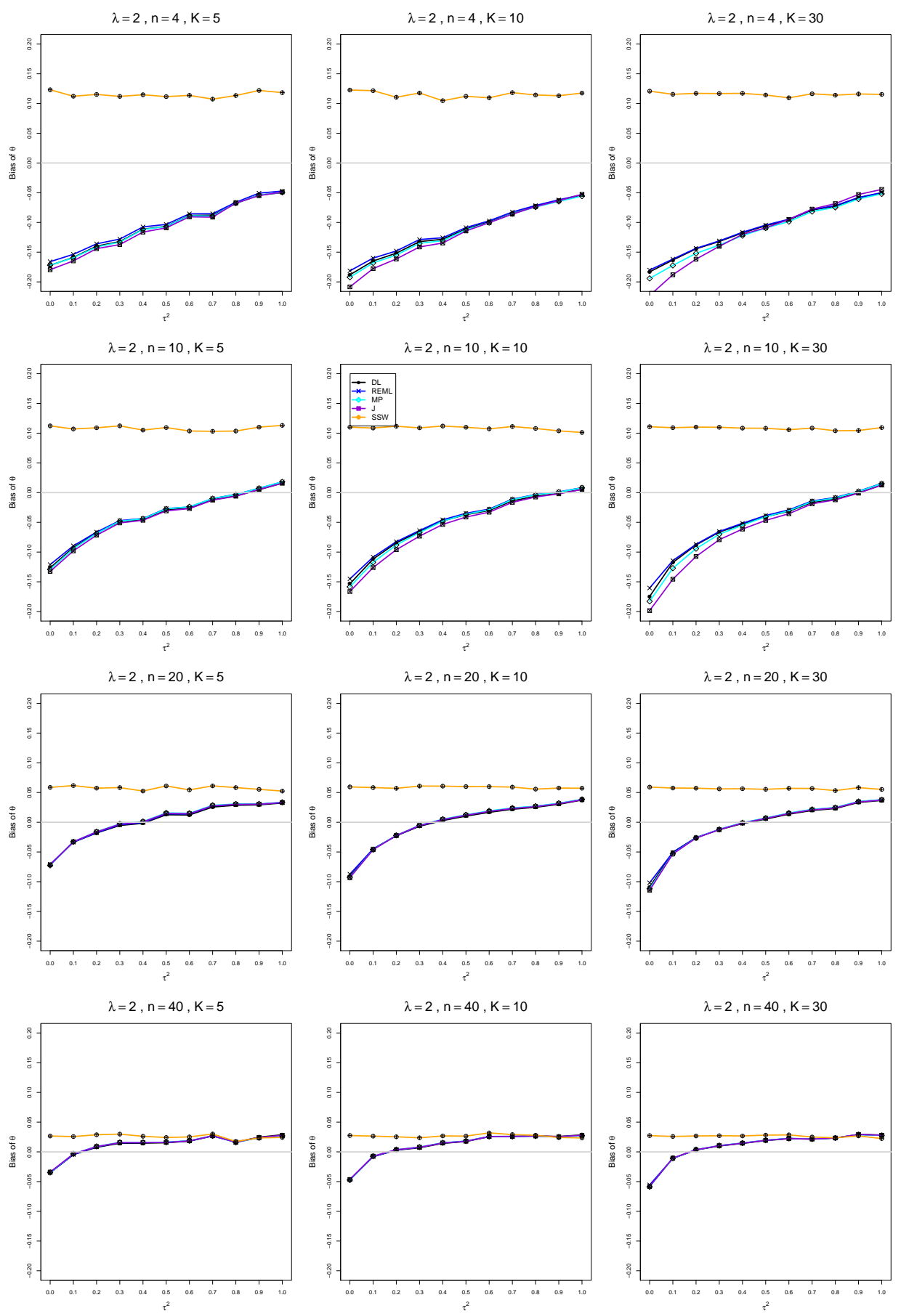

Figure B1.1.5: Bias of estimators of $\log (\operatorname{RoM}) \lambda$ when $\mu_{C}=1, \lambda=2, n=4,10,20,40$, and $K=5,10,30$ 


\section{B1.2 Coverage of interval estimators of $\lambda$}

Each figure corresponds to a value of $\lambda(=0,0.2,0.5,1,2)$, a set of values of $n(=4$, $10,20,40)$, and a set of values of $K(=5,10,30)$.

Each panel corresponds to a value of $n$ and a value of $K$ and has $\tau^{2}=0.0(0.1) 1.0$ on the horizontal axis.

The interval estimators of $\lambda$ are the companions to the inverse-variance-weighted point estimators

- DL (DerSimonian-Laird)

- REML (restricted maximum likelihood)

- MP (Mandel-Paule)

- J (Jackson)

and

- HKSJ (Hartung-Knapp-Sidik-Jonkman)

- HKSJ MP (HKSJ with MP estimator of $\tau^{2}$ )

- SSW MP (SSW as center and half-width equal to critical value from $t_{K-1}$ times estimated standard deviation of SSW with $\hat{\tau}^{2}=\hat{\tau}_{M P}^{2}$ 

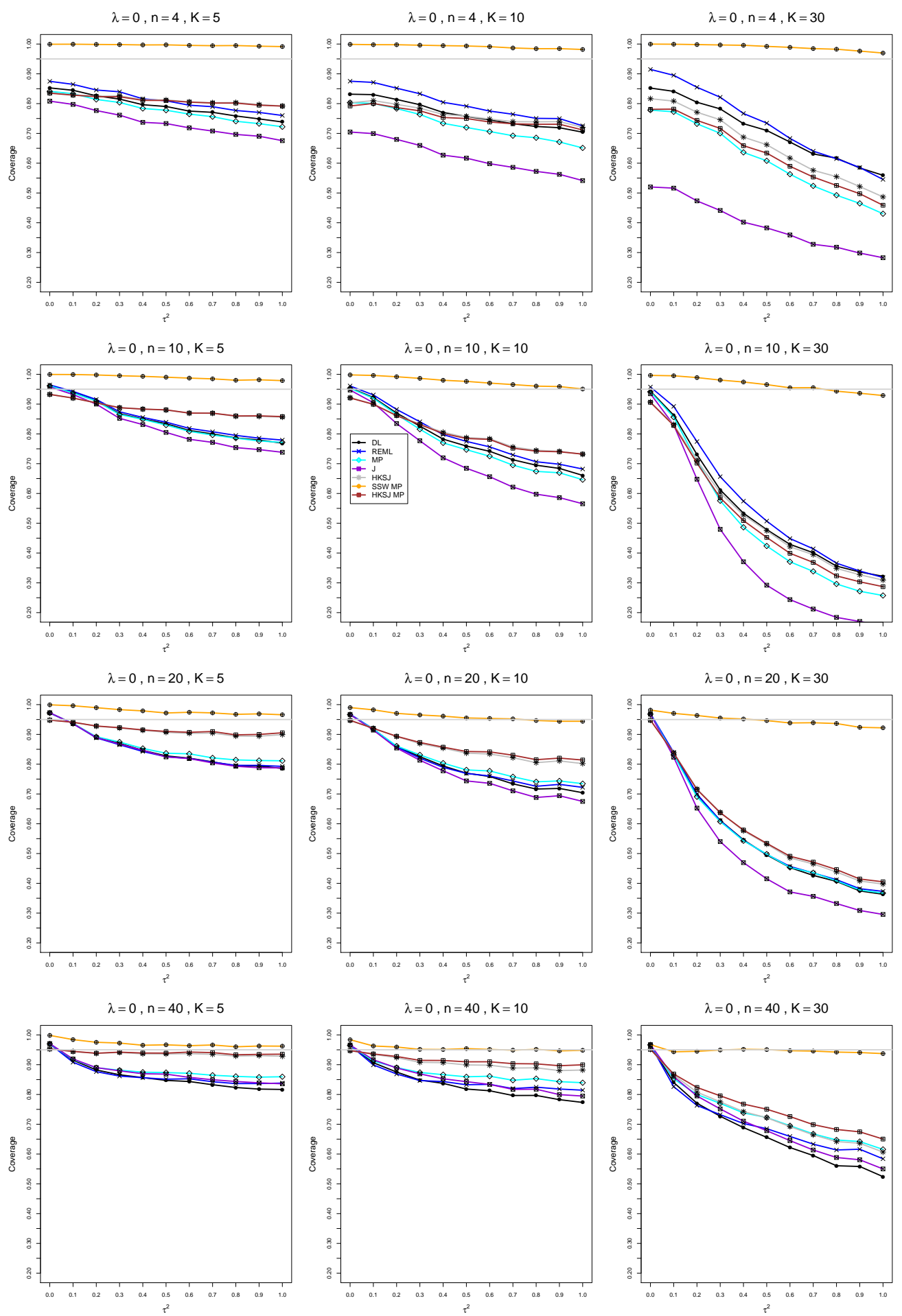

Figure B1.2.1: Coverage of $95 \%$ confidence intervals for $\lambda$ when $\mu_{C}=1, \lambda=0, n=$ 4, 10, 20, 40, and $K=5,10,30$ 

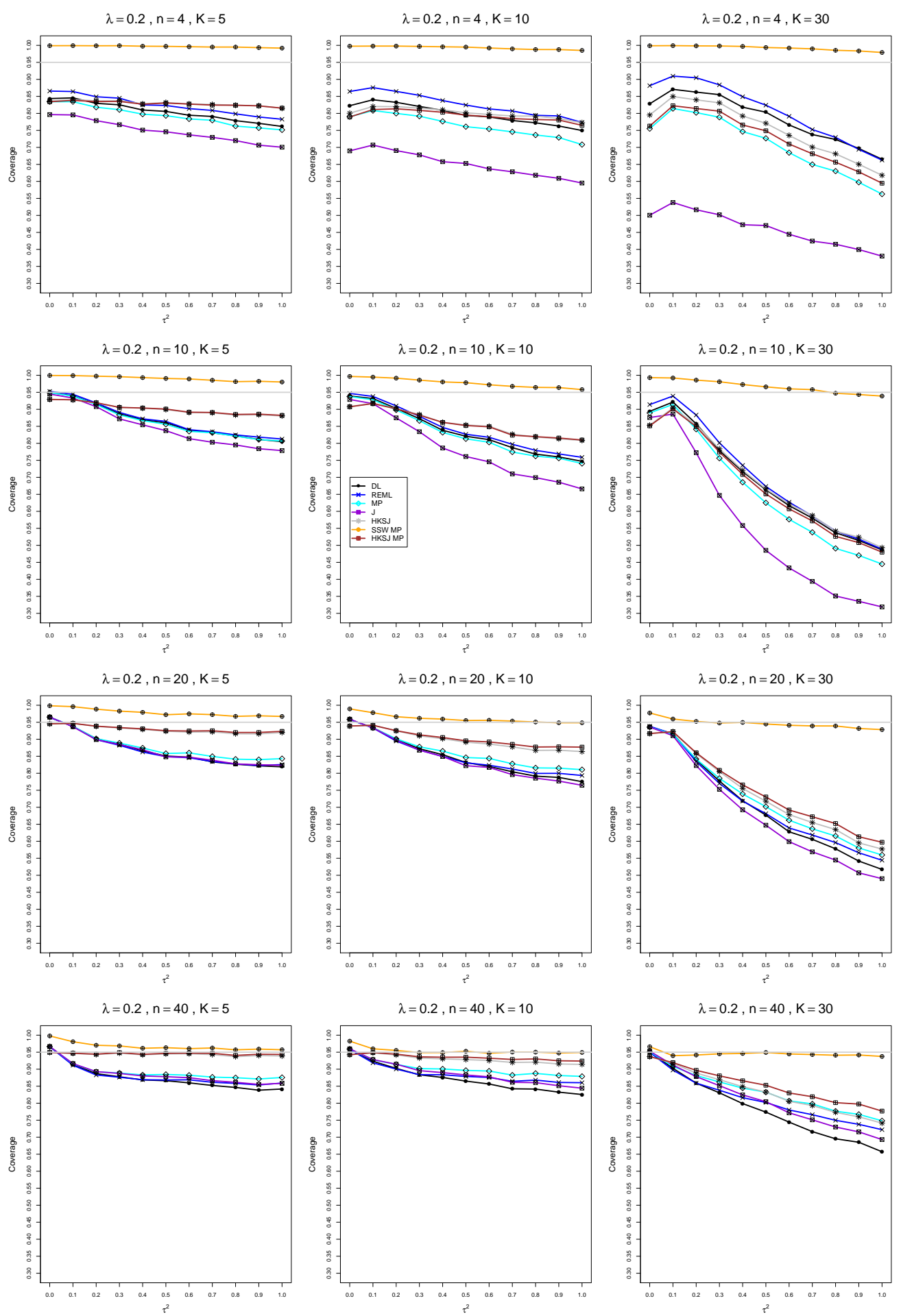

Figure B1.2.2: Coverage of $95 \%$ confidence intervals for $\lambda$ when $\mu_{C}=1, \lambda=0.2, n=$ 4, 10, 20, 40, and $K=5,10,30$ 

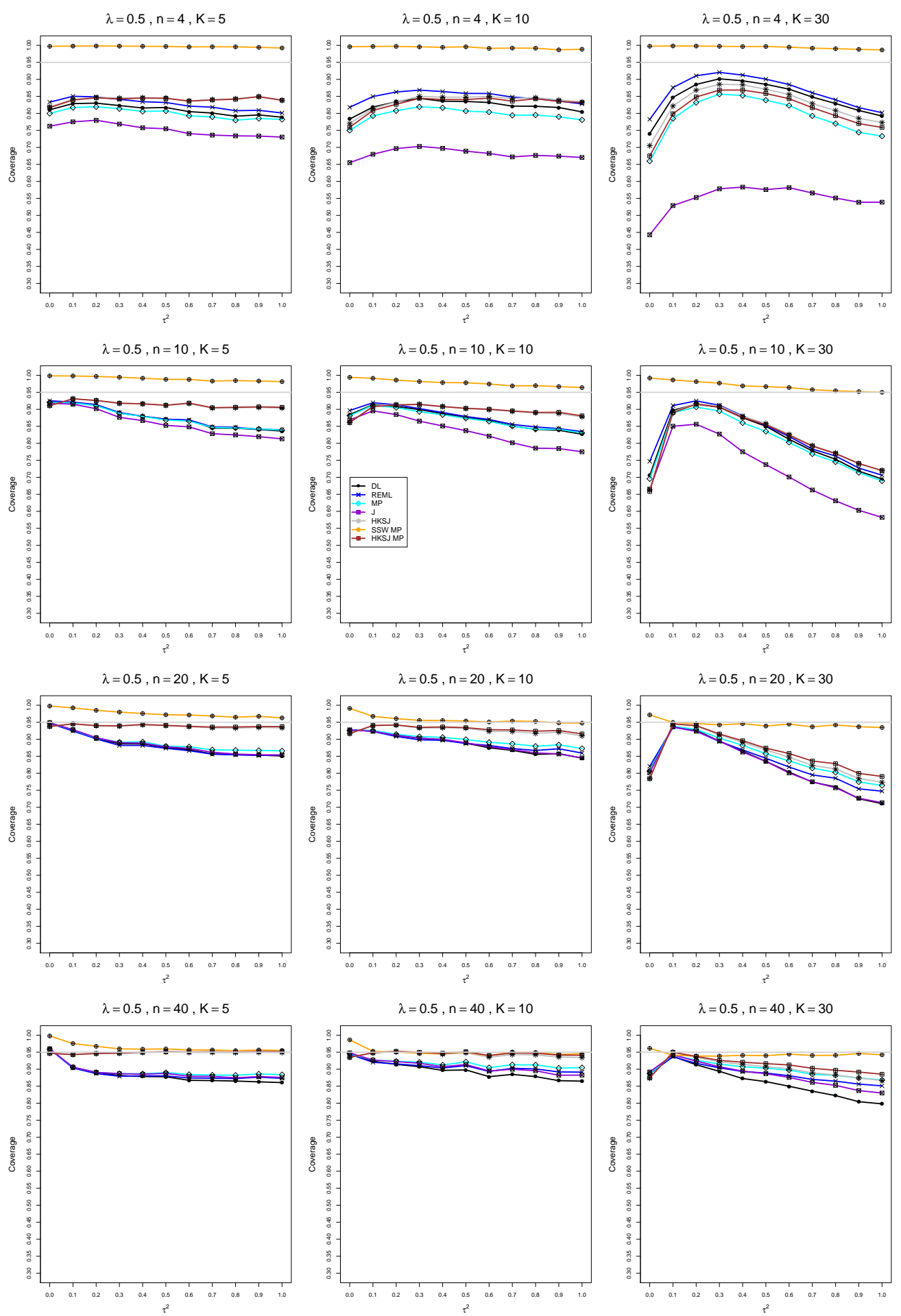

Figure B1.2.3: Coverage of $95 \%$ confidence intervals for $\lambda$ when $\mu_{C}=1, \lambda=0.5, n=$ 4, 10, 20, 40, and $K=5,10,30$ 

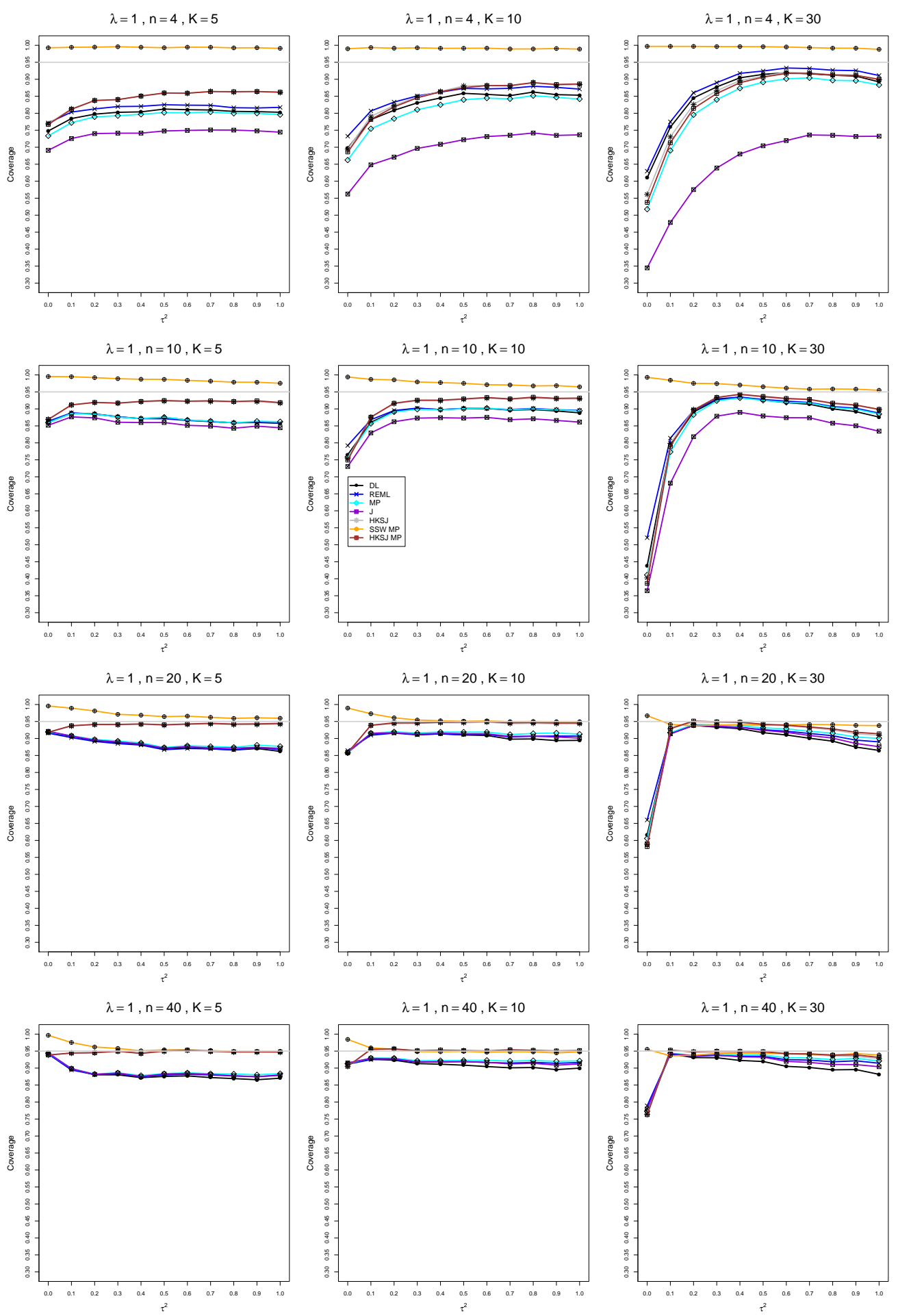

Figure B1.2.4: Coverage of $95 \%$ confidence intervals for $\lambda$ when $\mu_{C}=1, \lambda=1, n=$ 4, 10, 20, 40, and $K=5,10,30$ 

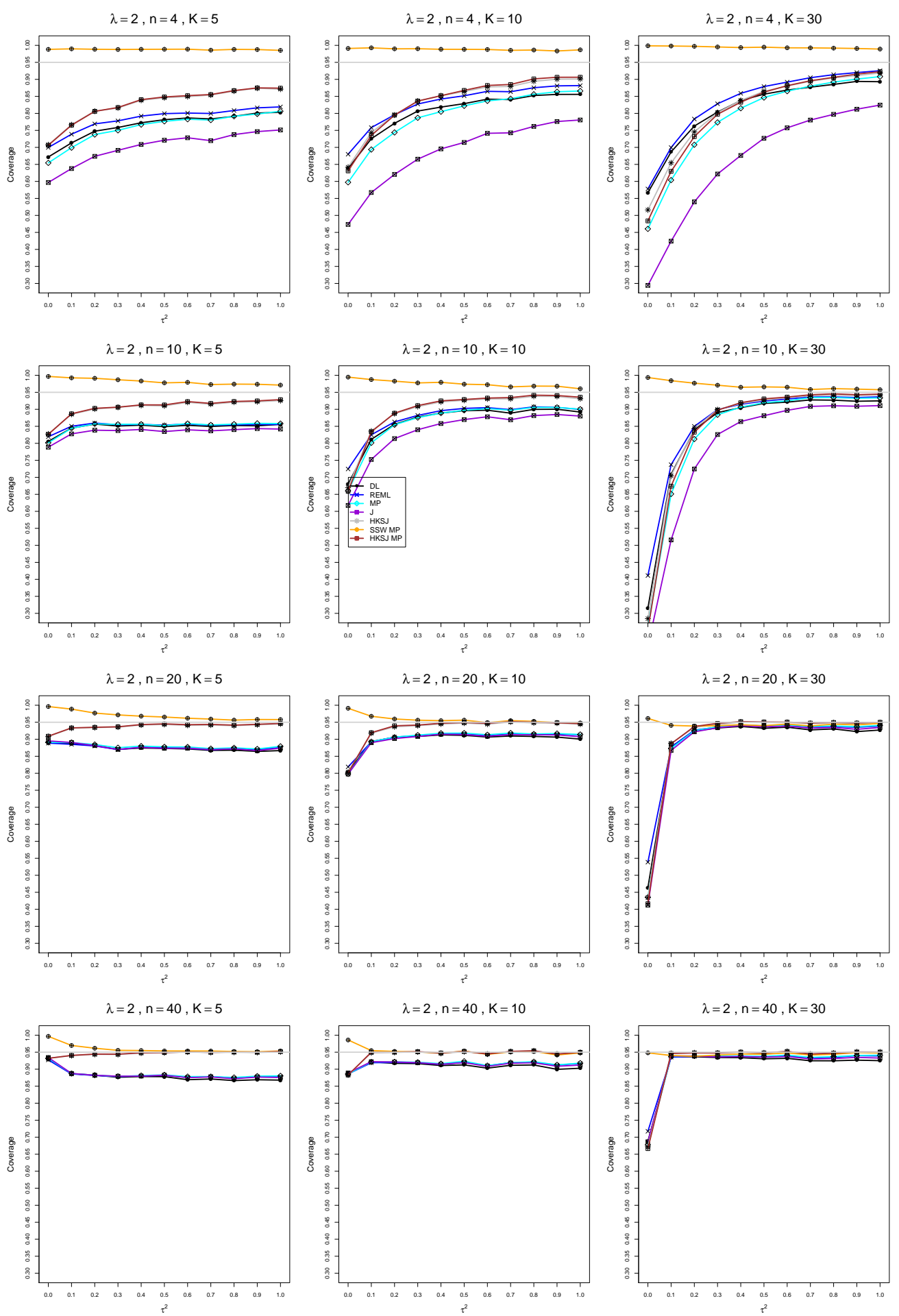

Figure B1.2.5: Coverage of $95 \%$ confidence intervals for $\lambda$ when $\mu_{C}=1, \lambda=2, n=$ 4, 10, 20, 40, and $K=5,10,30$ 
B2. Normal model, $\mu_{C}=4, n=4,10,20,40, K=$ $5,10,30$

\section{B2.1 Bias of point estimators of $\lambda$ when $\mu_{C}=4$}

Each figure corresponds to a value of $\lambda(=0,0.2,0.5,1,2)$, a set of values of $n(=4$, $10,20,40)$, and a set of values of $K(=5,10,30)$.

Each panel corresponds to a value of $n$ and a value of $K$ and has $\tau^{2}=0.0(0.1) 1.0$ on the horizontal axis.

The point estimators of $\lambda$ are

- DL (DerSimonian-Laird)

- REML (restricted maximum likelihood)

- MP (Mandel-Paule)

- J (Jackson)

- SSW (sample-size weighted) 

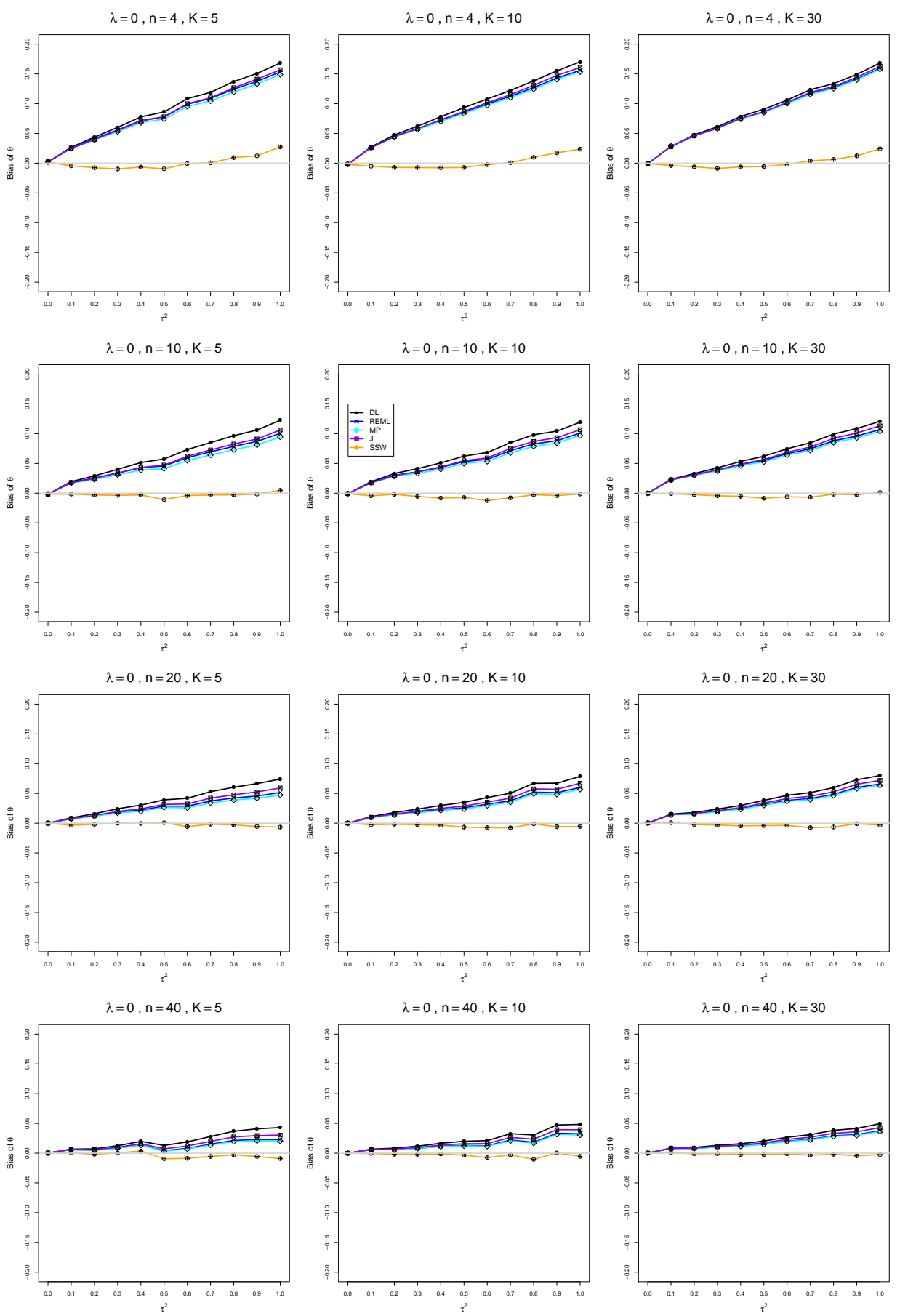

Figure B2.1.1: Bias of estimators of $\log (\operatorname{RoM}) \lambda$ when $\mu_{C}=4, \lambda=0, n=4,10,20,40$, and $K=5,10,30$ 

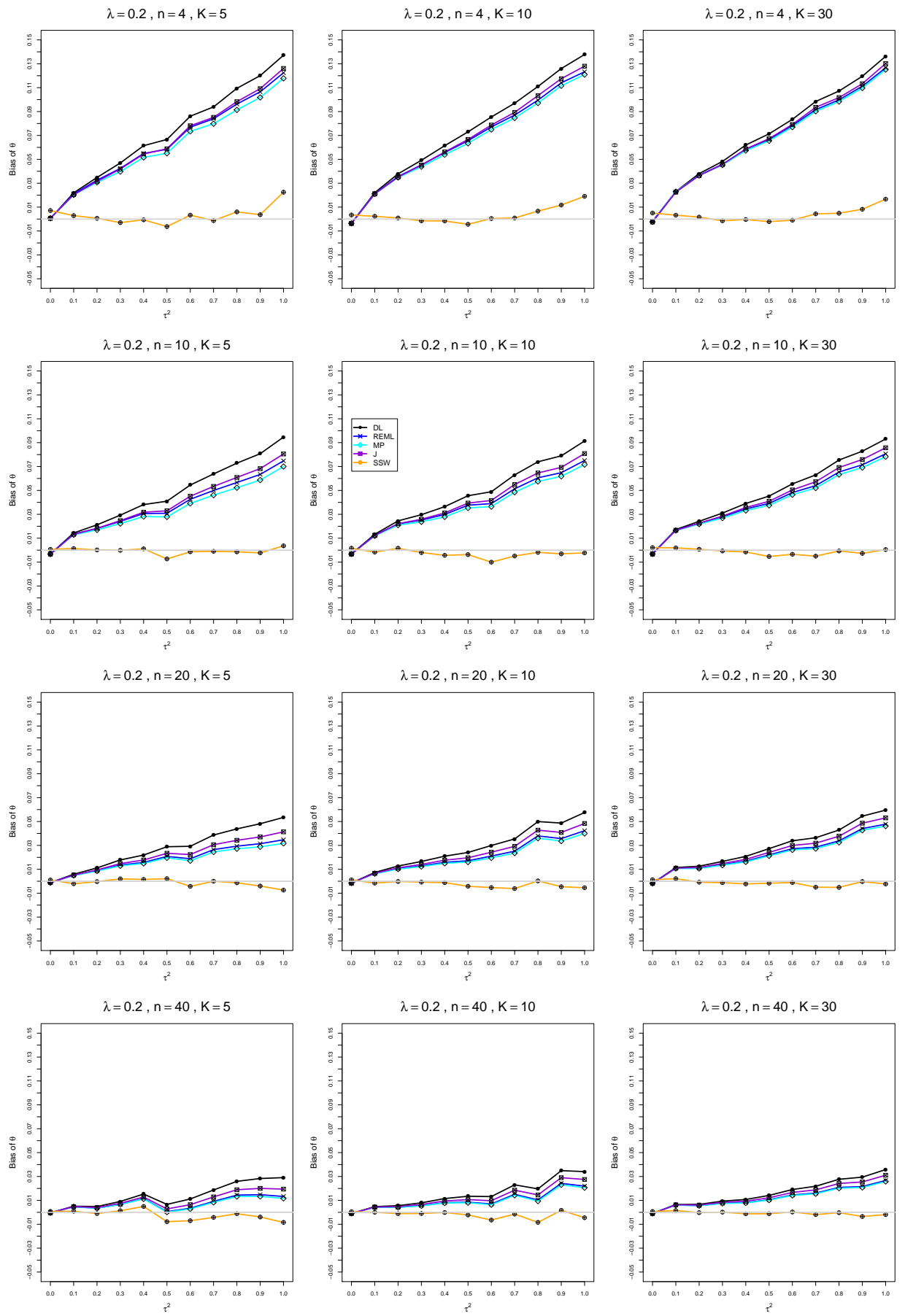

Figure B2.1.2: Bias of estimators of $\log (\operatorname{RoM}) \lambda$ when $\mu_{C}=4, \lambda=0.2, n=4,10,20,40$, and $K=5,10,30$ 

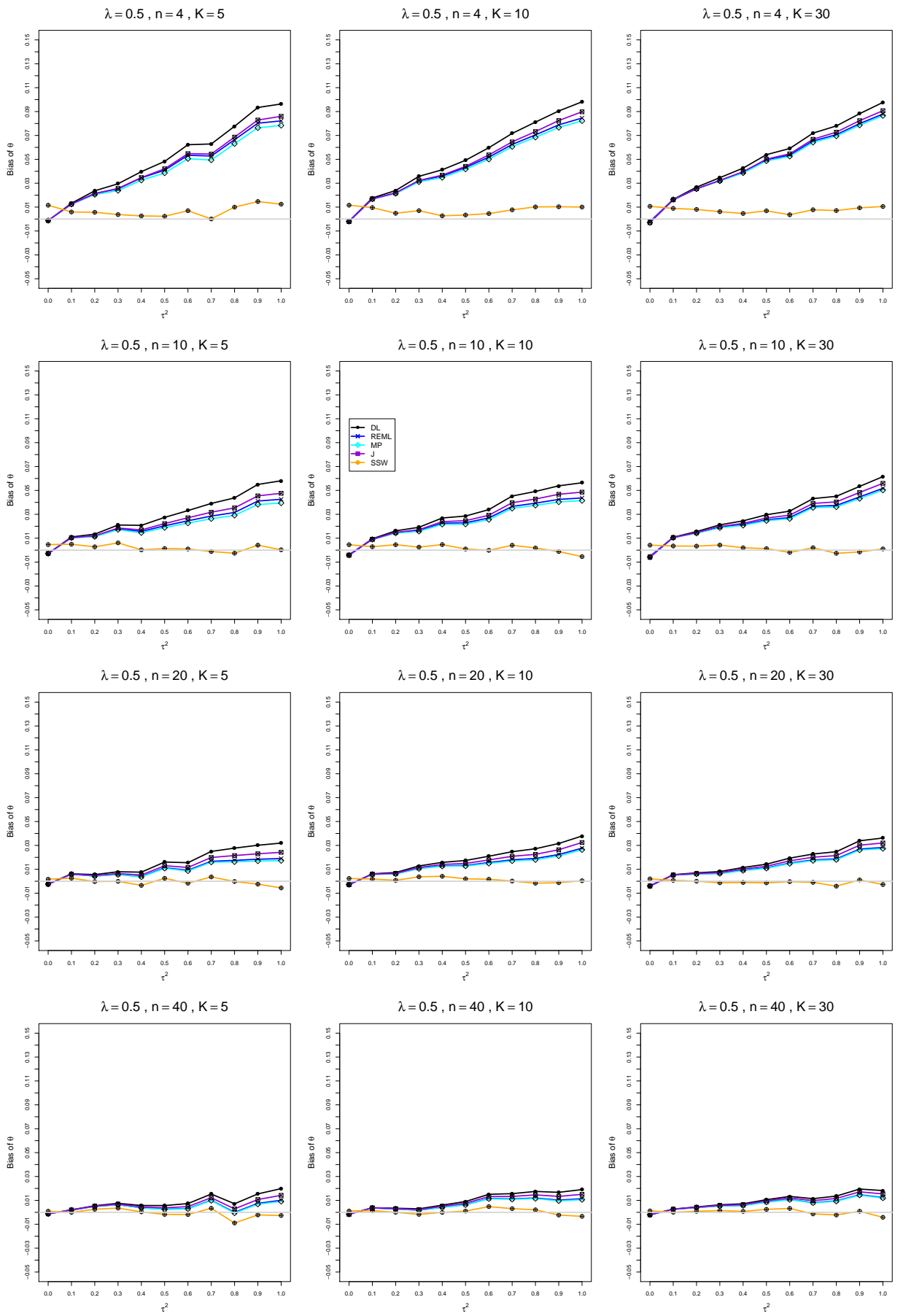

Figure B2.1.3: Bias of estimators of $\log (\operatorname{RoM}) \lambda$ when $\mu_{C}=4, \lambda=0.5, n=4,10,20,40$, and $K=5,10,30$ 

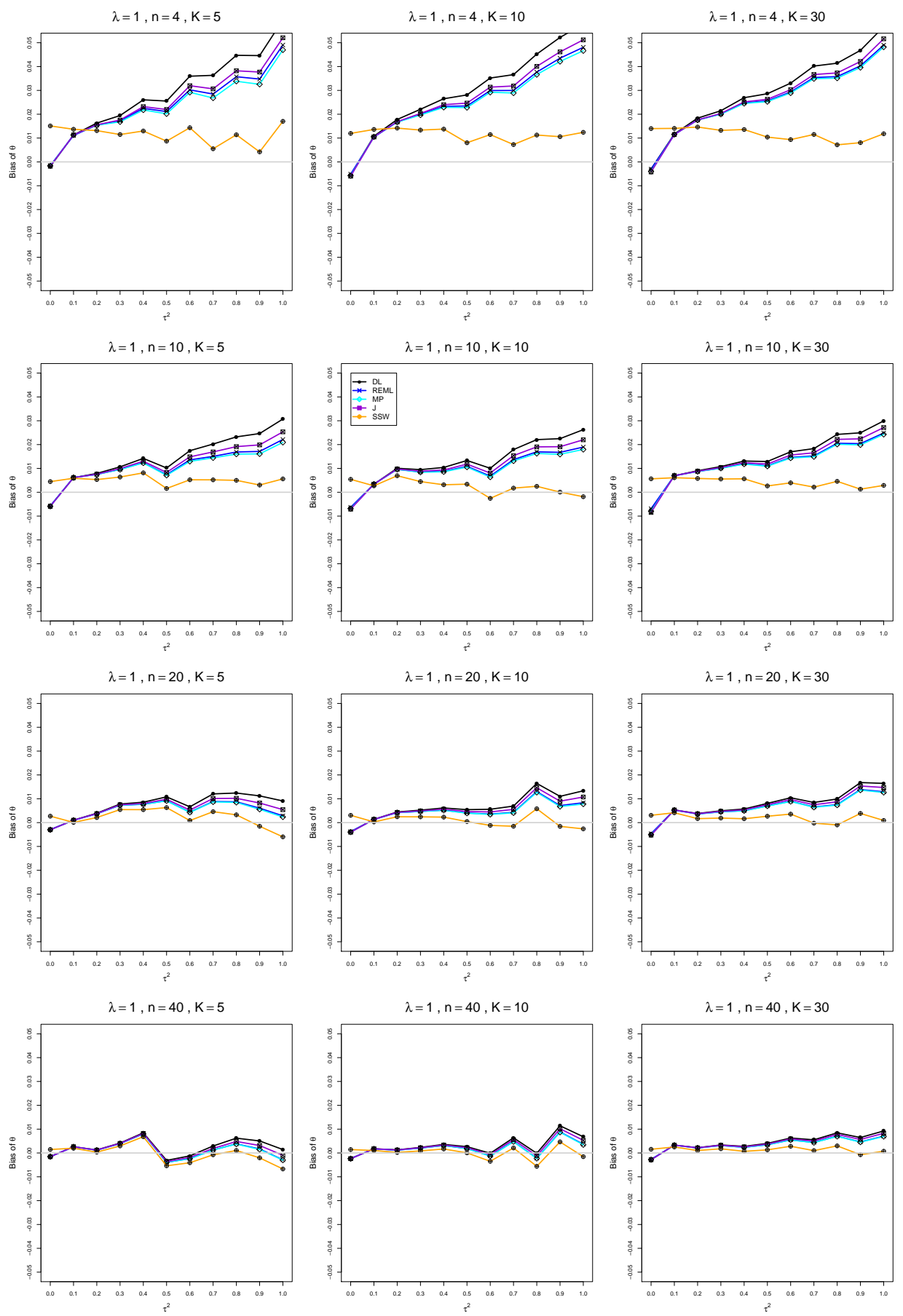

Figure B2.1.4: Bias of estimators of $\log (\operatorname{RoM}) \lambda$ when $\mu_{C}=4, \lambda=1, n=4,10,20,40$, and $K=5,10,30$ 

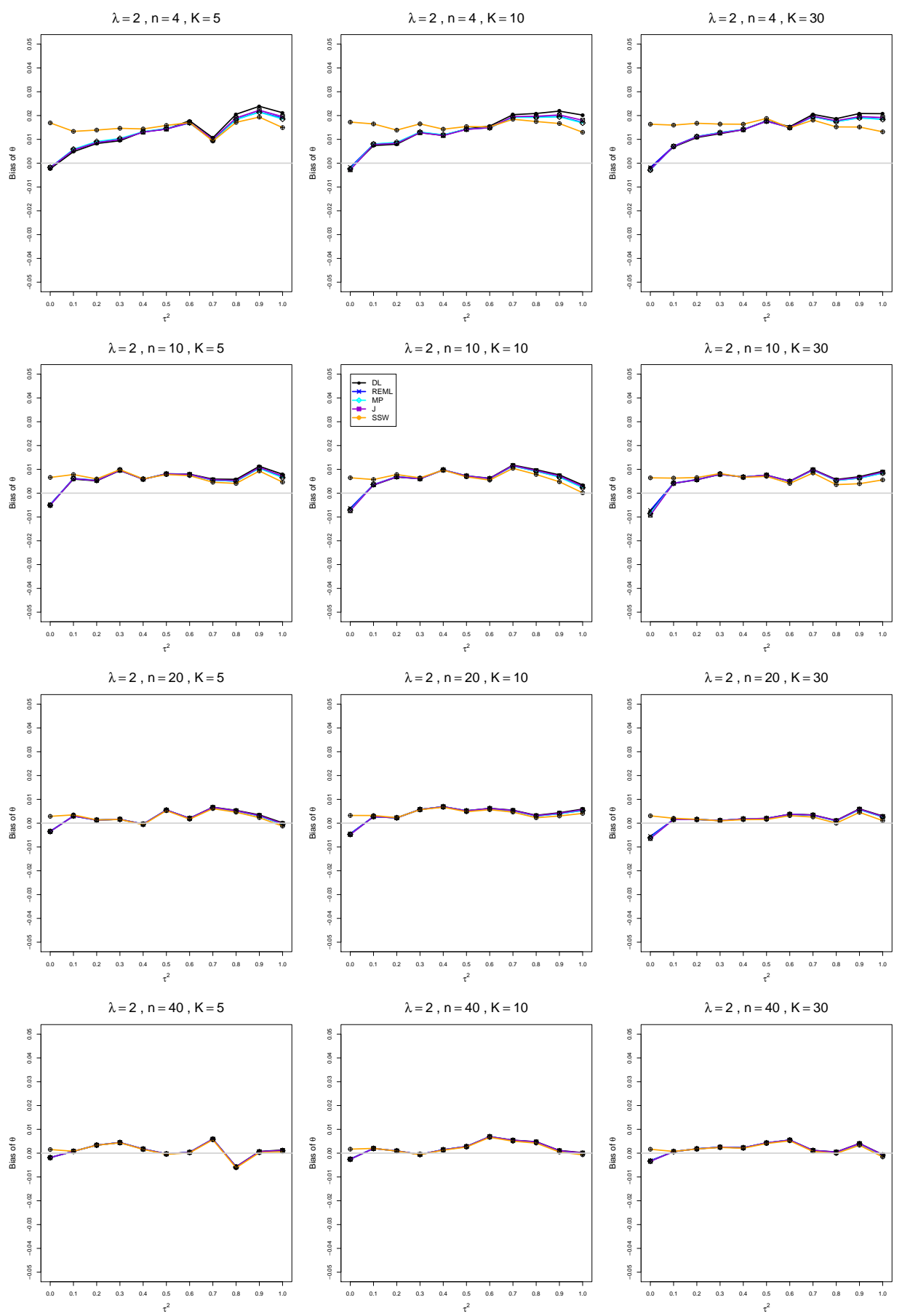

Figure B2.1.5: Bias of estimators of $\log (\operatorname{RoM}) \lambda$ when $\mu_{C}=4, \lambda=2, n=4,10,20,40$, and $K=5,10,30$ 


\section{B2.2 Coverage of interval estimators of $\lambda$}

Each figure corresponds to a value of $\lambda(=0,0.2,0.5,1,2)$, a set of values of $n(=4$, $10,20,40)$, and a set of values of $K(=5,10,30)$.

Each panel corresponds to a value of $n$ and a value of $K$ and has $\tau^{2}=0.0(0.1) 1.0$ on the horizontal axis.

The interval estimators of $\lambda$ are the companions to the inverse-variance-weighted point estimators

- DL (DerSimonian-Laird)

- REML (restricted maximum likelihood)

- MP (Mandel-Paule)

- J (Jackson)

and

- HKSJ (Hartung-Knapp-Sidik-Jonkman)

- HKSJ MP (HKSJ with MP estimator of $\tau^{2}$ )

- SSW MP (SSW as center and half-width equal to critical value from $t_{K-1}$ times estimated standard deviation of SSW with $\hat{\tau}^{2}=\hat{\tau}_{M P}^{2}$ 

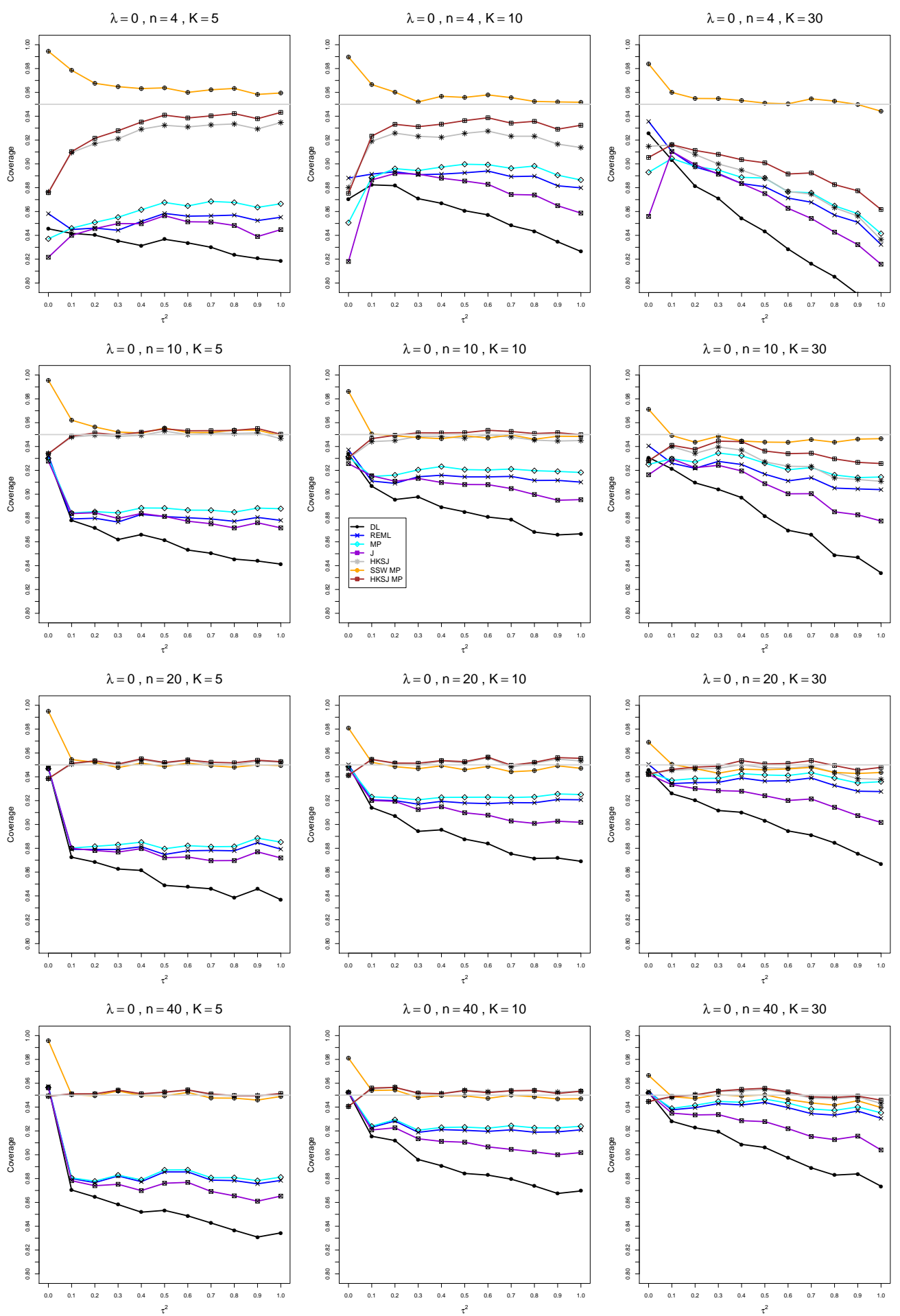

Figure B2.2.1: Coverage of $95 \%$ confidence intervals for $\lambda$ when $\mu_{C}=4, \lambda=0, n=$ 4, 10, 20, 40, and $K=5,10,30$ 

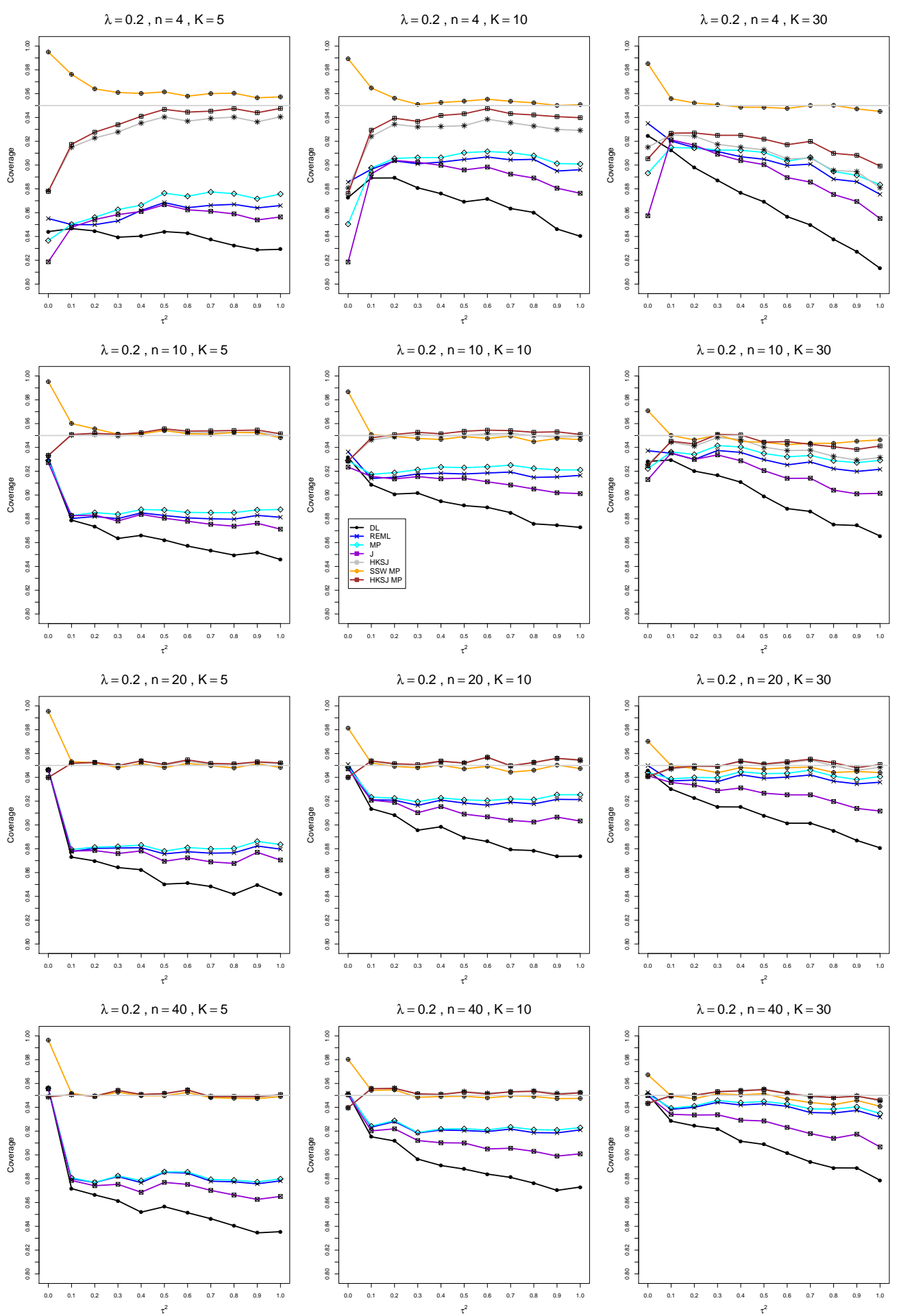

Figure B2.2.2: Coverage of $95 \%$ confidence intervals for $\lambda$ when $\mu_{C}=4, \lambda=0.2, n=$ 4, 10, 20, 40, and $K=5,10,30$ 

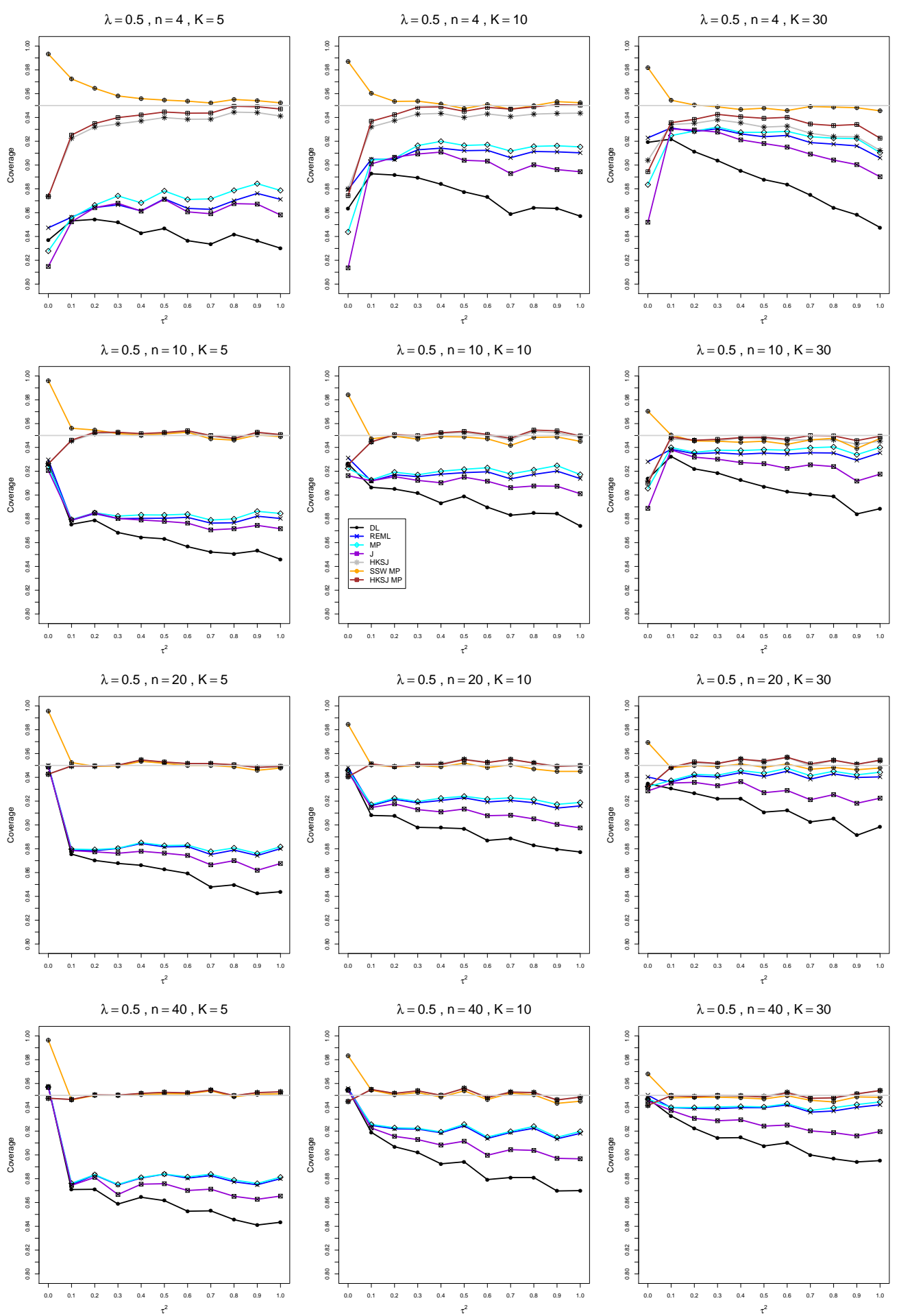

Figure B2.2.3: Coverage of $95 \%$ confidence intervals for $\lambda$ when $\mu_{C}=4, \lambda=0.5, n=$ 4, 10, 20, 40, and $K=5,10,30$ 

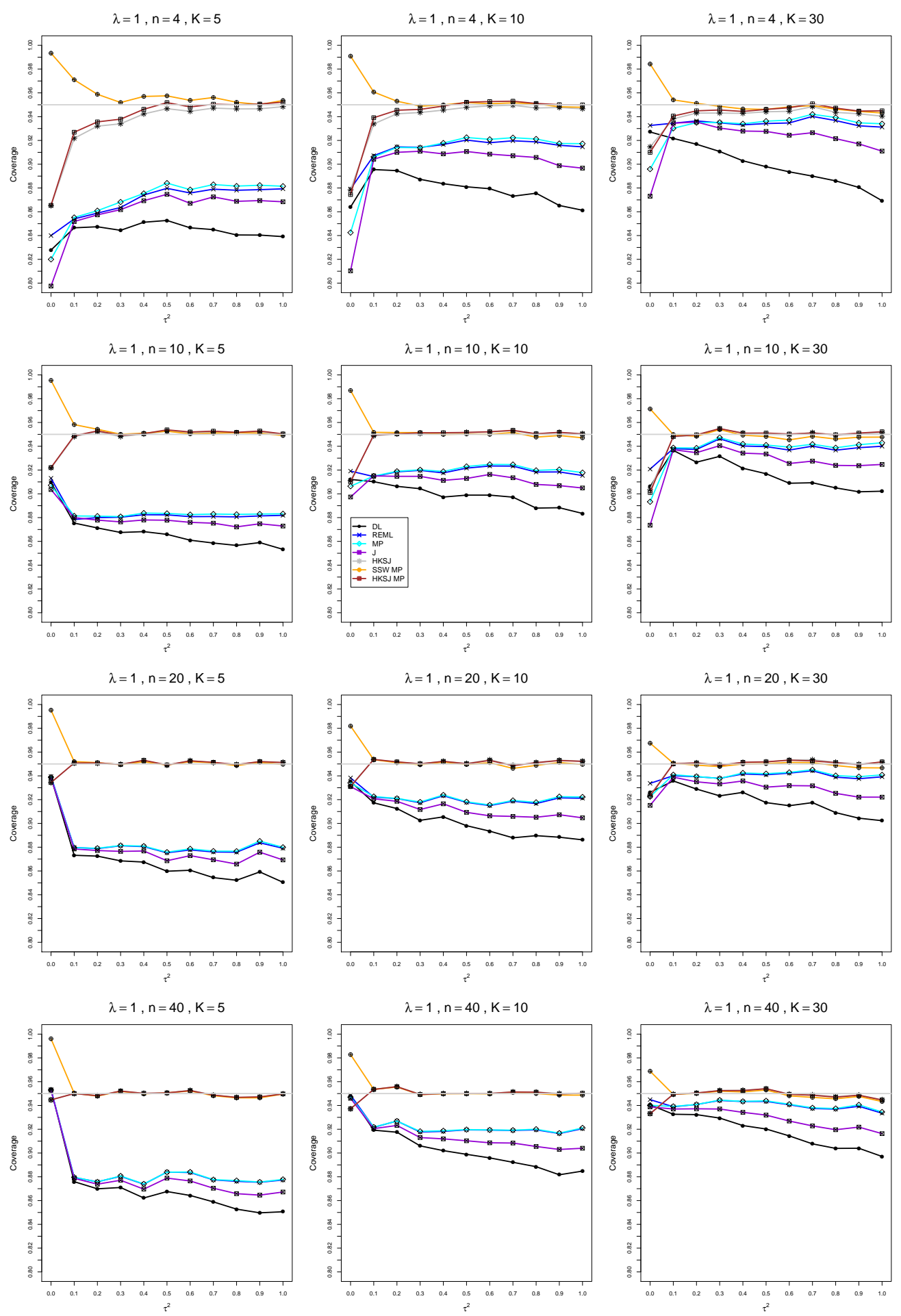

Figure B2.2.4: Coverage of $95 \%$ confidence intervals for $\lambda$ when $\mu_{C}=4, \lambda=1, n=$ 4, 10, 20, 40, and $K=5,10,30$ 

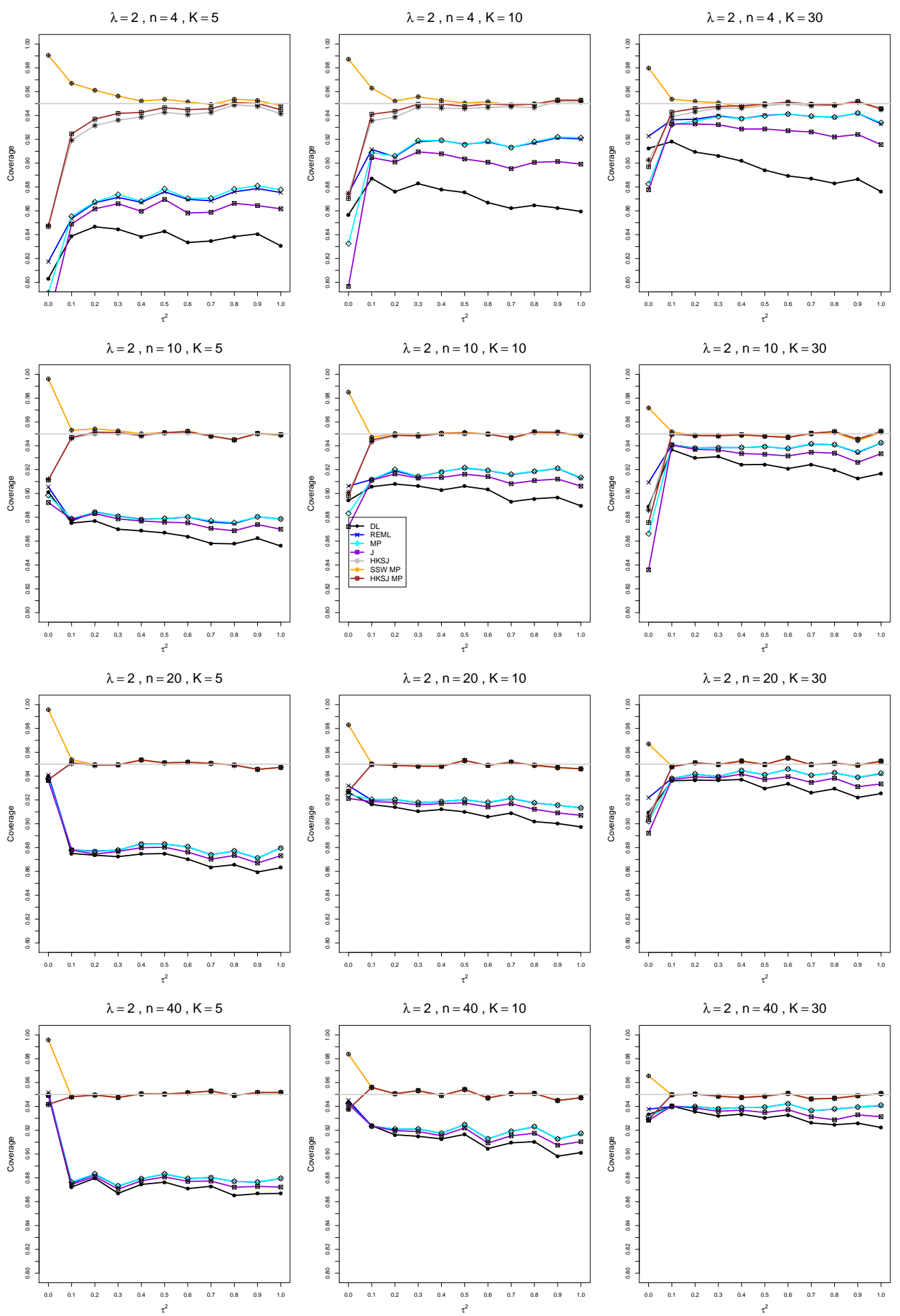

Figure B2.2.5: Coverage of $95 \%$ confidence intervals for $\lambda$ when $\mu_{C}=4, \lambda=2, n=$ 4, 10, 20, 40, and $K=5,10,30$ 
B3. Normal model, bias-corrected $\lambda, \mu_{C}=4, n=$ $4,10,20,40, K=5,10,30$

\section{B3.1 Bias of point estimators of $\lambda$ when $\mu_{C}=4$}

Each figure corresponds to a value of $\lambda(=0,0.2,0.5,1,2)$, a set of values of $n(=4$, $10,20,40)$, and a set of values of $K(=5,10,30)$.

Each panel corresponds to a value of $n$ and a value of $K$ and has $\tau^{2}=0.0(0.1) 1.0$ on the horizontal axis.

The point estimators of $\lambda$ are

- DL (DerSimonian-Laird)

- REML (restricted maximum likelihood)

- MP (Mandel-Paule)

- J (Jackson)

- SSW (sample-size weighted) 

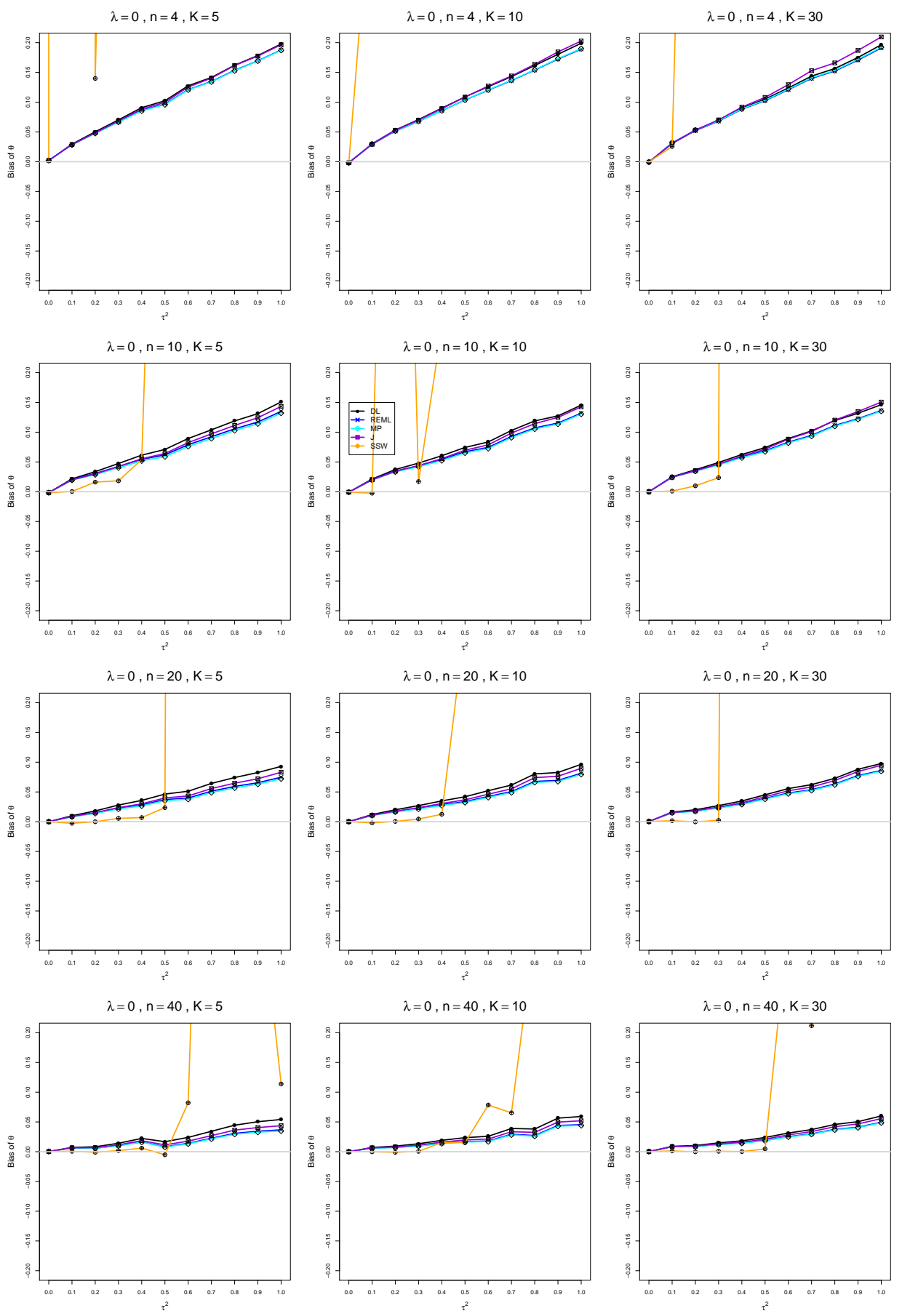

Figure B3.1.1: Bias of estimators of $\log (\operatorname{RoM}) \lambda$ when $\mu_{C}=4, \lambda=0, n=4,10,20,40$, and $K=5,10,30$ 

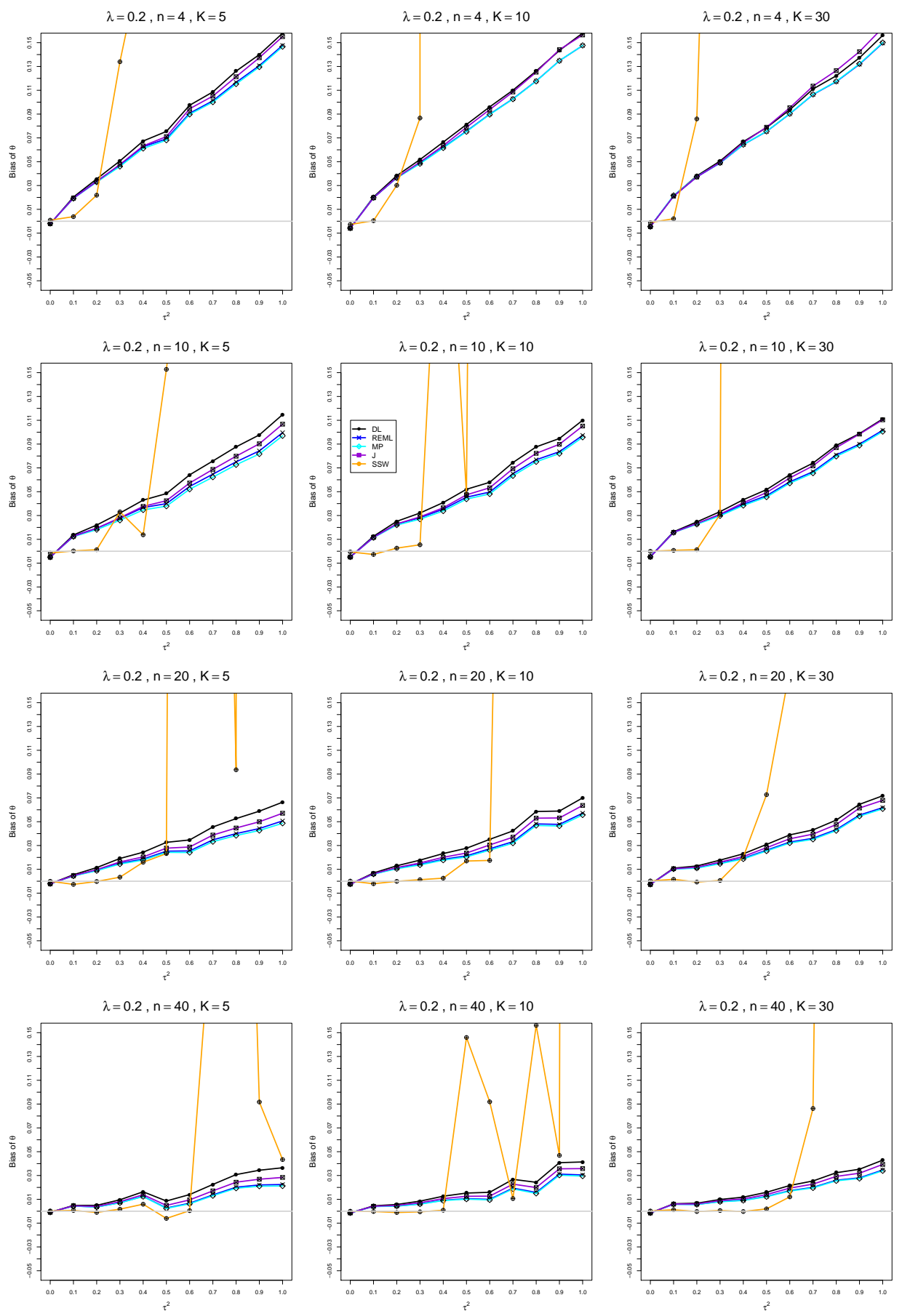

Figure B3.1.2: Bias of estimators of $\log (\mathrm{RoM}) \lambda$ when $\mu_{C}=4, \lambda=0.2, n=4,10,20,40$, and $K=5,10,30$ 

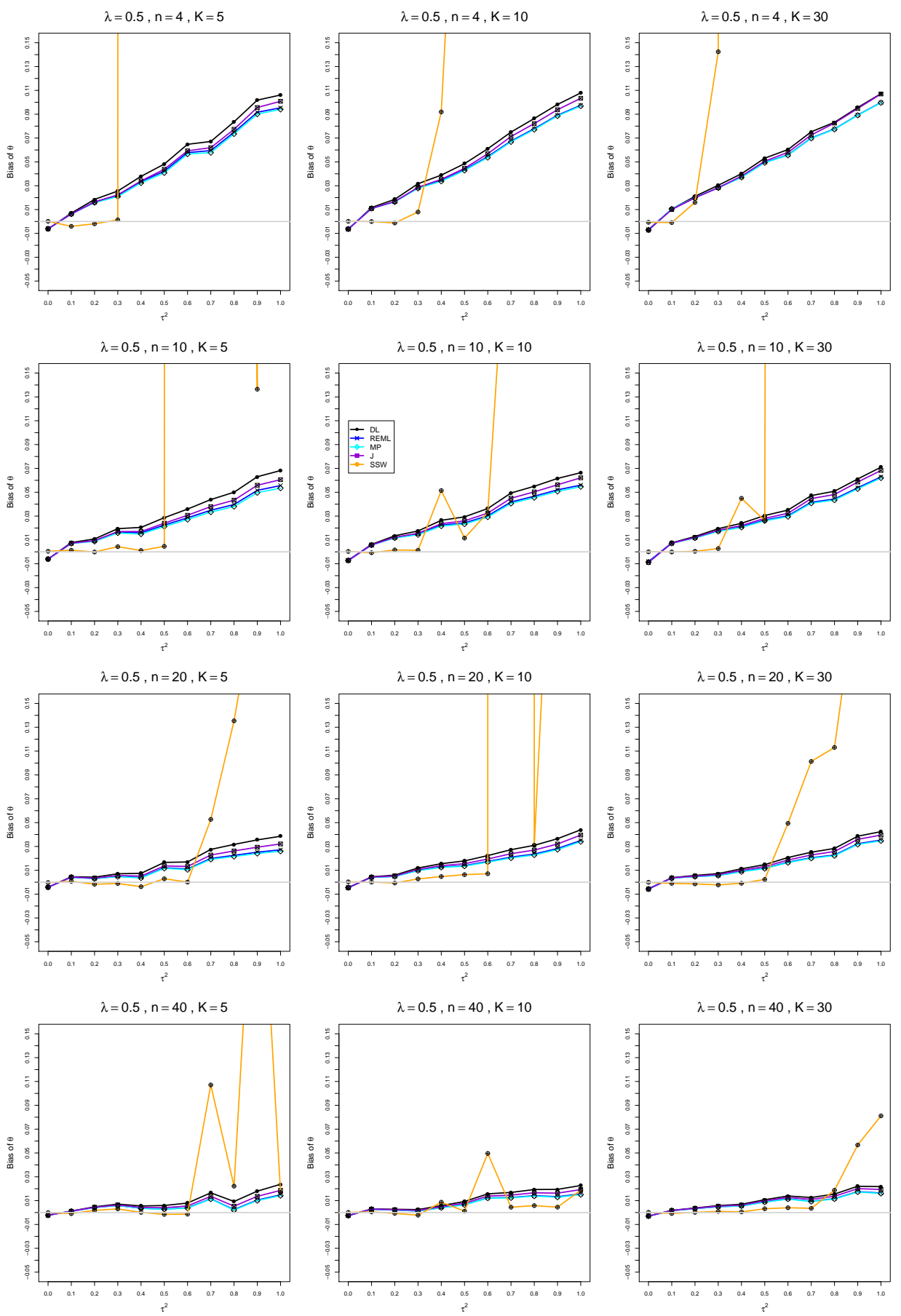

Figure B3.1.3: Bias of estimators of $\log (\operatorname{RoM}) \lambda$ when $\mu_{C}=4, \lambda=0.5, n=4,10,20,40$, and $K=5,10,30$ 

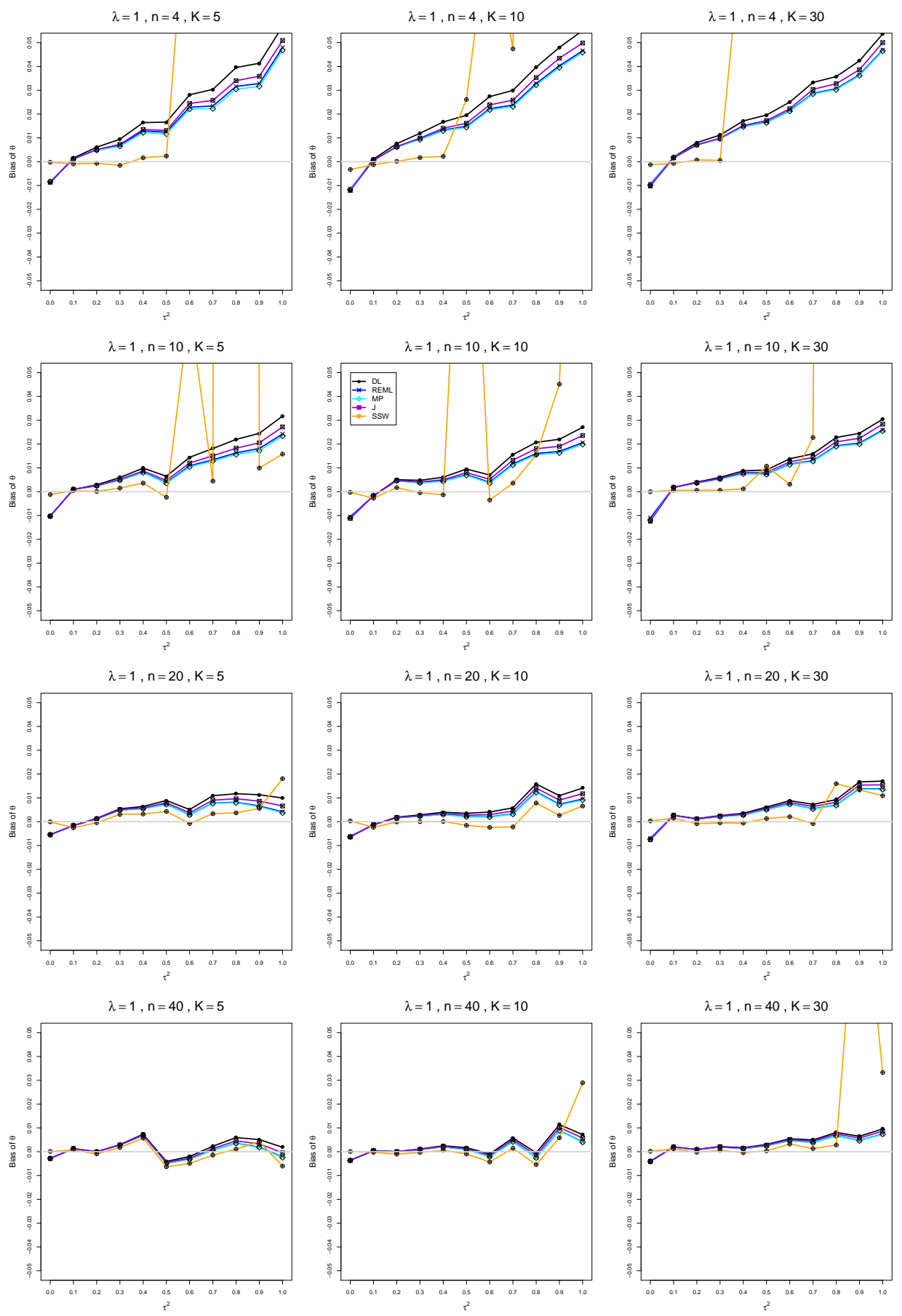

Figure B3.1.4: Bias of estimators of $\log (\operatorname{RoM}) \lambda$ when $\mu_{C}=4, \lambda=1, n=4,10,20,40$, and $K=5,10,30$ 

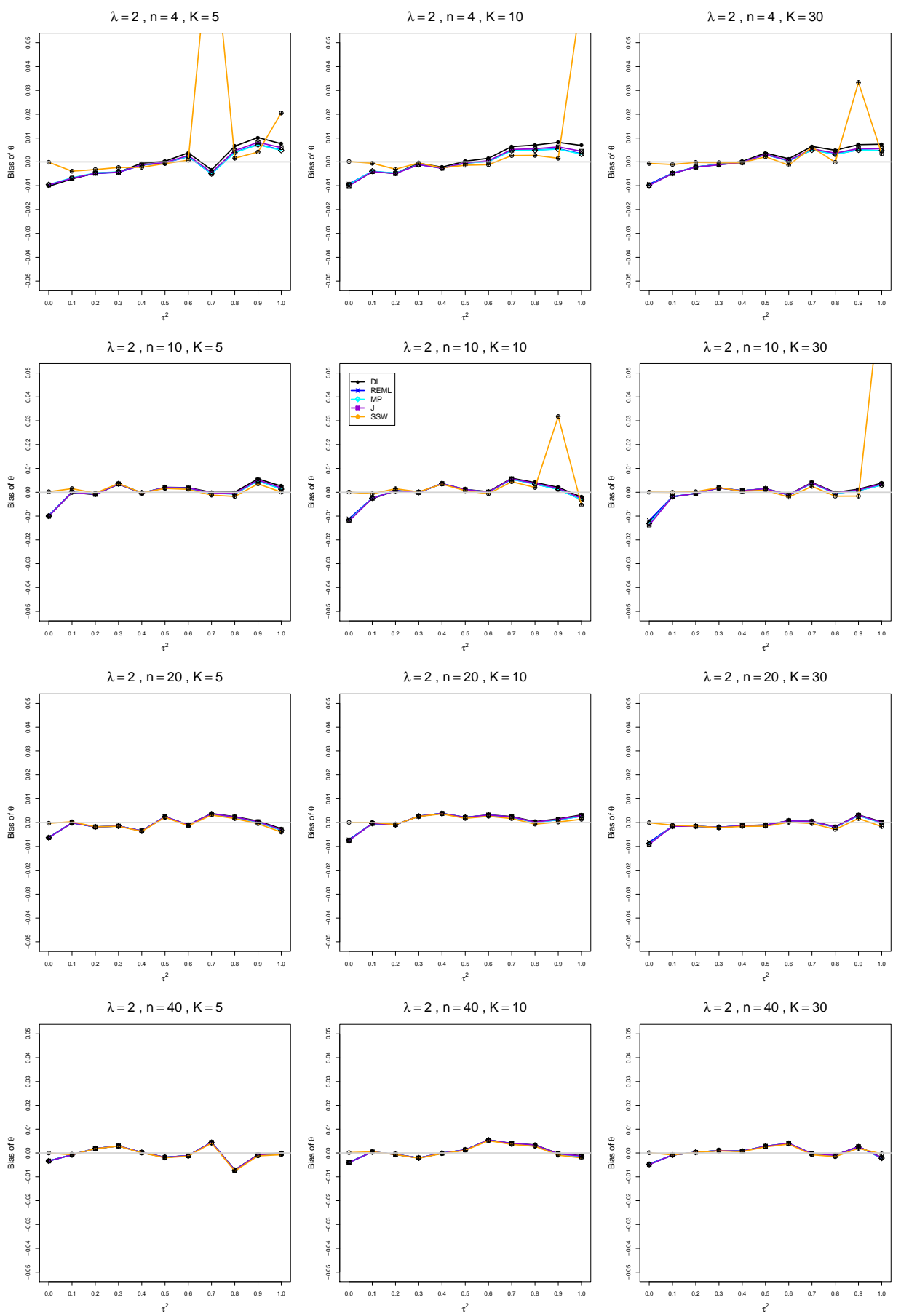

Figure B3.1.5: Bias of estimators of $\log (\operatorname{RoM}) \lambda$ when $\mu_{C}=4, \lambda=2, n=4,10,20,40$, and $K=5,10,30$ 


\section{B3.2 Coverage of interval estimators of $\lambda$}

Each figure corresponds to a value of $\lambda(=0,0.2,0.5,1,2)$, a set of values of $n(=4$, $10,20,40)$, and a set of values of $K(=5,10,30)$.

Each panel corresponds to a value of $n$ and a value of $K$ and has $\tau^{2}=0.0(0.1) 1.0$ on the horizontal axis.

The interval estimators of $\lambda$ are the companions to the inverse-variance-weighted point estimators

- DL (DerSimonian-Laird)

- REML (restricted maximum likelihood)

- MP (Mandel-Paule)

- J (Jackson)

and

- HKSJ (Hartung-Knapp-Sidik-Jonkman)

- HKSJ MP (HKSJ with MP estimator of $\tau^{2}$ )

- SSW MP (SSW as center and half-width equal to critical value from $t_{K-1}$ times estimated standard deviation of SSW with $\hat{\tau}^{2}=\hat{\tau}_{M P}^{2}$ 

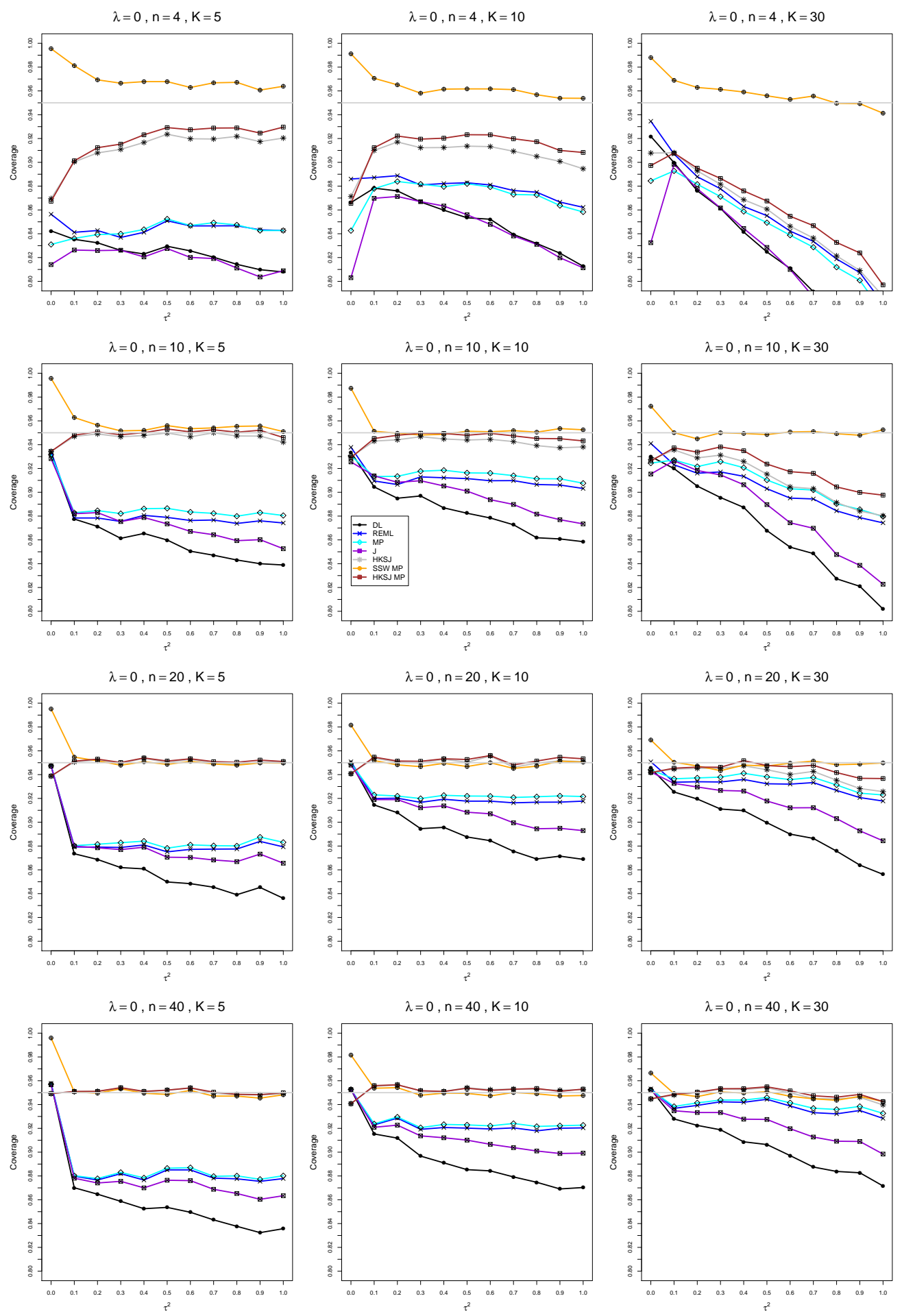

Figure B3.2.1: Coverage of $95 \%$ confidence intervals for $\lambda$ when $\mu_{C}=4, \lambda=0, n=$ 4, 10, 20, 40, and $K=5,10,30$ 

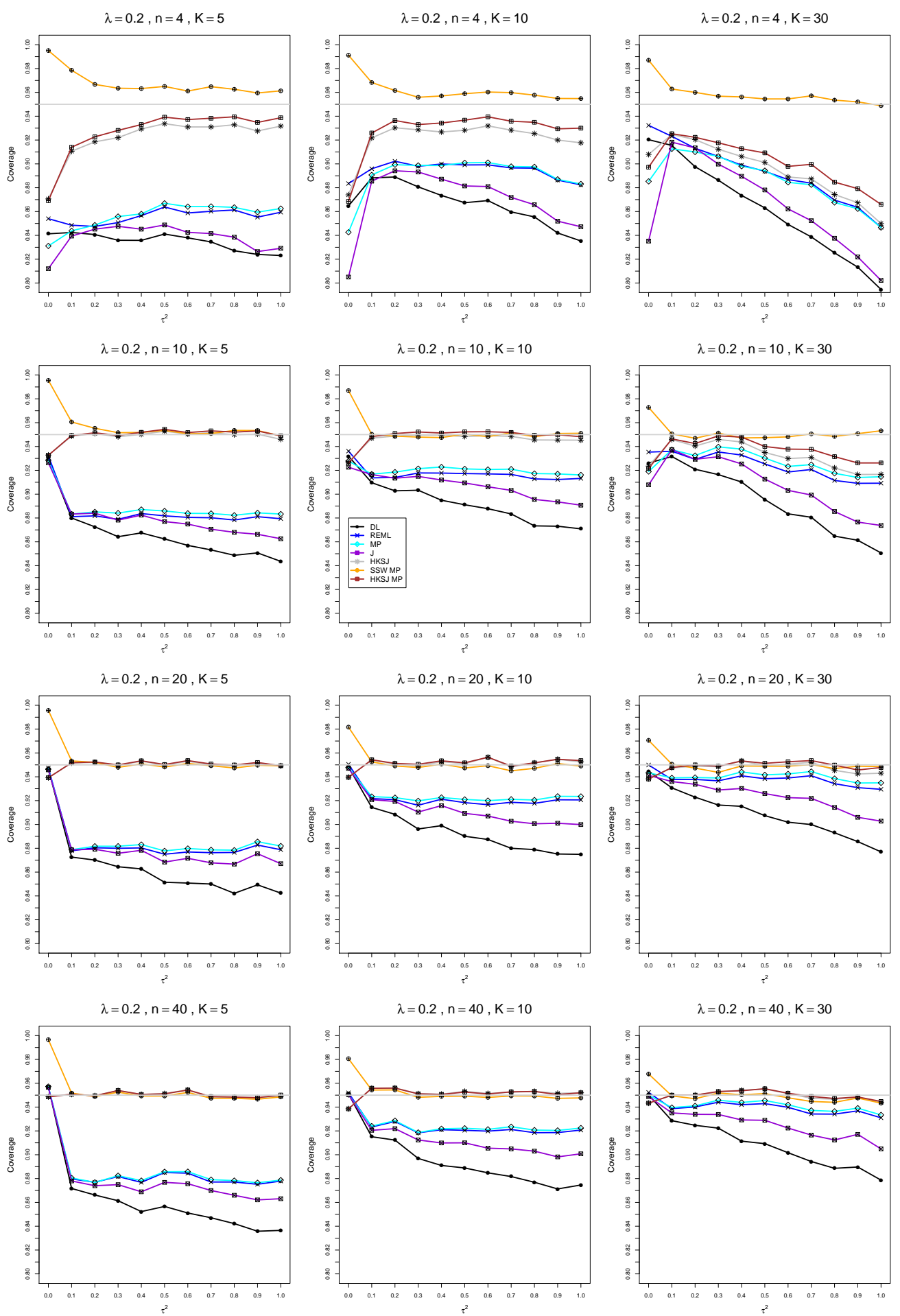

Figure B3.2.2: Coverage of $95 \%$ confidence intervals for $\lambda$ when $\mu_{C}=4, \lambda=0.2, n=$ 4, 10, 20, 40, and $K=5,10,30$ 

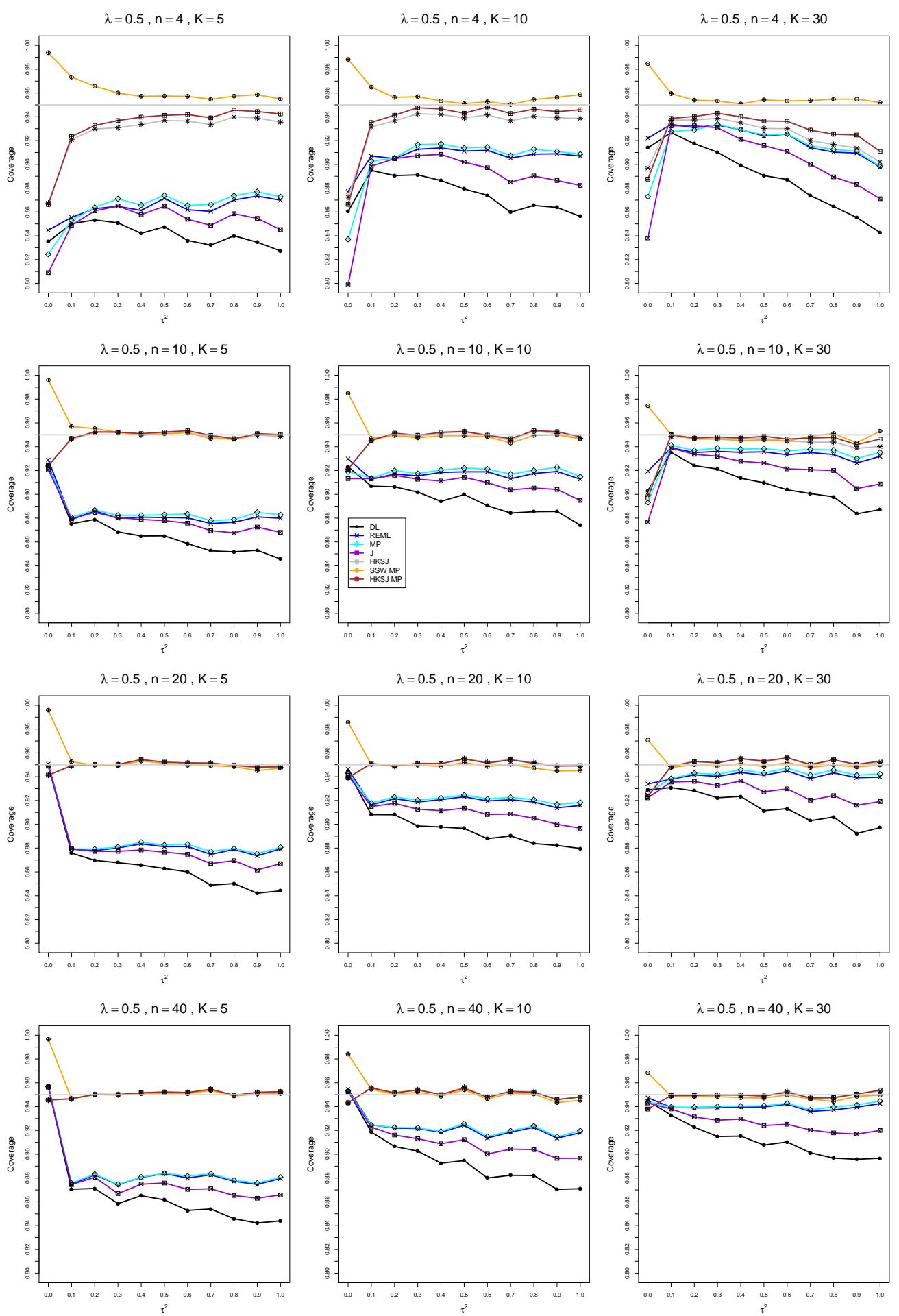

Figure B3.2.3: Coverage of $95 \%$ confidence intervals for $\lambda$ when $\mu_{C}=4, \lambda=0.5, n=$ 4, 10, 20, 40, and $K=5,10,30$ 

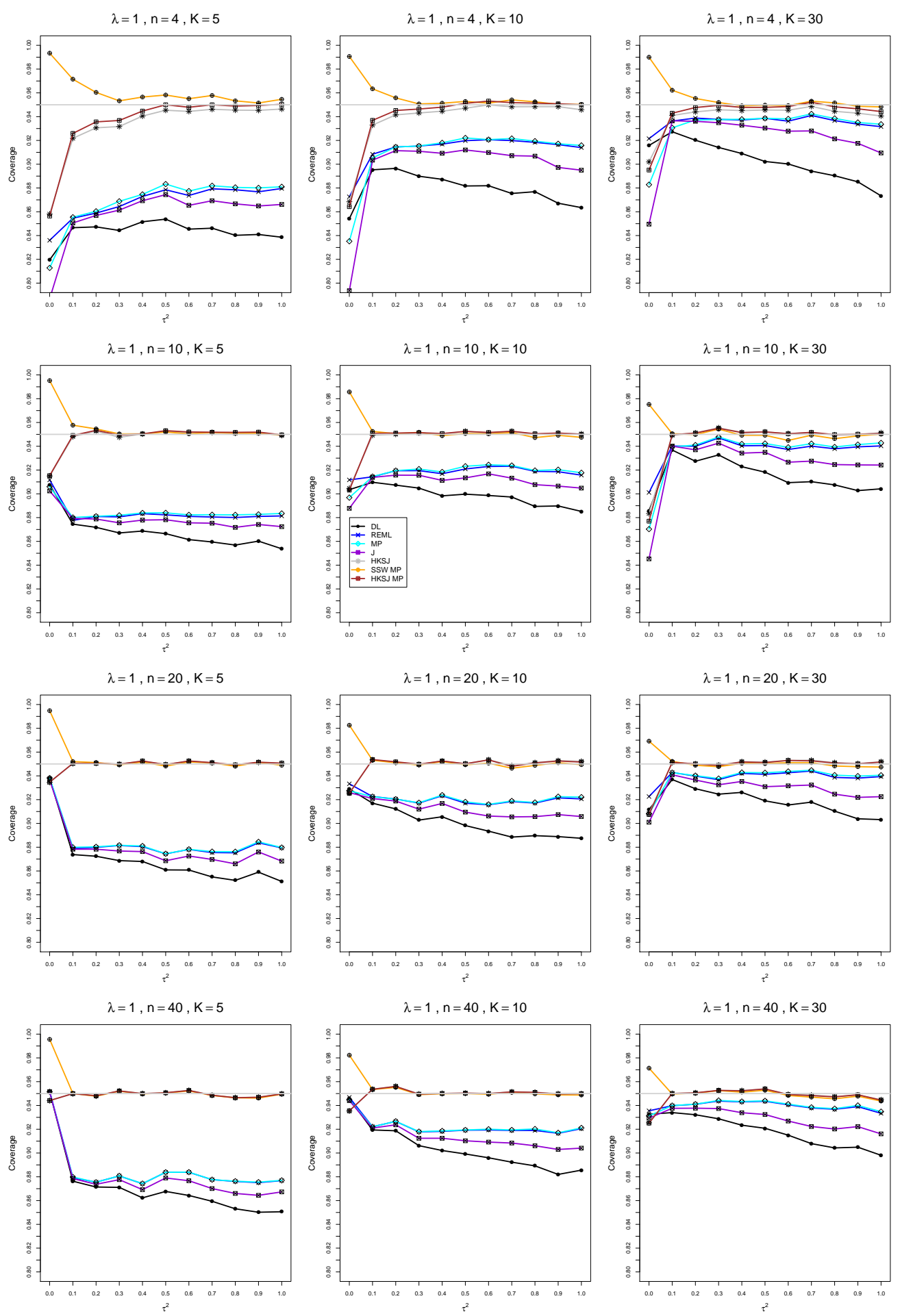

Figure B3.2.4: Coverage of $95 \%$ confidence intervals for $\lambda$ when $\mu_{C}=4, \lambda=1, n=$ 4, 10, 20, 40, and $K=5,10,30$ 

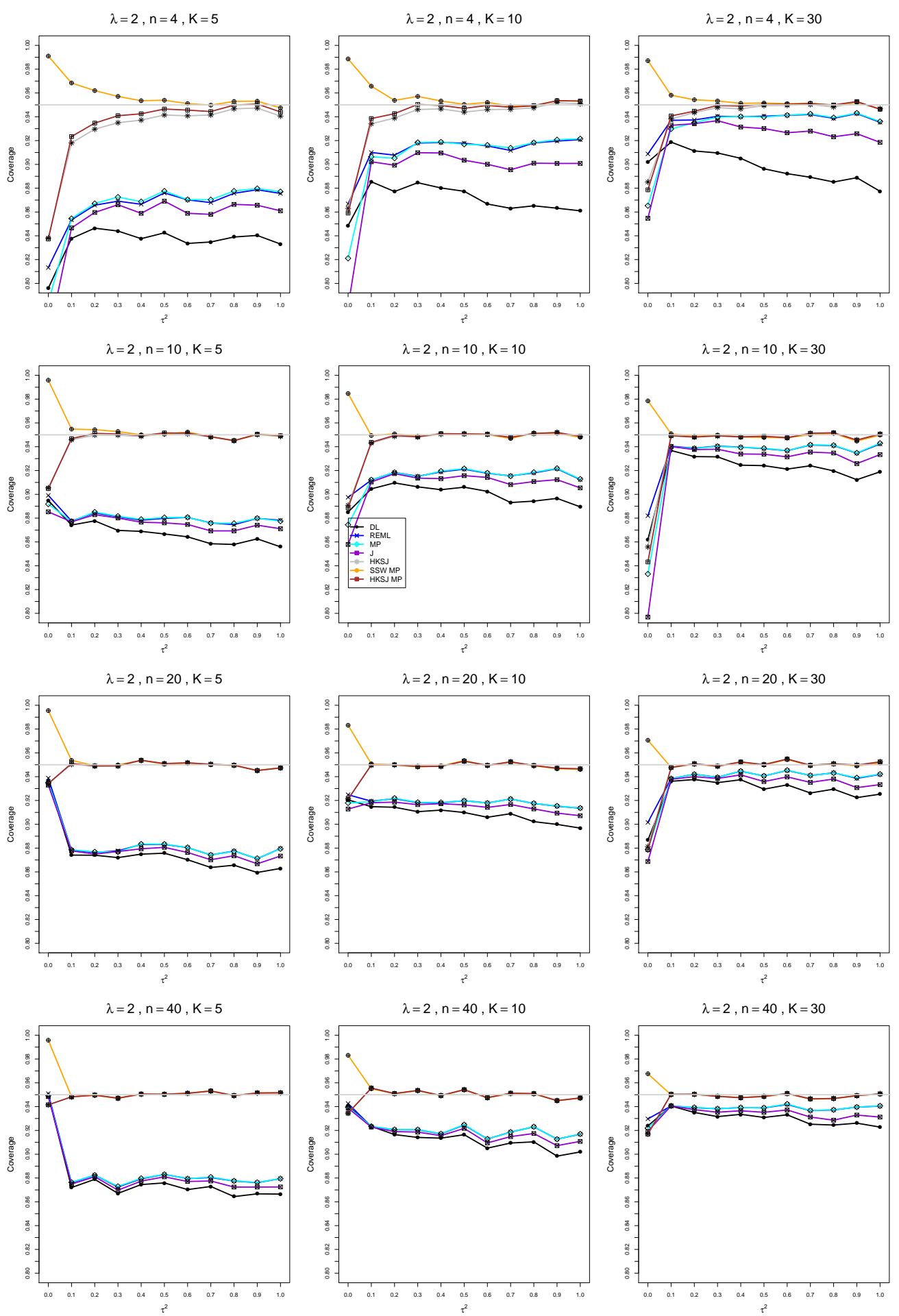

Figure B3.2.5: Coverage of $95 \%$ confidence intervals for $\lambda$ when $\mu_{C}=4, \lambda=2, n=$ 4, 10, 20, 40, and $K=5,10,30$ 
B4. Normal model, $\mu_{C}=1, n=4,10,20,40, K=$ $50,100,125$

\section{B4.1 Bias of point estimators of $\lambda$ when $\mu_{C}=1$}

Each figure corresponds to a value of $\lambda(=0,0.2,0.5,1,2)$, a set of values of $n(=4$, $10,20,40)$, and a set of values of $K(=50,100,125)$.

Each panel corresponds to a value of $n$ and a value of $K$ and has $\tau^{2}=0.0(0.1) 1.0$ on the horizontal axis.

The point estimators of $\lambda$ are

- DL (DerSimonian-Laird)

- REML (restricted maximum likelihood)

- MP (Mandel-Paule)

- J (Jackson)

- SSW (sample-size weighted) 

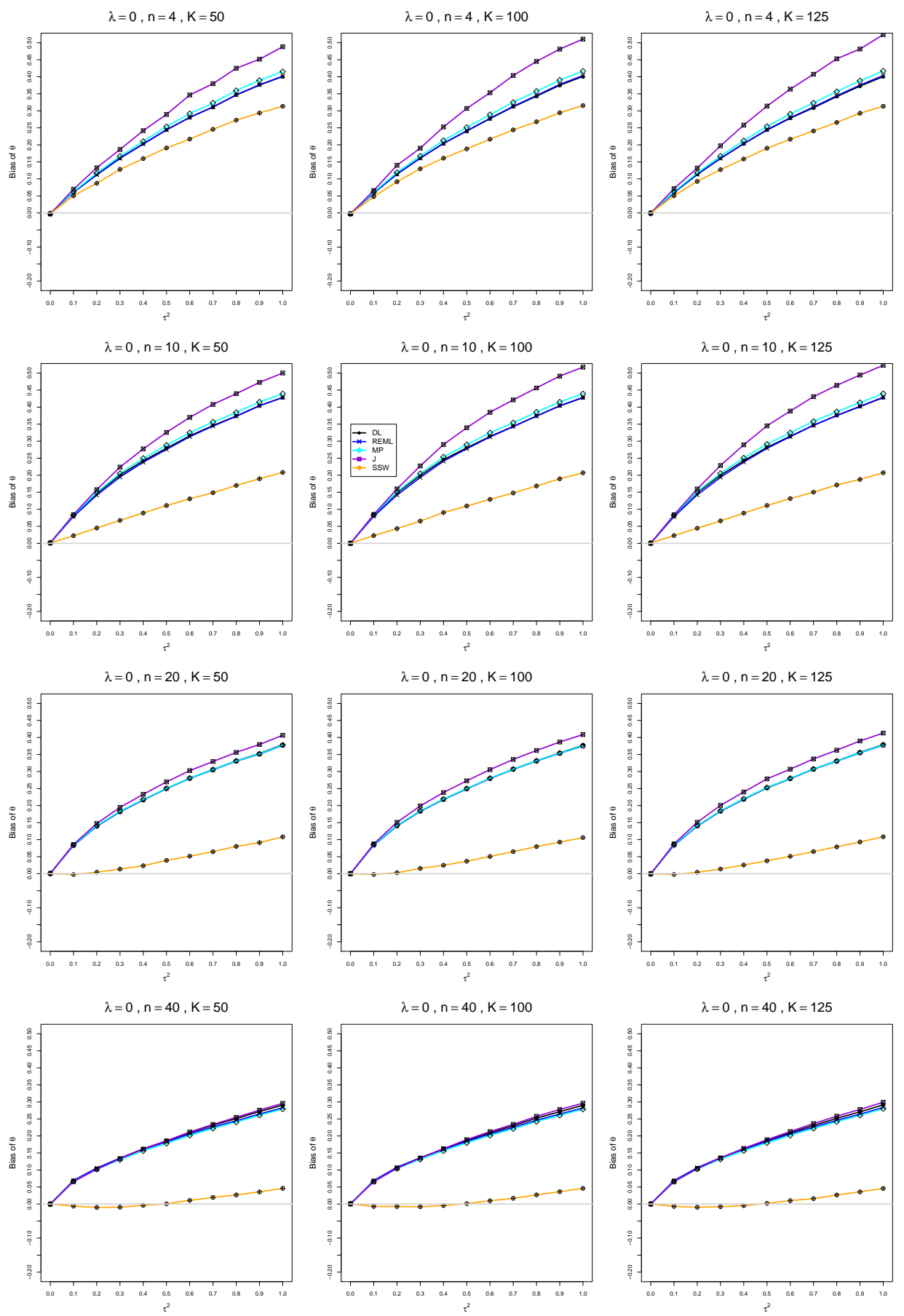

Figure B4.1.1: Bias of estimators of $\log (\operatorname{RoM}) \lambda$ when $\mu_{C}=1, \lambda=0, n=4,10,20,40$, and $K=50,100,125$ 

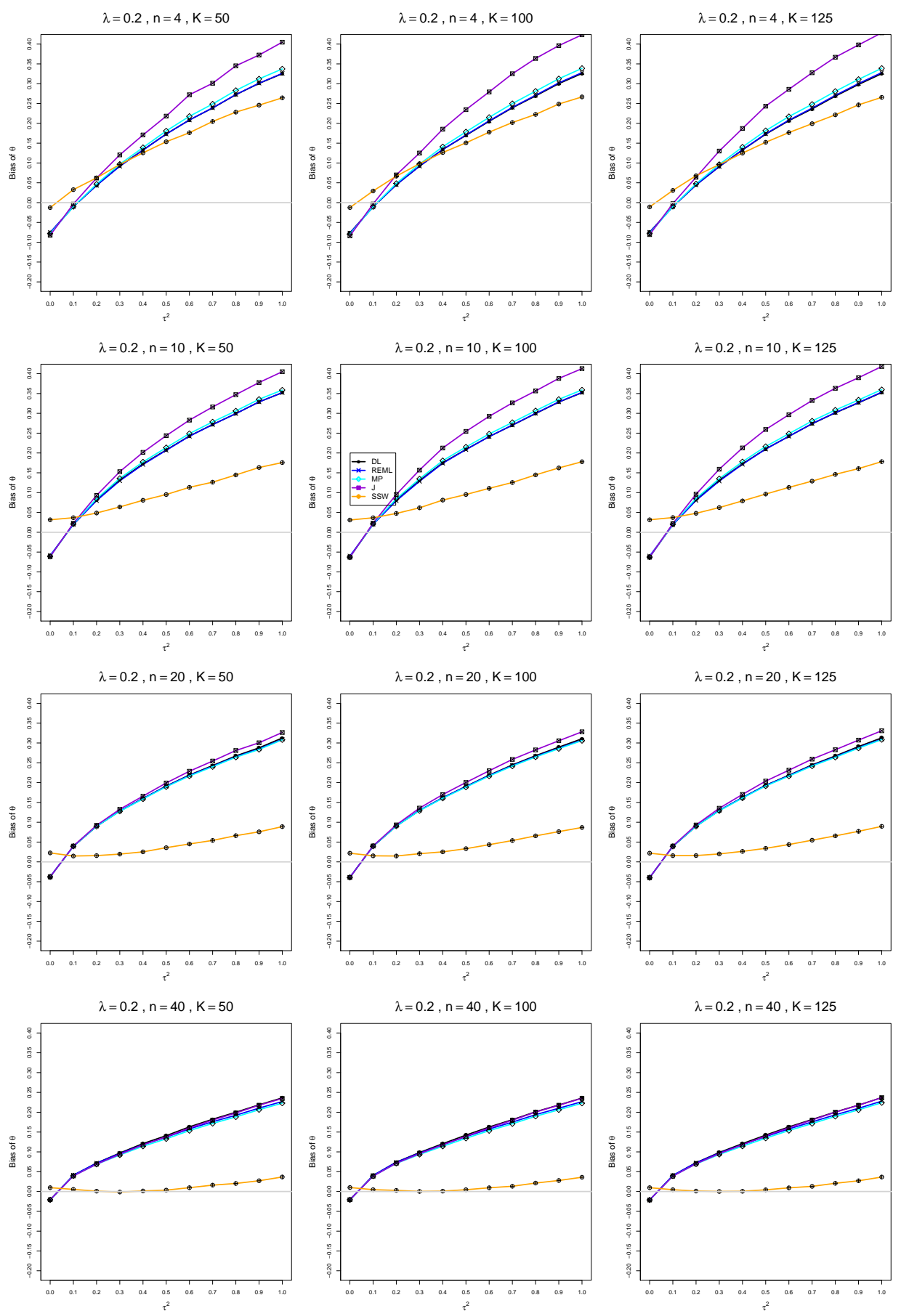

Figure B4.1.2: Bias of estimators of $\log (\mathrm{RoM}) \lambda$ when $\mu_{C}=1, \lambda=0.2, n=4,10,20,40$, and $K=50,100,125$ 

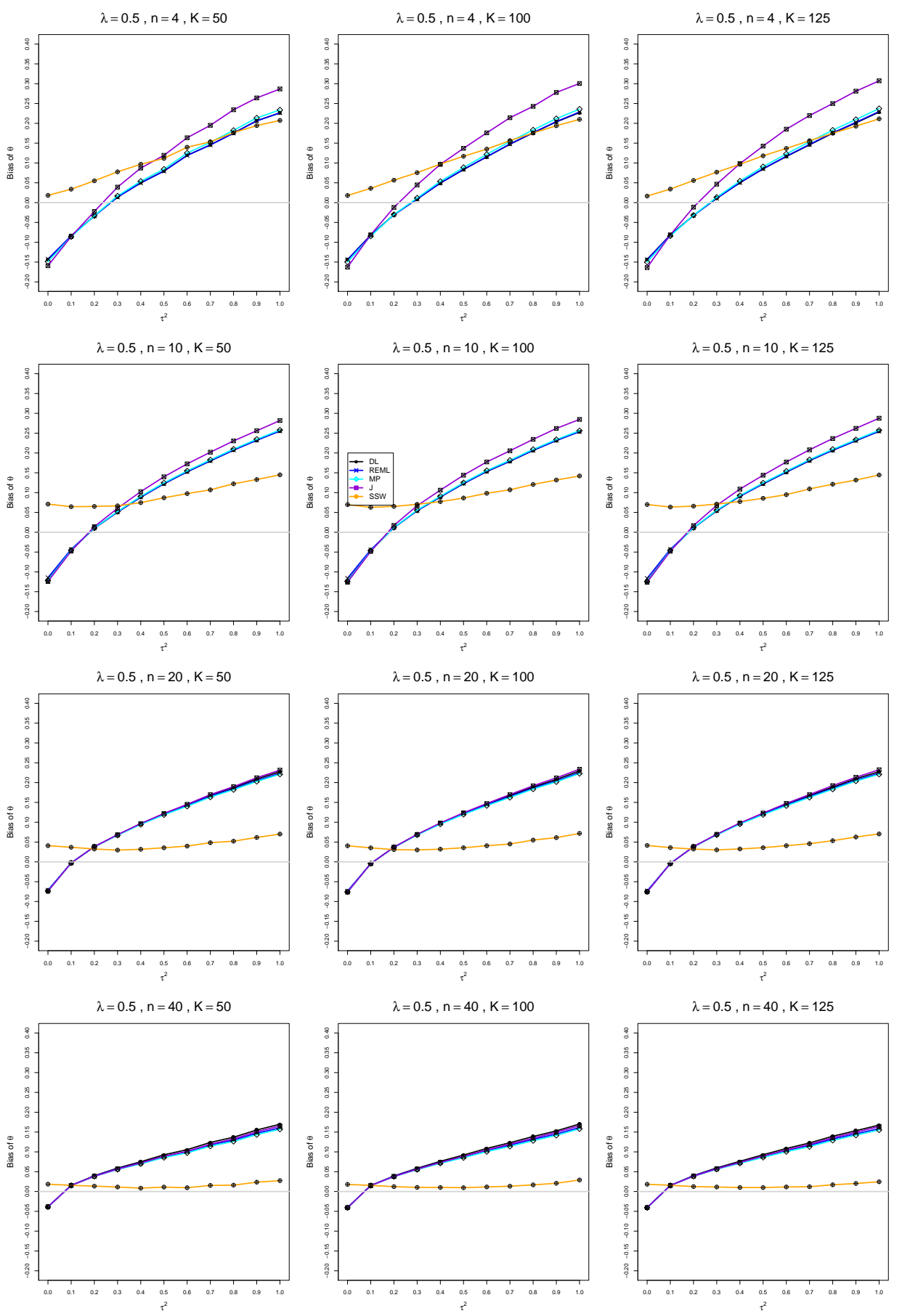

Figure B4.1.3: Bias of estimators of $\log (\mathrm{RoM}) \lambda$ when $\mu_{C}=1, \lambda=0.5, n=4,10,20,40$, and $K=50,100,125$ 

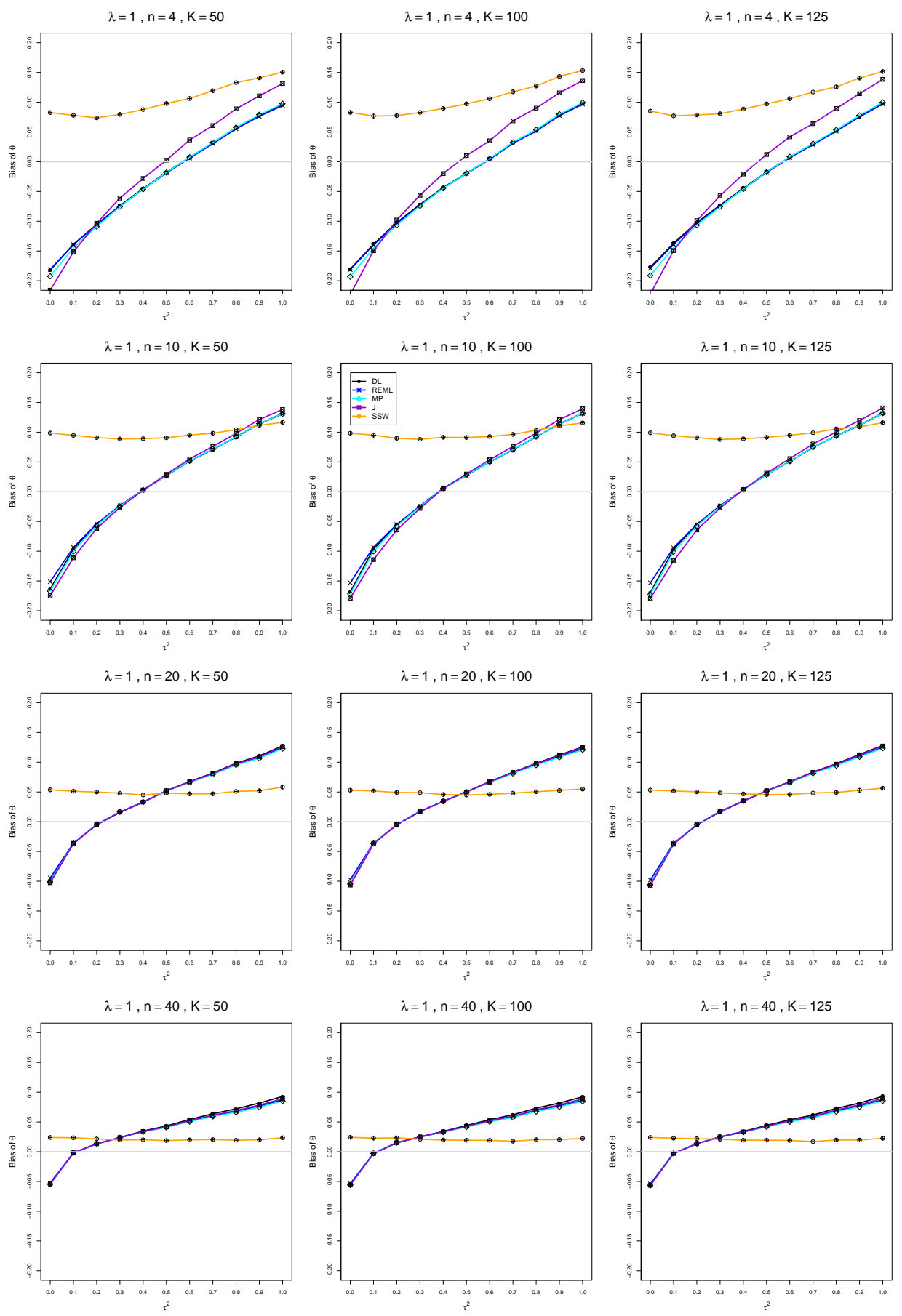

Figure B4.1.4: Bias of estimators of $\log (\operatorname{RoM}) \lambda$ when $\mu_{C}=1, \lambda=1, n=4,10,20,40$, and $K=50,100,125$ 

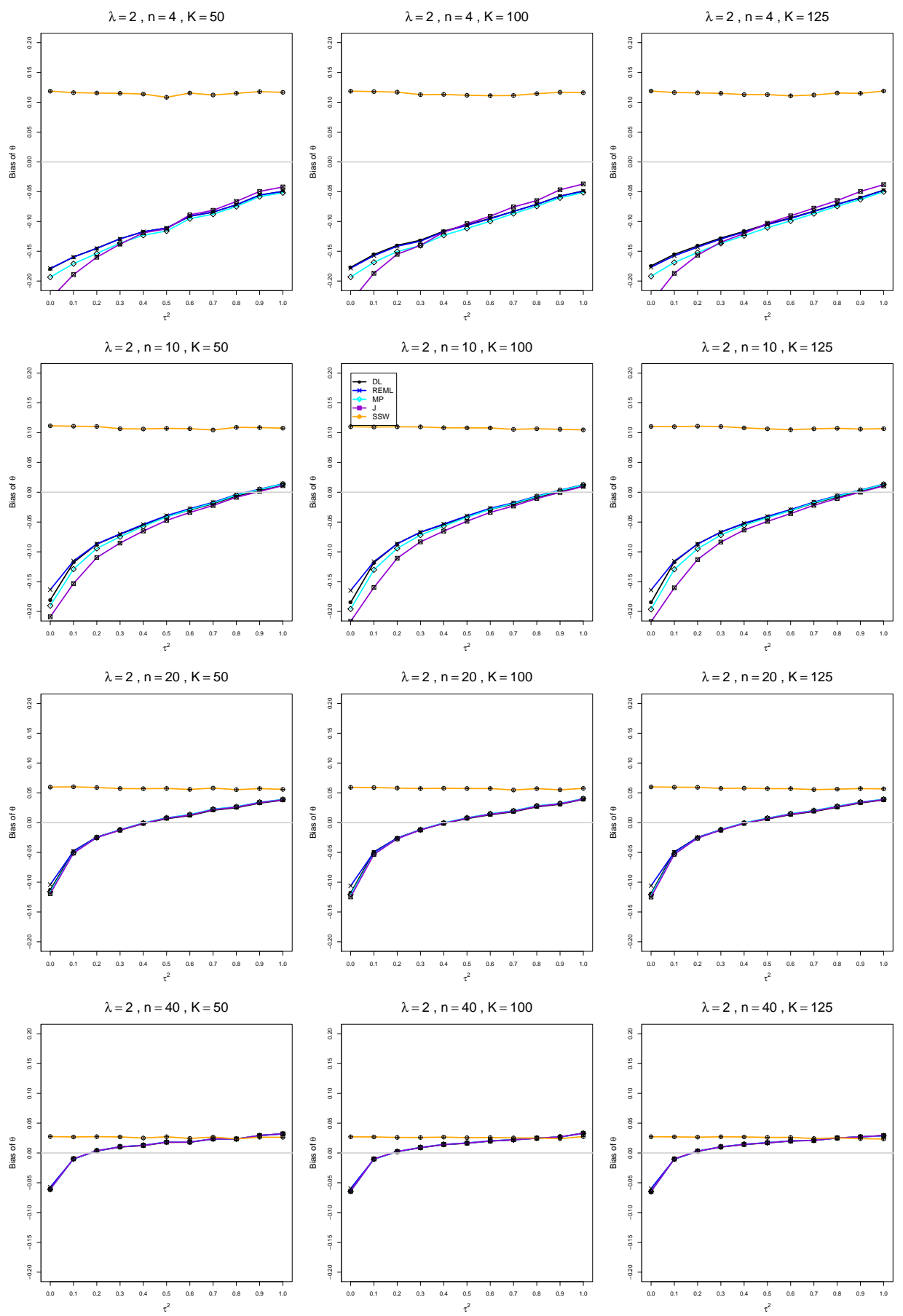

Figure B4.1.5: Bias of estimators of $\log (\operatorname{RoM}) \lambda$ when $\mu_{C}=1, \lambda=2, n=4,10,20,40$, and $K=50,100,125$ 


\section{B4.2 Coverage of interval estimators of $\lambda$}

Each figure corresponds to a value of $\lambda(=0,0.2,0.5,1,2)$, a set of values of $n(=4$, $10,20,40)$, and a set of values of $K(=50,100,125)$.

Each panel corresponds to a value of $n$ and a value of $K$ and has $\tau^{2}=0.0(0.1) 1.0$ on the horizontal axis.

The interval estimators of $\lambda$ are the companions to the inverse-variance-weighted point estimators

- DL (DerSimonian-Laird)

- REML (restricted maximum likelihood)

- MP (Mandel-Paule)

- J (Jackson)

and

- HKSJ (Hartung-Knapp-Sidik-Jonkman)

- HKSJ MP (HKSJ with MP estimator of $\tau^{2}$ )

- SSW MP (SSW as center and half-width equal to critical value from $t_{K-1}$ times estimated standard deviation of SSW with $\hat{\tau}^{2}=\hat{\tau}_{M P}^{2}$ 

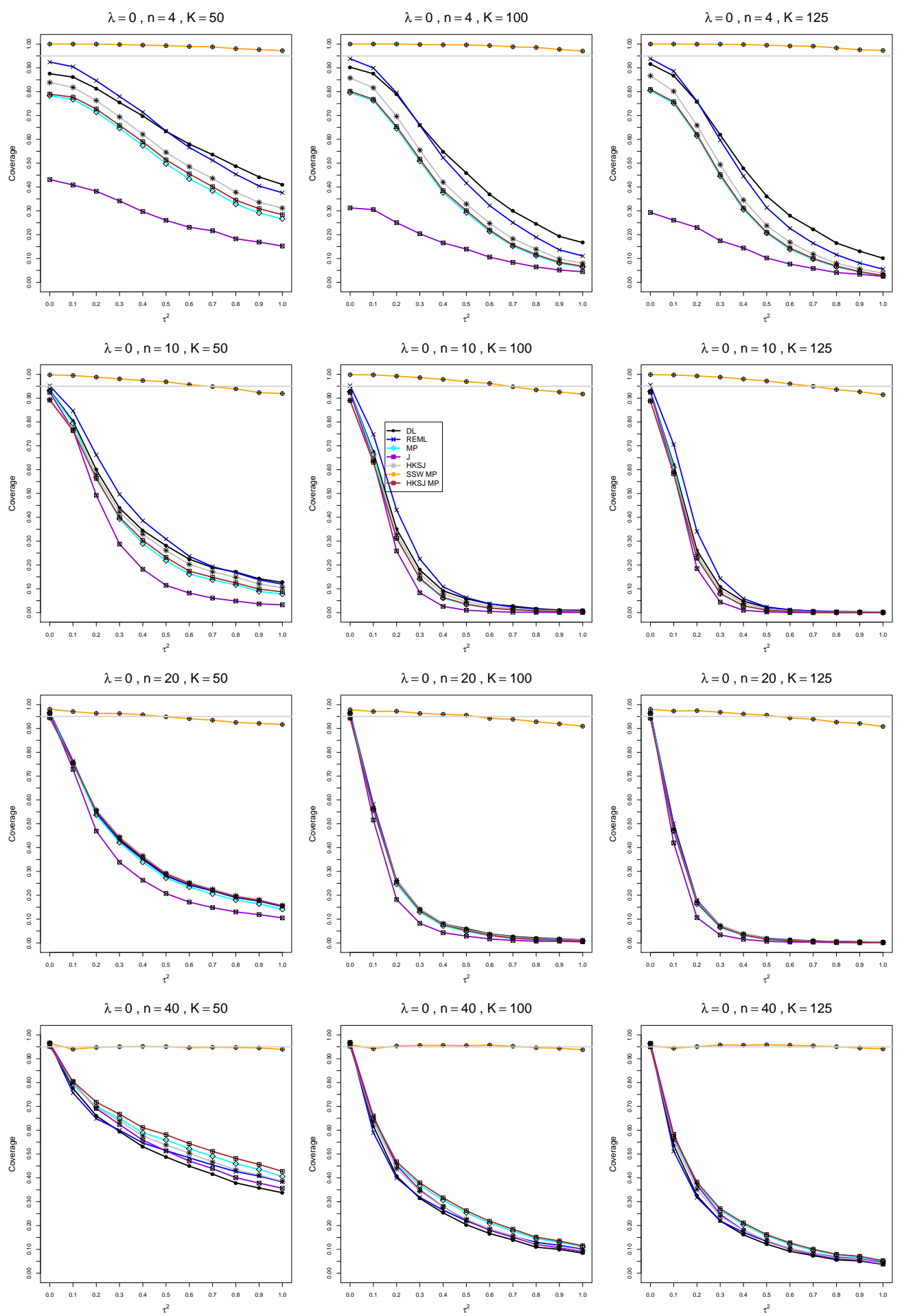

Figure B4.2.1: Coverage of $95 \%$ confidence intervals for $\lambda$ when $\mu_{C}=1, \lambda=0, n=$ 4, 10, 20, 40, and $K=50,100,125$ 

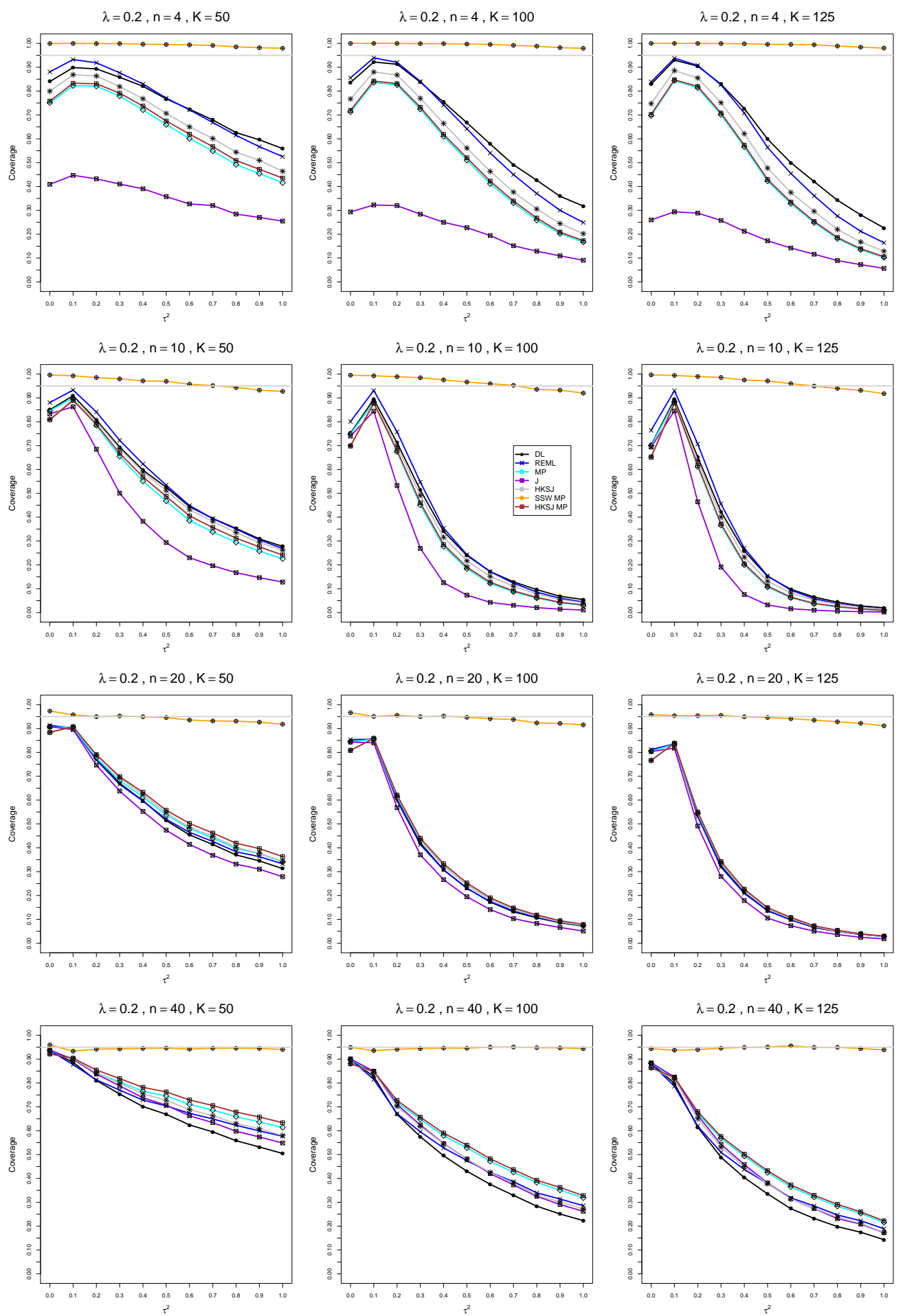

Figure B4.2.2: Coverage of $95 \%$ confidence intervals for $\lambda$ when $\mu_{C}=1, \lambda=0.2, n=$ 4, 10, 20, 40, and $K=50,100,125$ 

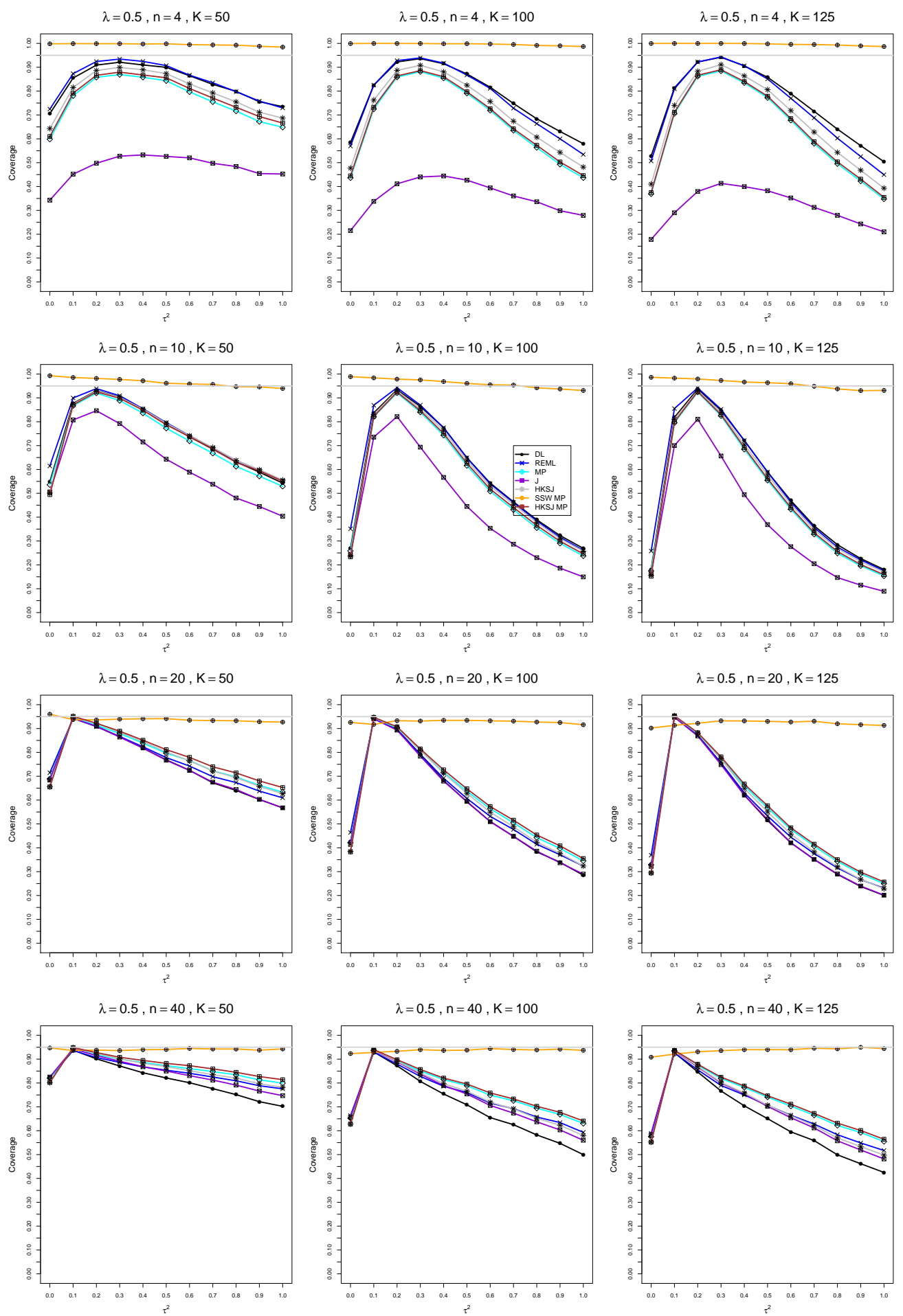

Figure B4.2.3: Coverage of $95 \%$ confidence intervals for $\lambda$ when $\mu_{C}=1, \lambda=0.5, n=$ 4, 10, 20, 40, and $K=50,100,125$ 

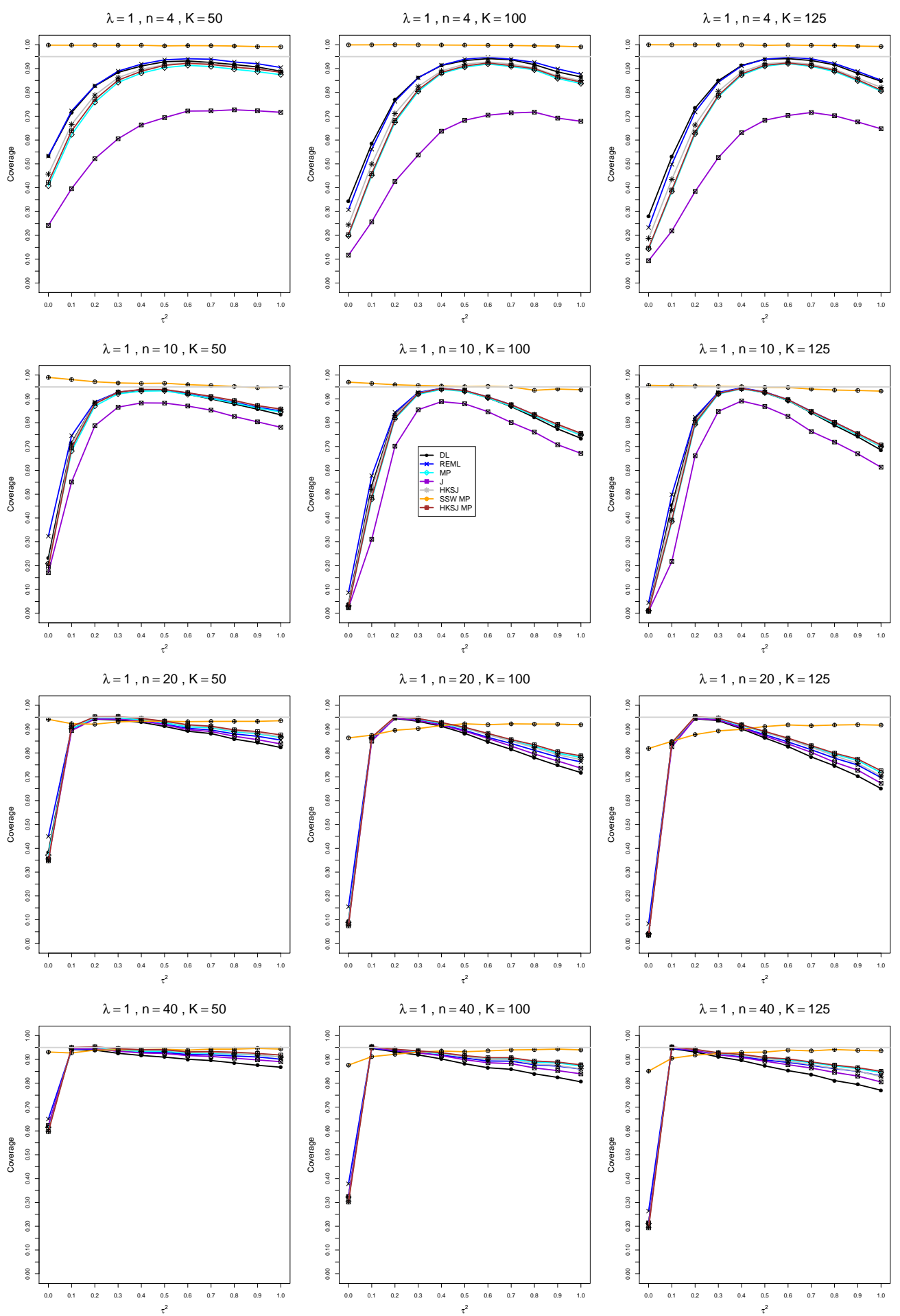

Figure B4.2.4: Coverage of $95 \%$ confidence intervals for $\lambda$ when $\mu_{C}=1, \lambda=1, n=$ 4, 10, 20, 40, and $K=50,100,125$ 

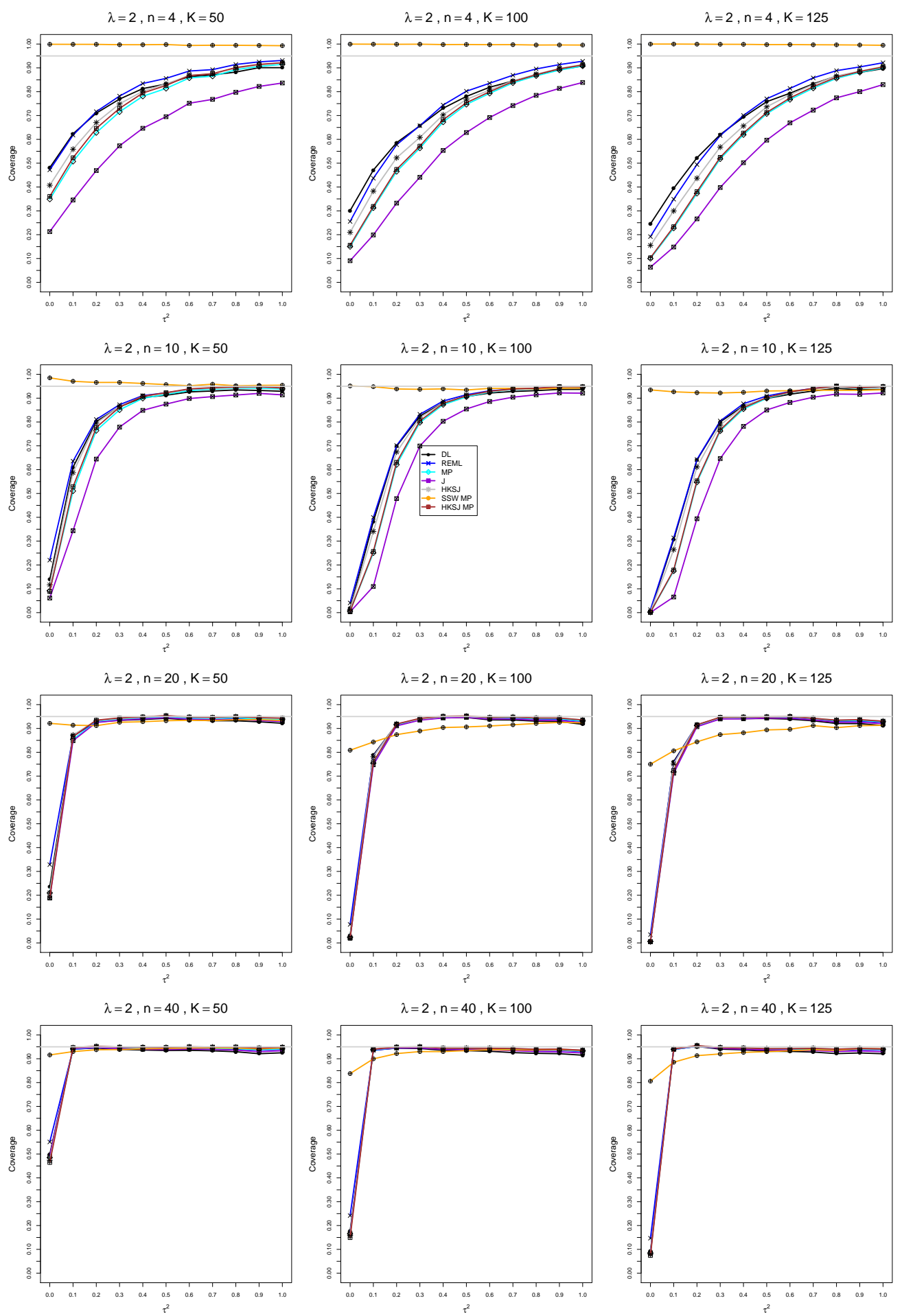

Figure B4.2.5: Coverage of $95 \%$ confidence intervals for $\lambda$ when $\mu_{C}=1, \lambda=2, n=$ 4, 10, 20, 40, and $K=50,100,125$ 
B5. Normal model, $\mu_{C}=4, n=4,10,20,40, K=$ $50,100,125$

\section{B5.1 Bias of point estimators of $\lambda$ when $\mu_{C}=4$}

Each figure corresponds to a value of $\lambda(=0,0.2,0.5,1,2)$, a set of values of $n(=4$, $10,20,40)$, and a set of values of $K(=50,100,125)$.

Each panel corresponds to a value of $n$ and a value of $K$ and has $\tau^{2}=0.0(0.1) 1.0$ on the horizontal axis.

The point estimators of $\lambda$ are

- DL (DerSimonian-Laird)

- REML (restricted maximum likelihood)

- MP (Mandel-Paule)

- J (Jackson)

- SSW (sample-size weighted) 

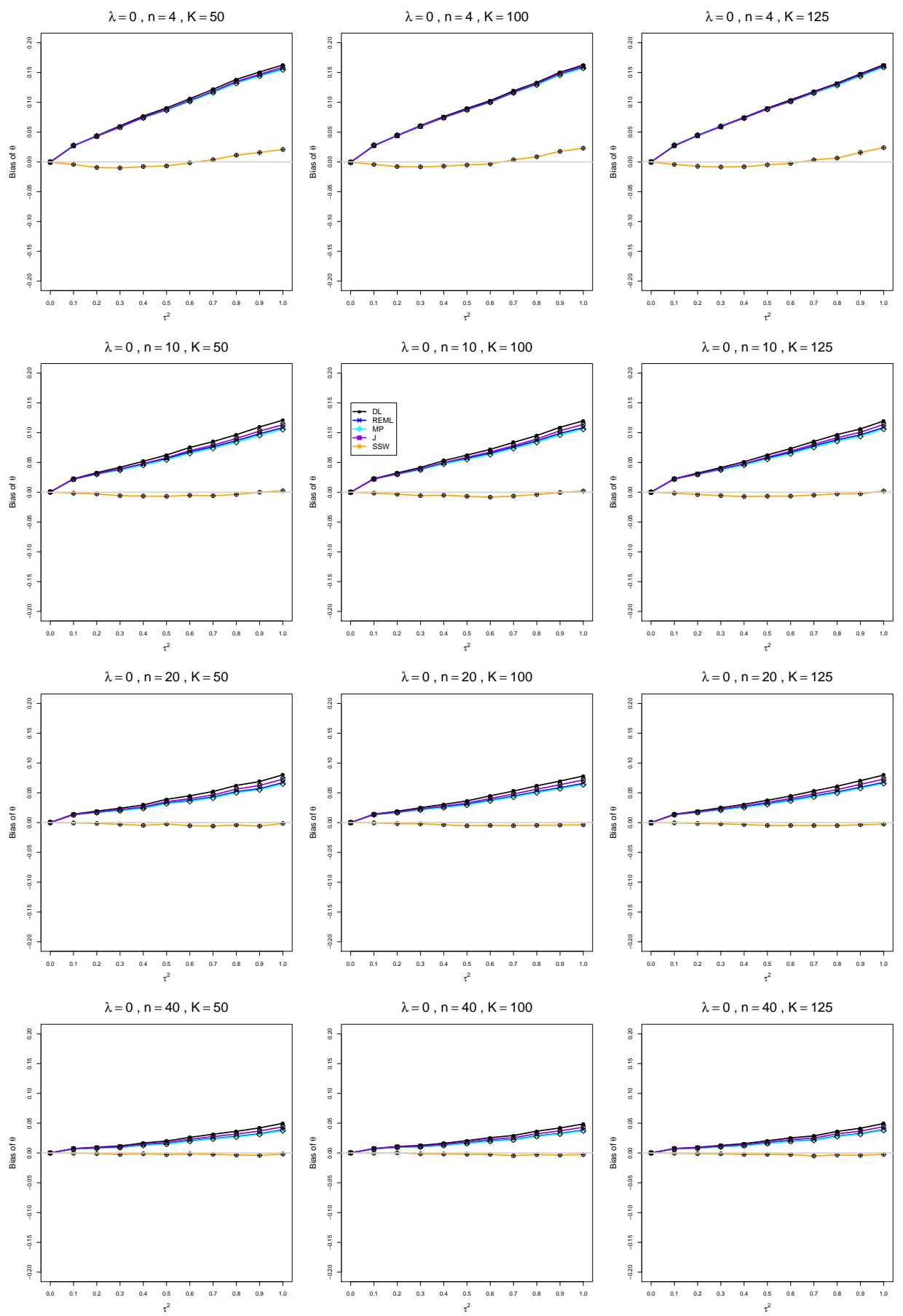

Figure B5.1.1: Bias of estimators of $\log (\operatorname{RoM}) \lambda$ when $\mu_{C}=4, \lambda=0, n=4,10,20,40$, and $K=50,100,125$ 

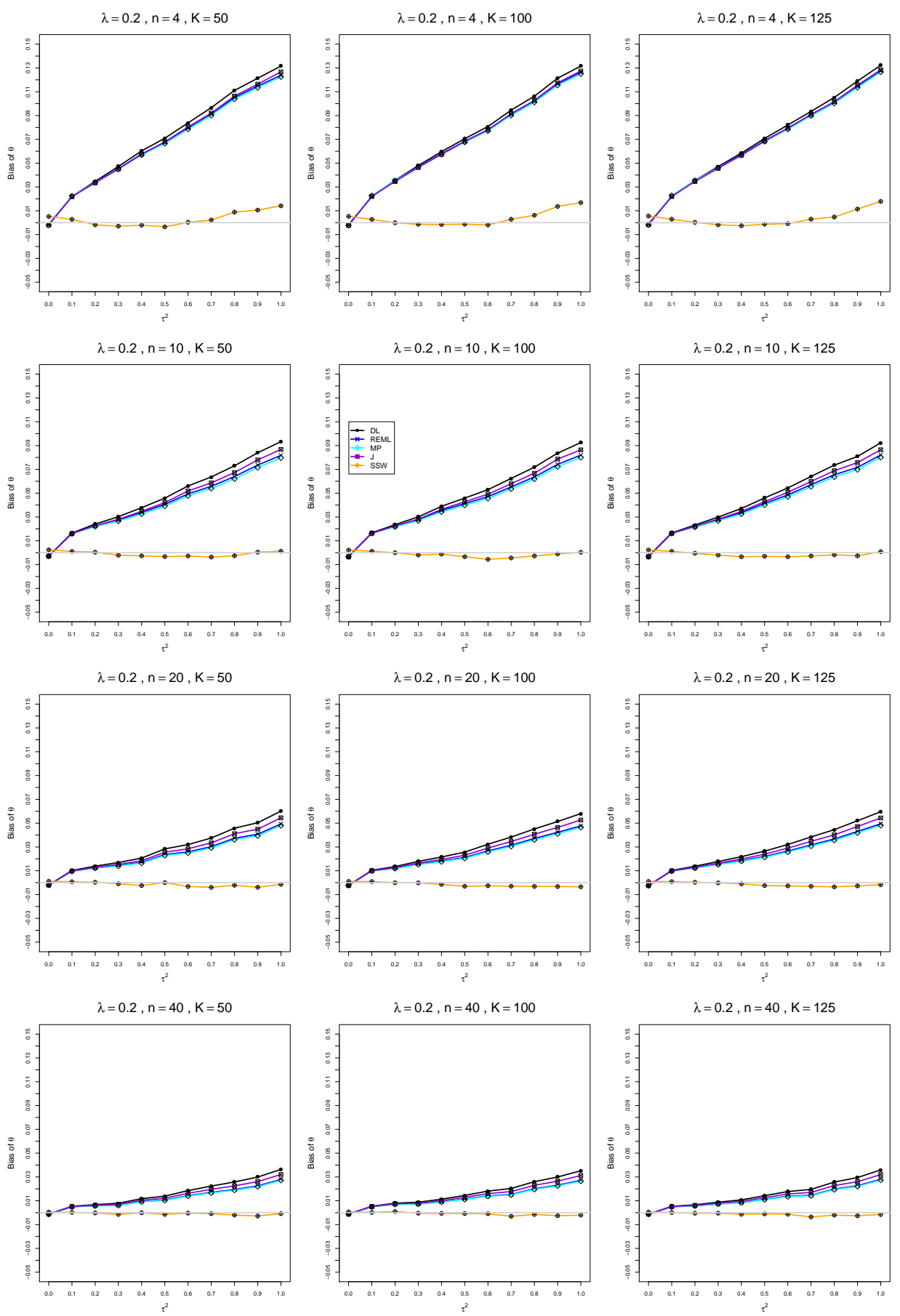

Figure B5.1.2: Bias of estimators of $\log (\mathrm{RoM}) \lambda$ when $\mu_{C}=4, \lambda=0.2, n=4,10,20,40$, and $K=50,100,125$ 

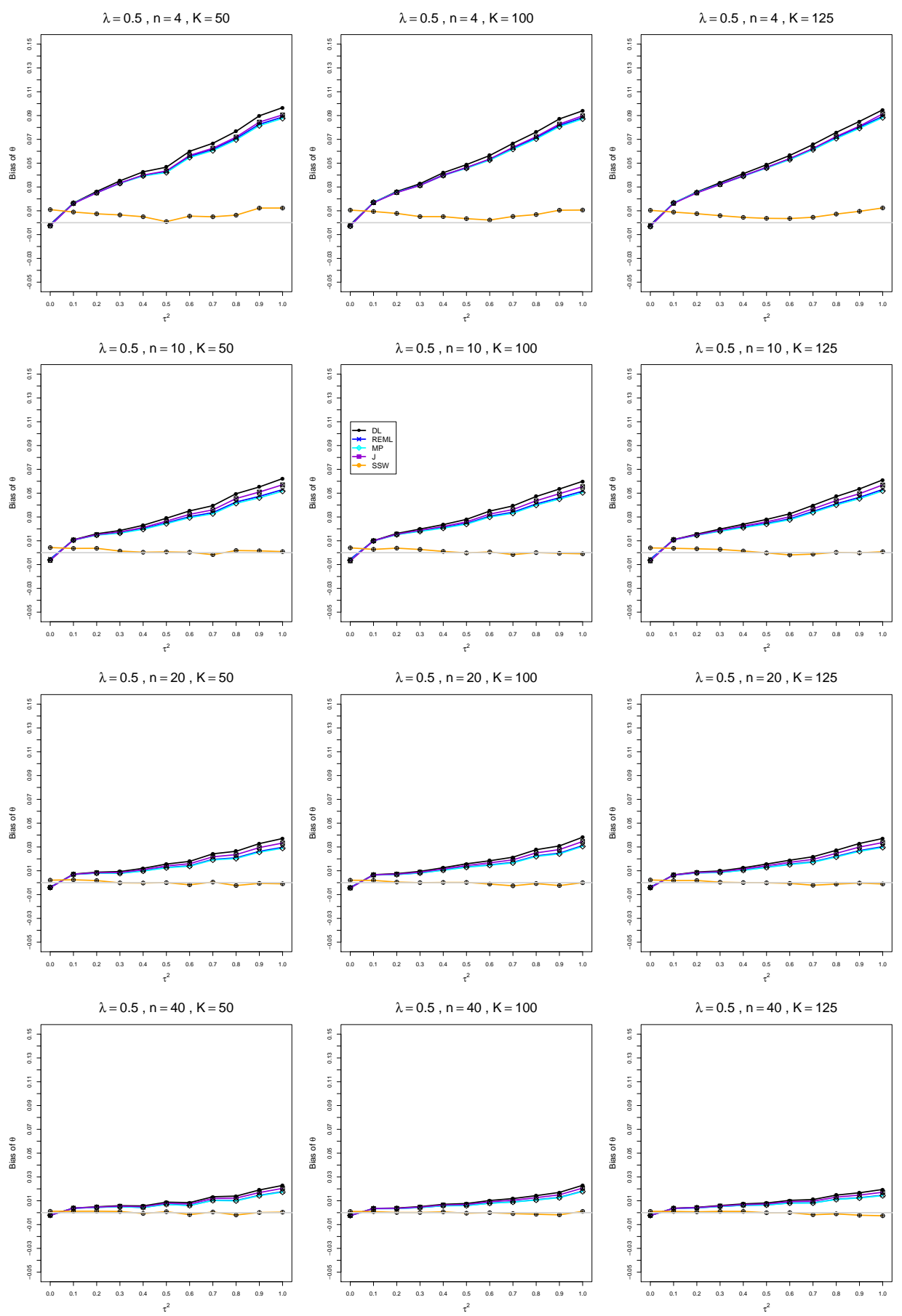

Figure B5.1.3: Bias of estimators of $\log (\operatorname{RoM}) \lambda$ when $\mu_{C}=4, \lambda=0.5, n=4,10,20,40$, and $K=50,100,125$ 

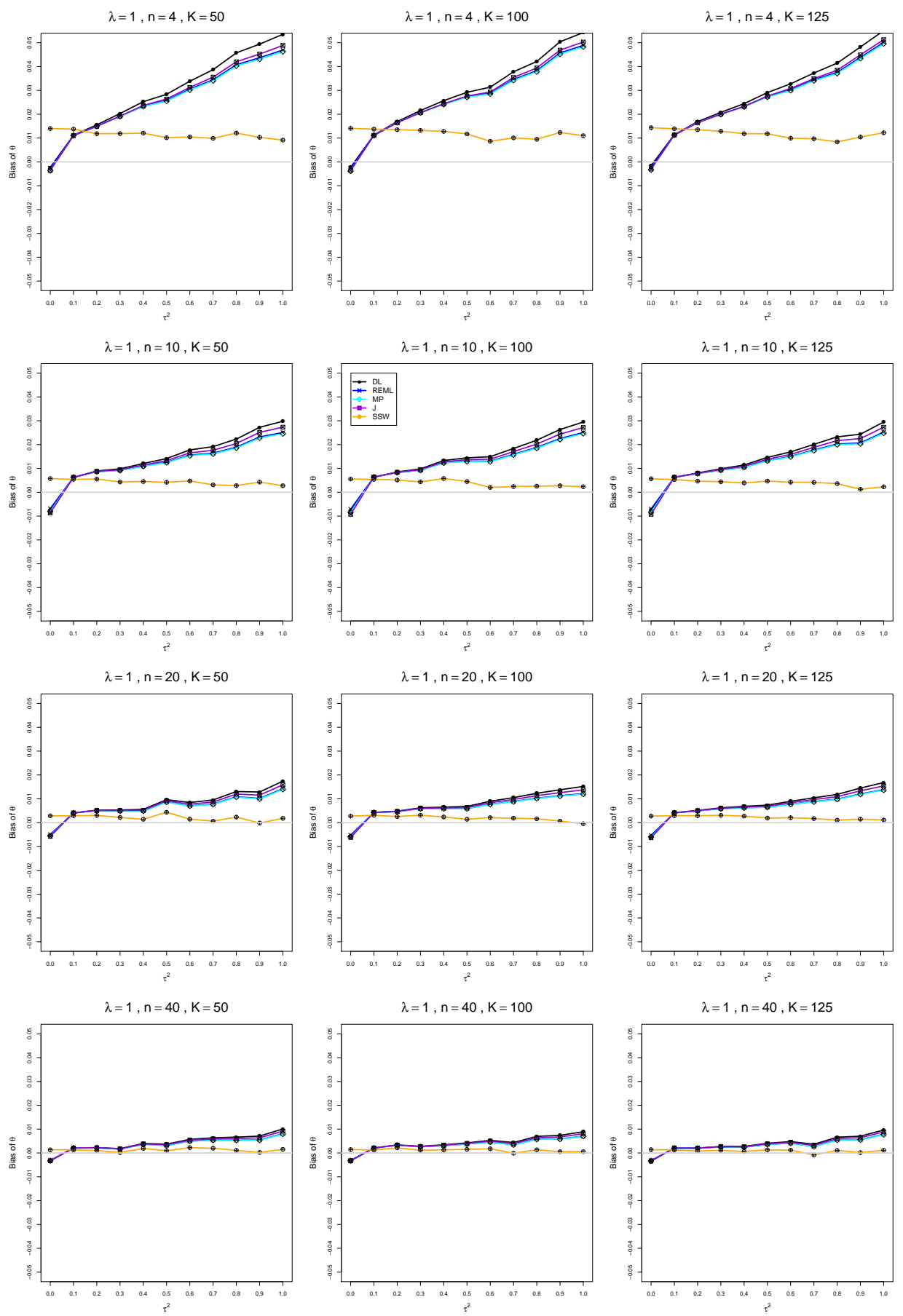

Figure B5.1.4: Bias of estimators of $\log (\operatorname{RoM}) \lambda$ when $\mu_{C}=4, \lambda=1, n=4,10,20,40$, and $K=50,100,125$ 

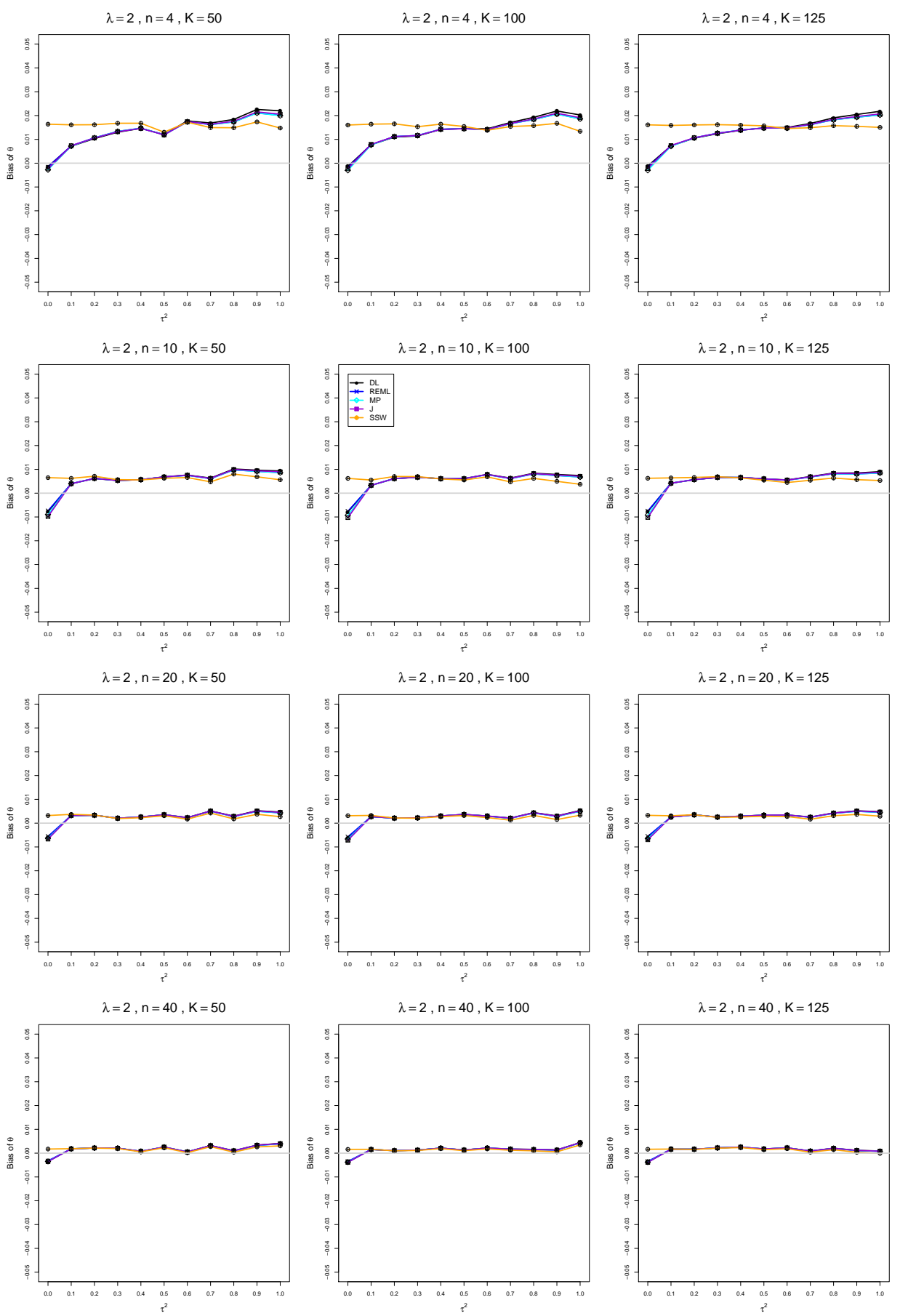

Figure B5.1.5: Bias of estimators of $\log (\operatorname{RoM}) \lambda$ when $\mu_{C}=4, \lambda=2, n=4,10,20,40$, and $K=50,100,125$ 


\section{B5.2 Coverage of interval estimators of $\lambda$}

Each figure corresponds to a value of $\lambda(=0,0.2,0.5,1,2)$, a set of values of $n(=4$, $10,20,40)$, and a set of values of $K(=50,100,125)$.

Each panel corresponds to a value of $n$ and a value of $K$ and has $\tau^{2}=0.0(0.1) 1.0$ on the horizontal axis.

The interval estimators of $\lambda$ are the companions to the inverse-variance-weighted point estimators

- DL (DerSimonian-Laird)

- REML (restricted maximum likelihood)

- MP (Mandel-Paule)

- J (Jackson)

and

- HKSJ (Hartung-Knapp-Sidik-Jonkman)

- HKSJ MP (HKSJ with MP estimator of $\tau^{2}$ )

- SSW MP (SSW as center and half-width equal to critical value from $t_{K-1}$ times estimated standard deviation of SSW with $\hat{\tau}^{2}=\hat{\tau}_{M P}^{2}$ 

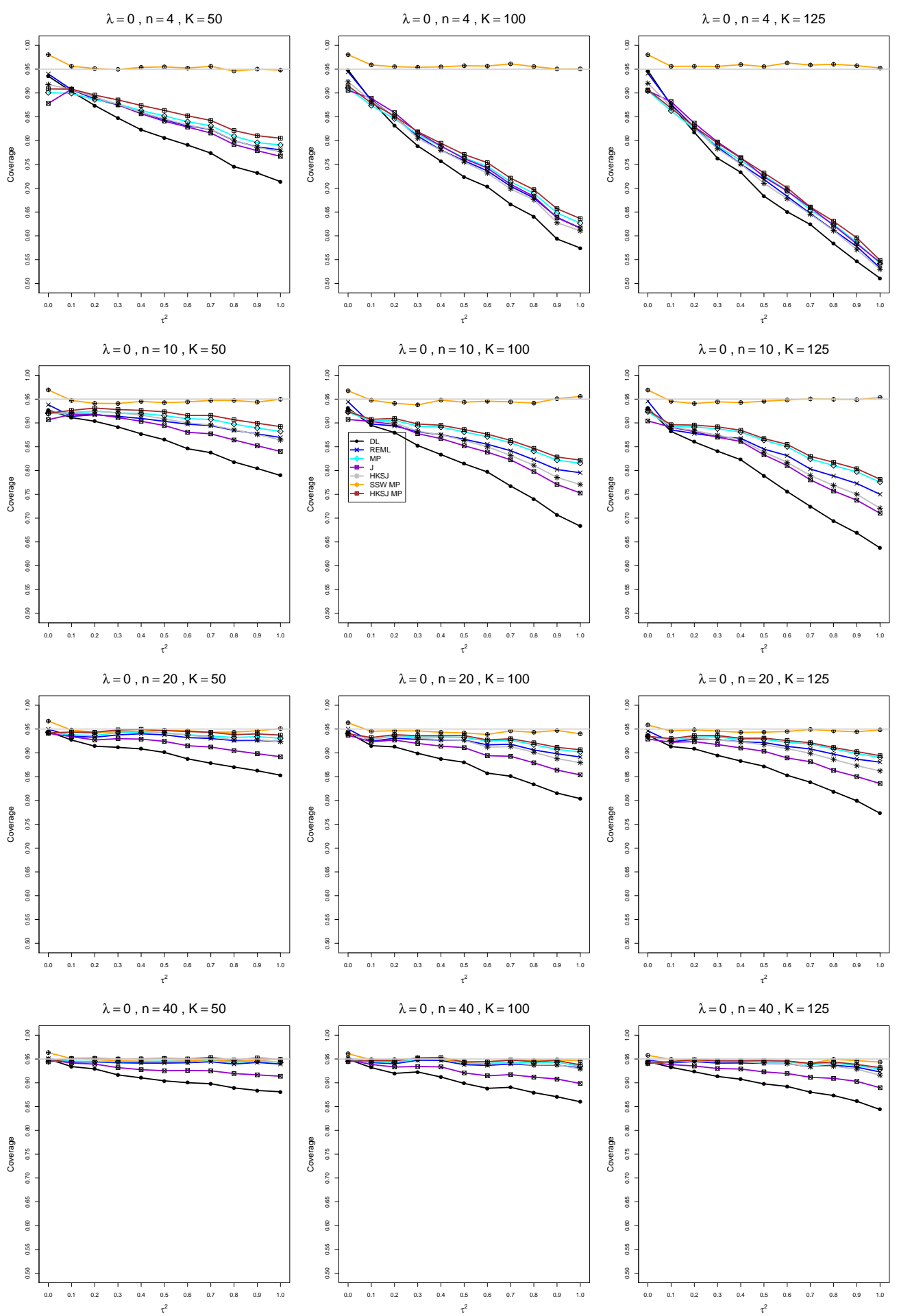

Figure B5.2.1: Coverage of $95 \%$ confidence intervals for $\lambda$ when $\mu_{C}=4, \lambda=0, n=$ 4, 10, 20, 40, and $K=50,100,125$ 

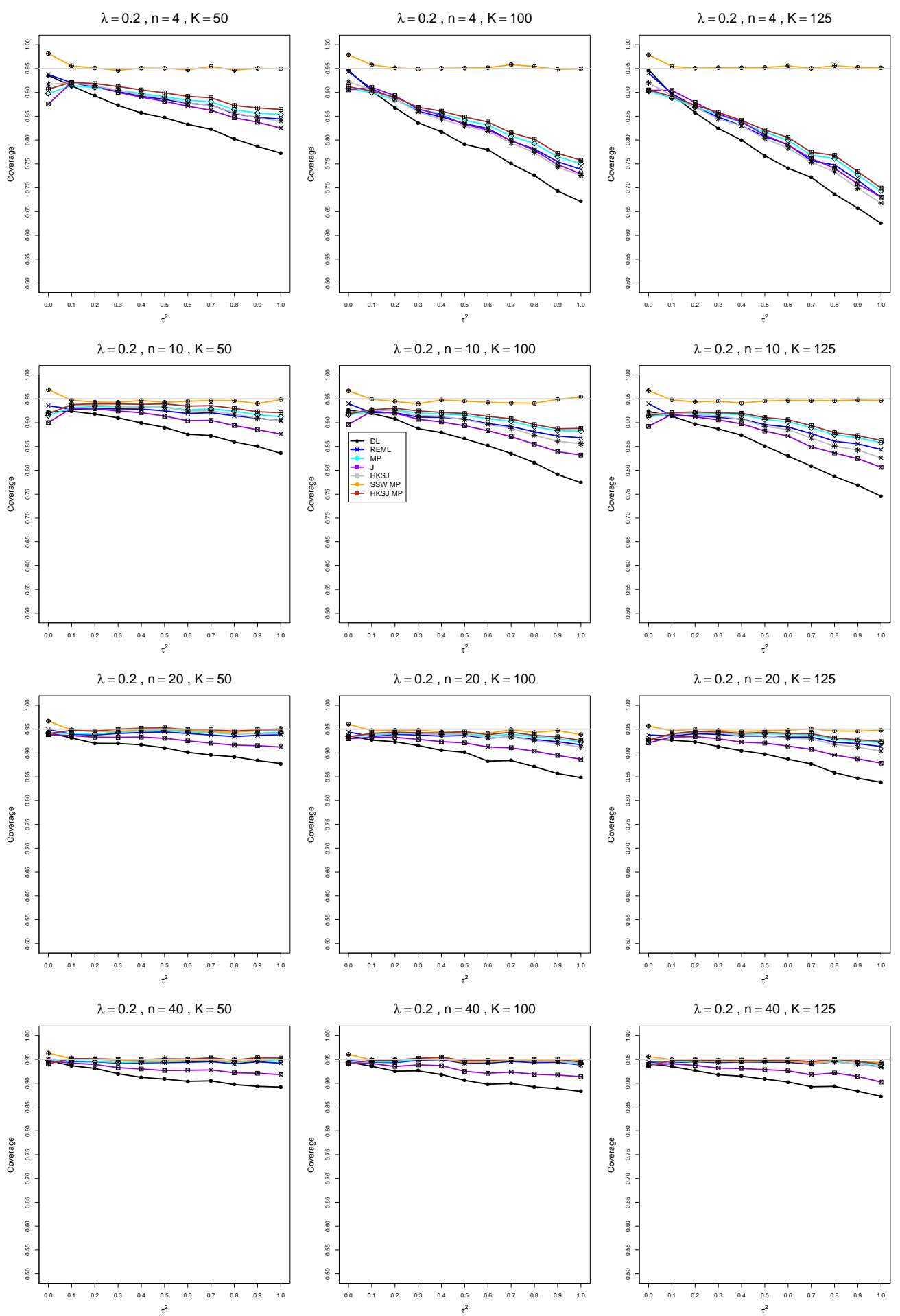

Figure B5.2.2: Coverage of $95 \%$ confidence intervals for $\lambda$ when $\mu_{C}=4, \lambda=0.2, n=$ 4, 10, 20, 40, and $K=50,100,125$ 

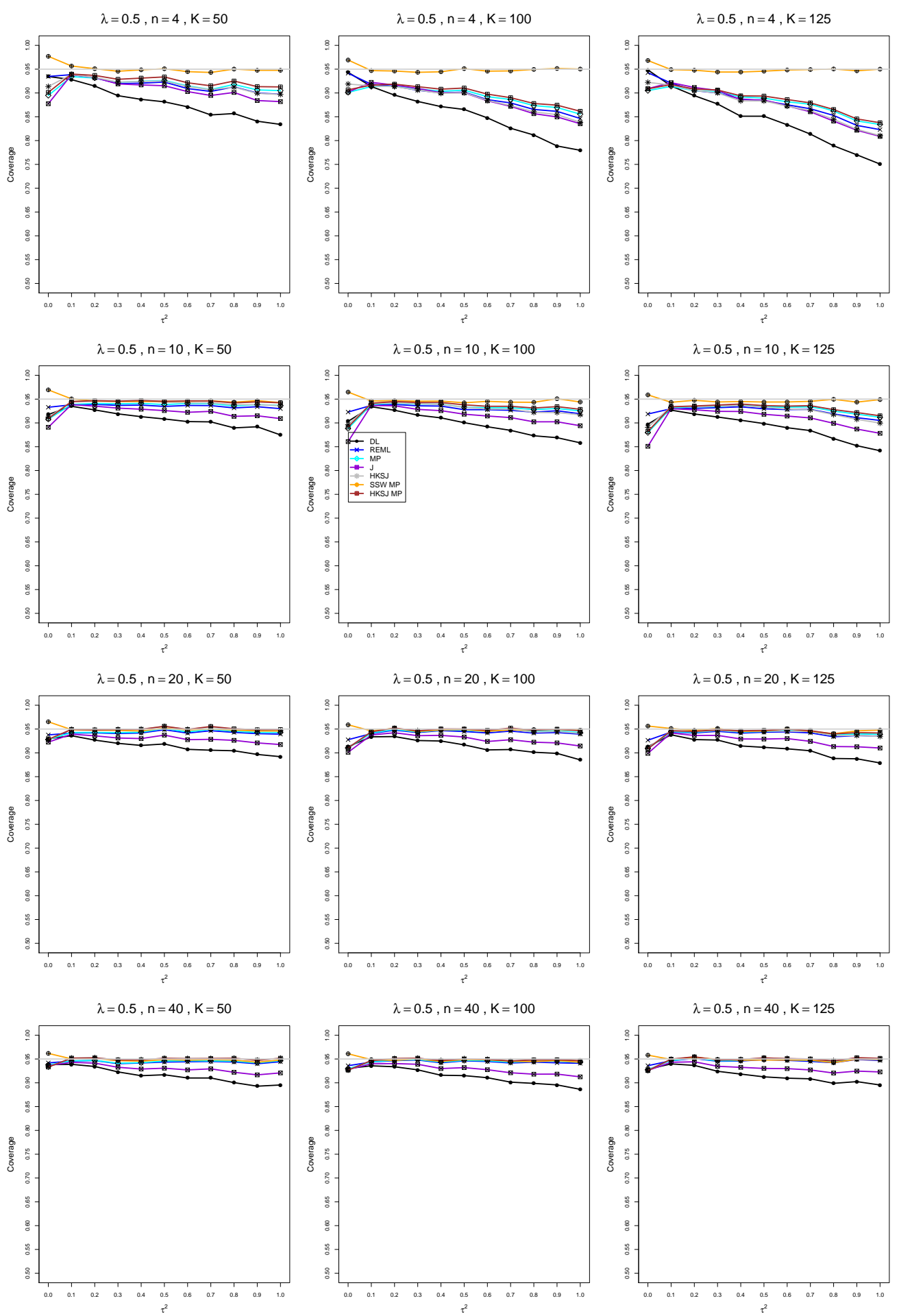

Figure B5.2.3: Coverage of $95 \%$ confidence intervals for $\lambda$ when $\mu_{C}=4, \lambda=0.5, n=$ 4, 10, 20, 40, and $K=50,100,125$ 

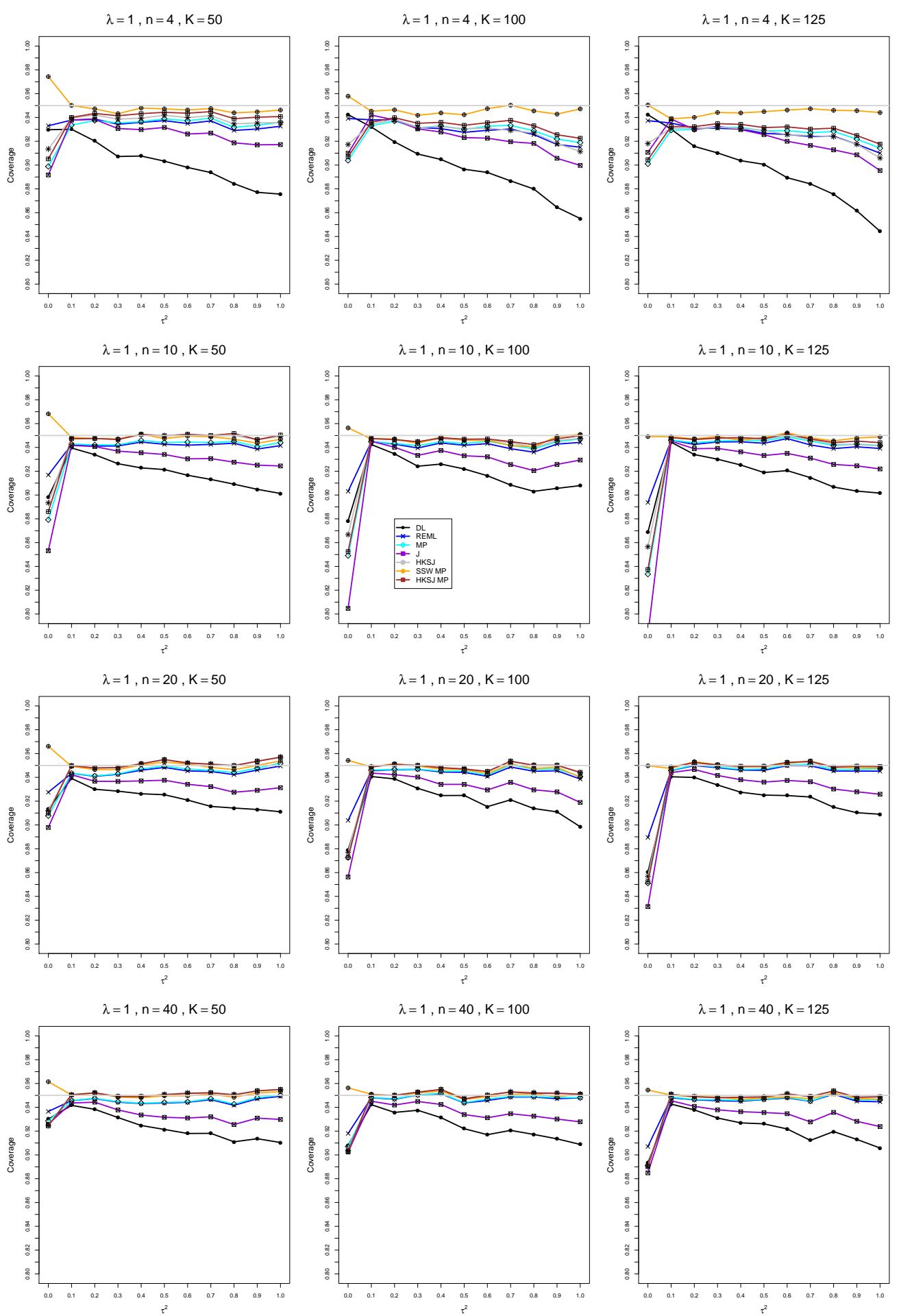

Figure B5.2.4: Coverage of $95 \%$ confidence intervals for $\lambda$ when $\mu_{C}=4, \lambda=1, n=$ 4, 10, 20, 40, and $K=50,100,125$ 

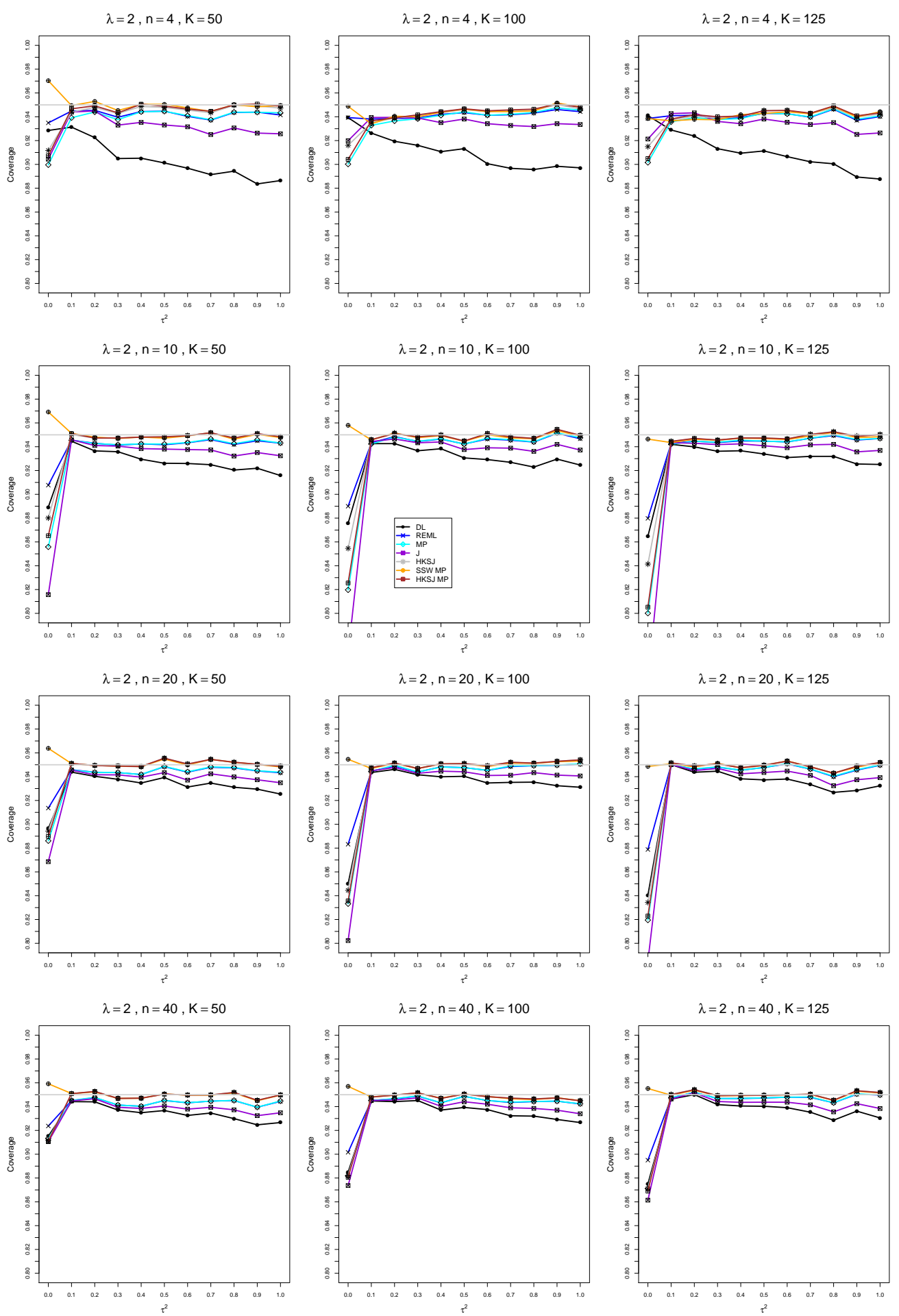

Figure B5.2.5: Coverage of $95 \%$ confidence intervals for $\lambda$ when $\mu_{C}=4, \lambda=2, n=$ 4, 10, 20, 40, and $K=50,100,125$ 
B6. Normal model, bias-corrected $\lambda, \mu_{C}=4$, $n=4,10,20,40, K=50,100,125$

B6.1 Bias of point estimators of $\lambda$ when $\mu_{C}=4$

Each figure corresponds to a value of $\lambda(=0,0.2,0.5,1,2)$, a set of values of $n(=4$, $10,20,40)$, and a set of values of $K(=50,100,125)$.

Each panel corresponds to a value of $n$ and a value of $K$ and has $\tau^{2}=0.0(0.1) 1.0$ on the horizontal axis.

The point estimators of $\lambda$ are

- DL (DerSimonian-Laird)

- REML (restricted maximum likelihood)

- MP (Mandel-Paule)

- J (Jackson)

- SSW (sample-size weighted) 

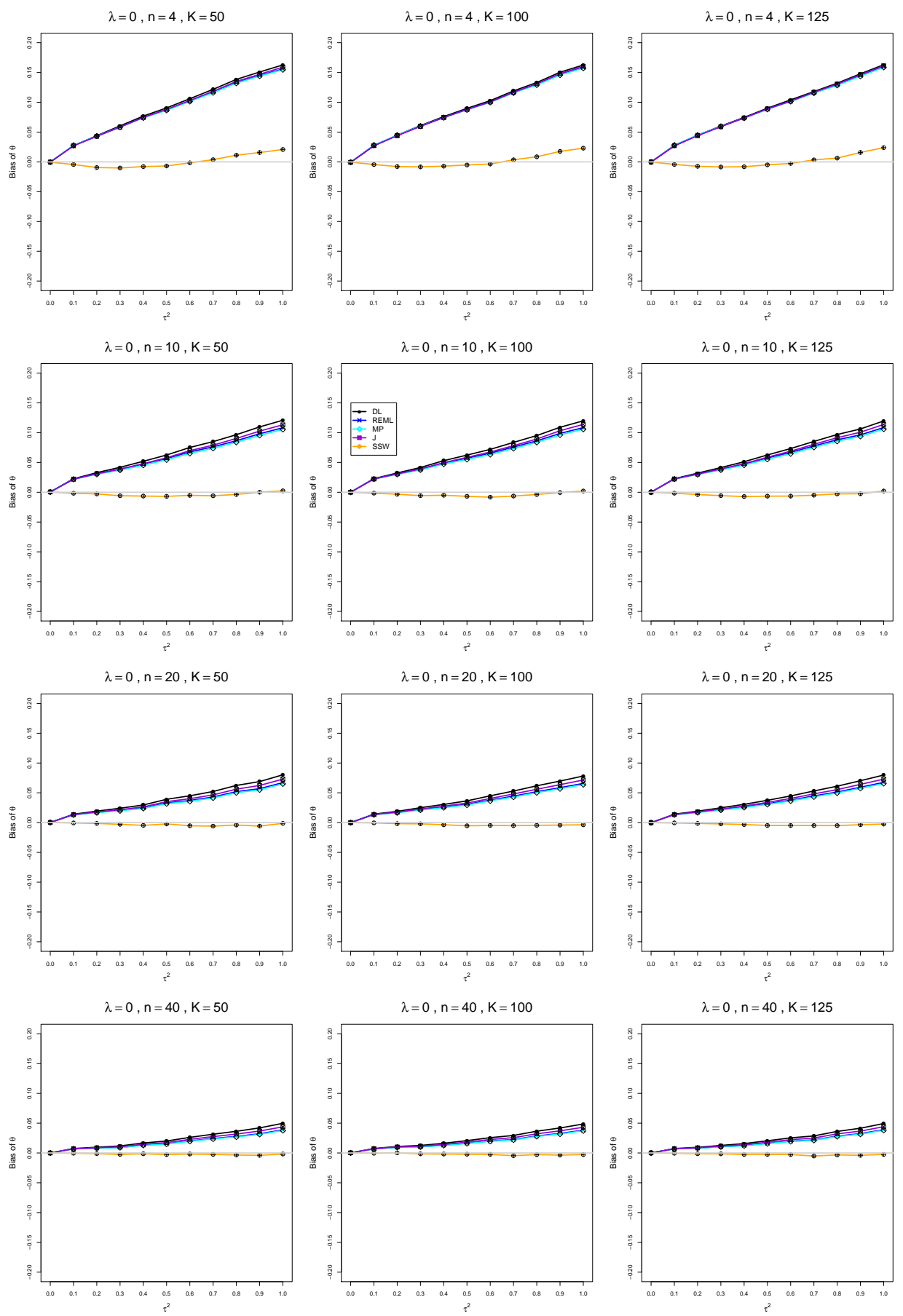

Figure B6.1.1: Bias of estimators of $\log (\operatorname{RoM}) \lambda$ when $\mu_{C}=4, \lambda=0, n=4,10,20,40$, and $K=50,100,125$ 

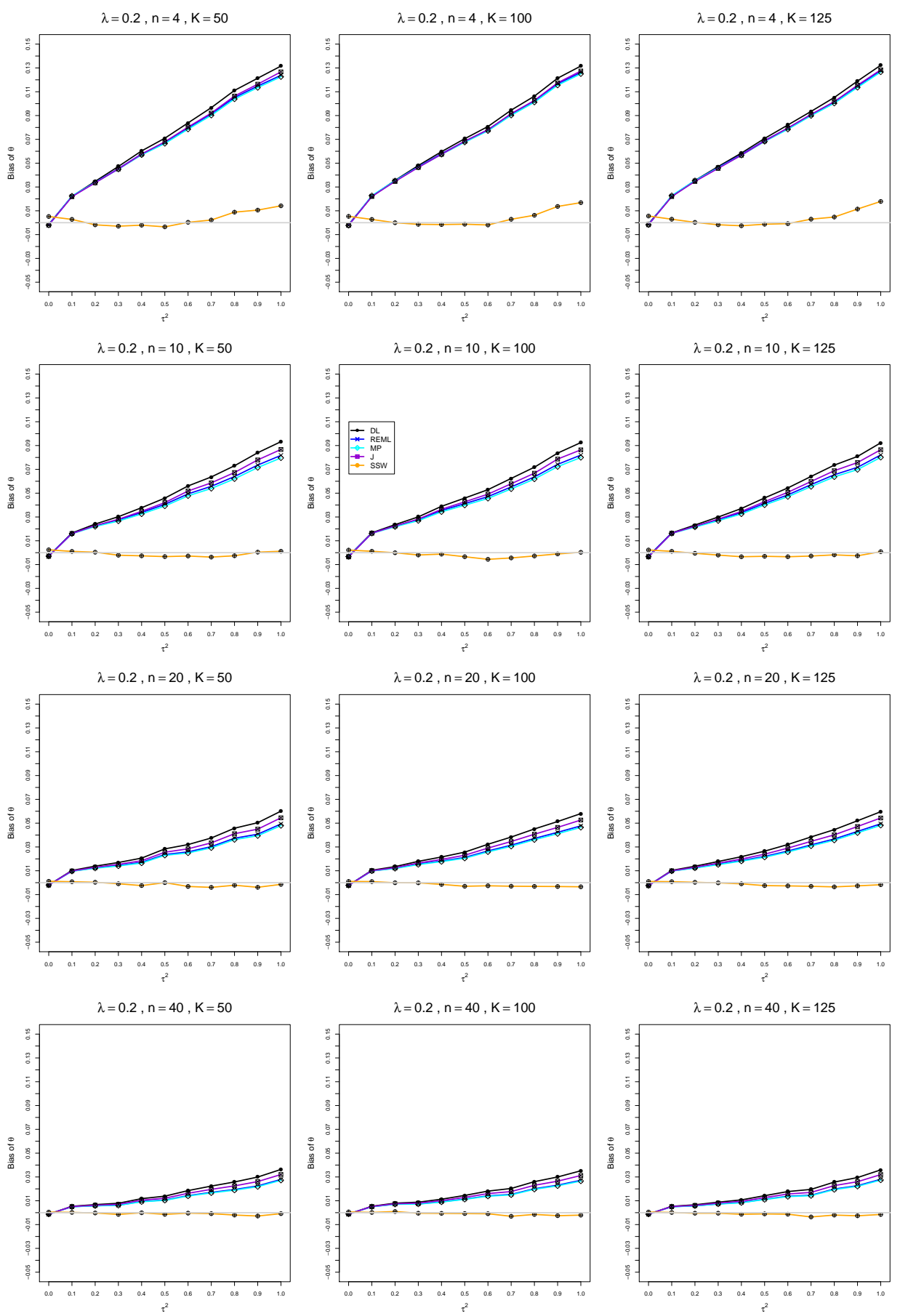

Figure B6.1.2: Bias of estimators of $\log (\operatorname{RoM}) \lambda$ when $\mu_{C}=4, \lambda=0.2, n=4,10,20,40$, and $K=50,100,125$ 

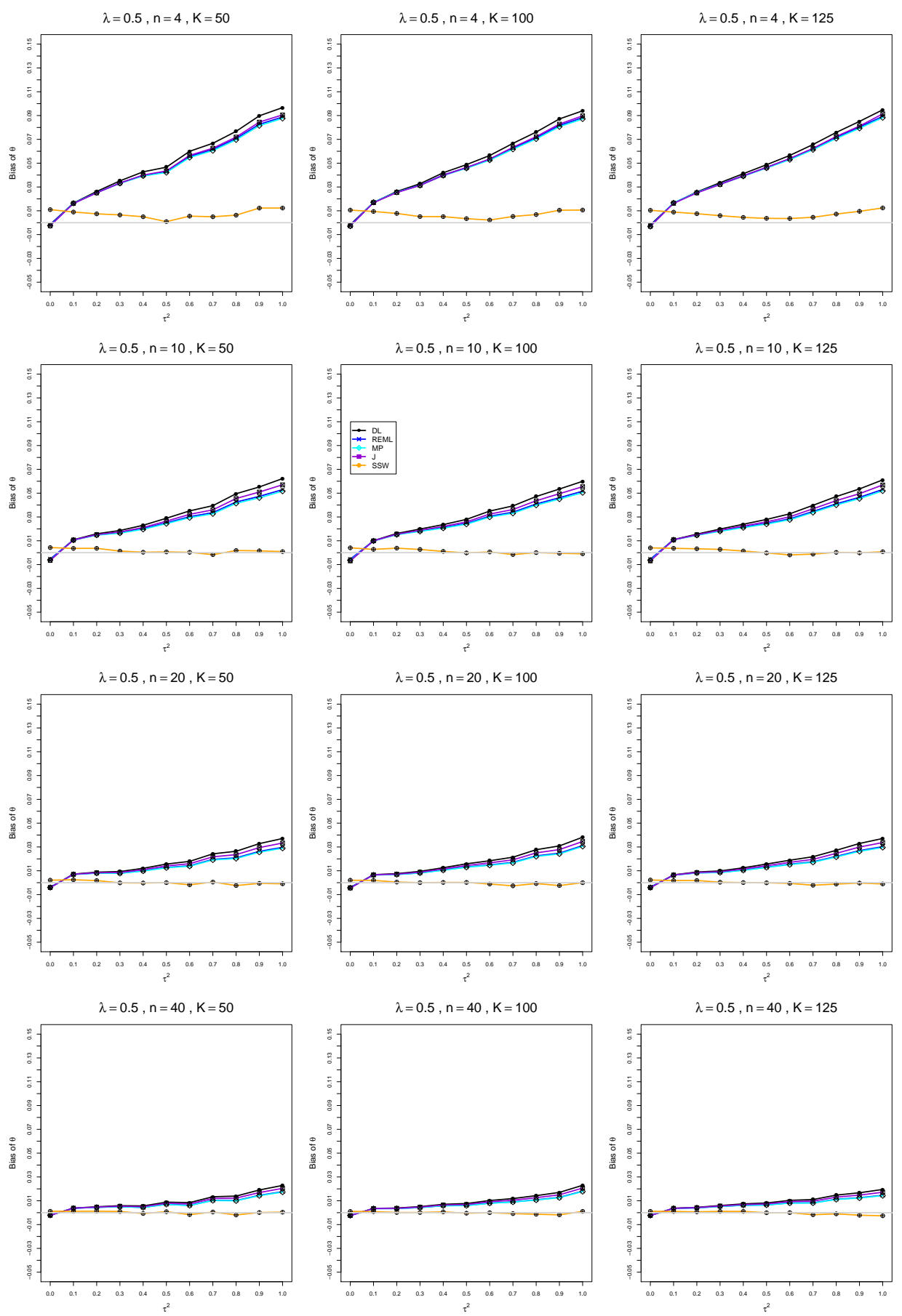

Figure B6.1.3: Bias of estimators of $\log (\operatorname{RoM}) \lambda$ when $\mu_{C}=4, \lambda=0.5, n=4,10,20,40$, and $K=50,100,125$ 

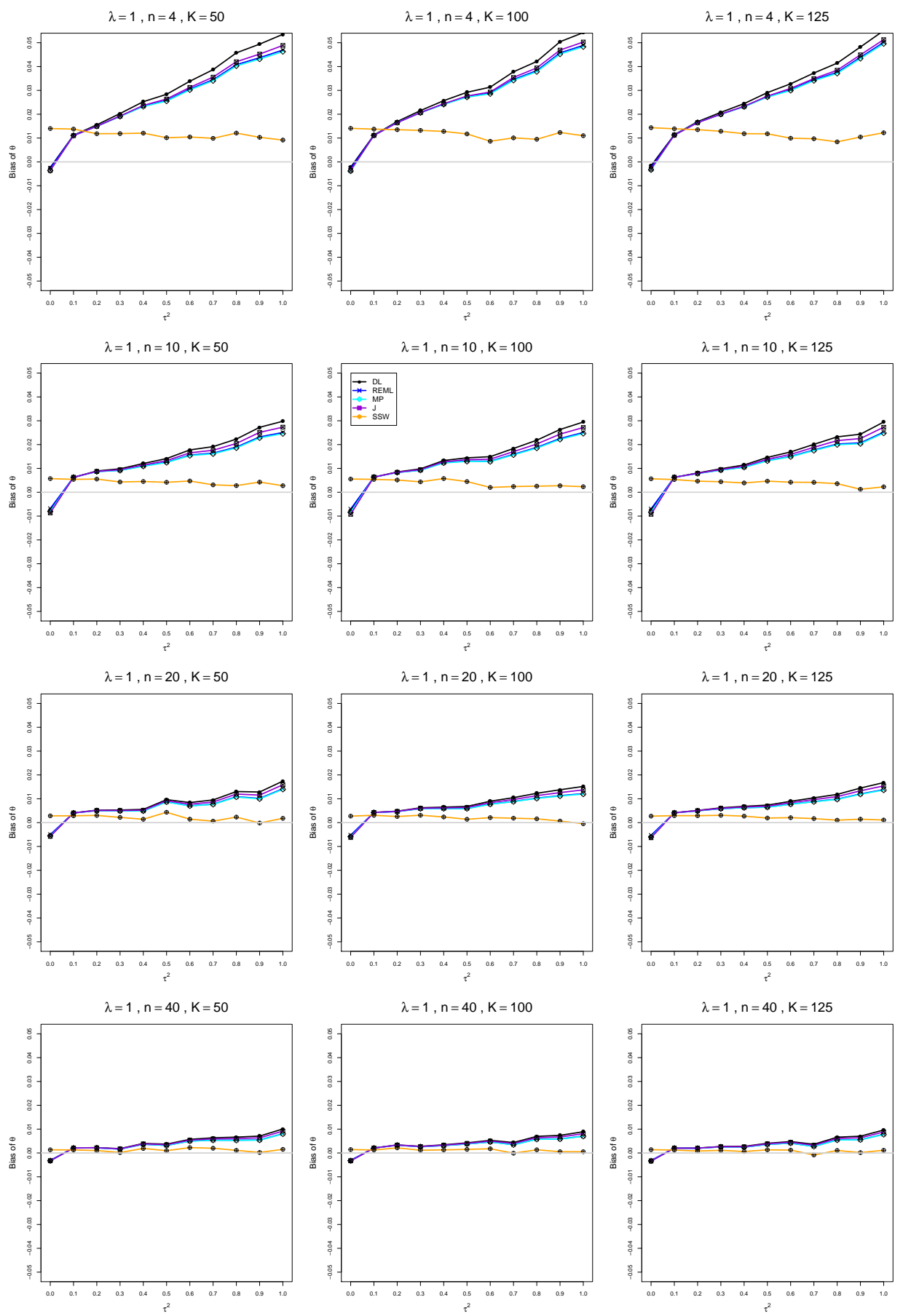

Figure B6.1.4: Bias of estimators of $\log (\operatorname{RoM}) \lambda$ when $\mu_{C}=4, \lambda=1, n=4,10,20,40$, and $K=50,100,125$ 

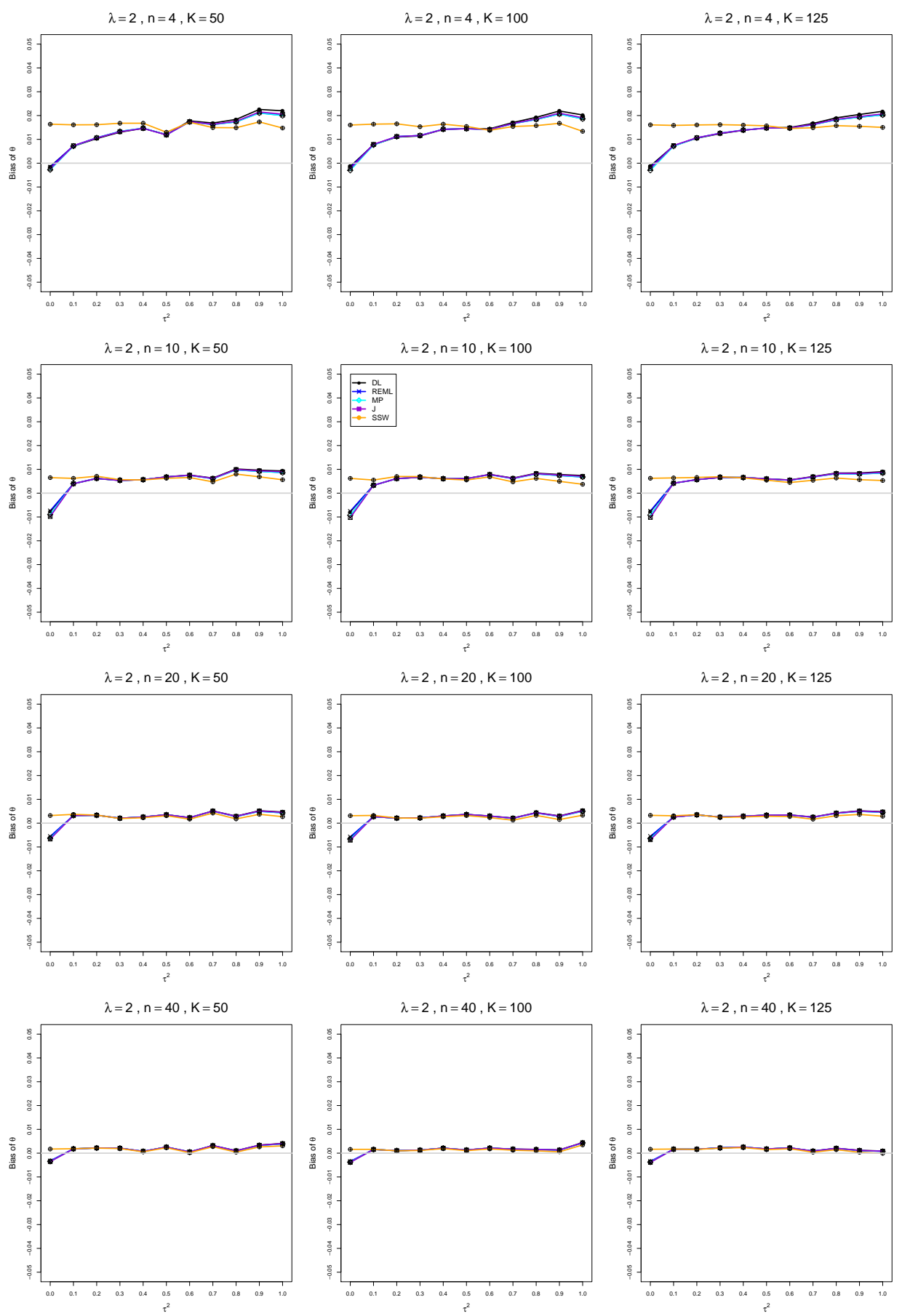

Figure B6.1.5: Bias of estimators of $\log (\operatorname{RoM}) \lambda$ when $\mu_{C}=4, \lambda=2, n=4,10,20,40$, and $K=50,100,125$ 


\section{B6.2 Coverage of interval estimators of $\lambda$}

Each figure corresponds to a value of $\lambda(=0,0.2,0.5,1,2)$, a set of values of $n(=4$, $10,20,40)$, and a set of values of $K(=50,100,125)$.

Each panel corresponds to a value of $n$ and a value of $K$ and has $\tau^{2}=0.0(0.1) 1.0$ on the horizontal axis.

The interval estimators of $\lambda$ are the companions to the inverse-variance-weighted point estimators

- DL (DerSimonian-Laird)

- REML (restricted maximum likelihood)

- MP (Mandel-Paule)

- J (Jackson)

and

- HKSJ (Hartung-Knapp-Sidik-Jonkman)

- HKSJ MP (HKSJ with MP estimator of $\tau^{2}$ )

- SSW MP (SSW as center and half-width equal to critical value from $t_{K-1}$ times estimated standard deviation of SSW with $\hat{\tau}^{2}=\hat{\tau}_{M P}^{2}$ 

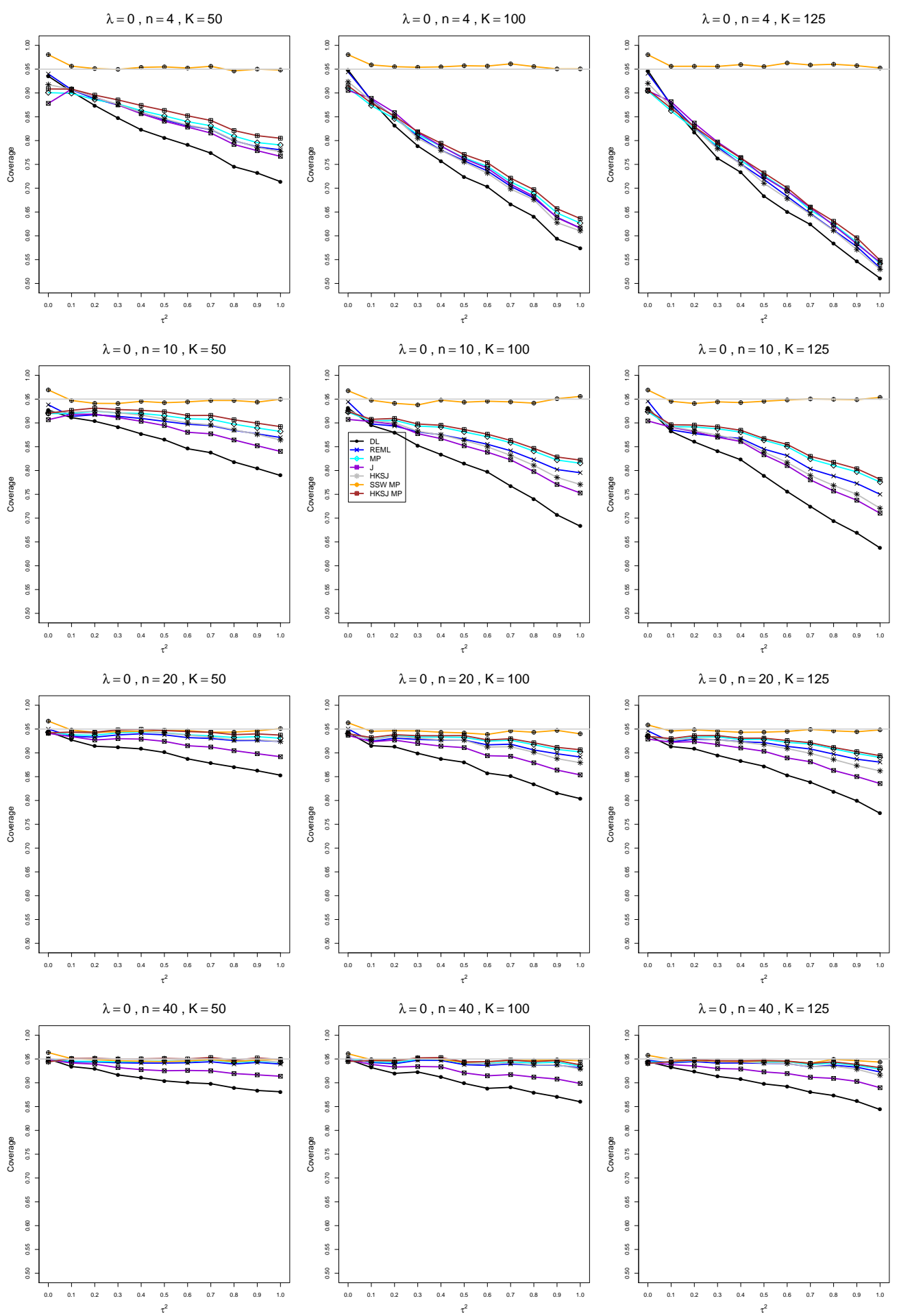

Figure B6.2.1: Coverage of $95 \%$ confidence intervals for $\lambda$ when $\mu_{C}=4, \lambda=0, n=$ 4, 10, 20, 40, and $K=50,100,125$ 

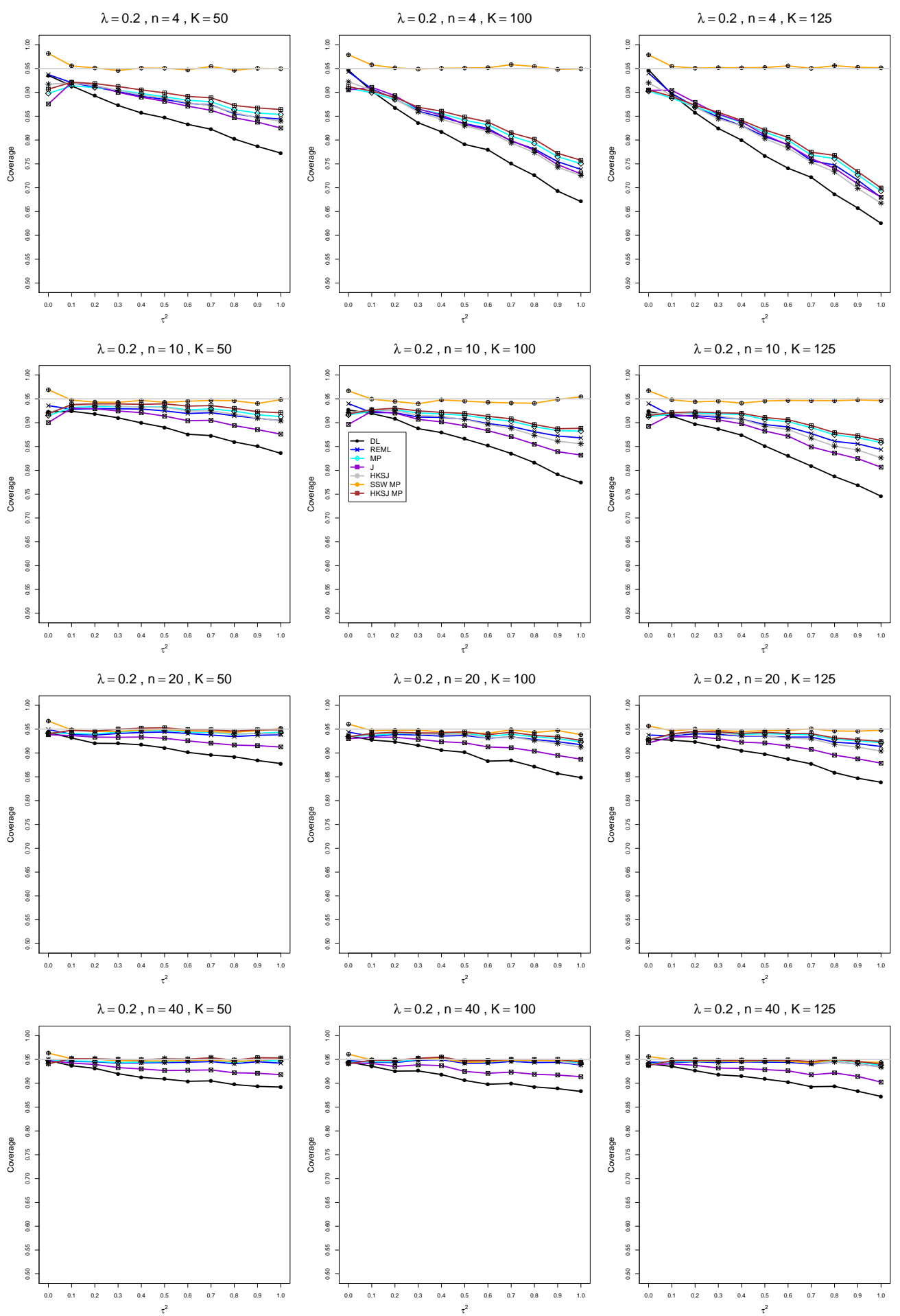

Figure B6.2.2: Coverage of $95 \%$ confidence intervals for $\lambda$ when $\mu_{C}=4, \lambda=0.2, n=$ 4, 10, 20, 40, and $K=50,100,125$ 

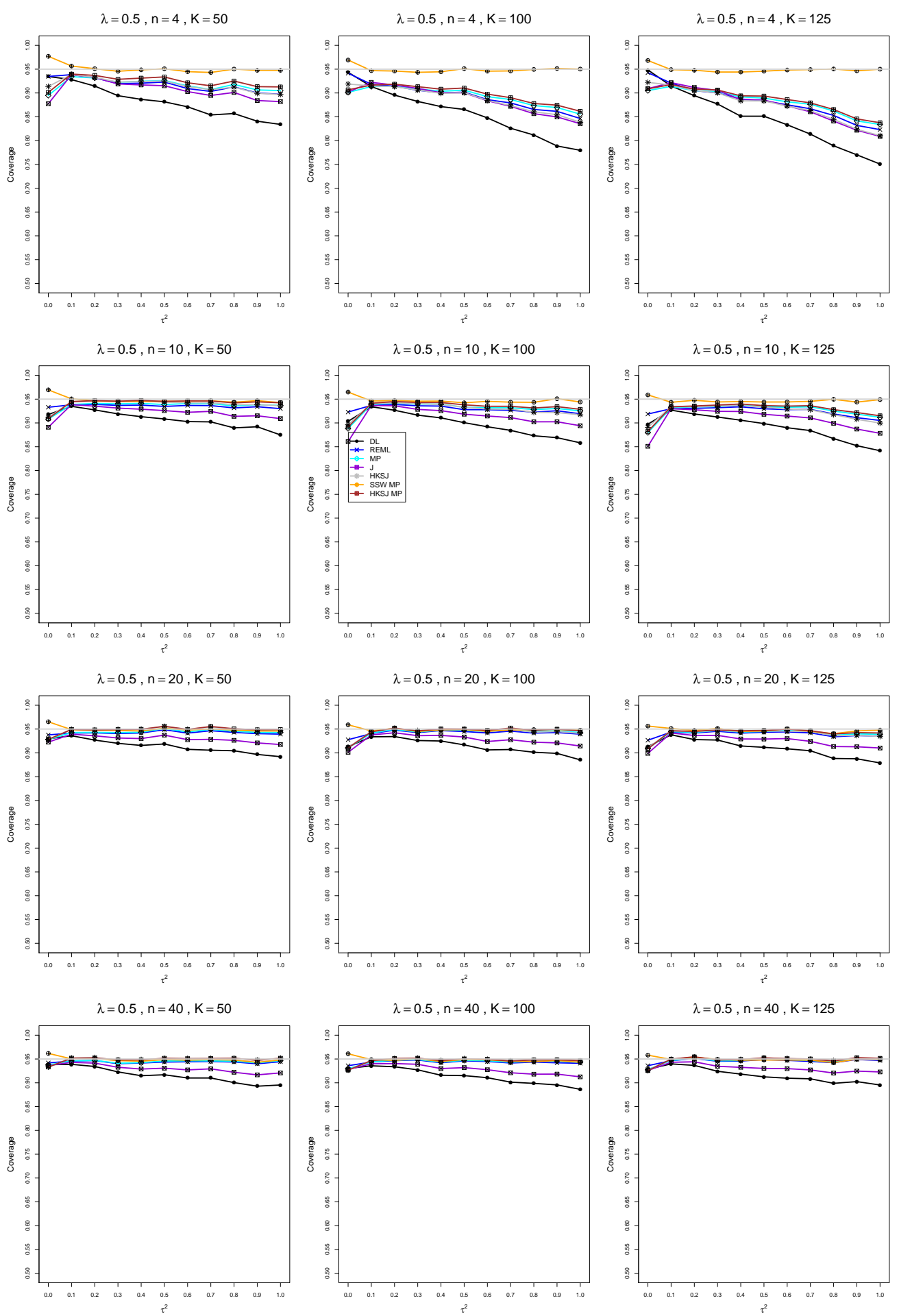

Figure B6.2.3: Coverage of $95 \%$ confidence intervals for $\lambda$ when $\mu_{C}=4, \lambda=0.5, n=$ 4, 10, 20, 40, and $K=50,100,125$ 

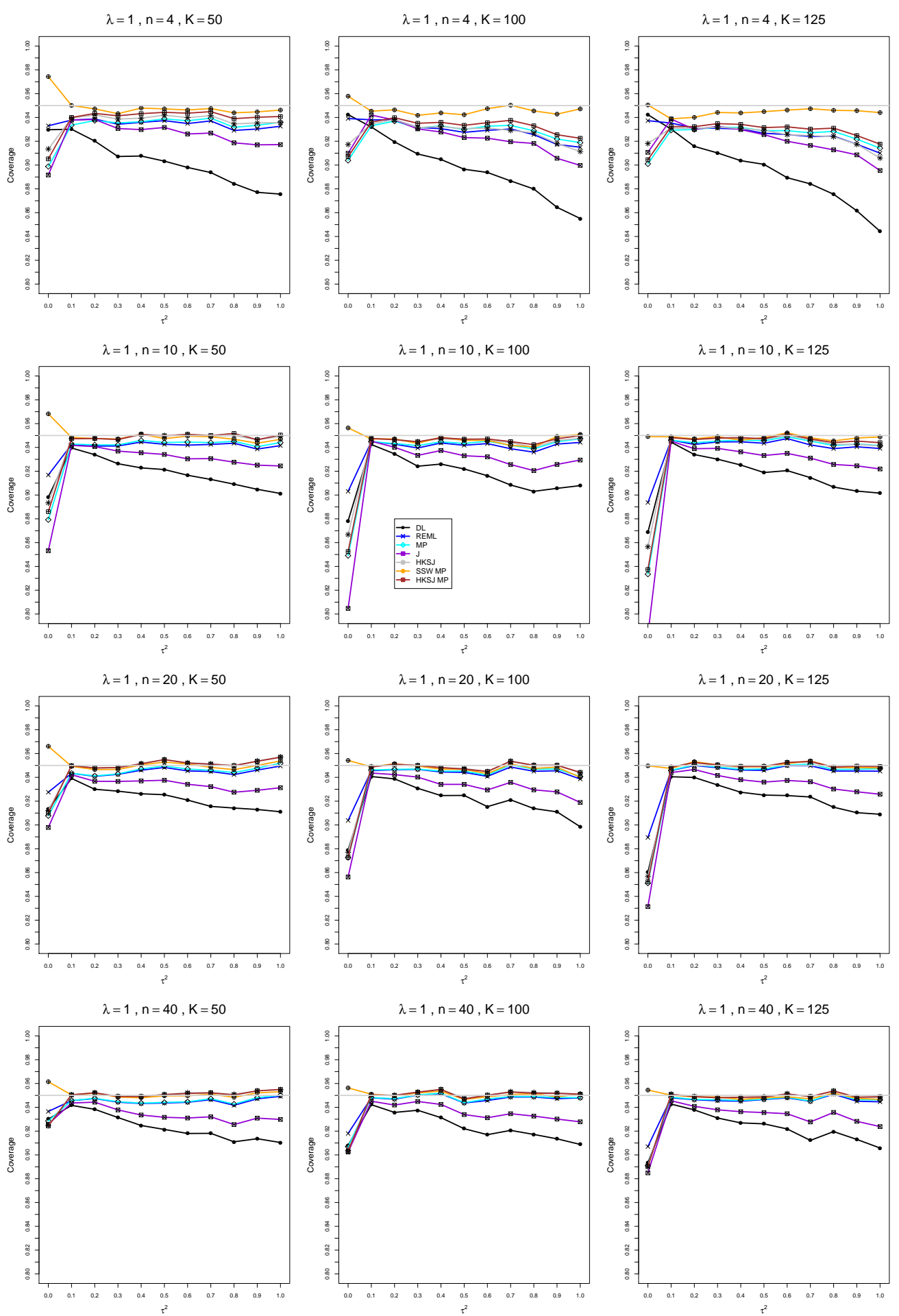

Figure B6.2.4: Coverage of $95 \%$ confidence intervals for $\lambda$ when $\mu_{C}=4, \lambda=1, n=$ 4, 10, 20, 40, and $K=50,100,125$ 

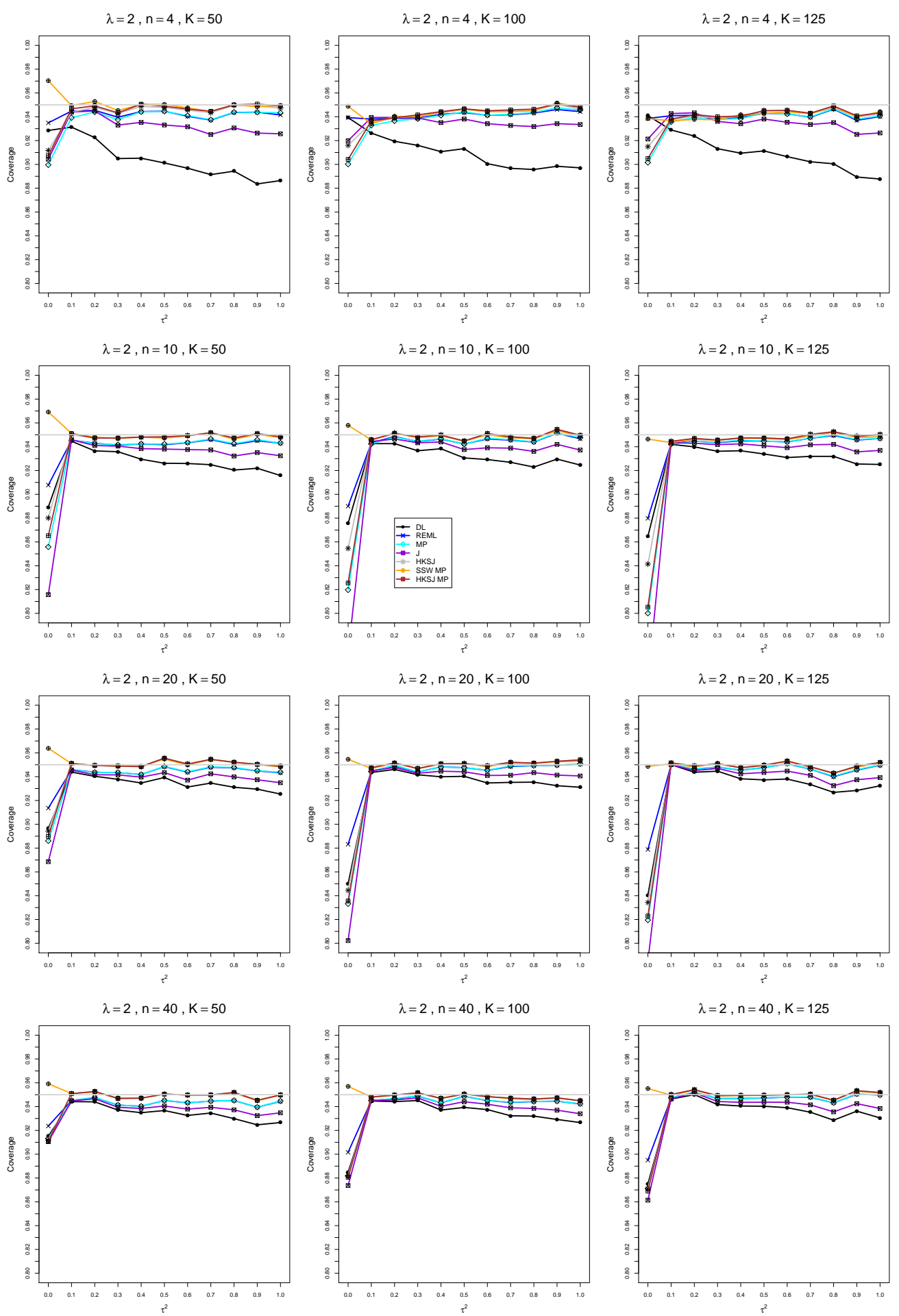

Figure B6.2.5: Coverage of $95 \%$ confidence intervals for $\lambda$ when $\mu_{C}=4, \lambda=2, n=$ 4, 10, 20, 40, and $K=50,100,125$ 


\section{C: Plots for bias and coverage of estimators of $\tau^{2}$ for RoM with $n=100,250,640,1000$}

- C1. Normal model, $\mu_{C}=1, K=5,10,30$

- C2. Normal model, $\mu_{C}=4, K=5,10,30$

- C3. Normal model, bias-corrected $\lambda, \mu_{C}=4, K=5,10,30$

- C4. Normal model, $\mu_{C}=1, K=50,100,125$

- C5. Normal model, $\mu_{C}=4, K=50,100,125$

- C6. Normal model, bias-corrected $\lambda, \mu_{C}=4, K=50,100,125$ 
C1. Normal model, $\mu_{C}=1, n=100,250,640,1000$, $K=5,10,30$

C1.1 Bias of point estimators of $\tau^{2}$ when $\mu_{C}=1$

Each figure corresponds to a value of $\lambda(=0,0.2,0.5,1,2)$, a set of values of $n(=$ $100,250,640,1000)$, and a set of values of $K(=5,10,30)$.

Each panel corresponds to a value of $n$ and a value of $K$ and has $\tau^{2}=0.0(0.1) 1.0$ on the horizontal axis.

The point estimators of $\tau^{2}$ are

- DL (DerSimonian-Laird)

- REML (restricted maximum likelihood)

- MP (Mandel-Paule)

- J (Jackson) 

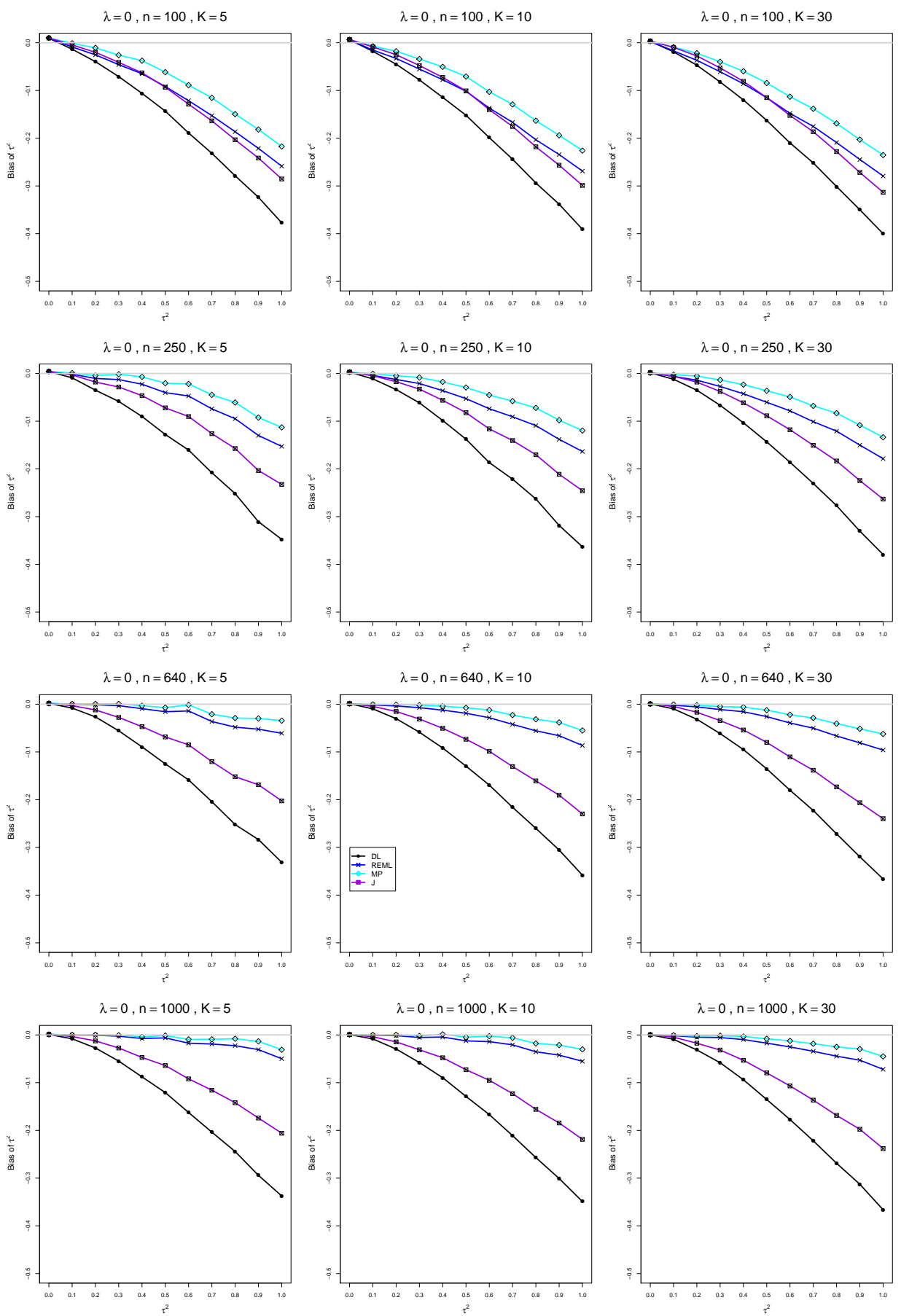

Figure C1.1.1: Bias of estimators of the between-studies variance $\tau^{2}$ when $\mu_{C}=1, \lambda=0$, $n=100,250,640,1000$, and $K=5,10,30$ 

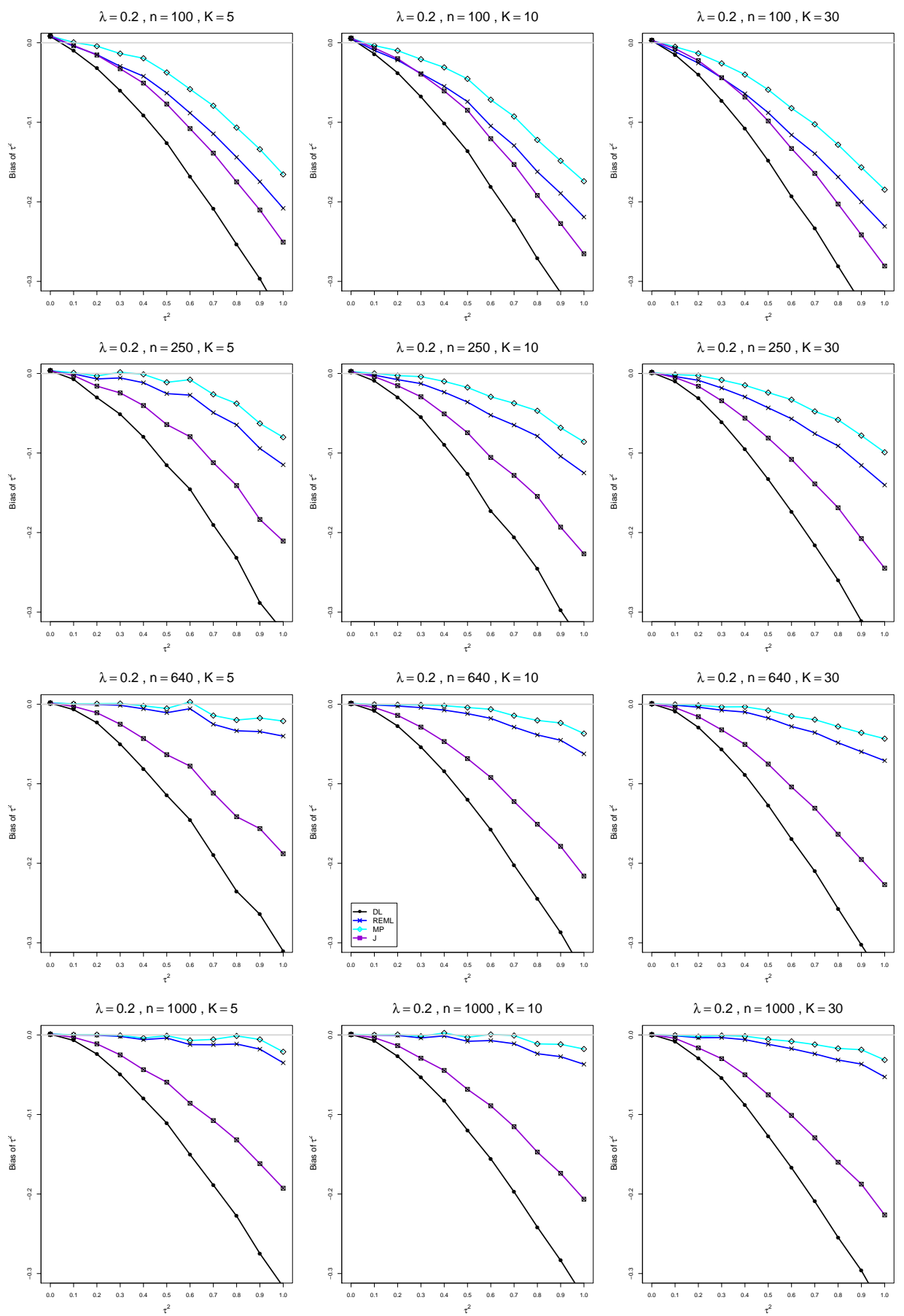

Figure C1.1.2: Bias of estimators of the between-studies variance $\tau^{2}$ when $\mu_{C}=1, \lambda=$ $0.2, n=100,250,640,1000$, and $K=5,10,30$ 

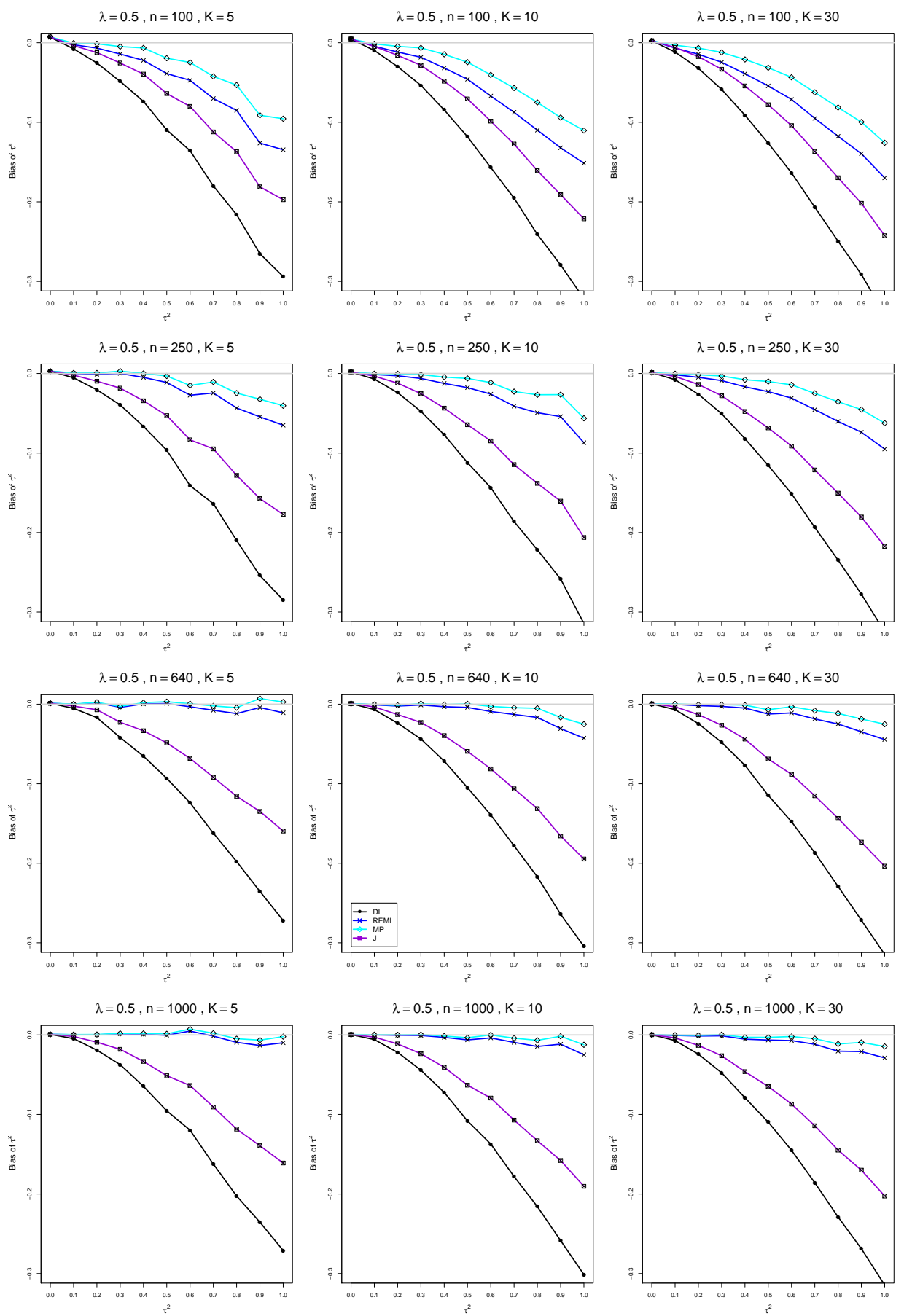

Figure C1.1.3: Bias of estimators of the between-studies variance $\tau^{2}$ when $\mu_{C}=1, \lambda=$ $0.5, n=100,250,640,1000$, and $K=5,10,30$ 

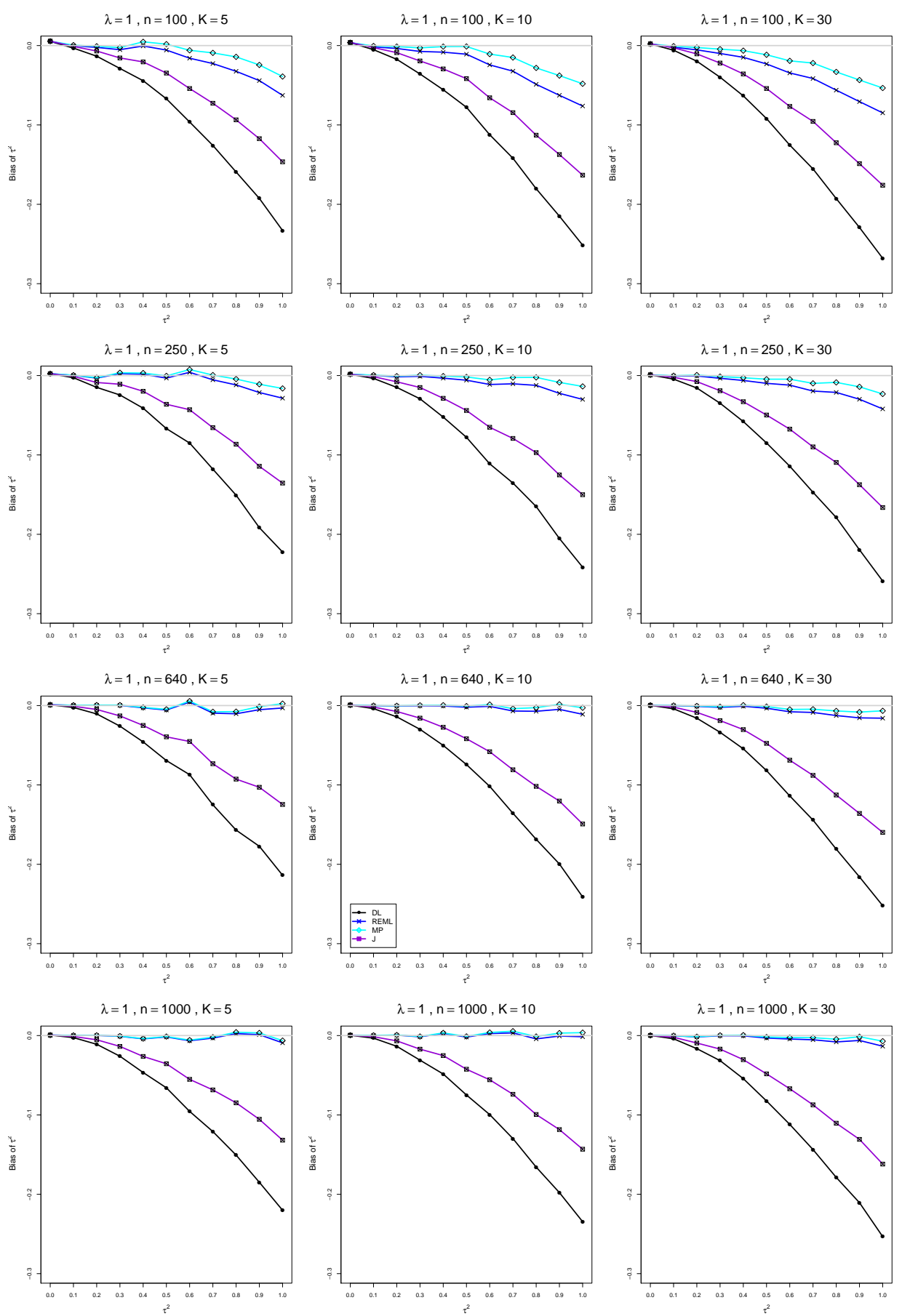

Figure C1.1.4: Bias of estimators of the between-studies variance $\tau^{2}$ when $\mu_{C}=1, \lambda=1$, $n=100,250,640,1000$, and $K=5,10,30$ 

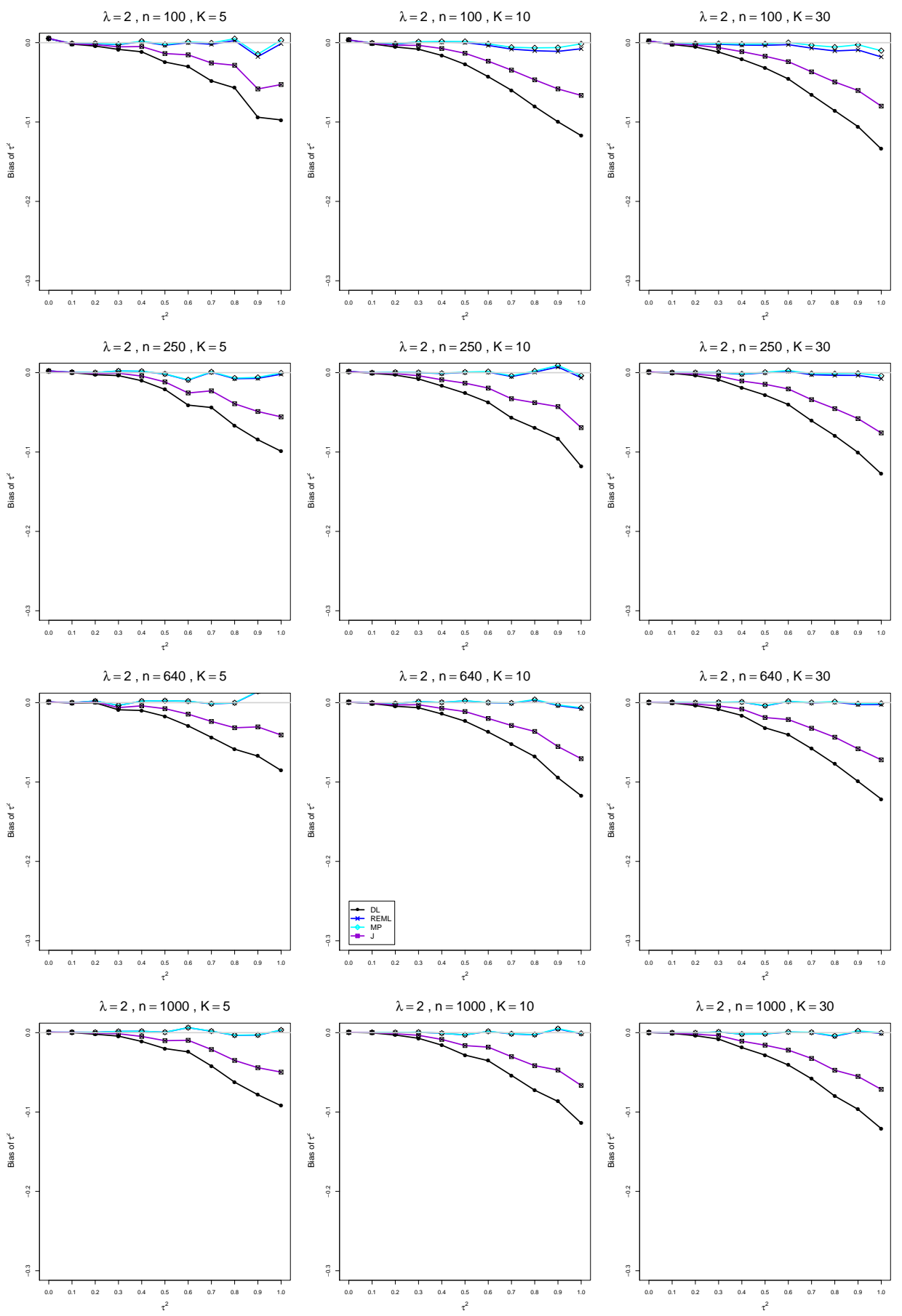

Figure C1.1.5: Bias of estimators of the between-studies variance $\tau^{2}$ when $\mu_{C}=1, \lambda=2$, $n=100,250,640,1000$, and $K=5,10,30$ 


\section{C1.2 Coverage of interval estimators of $\tau^{2}$ when $\mu_{C}=1$}

Each figure corresponds to a value of $\lambda(=0,0.2,0.5,1,2)$, a set of values of $n(=$ $100,250,640,1000)$, and a set of values of $K(=5,10,30)$.

Each panel corresponds to a value of $n$ and a value of $K$ and has $\tau^{2}=0.0(0.1) 1.0$ on the horizontal axis.

The interval estimators of $\tau^{2}$ are

- QP (Q-profile confidence interval)

- BJ (Biggerstaff and Jackson interval)

- PL (Profile likelihood interval)

- J (Jackson's interval) 

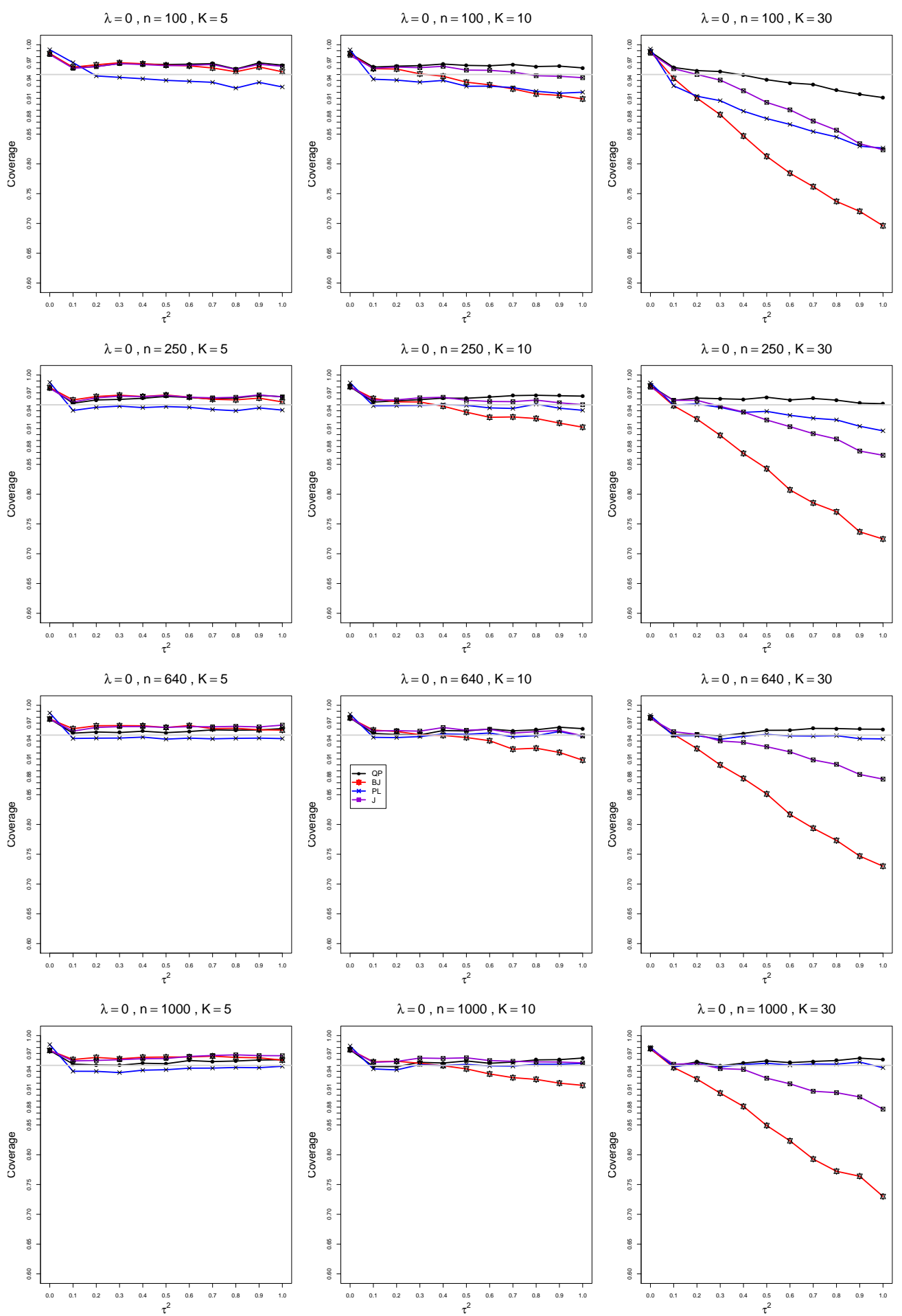

Figure C1.2.1: Coverage of 95\% confidence intervals for the between-studies variance $\tau^{2}$ when $\mu_{C}=1, \lambda=0, n=100,250,640,1000$, and $K=5,10,30$ 

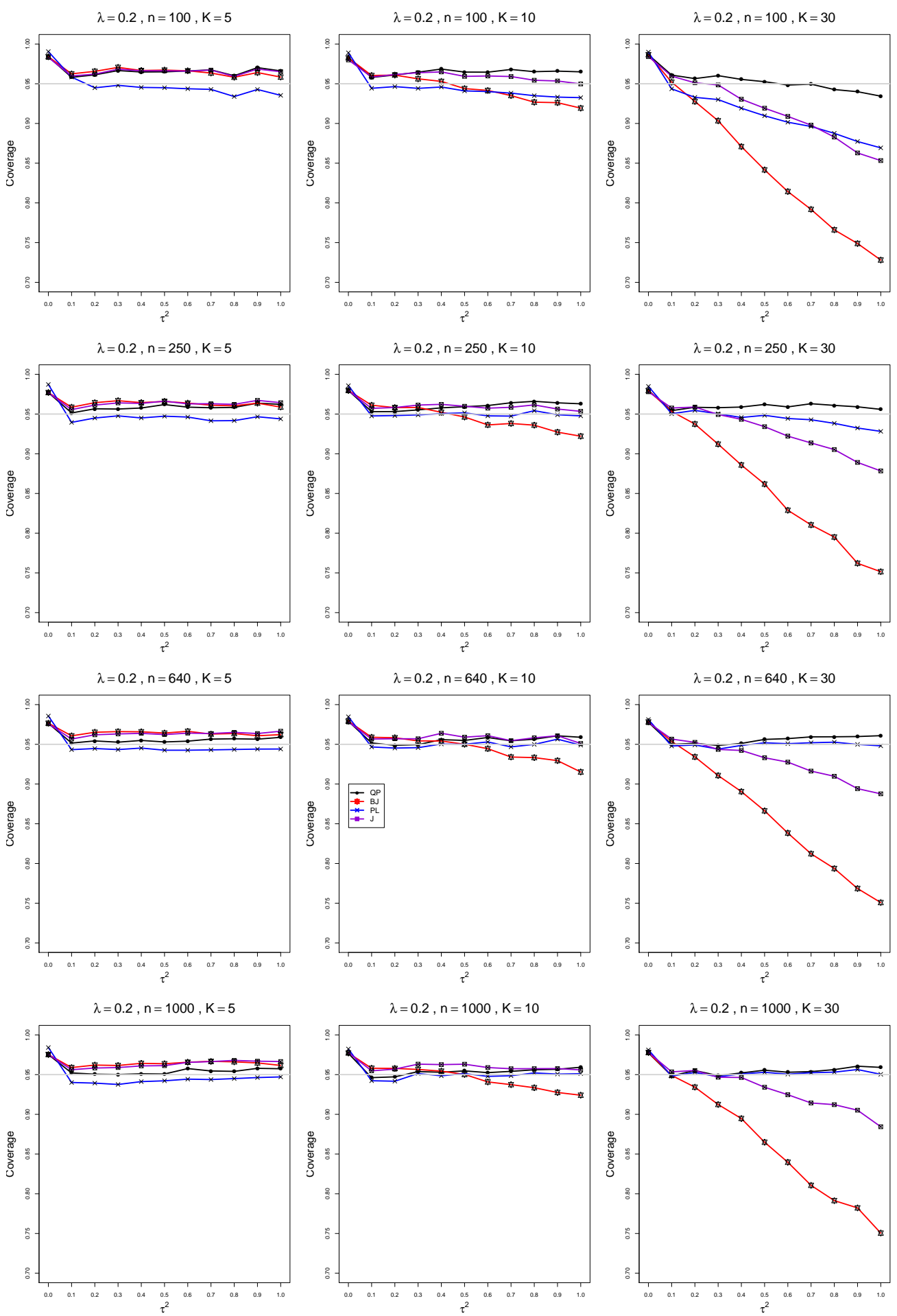

Figure C1.2.2: Coverage of 95\% confidence intervals for the between-studies variance $\tau^{2}$ when $\mu_{C}=1, \lambda=0.2, n=100,250,640,1000$, and $K=5,10,30$ 

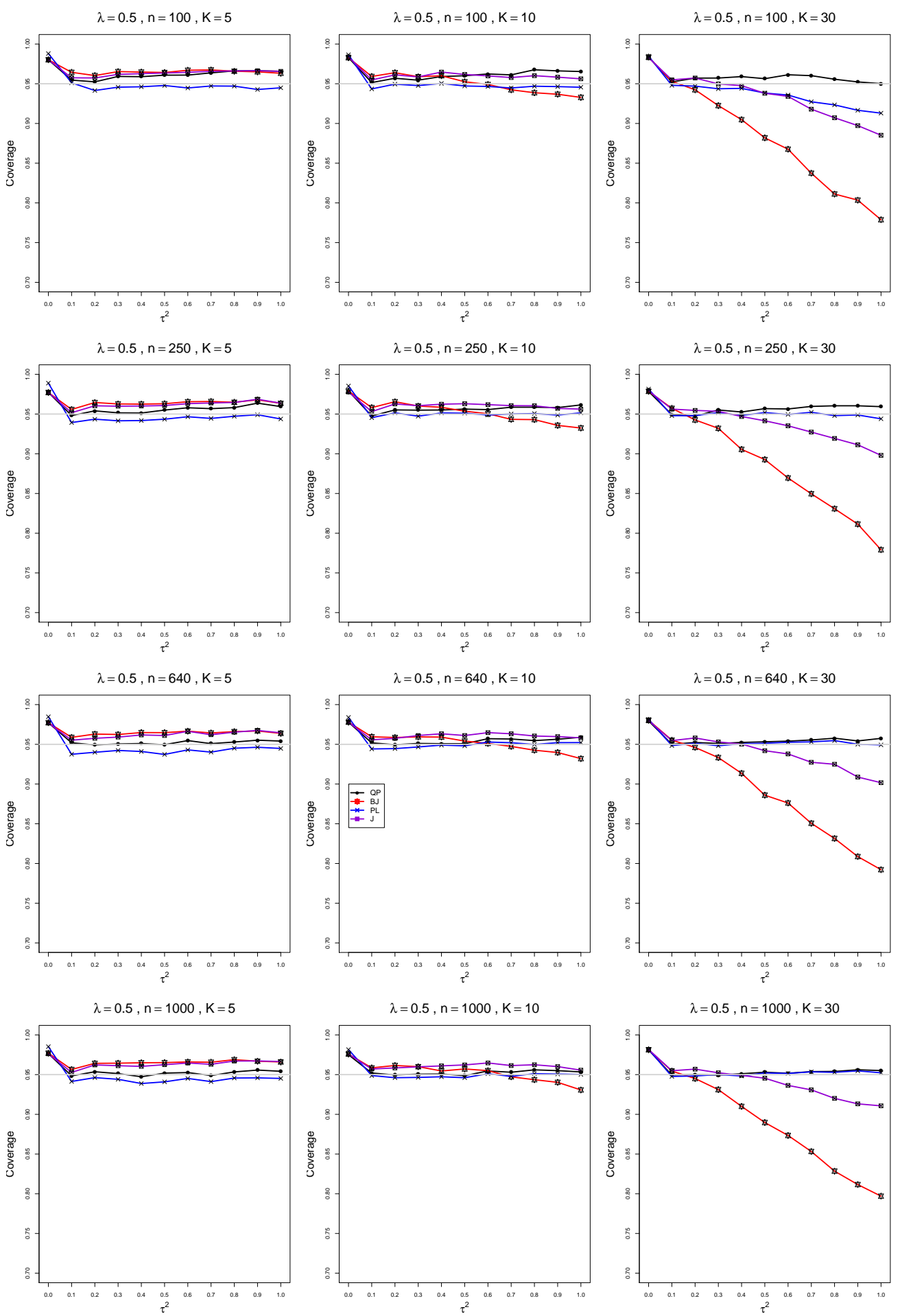

Figure C1.2.3: Coverage of 95\% confidence intervals for the between-studies variance $\tau^{2}$ when $\mu_{C}=1, \lambda=0.5, n=100,250,640,1000$, and $K=5,10,30$ 

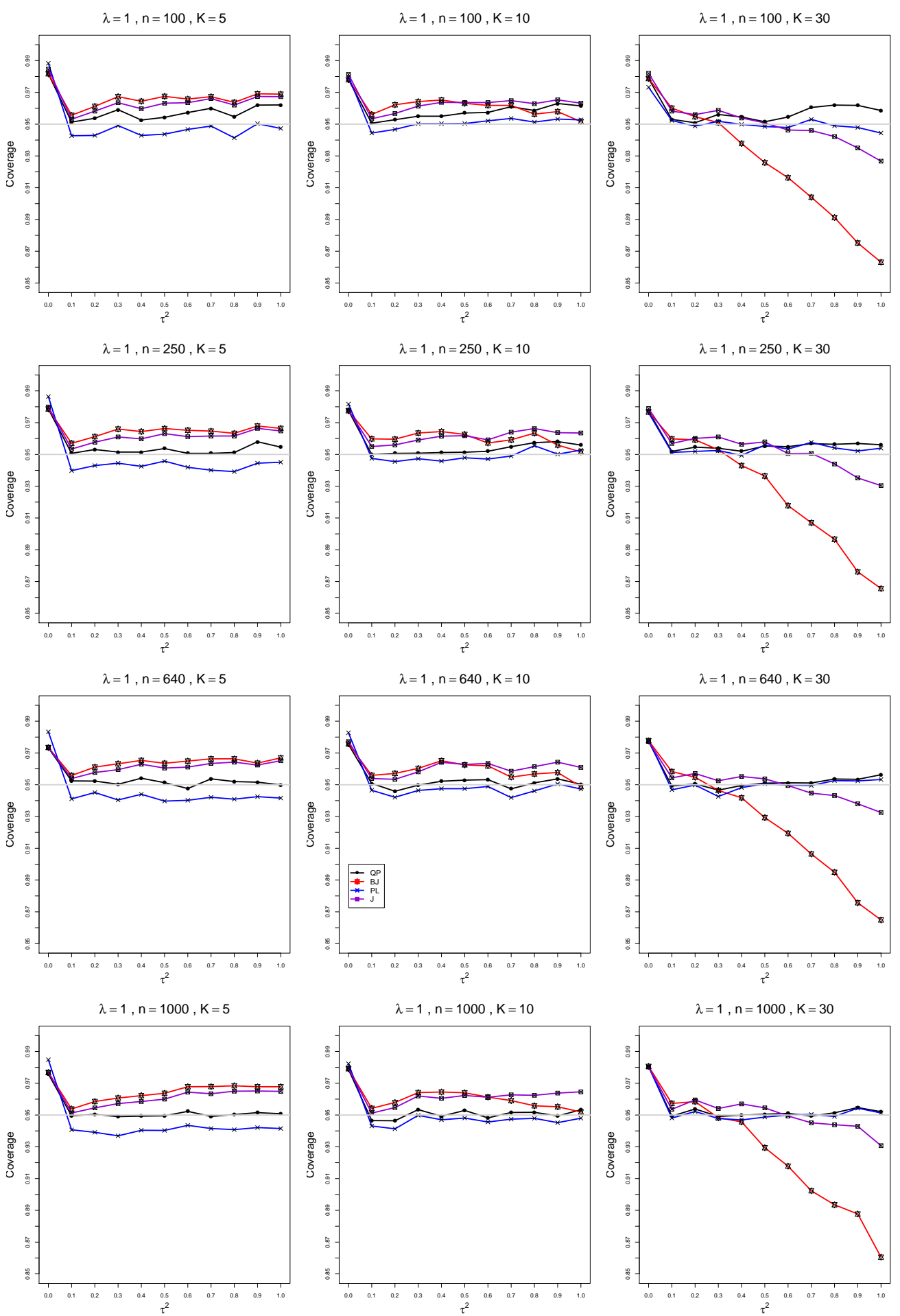

Figure C1.2.4: Coverage of 95\% confidence intervals for the between-studies variance $\tau^{2}$ when $\mu_{C}=1, \lambda=1, n=100,250,640,1000$, and $K=5,10,30$ 

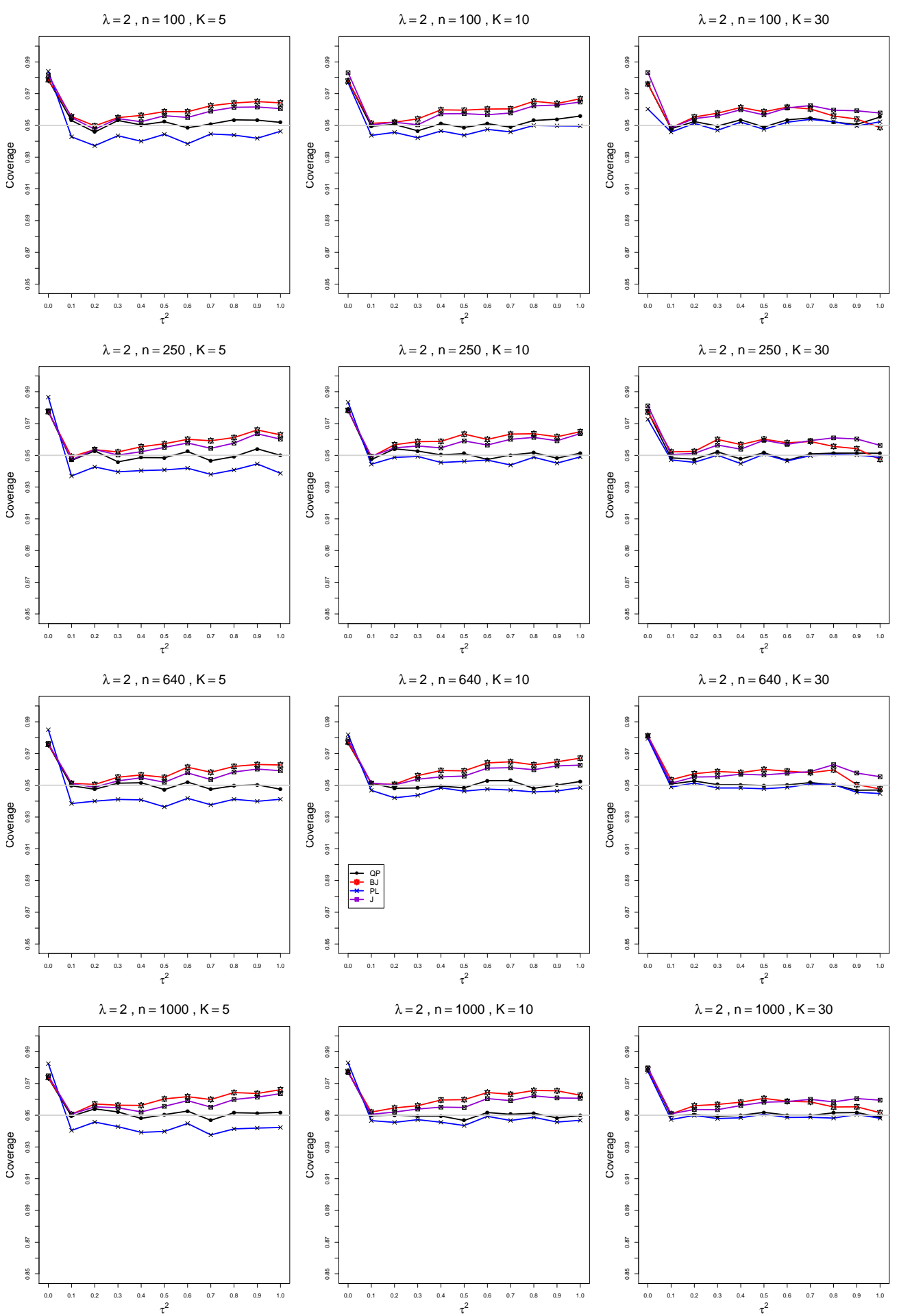

Figure C1.2.5: Coverage of 95\% confidence intervals for the between-studies variance $\tau^{2}$ when $\mu_{C}=1, \lambda=2, n=100,250,640,1000$, and $K=5,10,30$ 
C2. Normal model, $\mu_{C}=4, n=100,250,640,1000$, $K=5,10,30$

C2.1 Bias of point estimators of $\tau^{2}$ when $\mu_{C}=4$

Each figure corresponds to a value of $\lambda(=0,0.2,0.5,1,2)$, a set of values of $n(=$ $100,250,640,1000)$, and a set of values of $K(=5,10,30)$.

Each panel corresponds to a value of $n$ and a value of $K$ and has $\tau^{2}=0.0(0.1) 1.0$ on the horizontal axis.

The point estimators of $\tau^{2}$ are

- DL (DerSimonian-Laird)

- REML (restricted maximum likelihood)

- MP (Mandel-Paule)

- J (Jackson) 

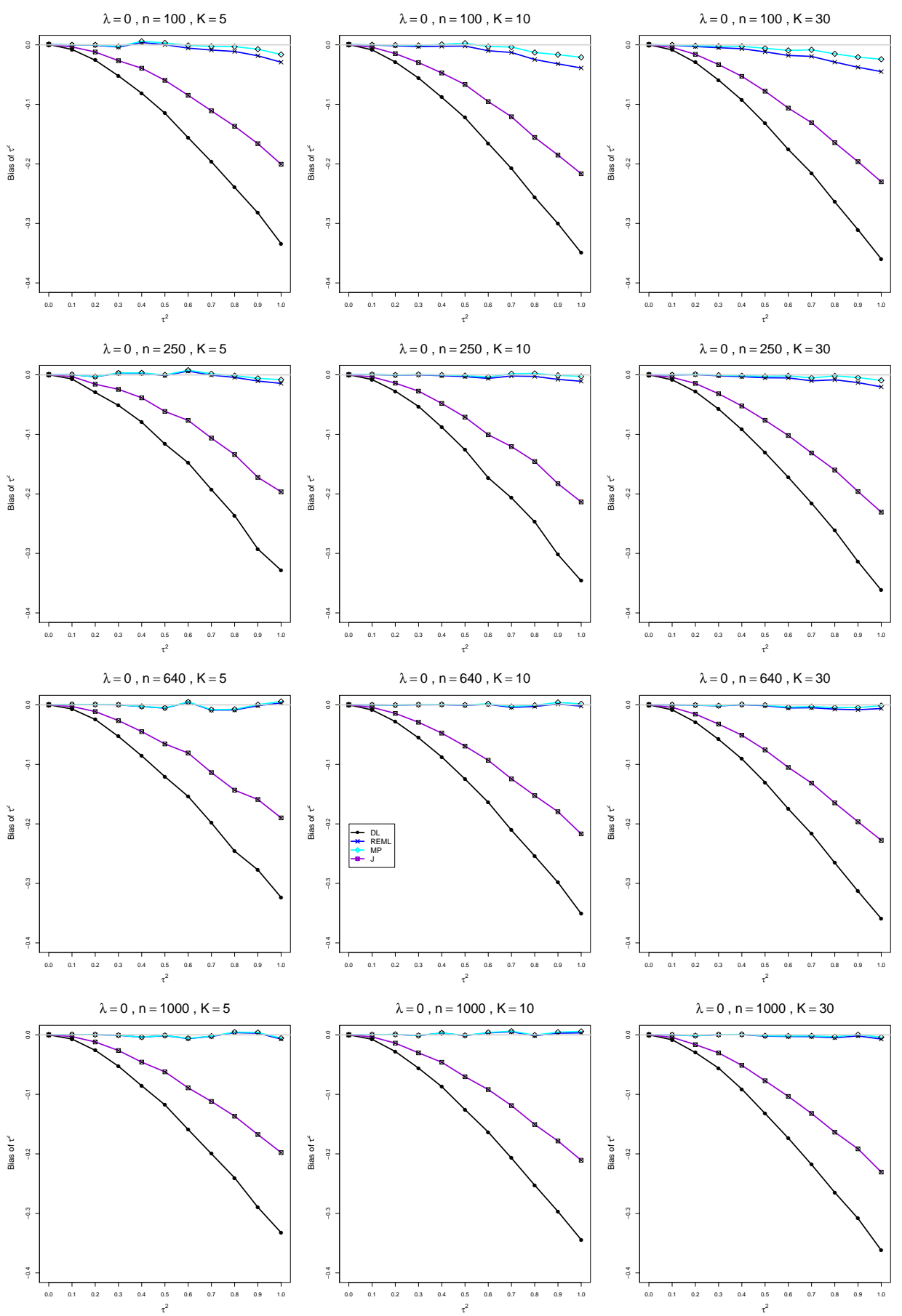

Figure C2.1.1: Bias of estimators of the between-studies variance $\tau^{2}$ when $\mu_{C}=4, \lambda=0$, $n=100,250,640,1000$, and $K=5,10,30$ 

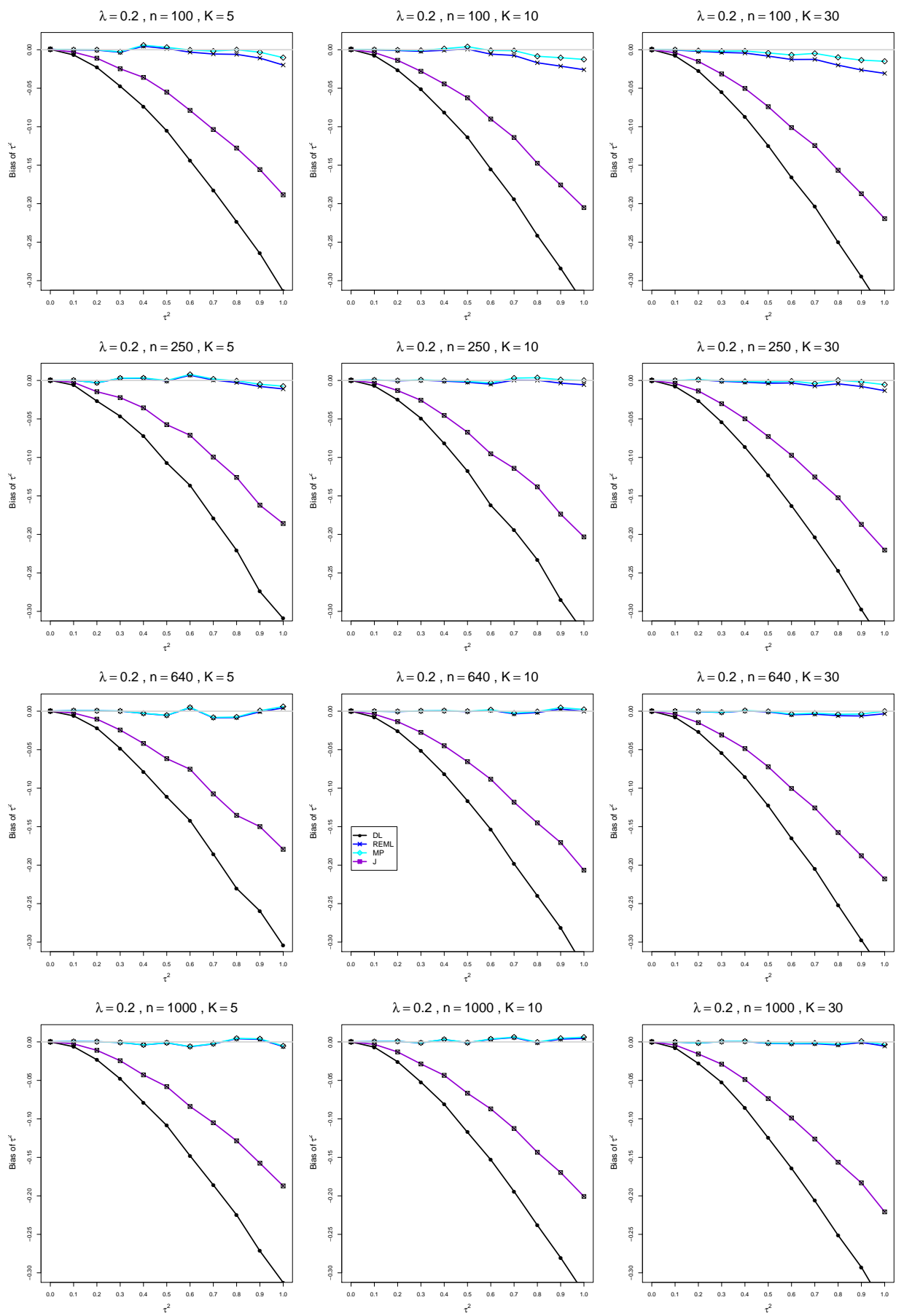

Figure C2.1.2: Bias of estimators of the between-studies variance $\tau^{2}$ when $\mu_{C}=4, \lambda=$ $0.2, n=100,250,640,1000$, and $K=5,10,30$ 

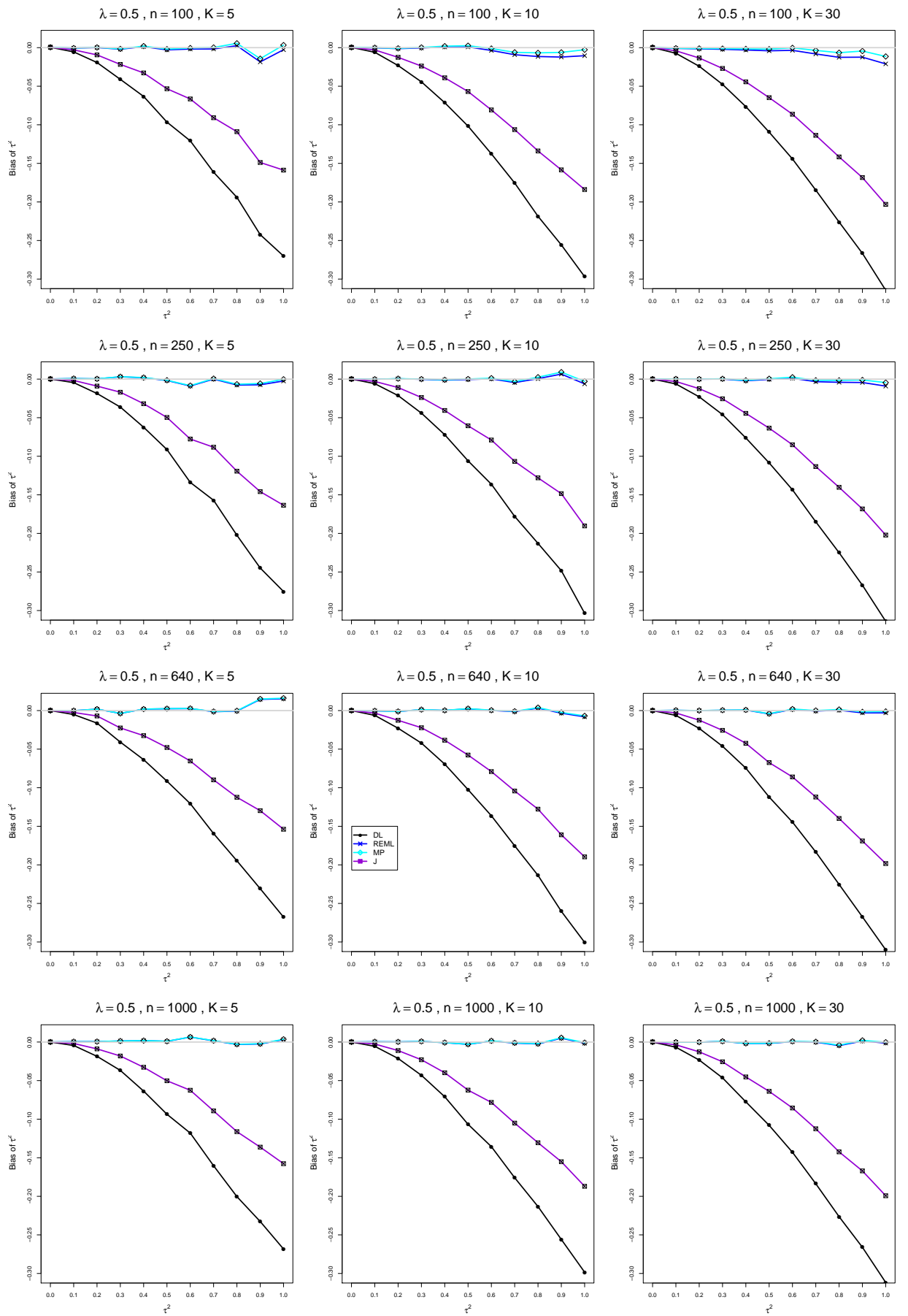

Figure C2.1.3: Bias of estimators of the between-studies variance $\tau^{2}$ when $\mu_{C}=4, \lambda=$ $0.5, n=100,250,640,1000$, and $K=5,10,30$ 

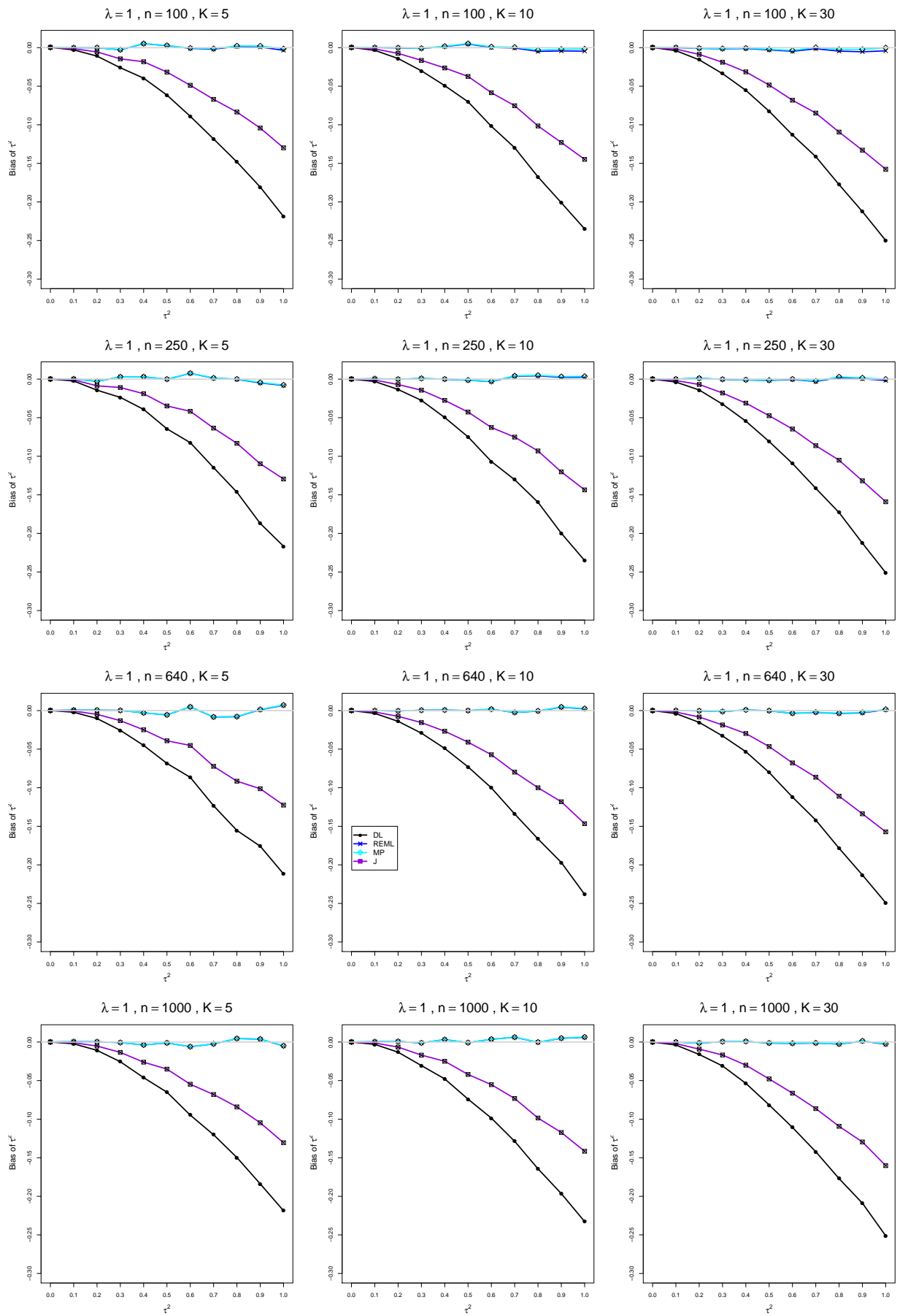

Figure C2.1.4: Bias of estimators of the between-studies variance $\tau^{2}$ when $\mu_{C}=4, \lambda=1$, $n=100,250,640,1000$, and $K=5,10,30$ 

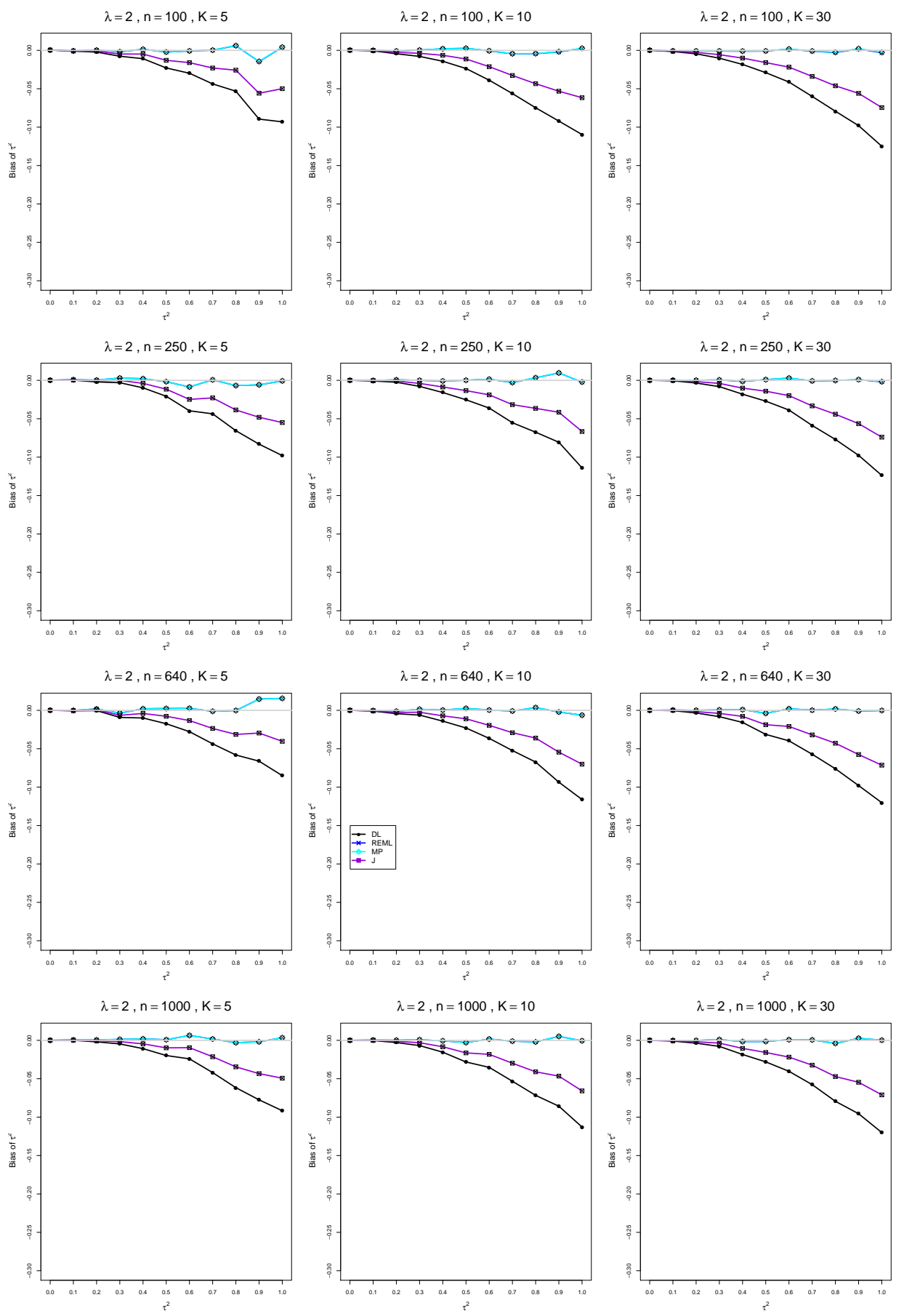

Figure C2.1.5: Bias of estimators of the between-studies variance $\tau^{2}$ when $\mu_{C}=4, \lambda=2$, $n=100,250,640,1000$, and $K=5,10,30$ 


\section{C2.2 Coverage of interval estimators of $\tau^{2}$ when $\mu_{C}=4$}

Each figure corresponds to a value of $\lambda(=0,0.2,0.5,1,2)$, a set of values of $n(=$ $100,250,640,1000)$, and a set of values of $K(=5,10,30)$.

Each panel corresponds to a value of $n$ and a value of $K$ and has $\tau^{2}=0.0(0.1) 1.0$ on the horizontal axis.

The interval estimators of $\tau^{2}$ are

- QP (Q-profile confidence interval)

- BJ (Biggerstaff and Jackson interval)

- PL (Profile likelihood interval)

- J (Jackson's interval) 

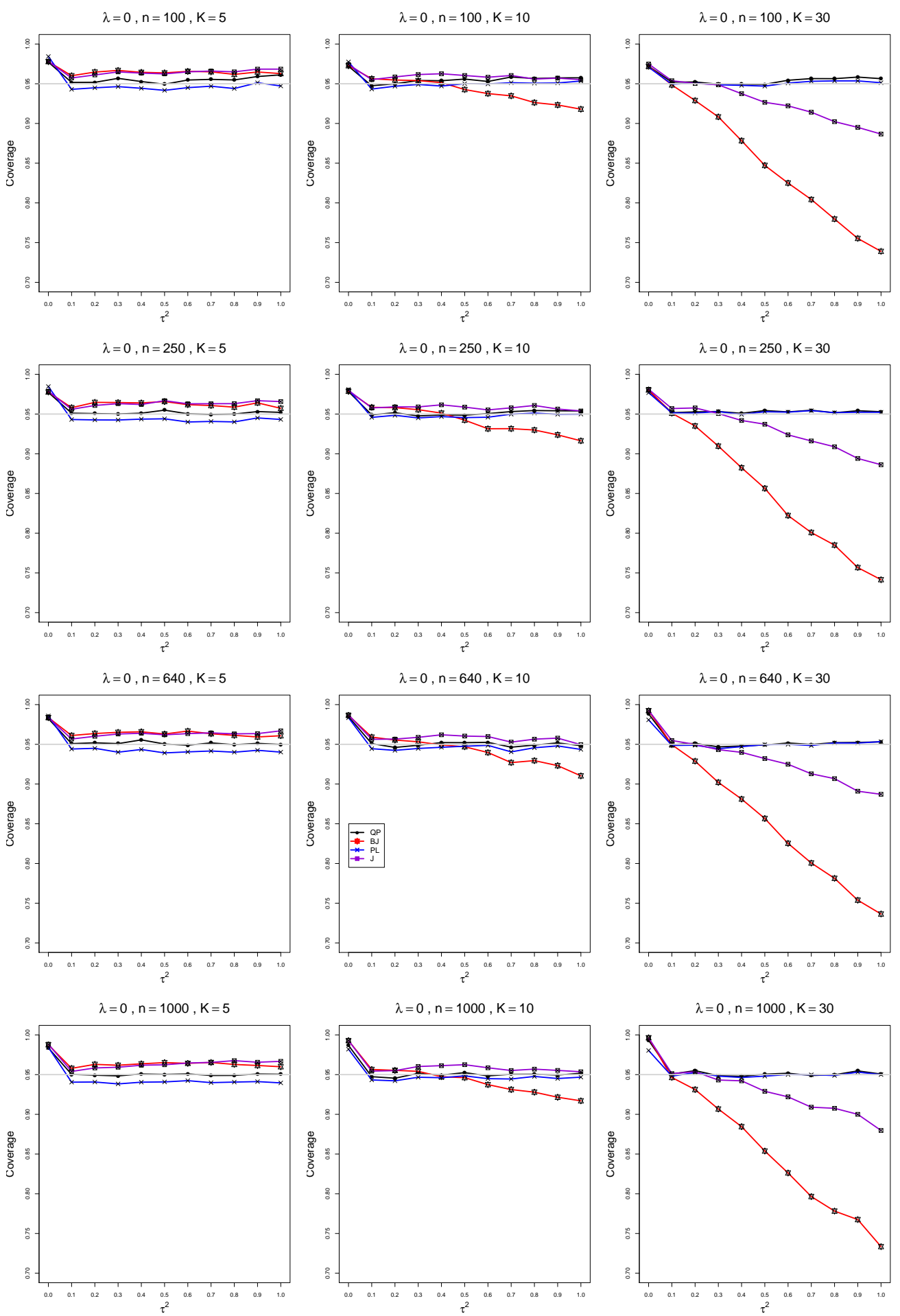

Figure C2.2.1: Coverage of 95\% confidence intervals for the between-studies variance $\tau^{2}$ when $\mu_{C}=4, \lambda=0, n=100,250,640,1000$, and $K=5,10,30$ 

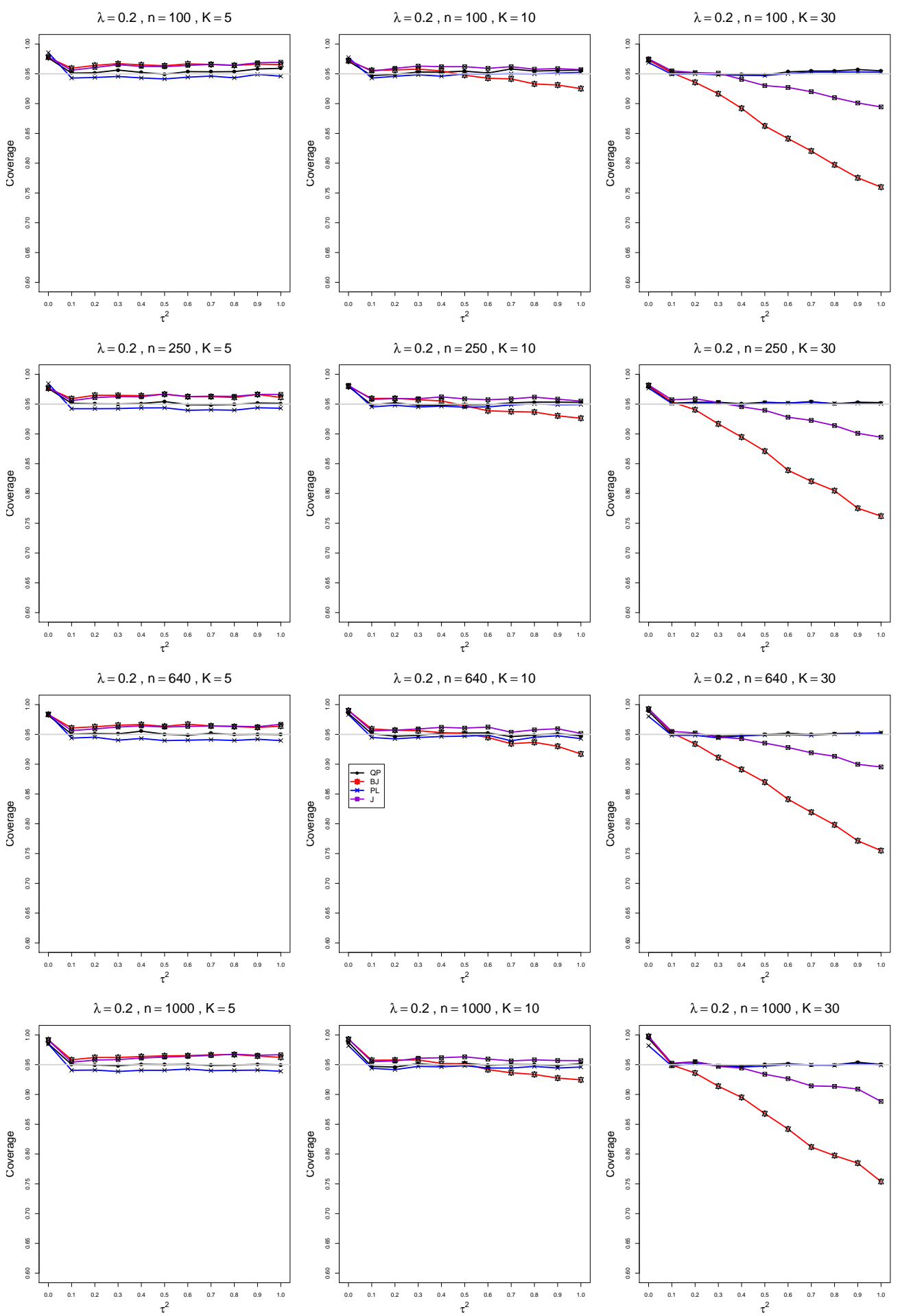

Figure C2.2.2: Coverage of 95\% confidence intervals for the between-studies variance $\tau^{2}$ when $\mu_{C}=4, \lambda=0.2, n=100,250,640,1000$, and $K=5,10,30$ 

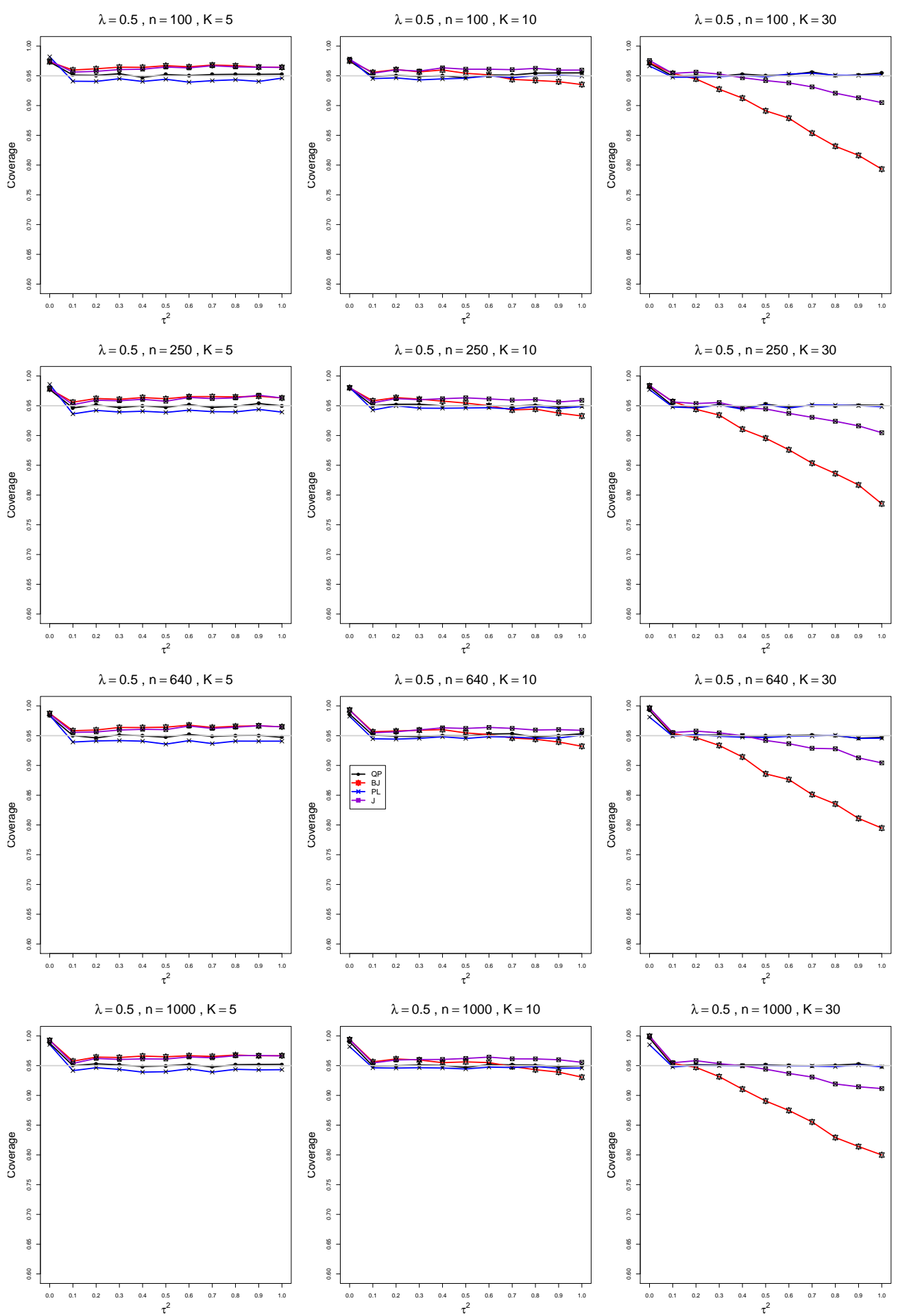

Figure C2.2.3: Coverage of 95\% confidence intervals for the between-studies variance $\tau^{2}$ when $\mu_{C}=4, \lambda=0.5, n=100,250,640,1000$, and $K=5,10,30$ 

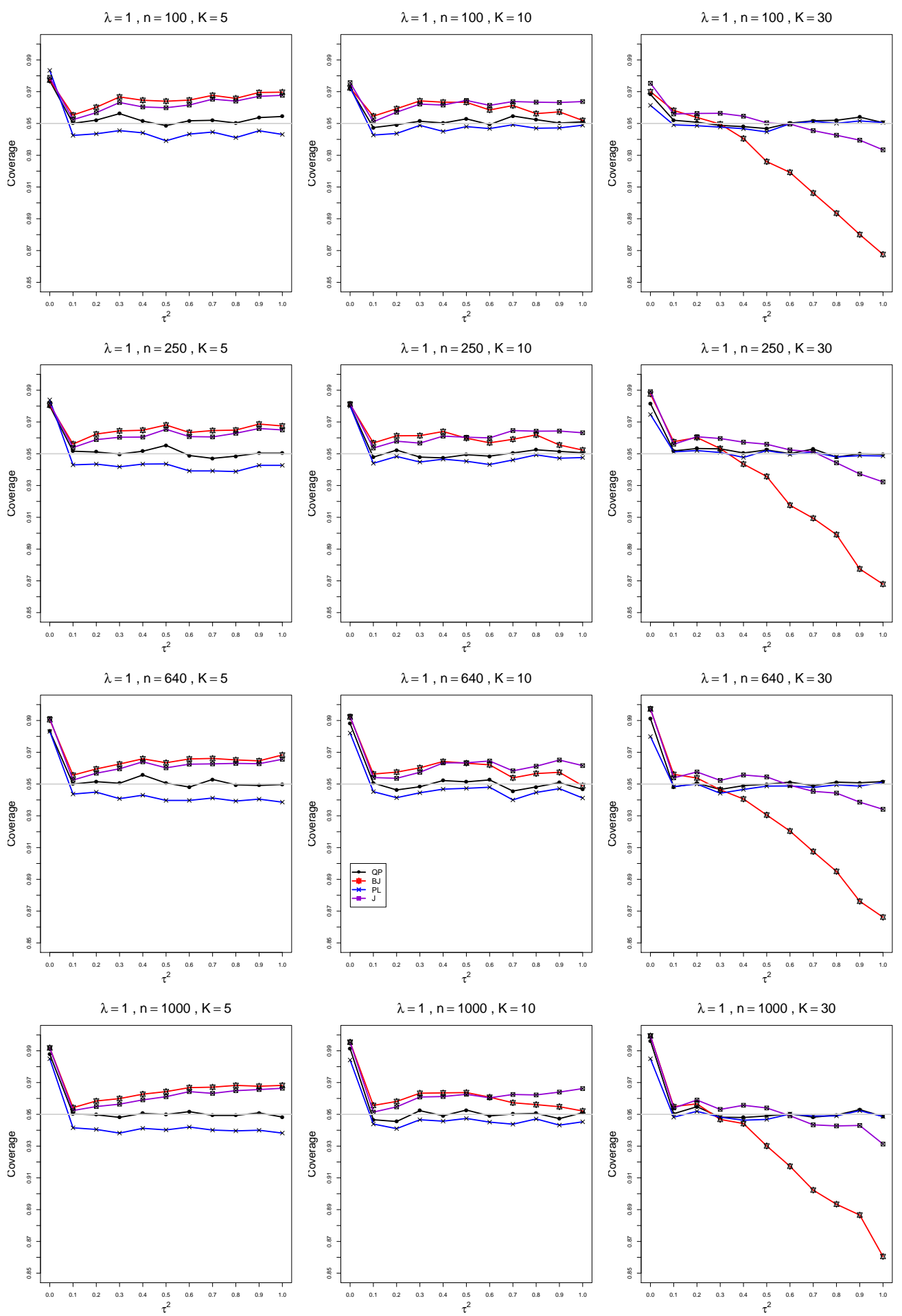

Figure C2.2.4: Coverage of 95\% confidence intervals for the between-studies variance $\tau^{2}$ when $\mu_{C}=4, \lambda=1, n=100,250,640,1000$, and $K=5,10,30$ 

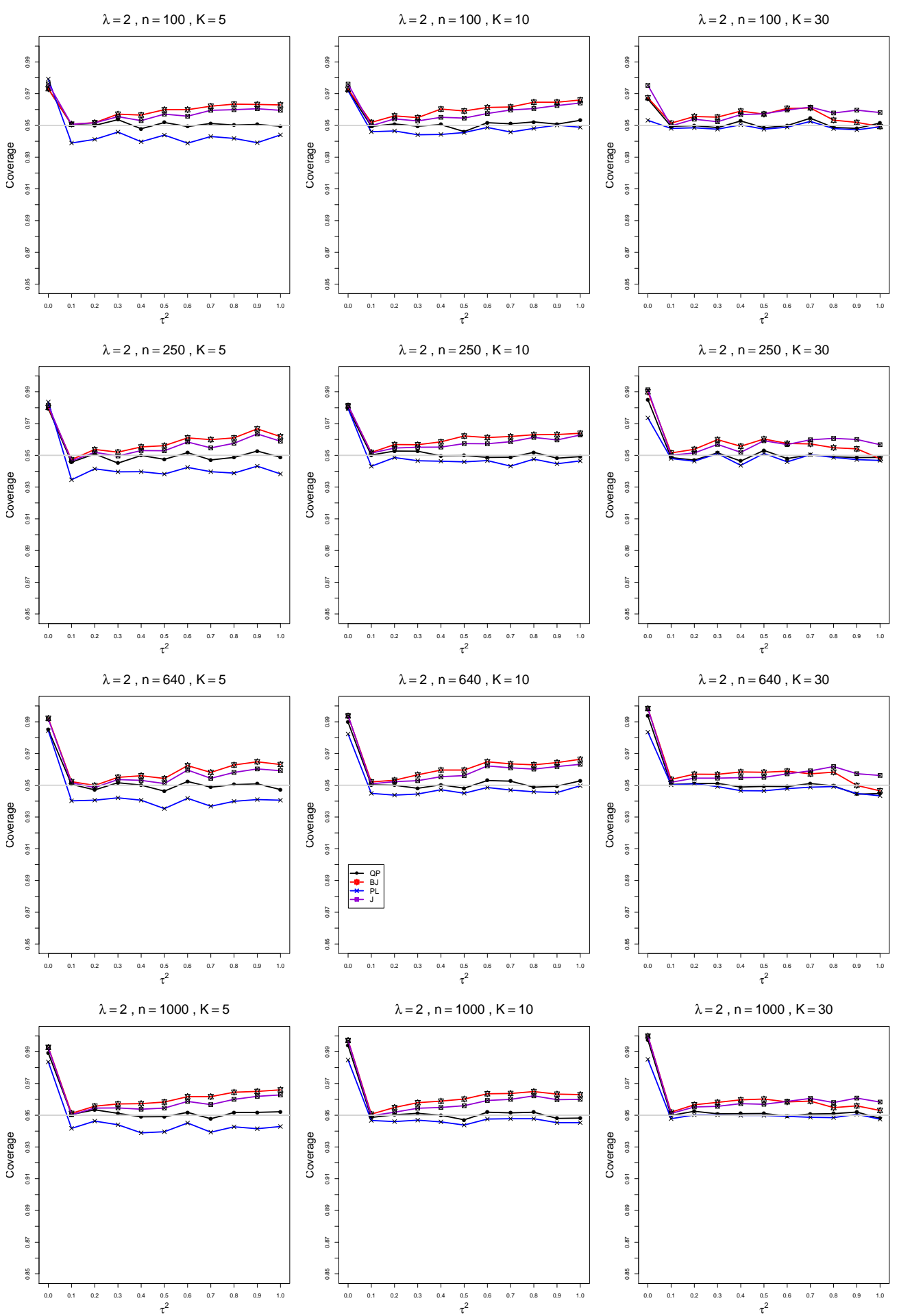

Figure C2.2.5: Coverage of 95\% confidence intervals for the between-studies variance $\tau^{2}$ when $\mu_{C}=4, \lambda=2, n=100,250,640,1000$, and $K=5,10,30$ 


\section{C3. Normal model, bias-corrected $\lambda, \mu_{C}=4$,}

$n=100,250,640,1000, K=5,10,30$

C3.1 Bias of point estimators of $\tau^{2}$ when $\mu_{C}=4$

Each figure corresponds to a value of $\lambda(=0,0.2,0.5,1,2)$, a set of values of $n(=$ $100,250,640,1000)$, and a set of values of $K(=5,10,30)$.

Each panel corresponds to a value of $n$ and a value of $K$ and has $\tau^{2}=0.0(0.1) 1.0$ on the horizontal axis.

The point estimators of $\tau^{2}$ are

- DL (DerSimonian-Laird)

- REML (restricted maximum likelihood)

- MP (Mandel-Paule)

- J (Jackson) 

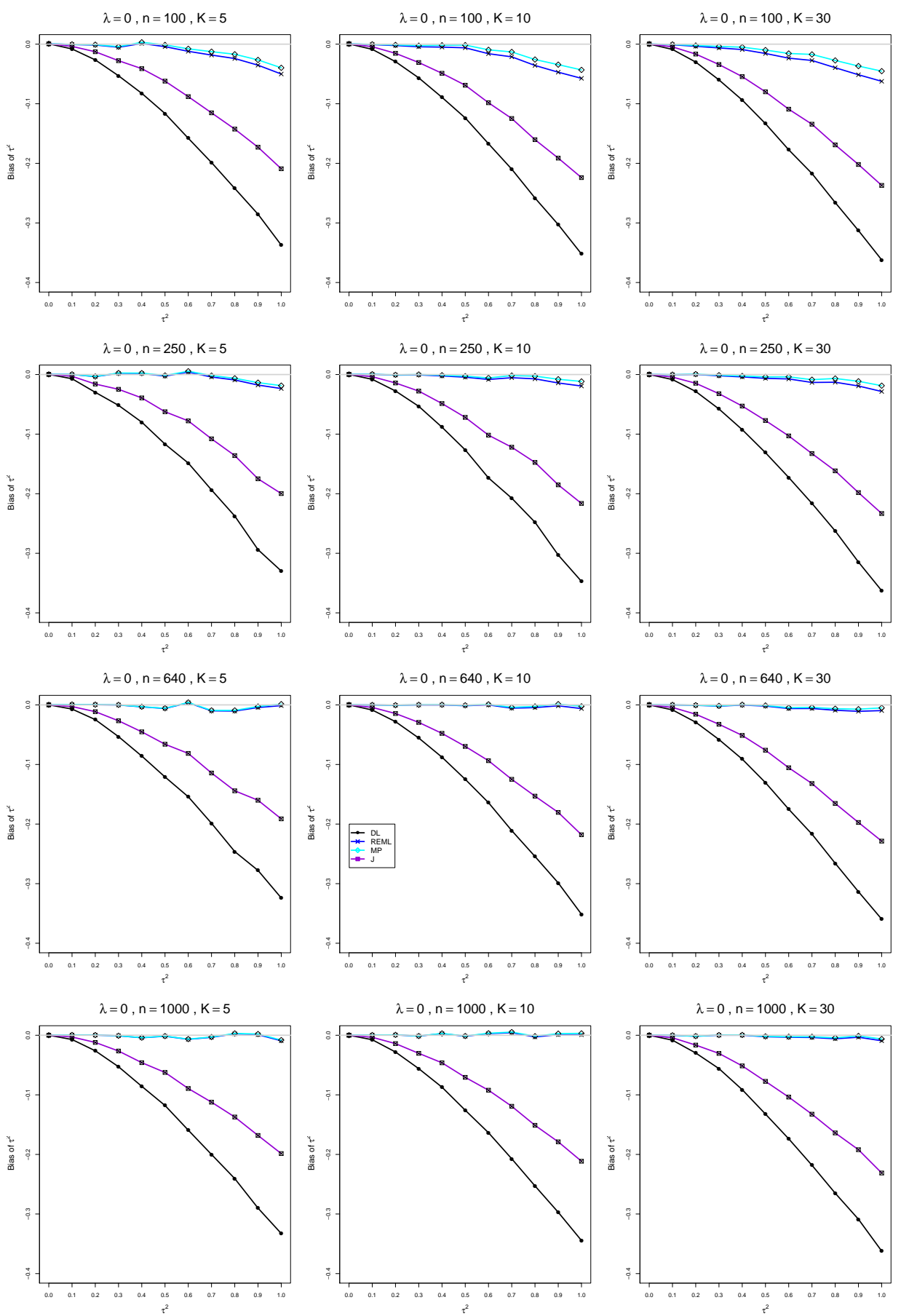

Figure C3.1.1: Bias of estimators of the between-studies variance $\tau^{2}$ when $\mu_{C}=4, \lambda=0$, $n=100,250,640,1000$, and $K=5,10,30$ 

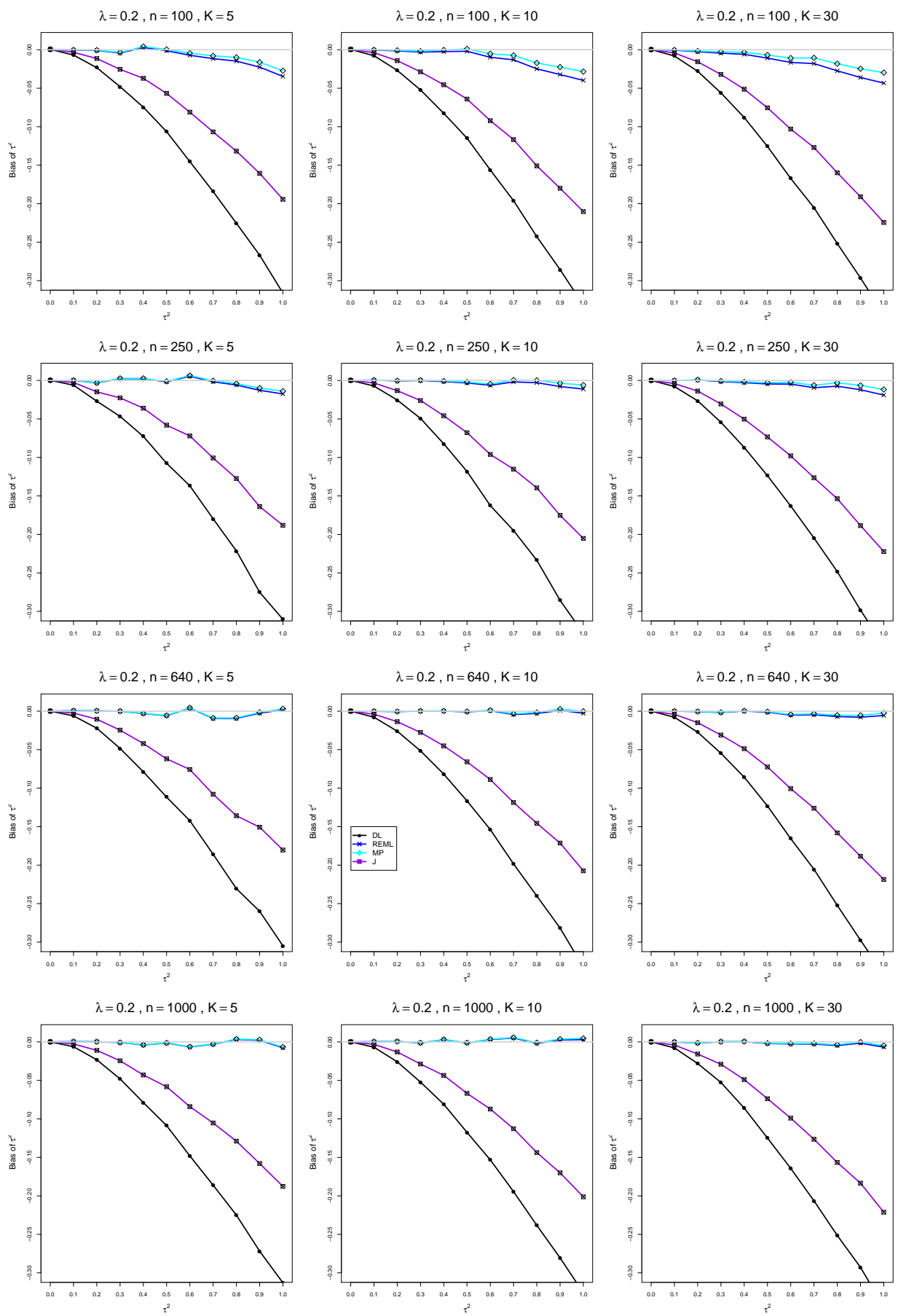

Figure C3.1.2: Bias of estimators of the between-studies variance $\tau^{2}$ when $\mu_{C}=4, \lambda=$ $0.2, n=100,250,640,1000$, and $K=5,10,30$ 

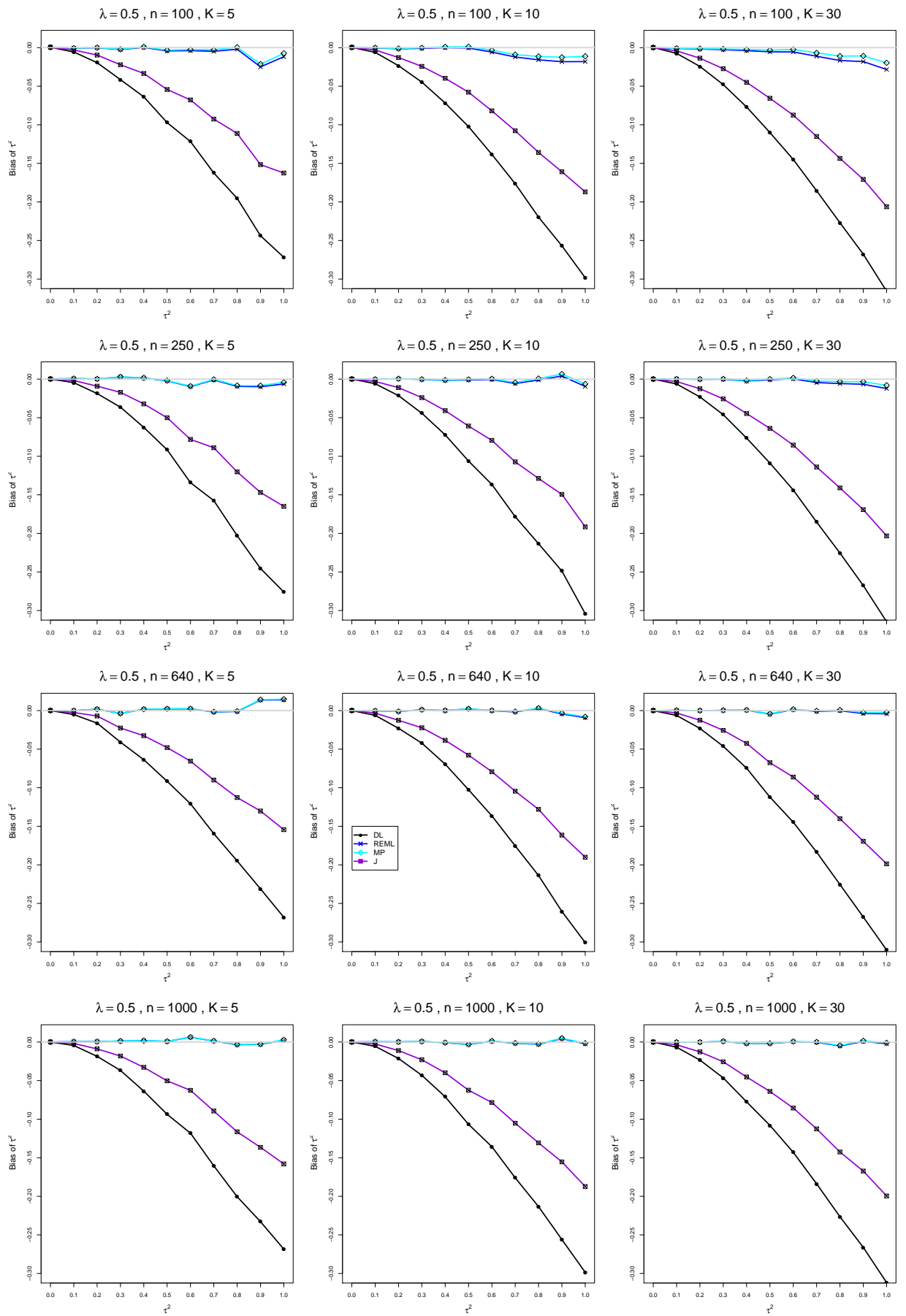

Figure C3.1.3: Bias of estimators of the between-studies variance $\tau^{2}$ when $\mu_{C}=4, \lambda=$ $0.5, n=100,250,640,1000$, and $K=5,10,30$ 

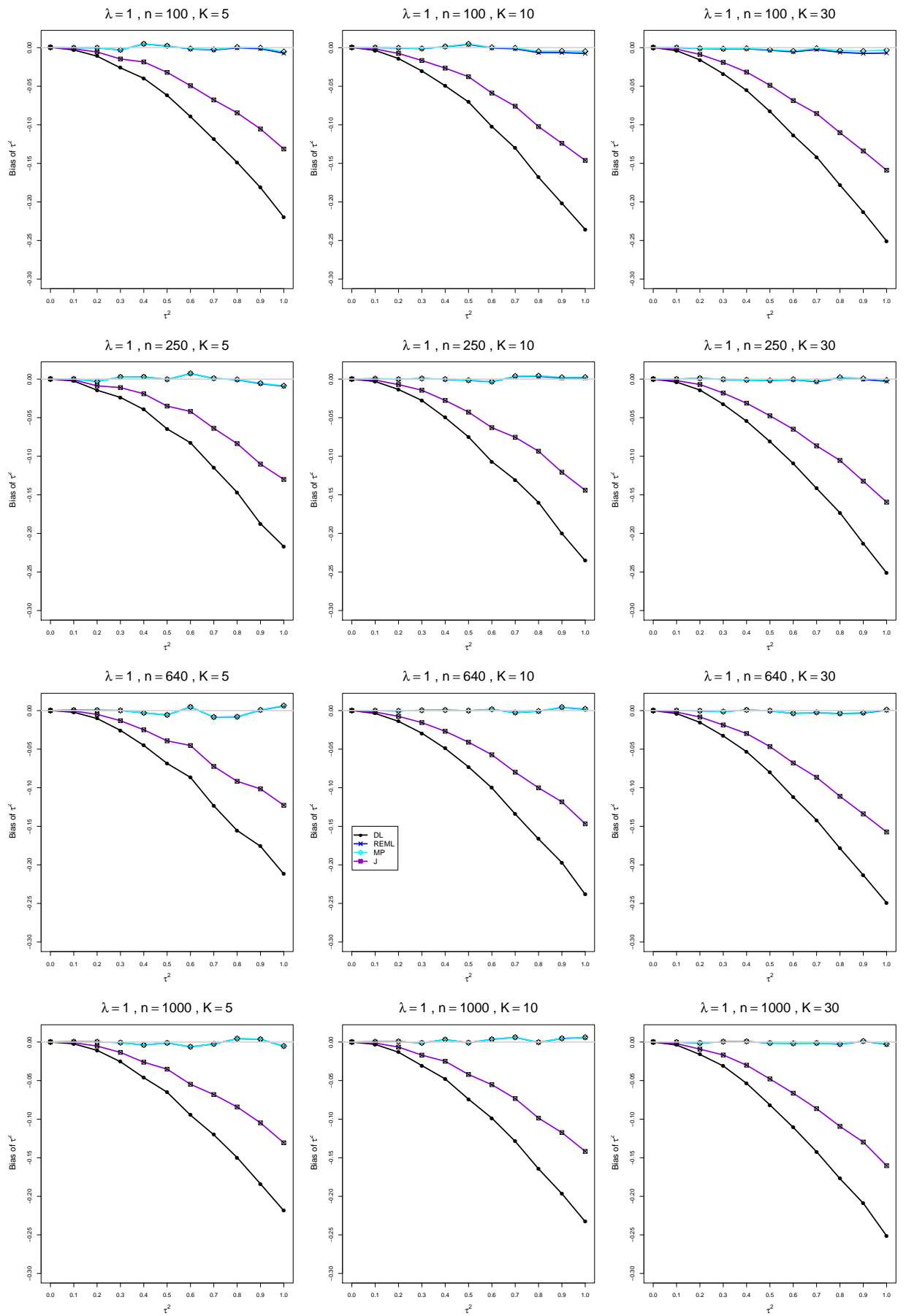

Figure C3.1.4: Bias of estimators of the between-studies variance $\tau^{2}$ when $\mu_{C}=4, \lambda=1$, $n=100,250,640,1000$, and $K=5,10,30$ 

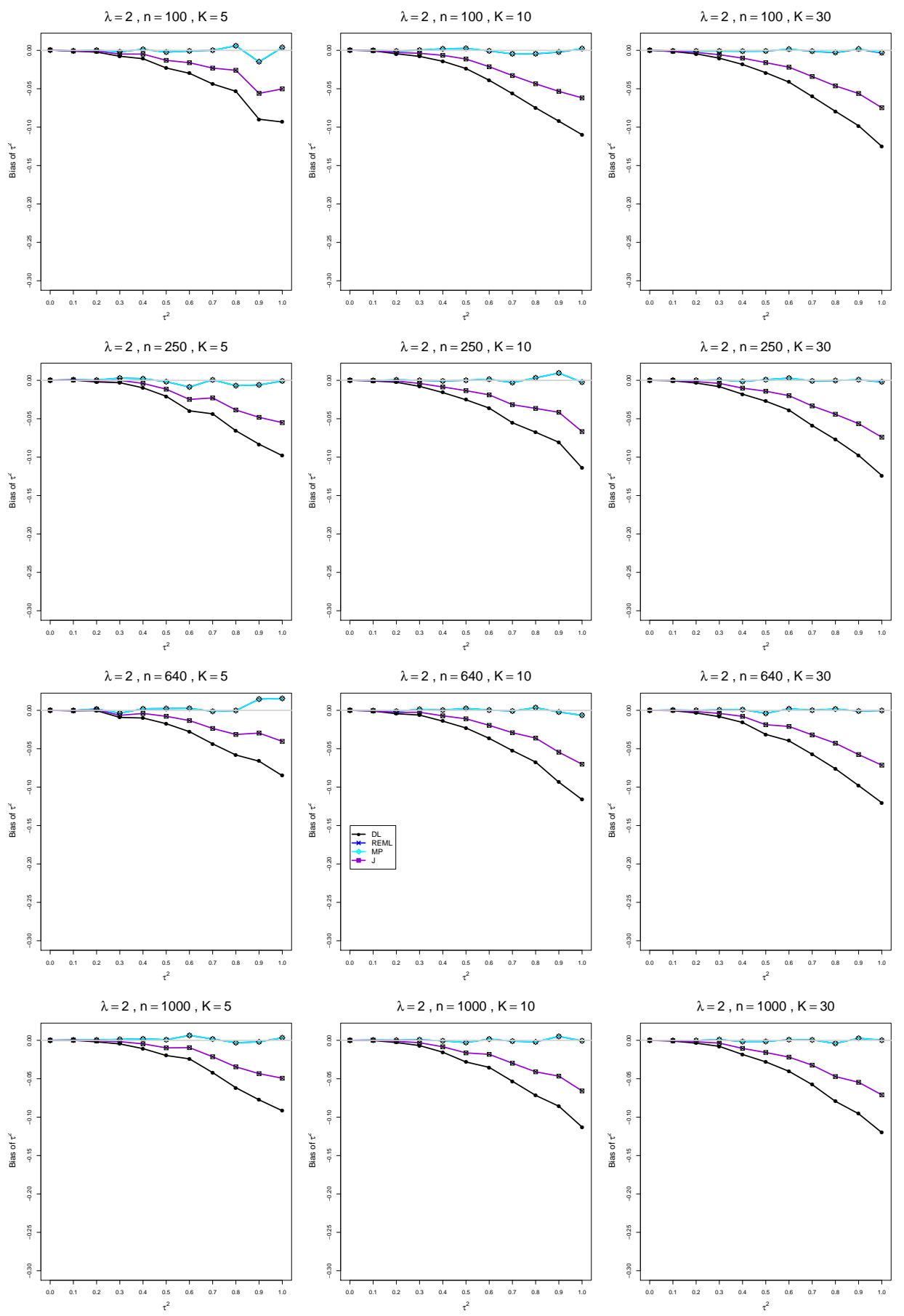

Figure C3.1.5: Bias of estimators of the between-studies variance $\tau^{2}$ when $\mu_{C}=4, \lambda=2$, $n=100,250,640,1000$, and $K=5,10,30$ 


\section{C3.2 Coverage of interval estimators of $\tau^{2}$ when $\mu_{C}=4$}

Each figure corresponds to a value of $\lambda(=0,0.2,0.5,1,2)$, a set of values of $n(=$ $100,250,640,1000)$, and a set of values of $K(=5,10,30)$.

Each panel corresponds to a value of $n$ and a value of $K$ and has $\tau^{2}=0.0(0.1) 1.0$ on the horizontal axis.

The interval estimators of $\tau^{2}$ are

- QP (Q-profile confidence interval)

- BJ (Biggerstaff and Jackson interval)

- PL (Profile likelihood interval)

- J (Jackson's interval) 

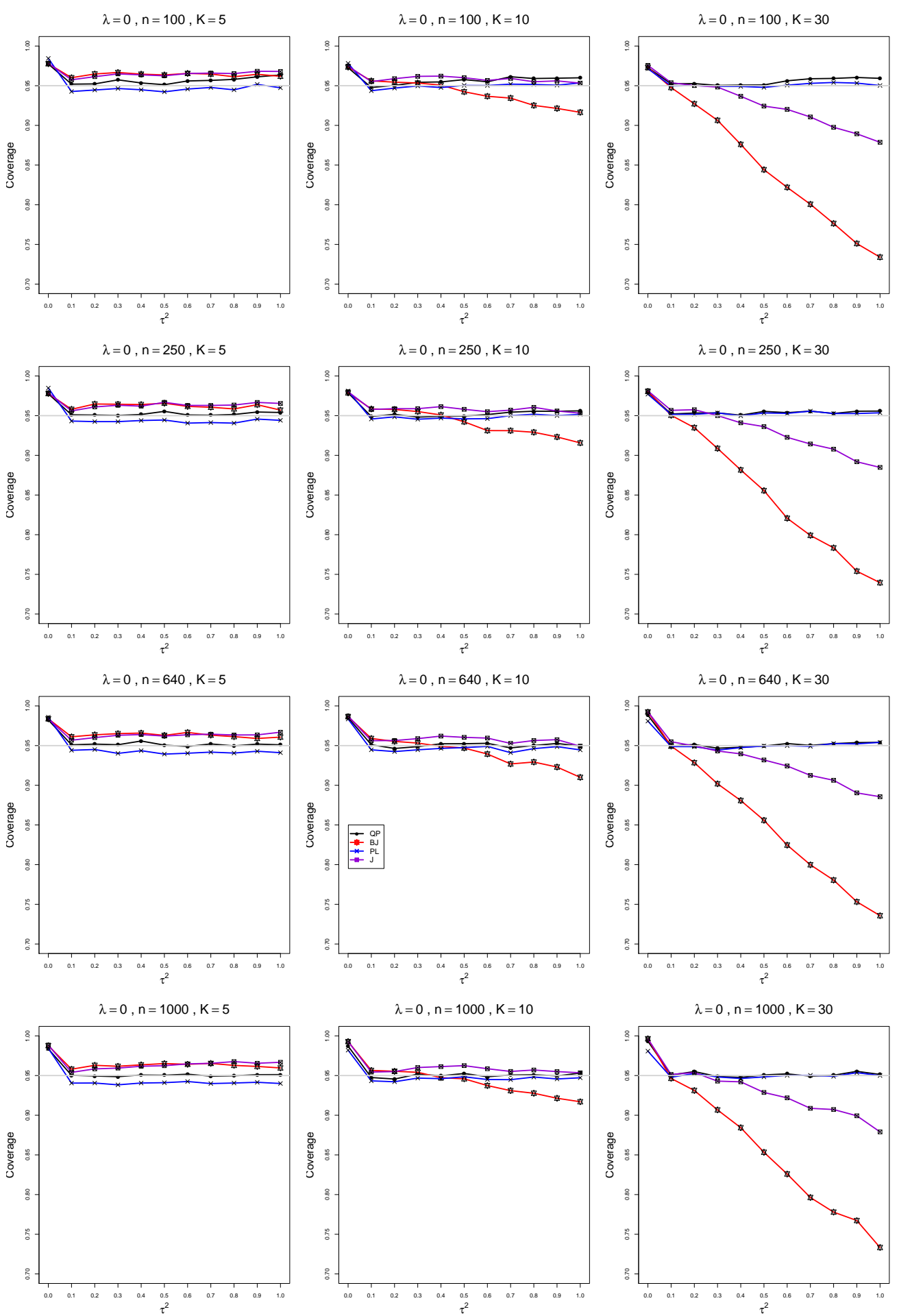

Figure C3.2.1: Coverage of 95\% confidence intervals for the between-studies variance $\tau^{2}$ when $\mu_{C}=4, \lambda=0, n=100,250,640,1000$, and $K=5,10,30$ 

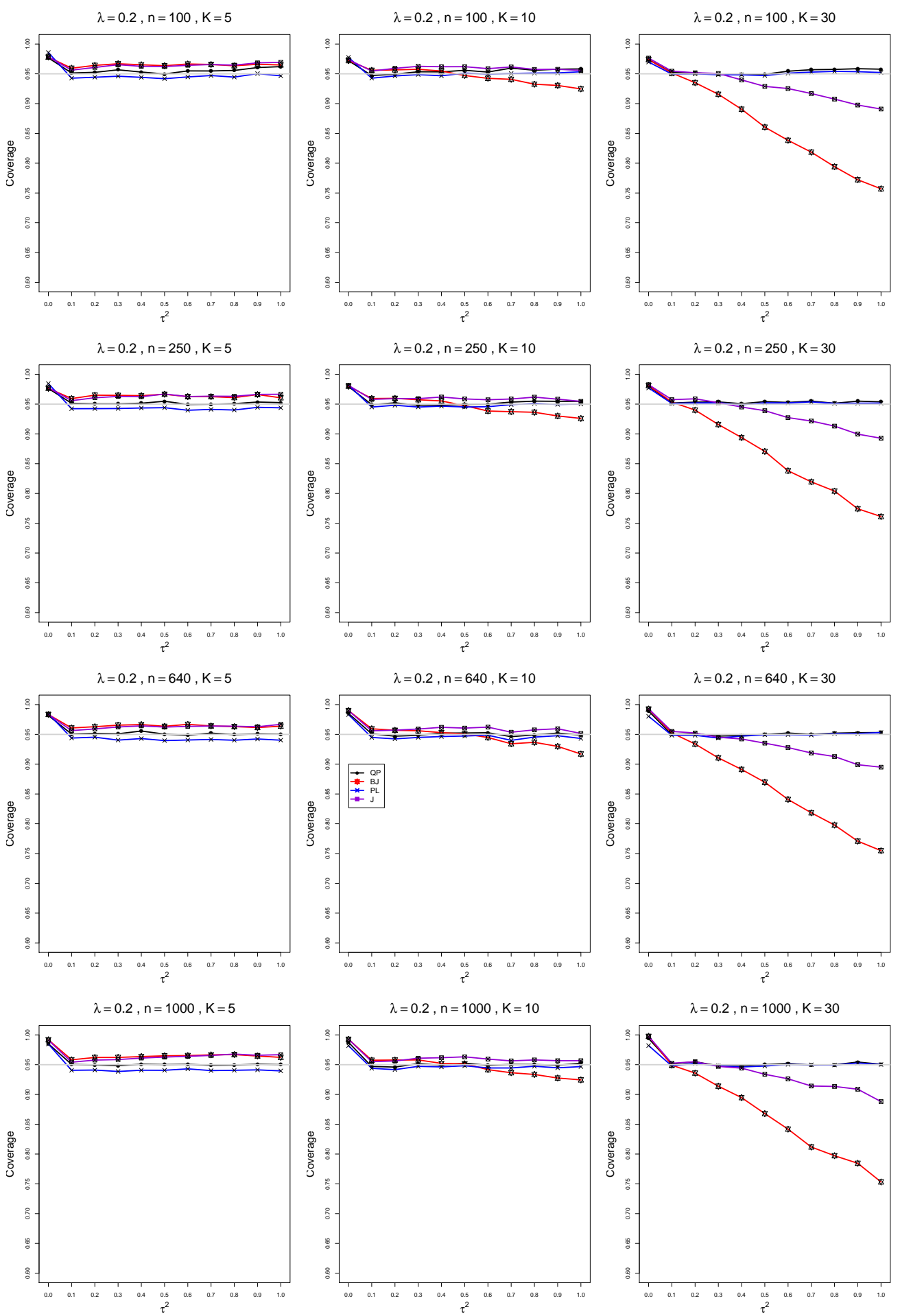

Figure C3.2.2: Coverage of 95\% confidence intervals for the between-studies variance $\tau^{2}$ when $\mu_{C}=4, \lambda=0.2, n=100,250,640,1000$, and $K=5,10,30$ 

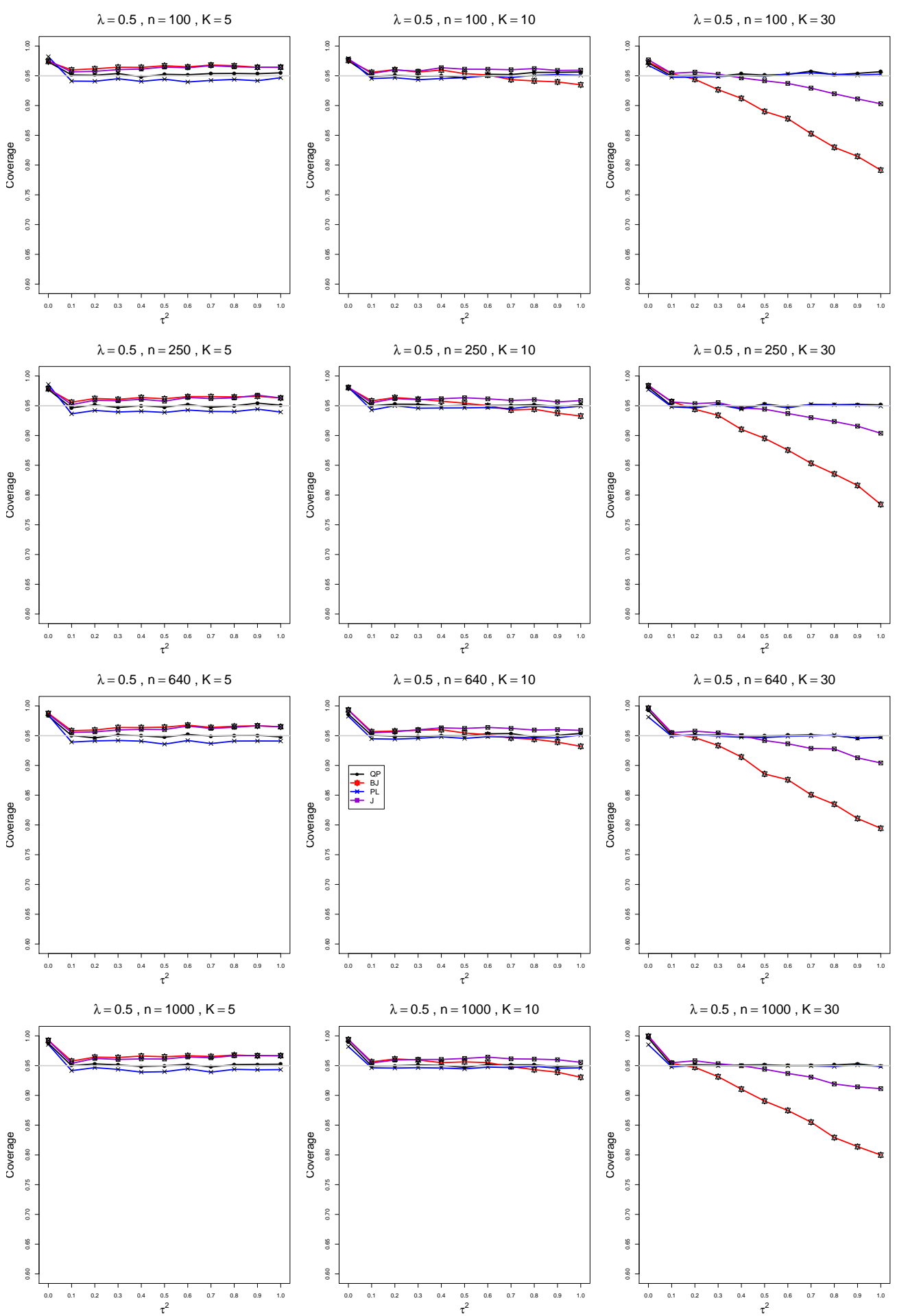

Figure C3.2.3: Coverage of 95\% confidence intervals for the between-studies variance $\tau^{2}$ when $\mu_{C}=4, \lambda=0.5, n=100,250,640,1000$, and $K=5,10,30$ 

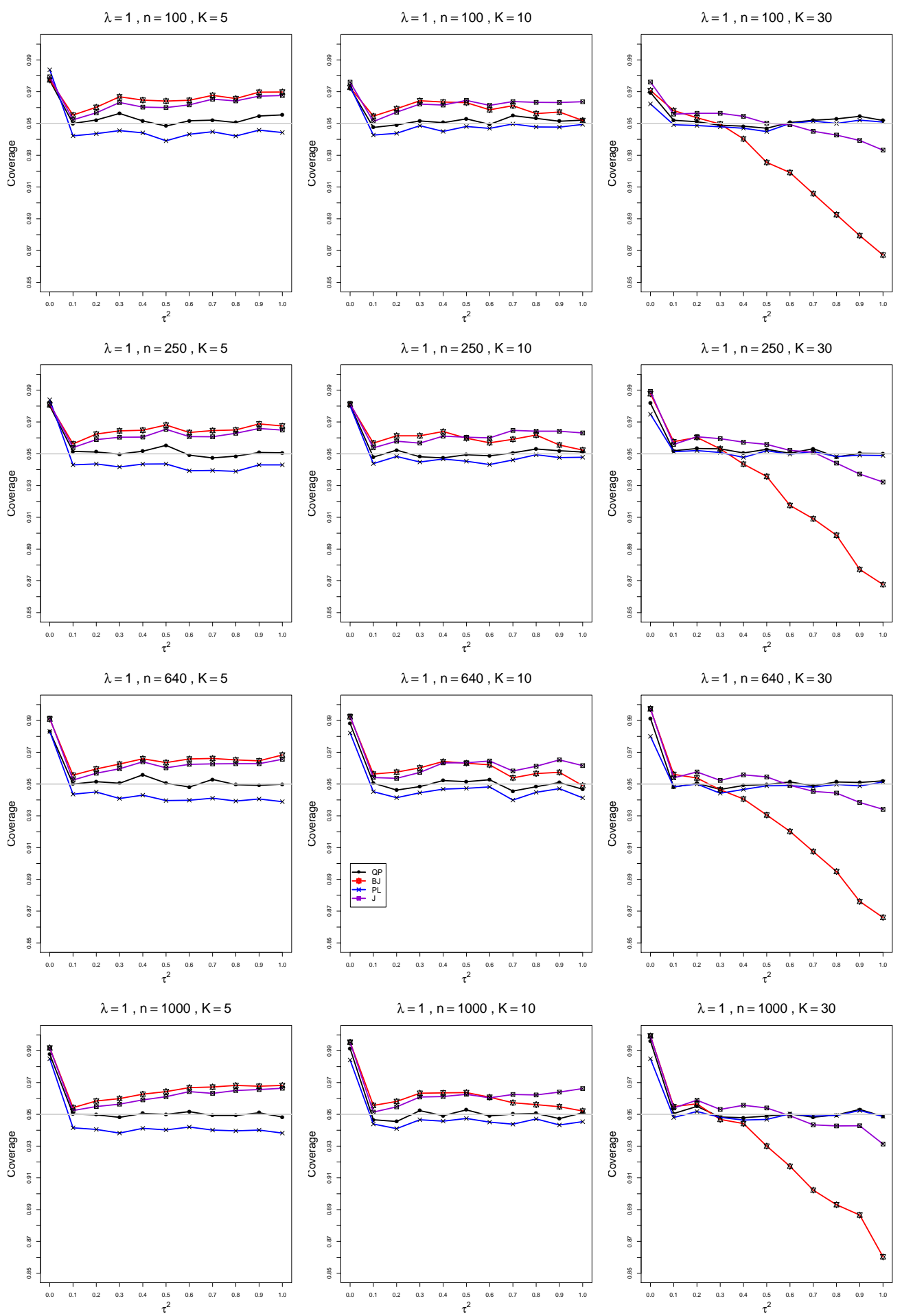

Figure C3.2.4: Coverage of 95\% confidence intervals for the between-studies variance $\tau^{2}$ when $\mu_{C}=4, \lambda=1, n=100,250,640,1000$, and $K=5,10,30$ 

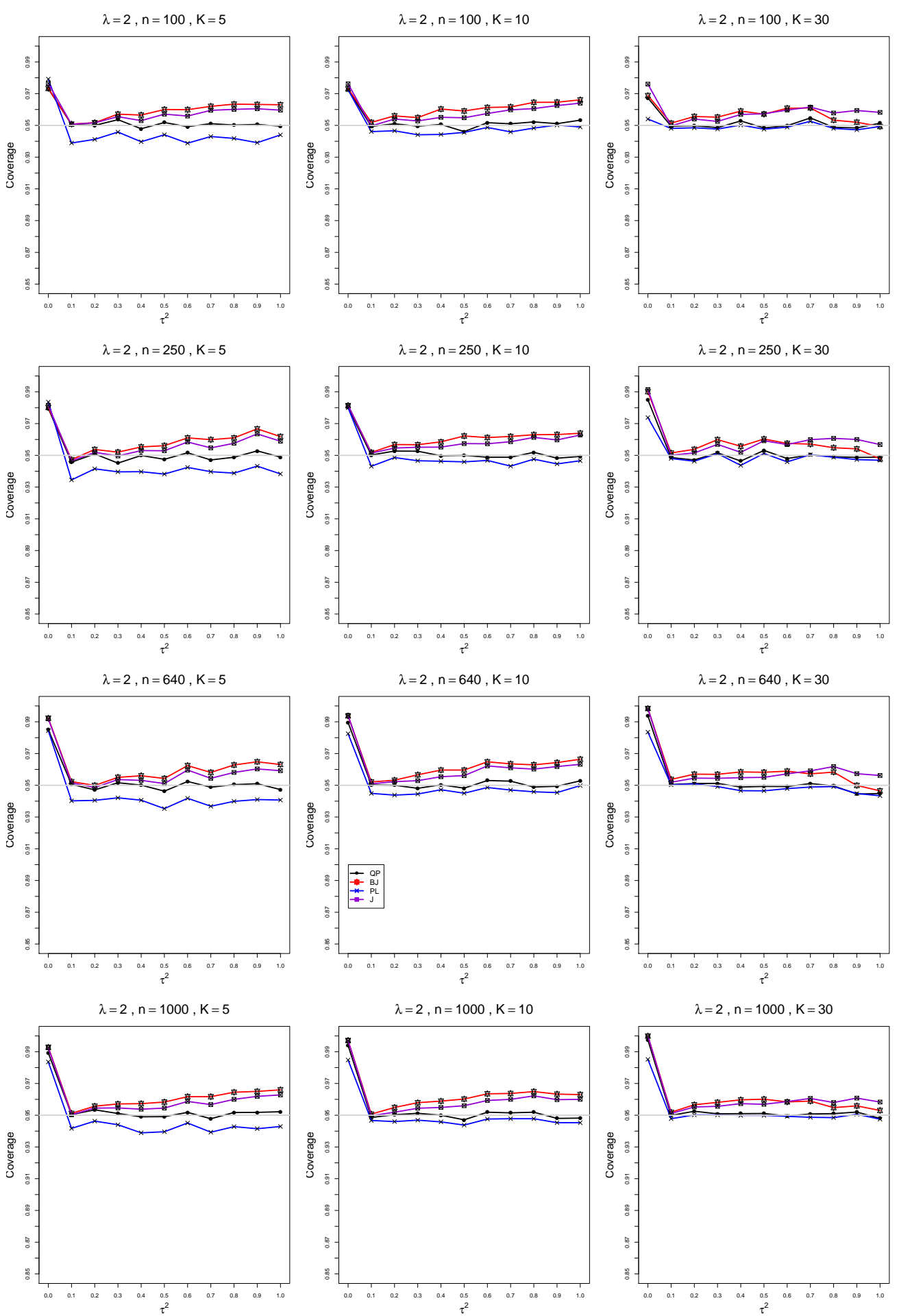

Figure C3.2.5: Coverage of 95\% confidence intervals for the between-studies variance $\tau^{2}$ when $\mu_{C}=4, \lambda=2, n=100,250,640,1000$, and $K=5,10,30$ 
C4. Normal model, $\mu_{C}=1, n=100,250,640,1000$, $K=50,100,125$

C4.1 Bias of point estimators of $\tau^{2}$ when $\mu_{C}=1$

Each figure corresponds to a value of $\lambda(=0,0.2,0.5,1,2)$, a set of values of $n(=$ $100,250,640,1000)$, and a set of values of $K(=50,100,125)$.

Each panel corresponds to a value of $n$ and a value of $K$ and has $\tau^{2}=0.0(0.1) 1.0$ on the horizontal axis.

The point estimators of $\tau^{2}$ are

- DL (DerSimonian-Laird)

- REML (restricted maximum likelihood)

- MP (Mandel-Paule)

- J (Jackson) 

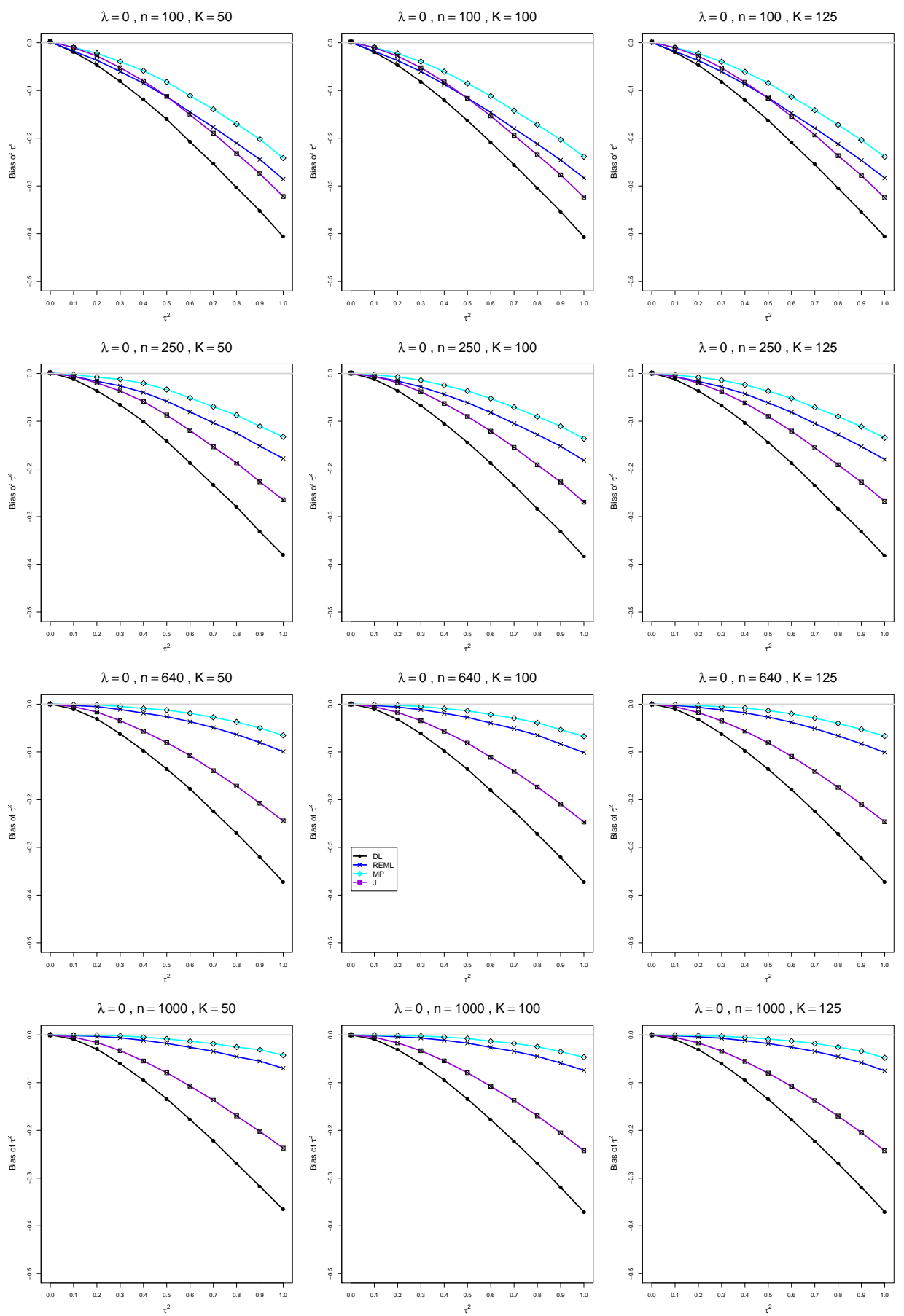

Figure C4.1.1: Bias of estimators of the between-studies variance $\tau^{2}$ when $\mu_{C}=1, \lambda=0$, $n=100,250,640,1000$, and $K=50,100,125$ 

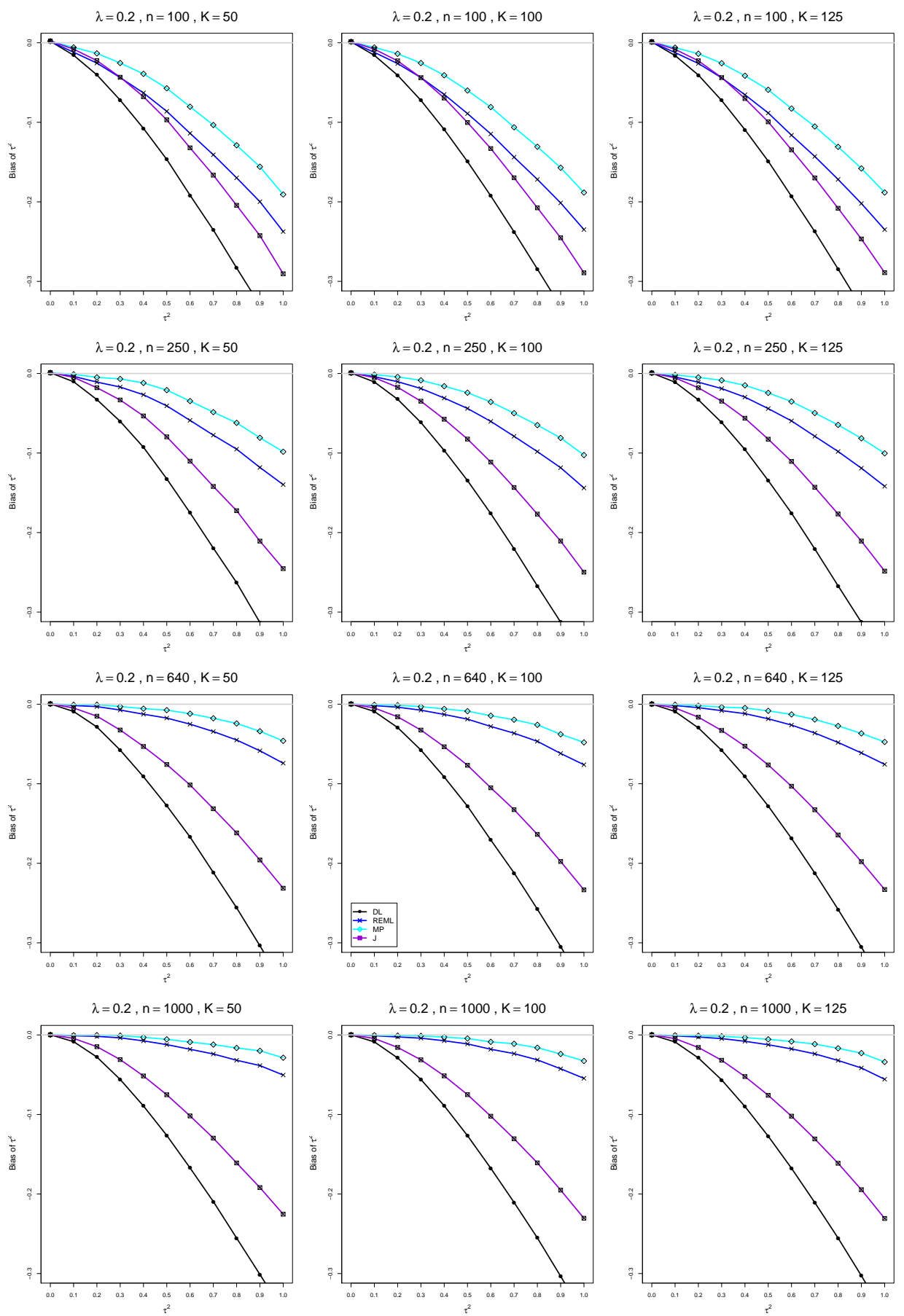

Figure C4.1.2: Bias of estimators of the between-studies variance $\tau^{2}$ when $\mu_{C}=1, \lambda=$ $0.2, n=100,250,640,1000$, and $K=50,100,125$ 

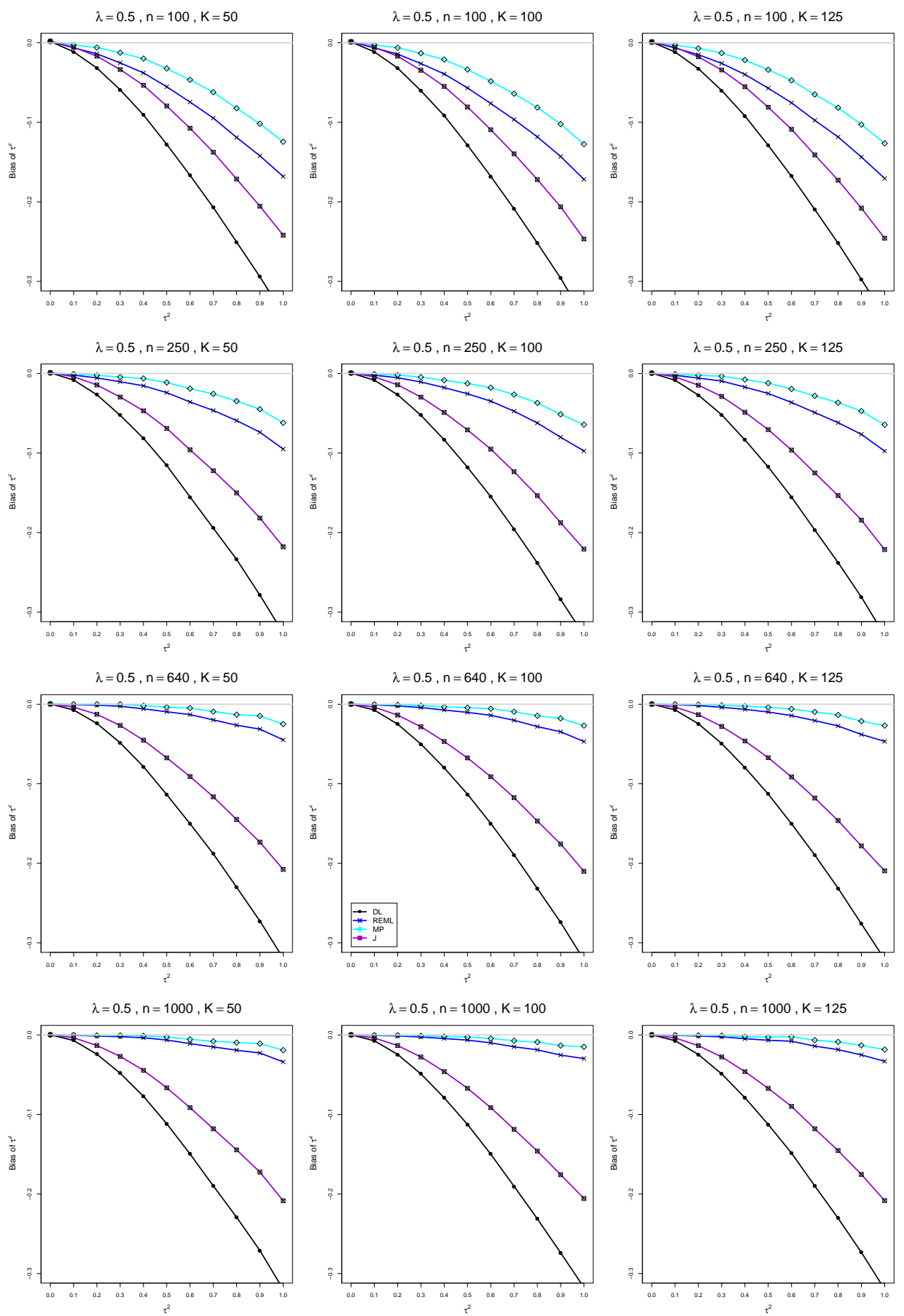

Figure C4.1.3: Bias of estimators of the between-studies variance $\tau^{2}$ when $\mu_{C}=1, \lambda=$ $0.5, n=100,250,640,1000$, and $K=50,100,125$ 

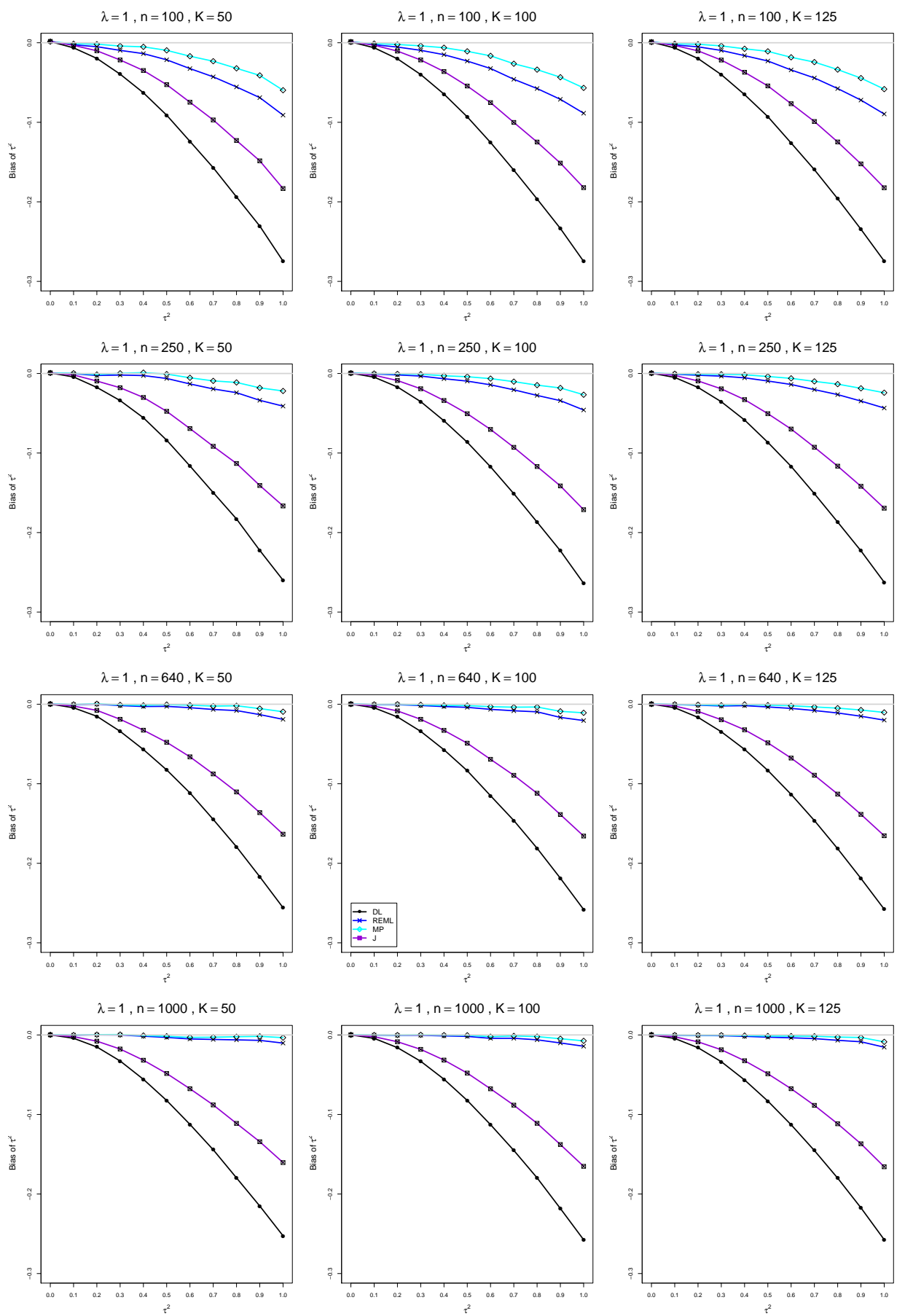

Figure C4.1.4: Bias of estimators of the between-studies variance $\tau^{2}$ when $\mu_{C}=1, \lambda=1$, $n=100,250,640,1000$, and $K=50,100,125$ 

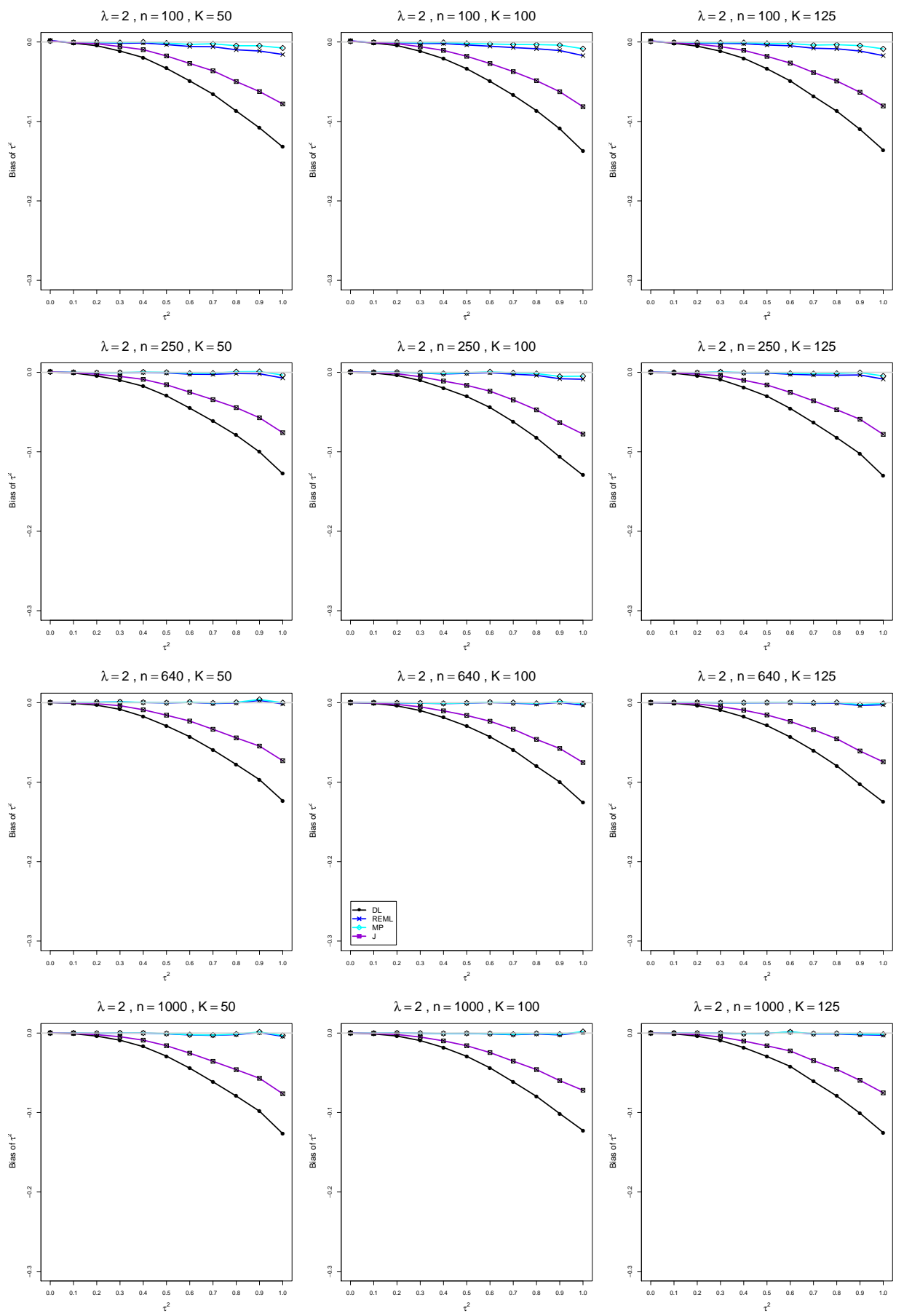

Figure C4.1.5: Bias of estimators of the between-studies variance $\tau^{2}$ when $\mu_{C}=1, \lambda=2$, $n=100,250,640,1000$, and $K=50,100,125$ 


\section{C4.2 Coverage of interval estimators of $\tau^{2}$ when $\mu_{C}=1$}

Each figure corresponds to a value of $\lambda(=0,0.2,0.5,1,2)$, a set of values of $n(=$ $100,250,640,1000)$, and a set of values of $K(=50,100,125)$.

Each panel corresponds to a value of $n$ and a value of $K$ and has $\tau^{2}=0.0(0.1) 1.0$ on the horizontal axis.

The interval estimators of $\tau^{2}$ are

- QP (Q-profile confidence interval)

- BJ (Biggerstaff and Jackson interval)

- PL (Profile likelihood interval)

- J (Jackson's interval) 

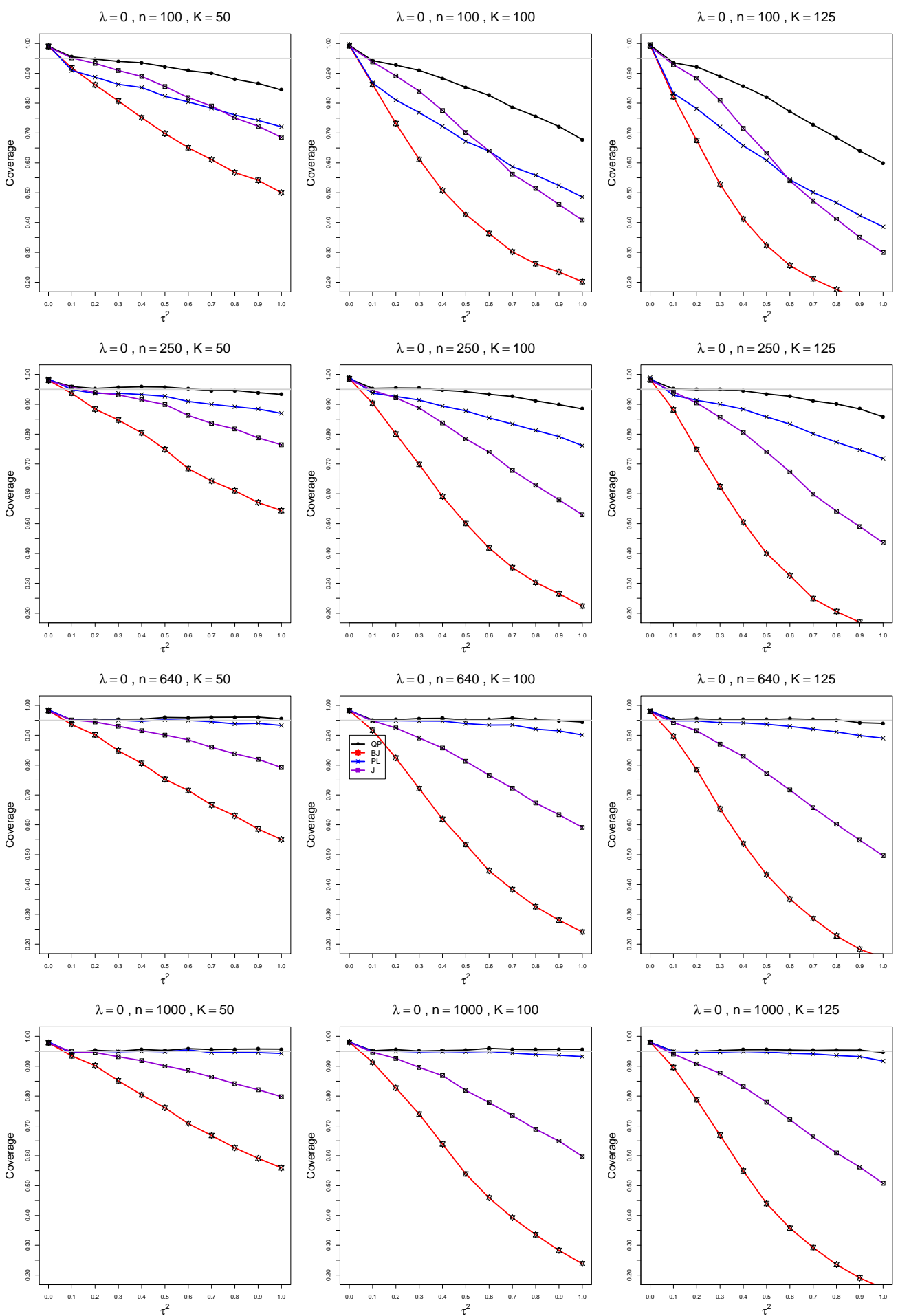

Figure C4.2.1: Coverage of 95\% confidence intervals for the between-studies variance $\tau^{2}$ when $\mu_{C}=1, \lambda=0, n=100,250,640,1000$, and $K=50,100,125$ 

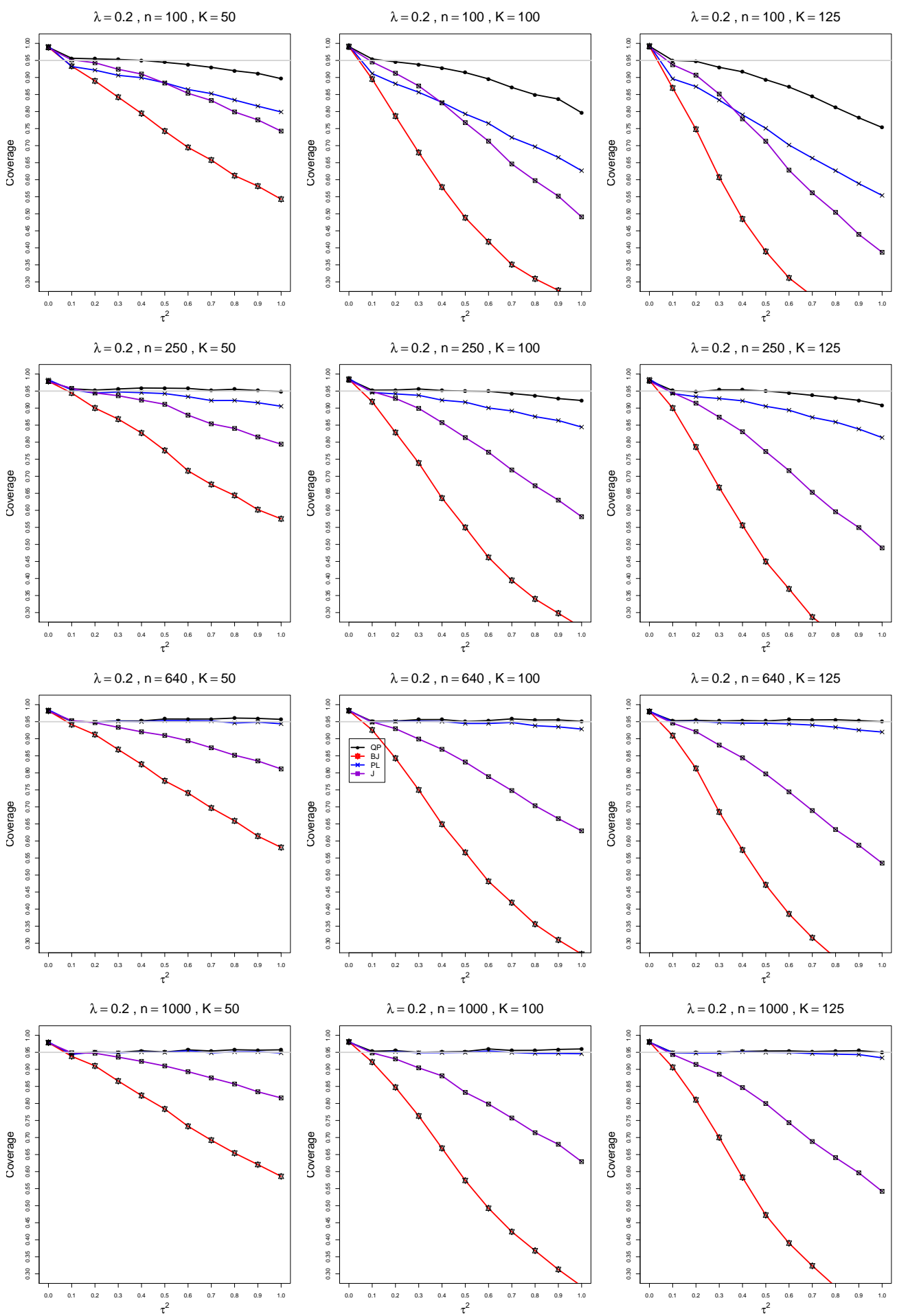

Figure C4.2.2: Coverage of 95\% confidence intervals for the between-studies variance $\tau^{2}$ when $\mu_{C}=1, \lambda=0.2, n=100,250,640,1000$, and $K=50,100,125$ 

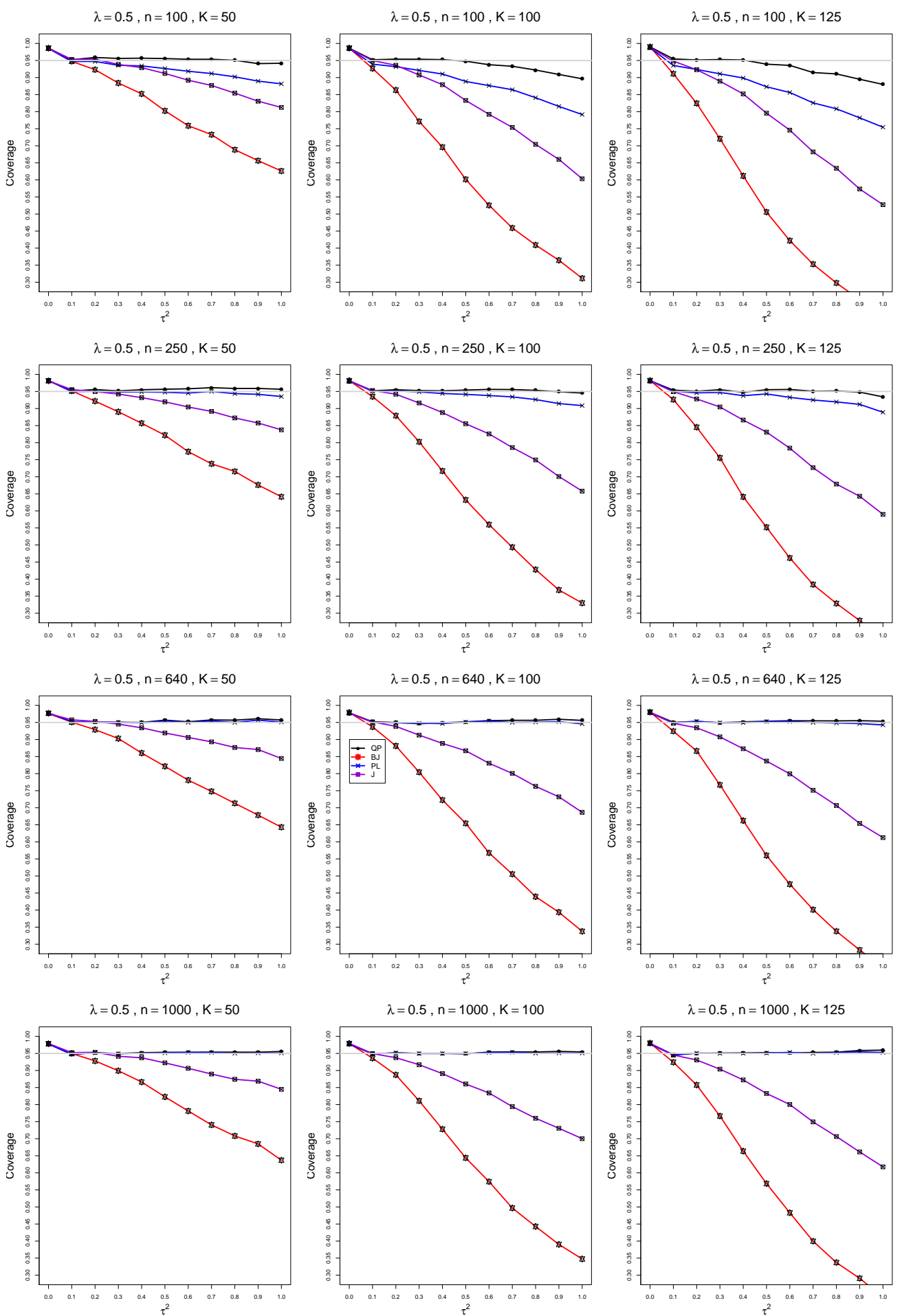

Figure C4.2.3: Coverage of 95\% confidence intervals for the between-studies variance $\tau^{2}$ when $\mu_{C}=1, \lambda=0.5, n=100,250,640,1000$, and $K=50,100,125$ 

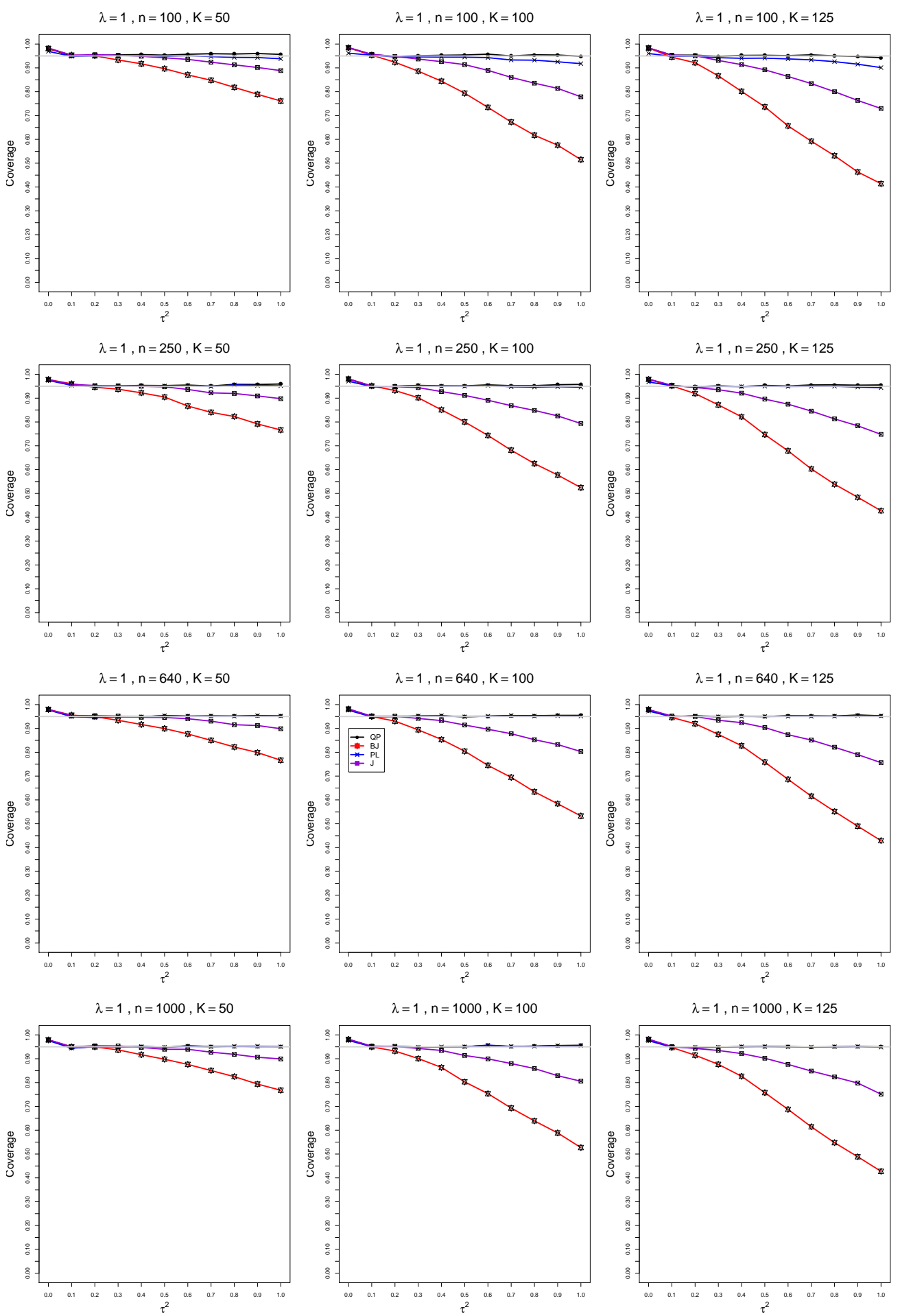

Figure C4.2.4: Coverage of 95\% confidence intervals for the between-studies variance $\tau^{2}$ when $\mu_{C}=1, \lambda=1, n=100,250,640,1000$, and $K=50,100,125$ 

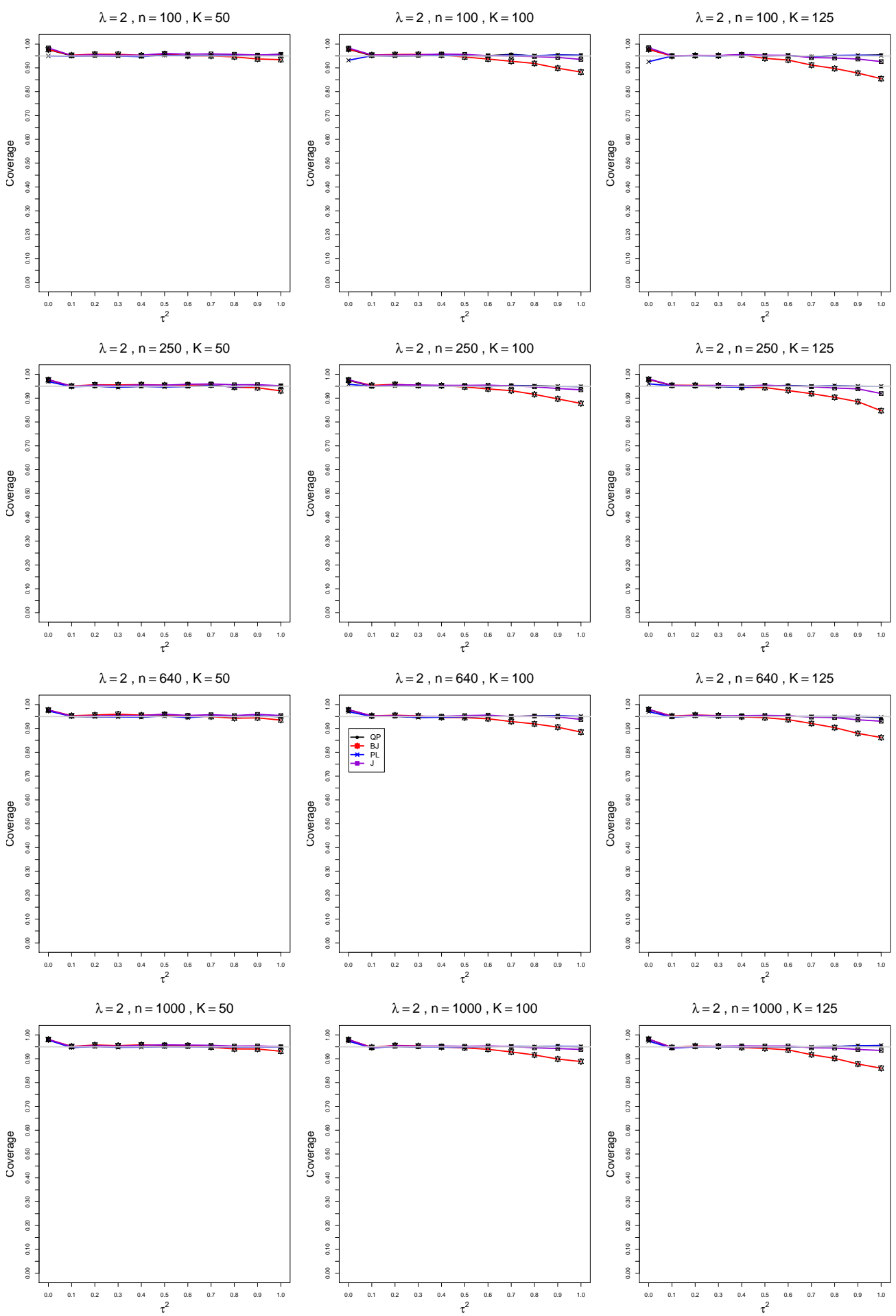

Figure C4.2.5: Coverage of 95\% confidence intervals for the between-studies variance $\tau^{2}$ when $\mu_{C}=1, \lambda=2, n=100,250,640,1000$, and $K=50,100,125$ 
C5. Normal model, $\mu_{C}=4, n=100,250,640,1000$, $K=50,100,125$

\section{C5.1 Bias of point estimators of $\tau^{2}$ when $\mu_{C}=4$}

Each figure corresponds to a value of $\lambda(=0,0.2,0.5,1,2)$, a set of values of $n(=$ $100,250,640,1000)$, and a set of values of $K(=50,100,125)$.

Each panel corresponds to a value of $n$ and a value of $K$ and has $\tau^{2}=0.0(0.1) 1.0$ on the horizontal axis.

The point estimators of $\tau^{2}$ are

- DL (DerSimonian-Laird)

- REML (restricted maximum likelihood)

- MP (Mandel-Paule)

- J (Jackson) 

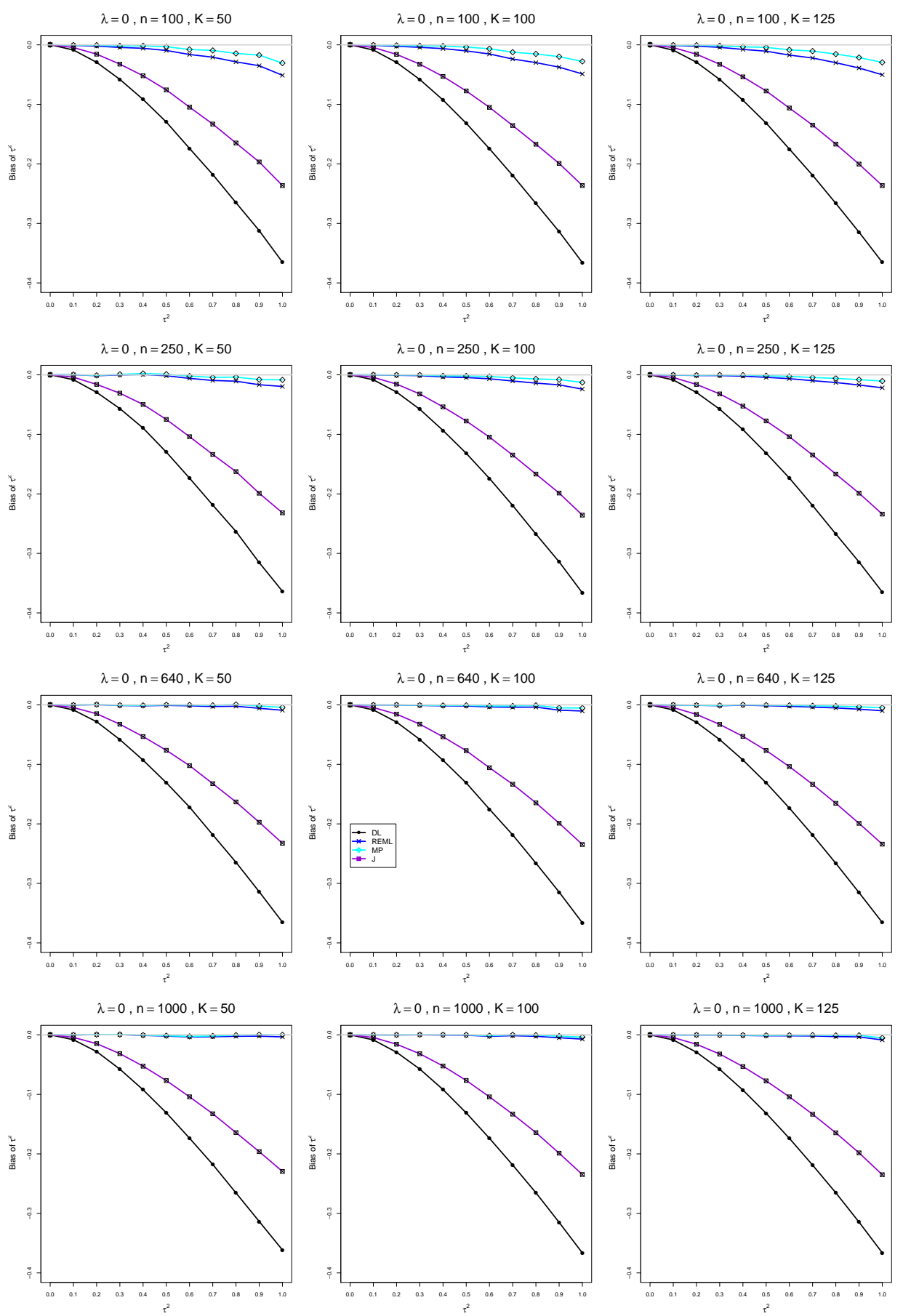

Figure C5.1.1: Bias of estimators of the between-studies variance $\tau^{2}$ when $\mu_{C}=4, \lambda=0$, $n=100,250,640,1000$, and $K=50,100,125$ 

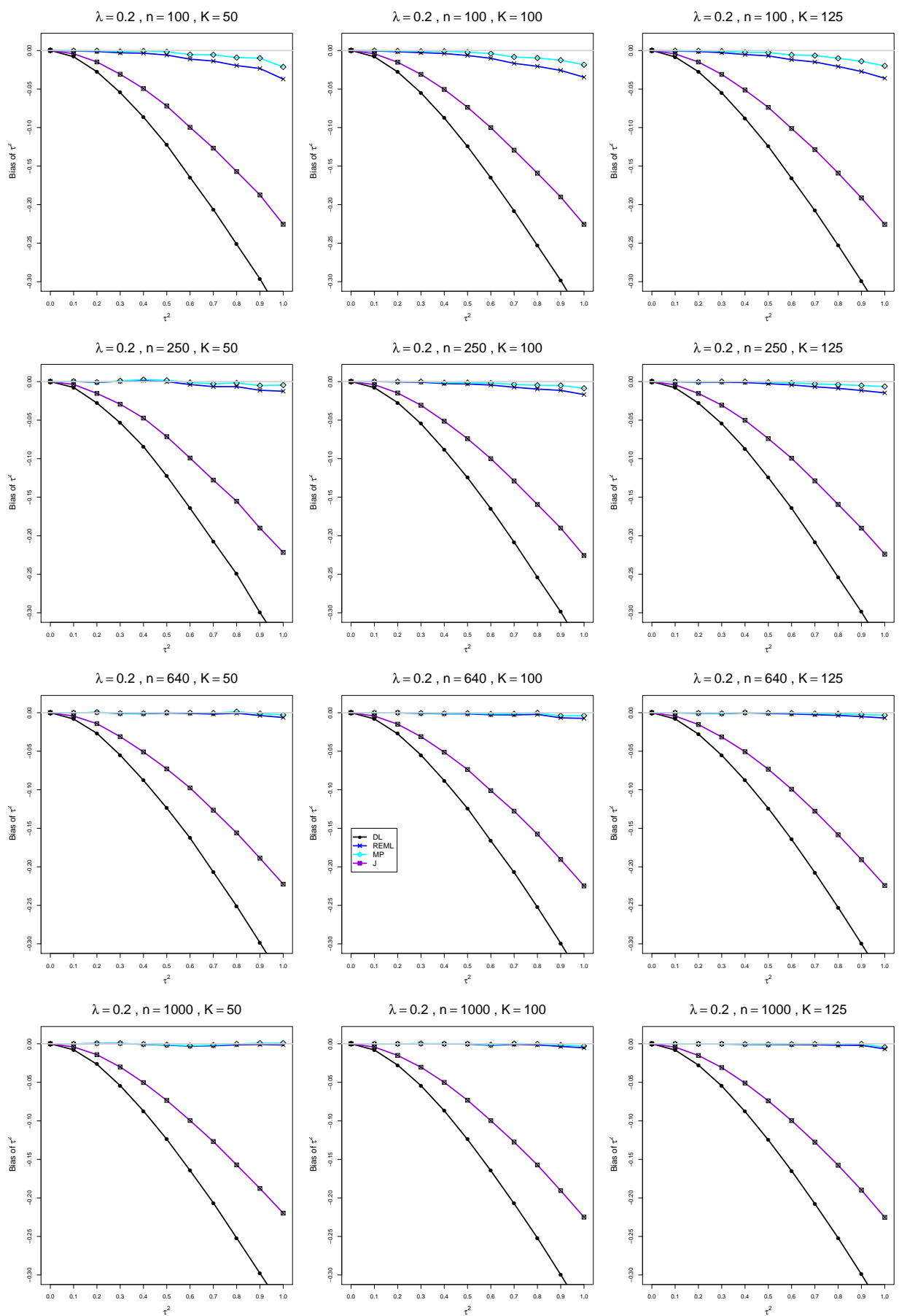

Figure C5.1.2: Bias of estimators of the between-studies variance $\tau^{2}$ when $\mu_{C}=4, \lambda=$ $0.2, n=100,250,640,1000$, and $K=50,100,125$ 

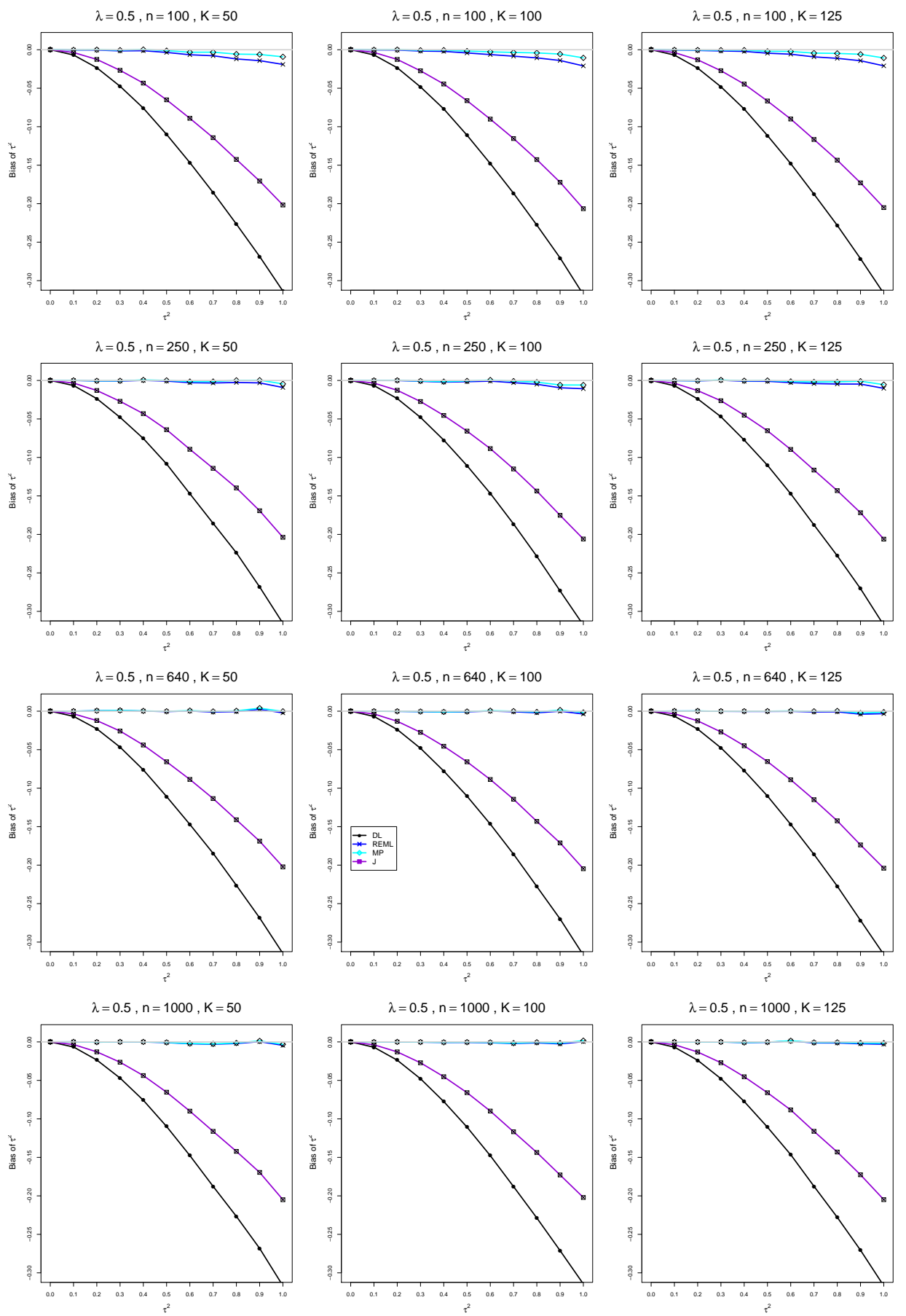

Figure C5.1.3: Bias of estimators of the between-studies variance $\tau^{2}$ when $\mu_{C}=4, \lambda=$ $0.5, n=100,250,640,1000$, and $K=50,100,125$ 

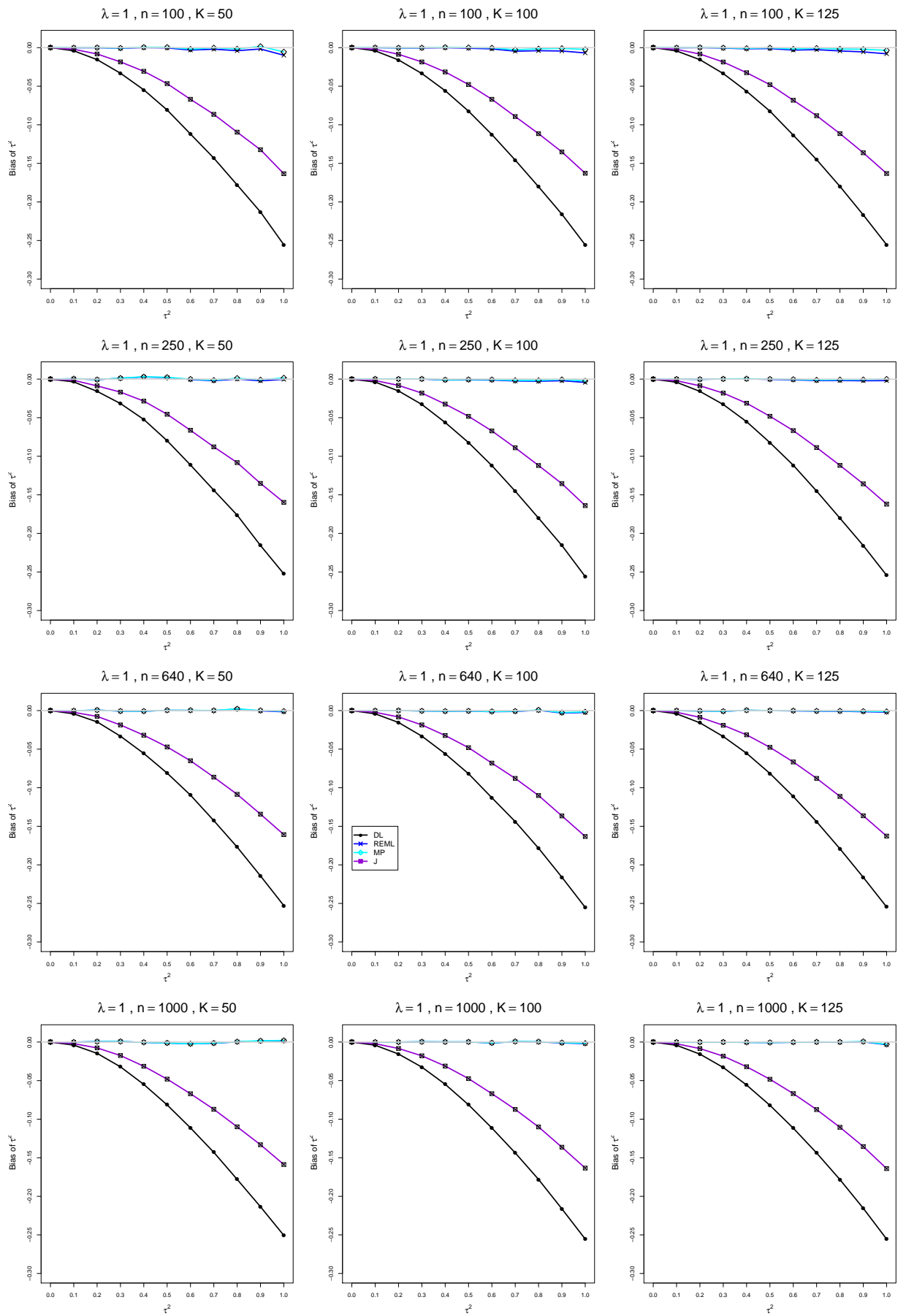

Figure C5.1.4: Bias of estimators of the between-studies variance $\tau^{2}$ when $\mu_{C}=4, \lambda=1$, $n=100,250,640,1000$, and $K=50,100,125$ 

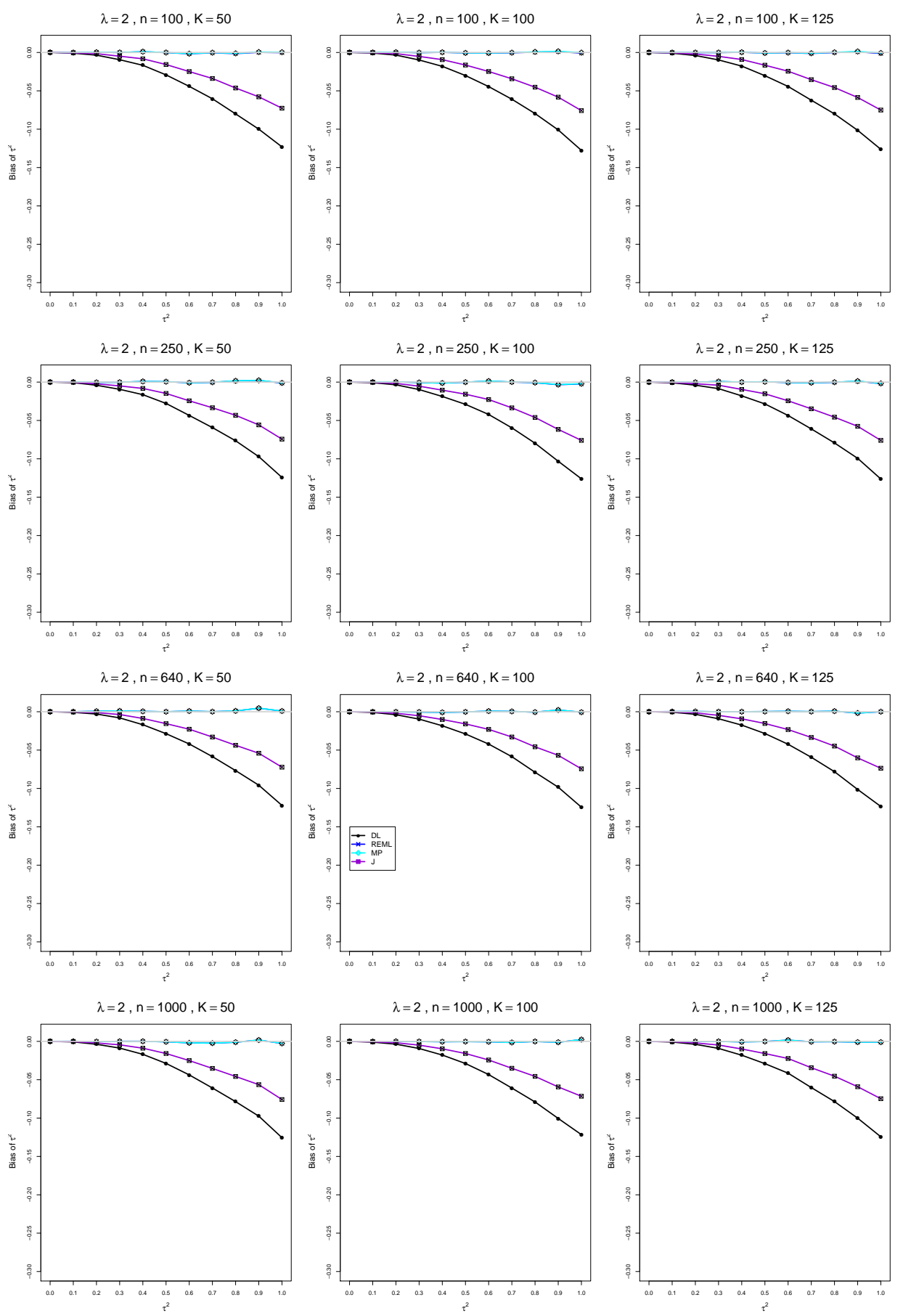

Figure C5.1.5: Bias of estimators of the between-studies variance $\tau^{2}$ when $\mu_{C}=4, \lambda=2$, $n=100,250,640,1000$, and $K=50,100,125$ 


\section{C5.2 Coverage of interval estimators of $\tau^{2}$ when $\mu_{C}=4$}

Each figure corresponds to a value of $\lambda(=0,0.2,0.5,1,2)$, a set of values of $n(=$ $100,250,640,1000)$, and a set of values of $K(=50,100,125)$.

Each panel corresponds to a value of $n$ and a value of $K$ and has $\tau^{2}=0.0(0.1) 1.0$ on the horizontal axis.

The interval estimators of $\tau^{2}$ are

- QP (Q-profile confidence interval)

- BJ (Biggerstaff and Jackson interval)

- PL (Profile likelihood interval)

- J (Jackson's interval) 

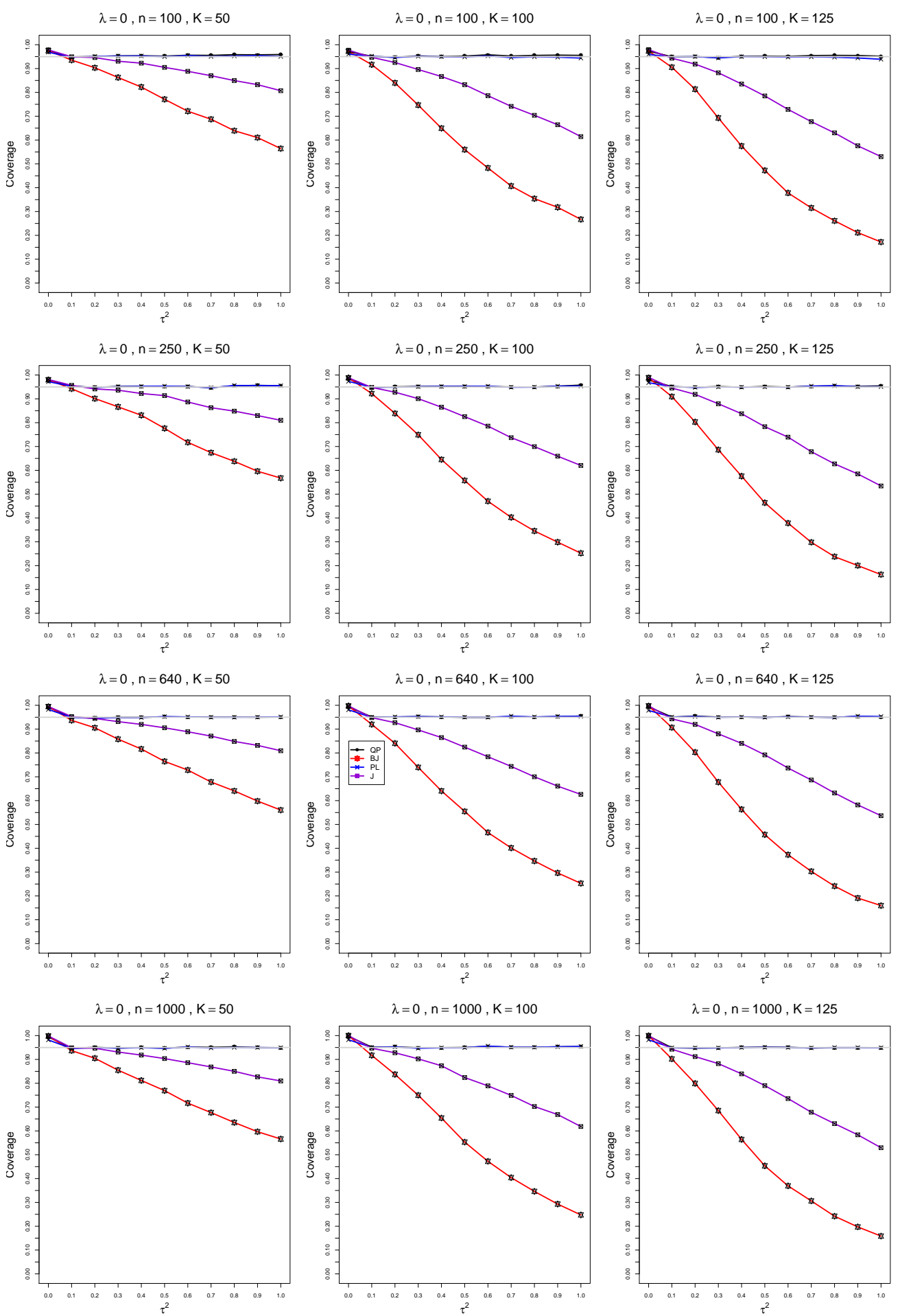

Figure C5.2.1: Coverage of 95\% confidence intervals for the between-studies variance $\tau^{2}$ when $\mu_{C}=4, \lambda=0, n=100,250,640,1000$, and $K=50,100,125$ 

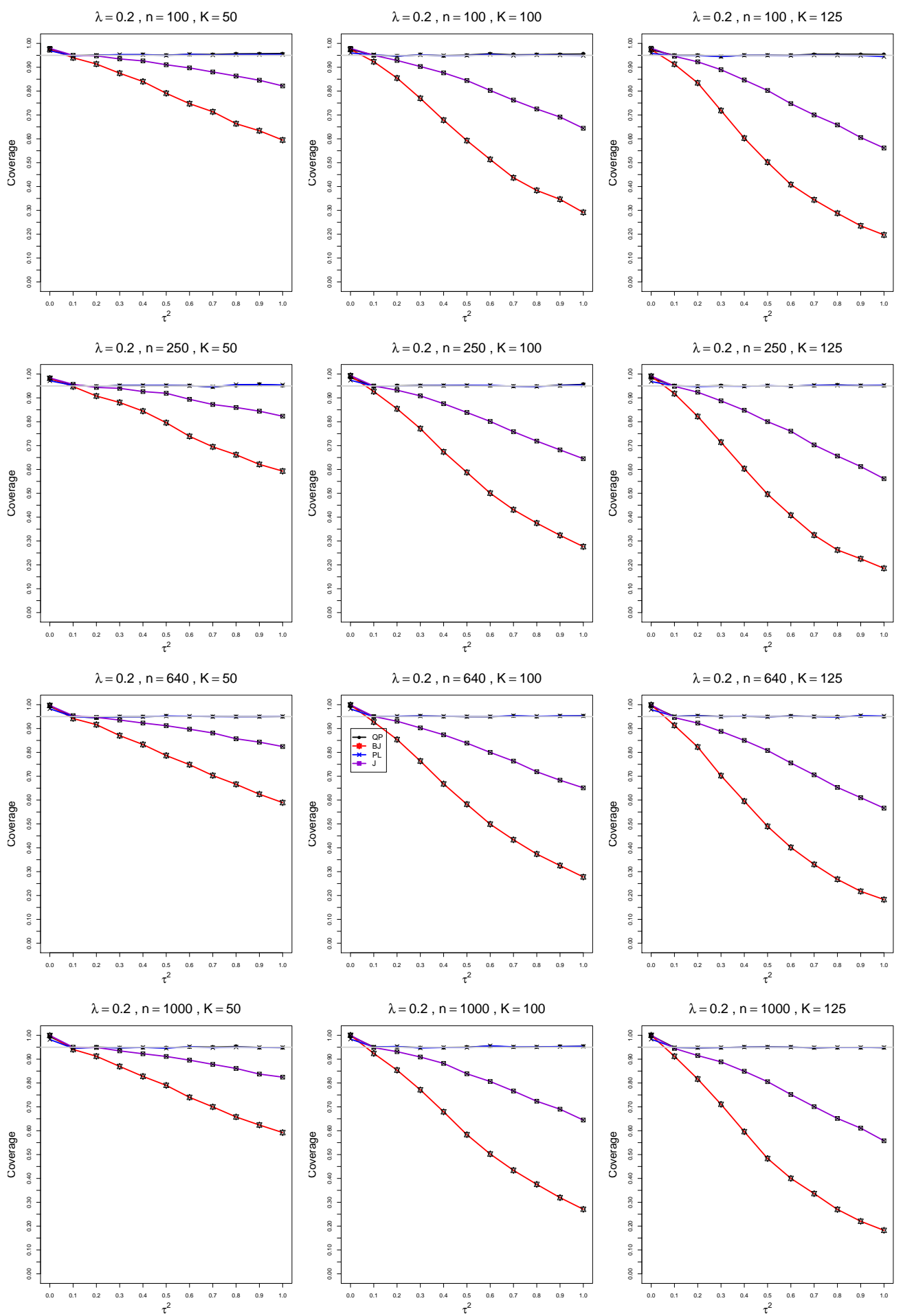

Figure C5.2.2: Coverage of 95\% confidence intervals for the between-studies variance $\tau^{2}$ when $\mu_{C}=4, \lambda=0.2, n=100,250,640,1000$, and $K=50,100,125$ 

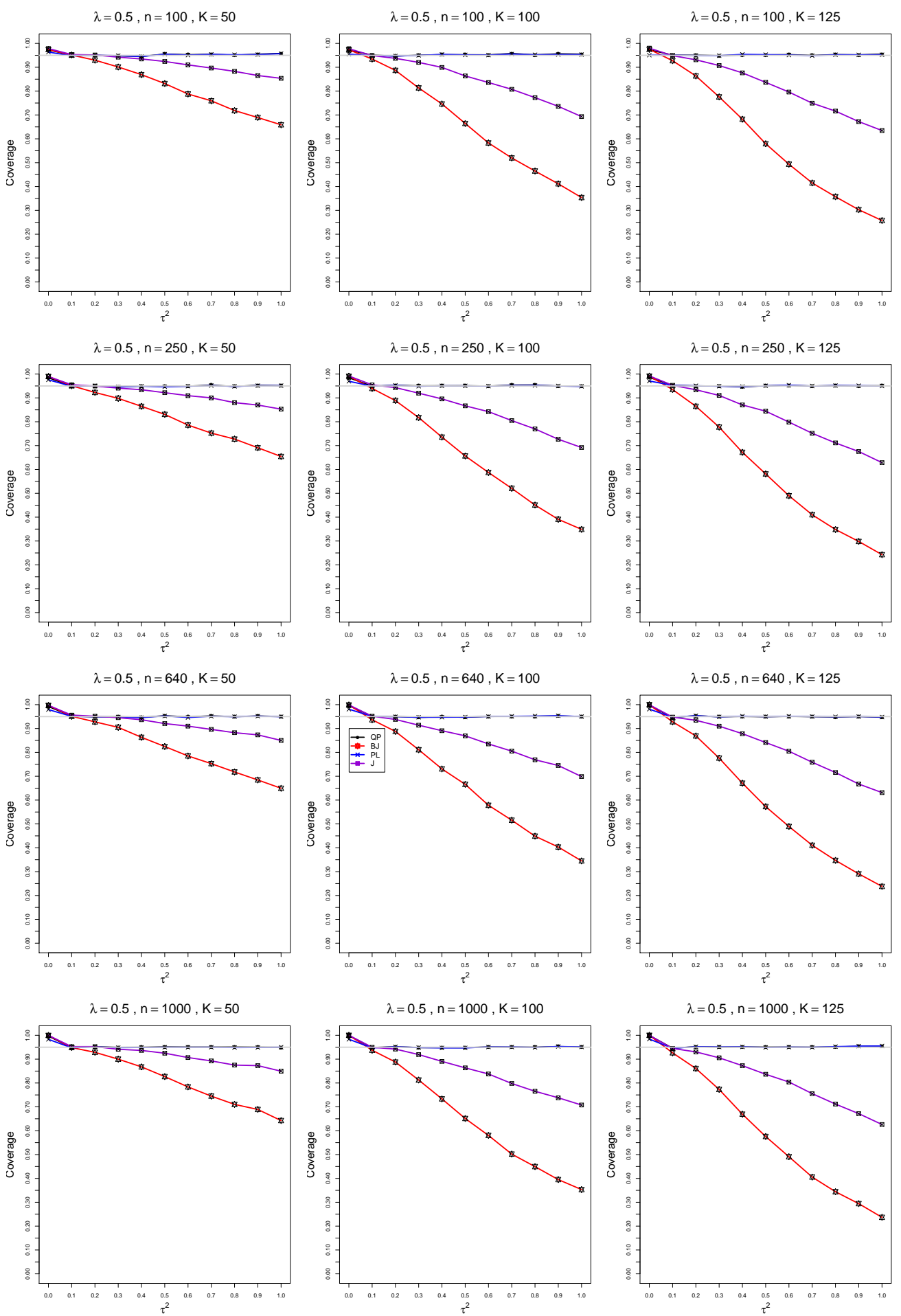

Figure C5.2.3: Coverage of 95\% confidence intervals for the between-studies variance $\tau^{2}$ when $\mu_{C}=4, \lambda=0.5, n=100,250,640,1000$, and $K=50,100,125$ 

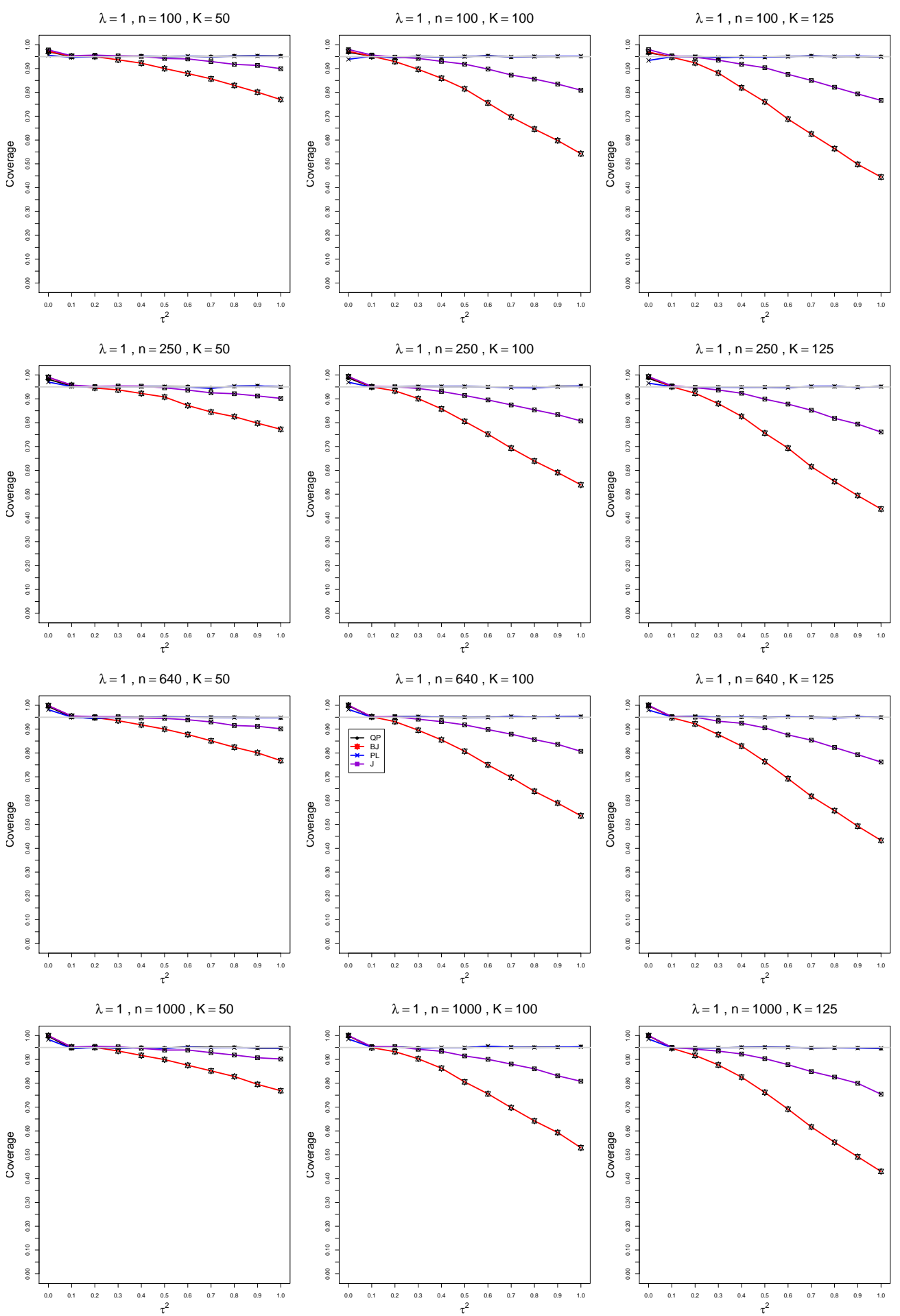

Figure C5.2.4: Coverage of 95\% confidence intervals for the between-studies variance $\tau^{2}$ when $\mu_{C}=4, \lambda=1, n=100,250,640,1000$, and $K=50,100,125$ 

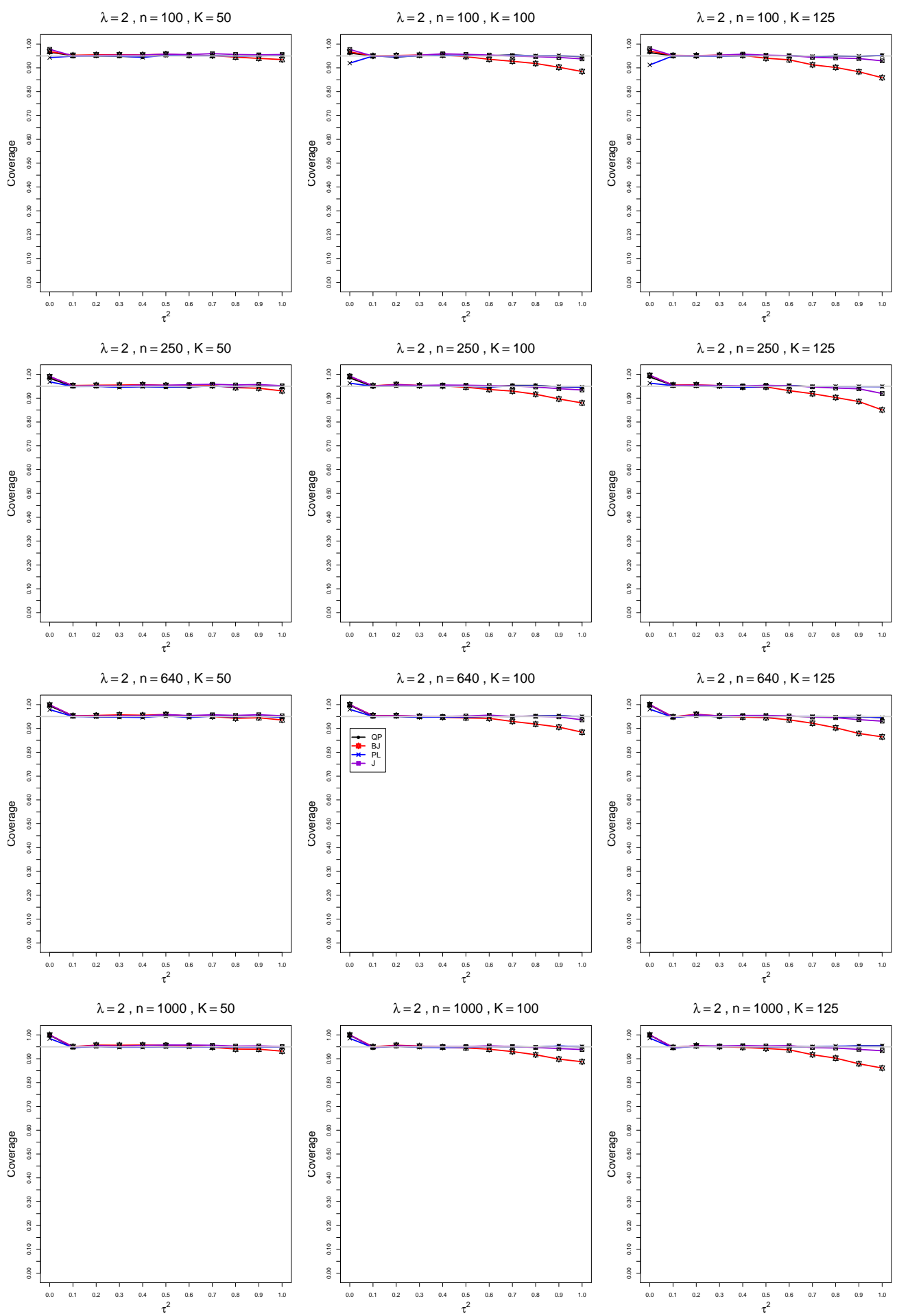

Figure C5.2.5: Coverage of 95\% confidence intervals for the between-studies variance $\tau^{2}$ when $\mu_{C}=4, \lambda=2, n=100,250,640,1000$, and $K=50,100,125$ 


\section{C6. Normal model, bias-corrected $\lambda, \mu_{C}=4$,}

$n=100,250,640,1000, K=50,100,125$

C6.1 Bias of point estimators of $\tau^{2}$ when $\mu_{C}=4$

Each figure corresponds to a value of $\lambda(=0,0.2,0.5,1,2)$, a set of values of $n(=$ $100,250,640,1000)$, and a set of values of $K(=50,100,125)$.

Each panel corresponds to a value of $n$ and a value of $K$ and has $\tau^{2}=0.0(0.1) 1.0$ on the horizontal axis.

The point estimators of $\tau^{2}$ are

- DL (DerSimonian-Laird)

- REML (restricted maximum likelihood)

- MP (Mandel-Paule)

- J (Jackson) 

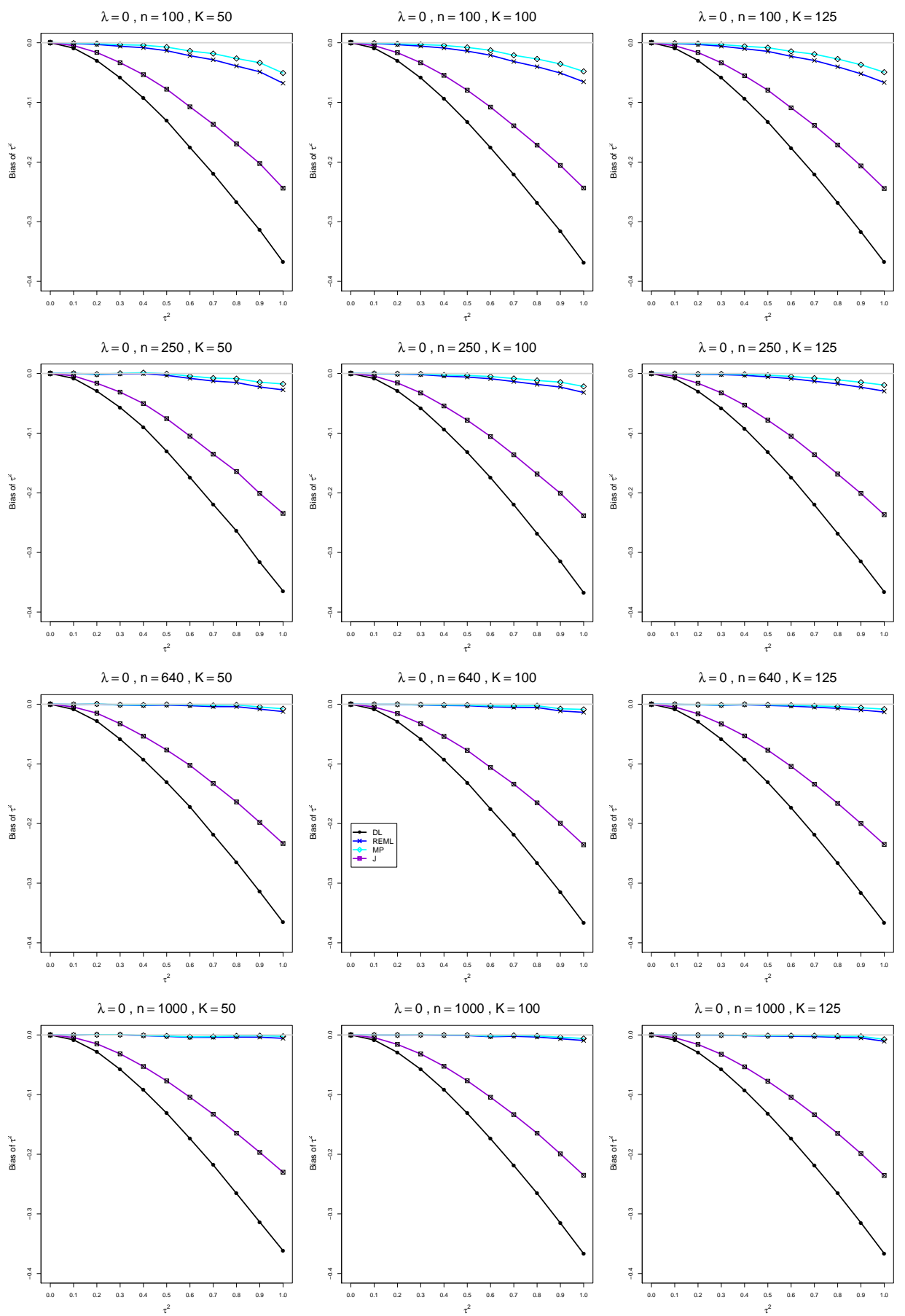

Figure C6.1.1: Bias of estimators of the between-studies variance $\tau^{2}$ when $\mu_{C}=4, \lambda=0$, $n=100,250,640,1000$, and $K=50,100,125$ 

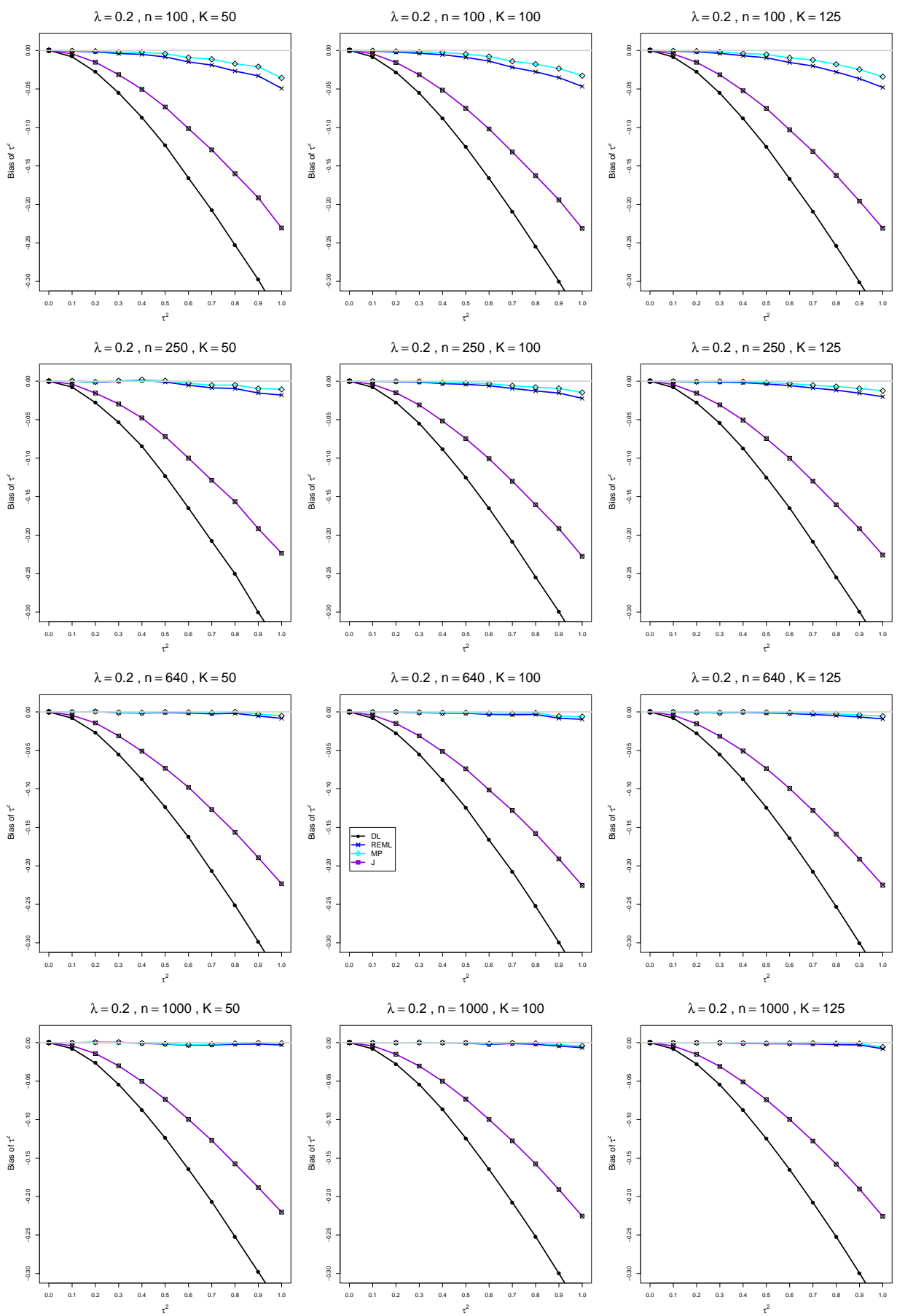

Figure C6.1.2: Bias of estimators of the between-studies variance $\tau^{2}$ when $\mu_{C}=4, \lambda=$ $0.2, n=100,250,640,1000$, and $K=50,100,125$ 

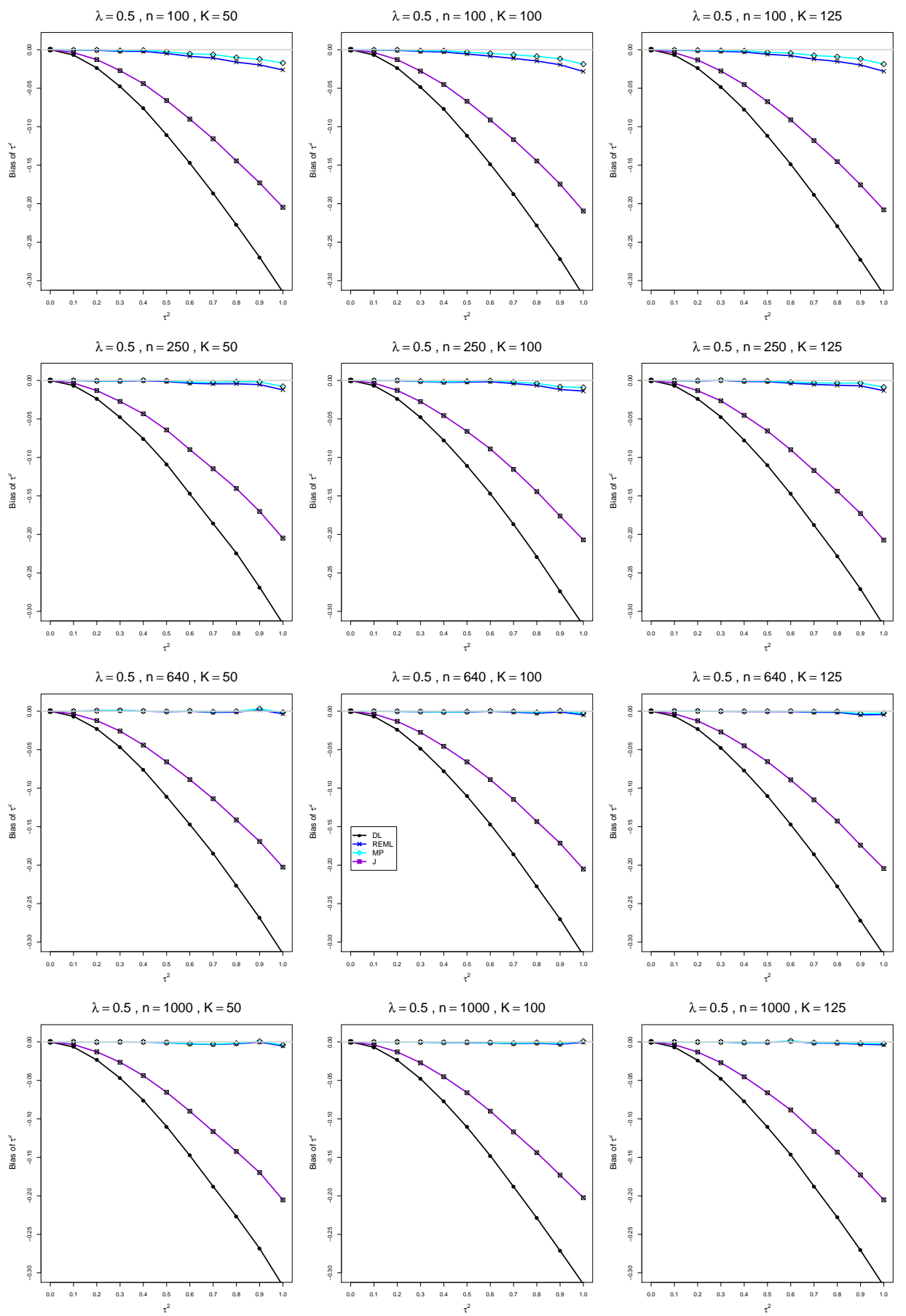

Figure C6.1.3: Bias of estimators of the between-studies variance $\tau^{2}$ when $\mu_{C}=4, \lambda=$ $0.5, n=100,250,640,1000$, and $K=50,100,125$ 

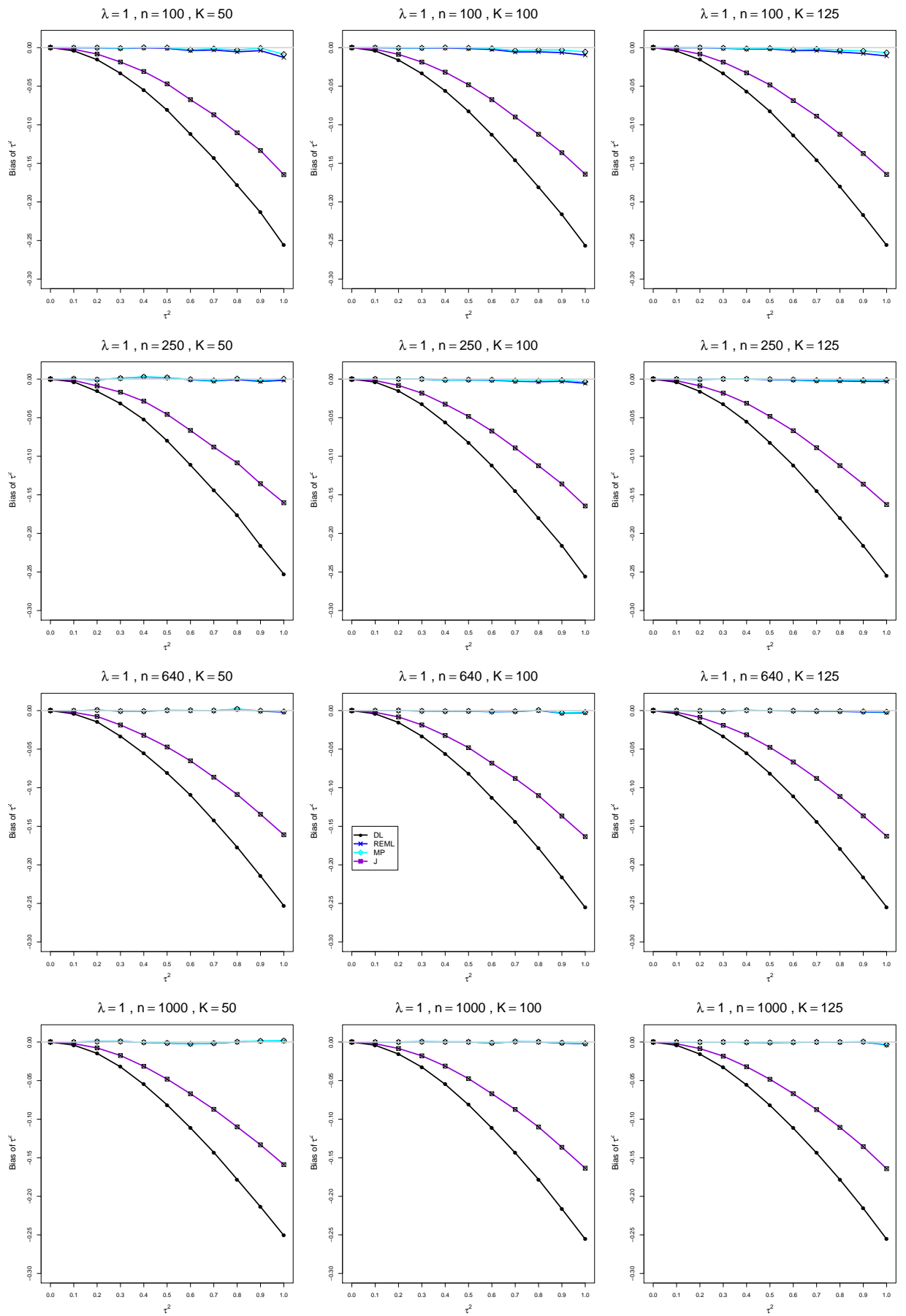

Figure C6.1.4: Bias of estimators of the between-studies variance $\tau^{2}$ when $\mu_{C}=4, \lambda=1$, $n=100,250,640,1000$, and $K=50,100,125$ 

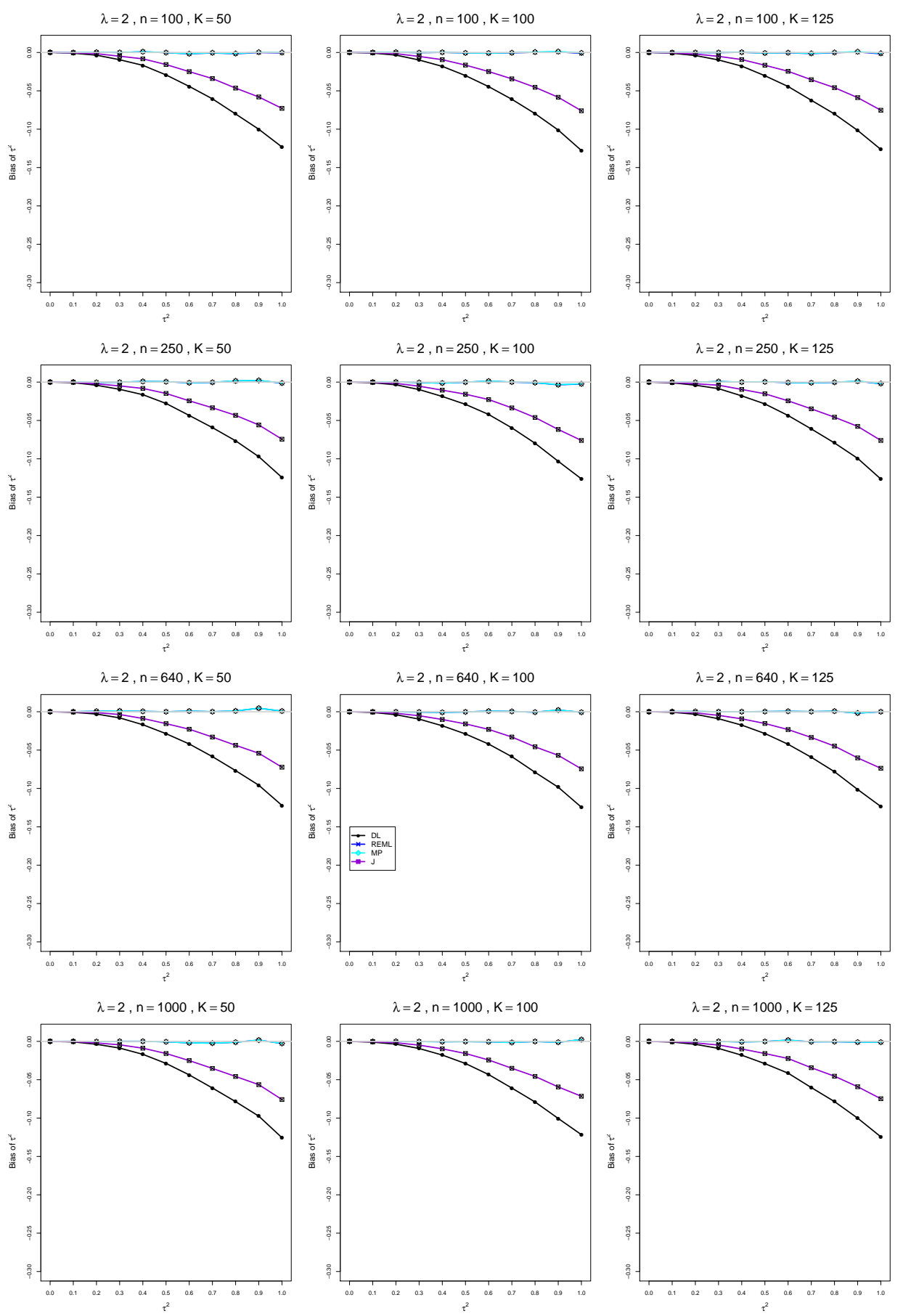

Figure C6.1.5: Bias of estimators of the between-studies variance $\tau^{2}$ when $\mu_{C}=4, \lambda=2$, $n=100,250,640,1000$, and $K=50,100,125$ 


\section{C6.2 Coverage of interval estimators of $\tau^{2}$ when $\mu_{C}=4$}

Each figure corresponds to a value of $\lambda(=0,0.2,0.5,1,2)$, a set of values of $n(=$ $100,250,640,1000)$, and a set of values of $K(=50,100,125)$.

Each panel corresponds to a value of $n$ and a value of $K$ and has $\tau^{2}=0.0(0.1) 1.0$ on the horizontal axis.

The interval estimators of $\tau^{2}$ are

- QP (Q-profile confidence interval)

- BJ (Biggerstaff and Jackson interval)

- PL (Profile likelihood interval)

- J (Jackson's interval) 

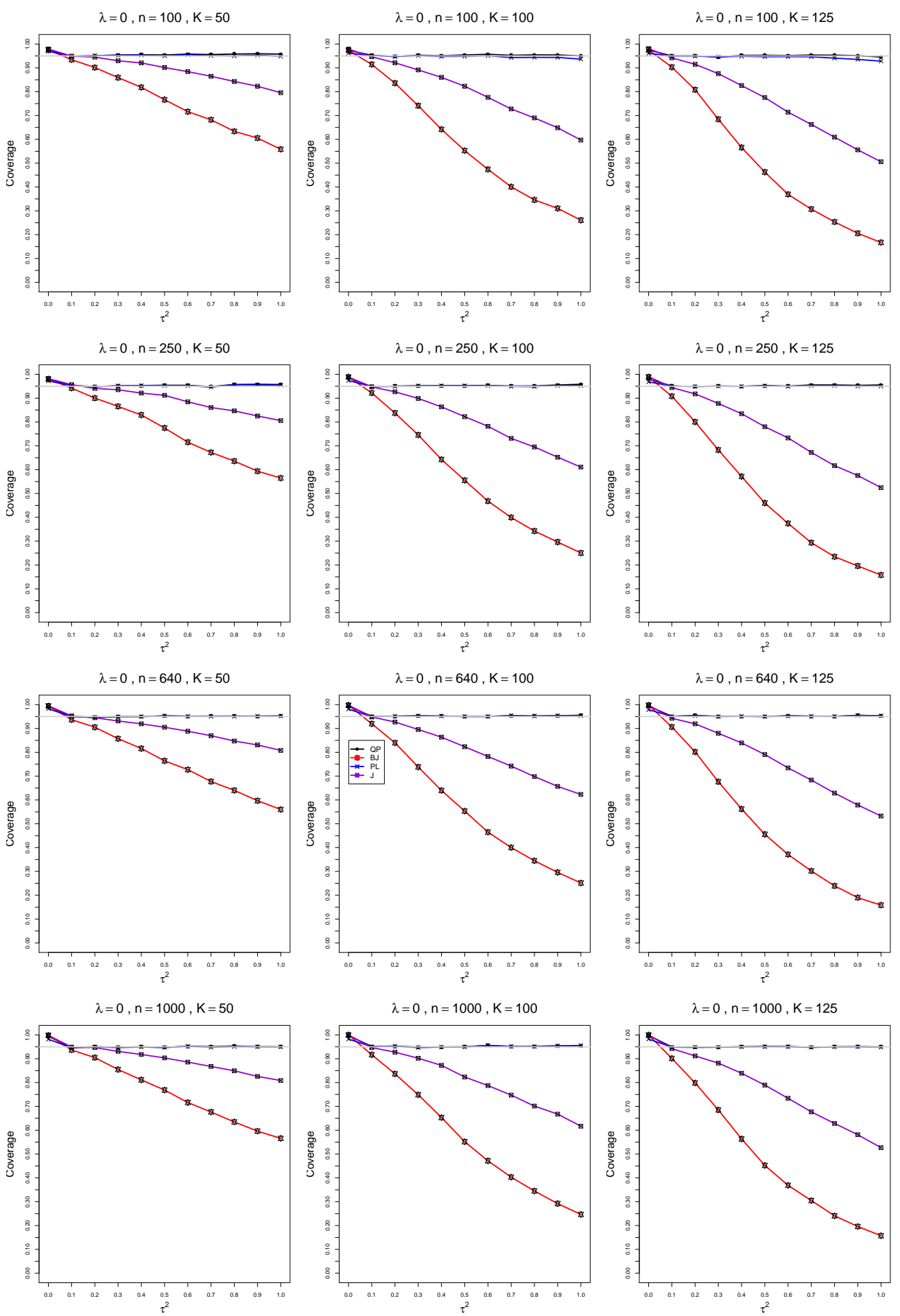

Figure C6.2.1: Coverage of 95\% confidence intervals for the between-studies variance $\tau^{2}$ when $\mu_{C}=4, \lambda=0, n=100,250,640,1000$, and $K=50,100,125$ 

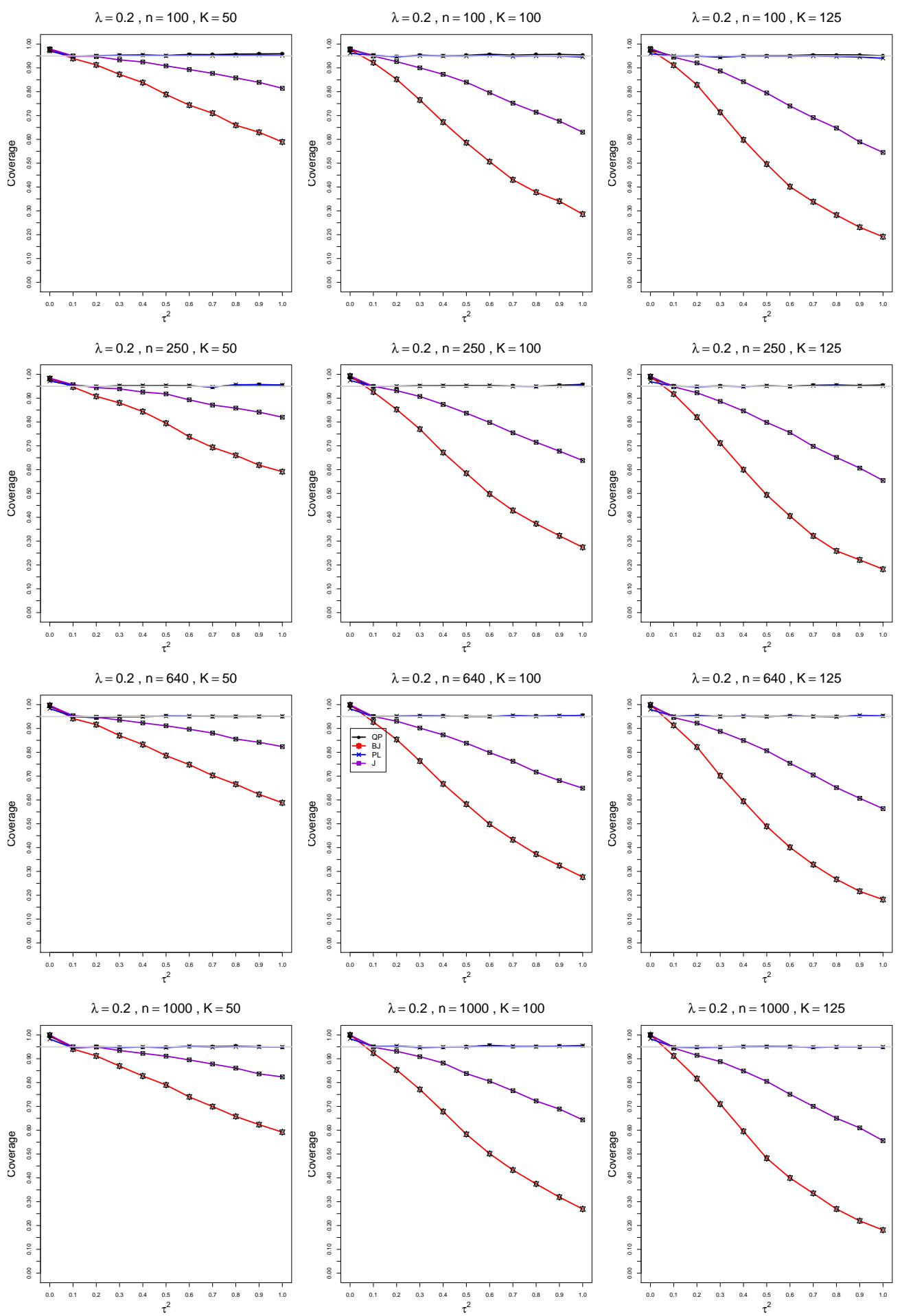

Figure C6.2.2: Coverage of 95\% confidence intervals for the between-studies variance $\tau^{2}$ when $\mu_{C}=4, \lambda=0.2, n=100,250,640,1000$, and $K=50,100,125$ 

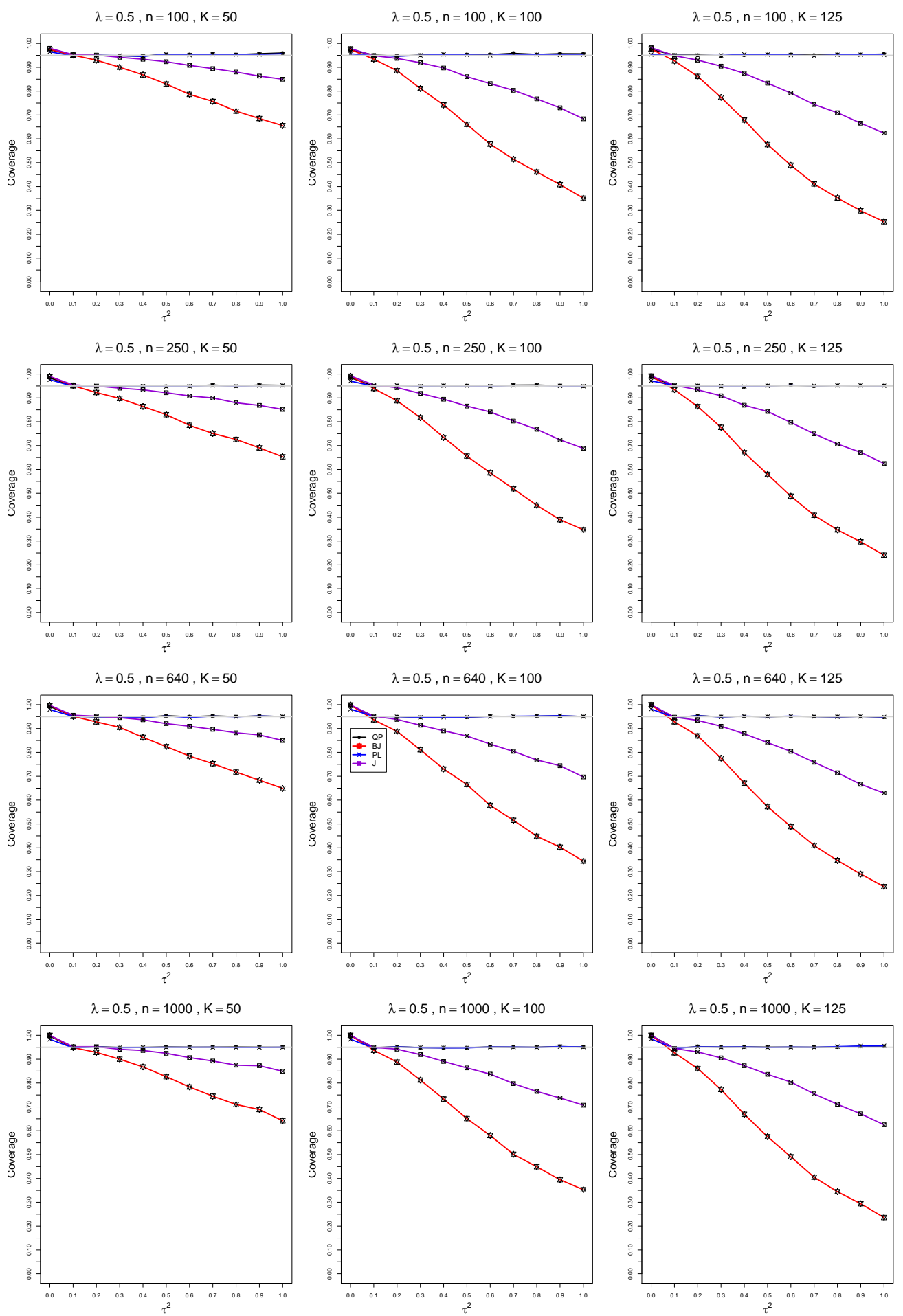

Figure C6.2.3: Coverage of 95\% confidence intervals for the between-studies variance $\tau^{2}$ when $\mu_{C}=4, \lambda=0.5, n=100,250,640,1000$, and $K=50,100,125$ 

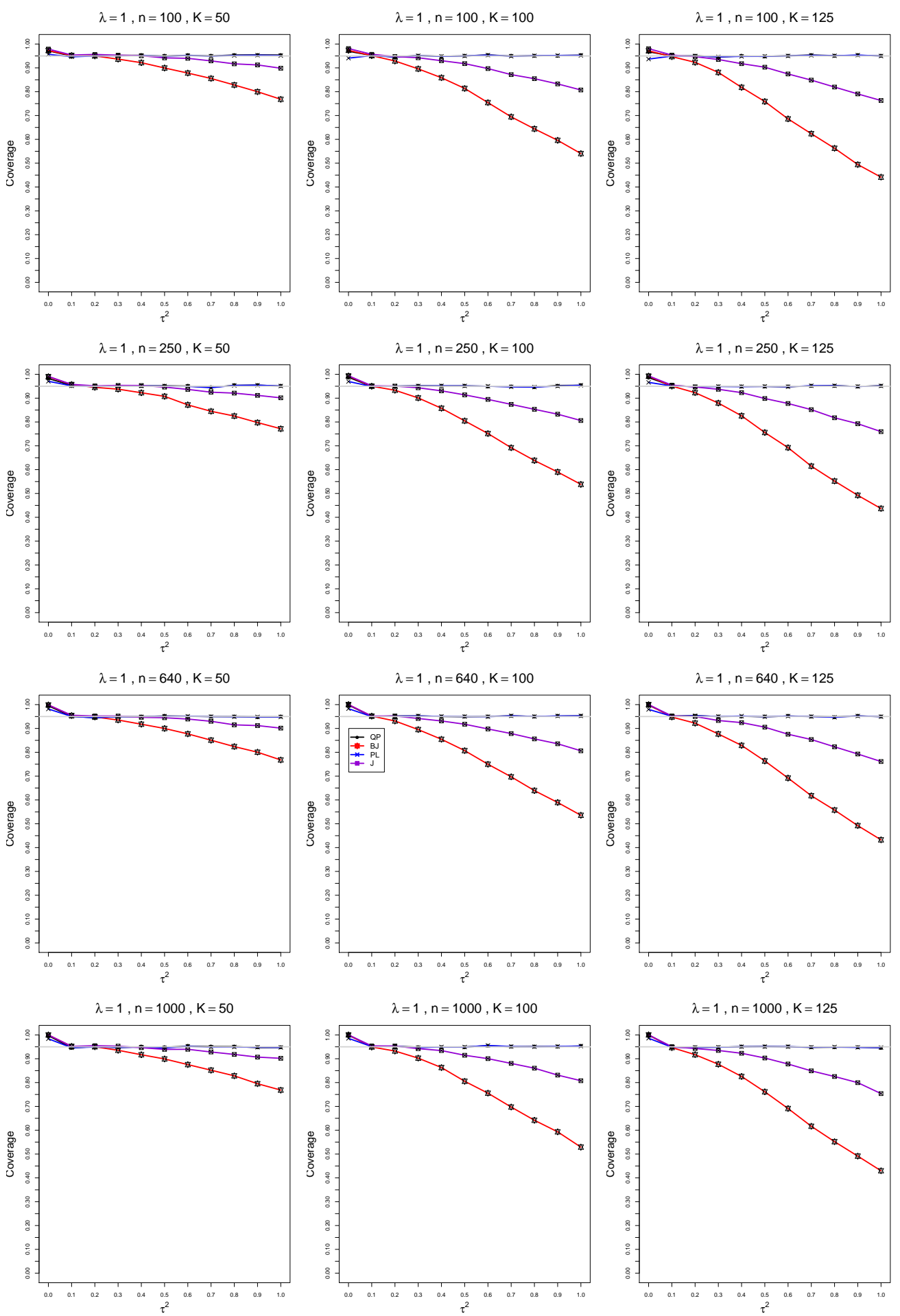

Figure C6.2.4: Coverage of 95\% confidence intervals for the between-studies variance $\tau^{2}$ when $\mu_{C}=4, \lambda=1, n=100,250,640,1000$, and $K=50,100,125$ 

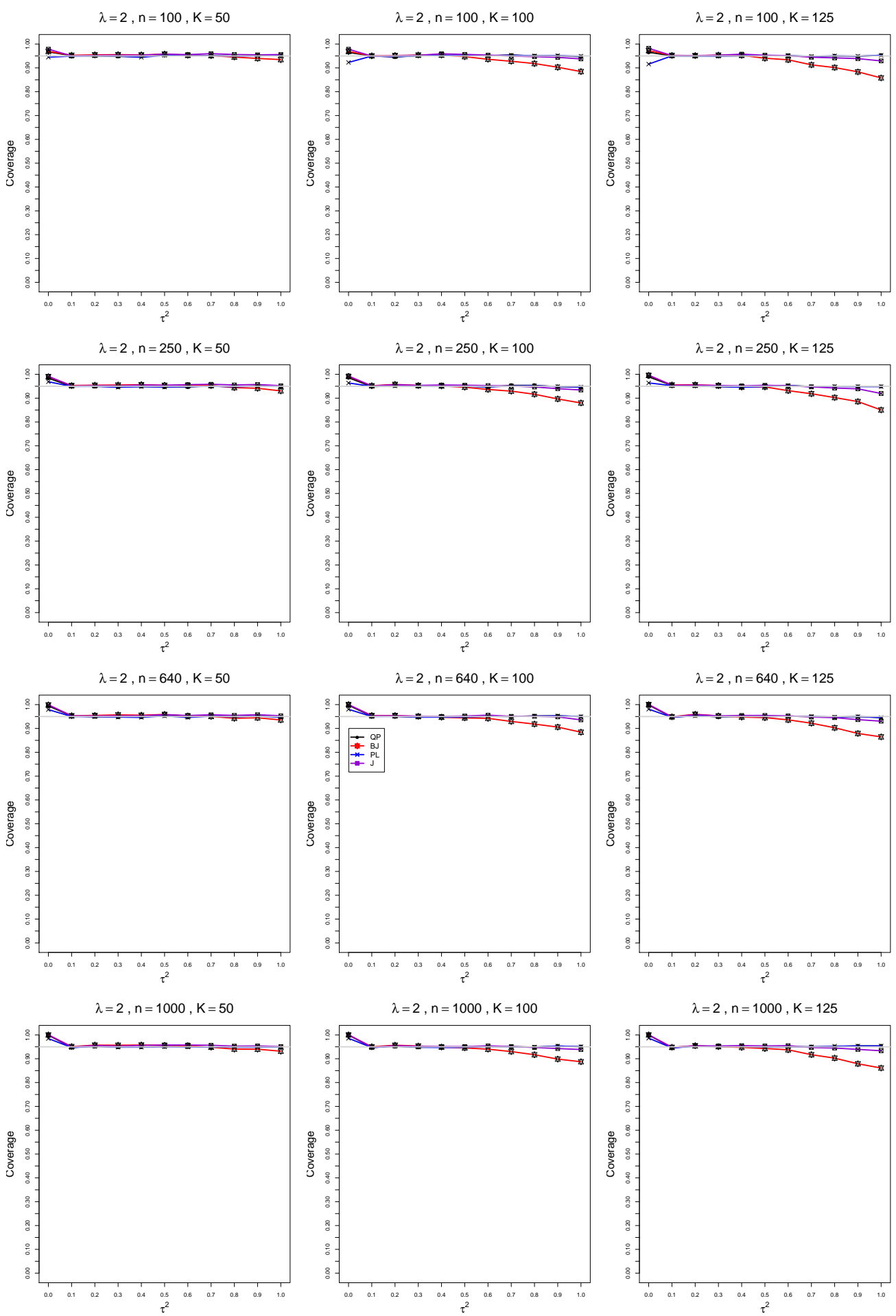

Figure C6.2.5: Coverage of 95\% confidence intervals for the between-studies variance $\tau^{2}$ when $\mu_{C}=4, \lambda=2, n=100,250,640,1000$, and $K=50,100,125$ 


\section{D: Plots of bias and coverage of estimators of $\lambda$ with $n=100,250,640,1000$}

- D1. Normal model, $\mu_{C}=1, K=5,10,30$

- D2. Normal model, $\mu_{C}=4, K=5,10,30$

- D3. Normal model, bias-corrected $\lambda, \mu_{C}=4, K=5,10,30$

- D4. Normal model, $\mu_{C}=1, K=50,100,125$

- D5. Normal model, $\mu_{C}=4, K=50,100,125$

- D3. Normal model, bias-corrected $\lambda, \mu_{C}=4, K=50,100,125$ 
D1. Normal model, $\mu_{C}=1, n=100,250,640$, $1000, K=5,10,30$

\section{D1.1 Bias of point estimators of $\lambda$ when $\mu_{C}=1$}

Each figure corresponds to a value of $\lambda(=0,0.2,0.5,1,2)$, a set of values of $n(=$ $100,250,640,1000)$, and a set of values of $K(=5,10,30)$.

Each panel corresponds to a value of $n$ and a value of $K$ and has $\tau^{2}=0.0(0.1) 1.0$ on the horizontal axis.

The point estimators of $\lambda$ are

- DL (DerSimonian-Laird)

- REML (restricted maximum likelihood)

- MP (Mandel-Paule)

- J (Jackson)

- SSW (sample-size weighted) 

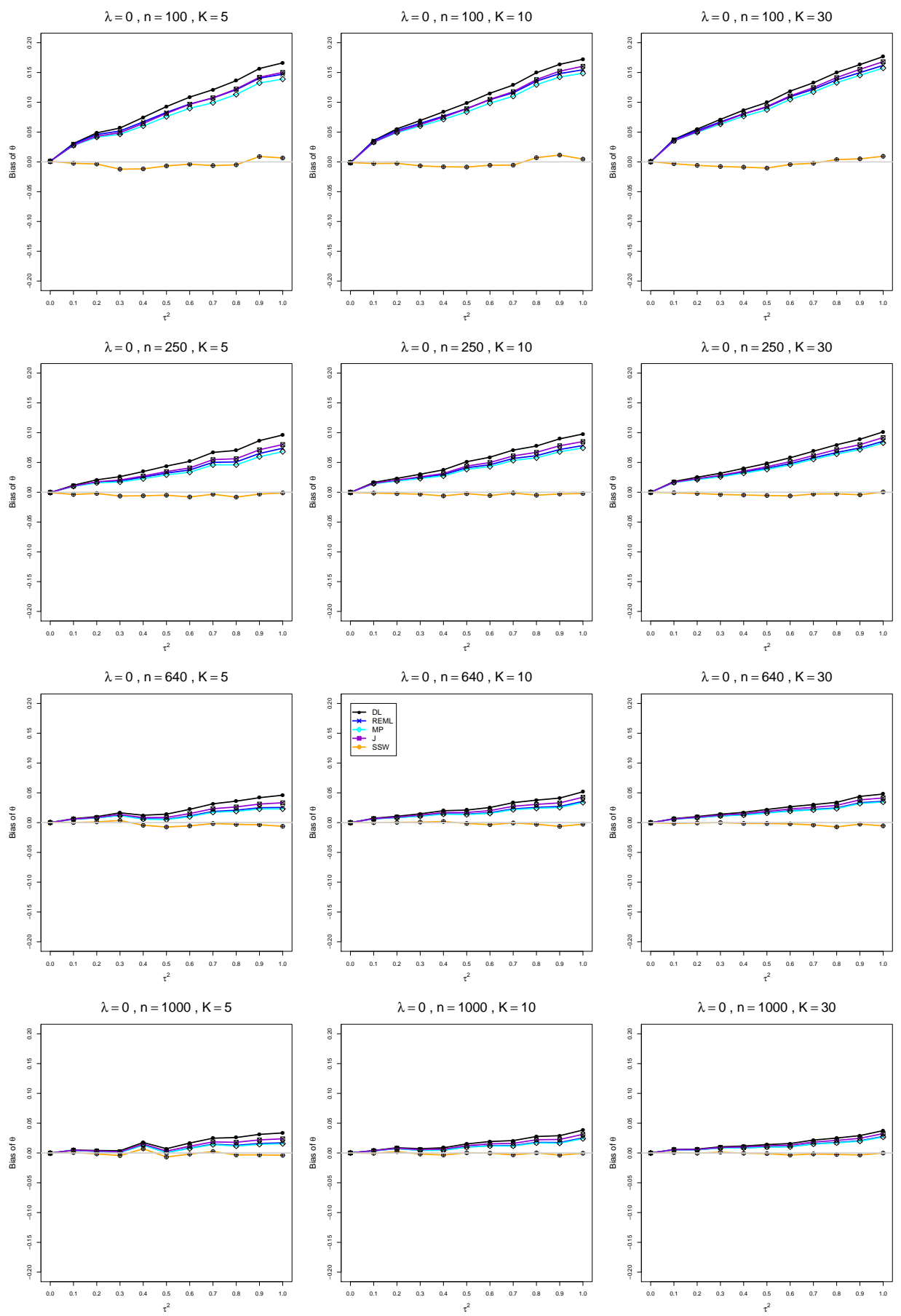

Figure D1.1.1: Bias of estimators of $\log (\operatorname{RoM}) \lambda$ when $\mu_{C}=1, \lambda=0, n=$ 100, 250, 640, 1000, and $K=5,10,30$ 

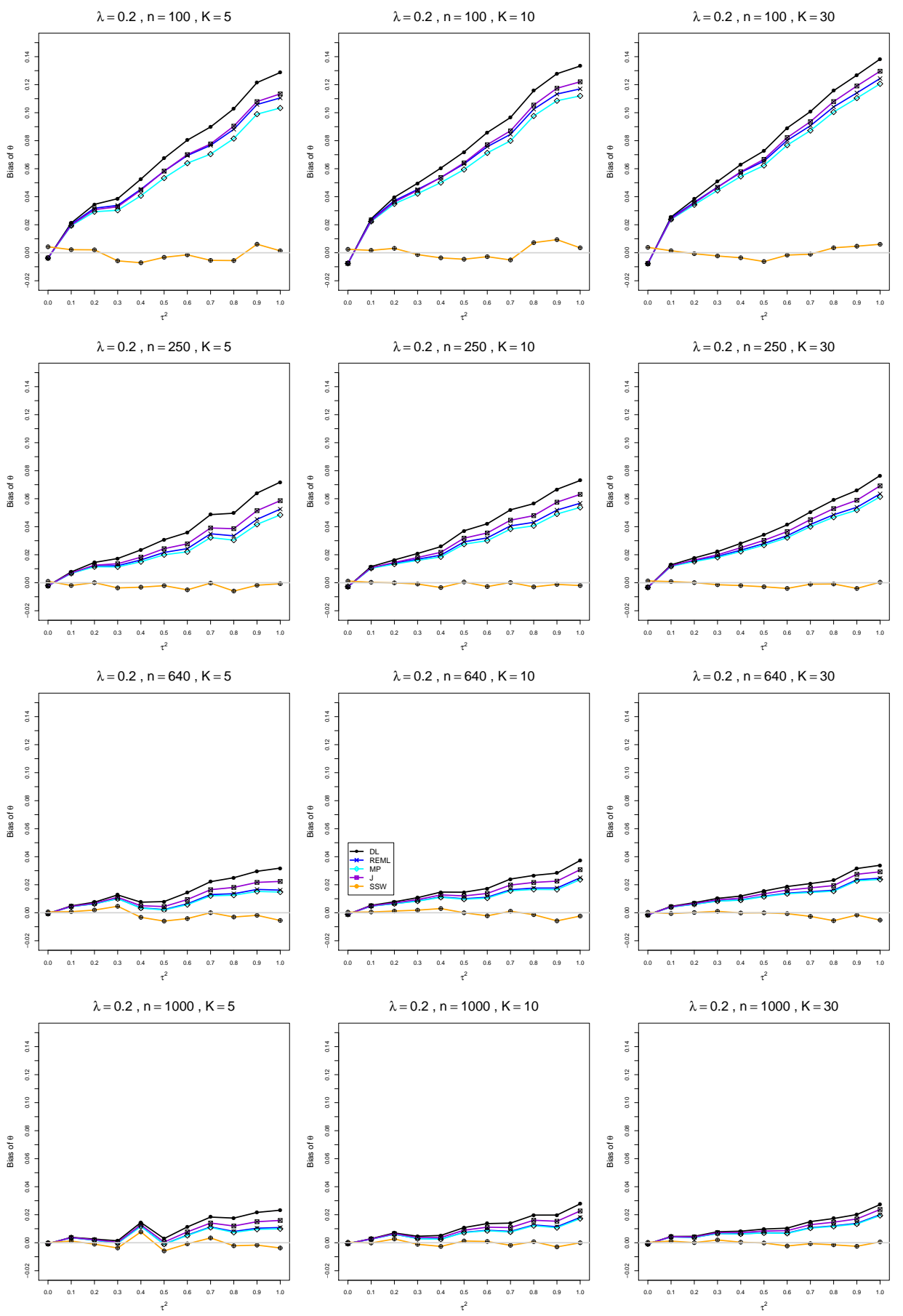

Figure D1.1.2: Bias of estimators of $\log (\mathrm{RoM}) \lambda$ when $\mu_{C}=1, \lambda=0.2, n=$ $100,250,640,1000$, and $K=5,10,30$ 

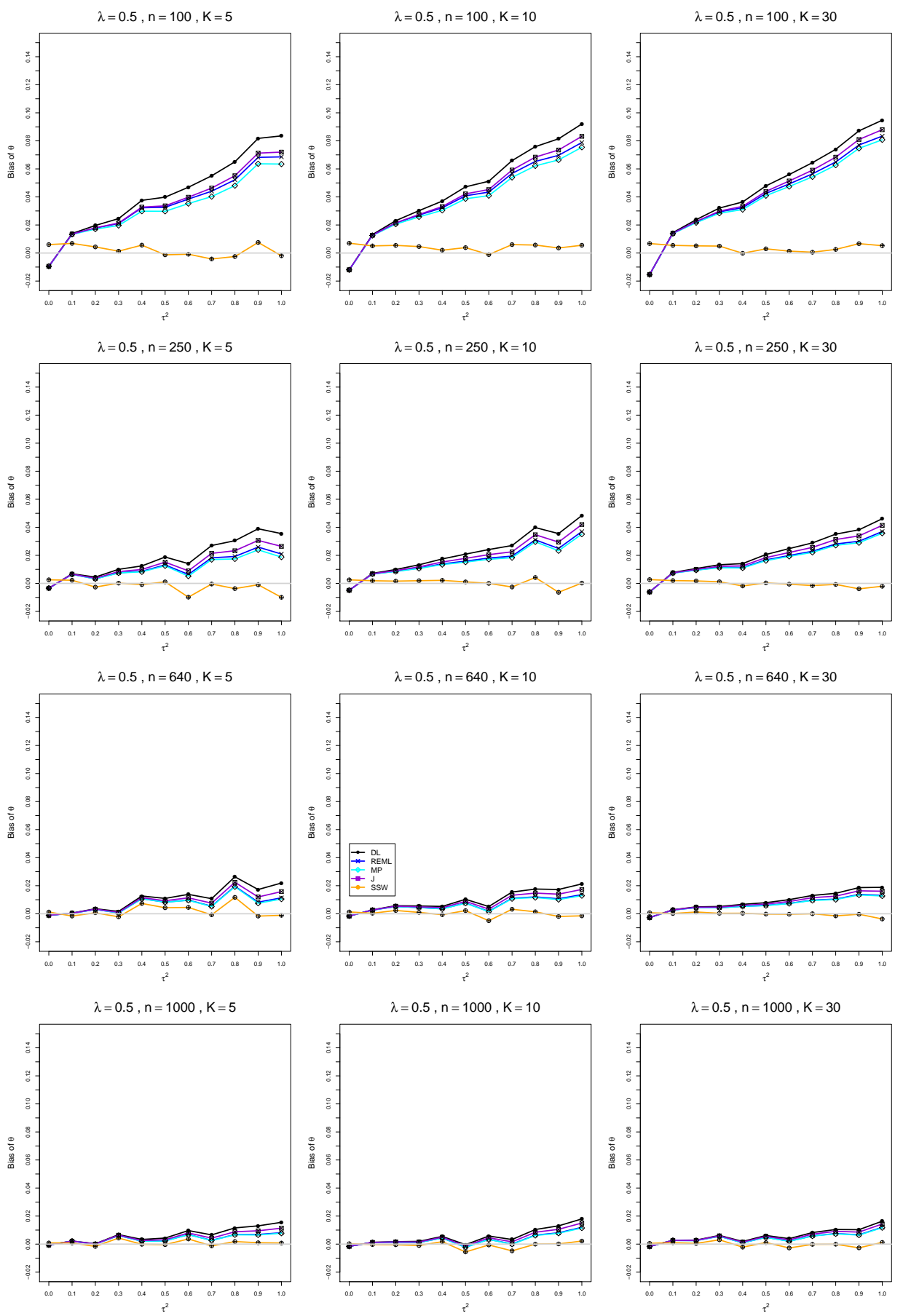

Figure D1.1.3: Bias of estimators of $\log (\operatorname{RoM}) \lambda$ when $\mu_{C}=1, \lambda=0.5, n=$ 100, 250, 640, 1000, and $K=5,10,30$ 

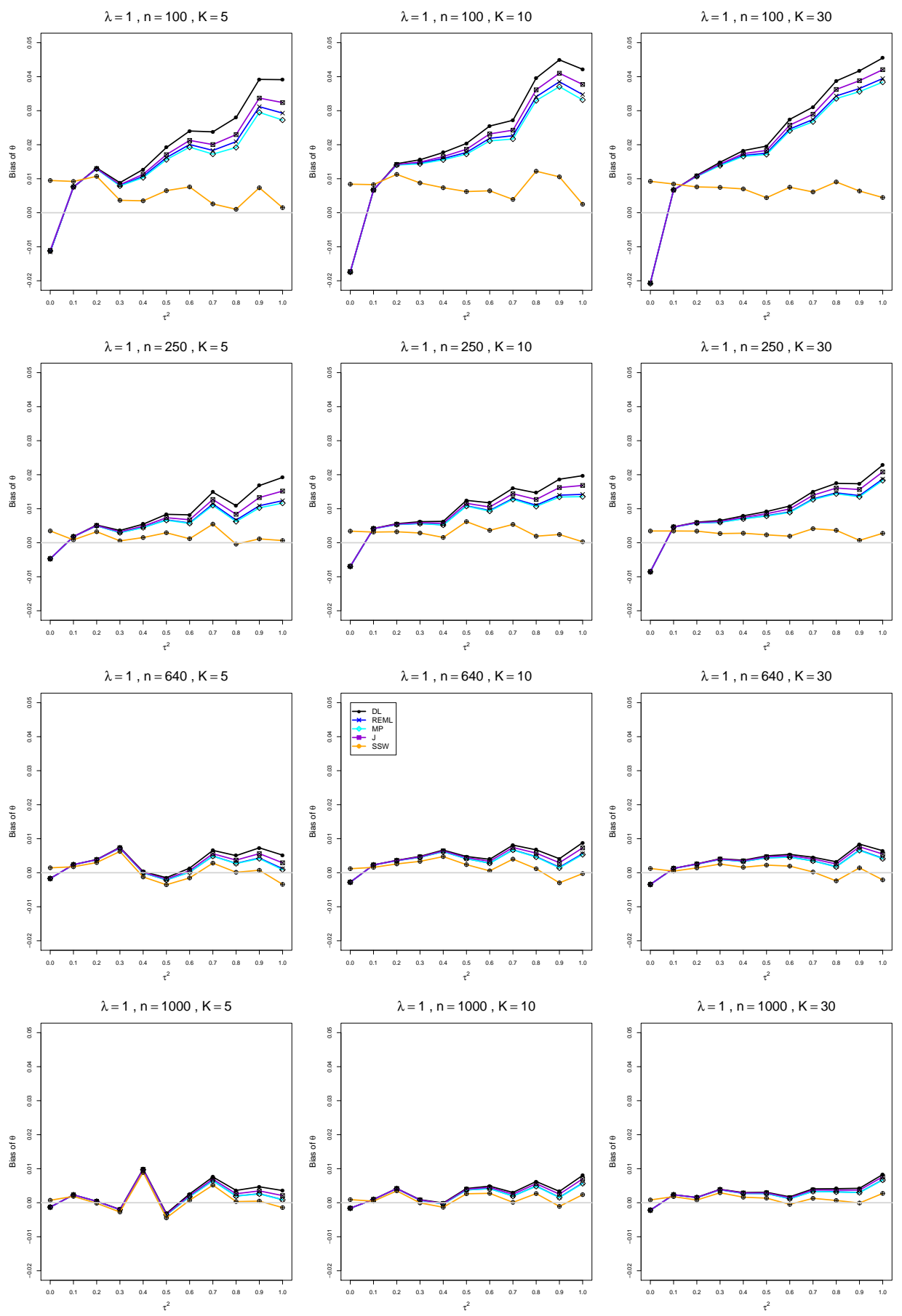

Figure D1.1.4: Bias of estimators of $\log (\operatorname{RoM}) \lambda$ when $\mu_{C}=1, \lambda=1, n=$ 100, 250, 640, 1000, and $K=5,10,30$ 

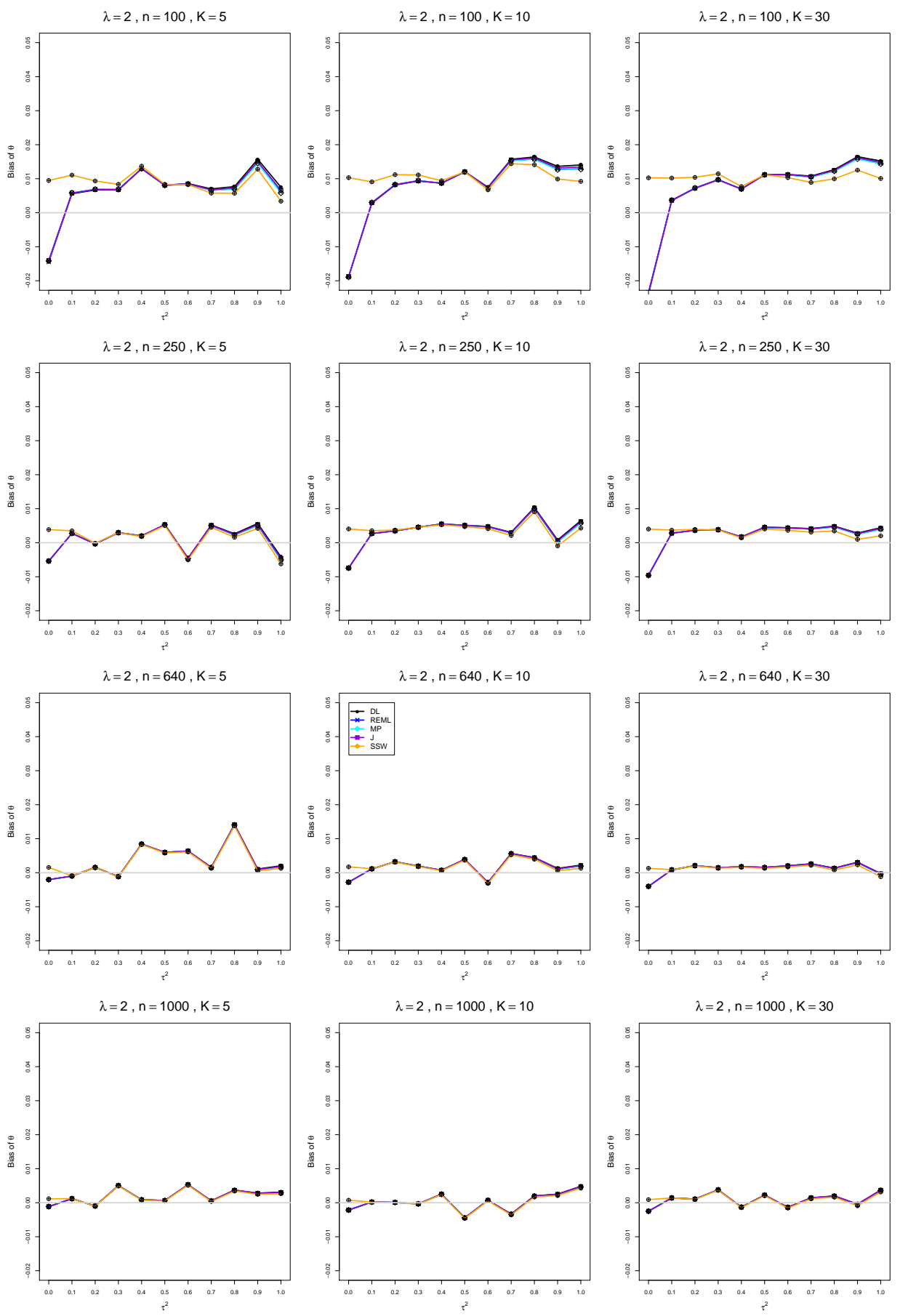

Figure D1.1.5: Bias of estimators of $\log (\operatorname{RoM}) \lambda$ when $\mu_{C}=1, \lambda=2, n=$ 100, 250, 640, 1000, and $K=5,10,30$ 


\section{D1.2 Coverage of interval estimators of $\lambda$}

Each figure corresponds to a value of $\lambda(=0,0.2,0.5,1,2)$, a set of values of $n(=$ $100,250,640,1000)$, and a set of values of $K(=5,10,30)$.

Each panel corresponds to a value of $n$ and a value of $K$ and has $\tau^{2}=0.0(0.1) 1.0$ on the horizontal axis.

The interval estimators of $\lambda$ are the companions to the inverse-variance-weighted point estimators

- DL (DerSimonian-Laird)

- REML (restricted maximum likelihood)

- MP (Mandel-Paule)

- J (Jackson)

and

- HKSJ (Hartung-Knapp-Sidik-Jonkman)

- HKSJ MP (HKSJ with MP estimator of $\tau^{2}$ )

- SSW (SSW as center and half-width equal to critical value from $t_{K-1}$ times estimated standard deviation of SSW with $\hat{\tau}^{2}=\hat{\tau}_{M P}^{2}$ 

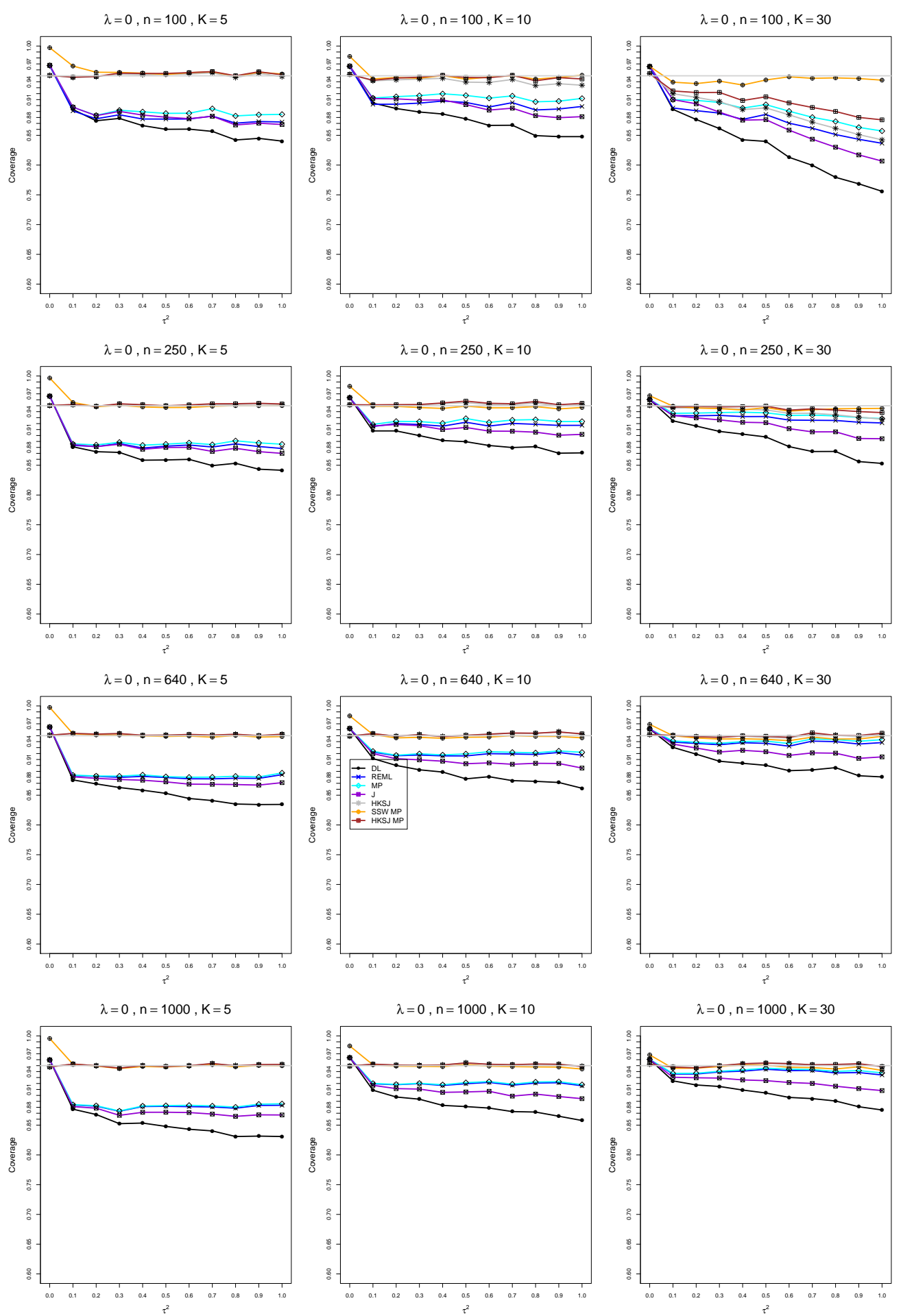

Figure D1.2.1: Coverage of $95 \%$ confidence intervals for $\lambda$ when $\mu_{C}=1, \lambda=0, n=$ 100, 250, 640, 1000, and $K=5,10,30$ 

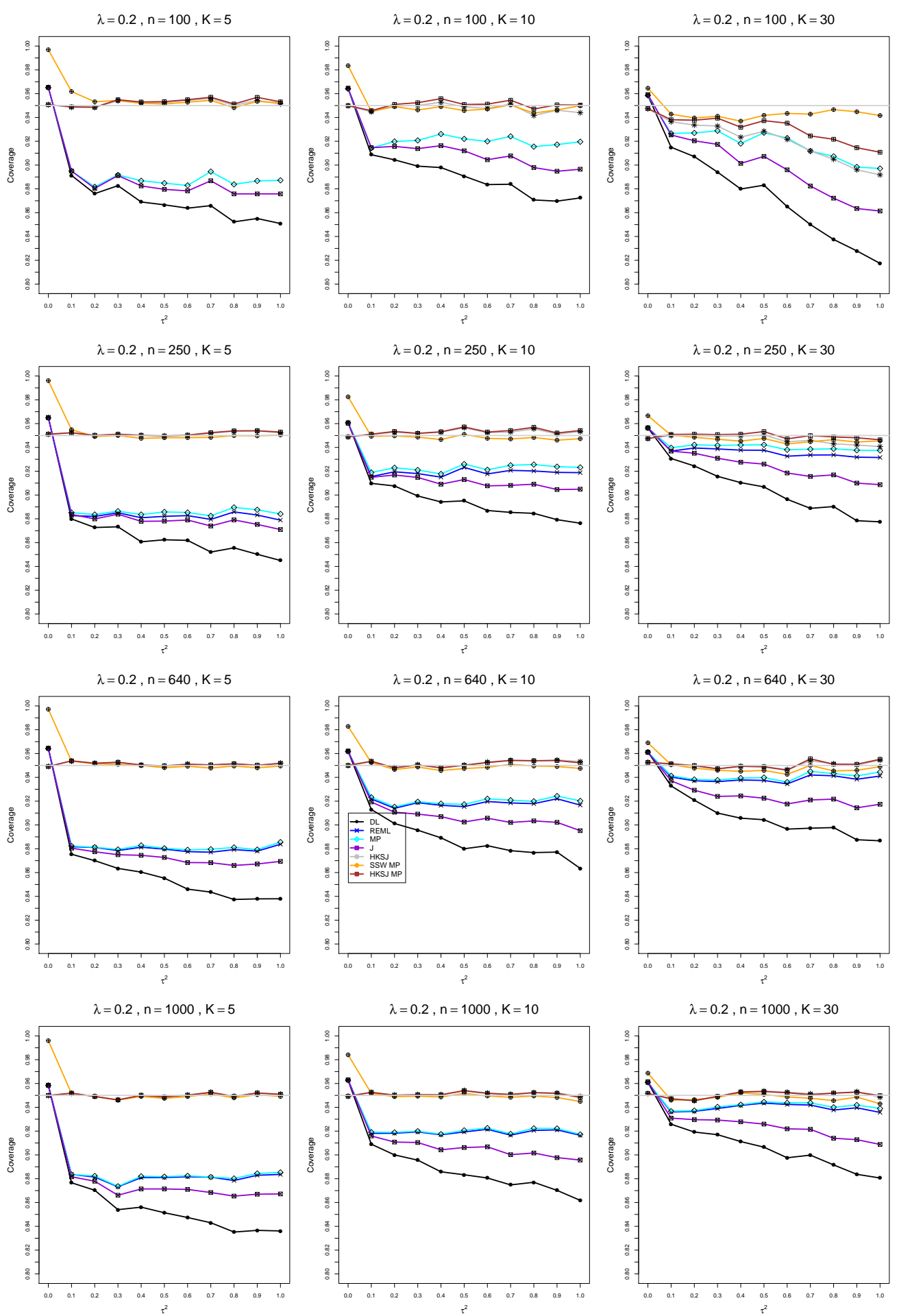

Figure D1.2.2: Coverage of $95 \%$ confidence intervals for $\lambda$ when $\mu_{C}=1, \lambda=0.2, n=$ 100, 250, 640, 1000, and $K=5,10,30$ 

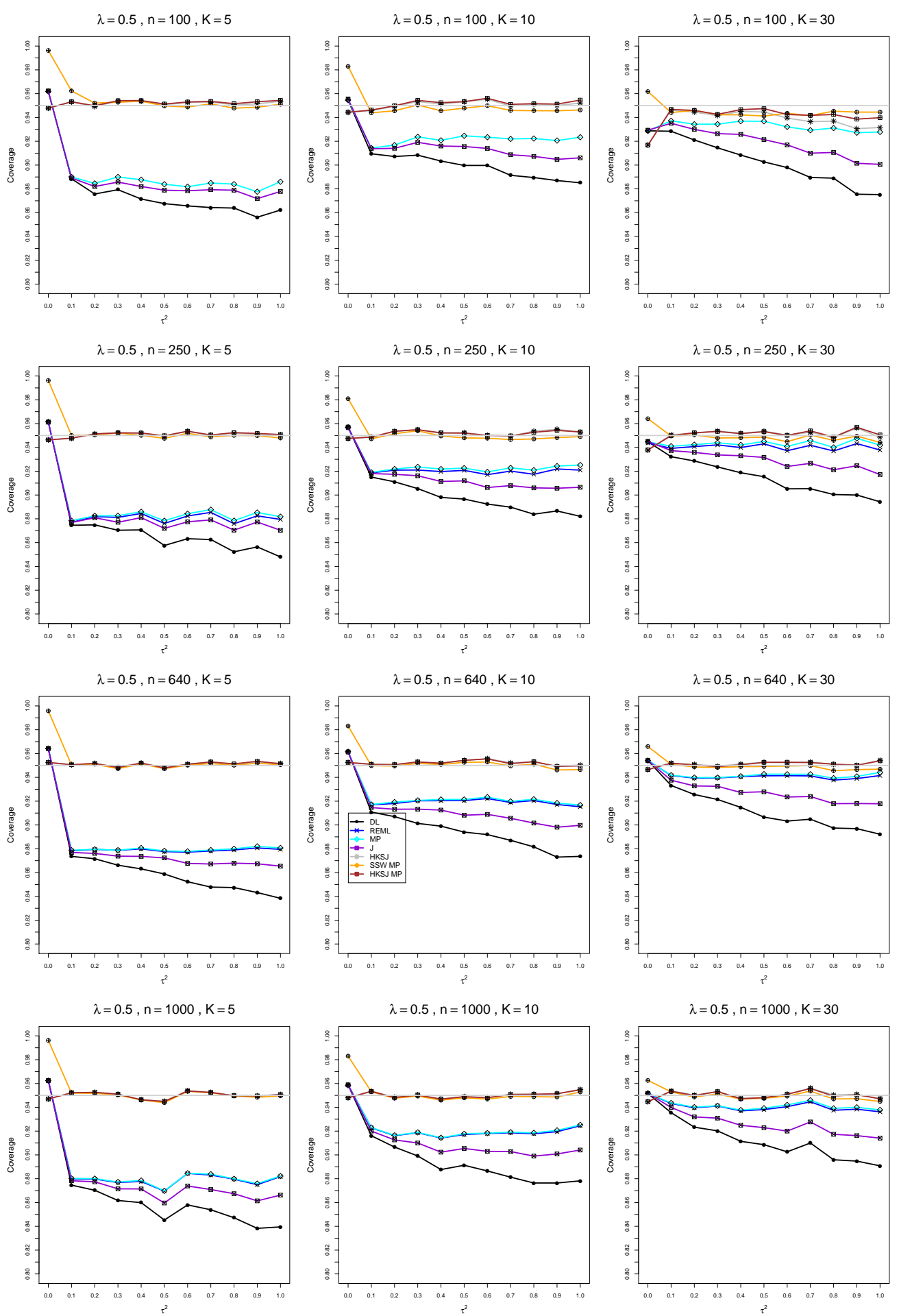

Figure D1.2.3: Coverage of $95 \%$ confidence intervals for $\lambda$ when $\mu_{C}=1, \lambda=0.5, n=$ 100, 250, 640, 1000, and $K=5,10,30$ 

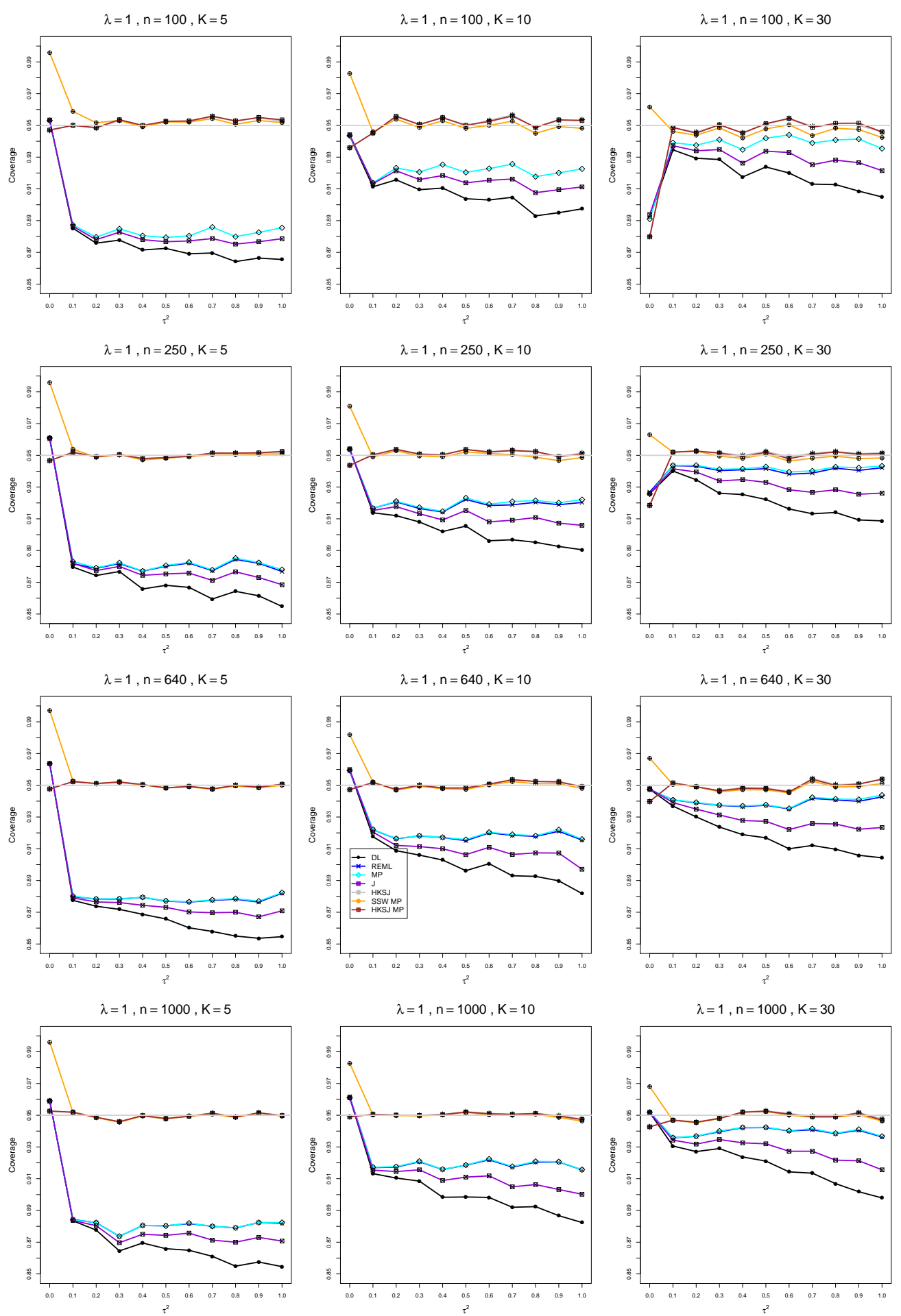

Figure D1.2.4: Coverage of $95 \%$ confidence intervals for $\lambda$ when $\mu_{C}=1, \lambda=1, n=$ 100, 250, 640, 1000, and $K=5,10,30$ 

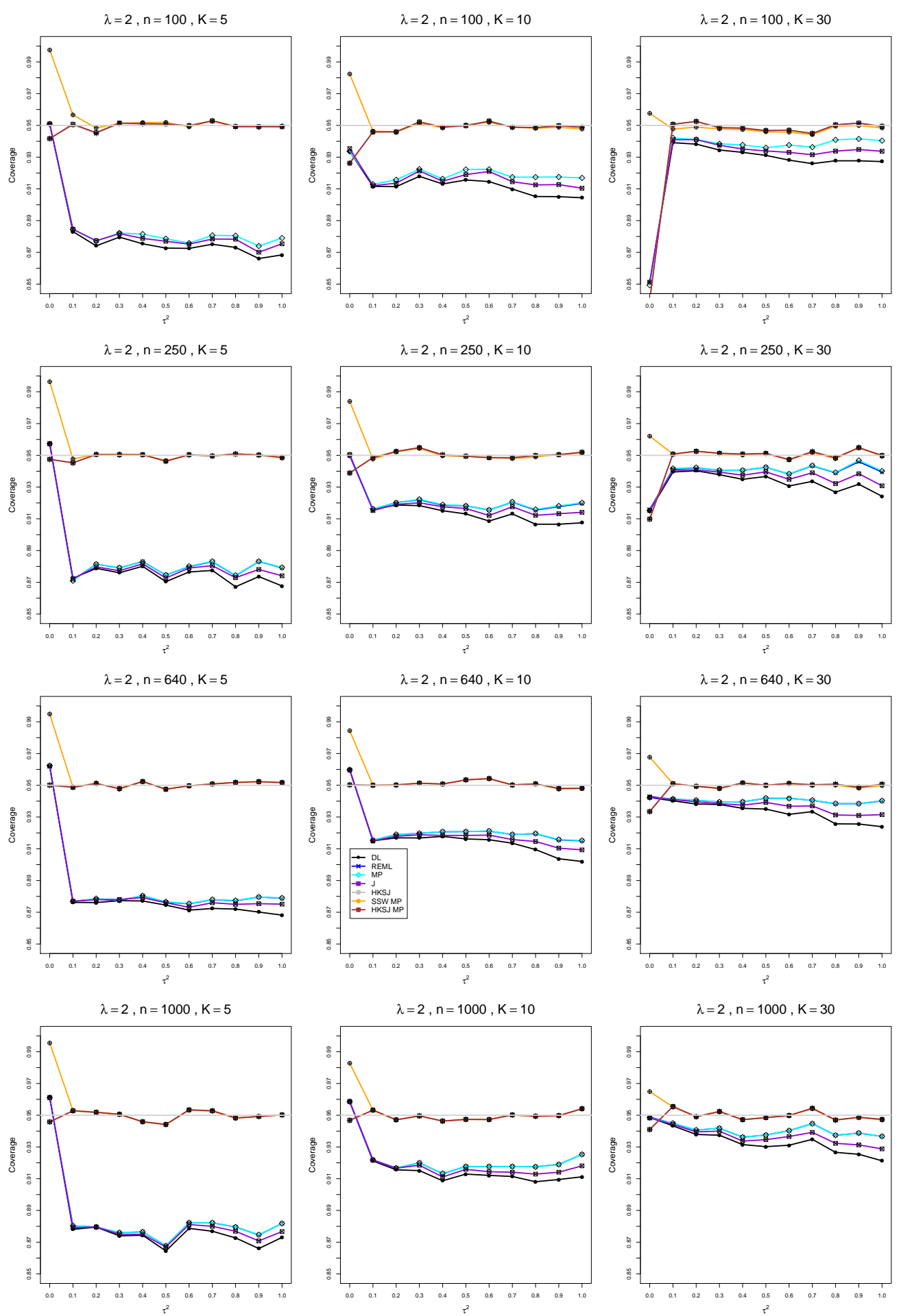

Figure D1.2.5: Coverage of $95 \%$ confidence intervals for $\lambda$ when $\mu_{C}=1, \lambda=2, n=$ 100, 250, 640, 1000, and $K=5,10,30$ 
D2. Normal model, $\mu_{C}=4, n=100,250,640$, $1000, K=5,10,30$

\section{D2.1 Bias of point estimators of $\lambda$ when $\mu_{C}=4$}

Each figure corresponds to a value of $\lambda(=0,0.2,0.5,1,2)$, a set of values of $n(=$ $100,250,640,1000)$, and a set of values of $K(=5,10,30)$.

Each panel corresponds to a value of $n$ and a value of $K$ and has $\tau^{2}=0.0(0.1) 1.0$ on the horizontal axis.

The point estimators of $\lambda$ are

- DL (DerSimonian-Laird)

- REML (restricted maximum likelihood)

- MP (Mandel-Paule)

- J (Jackson)

- SSW (sample-size weighted) 

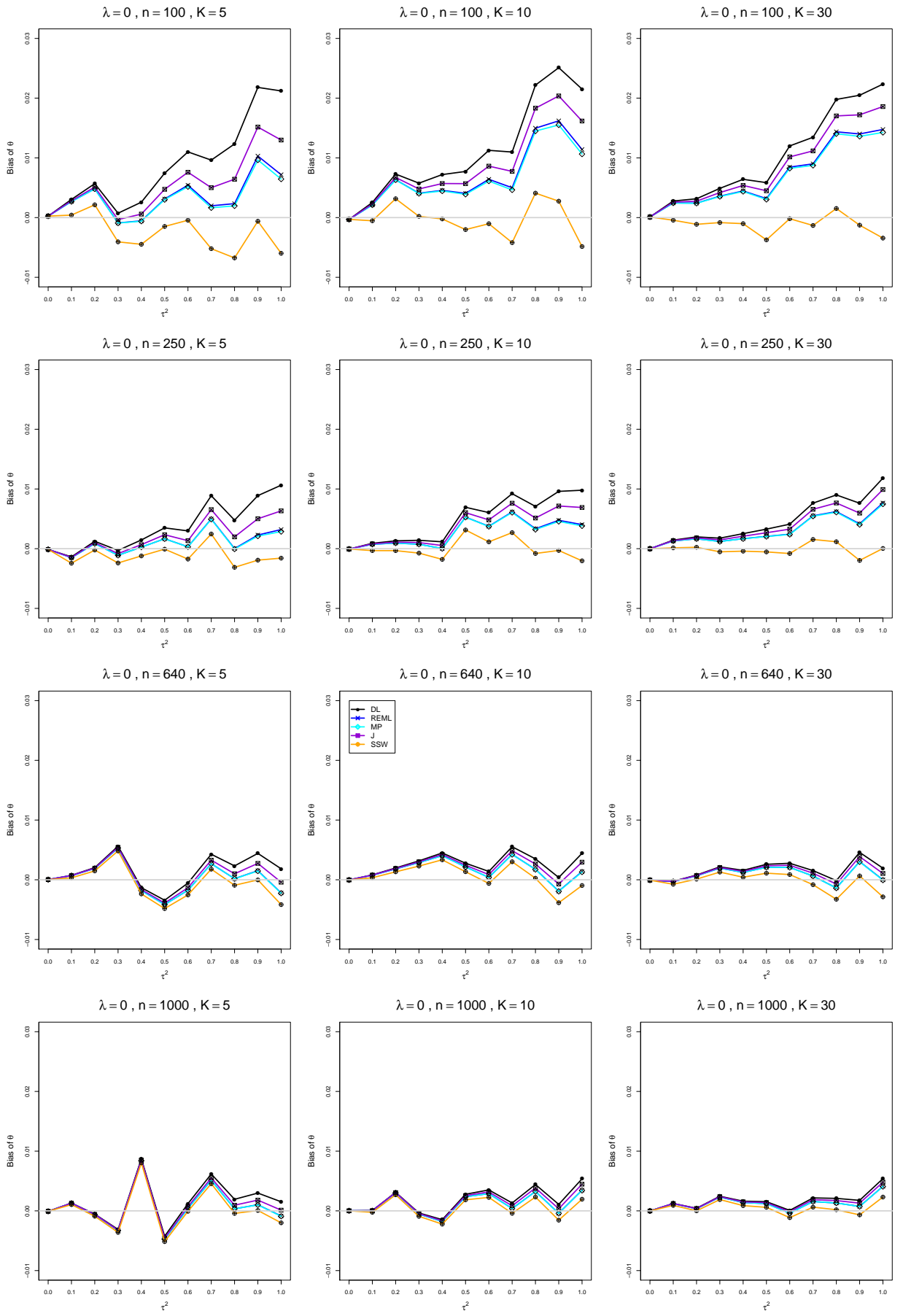

Figure D2.1.1: Bias of estimators of $\log (\operatorname{RoM}) \lambda$ when $\mu_{C}=4, \lambda=0, n=$ $100,250,640,1000$, and $K=5,10,30$ 

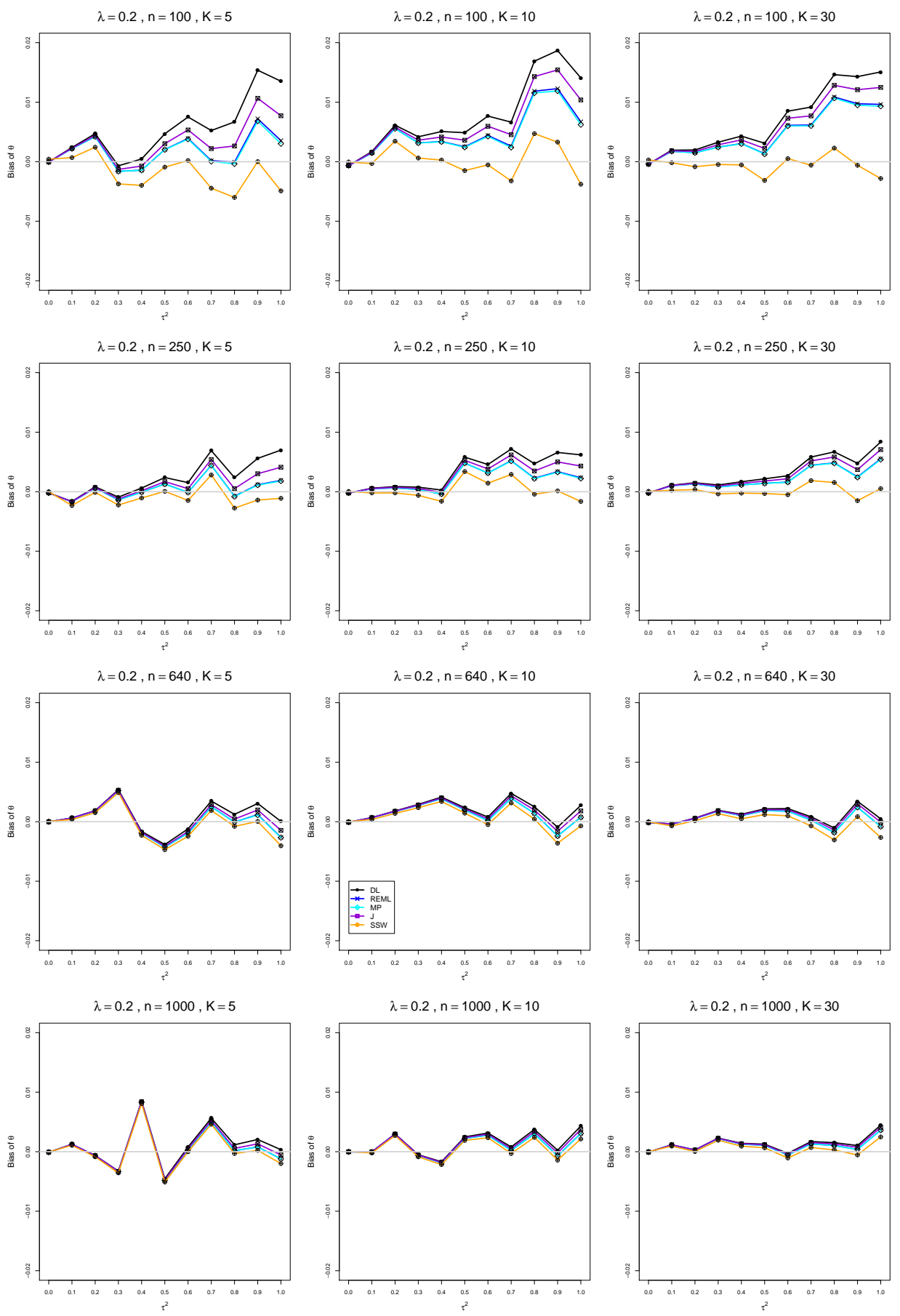

Figure D2.1.2: Bias of estimators of $\log (\mathrm{RoM}) \lambda$ when $\mu_{C}=4, \lambda=0.2, n=$ $100,250,640,1000$, and $K=5,10,30$ 

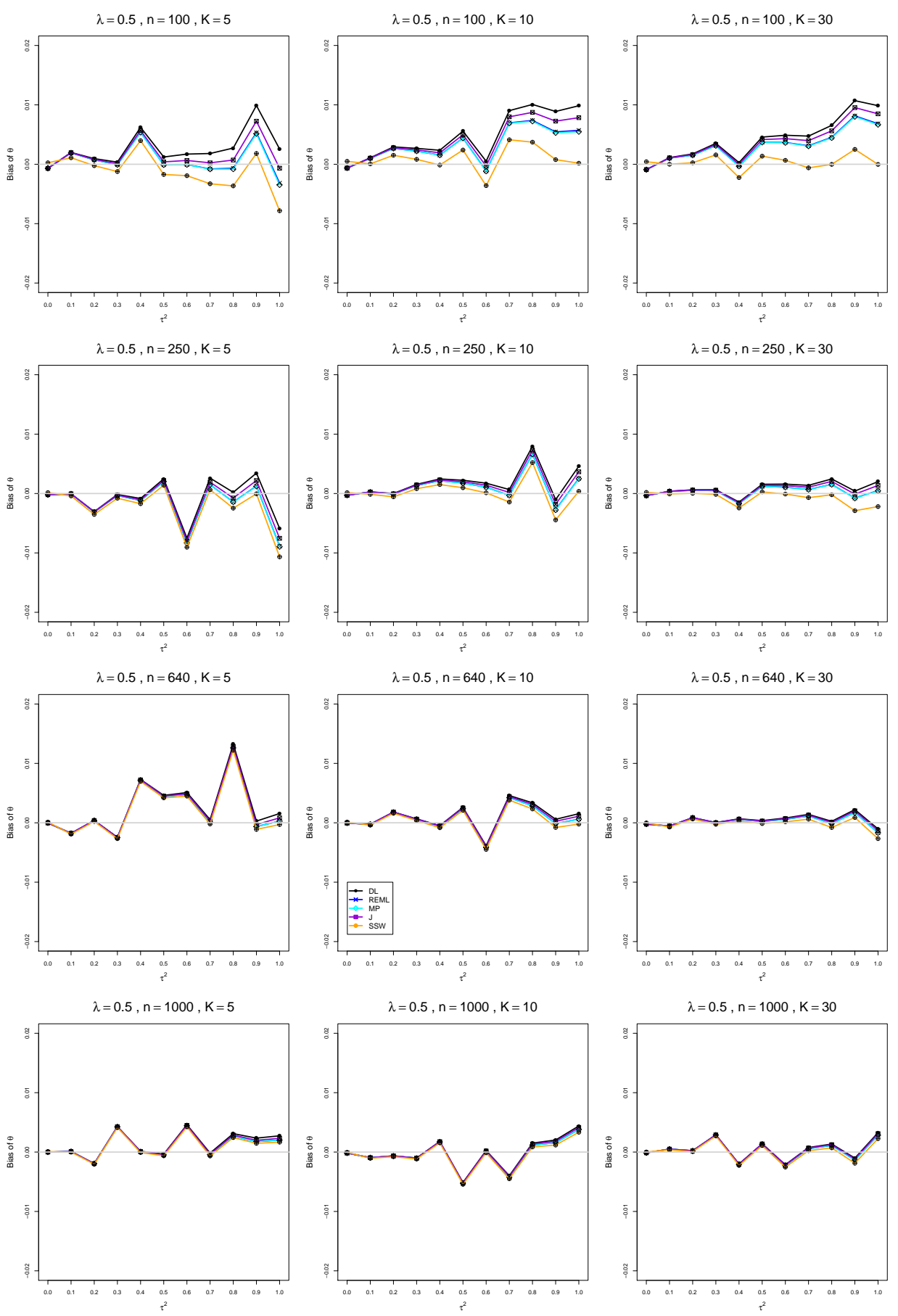

Figure D2.1.3: Bias of estimators of $\log (\mathrm{RoM}) \lambda$ when $\mu_{C}=4, \lambda=0.5, n=$ $100,250,640,1000$, and $K=5,10,30$ 

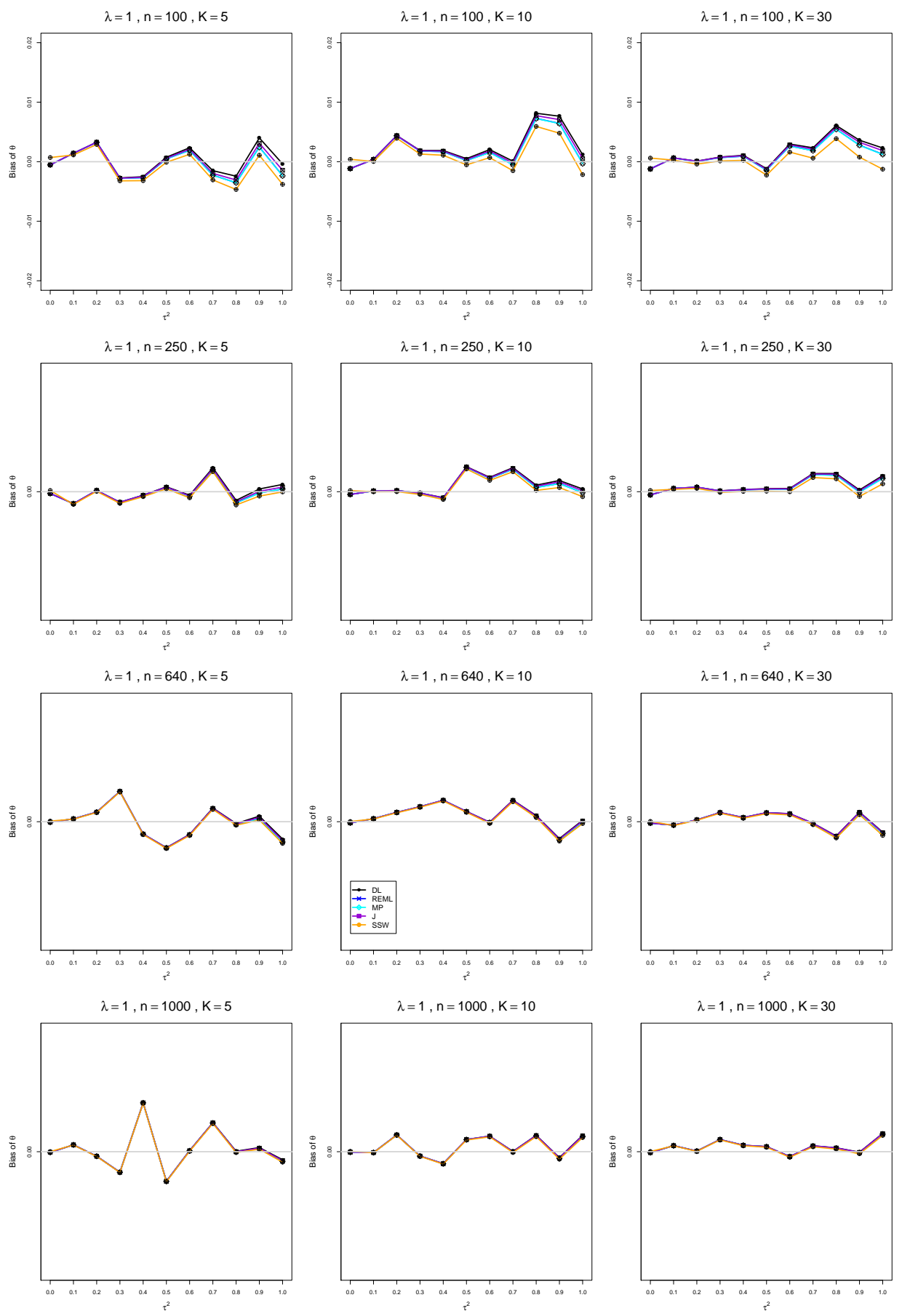

Figure D2.1.4: Bias of estimators of $\log (\operatorname{RoM}) \lambda$ when $\mu_{C}=4, \lambda=1, n=$ 100, 250, 640, 1000, and $K=5,10,30$ 

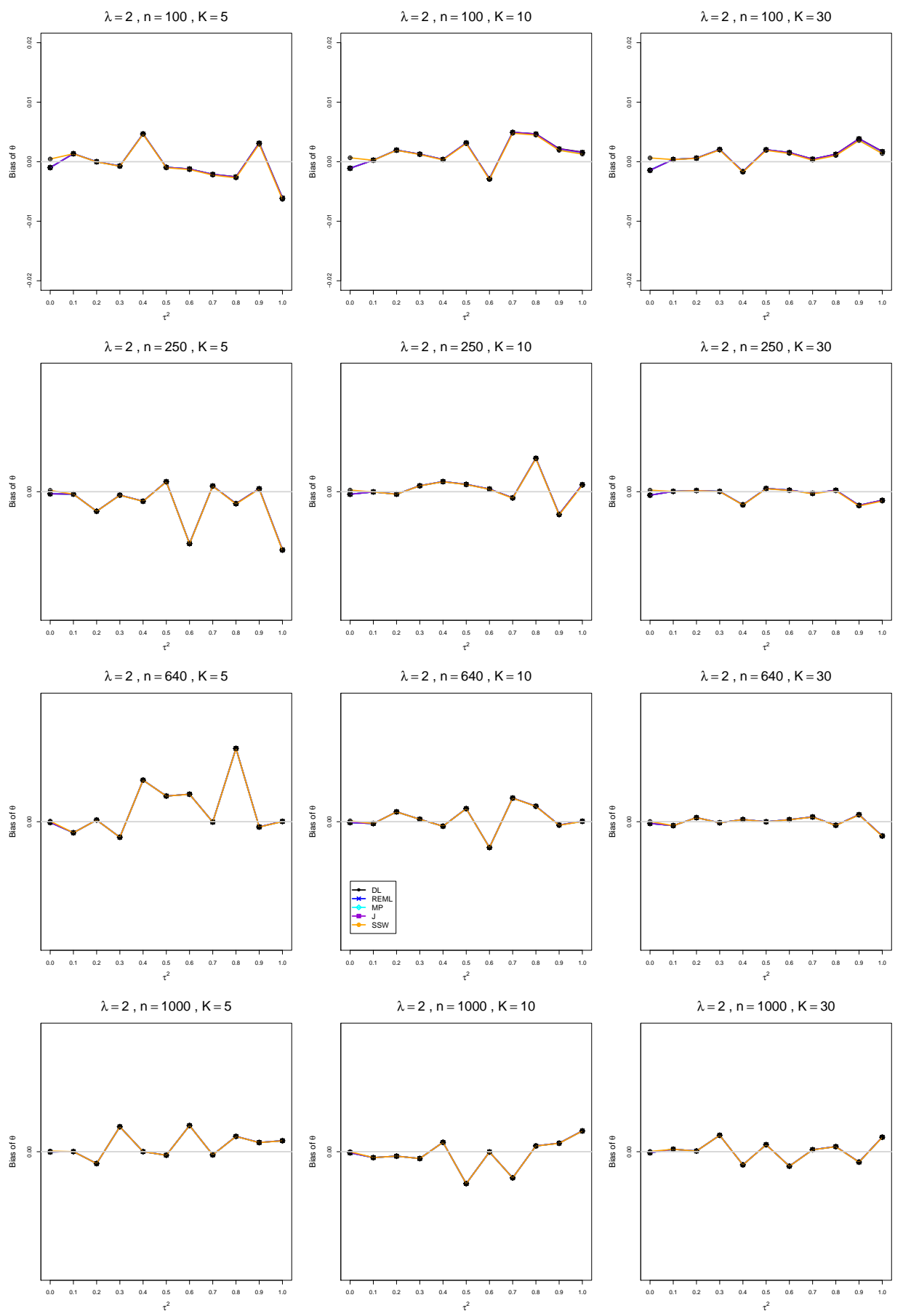

Figure D2.1.5: Bias of estimators of $\log (\operatorname{RoM}) \lambda$ when $\mu_{C}=4, \lambda=2, n=$ 100, 250, 640, 1000, and $K=5,10,30$ 


\section{D2.2 Coverage of interval estimators of $\lambda$}

Each figure corresponds to a value of $\lambda(=0,0.2,0.5,1,2)$, a set of values of $n(=$ $100,250,640,1000)$, and a set of values of $K(=5,10,30)$.

Each panel corresponds to a value of $n$ and a value of $K$ and has $\tau^{2}=0.0(0.1) 1.0$ on the horizontal axis.

The interval estimators of $\lambda$ are the companions to the inverse-variance-weighted point estimators

- DL (DerSimonian-Laird)

- REML (restricted maximum likelihood)

- MP (Mandel-Paule)

- J (Jackson)

and

- HKSJ (Hartung-Knapp-Sidik-Jonkman)

- HKSJ MP (HKSJ with MP estimator of $\tau^{2}$ )

- SSW (SSW as center and half-width equal to critical value from $t_{K-1}$ times estimated standard deviation of SSW with $\hat{\tau}^{2}=\hat{\tau}_{M P}^{2}$ 

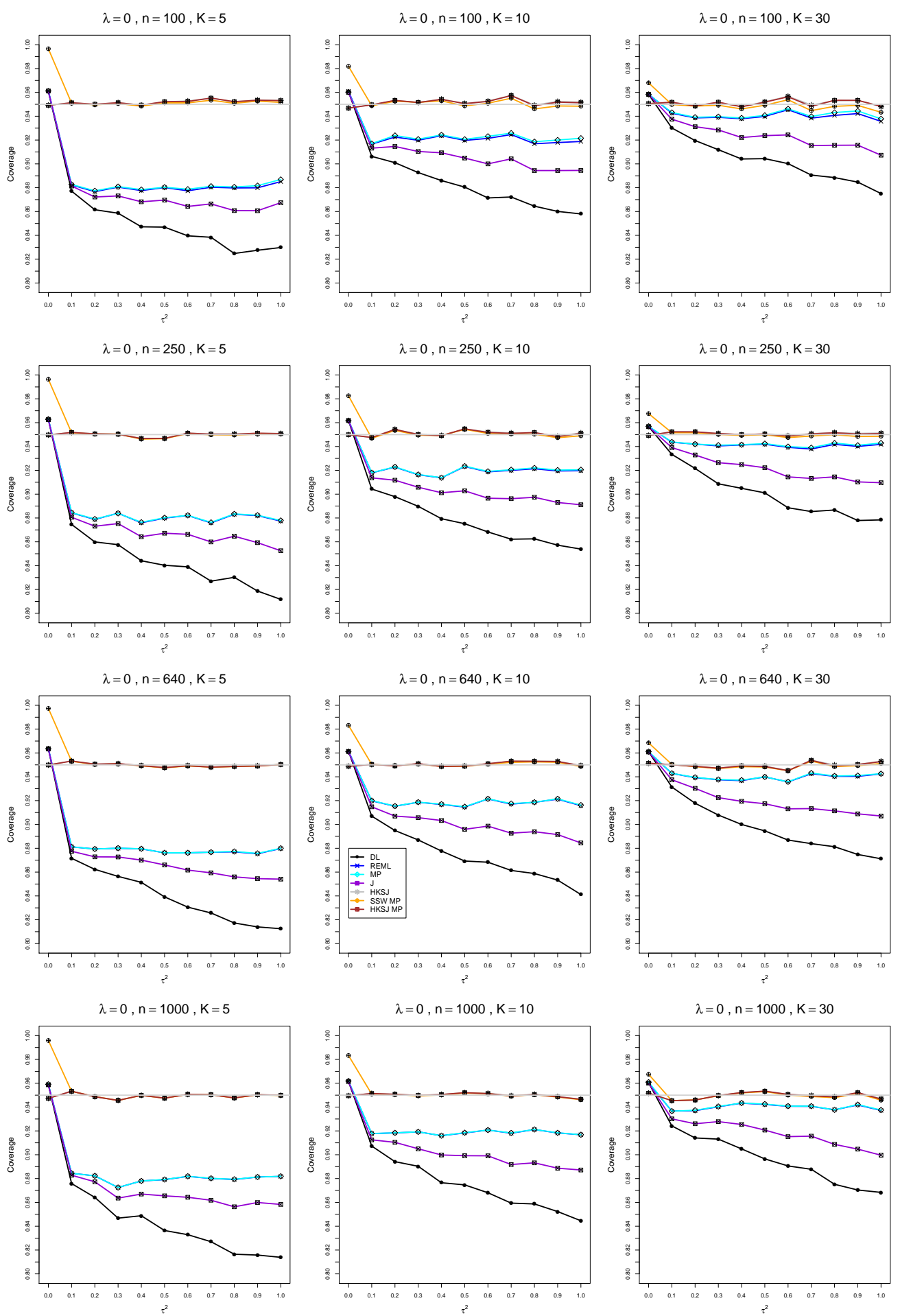

Figure D2.2.1: Coverage of $95 \%$ confidence intervals for $\lambda$ when $\mu_{C}=4, \lambda=0, n=$ 100, 250, 640, 1000, and $K=5,10,30$ 

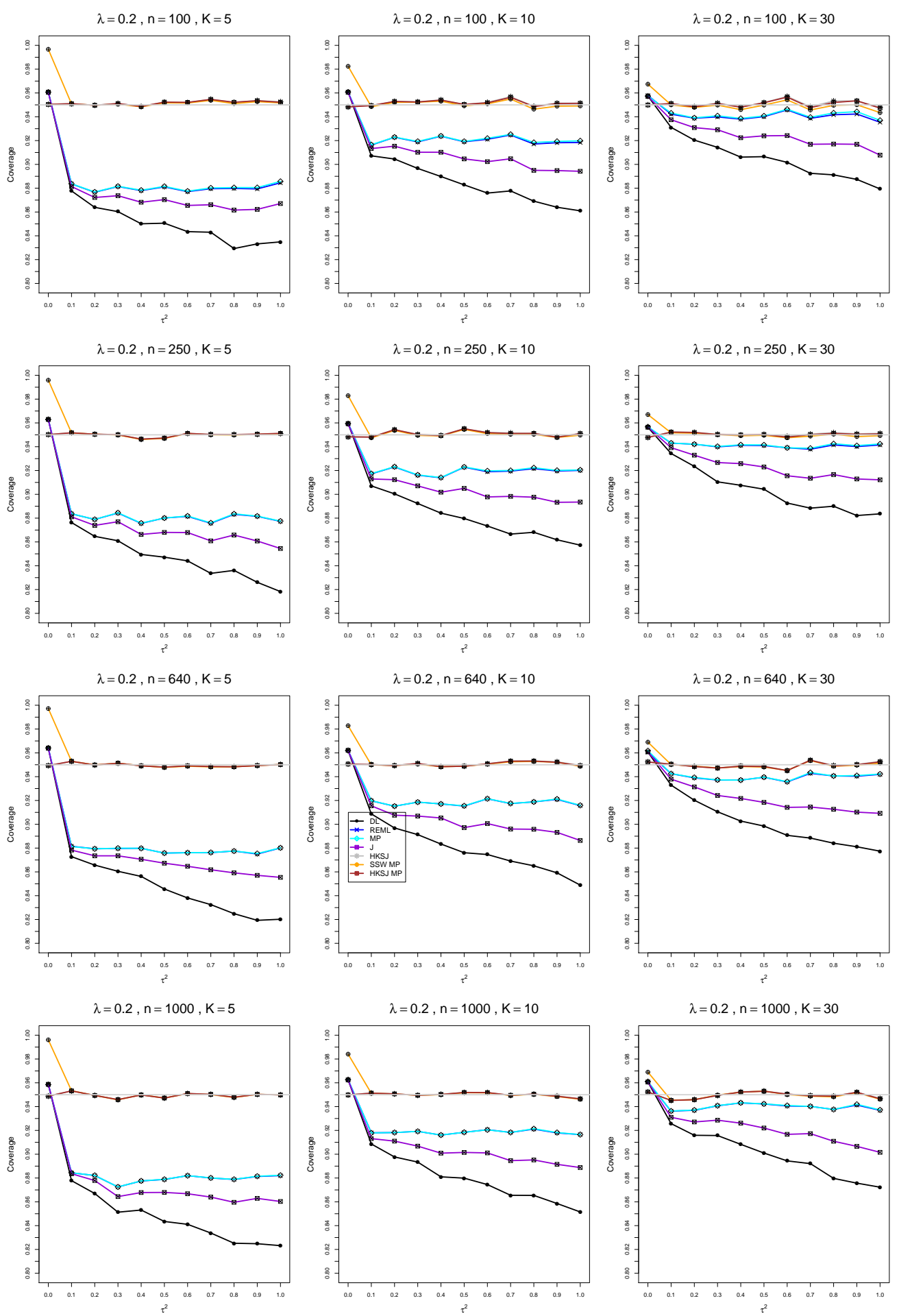

Figure D2.2.2: Coverage of $95 \%$ confidence intervals for $\lambda$ when $\mu_{C}=4, \lambda=0.2, n=$ 100, 250, 640, 1000, and $K=5,10,30$ 

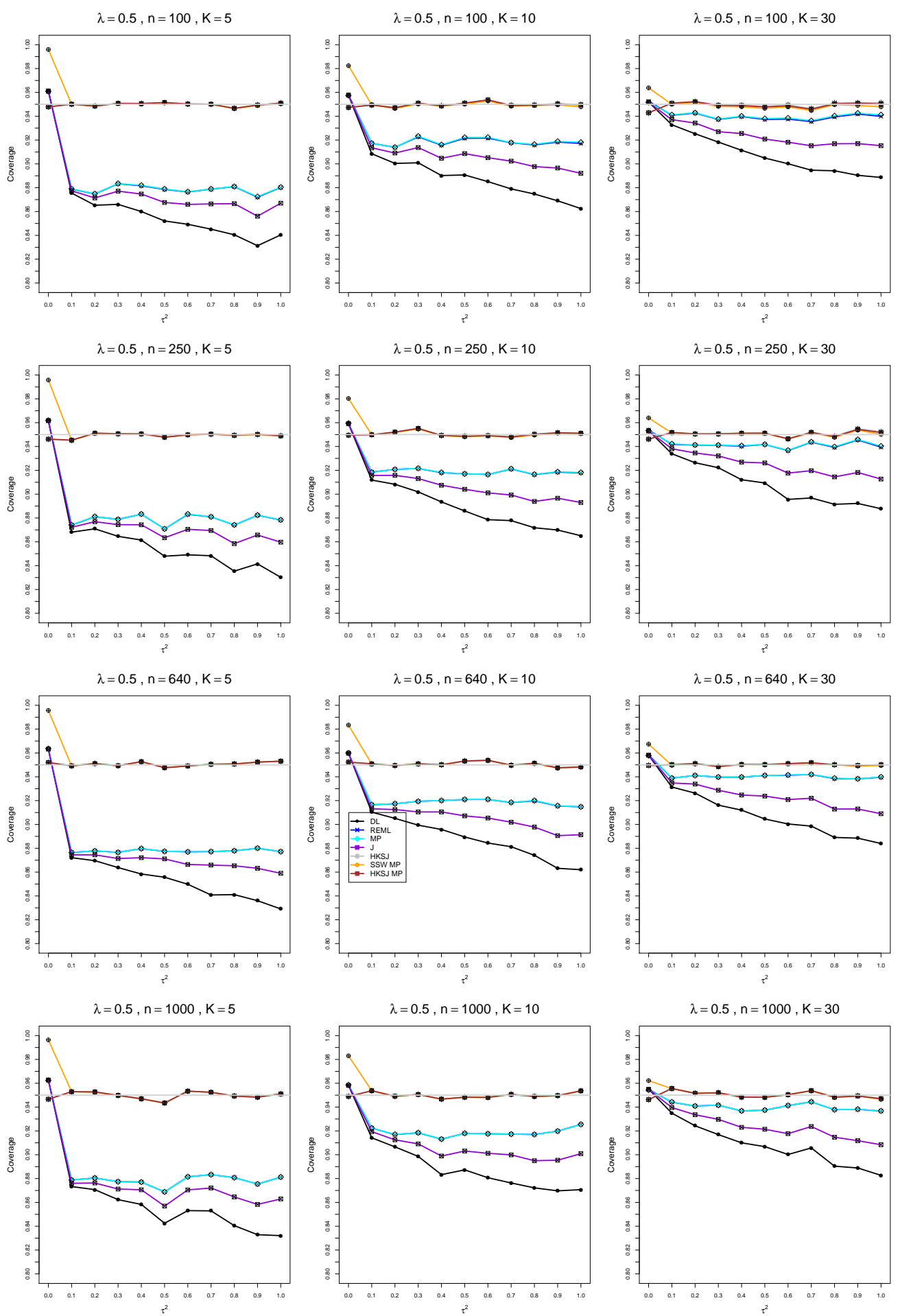

Figure D2.2.3: Coverage of $95 \%$ confidence intervals for $\lambda$ when $\mu_{C}=4, \lambda=0.5, n=$ 100, 250, 640, 1000, and $K=5,10,30$ 

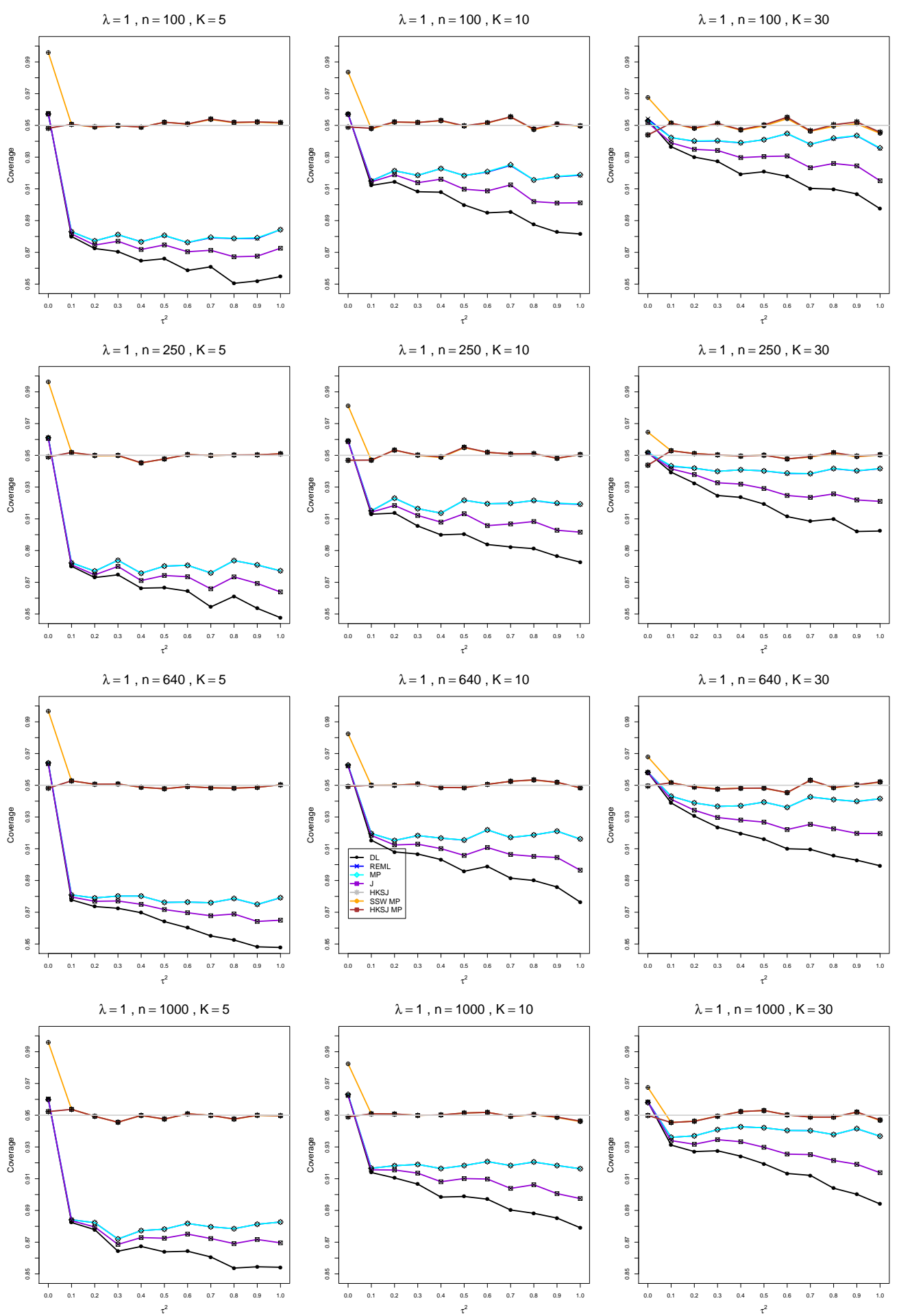

Figure D2.2.4: Coverage of $95 \%$ confidence intervals for $\lambda$ when $\mu_{C}=4, \lambda=1, n=$ 100, 250, 640, 1000, and $K=5,10,30$ 

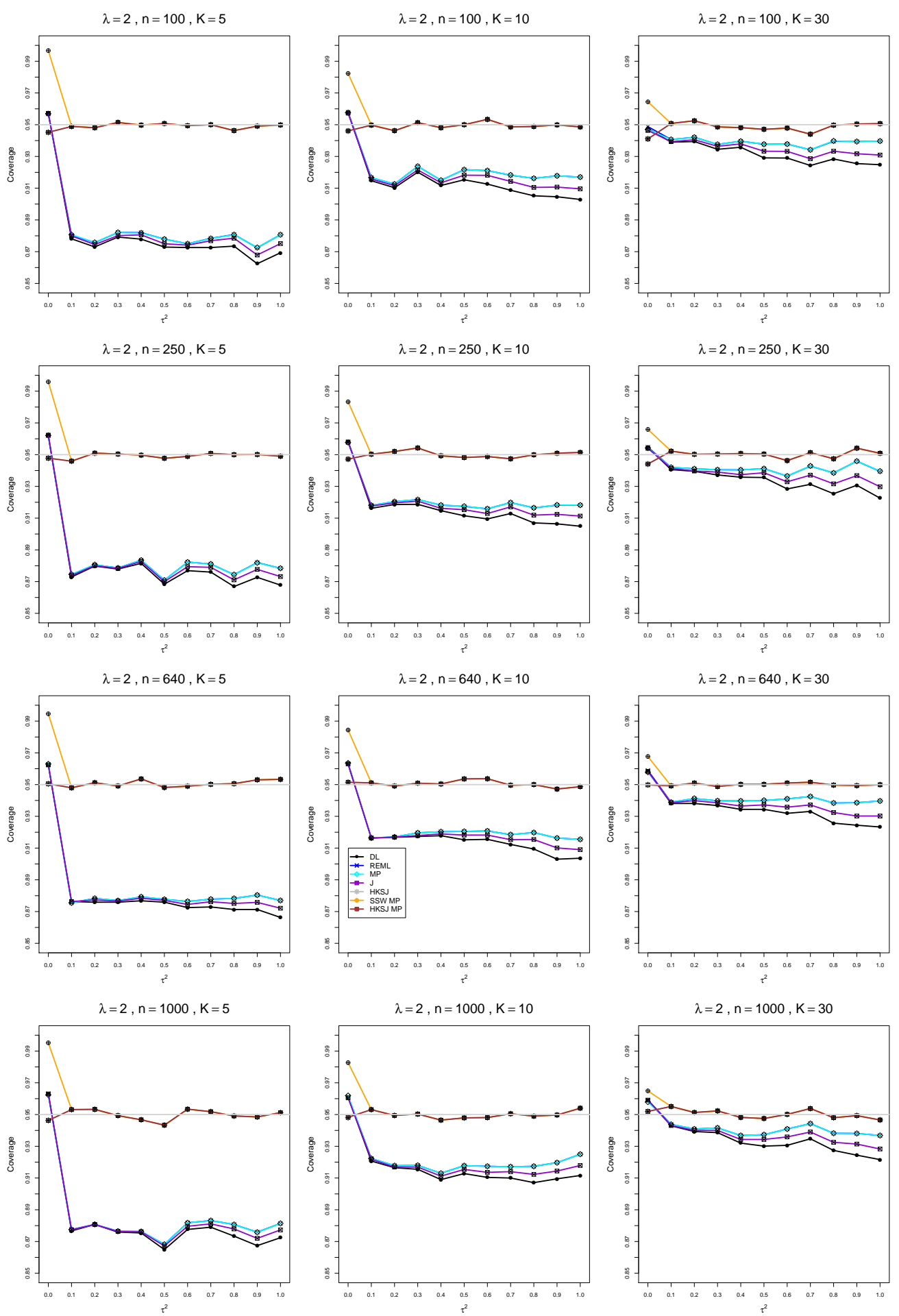

Figure D2.2.5: Coverage of $95 \%$ confidence intervals for $\lambda$ when $\mu_{C}=4, \lambda=2, n=$ 100, 250, 640, 1000, and $K=5,10,30$ 
D3. Normal model, bias-corrected $\lambda, \mu_{C}=4, n$ $=100,250,640,1000, K=5,10,30$

\section{D3.1 Bias of point estimators of $\lambda$ when $\mu_{C}=4$}

Each figure corresponds to a value of $\lambda(=0,0.2,0.5,1,2)$, a set of values of $n(=$ $100,250,640,1000)$, and a set of values of $K(=5,10,30)$.

Each panel corresponds to a value of $n$ and a value of $K$ and has $\tau^{2}=0.0(0.1) 1.0$ on the horizontal axis.

The point estimators of $\lambda$ are

- DL (DerSimonian-Laird)

- REML (restricted maximum likelihood)

- MP (Mandel-Paule)

- J (Jackson)

- SSW (sample-size weighted) 

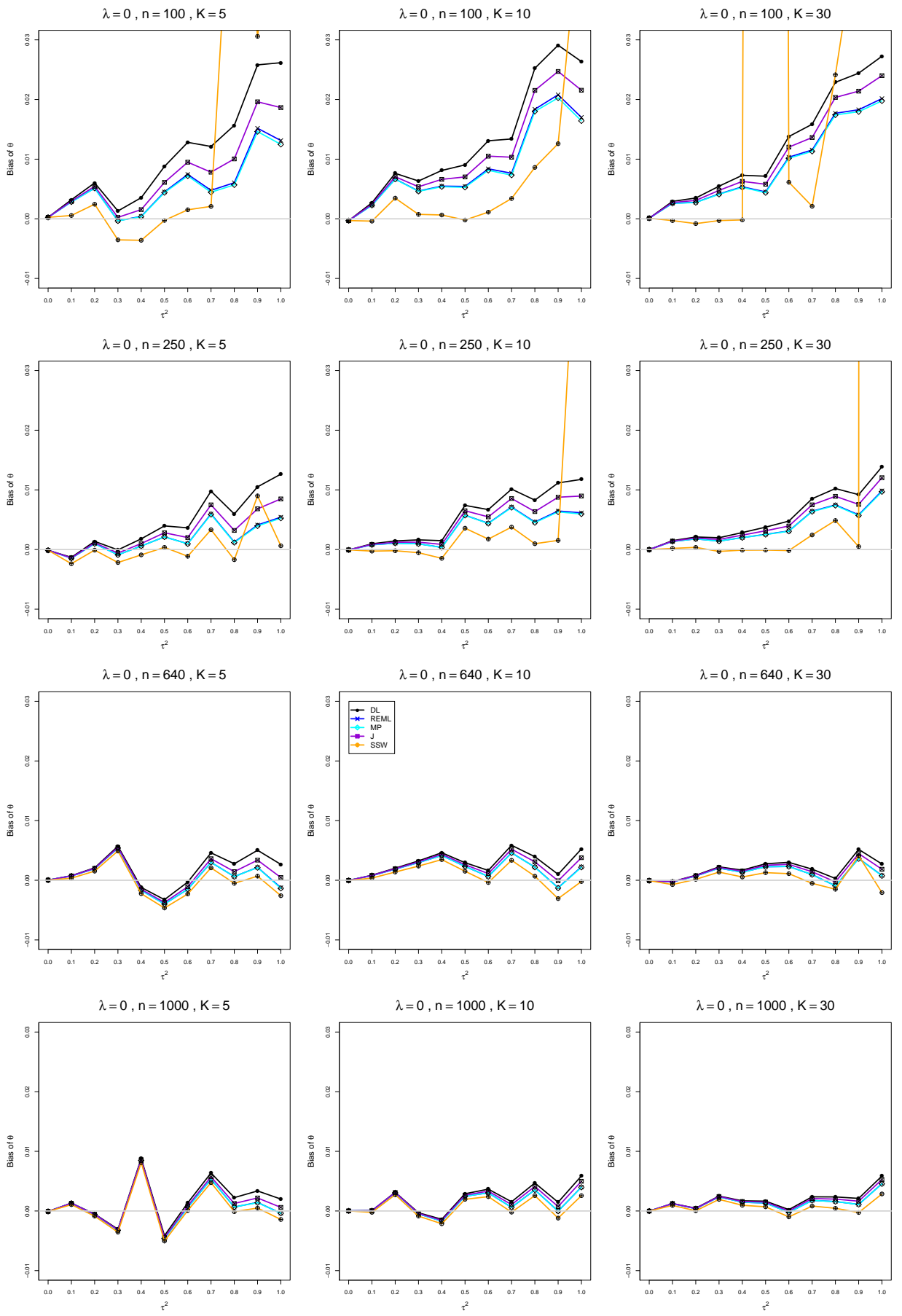

Figure D3.1.1: Bias of estimators of $\log (\operatorname{RoM}) \lambda$ when $\mu_{C}=4, \lambda=0, n=$ $100,250,640,1000$, and $K=5,10,30$ 

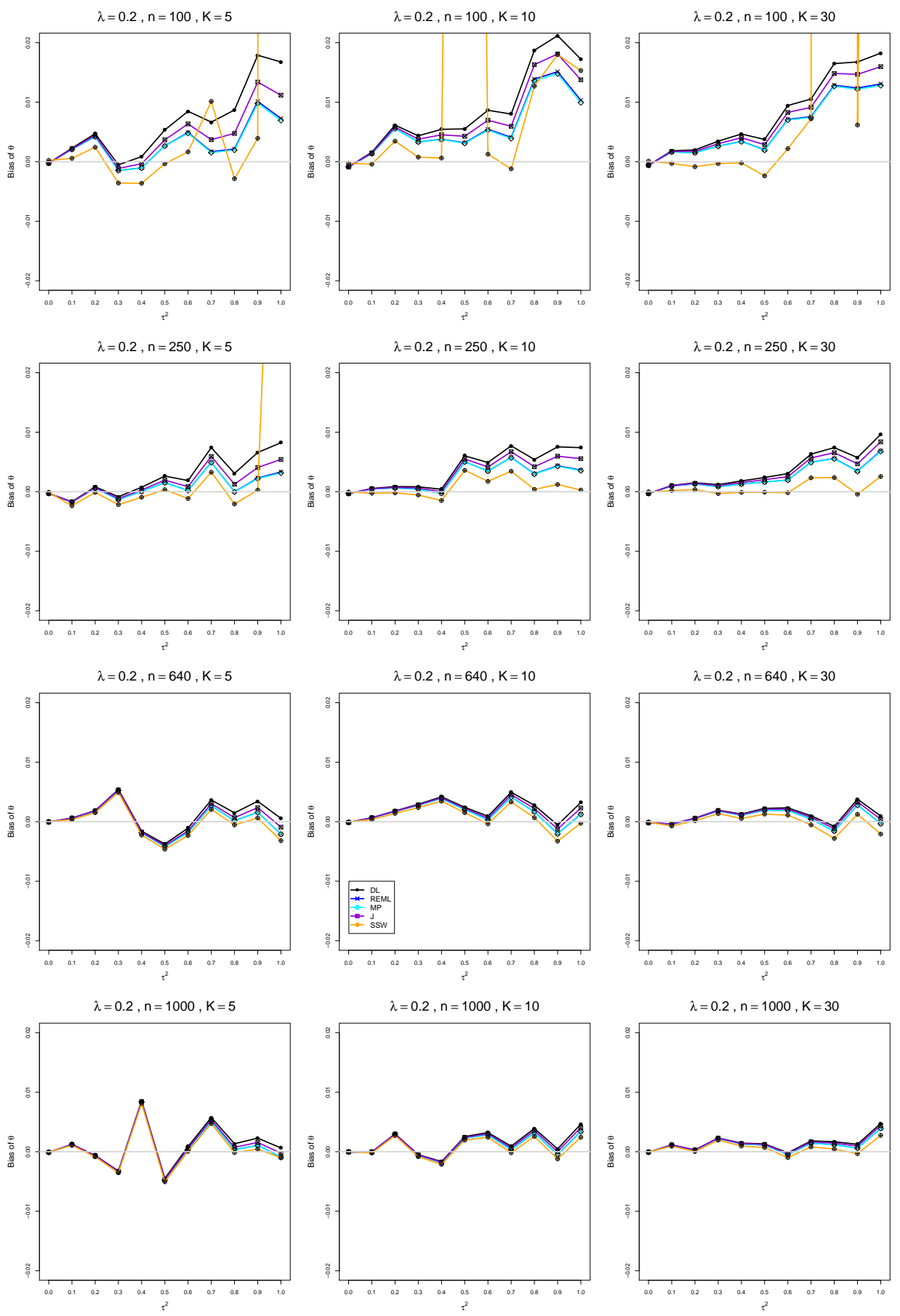

Figure D3.1.2: Bias of estimators of $\log (\mathrm{RoM}) \lambda$ when $\mu_{C}=4, \lambda=0.2, n=$ $100,250,640,1000$, and $K=5,10,30$ 

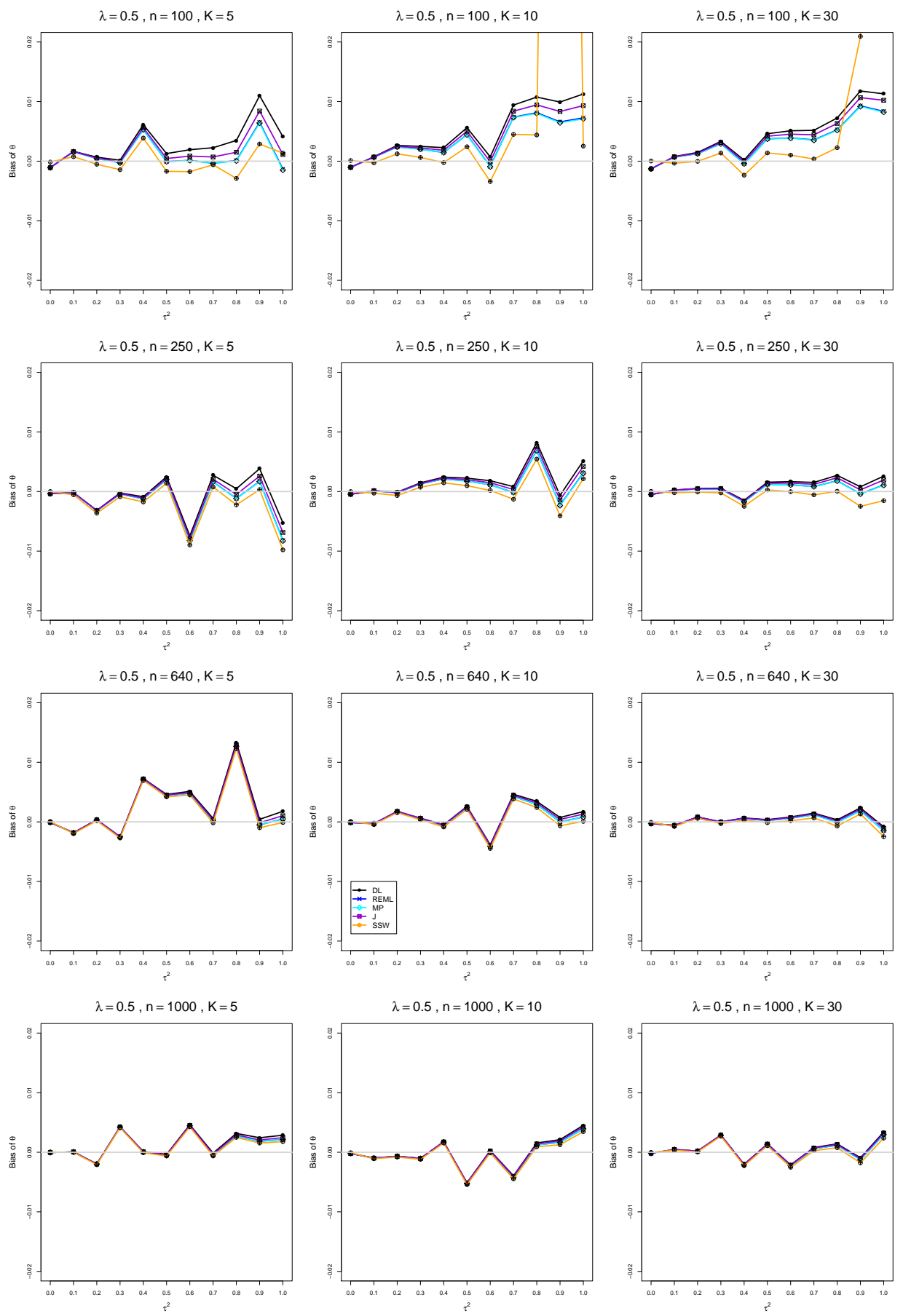

Figure D3.1.3: Bias of estimators of $\log (\mathrm{RoM}) \lambda$ when $\mu_{C}=4, \lambda=0.5, n=$ $100,250,640,1000$, and $K=5,10,30$ 

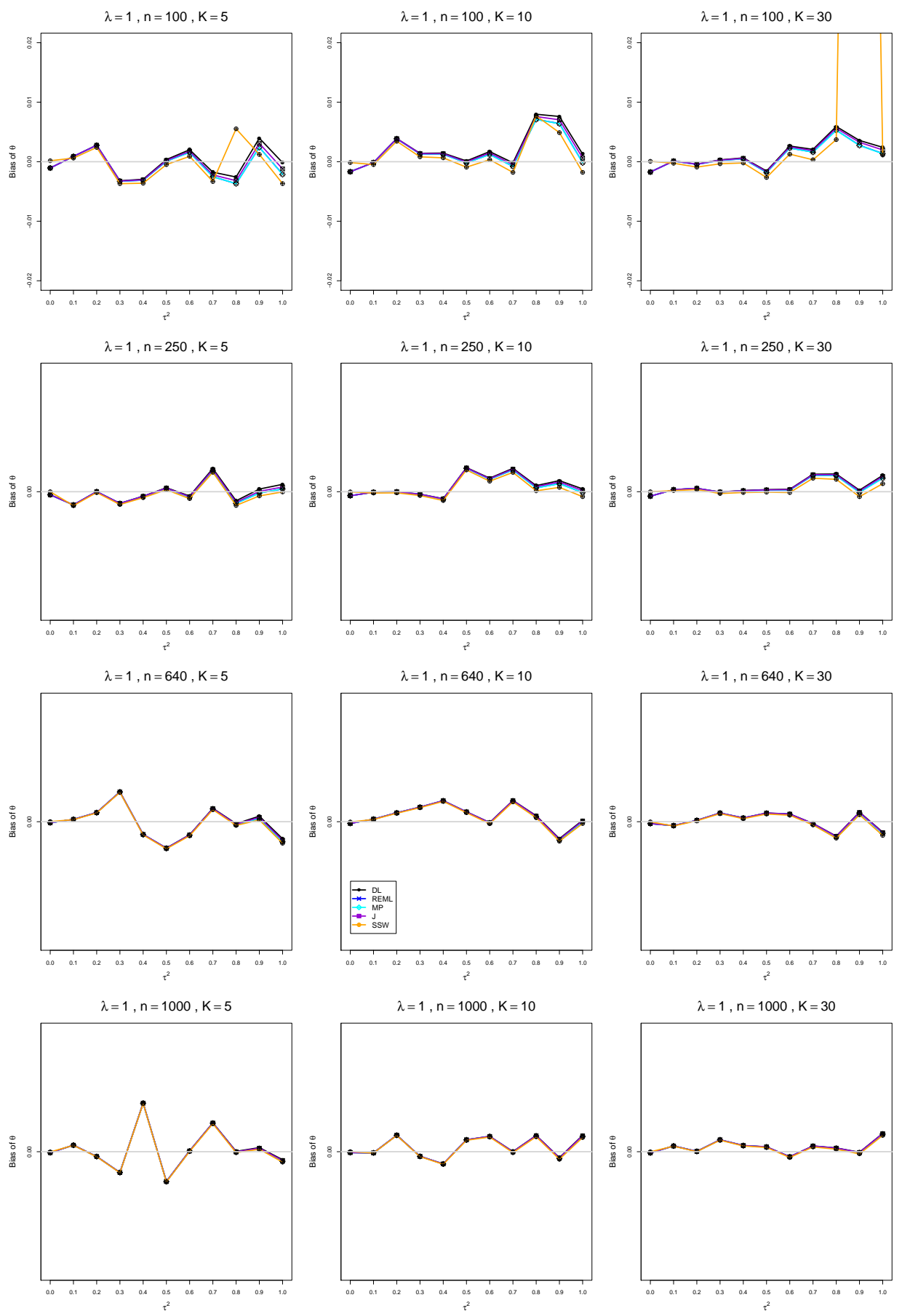

Figure D3.1.4: Bias of estimators of $\log (\operatorname{RoM}) \lambda$ when $\mu_{C}=4, \lambda=1, n=$ 100, 250, 640, 1000, and $K=5,10,30$ 

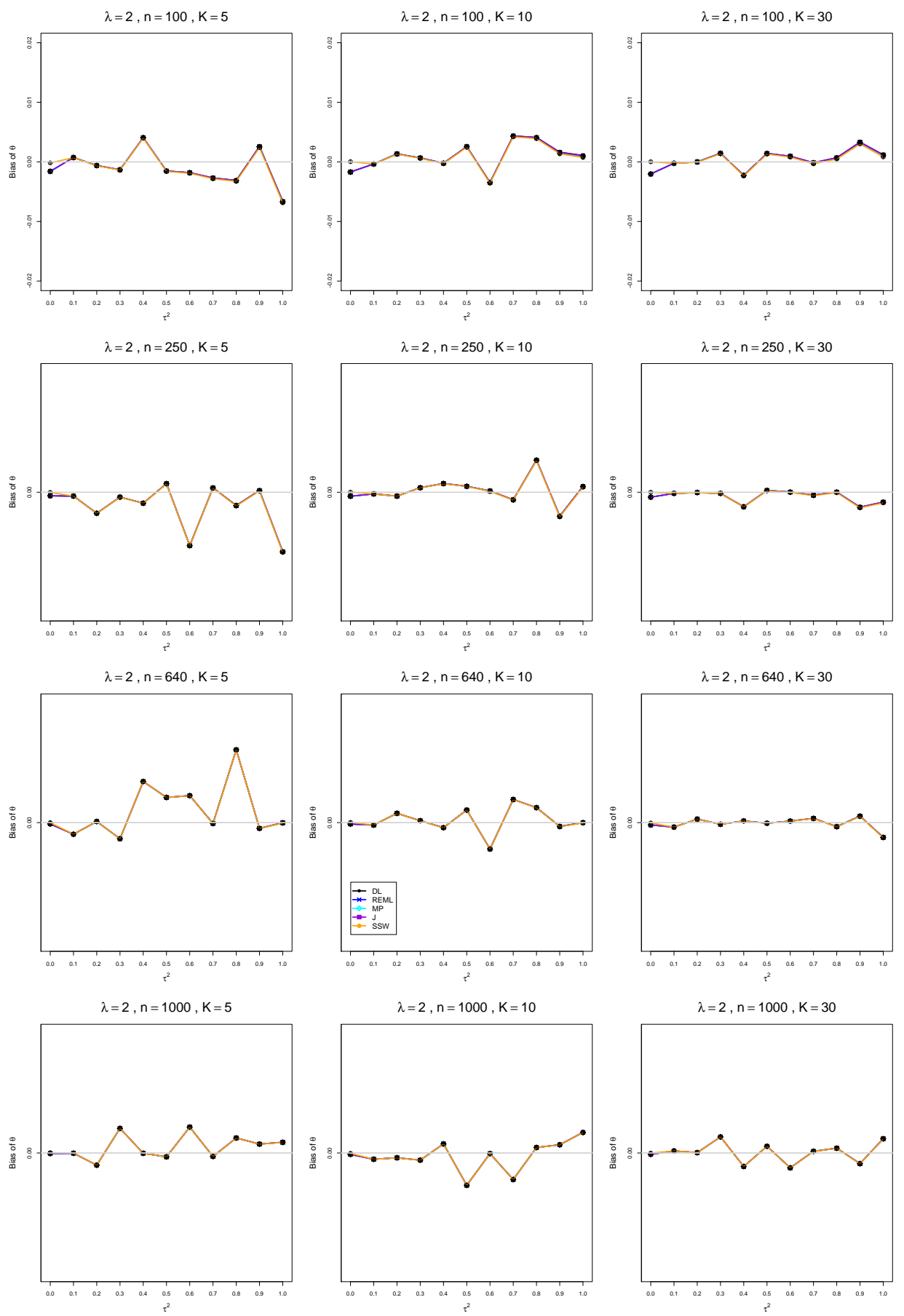

Figure D3.1.5: Bias of estimators of $\log (\operatorname{RoM}) \lambda$ when $\mu_{C}=4, \lambda=2, n=$ 100, 250, 640, 1000, and $K=5,10,30$ 


\section{D3.2 Coverage of interval estimators of $\lambda$}

Each figure corresponds to a value of $\lambda(=0,0.2,0.5,1,2)$, a set of values of $n(=$ $100,250,640,1000)$, and a set of values of $K(=5,10,30)$.

Each panel corresponds to a value of $n$ and a value of $K$ and has $\tau^{2}=0.0(0.1) 1.0$ on the horizontal axis.

The interval estimators of $\lambda$ are the companions to the inverse-variance-weighted point estimators

- DL (DerSimonian-Laird)

- REML (restricted maximum likelihood)

- MP (Mandel-Paule)

- J (Jackson)

and

- HKSJ (Hartung-Knapp-Sidik-Jonkman)

- HKSJ MP (HKSJ with MP estimator of $\tau^{2}$ )

- SSW (SSW as center and half-width equal to critical value from $t_{K-1}$ times estimated standard deviation of SSW with $\hat{\tau}^{2}=\hat{\tau}_{M P}^{2}$ 

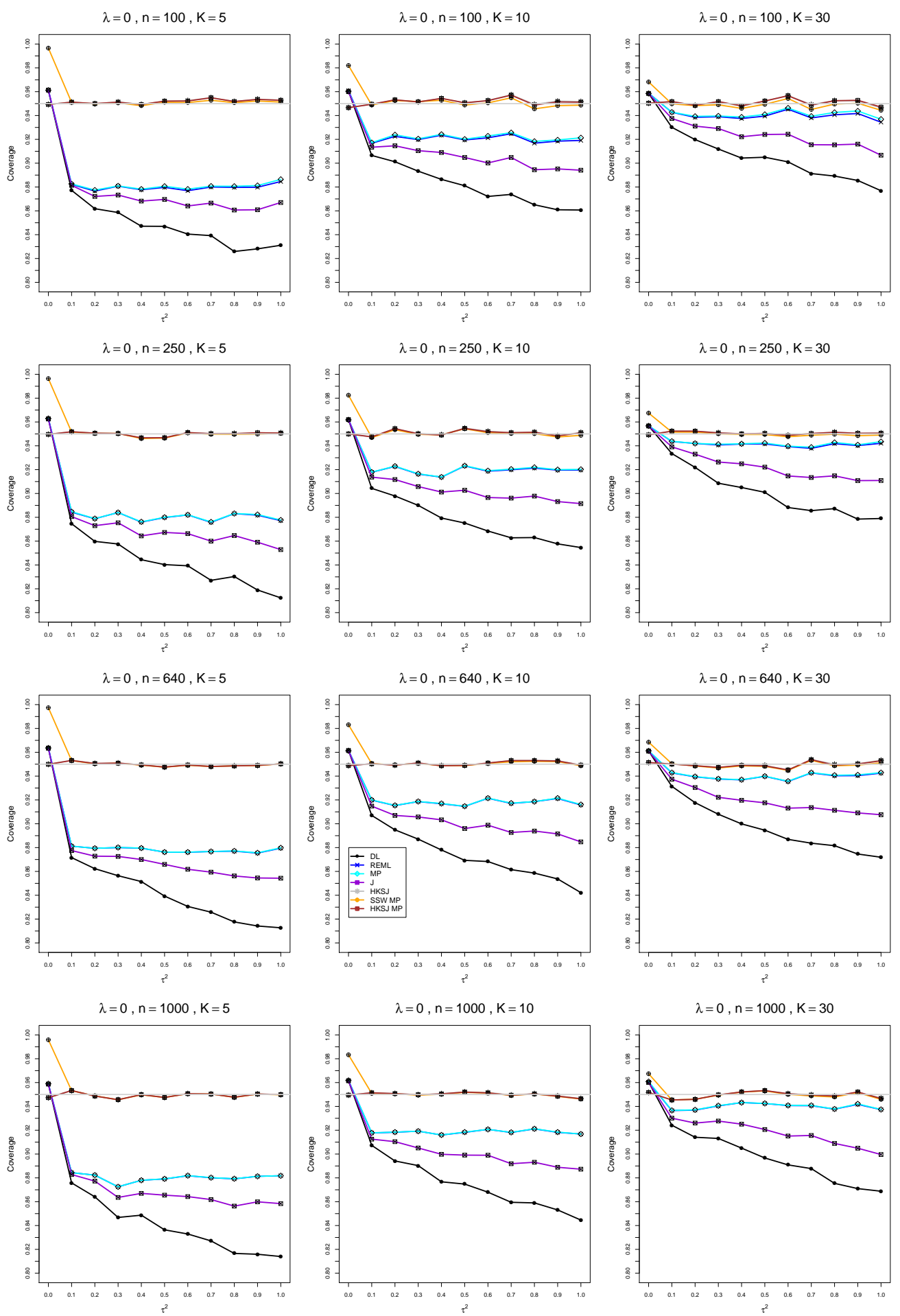

Figure D3.2.1: Coverage of $95 \%$ confidence intervals for $\lambda$ when $\mu_{C}=4, \lambda=0, n=$ 100, 250, 640, 1000, and $K=5,10,30$ 

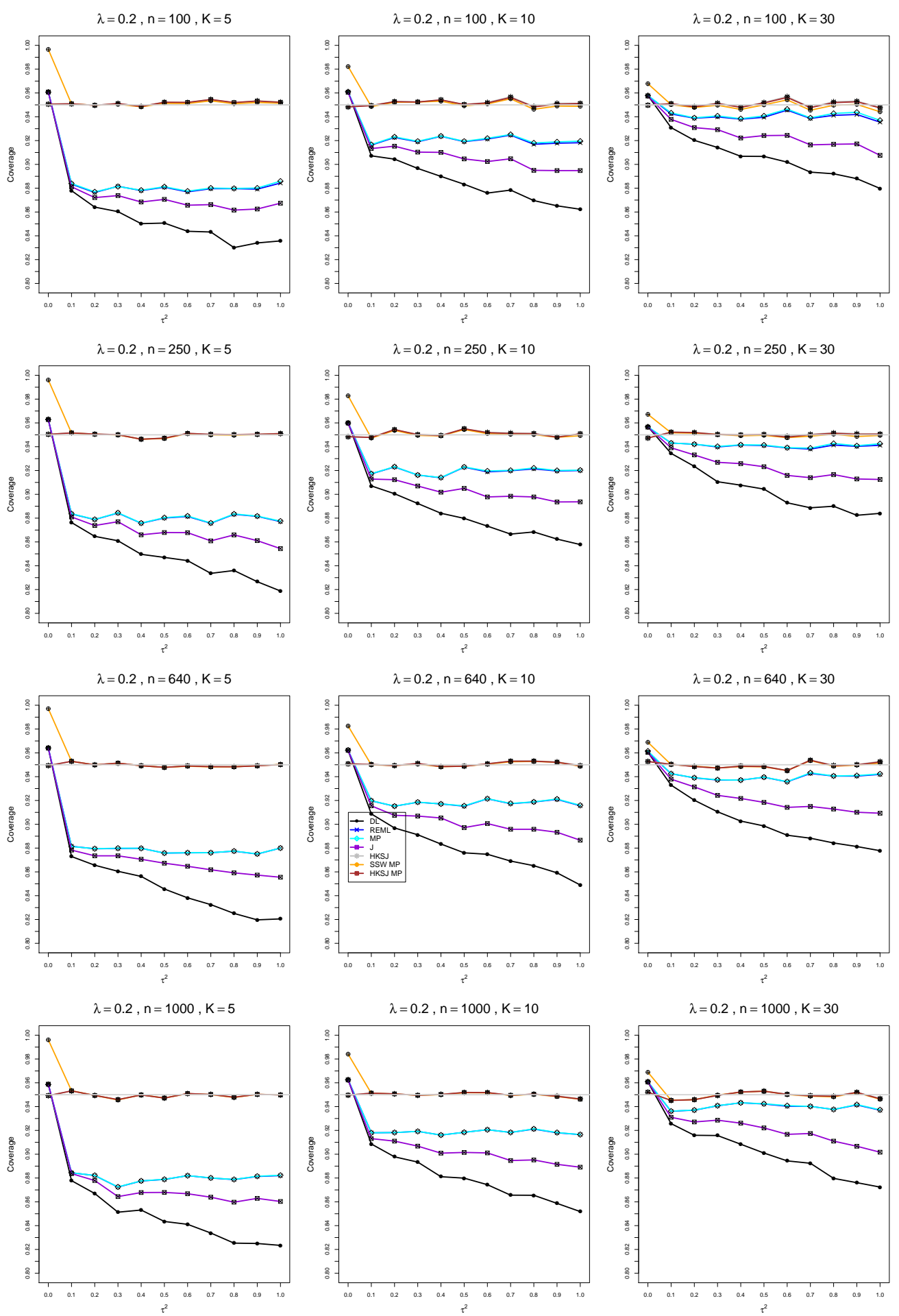

Figure D3.2.2: Coverage of $95 \%$ confidence intervals for $\lambda$ when $\mu_{C}=4, \lambda=0.2, n=$ 100, 250, 640, 1000, and $K=5,10,30$ 

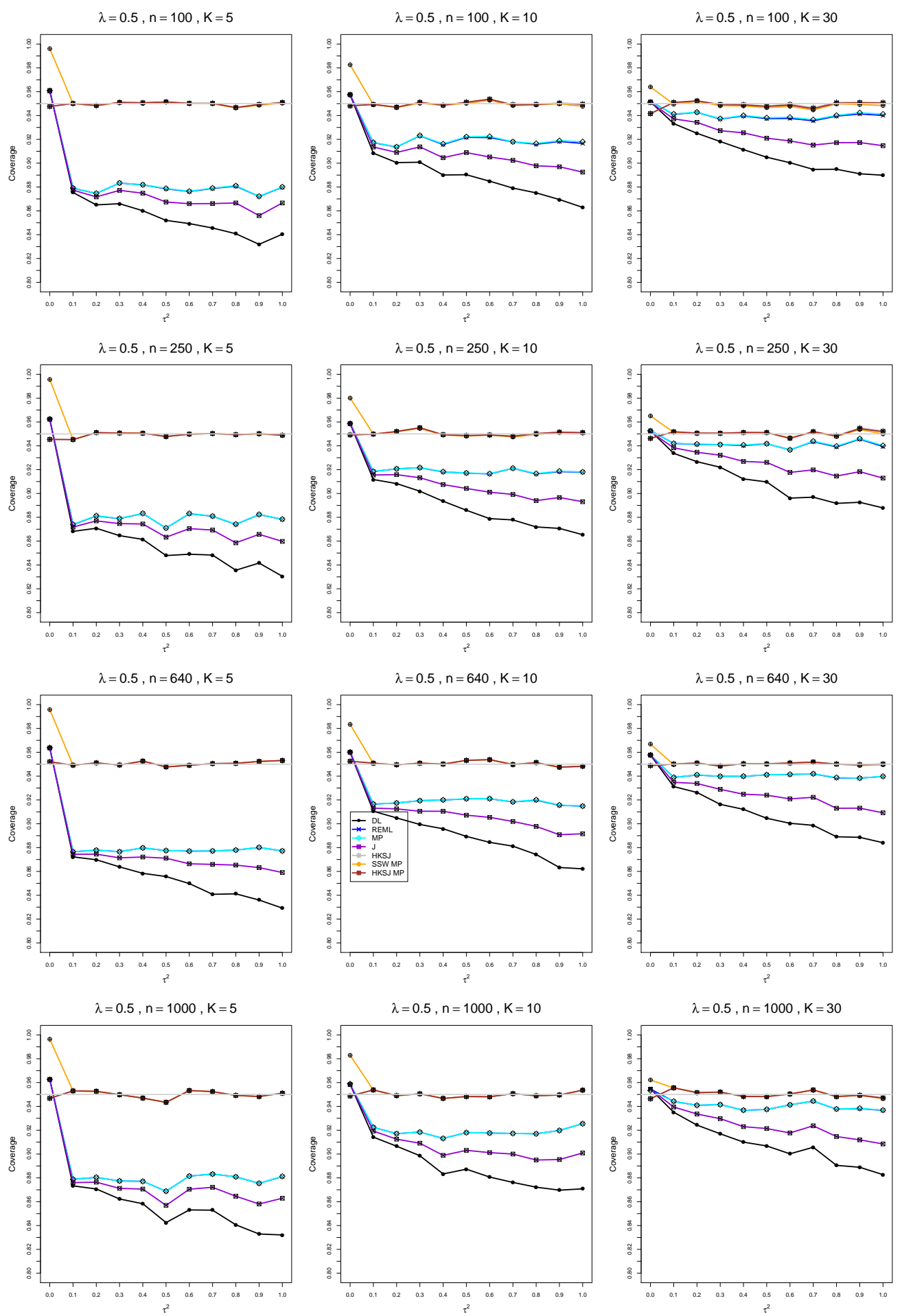

Figure D3.2.3: Coverage of $95 \%$ confidence intervals for $\lambda$ when $\mu_{C}=4, \lambda=0.5, n=$ 100, 250, 640, 1000, and $K=5,10,30$ 

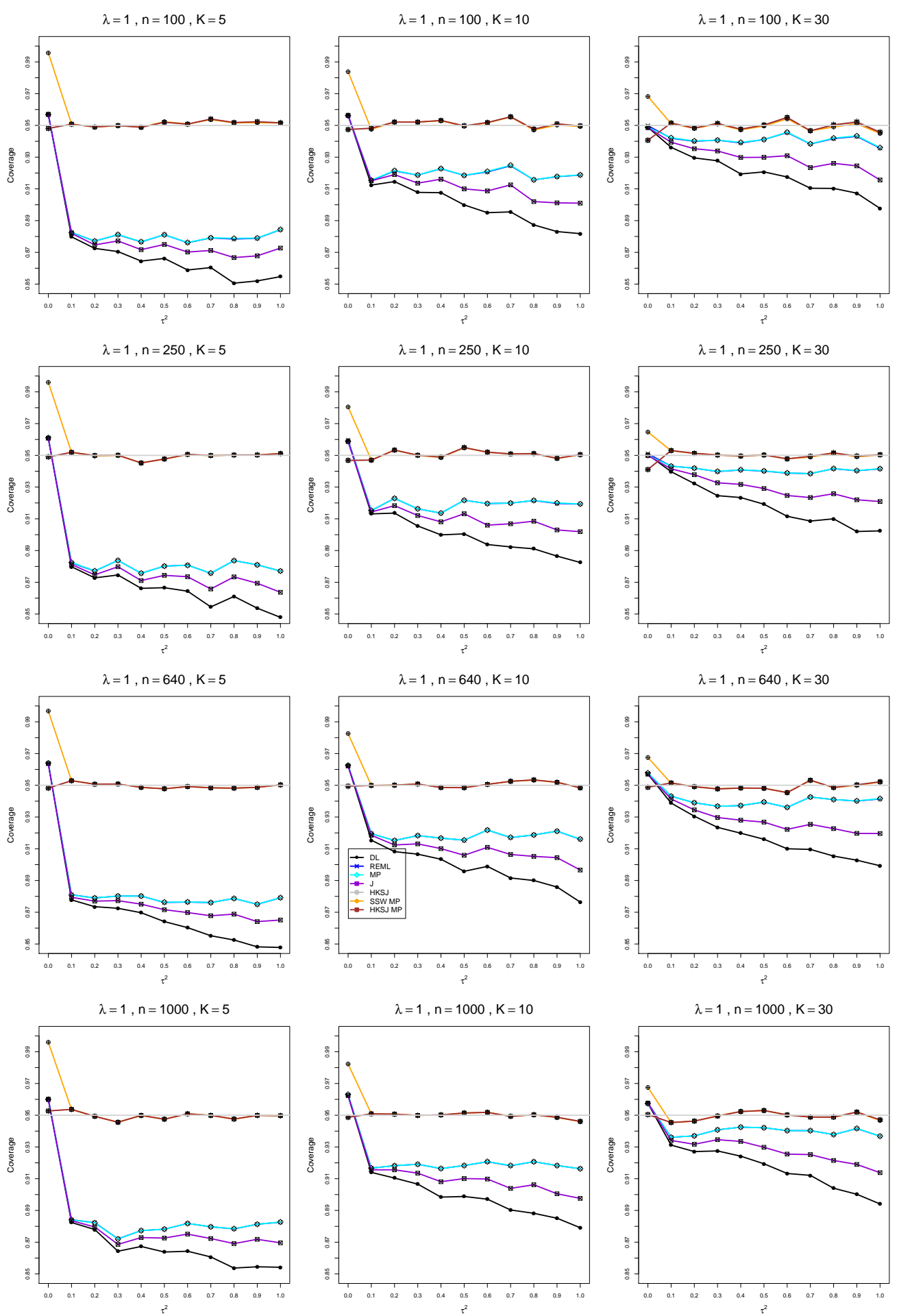

Figure D3.2.4: Coverage of $95 \%$ confidence intervals for $\lambda$ when $\mu_{C}=4, \lambda=1, n=$ 100, 250, 640, 1000, and $K=5,10,30$ 

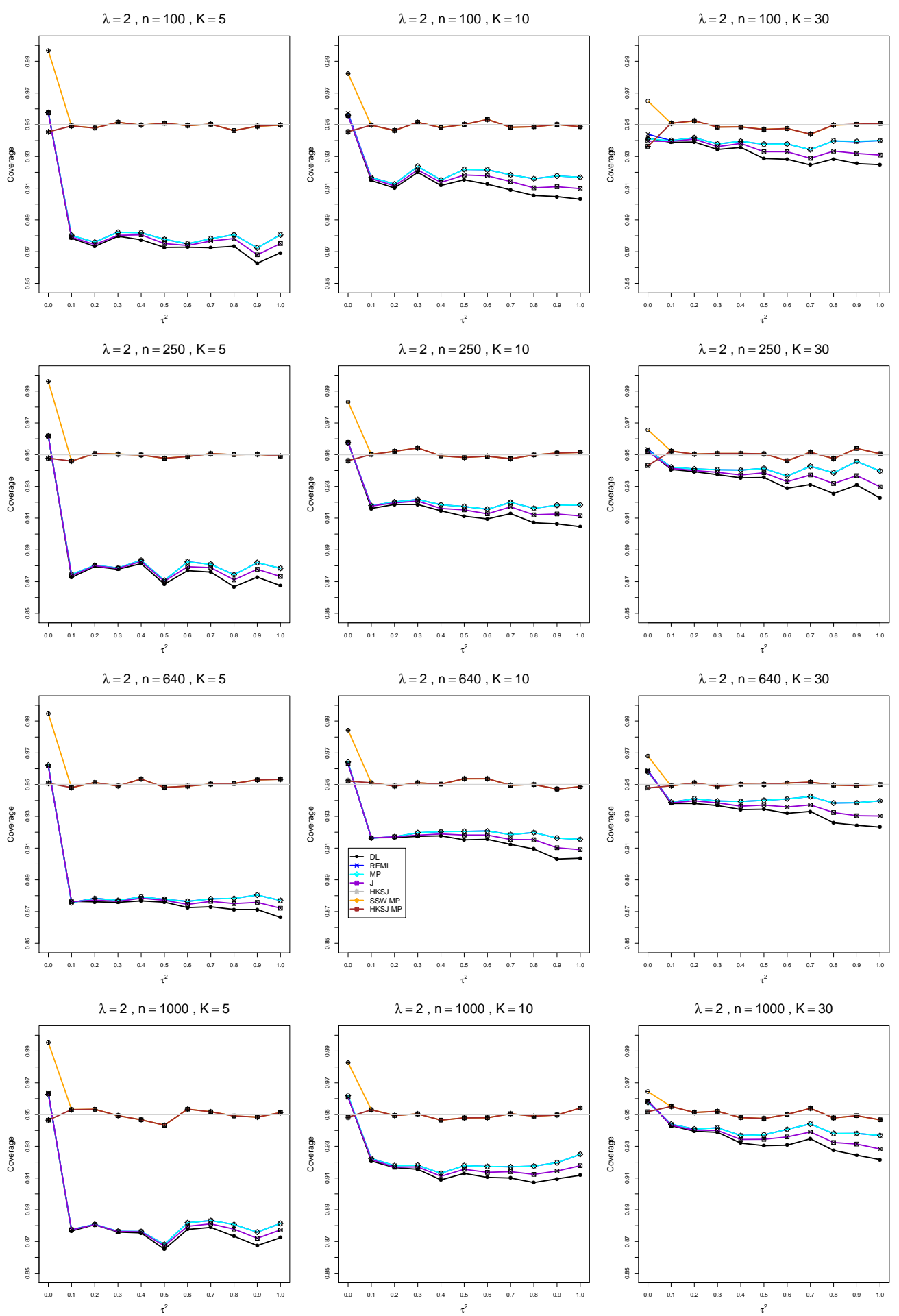

Figure D3.2.5: Coverage of $95 \%$ confidence intervals for $\lambda$ when $\mu_{C}=4, \lambda=2, n=$ 100, 250, 640, 1000, and $K=5,10,30$ 
D4. Normal model, $\mu_{C}=1, n=100,250,640$, $1000, K=50,100,125$

\section{D4.1 Bias of point estimators of $\lambda$ when $\mu_{C}=1$}

Each figure corresponds to a value of $\lambda(=0,0.2,0.5,1,2)$, a set of values of $n(=$ $100,250,640,1000)$, and a set of values of $K(=50,100,125)$.

Each panel corresponds to a value of $n$ and a value of $K$ and has $\tau^{2}=0.0(0.1) 1.0$ on the horizontal axis.

The point estimators of $\lambda$ are

- DL (DerSimonian-Laird)

- REML (restricted maximum likelihood)

- MP (Mandel-Paule)

- J (Jackson)

- SSW (sample-size weighted) 

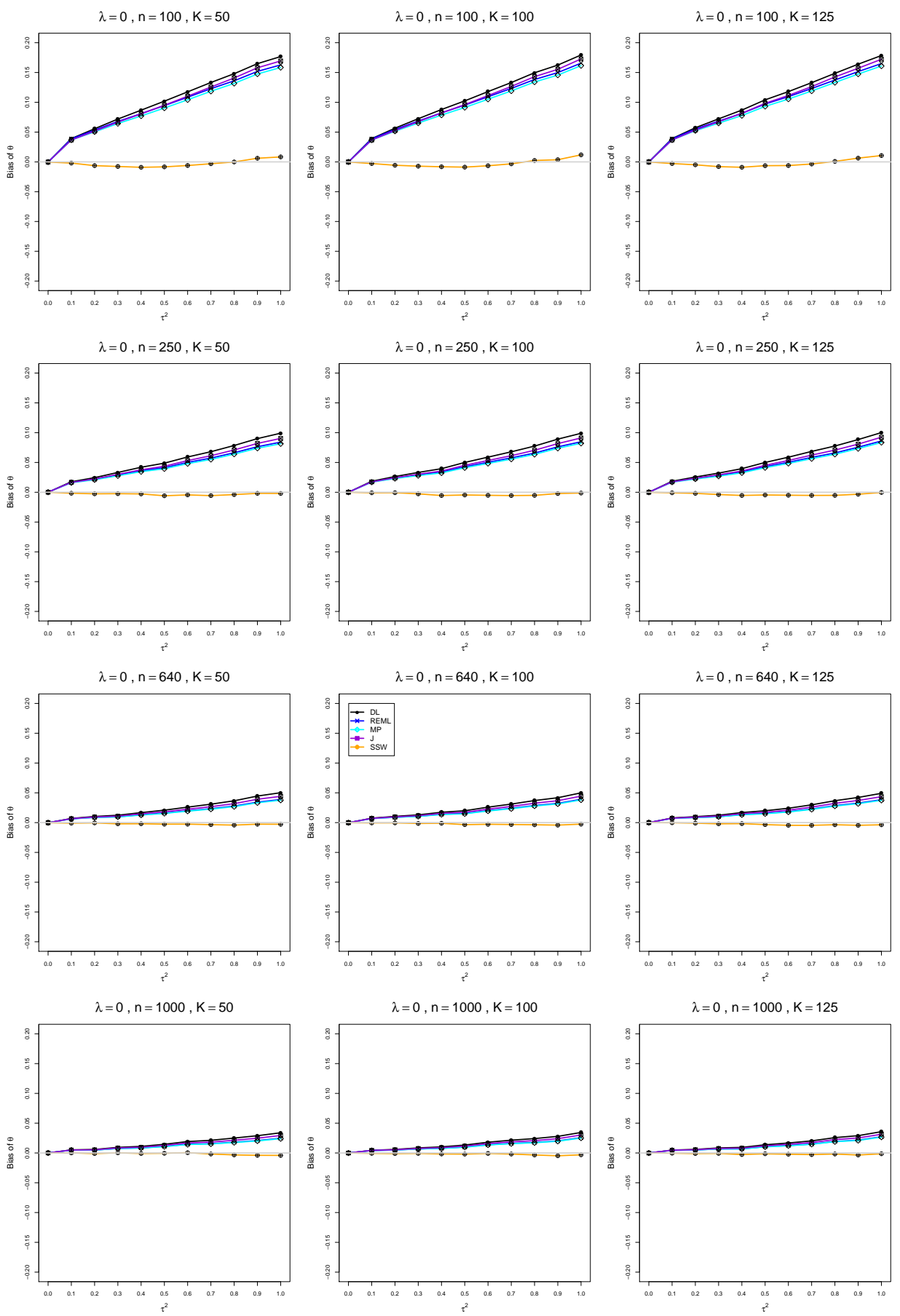

Figure D4.1.1: Bias of estimators of $\log (\operatorname{RoM}) \lambda$ when $\mu_{C}=1, \lambda=0, n=$ $100,250,640,1000$, and $K=50,100,125$ 

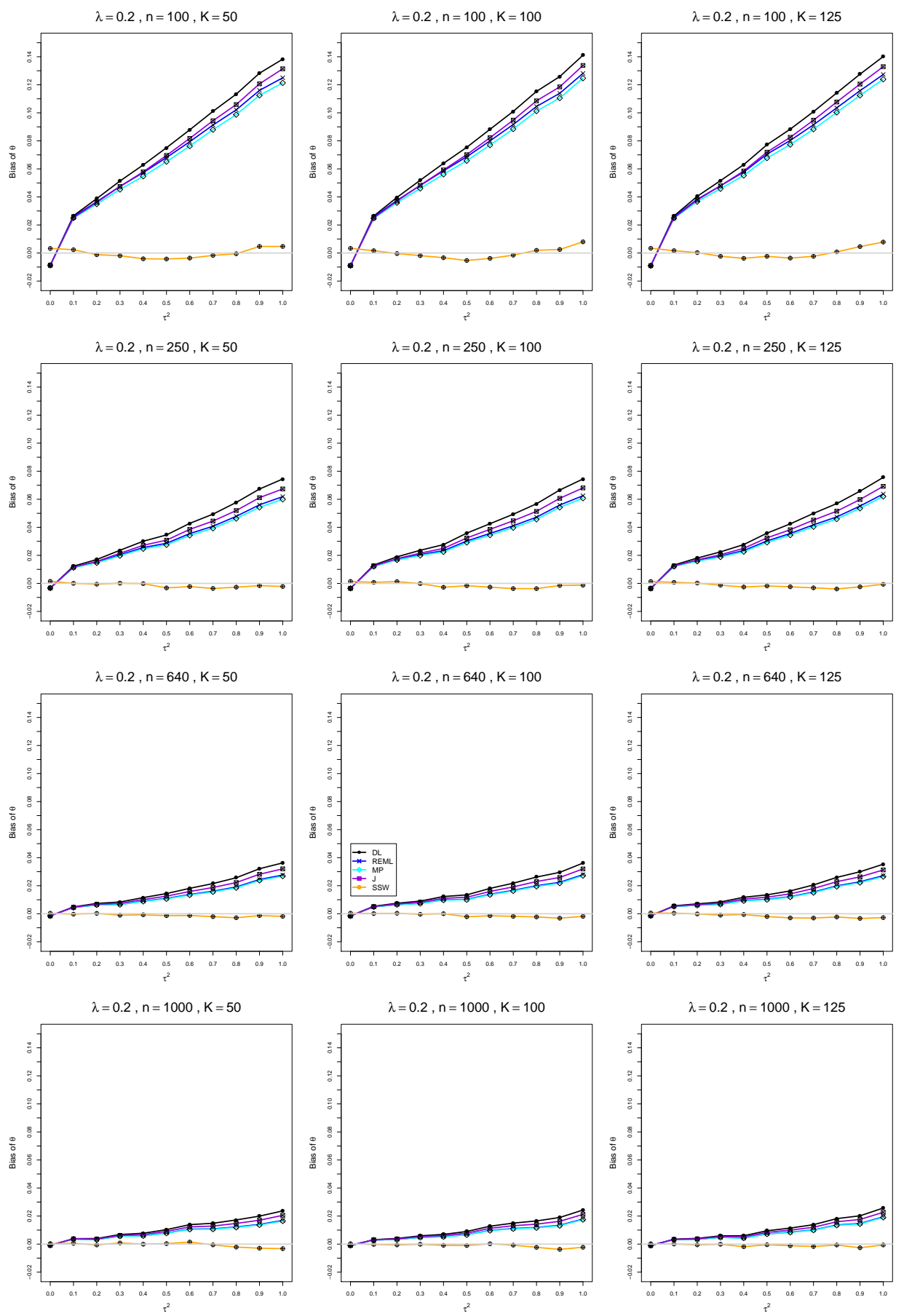

Figure D4.1.2: Bias of estimators of $\log (\mathrm{RoM}) \lambda$ when $\mu_{C}=1, \lambda=0.2, n=$ $100,250,640,1000$, and $K=50,100,125$ 

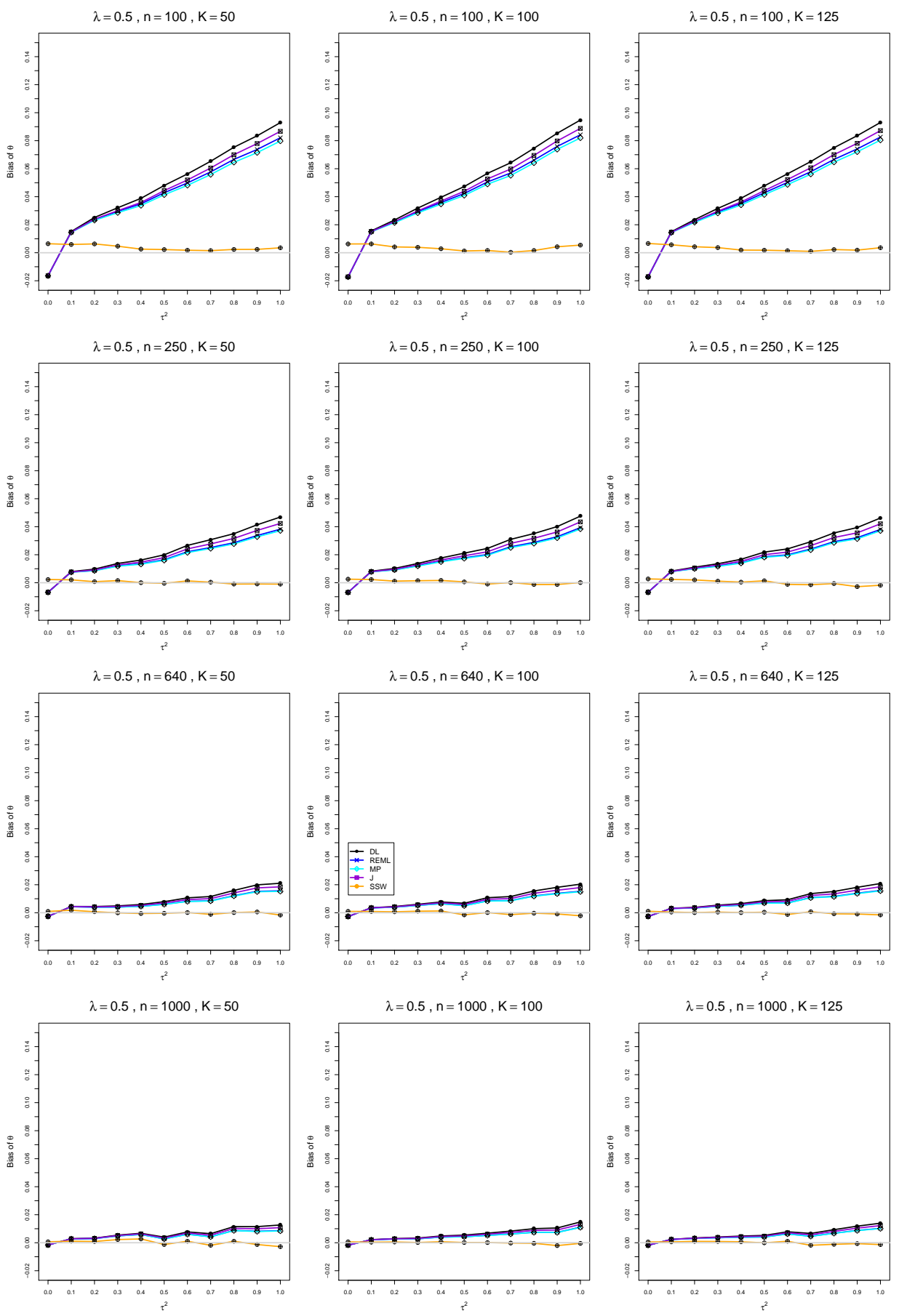

Figure D4.1.3: Bias of estimators of $\log (\mathrm{RoM}) \lambda$ when $\mu_{C}=1, \lambda=0.5, n=$ $100,250,640,1000$, and $K=50,100,125$ 

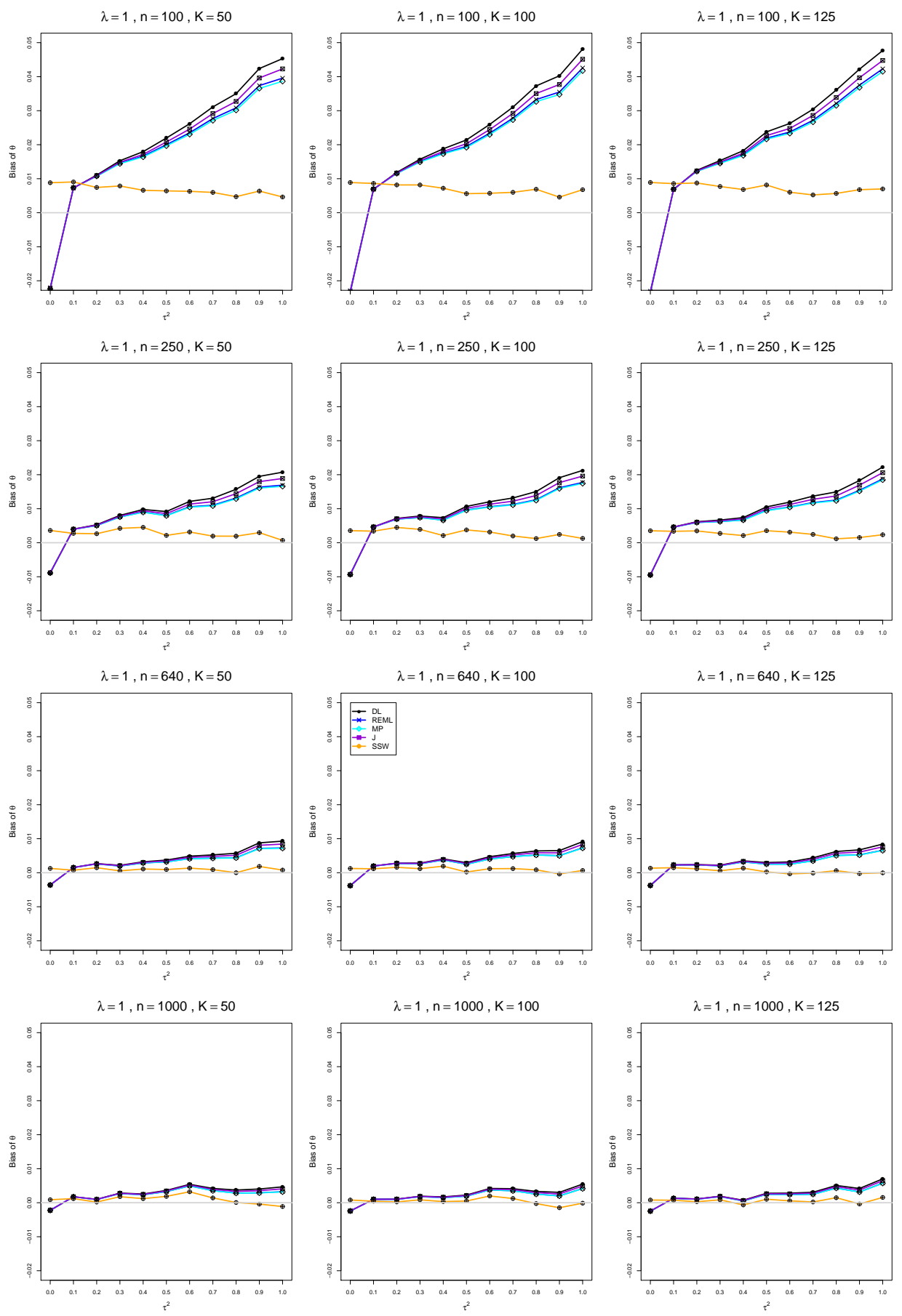

Figure D4.1.4: Bias of estimators of $\log (\operatorname{RoM}) \lambda$ when $\mu_{C}=1, \lambda=1, n=$ 100, 250, 640, 1000, and $K=50,100,125$ 

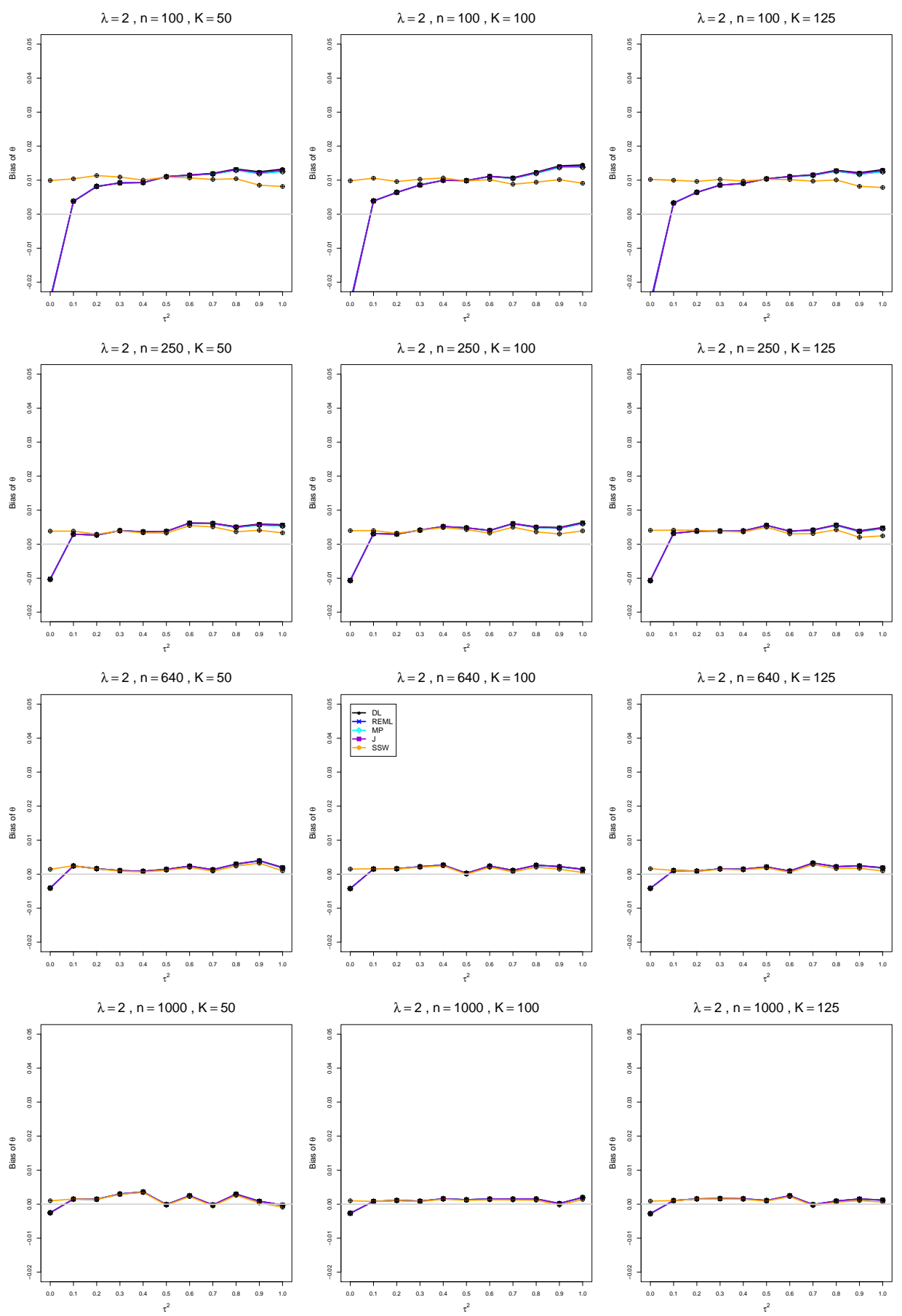

Figure D4.1.5: Bias of estimators of $\log (\operatorname{RoM}) \lambda$ when $\mu_{C}=1, \lambda=2, n=$ 100, 250, 640, 1000, and $K=50,100,125$ 


\section{D4.2 Coverage of interval estimators of $\lambda$}

Each figure corresponds to a value of $\lambda(=0,0.2,0.5,1,2)$, a set of values of $n(=$ $100,250,640,1000)$, and a set of values of $K(=50,100,125)$.

Each panel corresponds to a value of $n$ and a value of $K$ and has $\tau^{2}=0.0(0.1) 1.0$ on the horizontal axis.

The interval estimators of $\lambda$ are the companions to the inverse-variance-weighted point estimators

- DL (DerSimonian-Laird)

- REML (restricted maximum likelihood)

- MP (Mandel-Paule)

- J (Jackson)

and

- HKSJ (Hartung-Knapp-Sidik-Jonkman)

- HKSJ MP (HKSJ with MP estimator of $\tau^{2}$ )

- SSW (SSW as center and half-width equal to critical value from $t_{K-1}$ times estimated standard deviation of SSW with $\hat{\tau}^{2}=\hat{\tau}_{M P}^{2}$ 

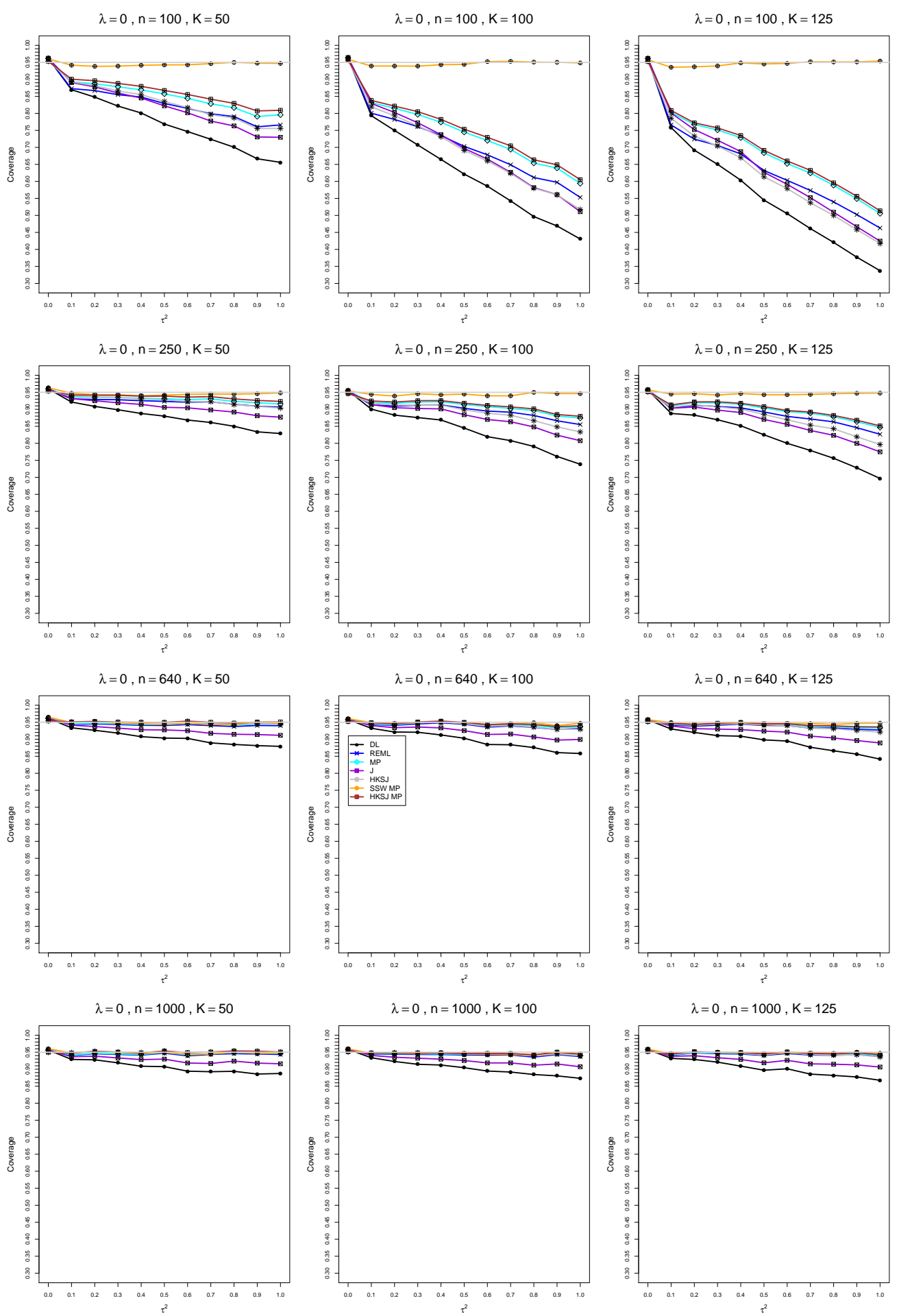

Figure D4.2.1: Coverage of $95 \%$ confidence intervals for $\lambda$ when $\mu_{C}=1, \lambda=0, n=$ 100, 250, 640, 1000, and $K=50,100,125$ 

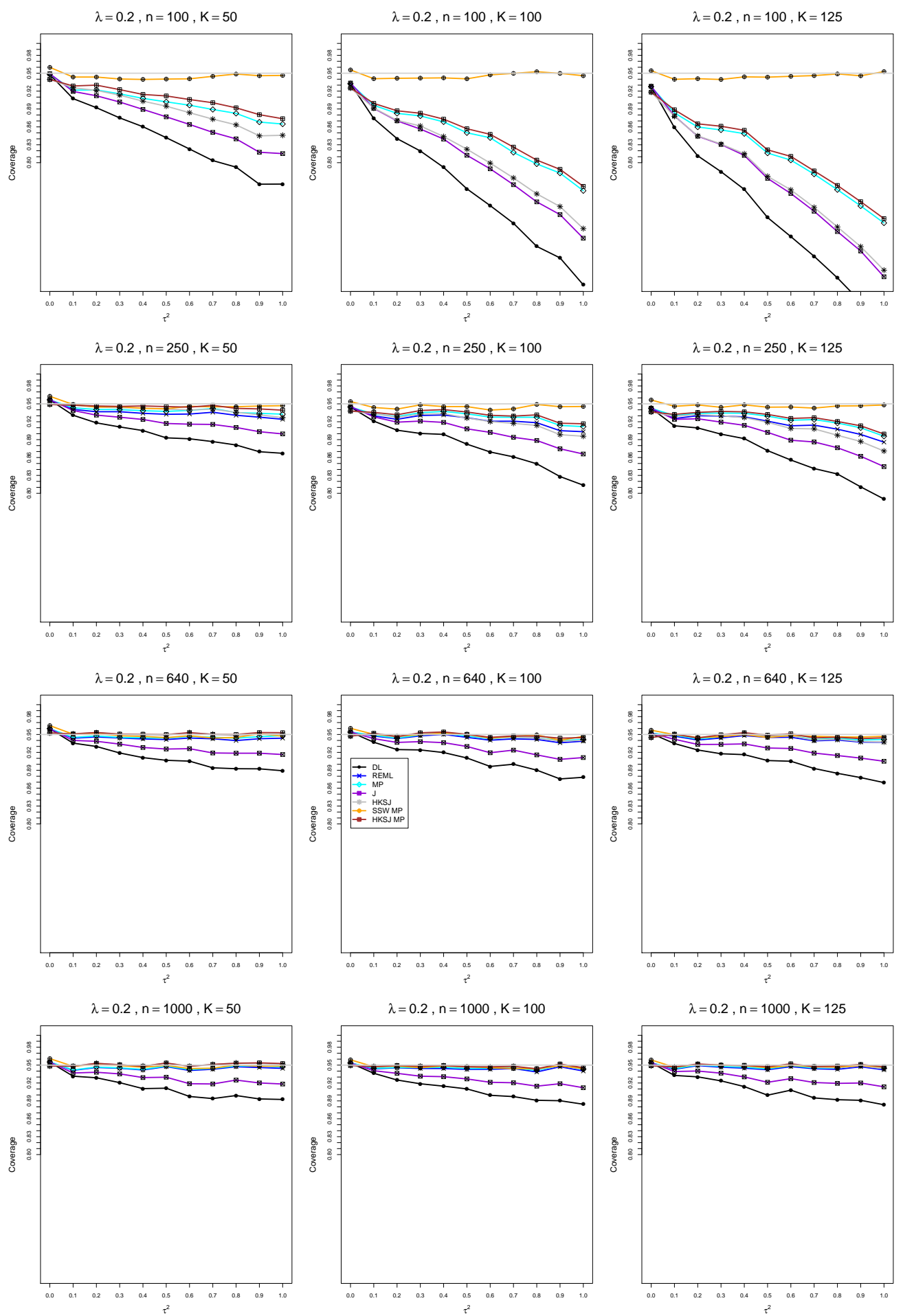

Figure D4.2.2: Coverage of $95 \%$ confidence intervals for $\lambda$ when $\mu_{C}=1, \lambda=0.2, n=$ 100, 250, 640, 1000, and $K=50,100,125$ 

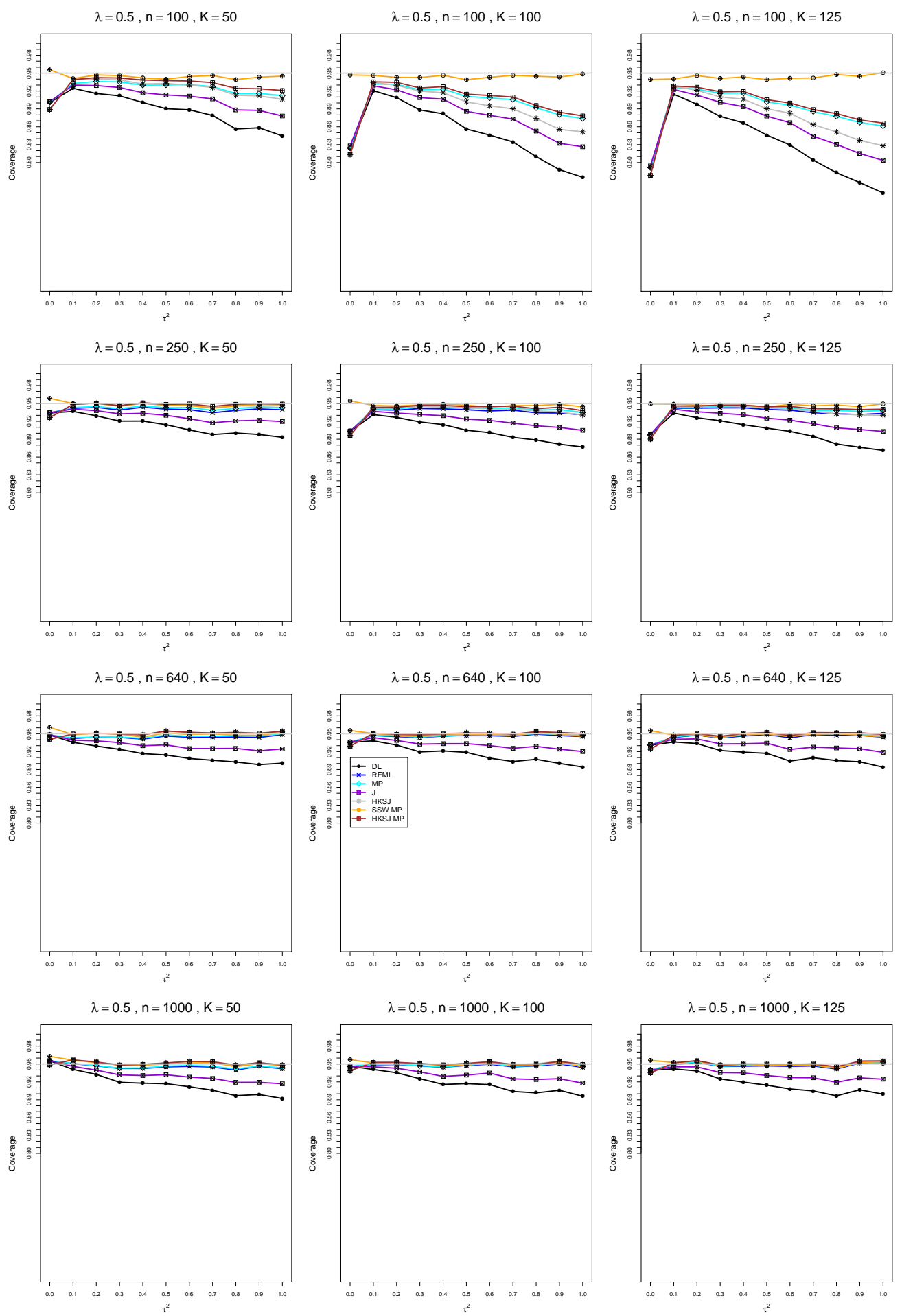

Figure D4.2.3: Coverage of $95 \%$ confidence intervals for $\lambda$ when $\mu_{C}=1, \lambda=0.5, n=$ 100, 250, 640, 1000, and $K=50,100,125$ 

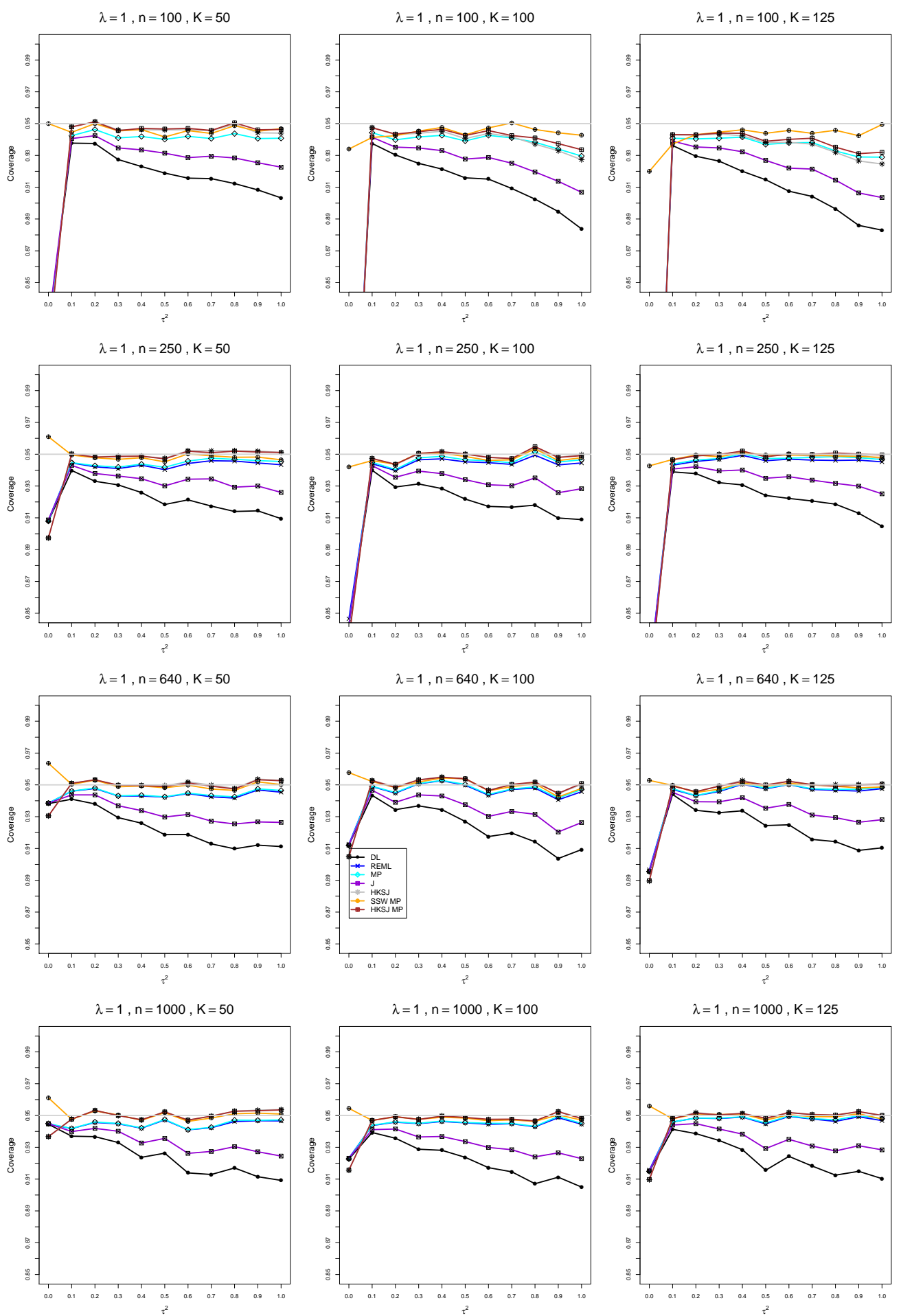

Figure D4.2.4: Coverage of $95 \%$ confidence intervals for $\lambda$ when $\mu_{C}=1, \lambda=1, n=$ 100, 250, 640, 1000, and $K=50,100,125$ 

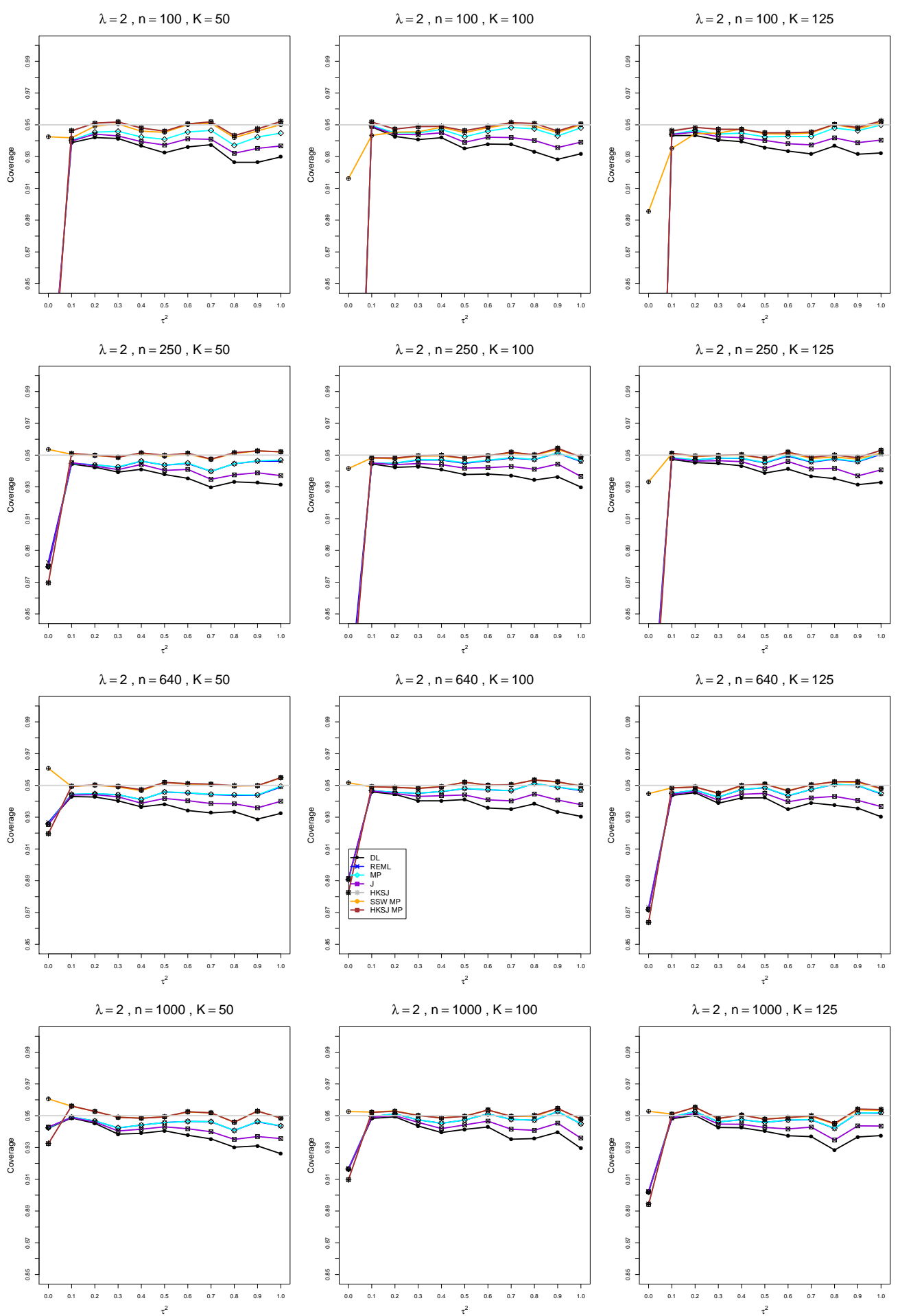

Figure D4.2.5: Coverage of $95 \%$ confidence intervals for $\lambda$ when $\mu_{C}=1, \lambda=2, n=$ 100, 250, 640, 1000, and $K=50,100,125$ 


\section{D5. Normal model, $\mu_{C}=4, n=100,250,640$, $1000, K=50,100,125$}

\section{D5.1 Bias of point estimators of $\lambda$ when $\mu_{C}=4$}

Each figure corresponds to a value of $\lambda(=0,0.2,0.5,1,2)$, a set of values of $n(=$ $100,250,640,1000)$, and a set of values of $K(=50,100,125)$.

Each panel corresponds to a value of $n$ and a value of $K$ and has $\tau^{2}=0.0(0.1) 1.0$ on the horizontal axis.

The point estimators of $\lambda$ are

- DL (DerSimonian-Laird)

- REML (restricted maximum likelihood)

- MP (Mandel-Paule)

- J (Jackson)

- SSW (sample-size weighted) 

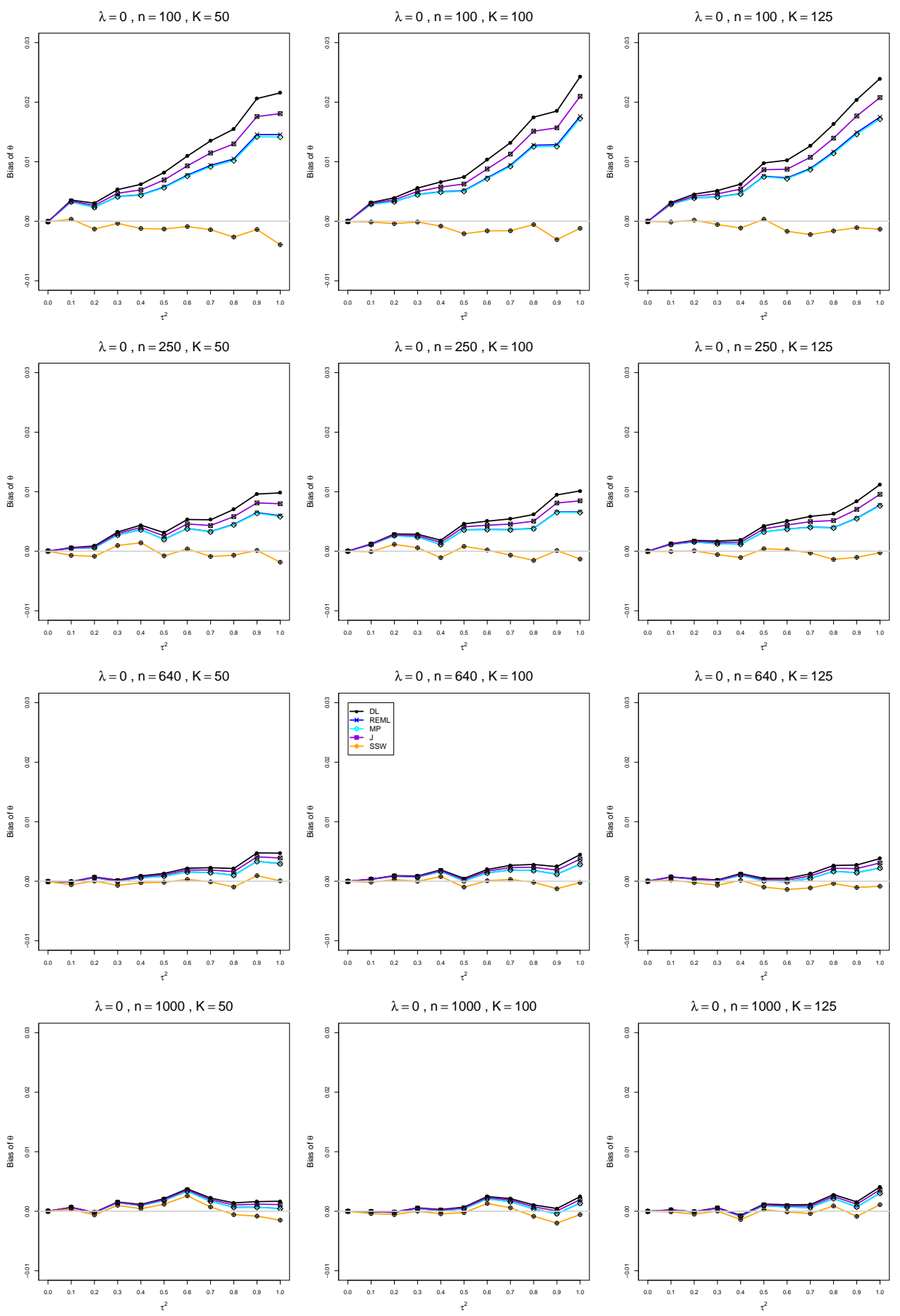

Figure D5.1.1: Bias of estimators of $\log (\operatorname{RoM}) \lambda$ when $\mu_{C}=4, \lambda=0, n=$ 100, 250, 640, 1000, and $K=50,100,125$ 

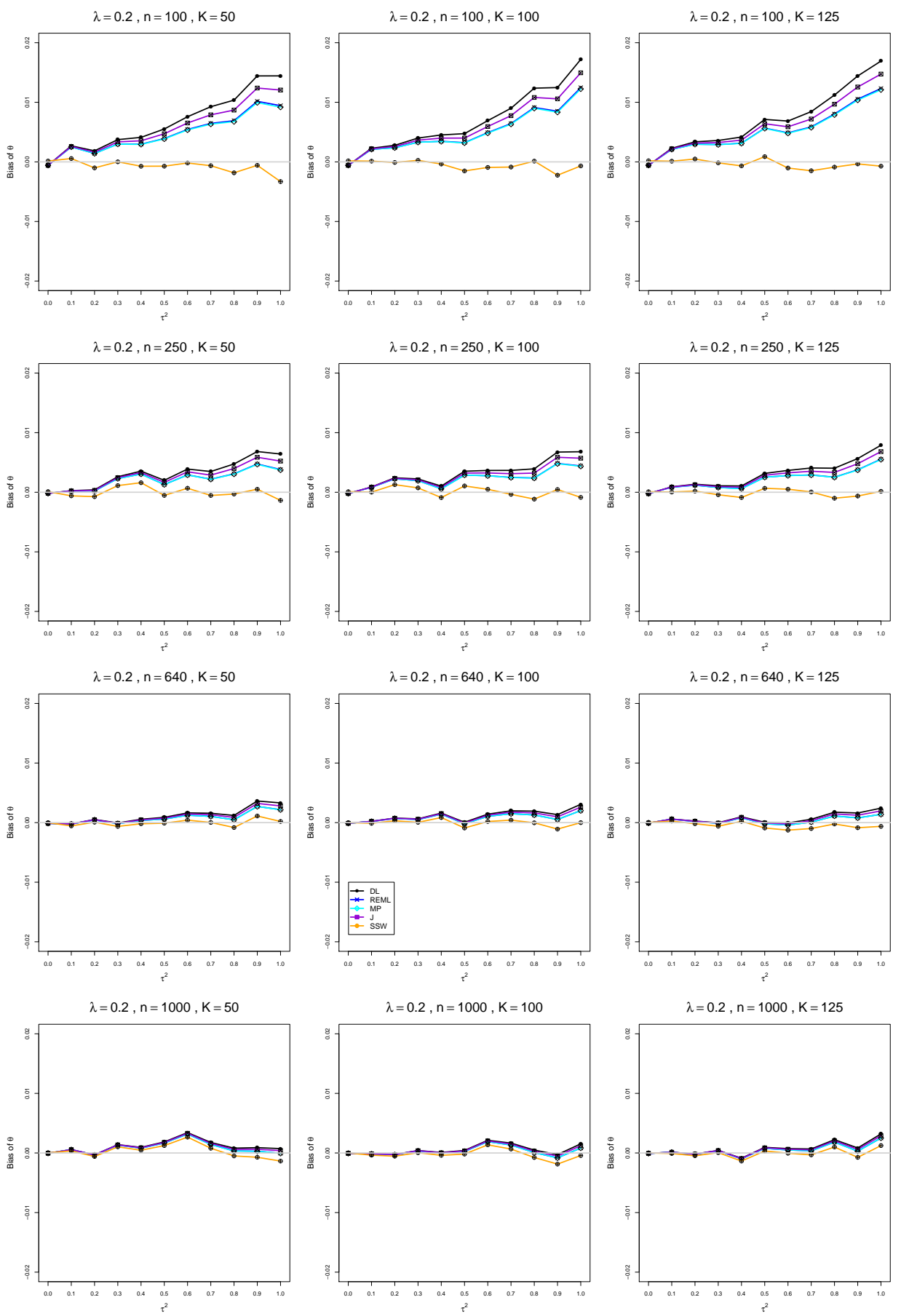

Figure D5.1.2: Bias of estimators of $\log (\mathrm{RoM}) \lambda$ when $\mu_{C}=4, \lambda=0.2, n=$ $100,250,640,1000$, and $K=50,100,125$ 

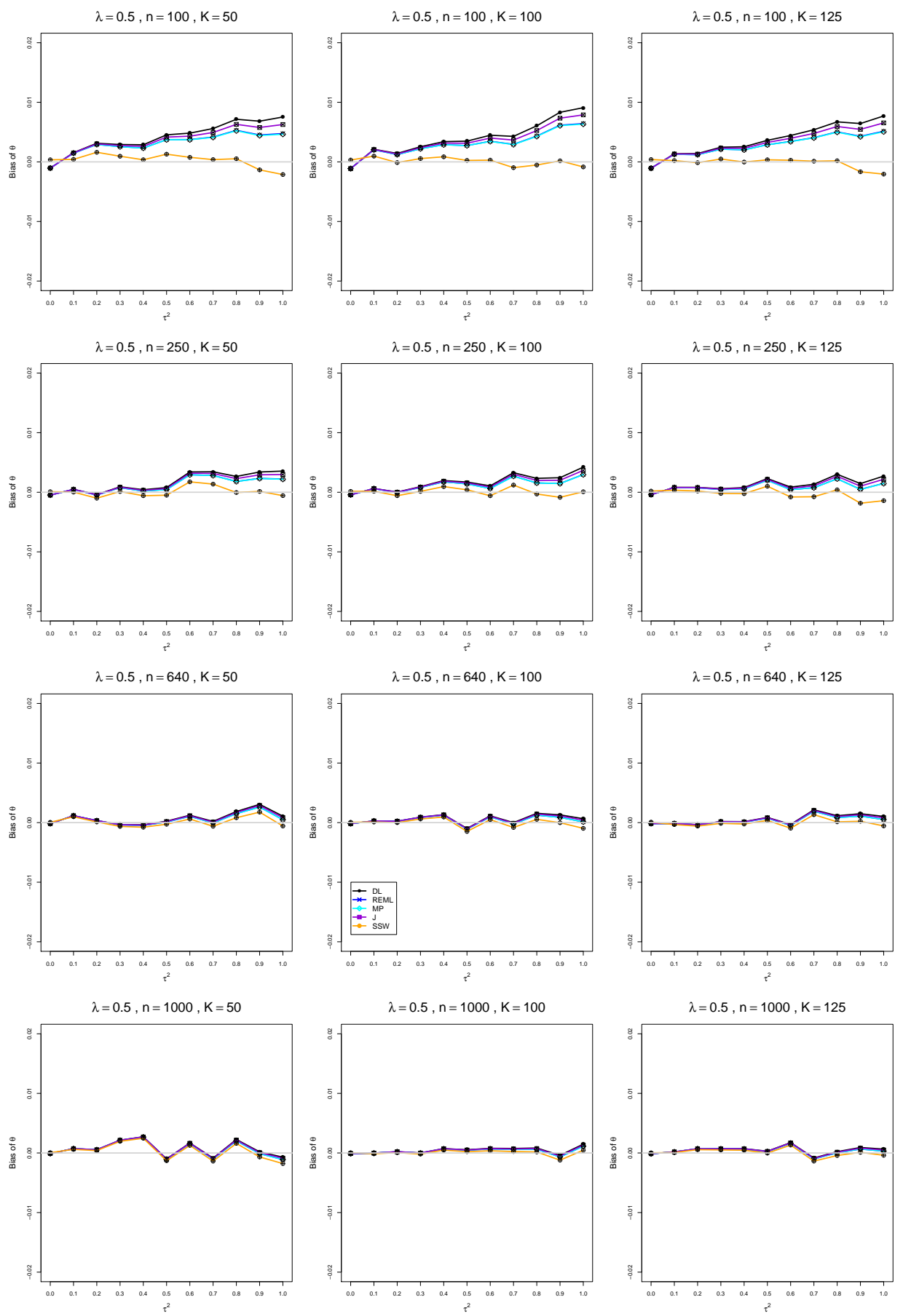

Figure D5.1.3: Bias of estimators of $\log (\mathrm{RoM}) \lambda$ when $\mu_{C}=4, \lambda=0.5, n=$ $100,250,640,1000$, and $K=50,100,125$ 

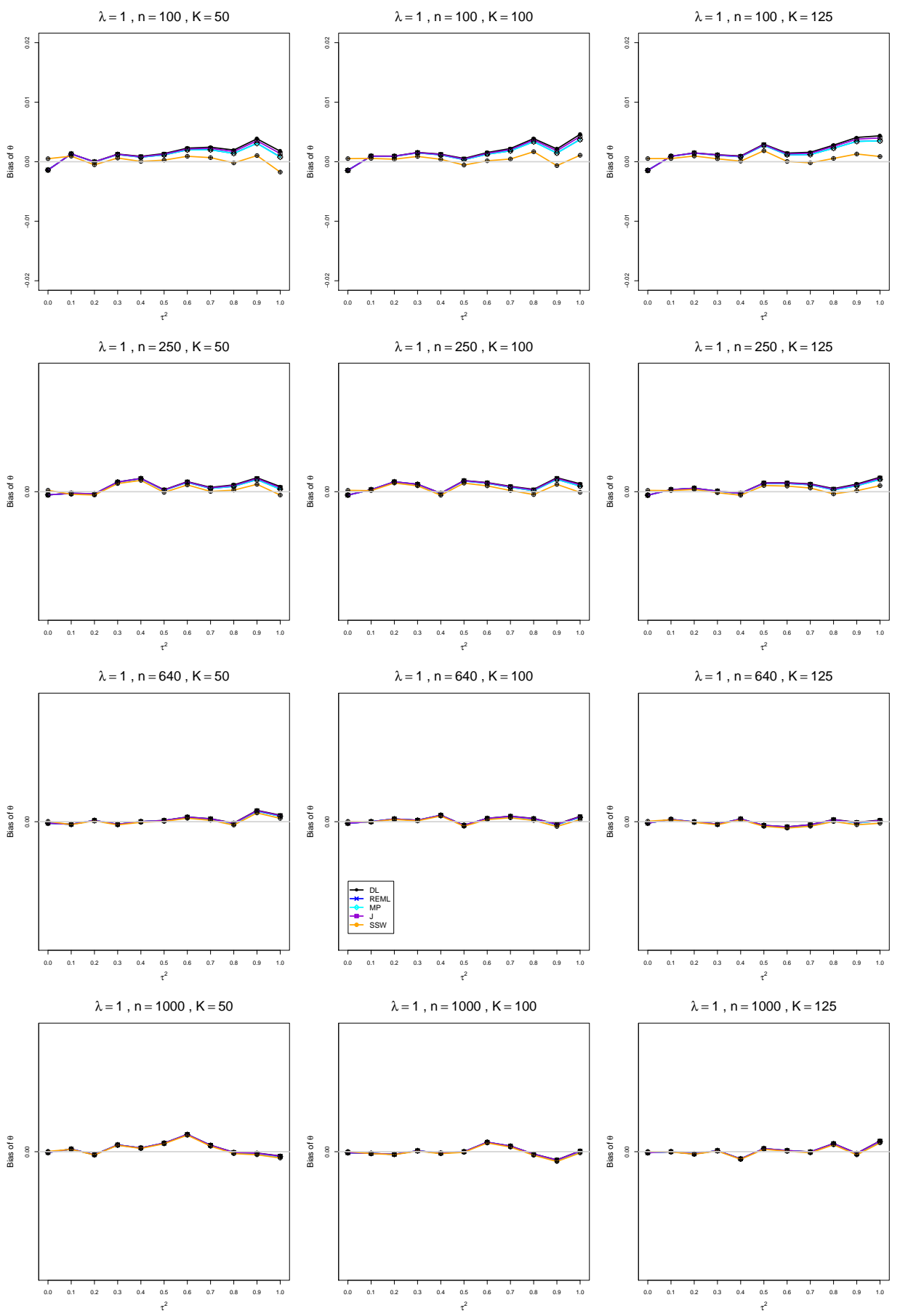

Figure D5.1.4: Bias of estimators of $\log (\operatorname{RoM}) \lambda$ when $\mu_{C}=4, \lambda=1, n=$ 100, 250, 640, 1000, and $K=50,100,125$ 

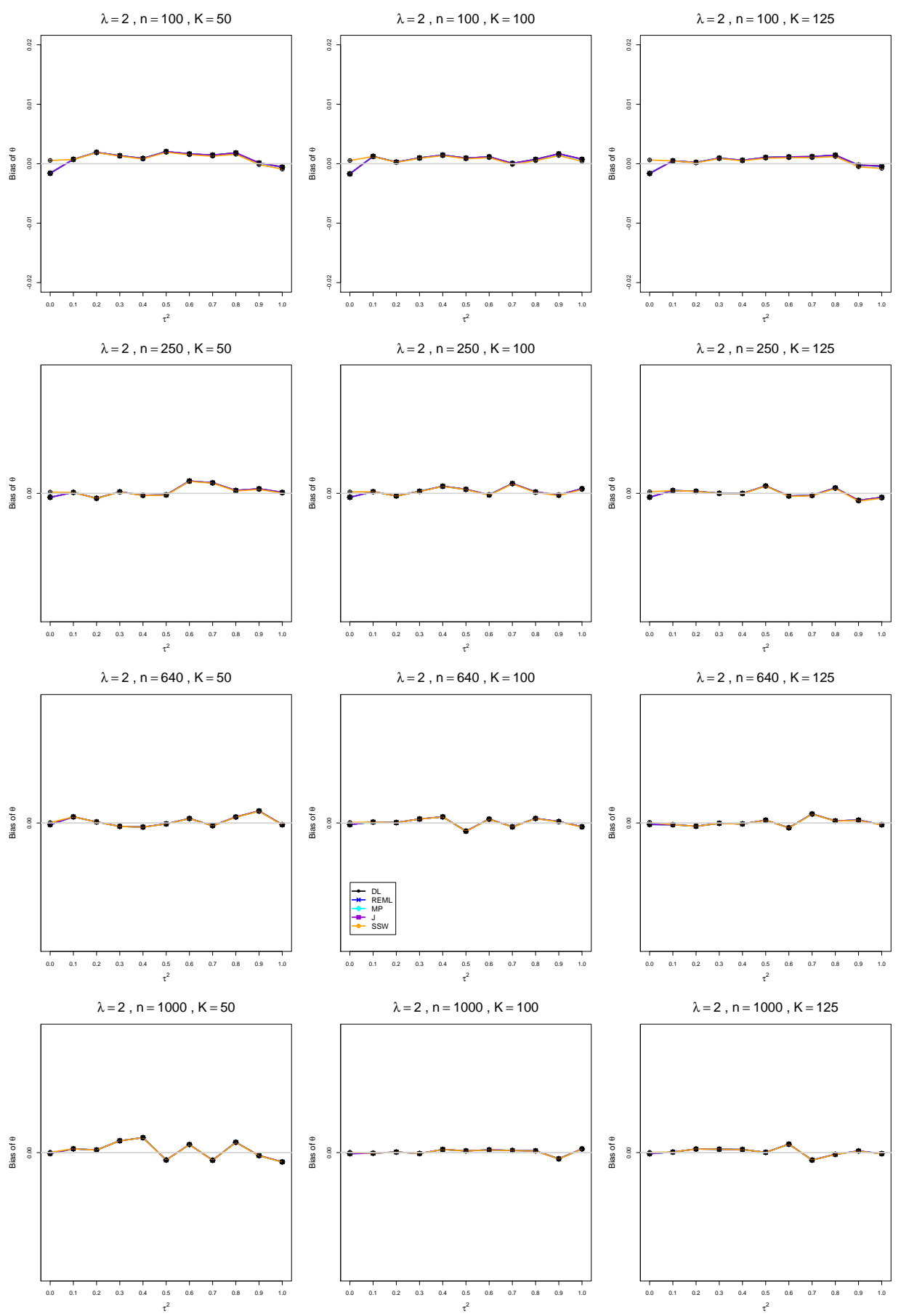

Figure D5.1.5: Bias of estimators of $\log (\operatorname{RoM}) \lambda$ when $\mu_{C}=4, \lambda=2, n=$ 100, 250, 640, 1000, and $K=50,100,125$ 


\section{D5.2 Coverage of interval estimators of $\lambda$}

Each figure corresponds to a value of $\lambda(=0,0.2,0.5,1,2)$, a set of values of $n(=$ $100,250,640,1000)$, and a set of values of $K(=50,100,125)$.

Each panel corresponds to a value of $n$ and a value of $K$ and has $\tau^{2}=0.0(0.1) 1.0$ on the horizontal axis.

The interval estimators of $\lambda$ are the companions to the inverse-variance-weighted point estimators

- DL (DerSimonian-Laird)

- REML (restricted maximum likelihood)

- MP (Mandel-Paule)

- J (Jackson)

and

- HKSJ (Hartung-Knapp-Sidik-Jonkman)

- HKSJ MP (HKSJ with MP estimator of $\tau^{2}$ )

- SSW (SSW as center and half-width equal to critical value from $t_{K-1}$ times estimated standard deviation of SSW with $\hat{\tau}^{2}=\hat{\tau}_{M P}^{2}$ 


\section{D6. Normal model, bias-corrected $\lambda, \mu_{C}=4, n$ $=100,250,640,1000, K=50,100,125$}

\section{D6.1 Bias of point estimators of $\lambda$ when $\mu_{C}=4$}

Each figure corresponds to a value of $\lambda(=0,0.2,0.5,1,2)$, a set of values of $n(=$ $100,250,640,1000)$, and a set of values of $K(=50,100,125)$.

Each panel corresponds to a value of $n$ and a value of $K$ and has $\tau^{2}=0.0(0.1) 1.0$ on the horizontal axis.

The point estimators of $\lambda$ are

- DL (DerSimonian-Laird)

- REML (restricted maximum likelihood)

- MP (Mandel-Paule)

- J (Jackson)

- SSW (sample-size weighted) 

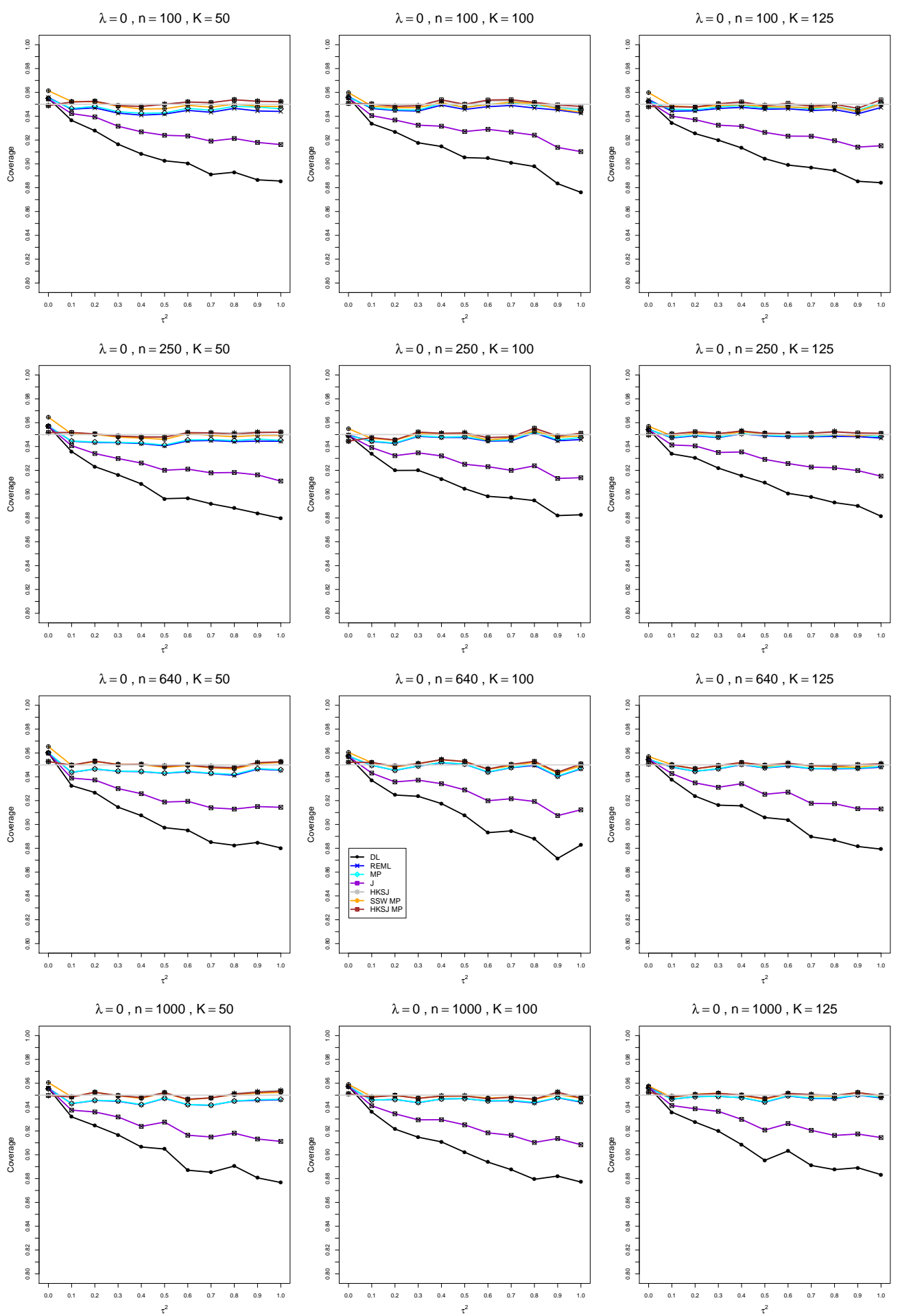

Figure D5.2.1: Coverage of $95 \%$ confidence intervals for $\lambda$ when $\mu_{C}=4, \lambda=0, n=$ 100, 250, 640, 1000, and $K=50,100,125$ 

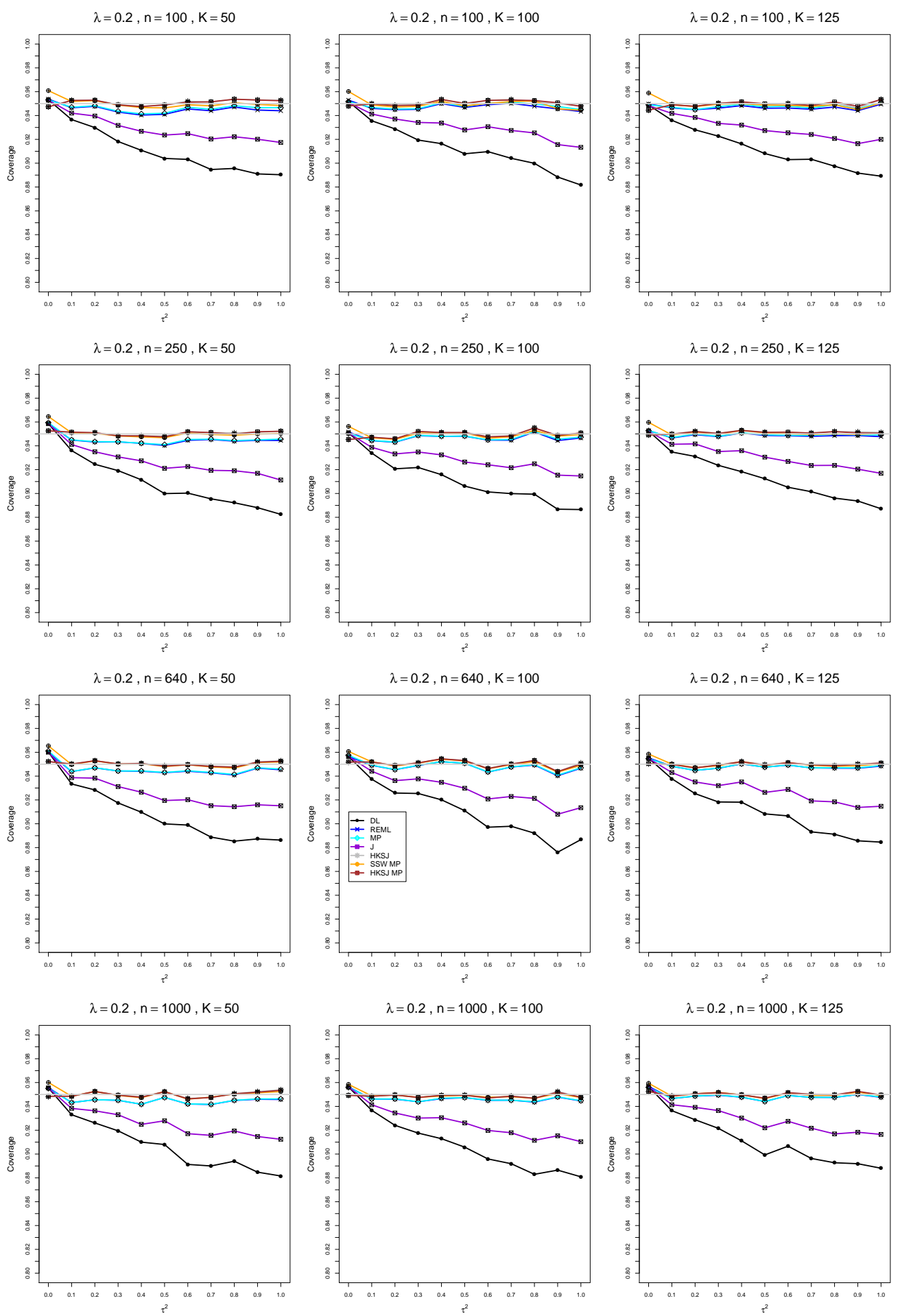

Figure D5.2.2: Coverage of $95 \%$ confidence intervals for $\lambda$ when $\mu_{C}=4, \lambda=0.2, n=$ 100, 250, 640, 1000, and $K=50,100,125$ 

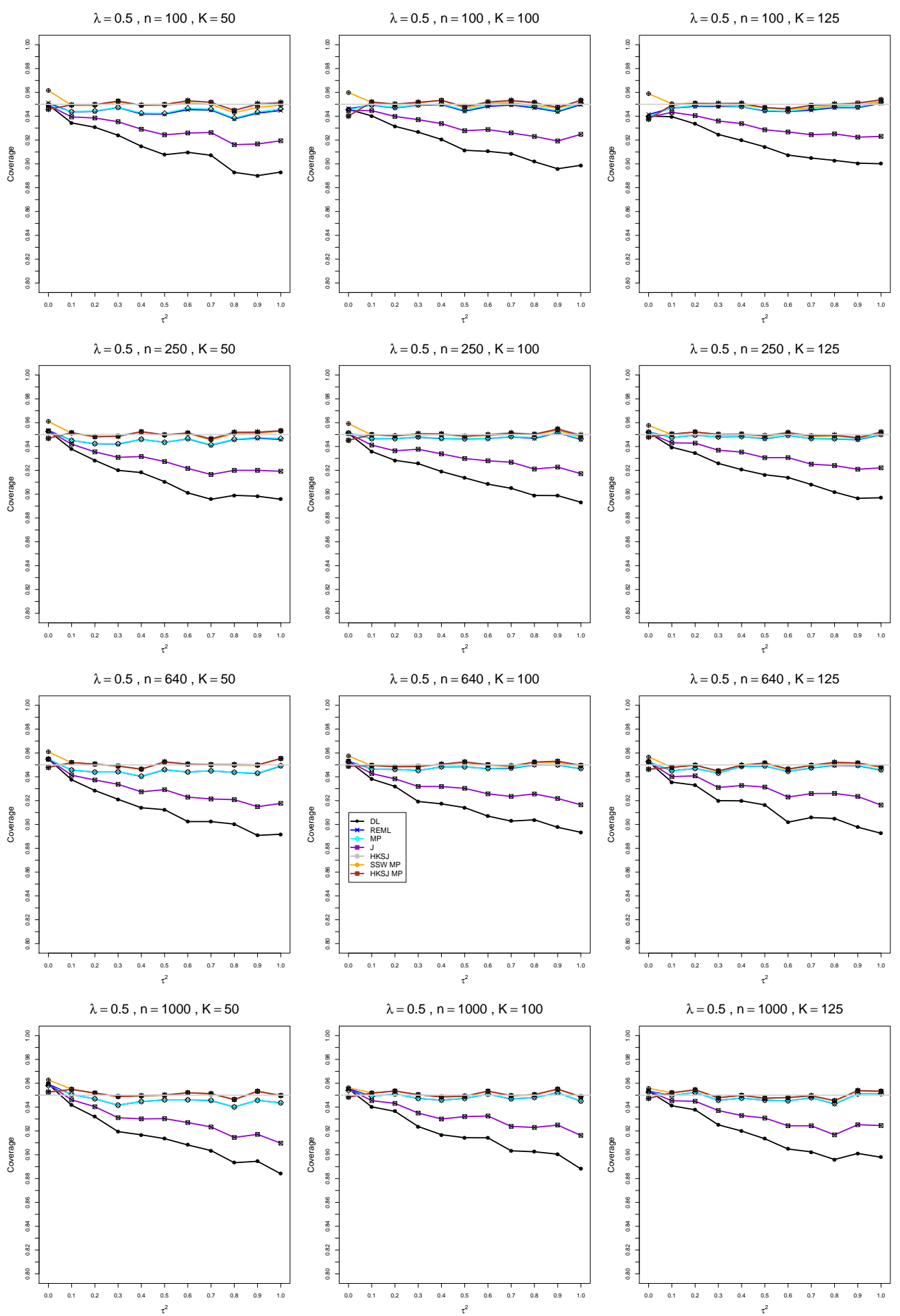

Figure D5.2.3: Coverage of $95 \%$ confidence intervals for $\lambda$ when $\mu_{C}=4, \lambda=0.5, n=$ 100, 250, 640, 1000, and $K=50,100,125$ 

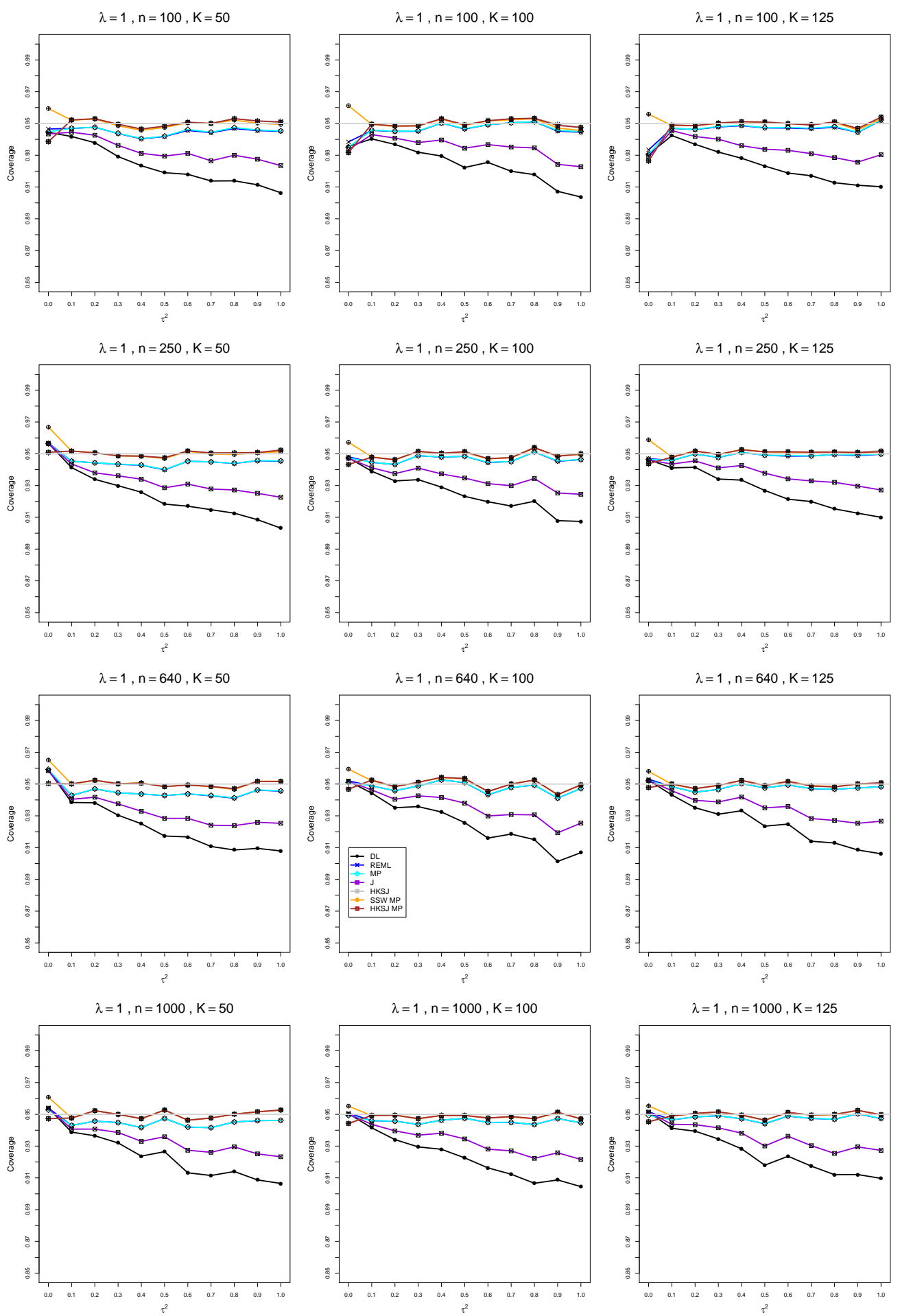

Figure D5.2.4: Coverage of $95 \%$ confidence intervals for $\lambda$ when $\mu_{C}=4, \lambda=1, n=$ 100, 250, 640, 1000, and $K=50,100,125$ 

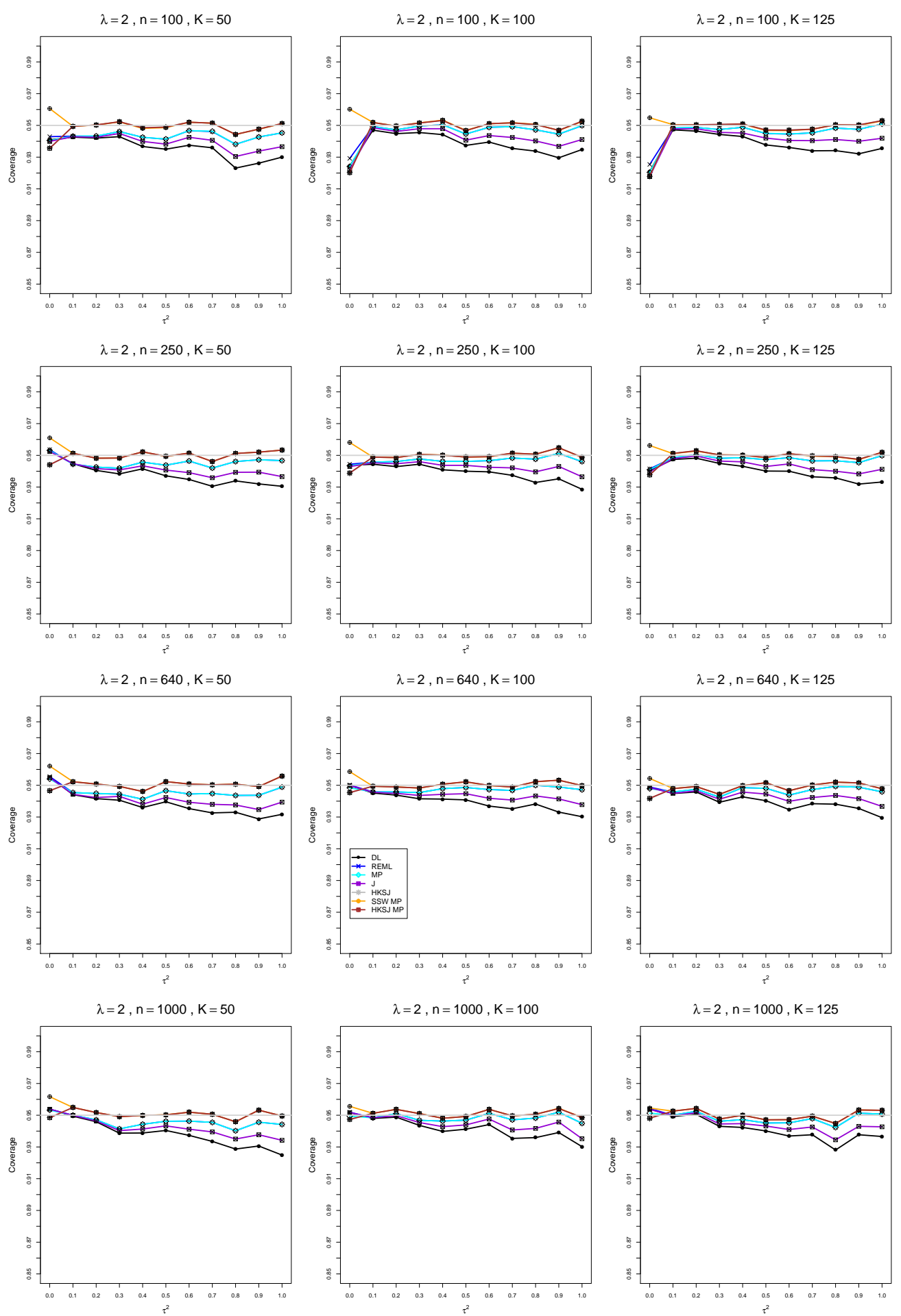

Figure D5.2.5: Coverage of $95 \%$ confidence intervals for $\lambda$ when $\mu_{C}=4, \lambda=2, n=$ 100, 250, 640, 1000, and $K=50,100,125$ 

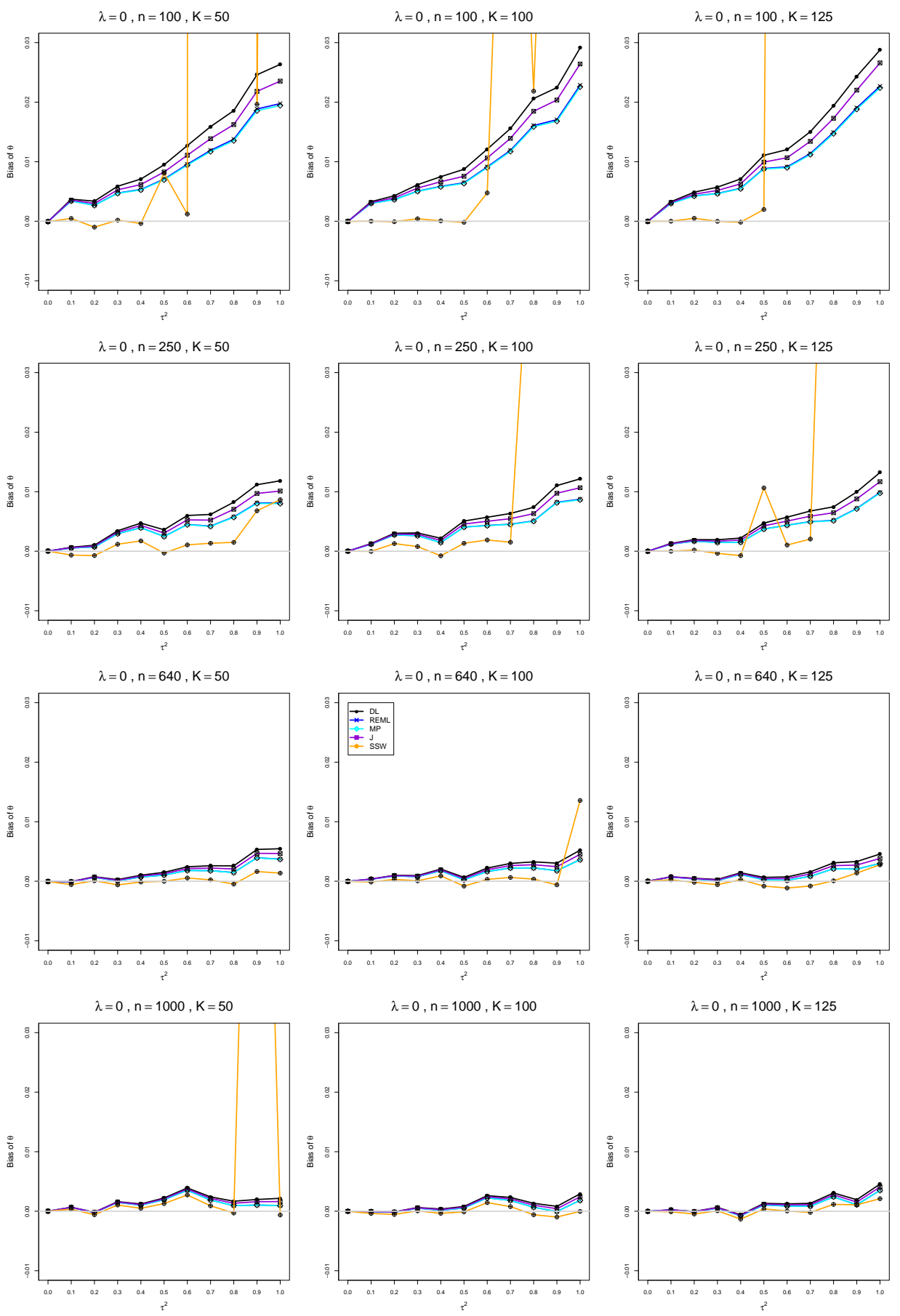

Figure D6.1.1: Bias of estimators of $\log (\operatorname{RoM}) \lambda$ when $\mu_{C}=4, \lambda=0, n=$ 100, 250, 640, 1000, and $K=50,100,125$ 

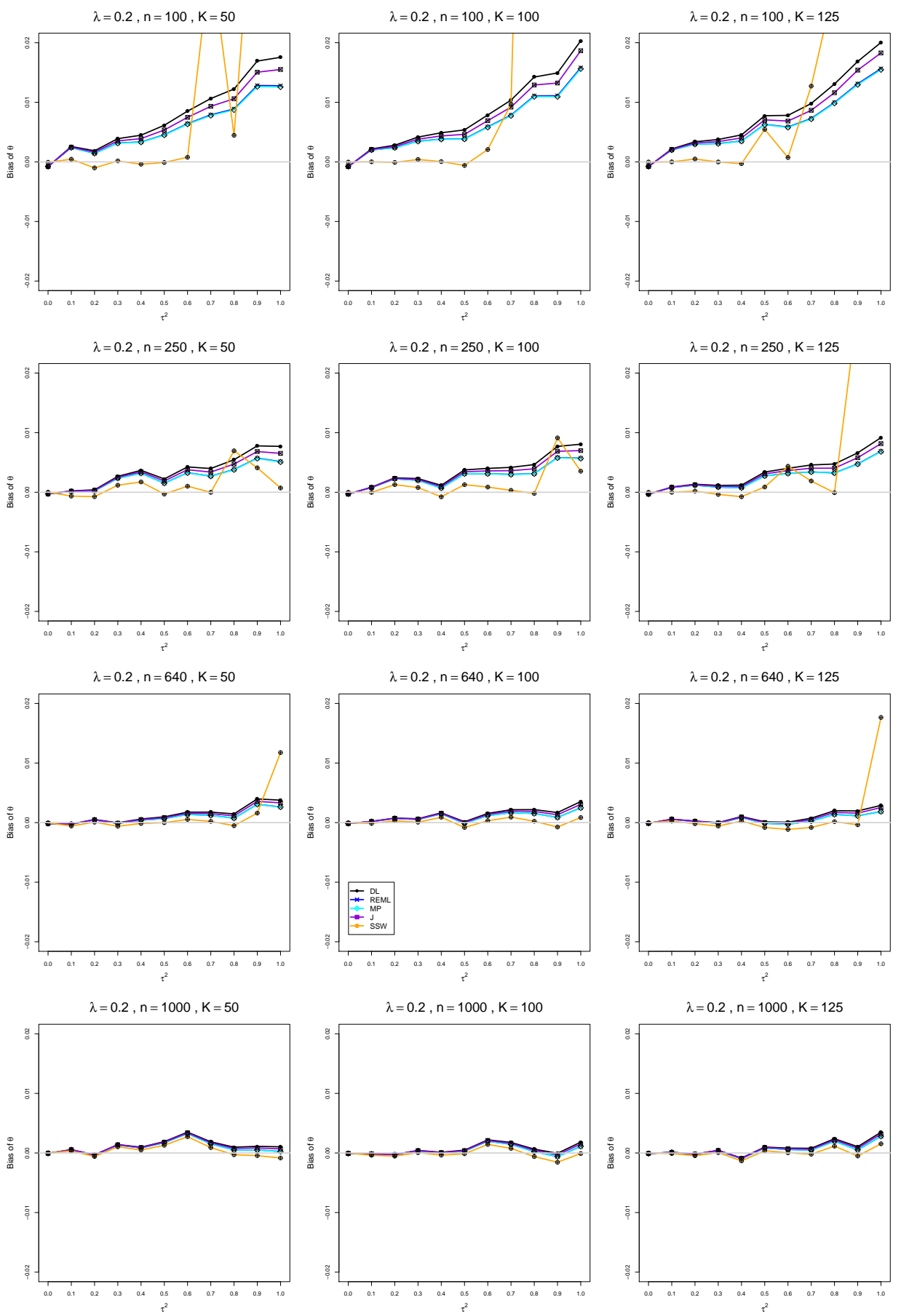

Figure D6.1.2: Bias of estimators of $\log (\mathrm{RoM}) \lambda$ when $\mu_{C}=4, \lambda=0.2, n=$ $100,250,640,1000$, and $K=50,100,125$ 

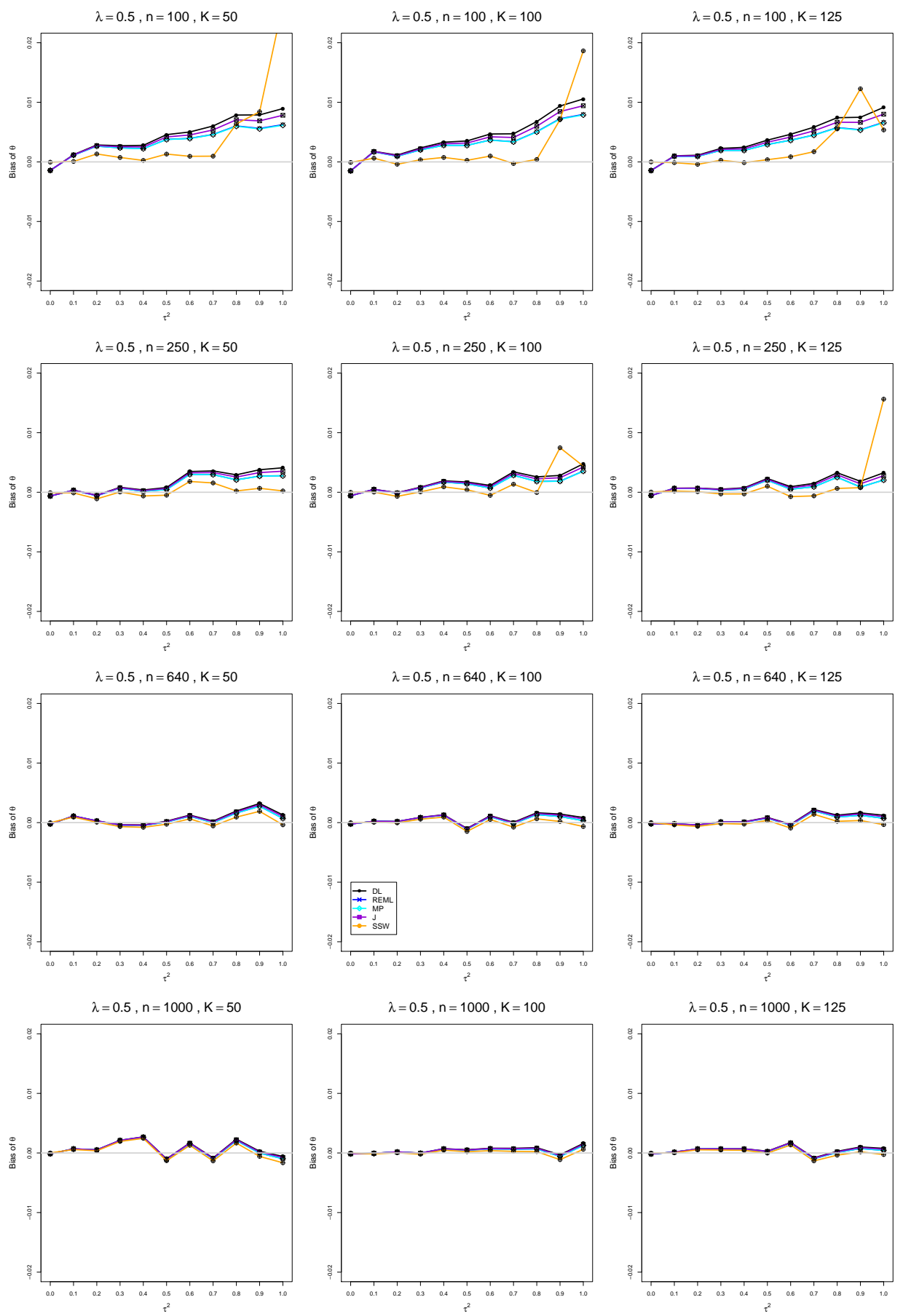

Figure D6.1.3: Bias of estimators of $\log (\mathrm{RoM}) \lambda$ when $\mu_{C}=4, \lambda=0.5, n=$ $100,250,640,1000$, and $K=50,100,125$ 

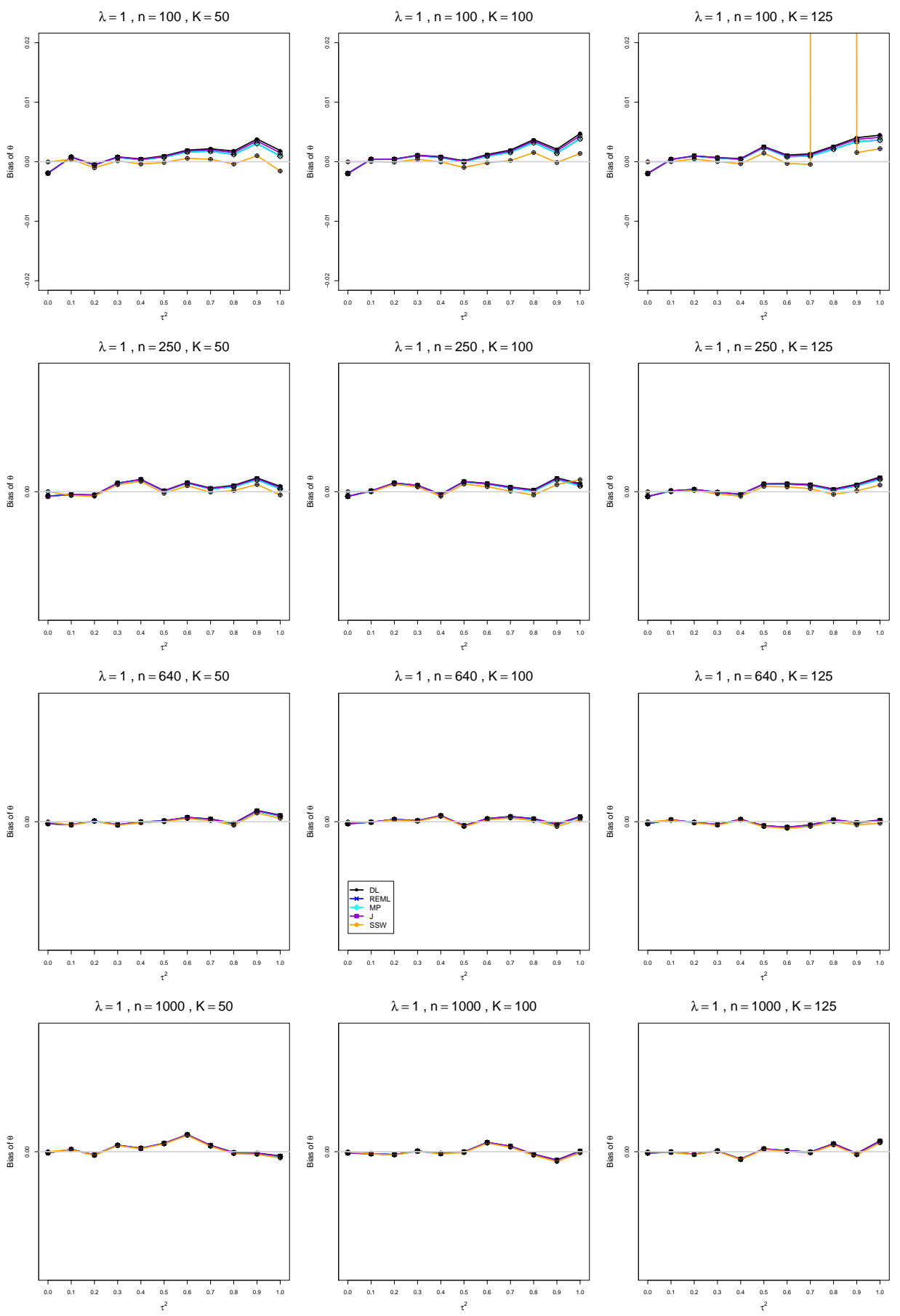

Figure D6.1.4: Bias of estimators of $\log (\operatorname{RoM}) \lambda$ when $\mu_{C}=4, \lambda=1, n=$ 100, 250, 640, 1000, and $K=50,100,125$ 

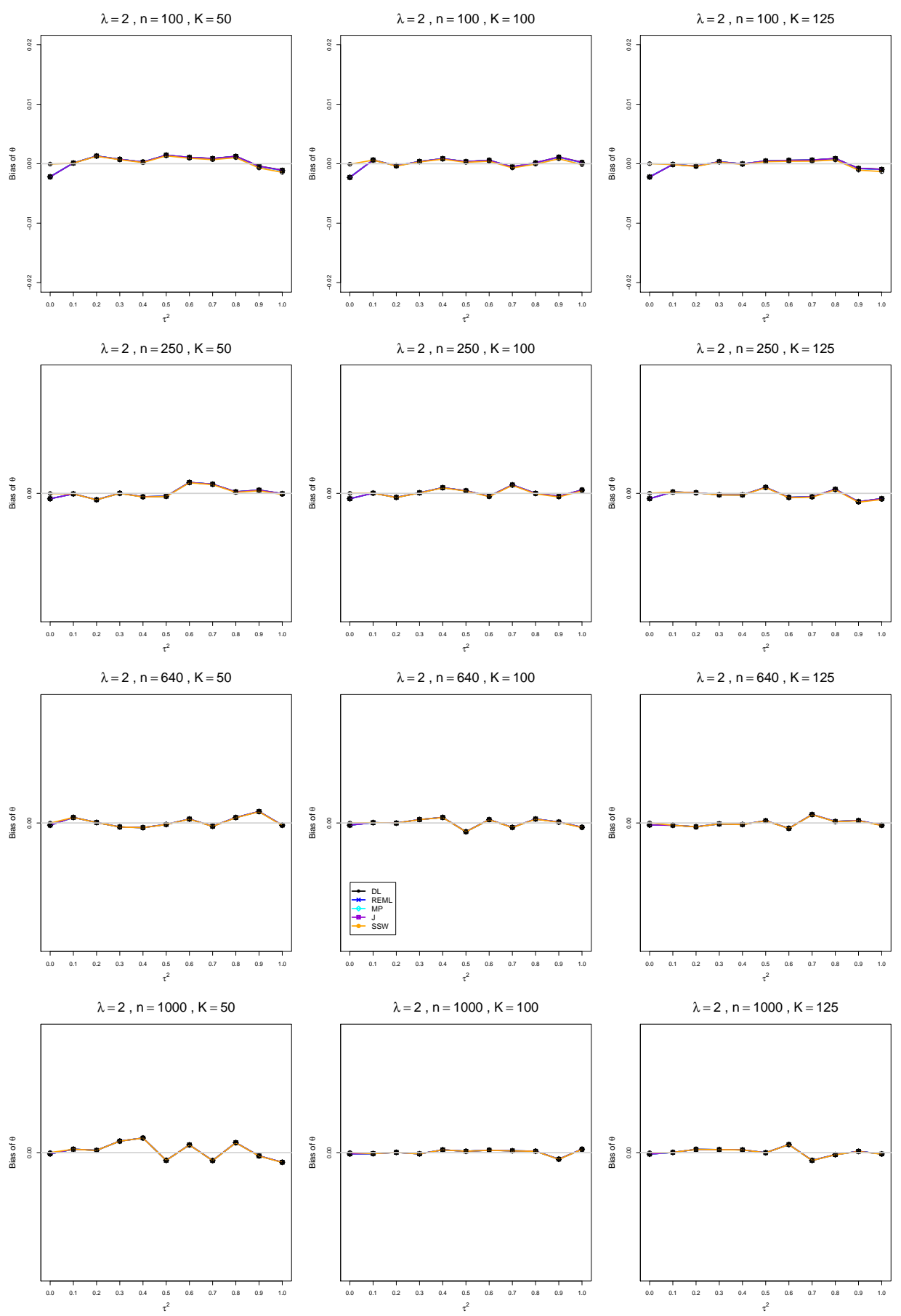

Figure D6.1.5: Bias of estimators of $\log (\operatorname{RoM}) \lambda$ when $\mu_{C}=4, \lambda=2, n=$ 100, 250, 640, 1000, and $K=50,100,125$ 


\section{D6.2 Coverage of interval estimators of $\lambda$}

Each figure corresponds to a value of $\lambda(=0,0.2,0.5,1,2)$, a set of values of $n(=$ $100,250,640,1000)$, and a set of values of $K(=50,100,125)$.

Each panel corresponds to a value of $n$ and a value of $K$ and has $\tau^{2}=0.0(0.1) 1.0$ on the horizontal axis.

The interval estimators of $\lambda$ are the companions to the inverse-variance-weighted point estimators

- DL (DerSimonian-Laird)

- REML (restricted maximum likelihood)

- MP (Mandel-Paule)

- J (Jackson)

and

- HKSJ (Hartung-Knapp-Sidik-Jonkman)

- HKSJ MP (HKSJ with MP estimator of $\tau^{2}$ )

- SSW (SSW as center and half-width equal to critical value from $t_{K-1}$ times estimated standard deviation of SSW with $\hat{\tau}^{2}=\hat{\tau}_{M P}^{2}$ 

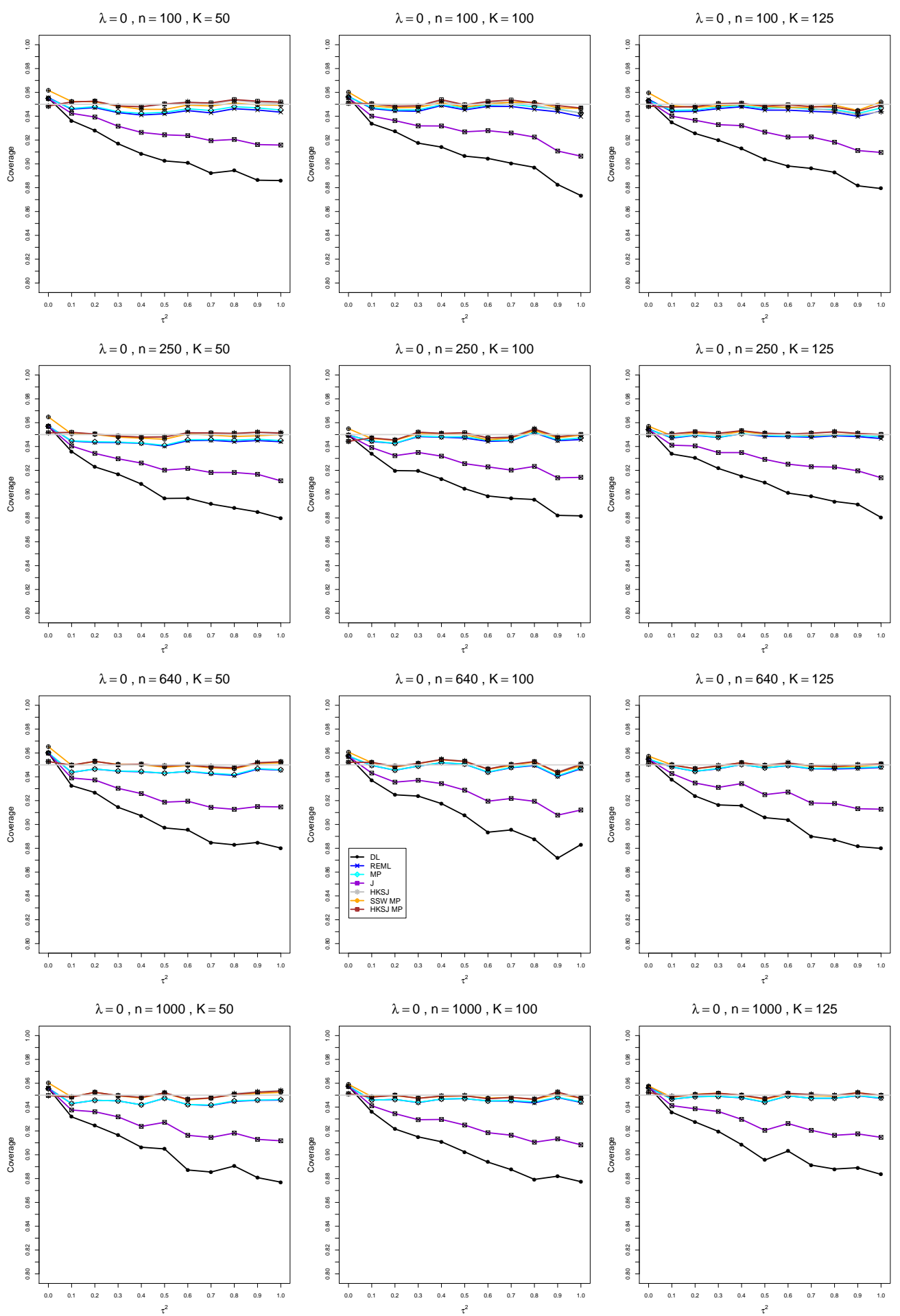

Figure D6.2.1: Coverage of $95 \%$ confidence intervals for $\lambda$ when $\mu_{C}=4, \lambda=0, n=$ 100, 250, 640, 1000, and $K=50,100,125$ 

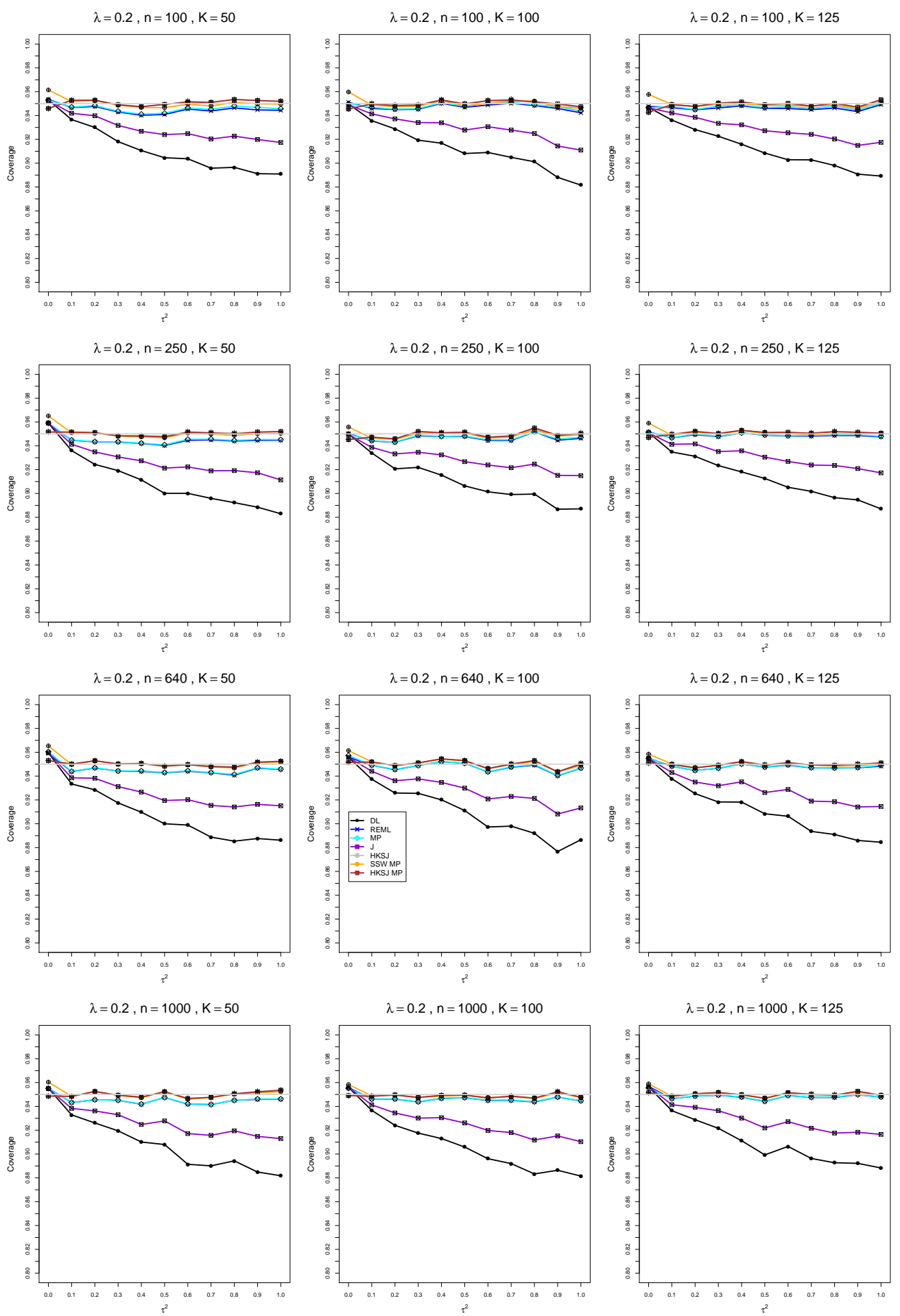

Figure D6.2.2: Coverage of $95 \%$ confidence intervals for $\lambda$ when $\mu_{C}=4, \lambda=0.2, n=$ 100, 250, 640, 1000, and $K=50,100,125$ 

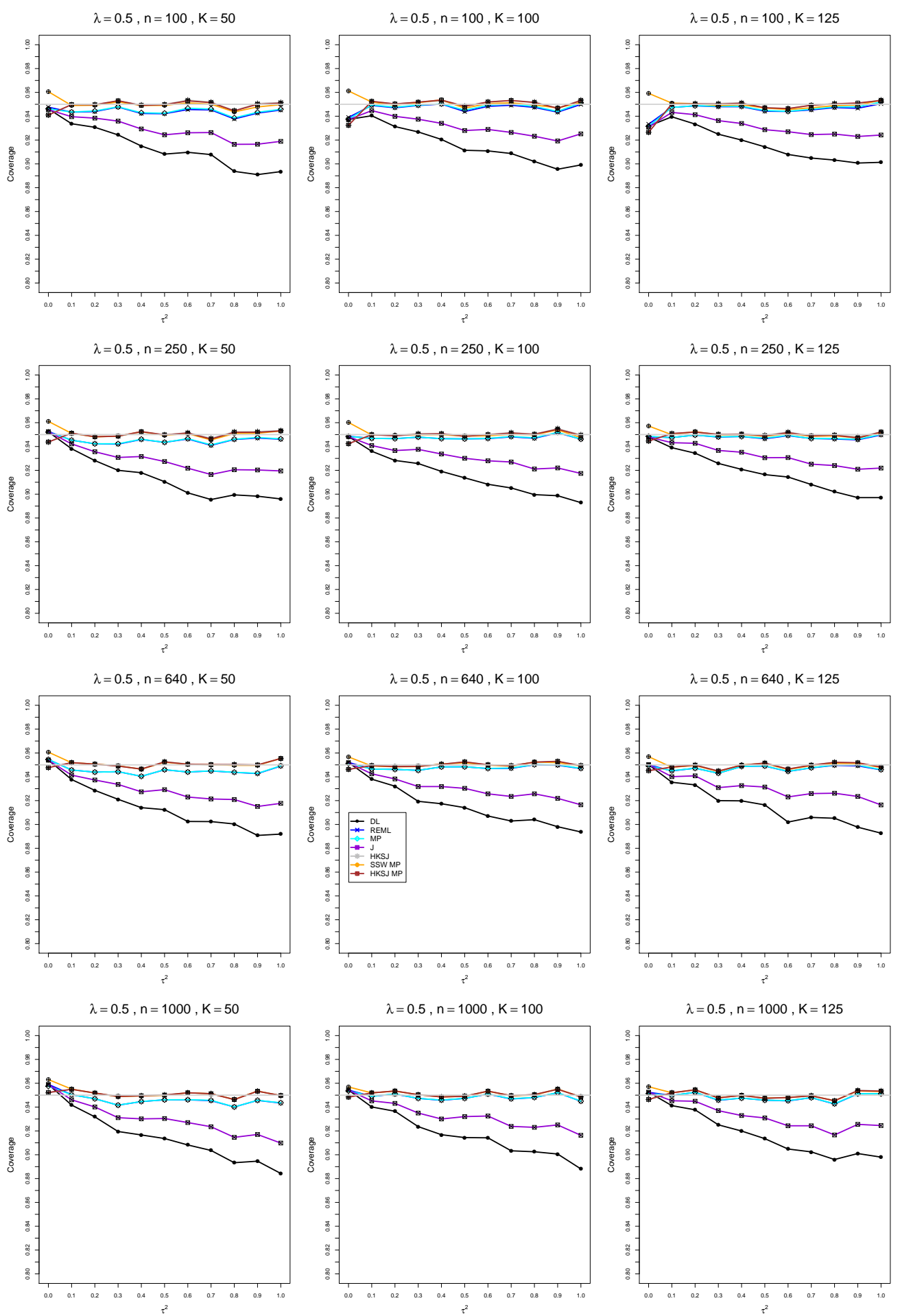

Figure D6.2.3: Coverage of $95 \%$ confidence intervals for $\lambda$ when $\mu_{C}=4, \lambda=0.5, n=$ 100, 250, 640, 1000, and $K=50,100,125$ 

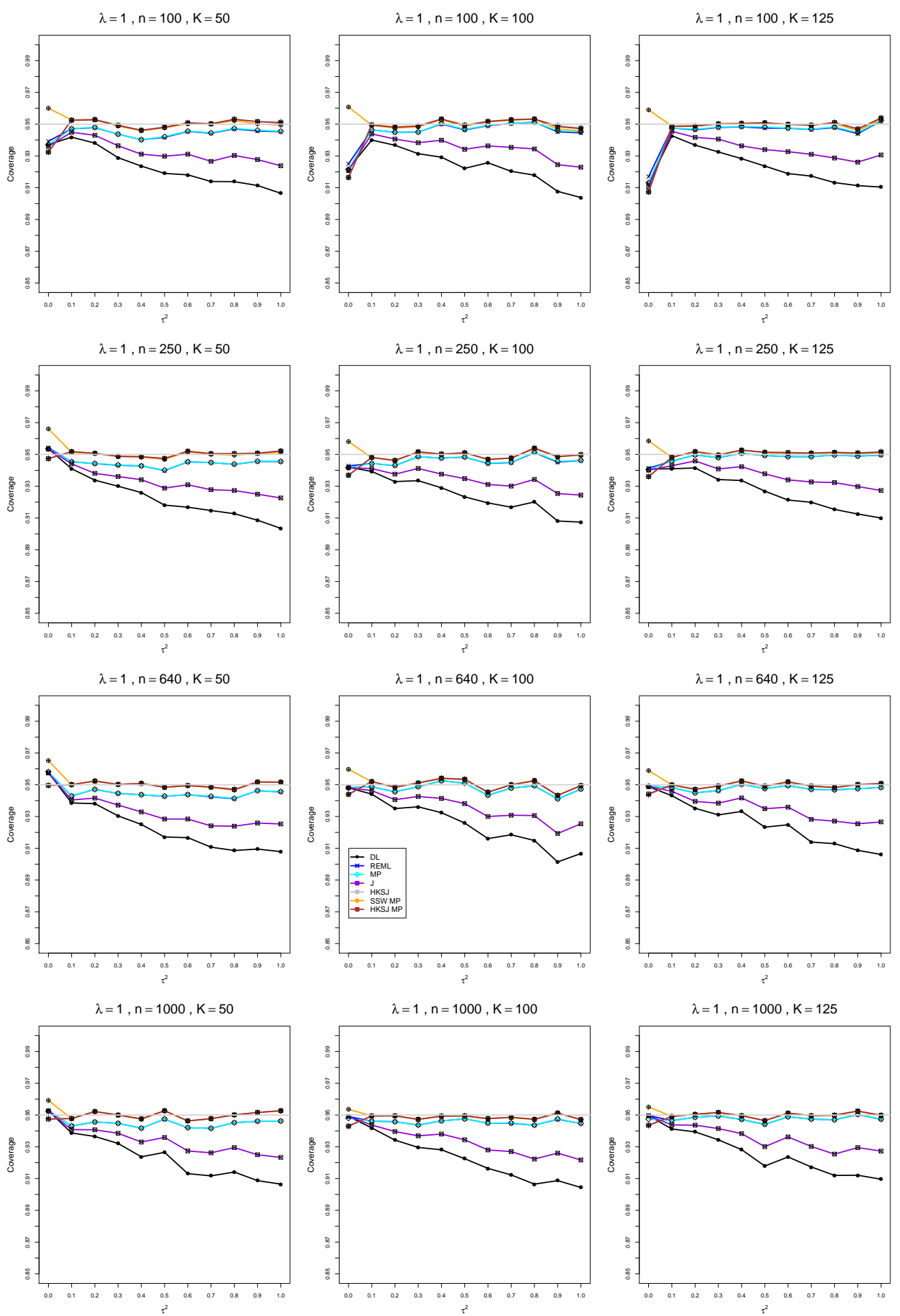

Figure D6.2.4: Coverage of $95 \%$ confidence intervals for $\lambda$ when $\mu_{C}=4, \lambda=1, n=$ 100, 250, 640, 1000, and $K=50,100,125$ 

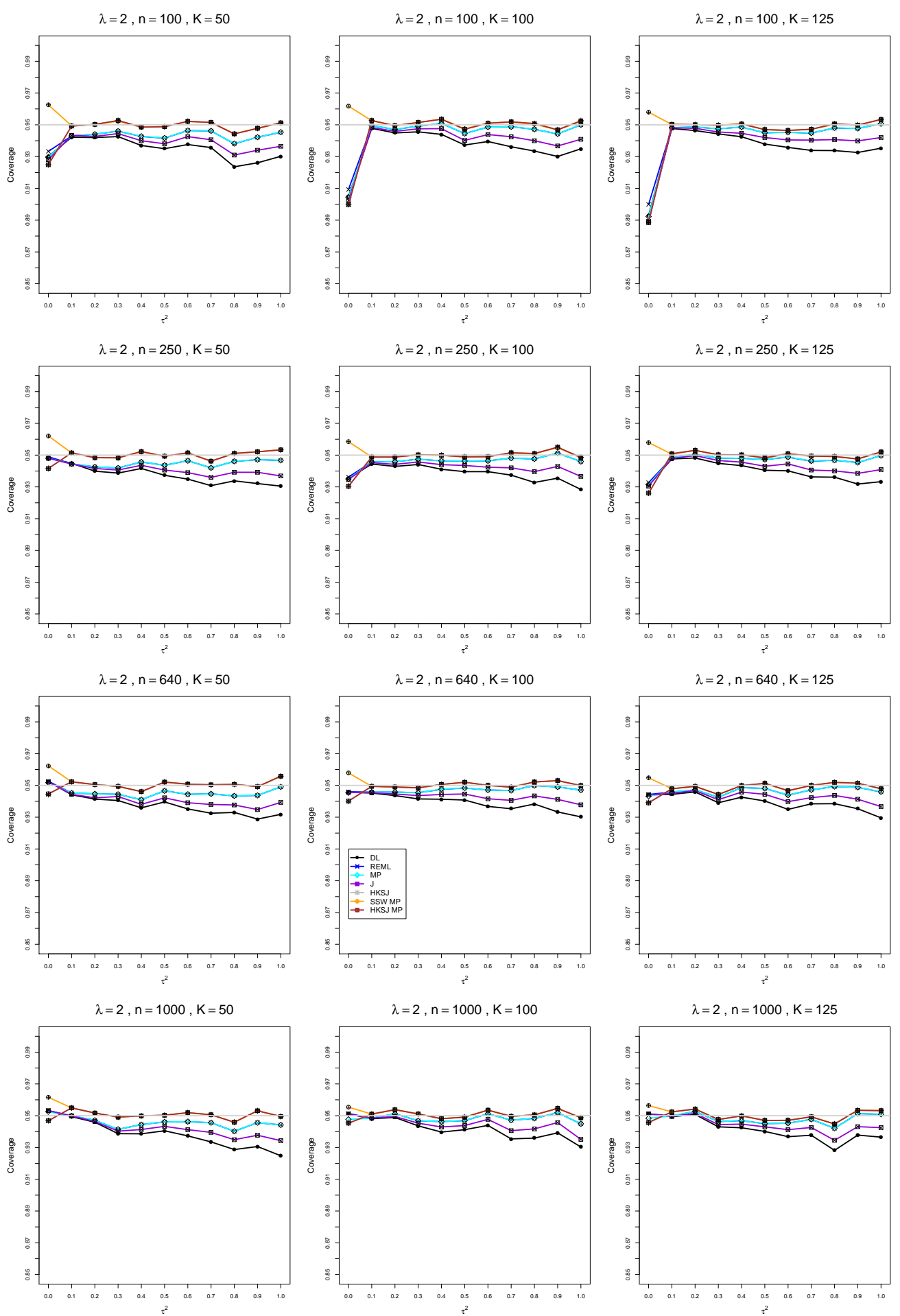

Figure D6.2.5: Coverage of $95 \%$ confidence intervals for $\lambda$ when $\mu_{C}=4, \lambda=2, n=$ 100, 250, 640, 1000, and $K=50,100,125$ 